2. To: (Receiving Organization)

Distribution

5. Proj./Prog./Dept./Div.:

SNE CSB

8. Originator Remarks:
3. From: (Originating Organization)

CSB subproject

6. Design Authority/Design Agent/Cog. Engr.:

S. A. Krieg

4. Related EDT No.:

$\mathrm{N} / \mathrm{A}$

7. Purchase Order No.:

$\mathrm{N} / \mathrm{A}$

9. Equlp./Component No.:

N/A

10. System/Bldg./Facility:

12. Major Assm. Dwg. No.:

$\mathrm{N} / \mathrm{A}$

13. Permit/Permit Application No.:

$\mathrm{N} / \mathrm{A}$

14. Required Response Date

$$
2-28-00
$$

15.

\begin{tabular}{|c|c}
\hline $\begin{array}{c}\text { (A) } \\
\text { leem } \\
\text { No. }\end{array}$ & (B) Document/Drawing No. \\
\hline 1 & SNF-5790 \\
\hline & \\
\hline & \\
\hline & \\
\hline & \\
\hline & \\
\hline
\end{tabular}

16.

Approval Designator $(F)$

E, S, Q, D OR N/A

(S'Ee'WHC-CM-3-5 Sec. 12.7)

1. Approval
2. Release
3. information

17.

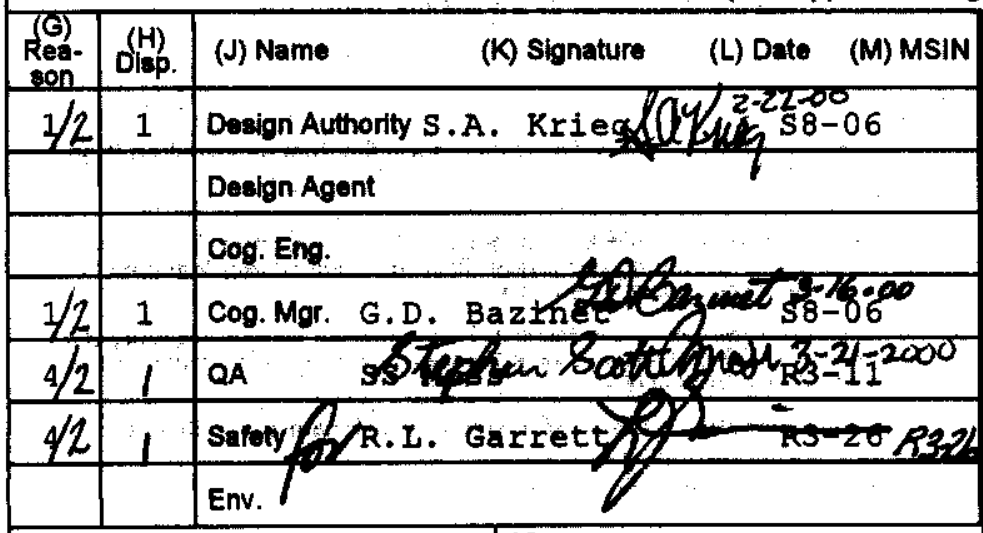

18.

Q. R. Krea

S.A Krieg

Signature of EDT

Originator

$2-22-\infty$

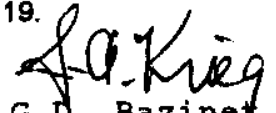

G.D. Bazinet

Authorized Representative for Recelving Organization
DATA TRANSMITTED

(C) Sheet
No. (D) Rev. 0
(E) Title or Description of Data Transmitted

Design Compliance

Matrices to ANSI and OSHA
(F)

(c)

(G)

Approval Reason

Desig- For Trane-

nator mittal

(H)

(I)

Origi-

nator
Dispo-
stion

Receiv-

nator mittal stion $\begin{gathered}\text { Dispo- } \\ \text { sition }\end{gathered}$

\begin{tabular}{l|l}
2 & 1
\end{tabular}

1

Reacon for Transmiltal (G)

4. Revlew

5. Post-Review

6. Dist. (Recelpt Acknow. Requlred)

KEY

SIGNATURE PISTRIBUTION

(See Approval Dealgnator for required eignatures)

\section{Approved \\ 4. Reviowed no/comment 5. Reviewed w/comment}

3. Dleapproved w/comment 6. Recelpt acknowledged
Disposition $(H) \&(I)$

\begin{tabular}{|l|l|llll}
\hline R(g) & (H) & (J) Name & (K) Signature & (L) Date & (M) MSIN
\end{tabular}

\begin{tabular}{ll|ll|}
\hline 3 & & D.M. Black & R3-86 \\
\hline 3 & & O.M. Serrano & R3-86 \\
\hline
\end{tabular}

3 RBendixsen B3-26 CSB Projectiles $58-06$ Centralfiles (2) B/-OZ

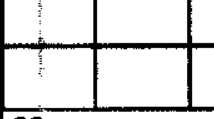

20

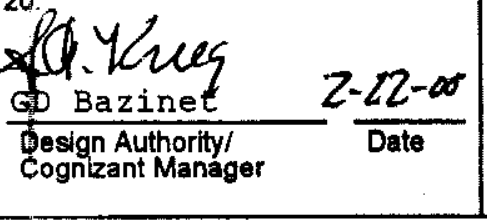

21. DOE APPROVAL (if required)

Ctr No. N/A

$\square$ Approved

$\square$ Approved w/comments

$\square$ Disapproved w/comments 


\section{INFORMATION CLEARANCE FORM}

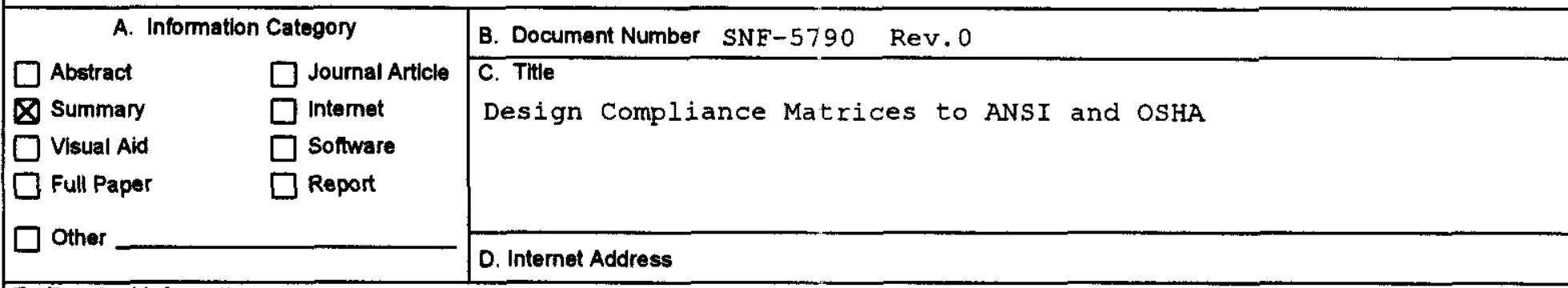

\section{E. Required Information}

1. Is document potentially Classified? $\bigotimes$ No $\square$ Yes (MANDATORY) 4. Does Information Contain the Following: (MANDATORY)

a. New or Novel (Patentable) Subject Matter? $\quad$ No $\square$ Yes

\section{Manager's Signature Required}

If Yes ADC Signature Required

\No $\square$ Yes Classiffed

2. Internal Revlew Required? $\square$ No $\square$ Yes if Yes, Document Signatures Below

Counsel

Program

3. References in the Information are Applied Technology $\bigotimes_{\text {No }} \square$ Yes Export Controlled Information XNo $\square$ Yos
If "Yes", Disclosure No.:

b. Information Recelved in Confldence, Such as Proprietary and/or Inventions?

$\square$ No $\square$ Yes If "Yes", Alix Appropriate Legends/Notices.

c. Copyrights? $\mathbb{X}$ No $\square$ Yes If "Yos", Attach Permission.

d. Trademarks? $\bigotimes$ No $\square$ Yes If "Yos", Identify in Document.

5. Is Information requiring submission to OSTI? $\quad$ No $\square$ Yes

If Yes $U C-\mathrm{N} / \mathrm{A}$ and $B \& R-\mathrm{N} / \mathrm{A}$

6. Release Level? $\square$ Public $\square$ Limited

7. Charge Code 105626

\section{F. Complete for a Joumal Article}

1. Title of Journal

G. Complete for a Presentation

1. Title for Conference or Meeting

2. Group Sponsoring

3. Date of Conference

5. WII Information be Published in Proceedings? $\bigotimes$ No $\square$ Yes

4. City/State

H. Author/Requestor

6. Will Material be Handed Out? $\mathrm{X}$ No $\square$ Yes

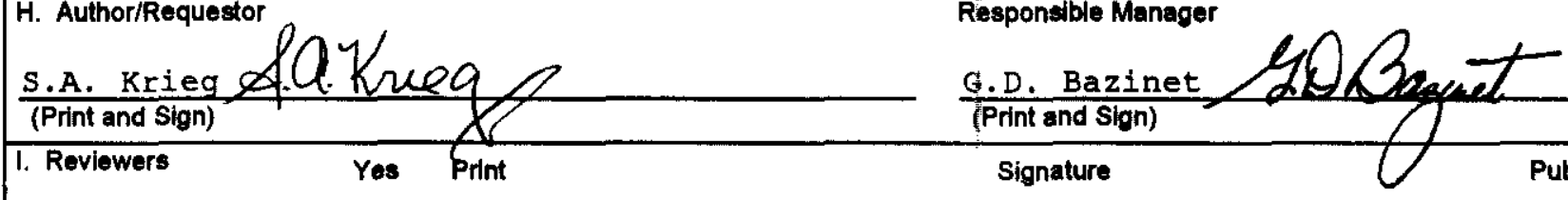

Public Y/N (If N, complete J)

General Counael

$\square$

Signature

$\mathrm{Y} / \mathrm{N}$

Ofice of External Affairs

DOE-RL
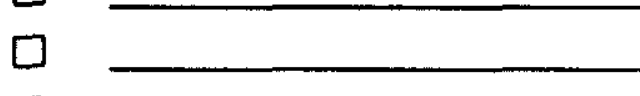

-

Other

E]

Other
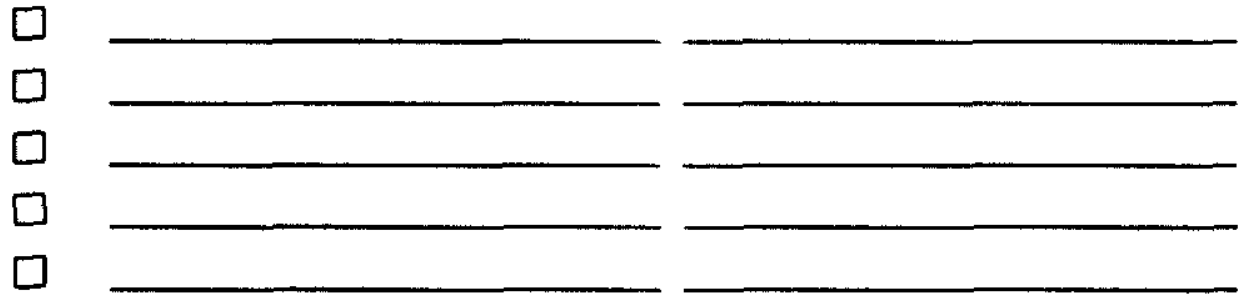

$\mathrm{Y} / \mathrm{N}$

$\mathrm{Y} / \mathrm{N}$

$\mathrm{Y} / \mathrm{N}$

$\mathrm{Y} / \mathrm{N}$

J. If Information Includes Sensitive Information and is not to be released to the Public indicate category below.
$\square$ Applied Technology
[ Protected CRADA
$\square$ Personalvivate
[ Export Controlled
$\square$ Proprietary
$\square$ Procurement-Sensitive
$\square$ Business-Sensitive
$\square$ Patentable
$\square$ Predecisional
$\square$ Other (Spectfy)
$\square$ UCNI

K. If Additional Comments, Please Attach Separate Sheet 
SNF-5790

Revision 0

\section{Design Compliance Matrices to ANSI and OSHA}

Prepared for the U.S. Department of Engrgy

Assistant Secretary for Environmental Management

Project Hanford Wonesmonent Contrector for tho

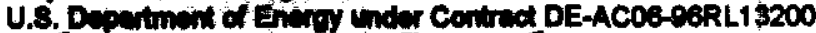

Finer Henford

P.O. Bax 1000

Richlend, Washington 
SNF-5790

Revision 0

ECN

\title{
Design Compliance Matrices to ANSI and OSHA
}

\author{
R. B. Bendixsen \\ Fluor Federal Services
}

Date Published

March 2000

Prepared for the U.S. Depertment of Enprgy

Assistant Secretary for Environmental Management

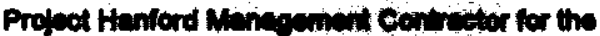

U.8. Departinent of Enwoy Unier Covinget DE-ACOs-00RL 13200

Fivor Hanford

P.O. Box 1000

Richlend, Woshington
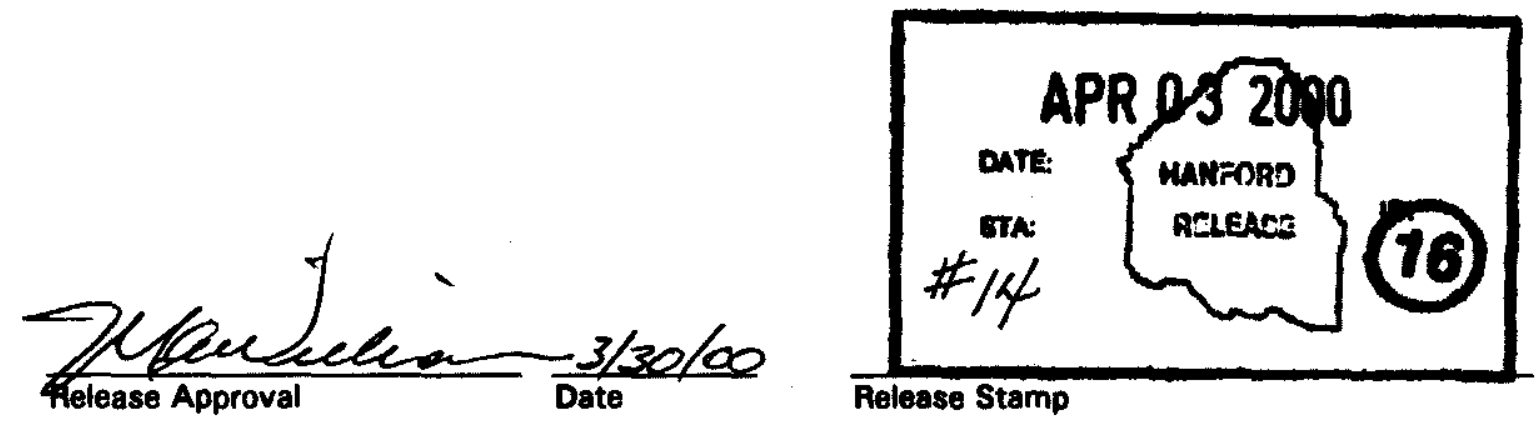

Release Stamp 
TRAREMANK DSCLLANER

Relerence herein to eny epecinc commercial product, proceses,

or earvice by trede name, trademak, manufi cturer, or

cthervies, dow not neceveartly conethete or imply the

endoreement, recommendation, of favoring ty the Uniked

state. Government or any agency thereof or its contractore or

wecontrectors.

The report hes been reproduced from the beid avallable copy.

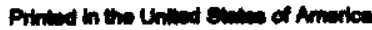

Total Pagen: 239 
SNF-5790, Rev. 0

Page 1 of 236

FDI RESPONSE TO ADDITIONAL CLARIFICATIONS TO FDH-9761261

\section{BACKGROUND}

This Supporting Document contains Design Compliance Matrices originally transmitted in DRAFT form by Fluor Daniel, Inc. to DE\&S Hanford, Inc. for review and comment under Transmittal No. FDT-110 dated May 28, 1998. To the best of the knowledge of the author, DE\&S Hanford, Inc. had no review comments on this transmittal. DE\&S Hanford, Inc. has requested the Fluor Daniel, Inc. prepare the contents of FDT-110 for issue as a Supporting Document. The words DRAFT have been removed from pages transmitted under FDT-110, each page has been sequentially numbered, and the supporting document number and revision have been placed on every page.

\section{ABSTRACT}

U.S. Department of Energy Letter 98-SFD-026 requested Fluor Daniel Hanford, Inc. to provide clarifications as to compliance with ANSI 57.1, 57.2, 57.9, and 29 CFR 1910.179 (OSHA), in the form of an item-by-item compliance matrix, for the CSB. This Supporting Document contains Fluor Daniel, Inc.'s response for use by Fluor Daniel Hanford, Inc. regarding the clarifications requested by the U.S. Department of Energy.

TABLE OF CONTENTS

ATTACHMENT 1 - FDI RESPONSE TO ADDITIONAL CLARIFICATIONS

Page No.

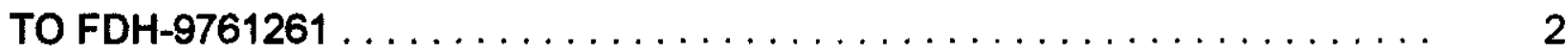

ATTACHMENT 2 - ANSI 57.1 COMPLIANCE MATRIX . . . . . . . . . . . 10

ATTACHMENT 3 - ANSI 57.2 COMPLIANCE MATRIX ........... 38

ATTACHMENT 4 - 29 CFR 1910.179 COMPLIANCE MATRIX . . . . . . . . . . 81

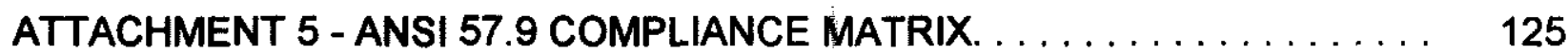


SNF-5790 Rev. 0

Page 2 of 236

\section{ATTACHMENT 1}

\section{FDI Response to Addifonal Clarifications} to FDH-9761261 


\section{SUBJECT: FDI RESPONSE TO ADDITIONAL CLARIFICATIONS TO FDH-9761261}

DESH had previously requested FDI to evaluate the safety classification of the Receiving Crane in letter DESH-9761243 (Reference 3.1). FDI assigned EDN-077 for evaluating the Receiving Crane for compliance with $10 \mathrm{CFR} 72$, ANSI 57.1, 57.2 and 57.9 for reasons other than release criteria. The report was transmitted to DESH on 1/6/98 via FDI Transmittal FDP-473 (Reference 3.2). The Service Station Gantry Crane and Hoist compliance was arrived at separately by DESH with input from FDI T3 personnel and, along with a similar evaluation for the MHM, included with the Receiving Crane evaluation in FDH letter report FDH-9761261 R4 (Reference 3.3) and submitted to DOE on $1 / 20 / 98$.

After review of that submittal, the DOE in letter 98-SFD-026 (Reference 3.4) dated 3/4/98 requested FDH to provide clarifications as to compliance with ANSI 57.1, 57.2 and 57.9 in the CSB SAR revision or separate formal submittal. The DOE requested an item-by-item compliance matrix and a discussion as to how each of the applicable ANSI standard items was incorporated into the handling devices. The complete list of action items required by DOE letter 98-SFD-026 (Reference 3.4) which require FDI actions are as follows.

* Item \#4 Receiving Crane. Provide mitigation feature design and safety classification feature design.

ACTION: FDI is to issue the specification package on the Receiving Pit impact absorbers. Classification of IA package is SC. Include description of the IAs and how the design meets the $\mathrm{SC}$ requirements in the CSB FSAR.

* Item \#11 Receiving Crane. Include results of seismic analysis to be performed on the existing RC design for the rated load of 60 tons. Provide a discussion in the CSB SAR of how ITS equipment meet $10 \mathrm{CFR} 72.122$ (b)(3)seismic requirements. FDI has previously supplied the $\mathrm{RC}$ vendor with spectra for the Truck Vestibule and LI/LO area.

ACTION: FDI to include results in CSB FSAR.

* Item Item \#13a CSB Receiving Crane. Provide an item by item discussion of ANSI 57.1 and ANSI 57.2 for the CSB receiving crane and how each item (requirement) was implemented. These were previously provided in FDP-473 by reference to an earlier work for compliance to the 29 NRC equivalency items contained in FDP-012.

ACTION: FDI to prepare an item by item discussion of ANSI 57.1 and ANSI 57.2 for the CSB receiving crane and how each item (requirement) was implemented.

* Item \#14 CSB Major Equipment-(Important to Safety-maybe safety class). Provide an item by item discussion of ANSI 57.9 compliance for the CSB receiving crane and all other CSB major equipment. This was previously provided in FDP-473 by reference to an earlier work for compliance contained in FRF-2792.

ACTION: Provide an item by item discussion of ANSI 57.9 compliance for the CSB receiving crane and all other CSB major equipment. Include design changes by DCN to the safety classification of the Service Station Gantry Crane and Hoist. Include DESH secondary confinement white paper information. Include discussion from CSB SAR Rev 7. Provide 
discussion in CSB FSAR.

* Item \#15 CSB. Provide discussion in the CSB SAR of facility features to mitigate MCO drops to acceptable limits.

ACTION: See item 4. Update drop discussion in the CSB FSAR,

As requested, FDI has completed Design Compliance Matrixes (DCM) to address the DOE clarification comments 13 and 14 to FDH-9761261 R4, Attachment 1 (Reference 3.2). The design compliance matrixes address the American National Standards (ANSI) 57.1, 57.2, and 57.9, and the Occupational Safety and Health Administration (OSHA) Regulation Standard 29 CFR 1910.179 to verify design compliance for the Canister Storage Building (CSB) receiving crane and major equipment and components with these standards. The ANSI 57.1, ANSI 57.2, and OSHA 29 CFR 1910.179 address only the design for the receiving crane, and the ANSI 57.9 addresses the CSB facility. These DCMs are issued for your review and concurrence.

\subsection{Summary:}

The DCMs provided a ANSI standard section review of the design for the receiving crane and major equipment and components. Each standard section was provided with a justification for compliance and a references to a design or project document that substantiated the determination.

The DCM for the OSHA compliance provided same review of the receiving crane design, and some sections were not applicable based on the type of crane provided for the CSB facility and facility operating requirements.

ANSI standards 57.1 and 57.2 address primarily pool type SNF storage facilities, and consequently some sections are not applicable to the CSB facility (and components). The CSB facility provides only interim storage of dry Multicanister Overpacks (MCO) in dry storage vaults for future retrieval for long term storage or processing.

The DCMs determined that some discrepancies exist in complying with the standards which are based on the project's design-to-cost decisions or other codes and standards relied on for the design of the facility. The following section provides the process used to determine design compliance with each standard.

\subsection{Design Compliance Matrixes}

The specific requests of DOE letter 98-SFD-026 (Reference 3.4) are repeated below in italics with a discussion of the process and specific compliance deviations for each standard DCM.

\section{$2.1 \quad$ Item 13}

Page 18: SNFP Path Fonward Additional NRC Requirments, HNF-SD-SNF-DB-003 invokes the requirements of ANSI 57.1 and 57.2 to the CSB and CVD. Many 
requirements from ANSI 57.1 and 57.2 do not appear to have been incorporated into the receiving crane specification or the cold vacuum facility hoist, e.g., ANSI 57.1, 6.1.3, 6.3.4: ANSI 57.2, 5.2.4.5, 6.2.1.1.a. FDH shall verify DB-003 has item \#13 been applied to these two cranes?

Action: Along with the CSB and CVD SAR revisions or separate formal document submittal matrix showing compliance with the ANSI 57.1 and 57.2 to both the CSB Receiving Crane and CVDF Hoist. Include the discussion how each of the applicable ANSI Standard items was incorporated into these handling devices. (Provide references).

\subsubsection{ANSI 57.1}

The ANSI 57.1 DCM for the receiving crane (Attachment 1) was conducted to determine compliance sections and deviations from the standard. The process for the evaluation is based on the following.

a) Base the design review on the Procurement Specification W-379-P-CSB-14620, CSB FSAR HNF-SD-SNF-RPT-004, Rev 7B, MCO Topical Report HNF-SDSNF-SARR-005, and the Receiving Crane Operating, Parts, \& Maintenance Manual MGW-SVX-452651.

b) Base the ANSI 57.1 review on Sections 5 and 6 of the standard.

c) Base the design review on the receiving crane only, and mention interfaces with the CSB facility design or operation as applicable.

The ANSI 57.1 DMC design deviations are as follows.

a) The crane design is not part of the primary reactor containment, pool handling equipment, building electrical power supply, storage pool equipment, grappling, fuel handling and refueling machines, upender, fuel transfer carriage or machine, handling tools, inspection equipment, temporary storage, transfer tube assembly, fuel elevator, and fuel preparation equipment.

b) The crane design does not move fuel units in storage pools or directly handle fuel units.

c) The crane design does not provide communication or building location.

d) The crane will not be in a galvanic or chemical corrosive environment.

\subsubsection{ANSI 57.2}


The ANSI 57.2 DCM for the receiving crane (Attachment 2) was conducted to determine compliance sections and deviations from the standard. The process for the evaluation is based on the following.

a) Base the design review on the Procurement Specification W-379-P-CSB-14620, CSB FSAR HNF-SD-SNF-RPT-004, Rev 7B, MCO Topical Report HNF-SDSNF-SARR-005, and the Receiving Crane Operating, Parts, \& Maintenance Manual MGW-SVX-452651.

b) Base the ANSI 57.2 review on Sections 5 and 6 of the standard.

c) Base the design review on the receiving crane only, and mention interfaces with the CSB facility design or operation as applicable.

The ANSI 57.2 DMC design deviations are as follows.

a) The crane design is not part of the cooling, cleanup and makeup systems, spent fuel storage racks, fuel storage building, cask coolent flush system, shipping cask, transportation vehicle, cask decontamination area, monitoring systems, and spent fuel storage assemblies.

b) The crane design does not move fuel units in or over storage pools, or directly handle fuel units.

c) The crane design does not provide electrical power, I\&C, health protection, and communication.

d) The crane design does not maintain MCOs.

e) The crane design does not directly transfer radioactive materials.

\subsubsection{OSHA 22 CER 1910.179}

The OSHA 29 CFR 1910.179 DCM for the receiving crane (Attachment 3) was conducted to determine compliance sections and deviations from the standard. Compliance to this standard was predicated by its inclusion as a reference in ANSI 57.9. The process for the evaluation is based on the following.

a) Base the design review on the Procurement Specification W-379-P-CSB-14620, CSB FSAR HNF-SD-SNF-RPT-004, Rev 7B, MCO Topical Report HNF-SDSNF-SARR-005, and the Receiving Crane Operating, Parts, \& Maintenance Manual MGW-SVX-452651.

b) Base the OSHA 29 CFR 1910.179 review on Sections (b) and (o) of the standard. 
c) Base the design review on the receiving crane only, and mention interfaces with the CSB facility design or operation as applicable.

The OSHA 29 CFR 1910.179 DCM design deviations are as follows.

a) The crane design does not have more than one trolley.

b) The crane design does not provide a cab.

c) The crane design is not parallel to other cranes.

d) The crane design is for an indoor crane.

e) The crane design is not for a modified crane.

f) The crane design does not provide sweeps, and bridge stops provided are provided by the CSB facility.

g) The crane design does not handle hot metal.

h) The crane design does not use foot-operated mechanical control brakes.

I) The crane design does not use a pendent station.

j) The crane design does not provide main power disconnect (provided by CSB facility).

k) The crane design does not use hoist chains.

1) The crane design does not provide operational testing, inspection, maintenance.

\section{$2.2 \quad$ Item 14}

Page 12: SNFP Path Forward Additional NRC Requirments, HNF-SD-SNF-DB-003 requires that the requirements of $A N S I 57.9$ be reviewed for incorporation into the design of the CSB. Many sections of ANSI 57.9 were not mentioned and not justification, e.g., $6.1 .1 .3 .3,6.1 .2 .3 .1$. FDH shall demonstrate that the additional considerations of $D B$ 003 , item $\# 28$ be applied to the CSB.

Action: Along with a CSB SAR revision or separate formal submittal, provide an item by item discussion of the compliance with ANSI 57.9 to the CSB design. Include in the discussion a description of how each of the applicable ANSI Standard items was implemented into the CSB design, include all major equipment in that discussion. 


\subsubsection{ANSI 57.9}

The ANSI 57.9 DCM for the CSB facility (Attachment 4) was conducted to determine compliance sections and deviations from the standard. The process for the evaluation is based on the following.

a) Base the design review on the CSB FSAR HNF-SD-SNF-RPT-004, Design Basis Document FDI 80460200, Procurement Specification W-379-P-CSB-14620 and W-379-C-CSB-14320, Rev 7B, MCO Topical Report HNF-SD-SNF-SARR-005, and the Receiving Crane Operating, Parts, \& Maintenance Manual MGW-SVX452651 .

b) Base the ANSI 57.9 review on Sections 5 and 6 of the standard.

c) Base the design review on the CSB facility only, and mention interfaces with the CSB facility operations, K-Basins and CVD design or operations, or MCO, MHM, and handling equipment as applicable

The ANSI 57.9 DMC design deviations are as follows.

a) The CSB facility design is not part of the CVD or K-Basins facilities..

b) The CSB facility design does not provide washdown areas, radwaste drainage, washdown of transportation packages, and decontamination facilities per designto-cost.

c) The CSB facility design does not require auxiliary cooling for MCOs.

d) The CSB facility design does not provide a shielded cell.

e) The CSB facility design does not handle fuel units or store uncanistered fuel units.

f) The CSB facility design does not provide operational requirements.

g) The CSB facility design does not provide the handling tools, MCO or MHM.

\subsection{References}

3.1 DE\&S Hanford Letter DESH-9761243, Evaluation of Receiving Crane, dated December 3, 1997, M.K. Mahaffey to P.J. Bedell.

3.2 Fluor Daniel Inc. Transmittal No. FDP-473, FDI Evaluation of the Receiving Crane, dated January 6, 1998, P.J. Bedell to M.K. Mahaffey. 
SNF-5790 Rev. 0

Page 9 of 236

3.3 Fluor Daniel Hanford Letter FDH-9761261 R4, Safety Classification of Cranes and Handling Equipment, dated January 20, 1998, N.H. Williams to E.D. Sellers.

3.4 DOE-RL Letter 98-SFD-026, Safety Classification and Designation as Important-toSafety of Spent Nuclear Fuel Project (SNFP) Cranes and Handling Equipment, E.D. Sellers to H.J. Hatch. 
SNF-5790 Rev. 0

Page 10 of 236

\title{
ATTACHMENT 2
}

\author{
ANSI 57.1
}

\section{COMPLIANCE MATRIX}




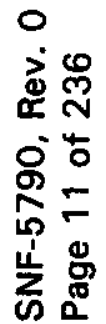

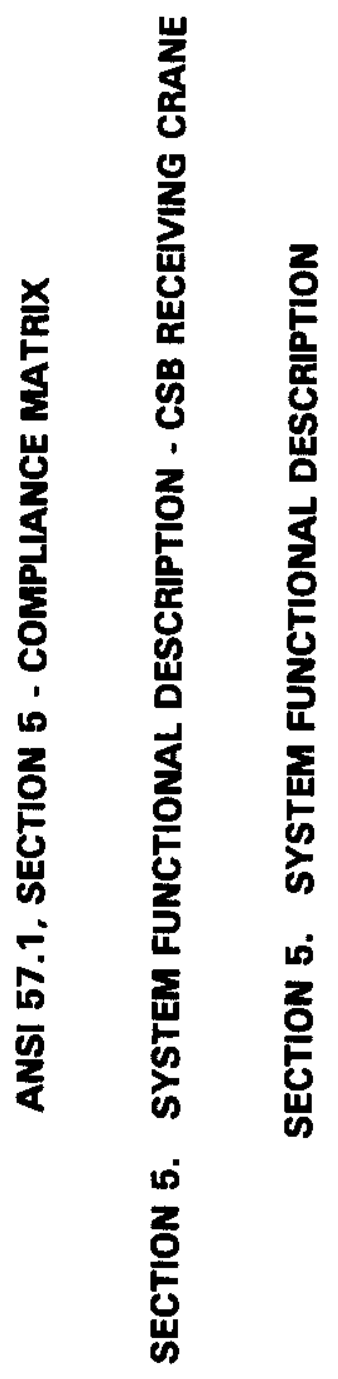

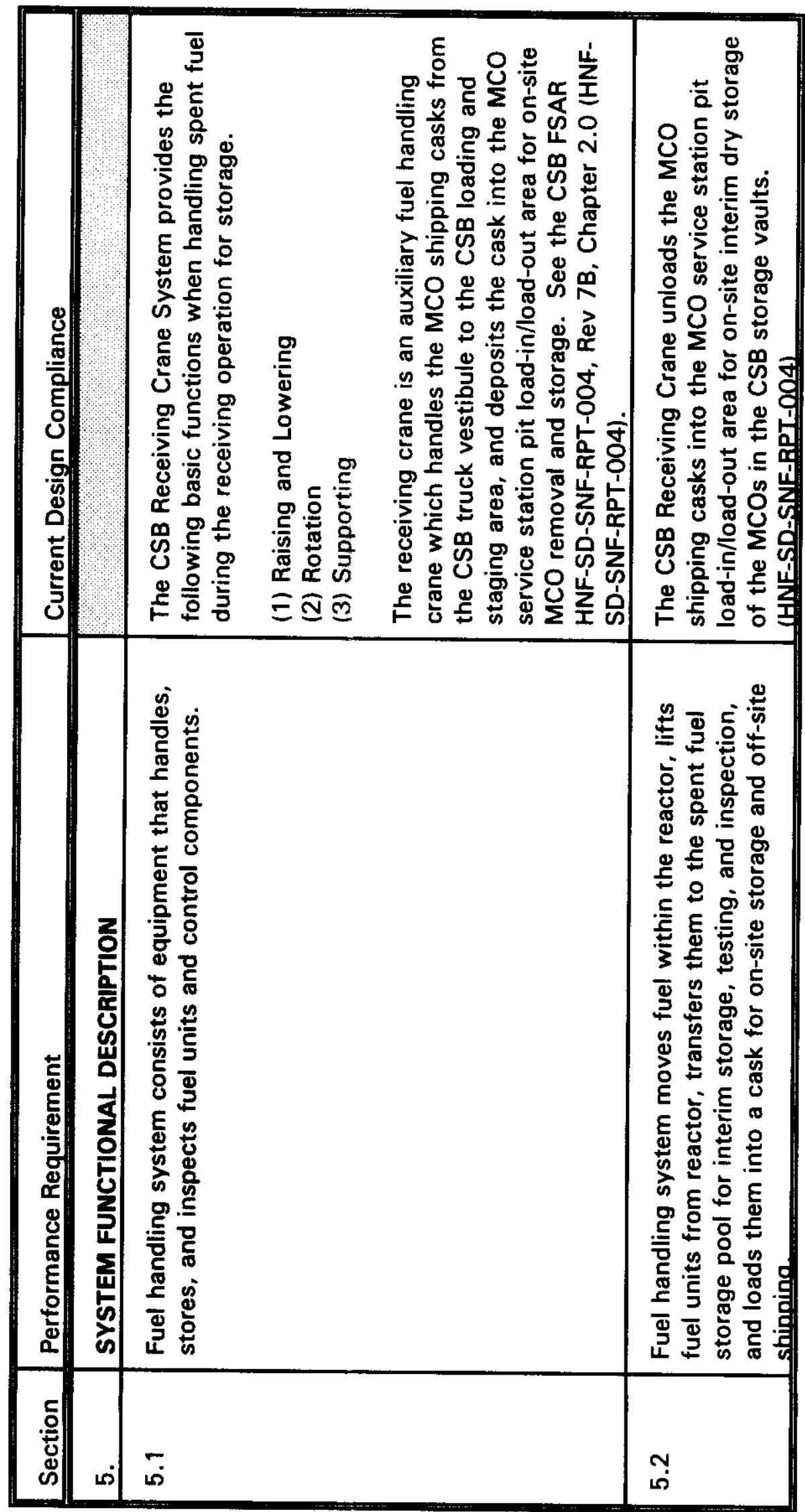




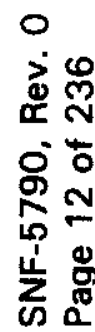

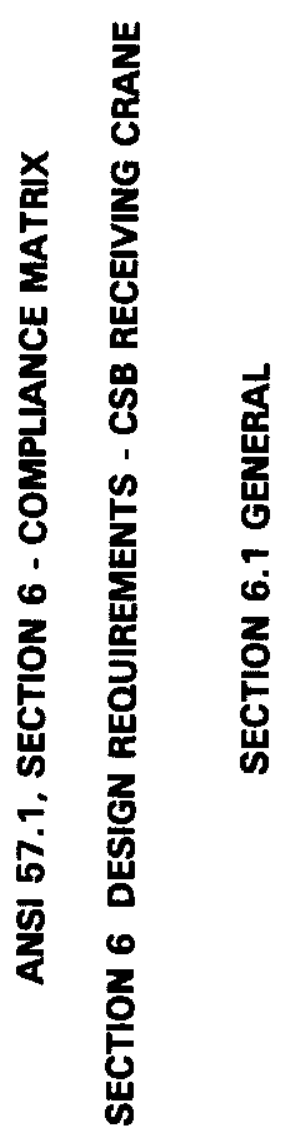

\begin{tabular}{|c|c|c|c|}
\hline 0 & 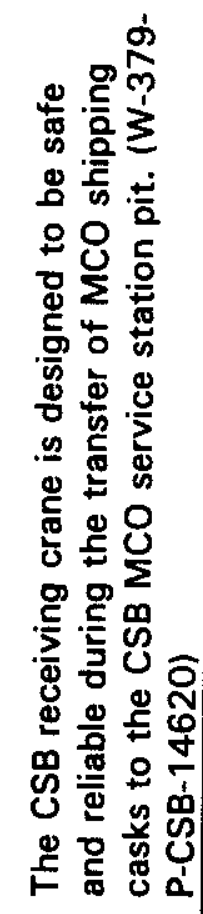 & 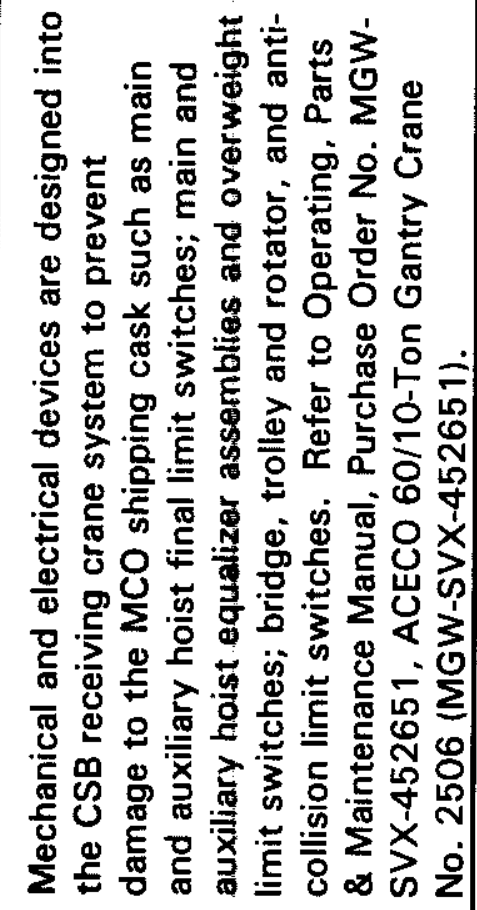 & 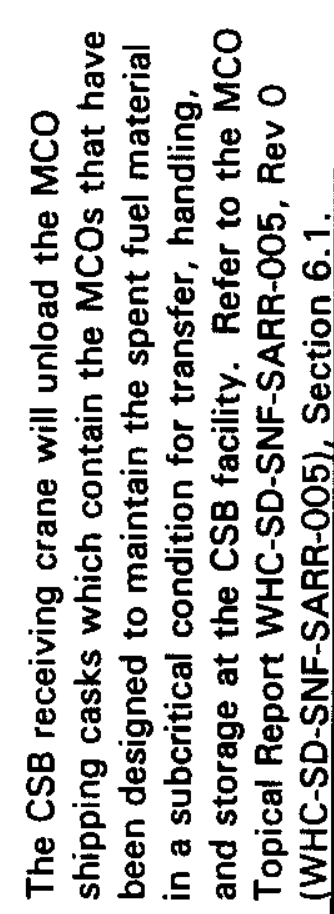 \\
\hline 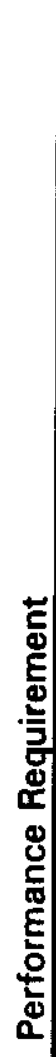 & 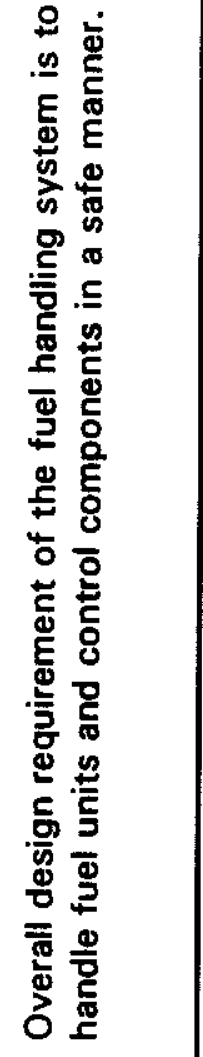 & 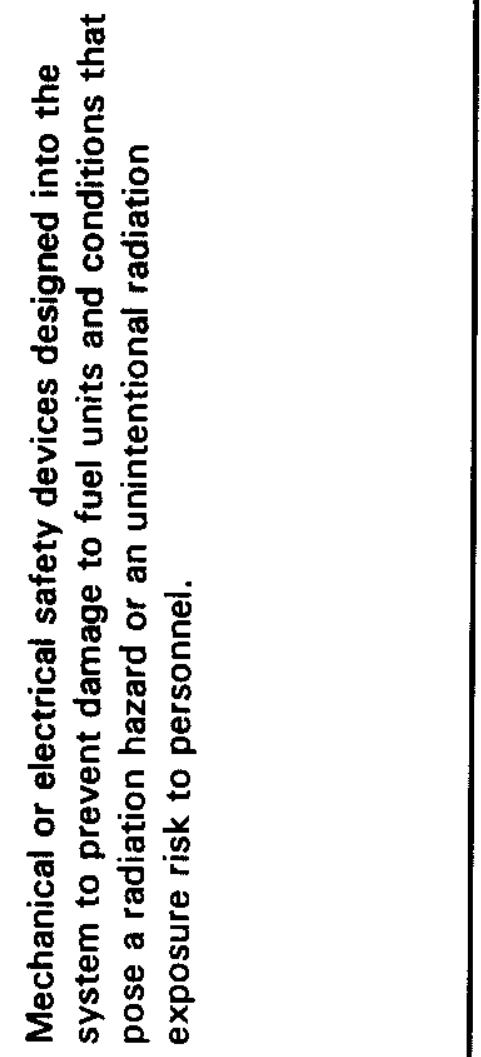 & 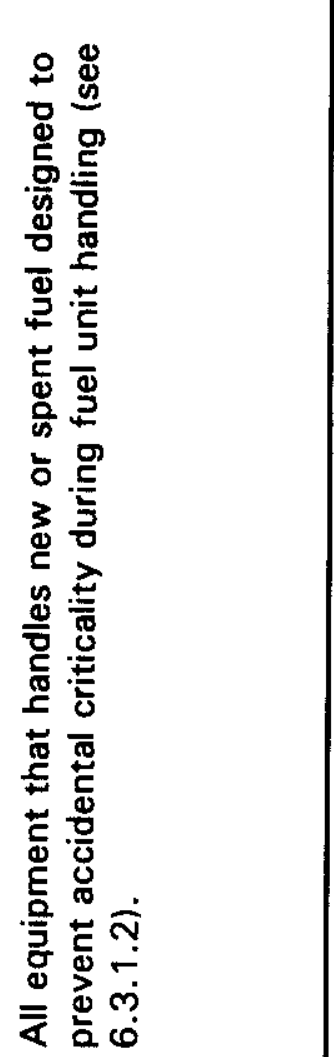 \\
\hline & $\check{\oplus}$ & 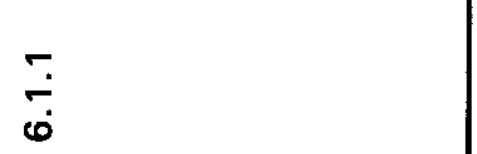 & \\
\hline
\end{tabular}




\begin{tabular}{|c|c|c|c|c|c|}
\hline 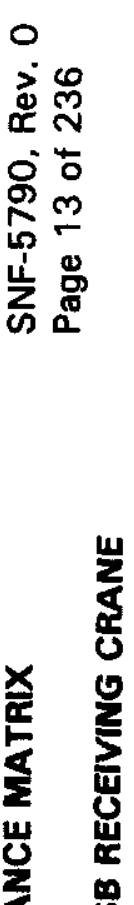 & 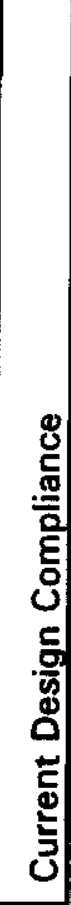 & 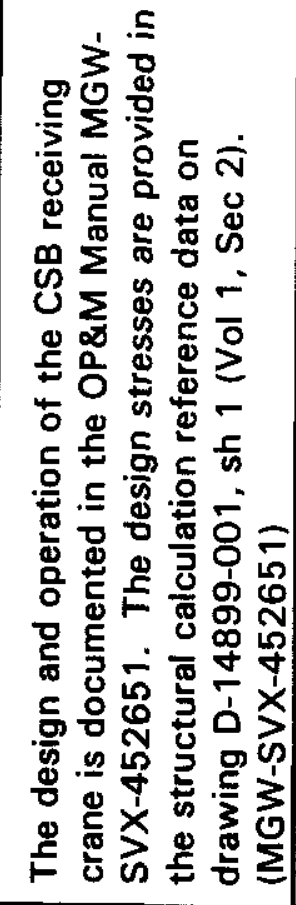 & 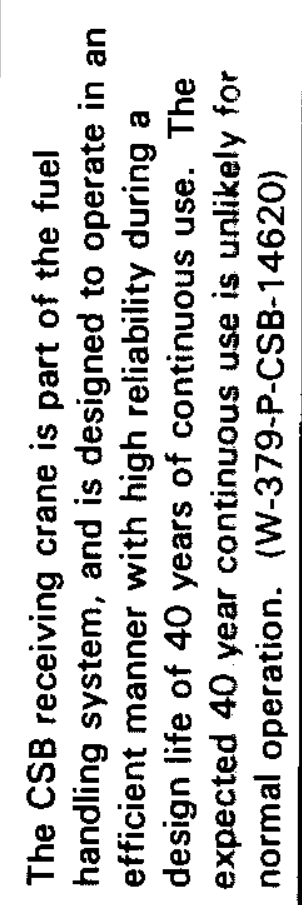 & 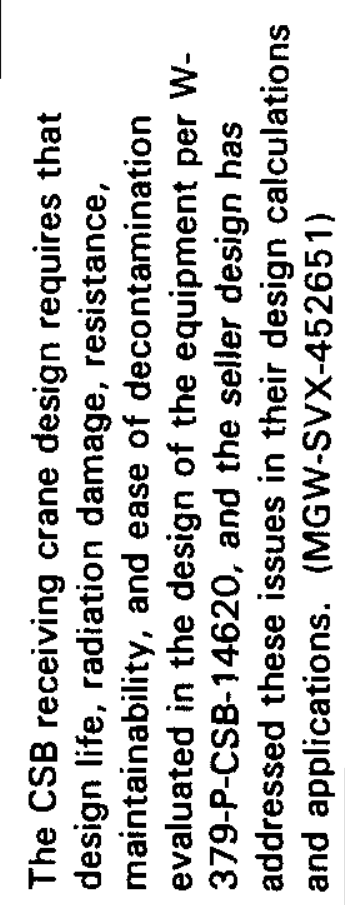 & 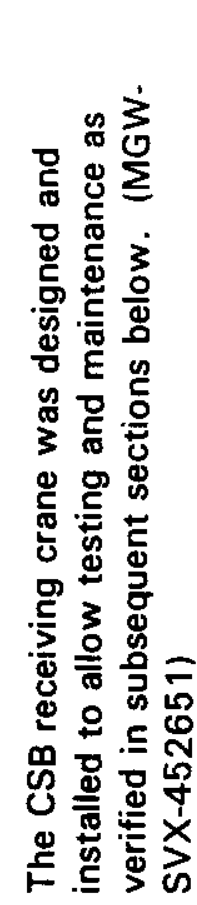 \\
\hline 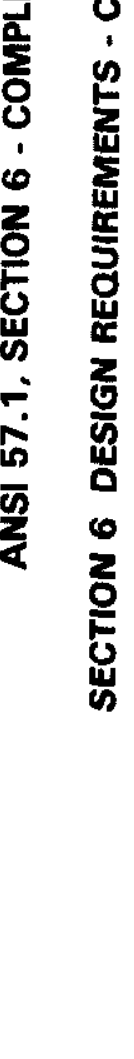 & 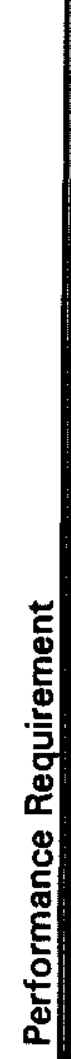 & 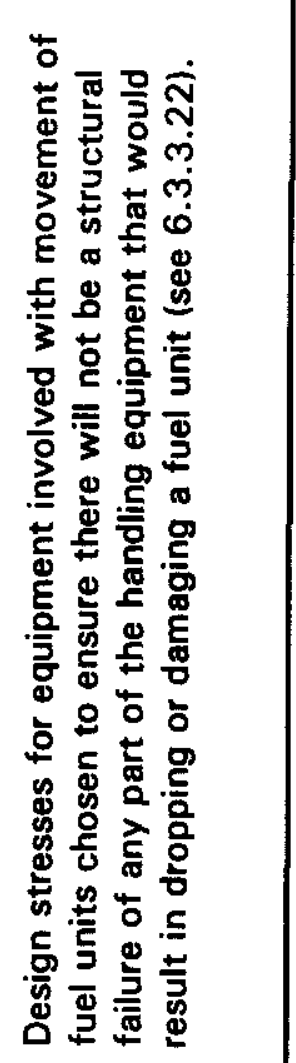 & 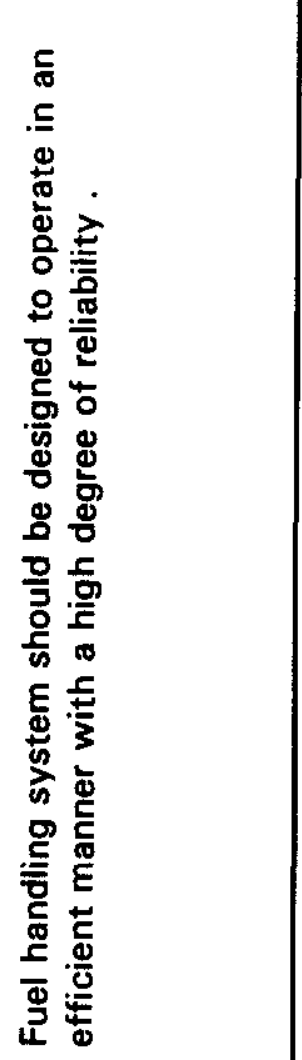 & 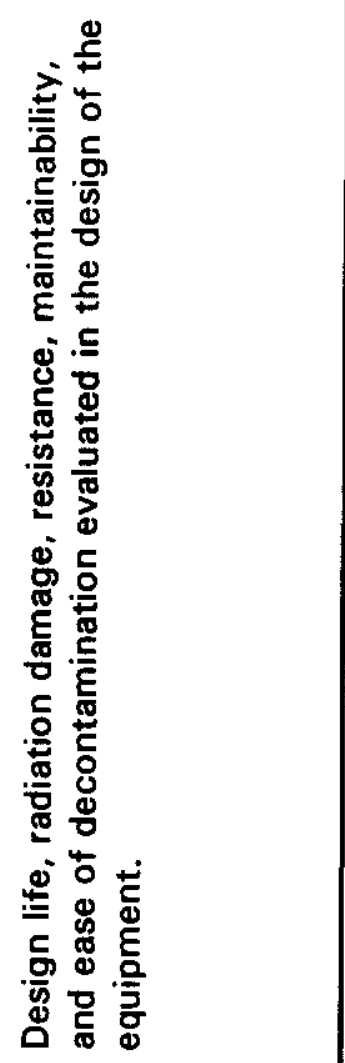 & 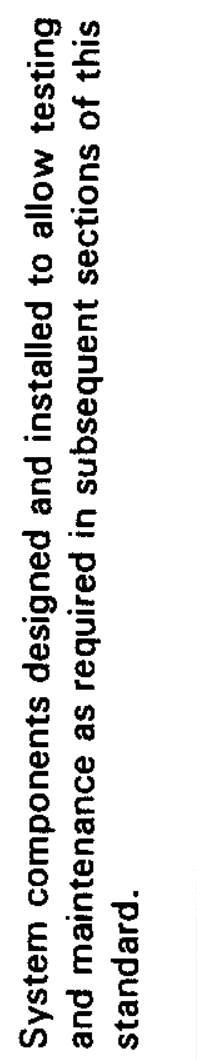 \\
\hline & 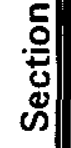 & $\stackrel{m}{\check{\varphi}}$ & $\stackrel{+}{\dot{\sigma}}$ & مُ مُ & $\frac{\varphi}{\dot{\varphi}}$ \\
\hline
\end{tabular}




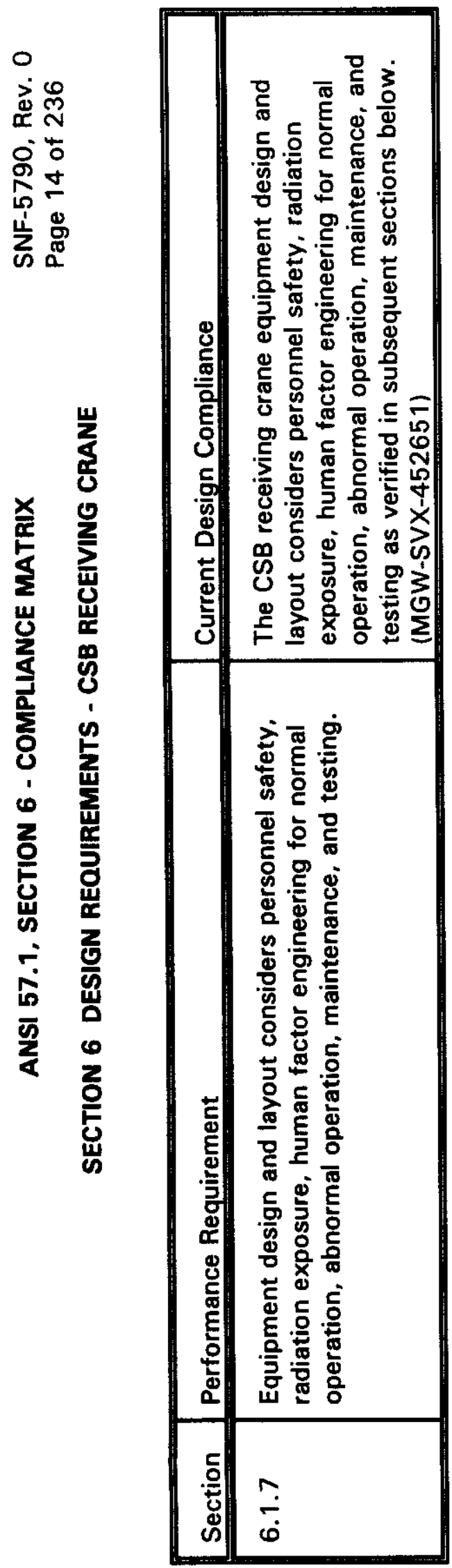




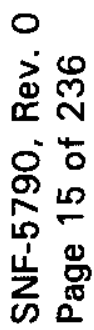

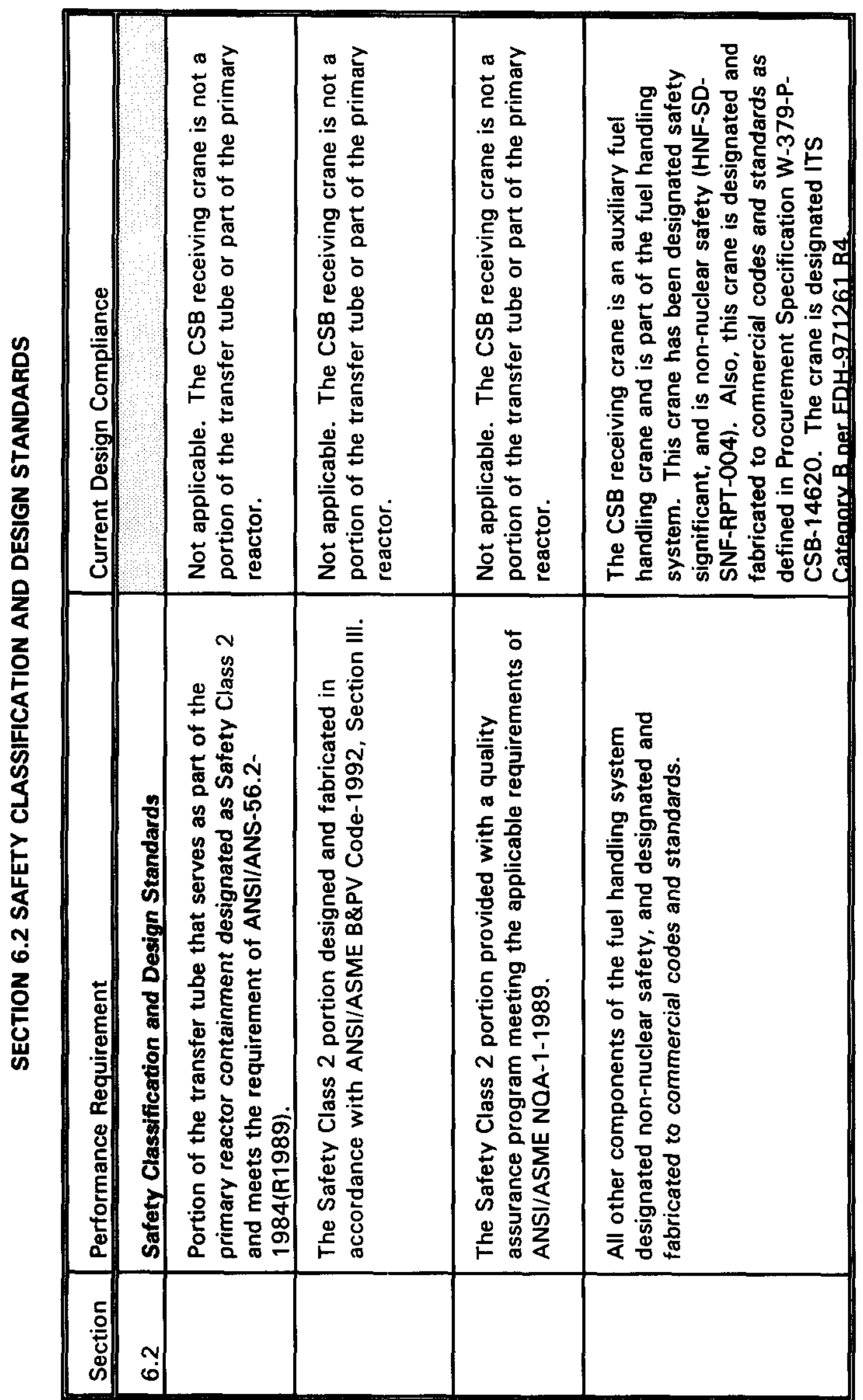




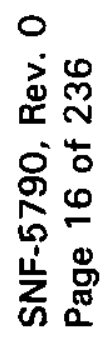
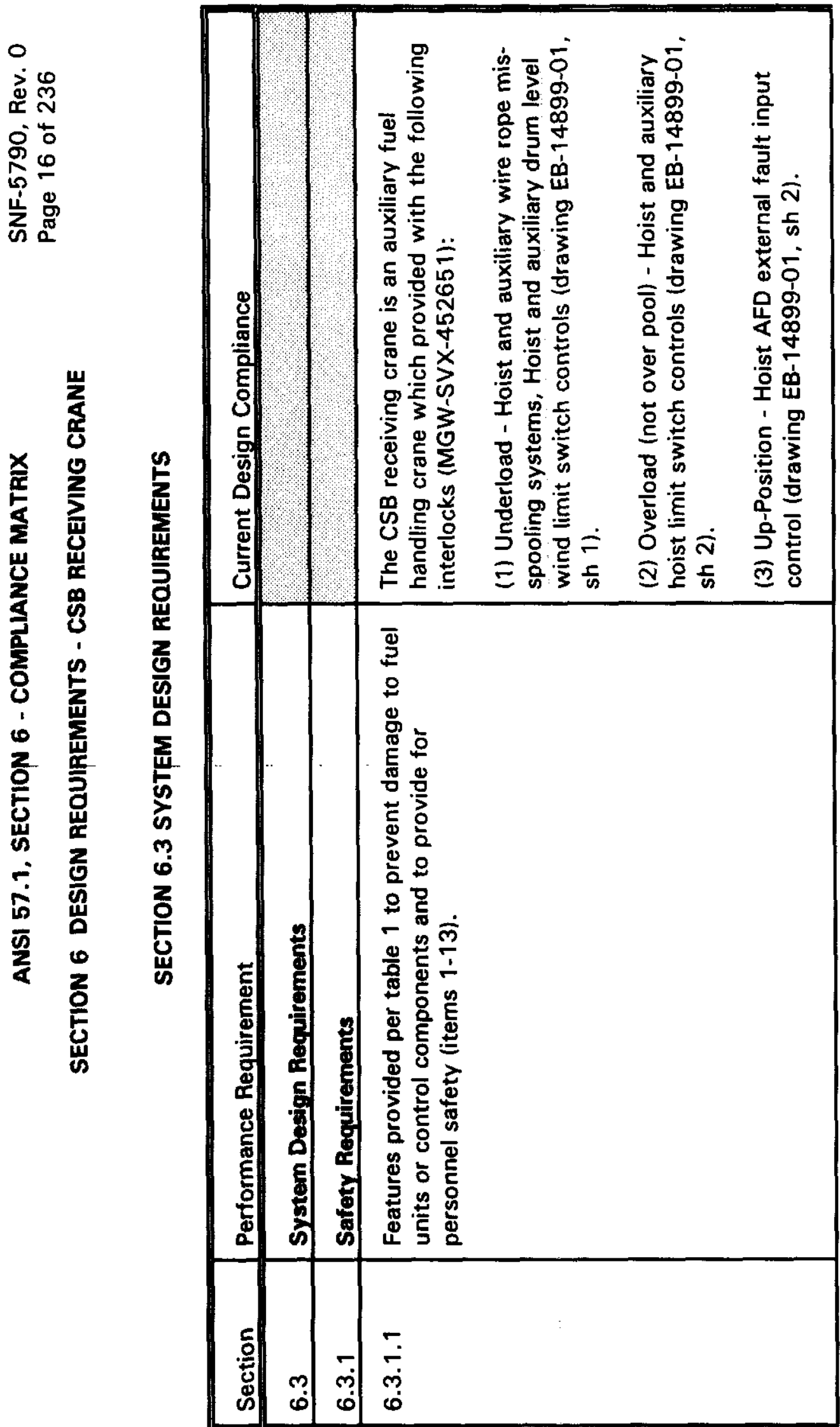


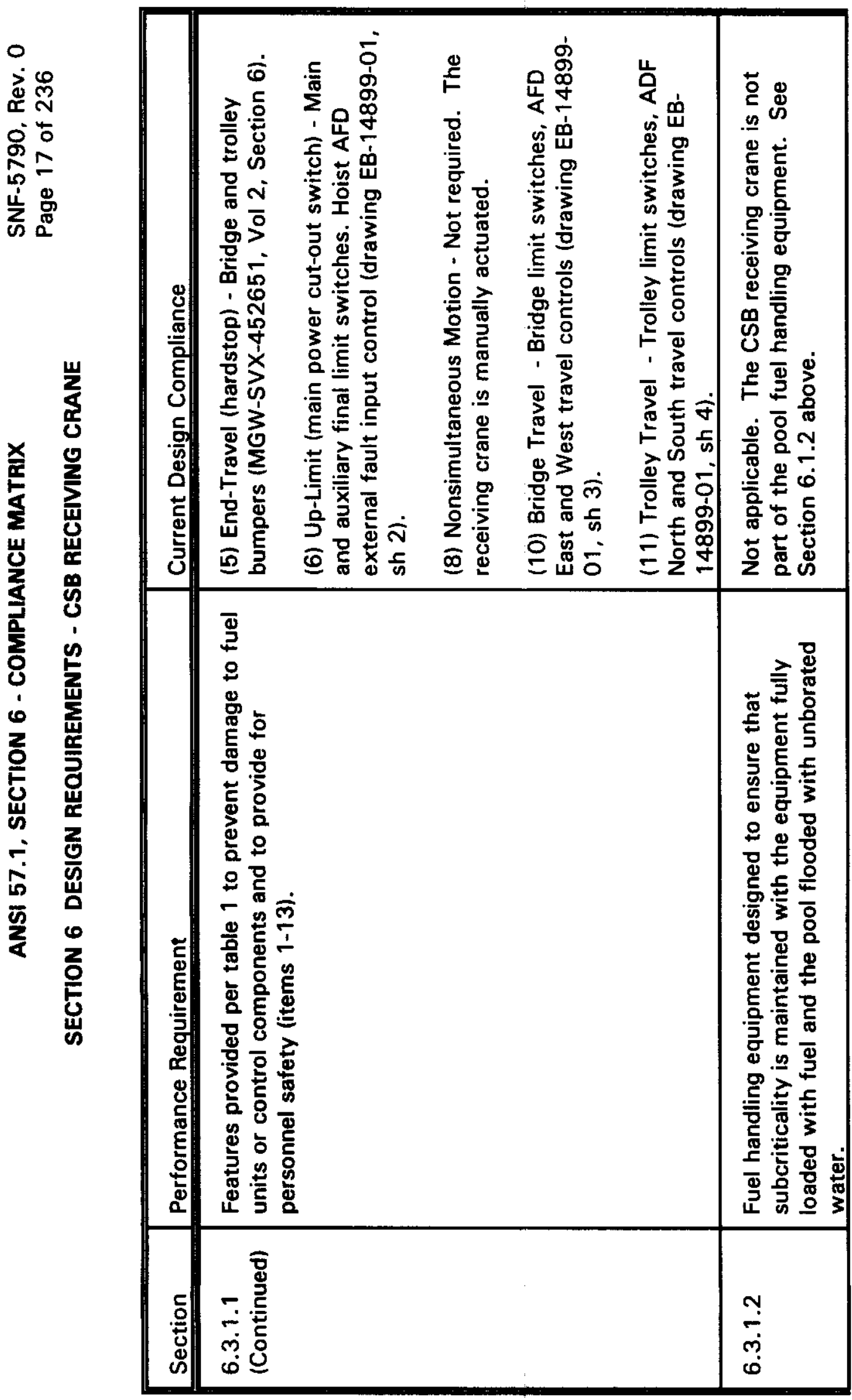




\begin{tabular}{|c|c|c|c|c|}
\hline 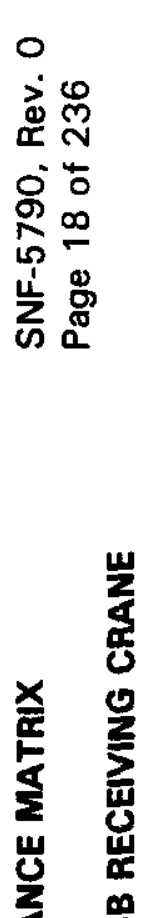 & 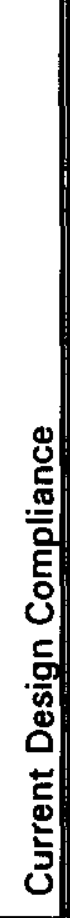 & 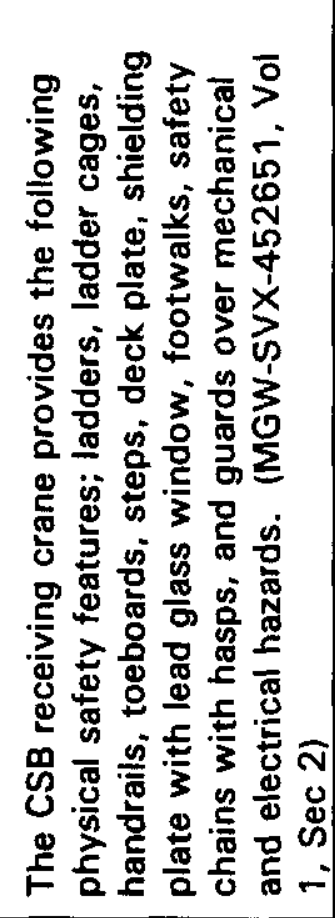 & 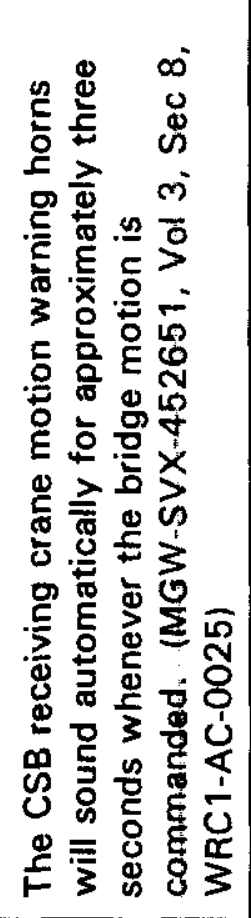 & 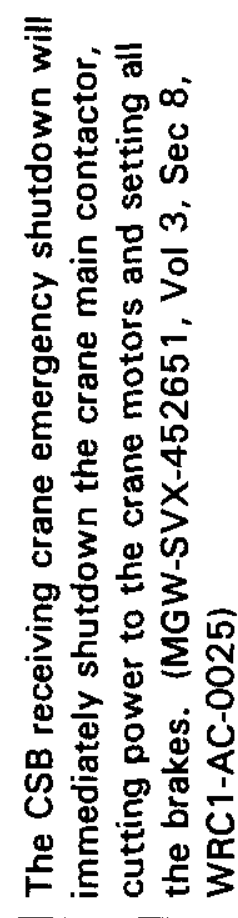 \\
\hline 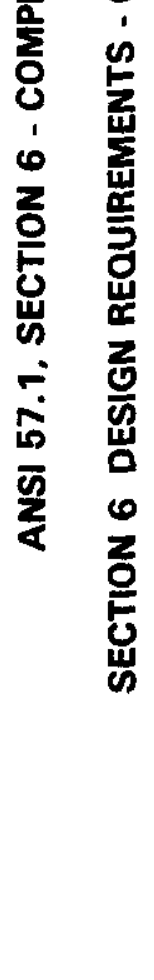 & 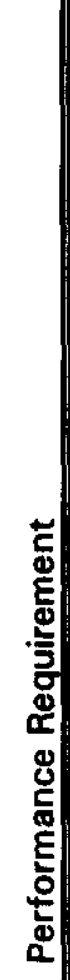 & 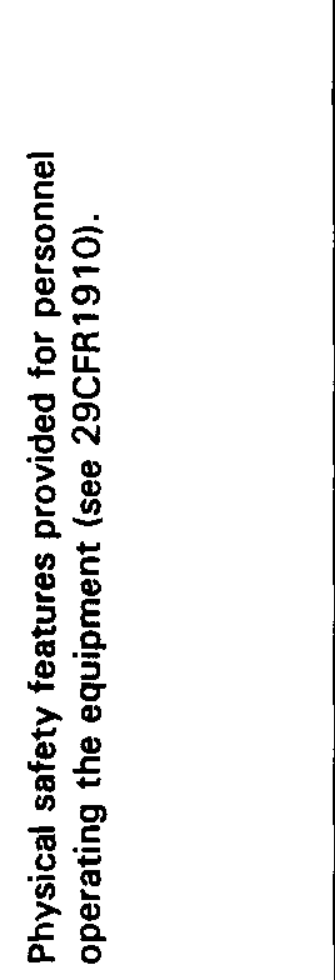 & 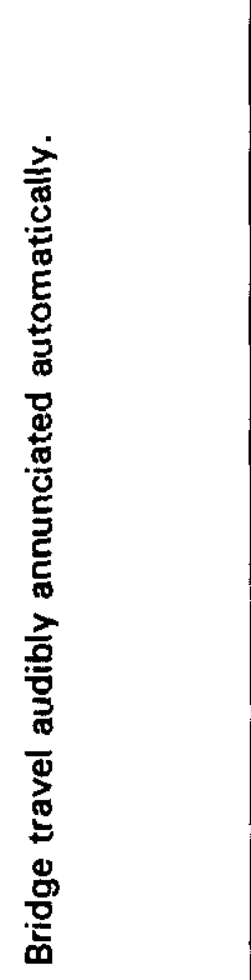 & 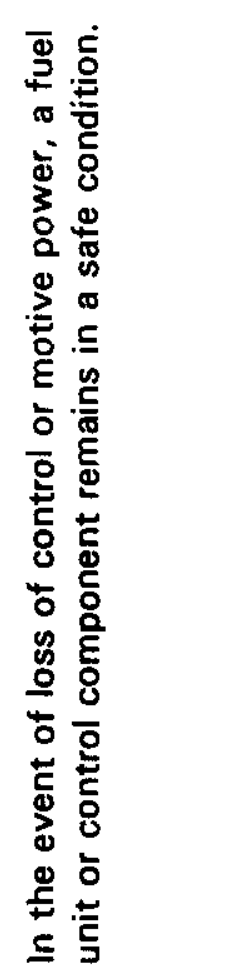 \\
\hline & 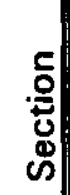 & $\begin{array}{l}? \\
\stackrel{?}{0} \\
\ddot{0}\end{array}$ & 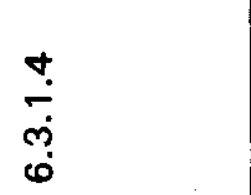 & $\begin{array}{l}\stackrel{0}{0} \\
\text { ம் }\end{array}$ \\
\hline
\end{tabular}




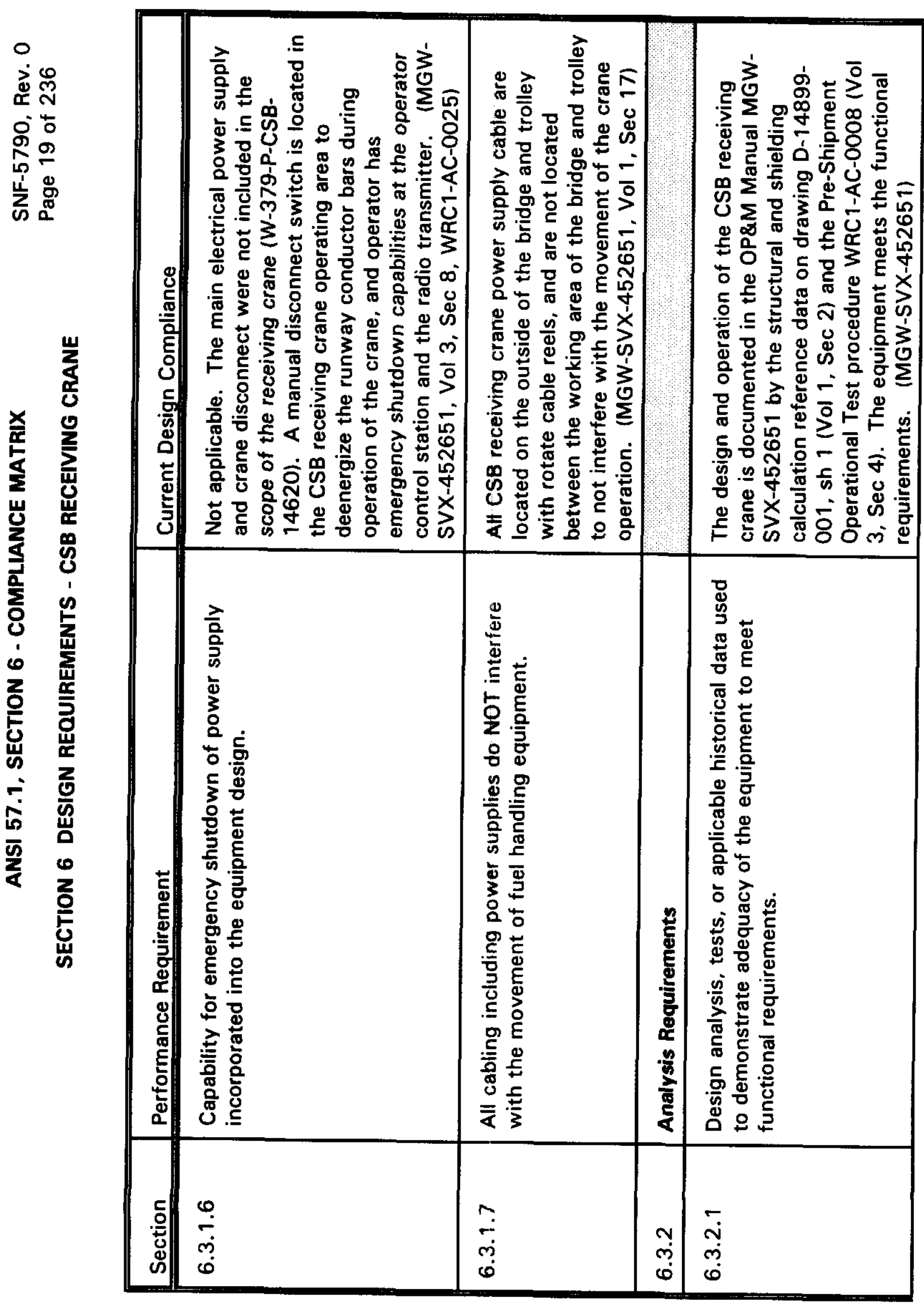




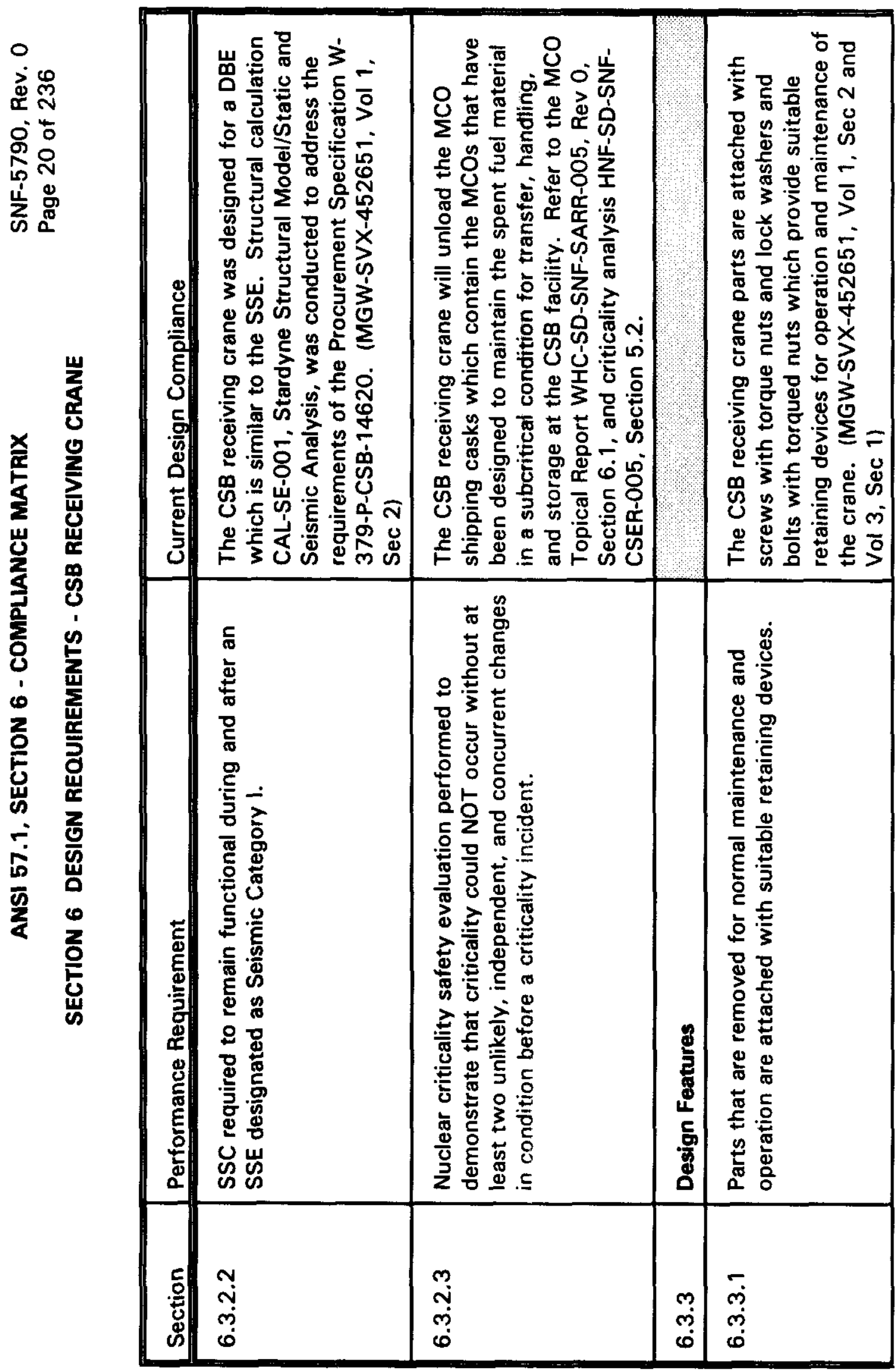




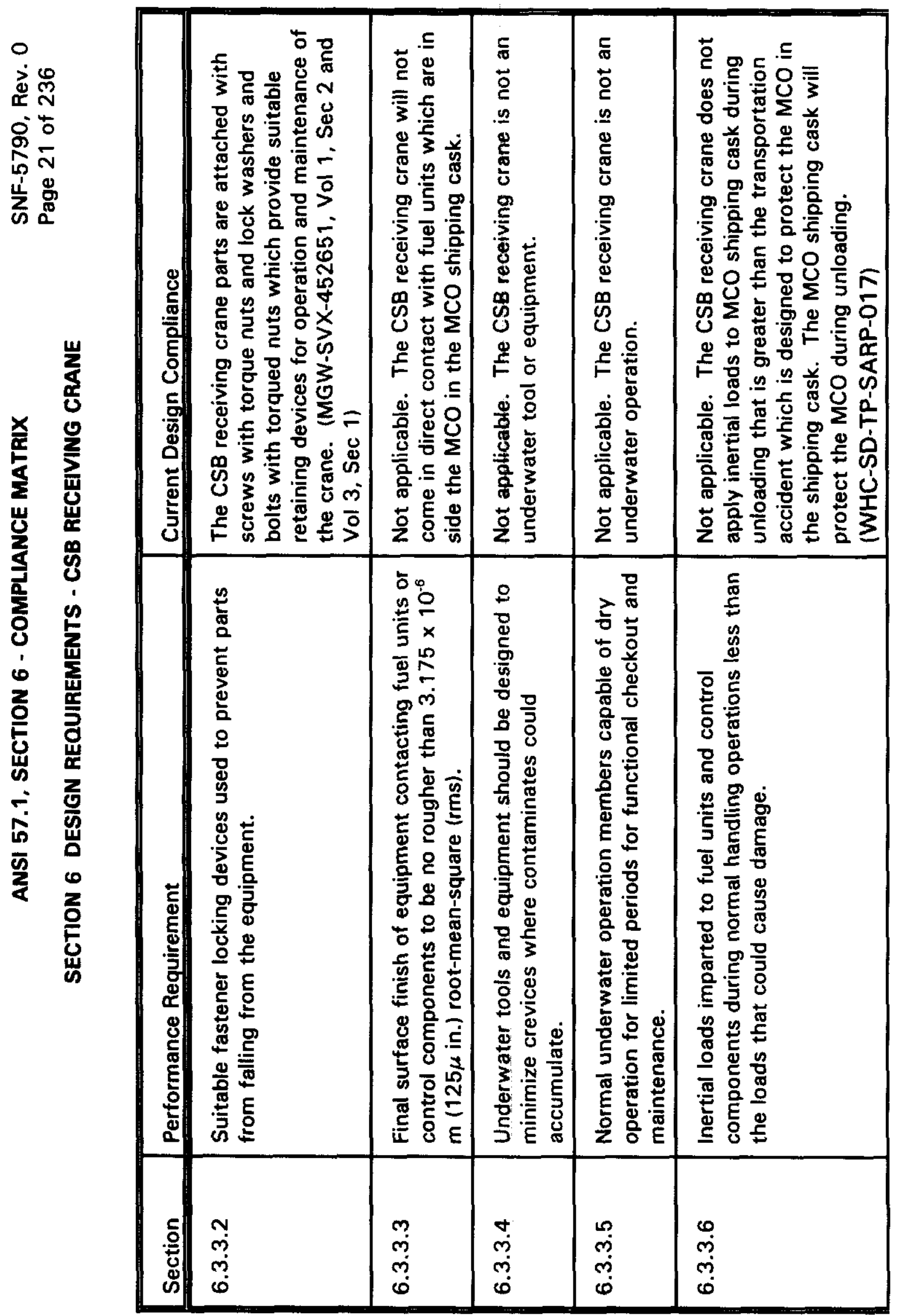




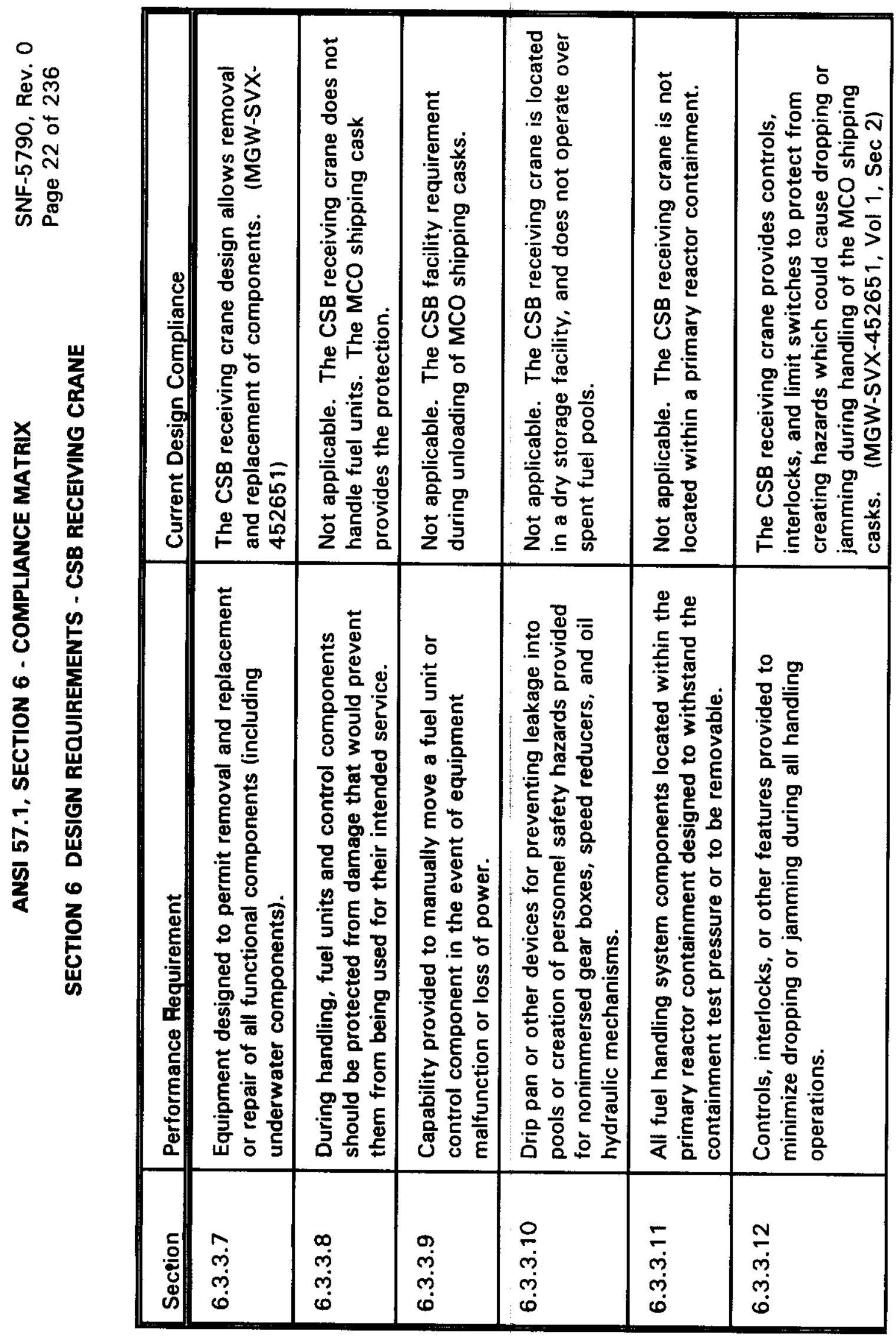




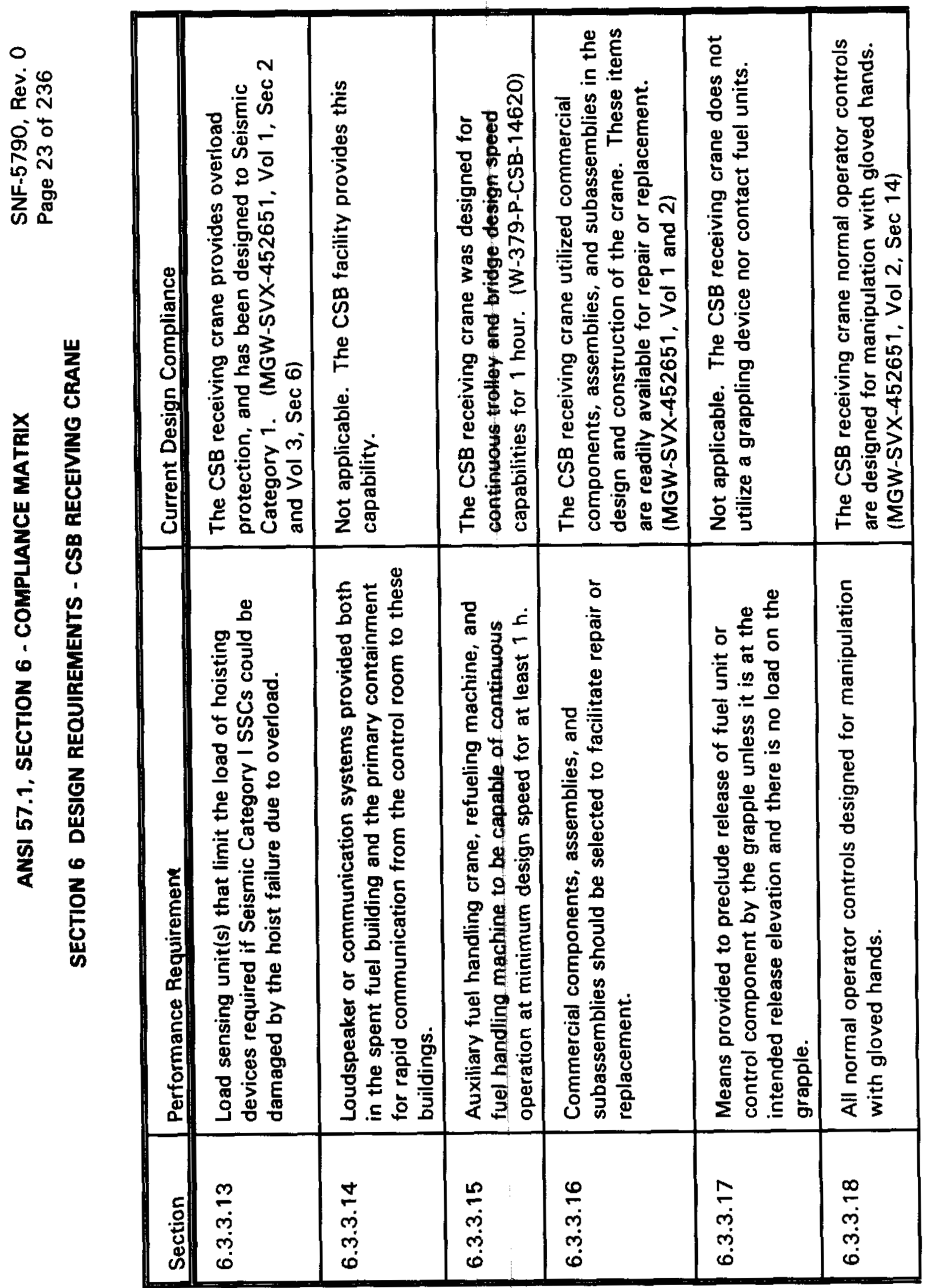




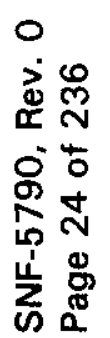

\begin{tabular}{|c|c|c|c|c|c|c|}
\hline 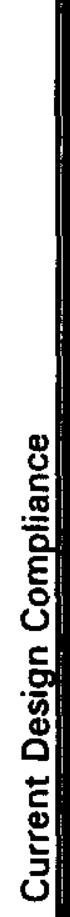 & 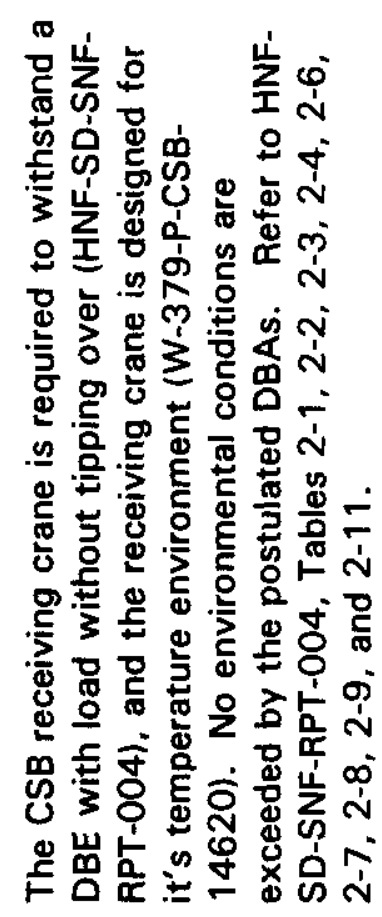 & 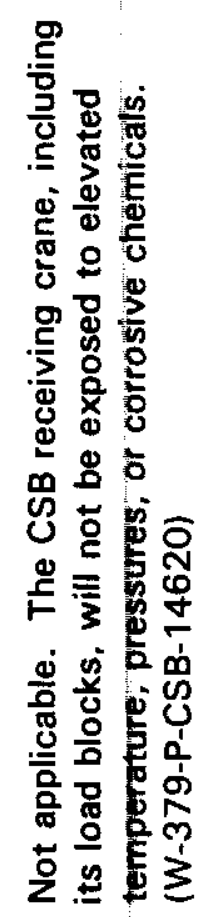 & 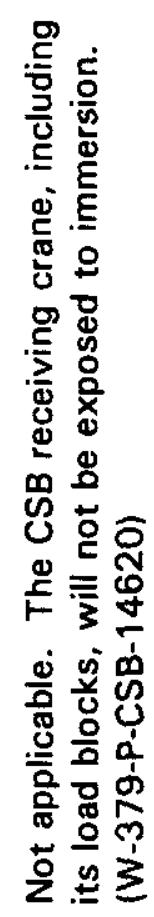 & 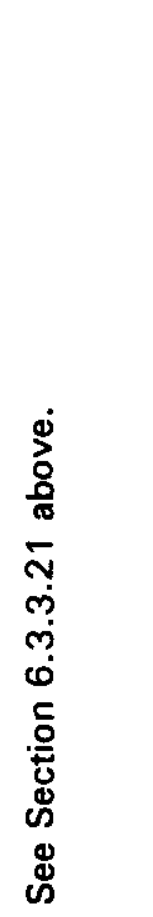 & 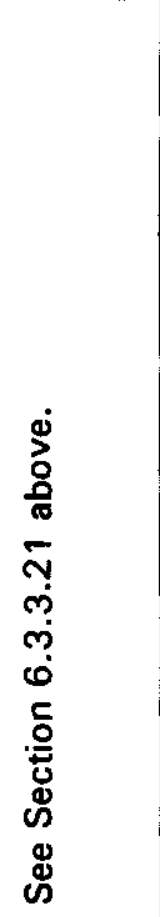 & 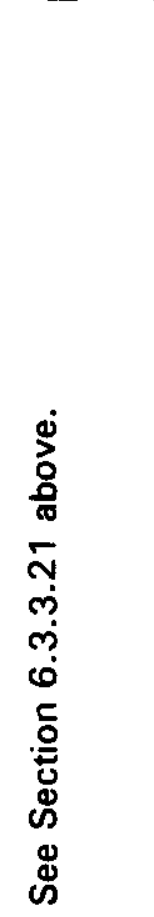 \\
\hline 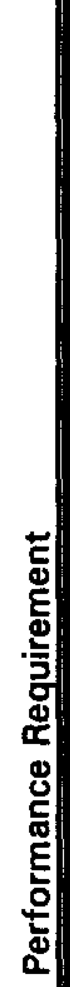 & 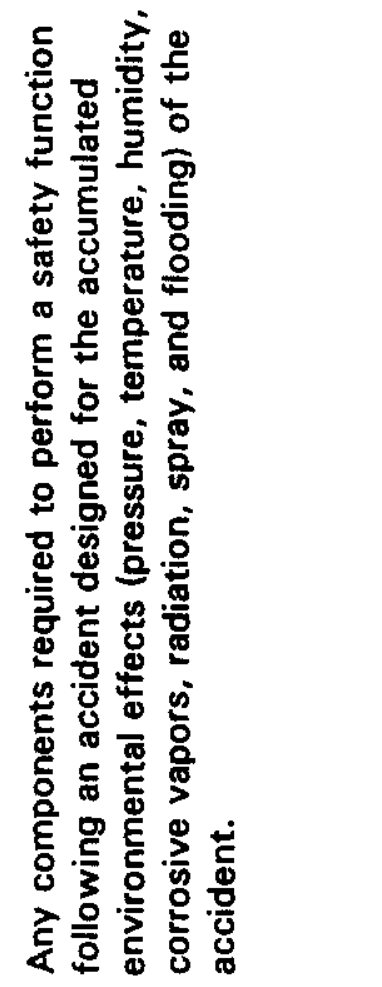 & 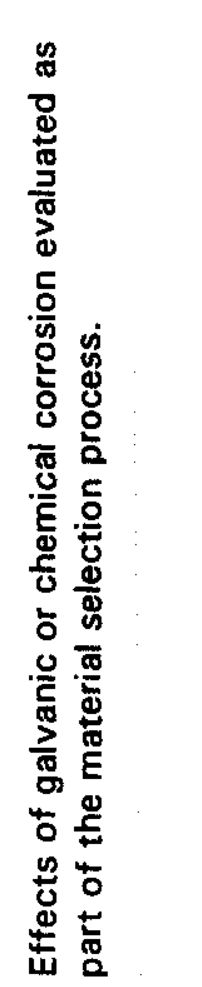 & 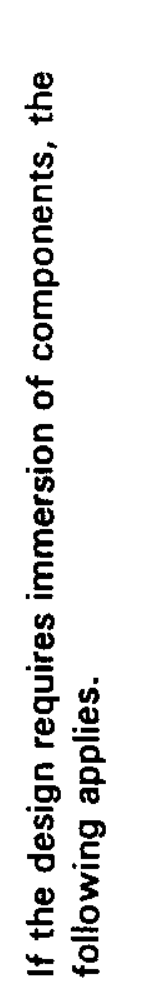 & 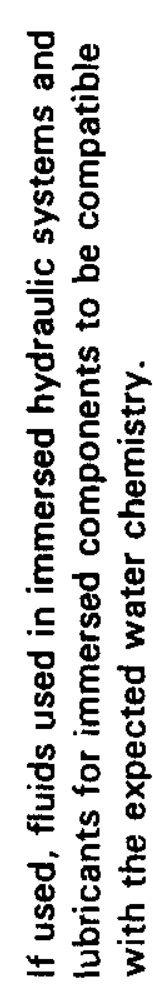 & 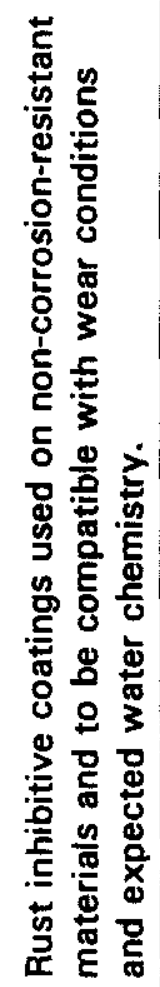 & 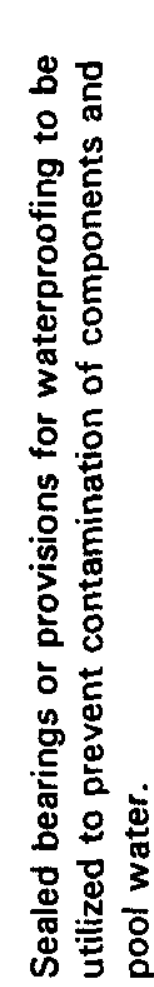 \\
\hline 잏ㅁ & $\begin{array}{l}\frac{\sigma}{\sigma} \\
\dot{m} \\
\dot{m} \\
\dot{\theta}\end{array}$ & $\begin{array}{l}\stackrel{P}{\text { }} \\
\text { ஸे } \\
\dot{\varphi}\end{array}$ & $\begin{array}{l}\bar{v} \\
\dot{m} \\
\dot{\varphi}\end{array}$ & $\begin{array}{l}\bar{N} \\
\dot{m} \\
\dot{\varphi}=\end{array}$ & $\begin{array}{l}\tilde{N} \\
\dot{\vartheta} \\
\dot{\theta} \\
\dot{0}\end{array}$ & $\begin{array}{l}\bar{\sim} \\
\dot{m} \\
\dot{\varphi} \\
\dot{\omega} \overline{9}\end{array}$ \\
\hline
\end{tabular}




\begin{tabular}{|c|c|c|c|c|c|c|c|}
\hline 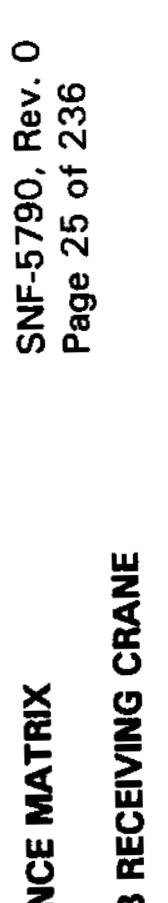 & 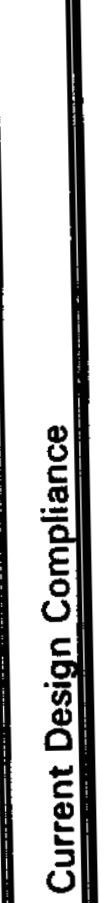 & 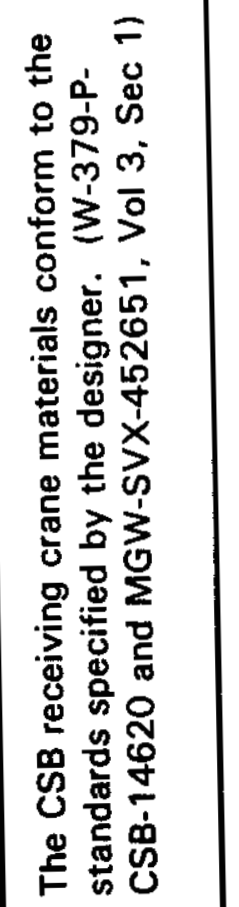 & 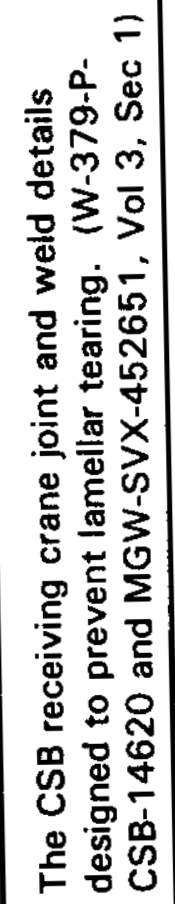 & 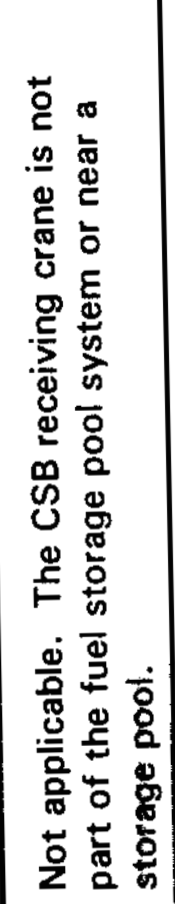 & 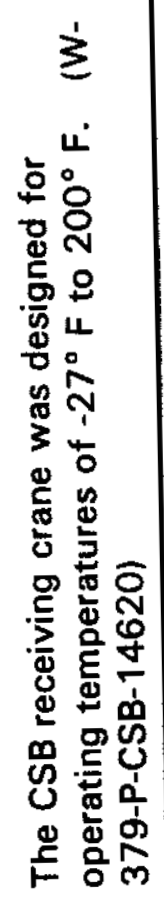 & 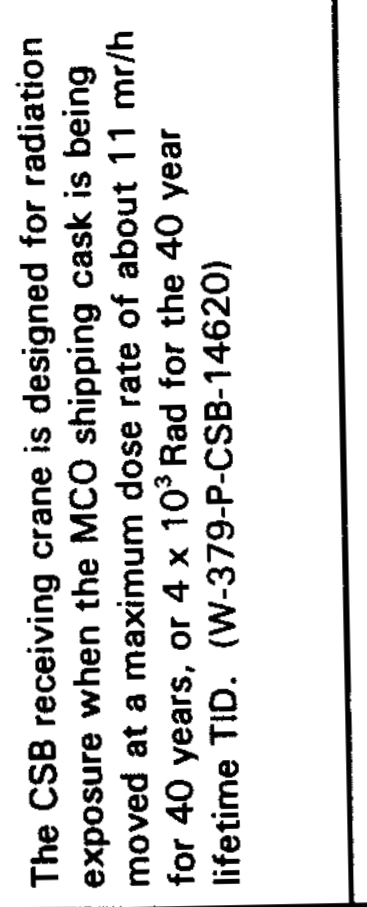 & 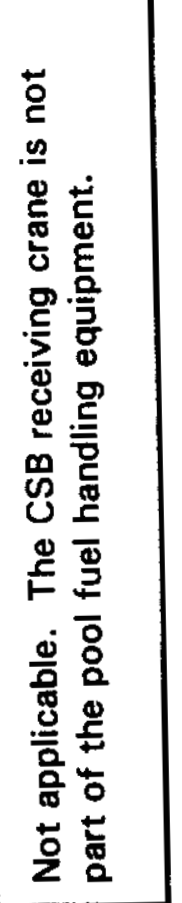 \\
\hline \multirow{2}{*}{ 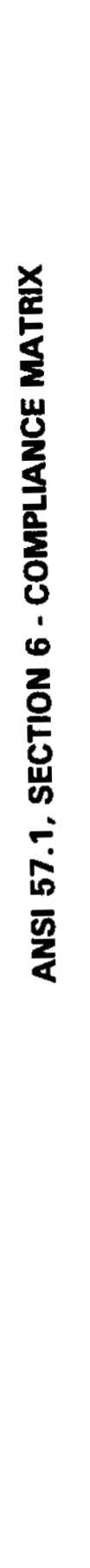 } & 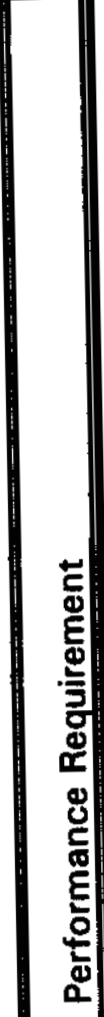 & 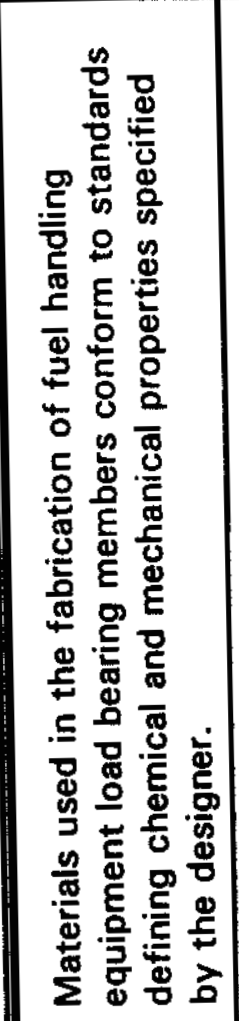 & 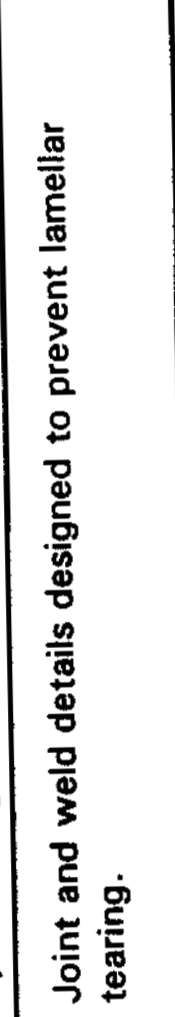 & 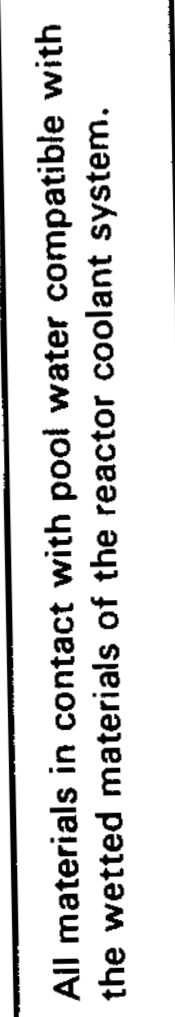 & 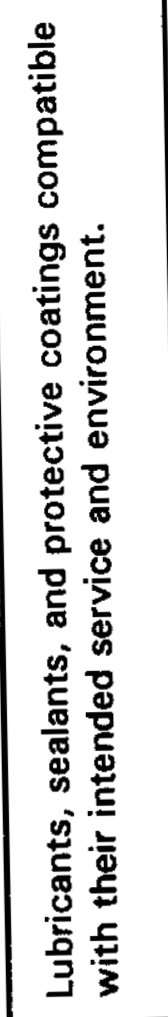 & 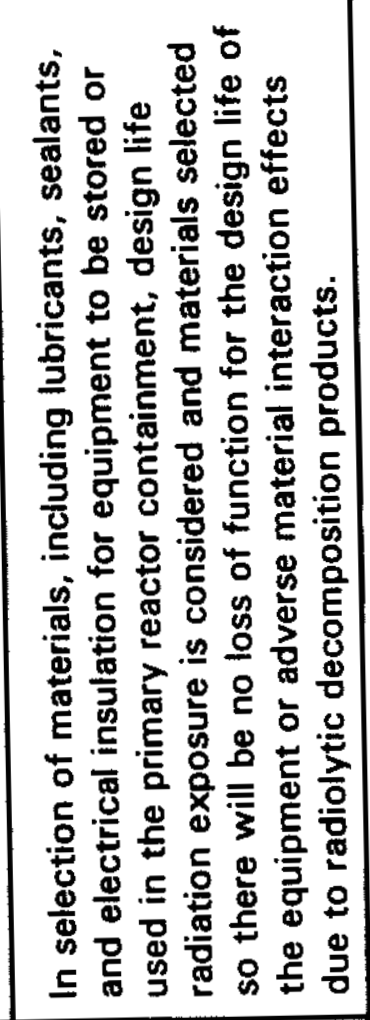 & 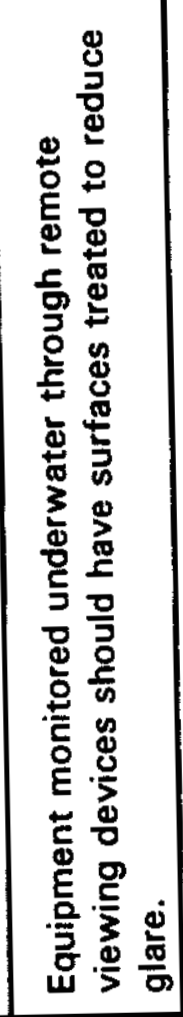 \\
\hline & 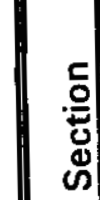 & ָָ & $\begin{array}{l}\text { ले } \\
\stackrel{m}{ }\end{array}$ & $\begin{array}{l}\text { ஸे } \\
\text { ஸे } \\
\dot{0}\end{array}$ & $\begin{array}{l}\text { ले } \\
\text { ले } \\
\dot{0}\end{array}$ & $\begin{array}{l}\bar{N} \\
\text { m. } \\
\dot{\varphi}\end{array}$ & 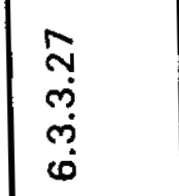 \\
\hline
\end{tabular}




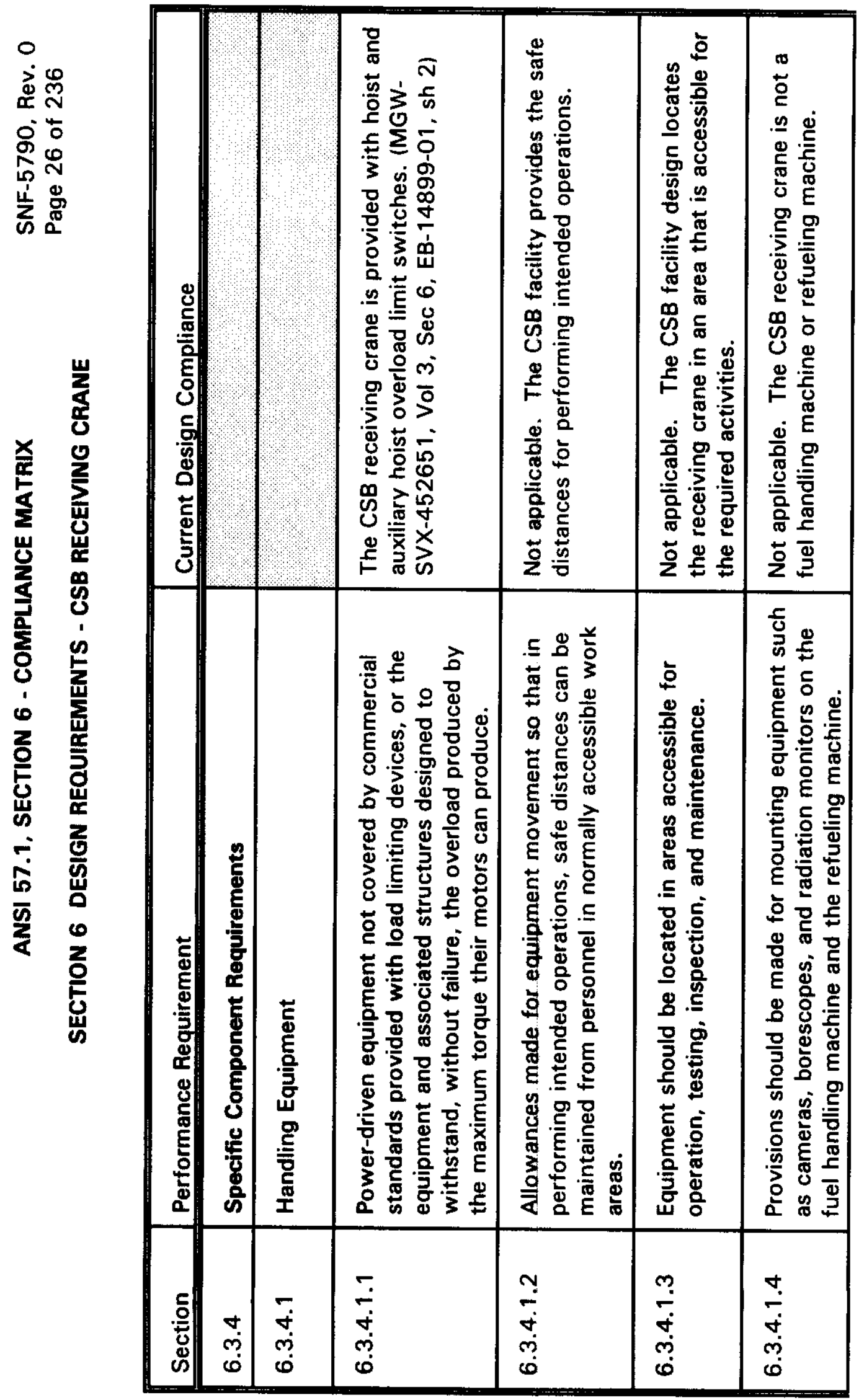




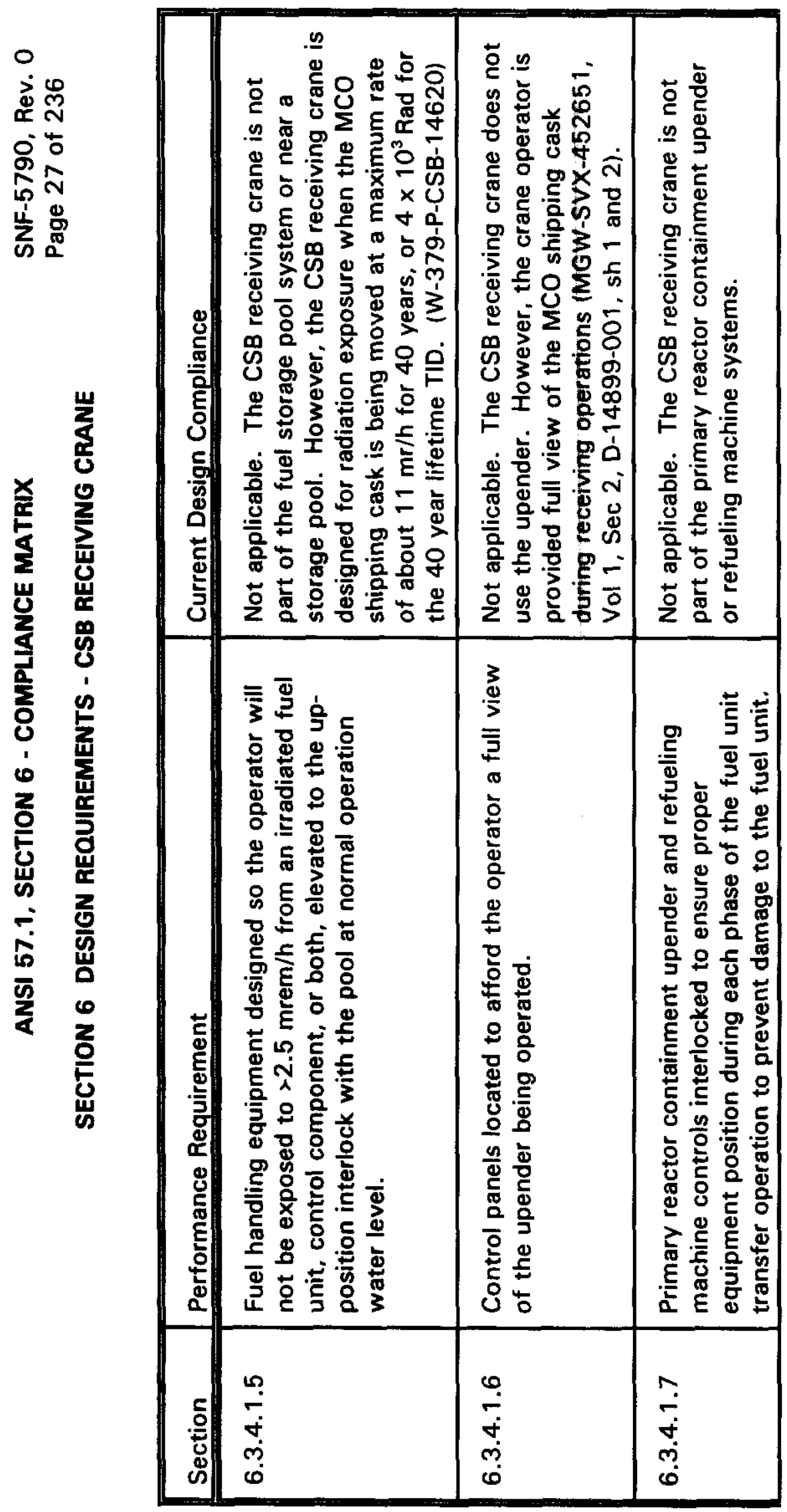




\begin{tabular}{|c|c|c|c|c|c|}
\hline 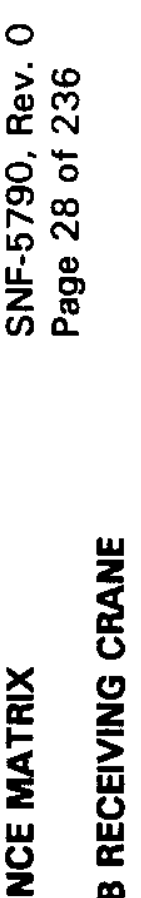 & 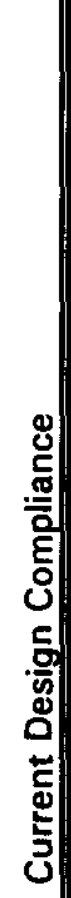 & 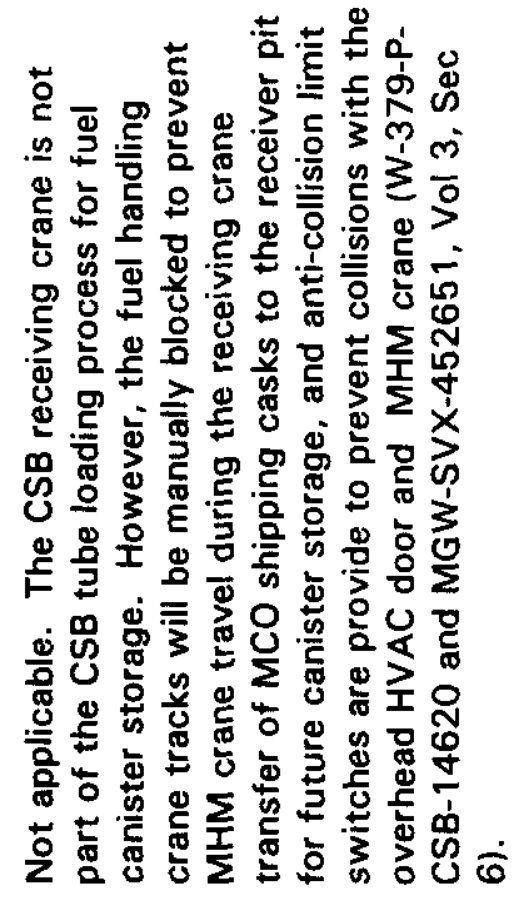 & 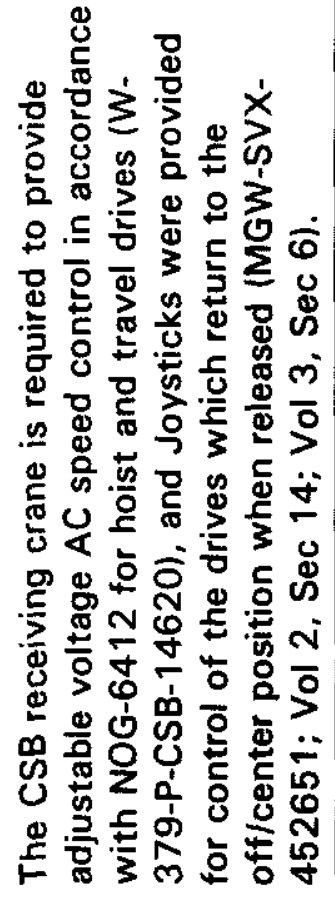 & 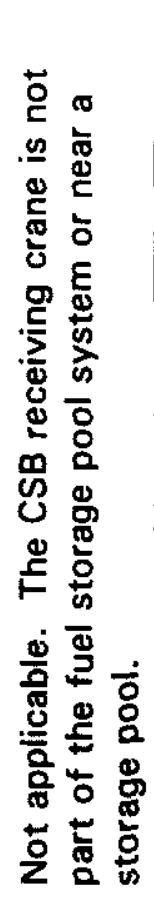 & 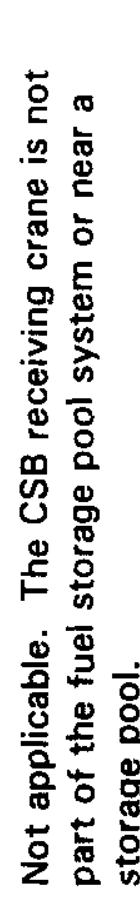 \\
\hline 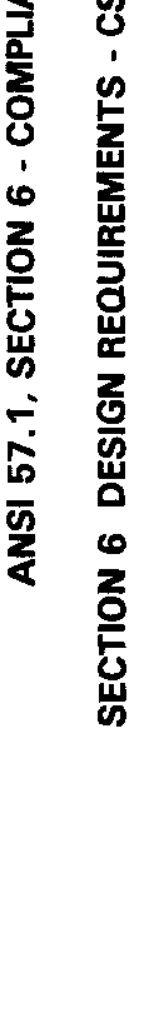 & 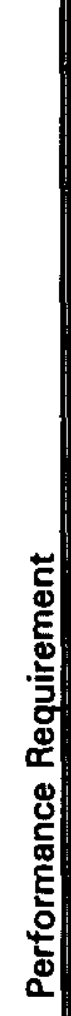 & 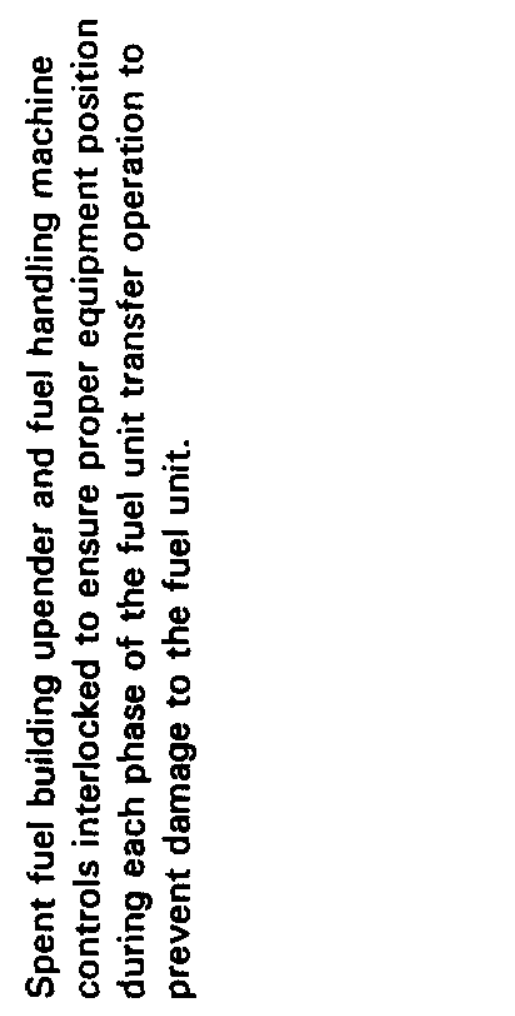 & 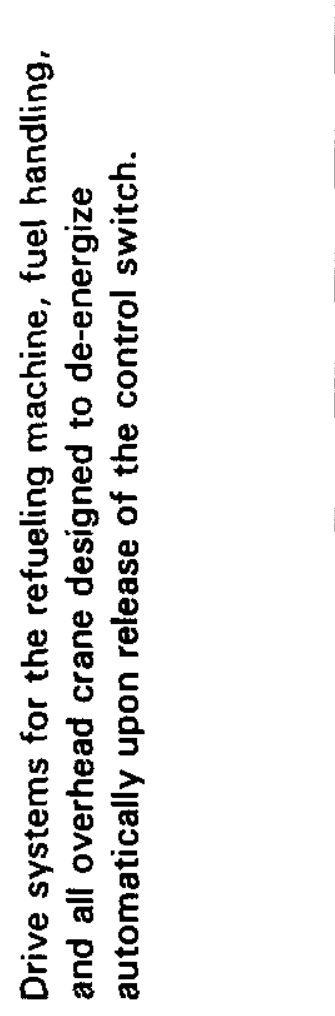 & 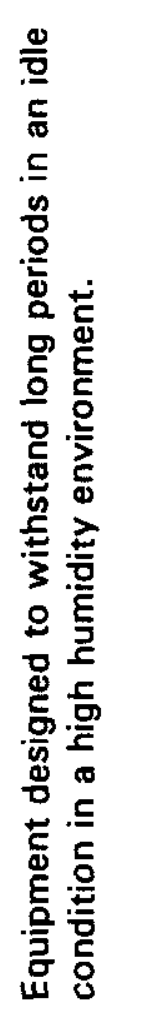 & 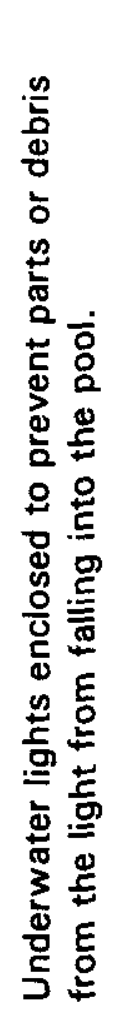 \\
\hline & 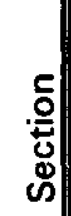 & $\begin{array}{l}\infty \\
\dot{+} \\
\dot{\sim} \\
\dot{\theta}\end{array}$ & 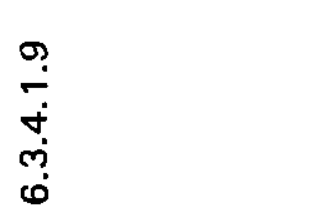 & $\begin{array}{l}\stackrel{0}{\check{r}} \\
\dot{\sigma} \\
\dot{\sim} \\
\dot{0}\end{array}$ & $\begin{array}{l}\check{F} \\
\check{J} \\
\dot{\sigma} \\
\dot{0}\end{array}$ \\
\hline
\end{tabular}




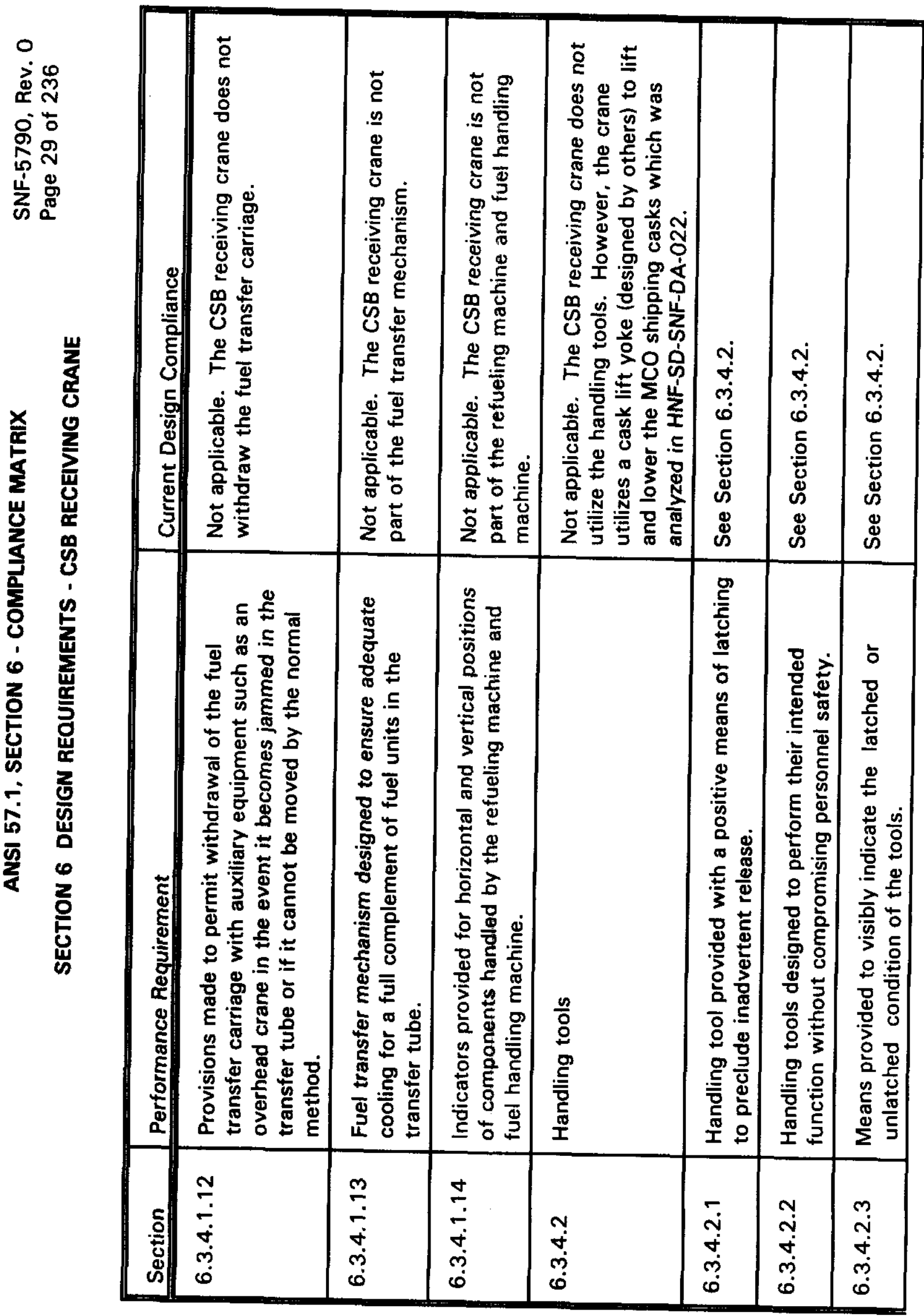




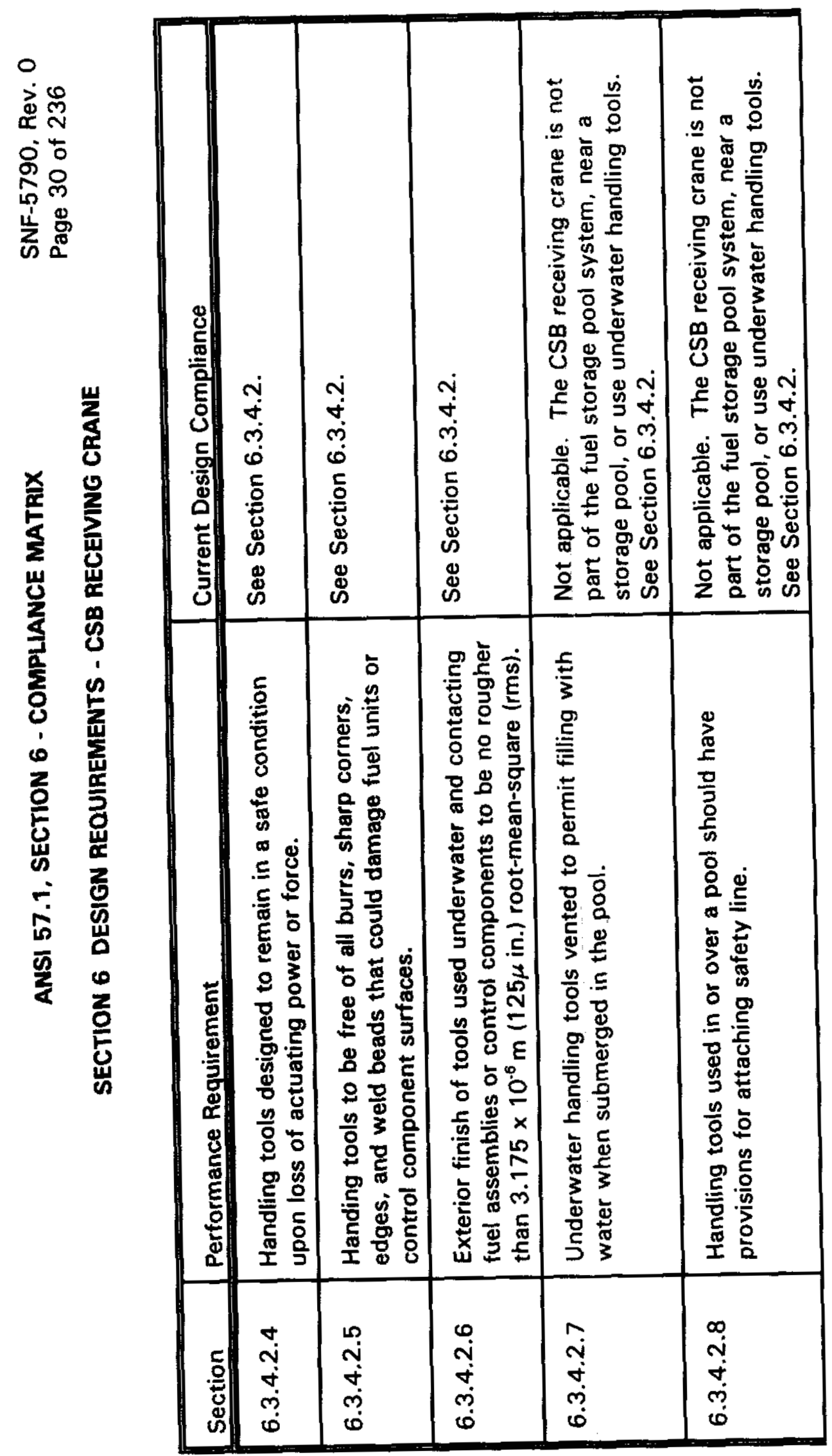




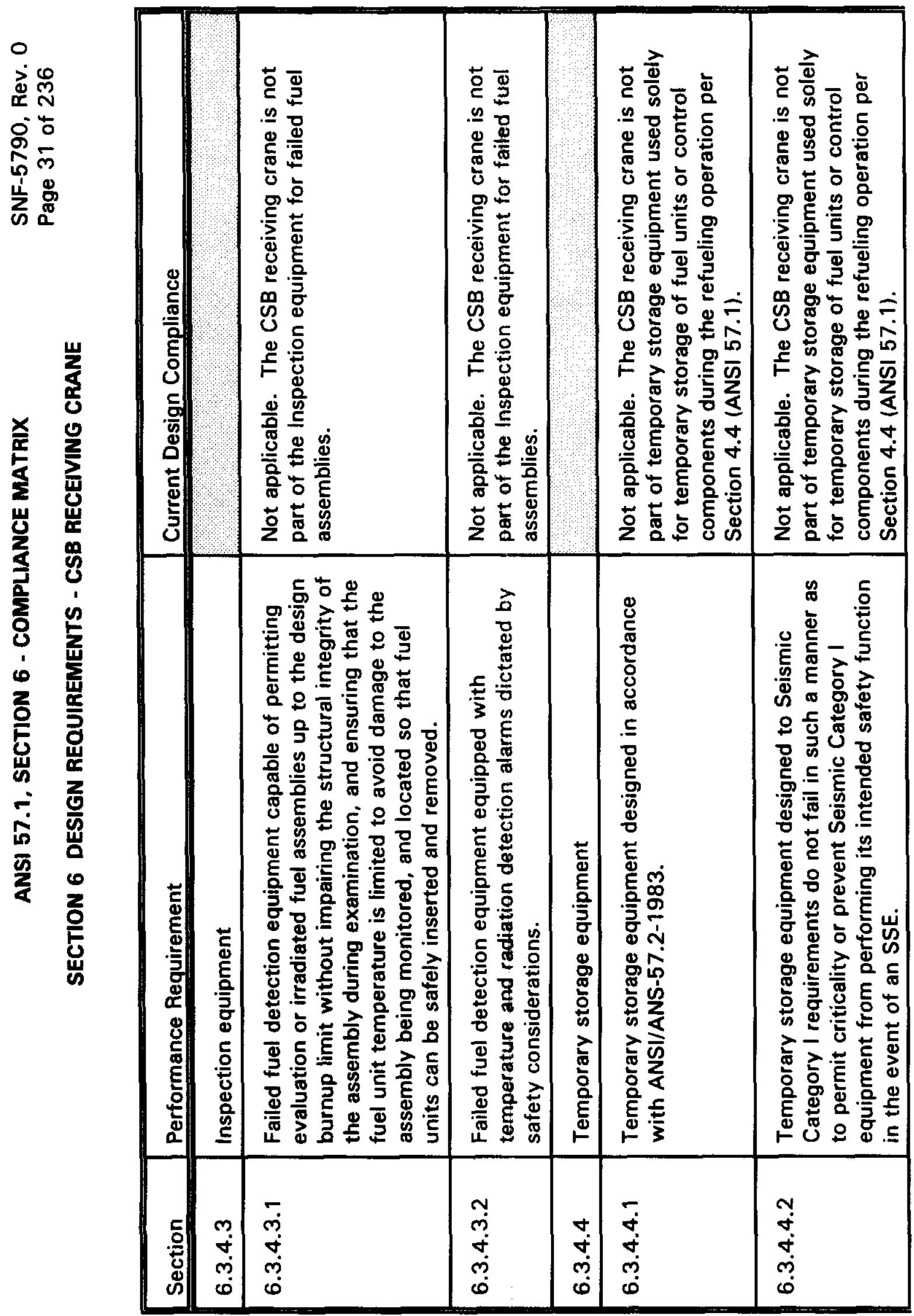




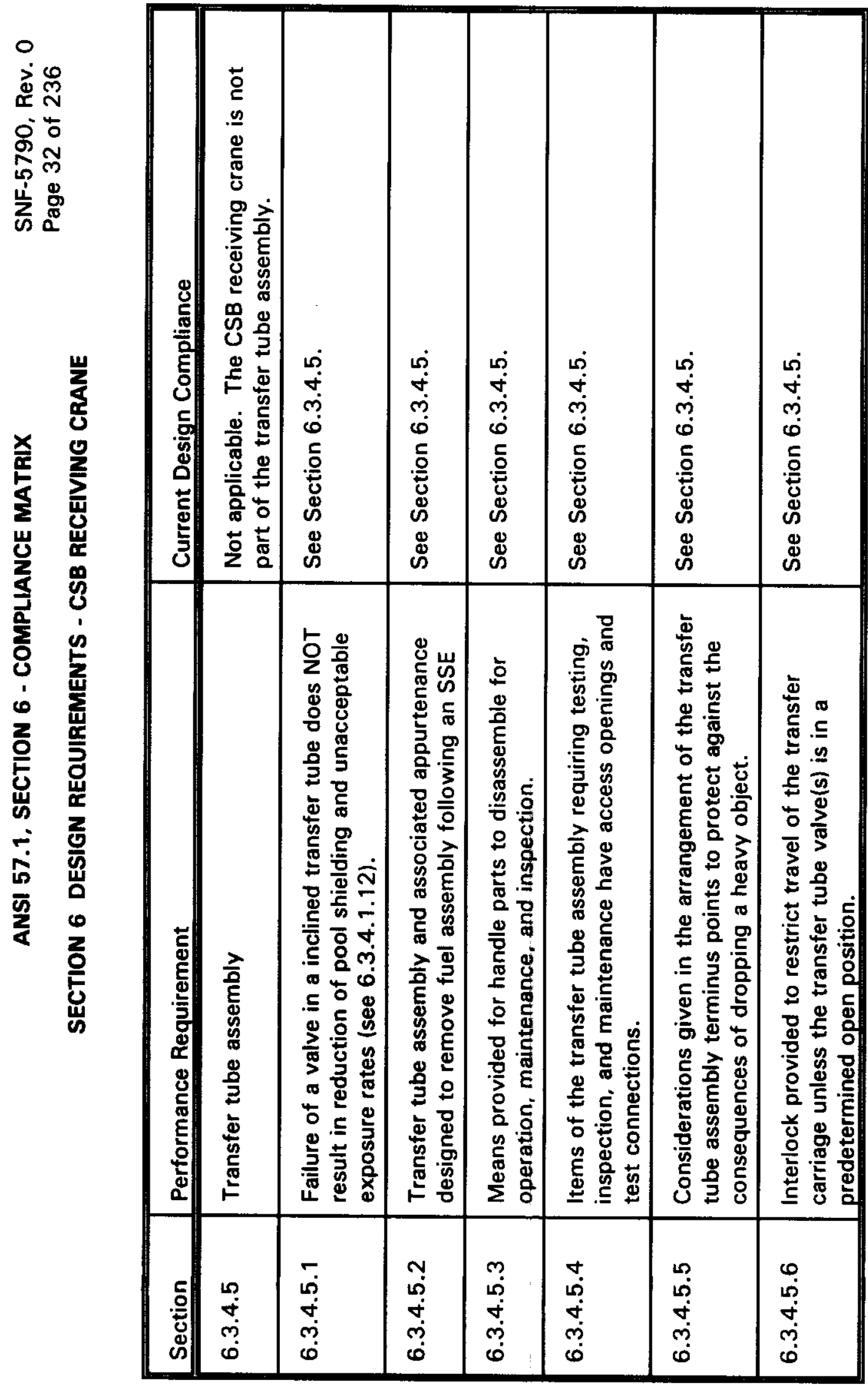




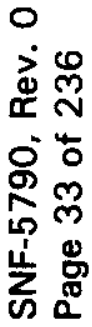

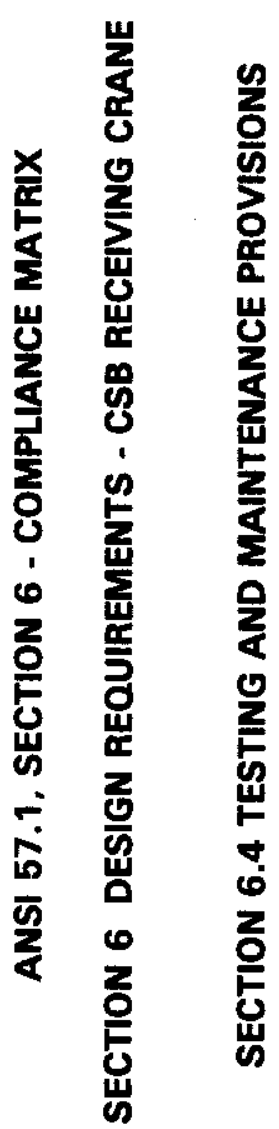

\begin{tabular}{|c|c|c|c|c|c|}
\hline 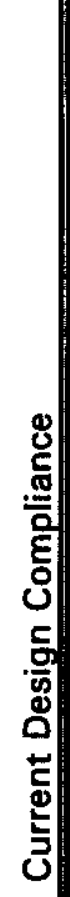 & & 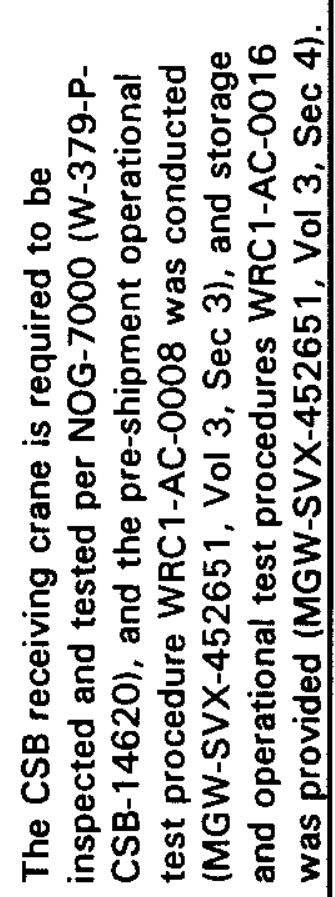 & 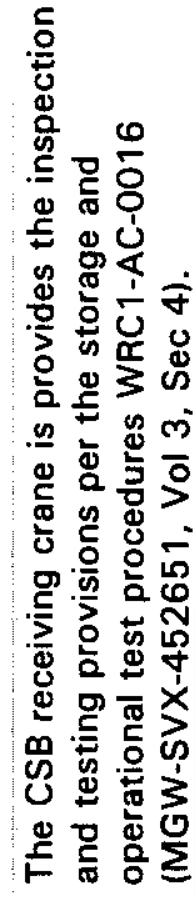 & 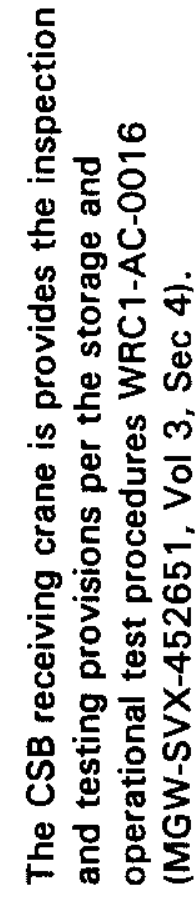 & 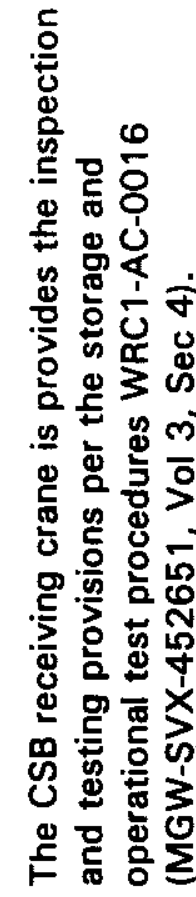 \\
\hline 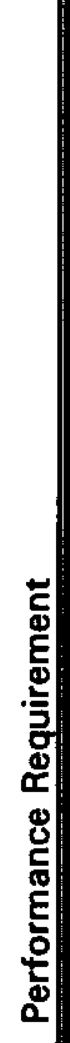 & 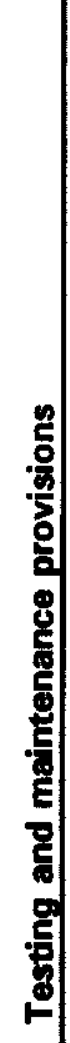 & 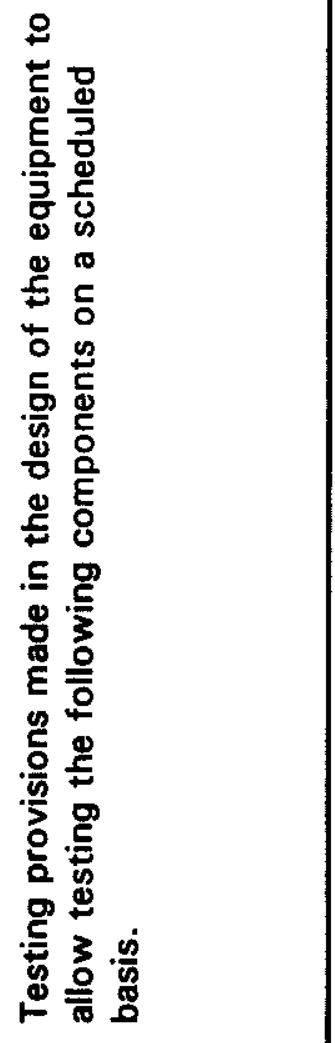 & 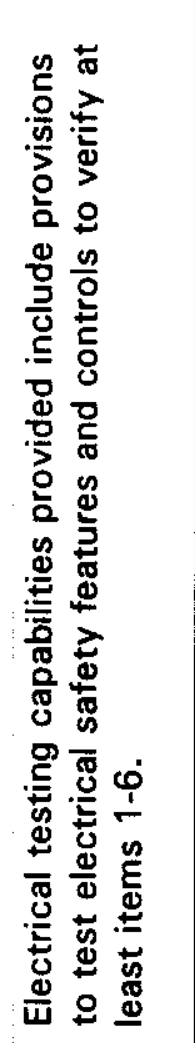 & 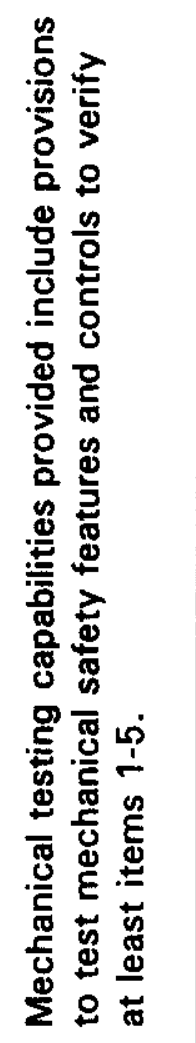 & 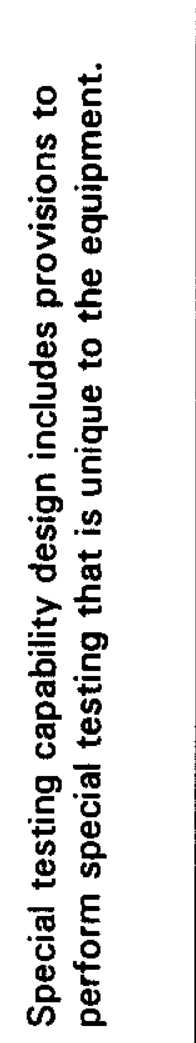 \\
\hline 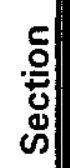 & $\begin{array}{l}\mathbf{y} \\
0\end{array}$ & $\underset{0}{\dot{0}}$ & $\begin{array}{r}\check{\sigma} \\
\check{\sigma} \\
\end{array}$ & $\begin{array}{l}\text { Vִ } \\
\dot{\sigma}\end{array}$ & $\begin{array}{l}n \\
\text { r } \\
0\end{array}$ \\
\hline
\end{tabular}




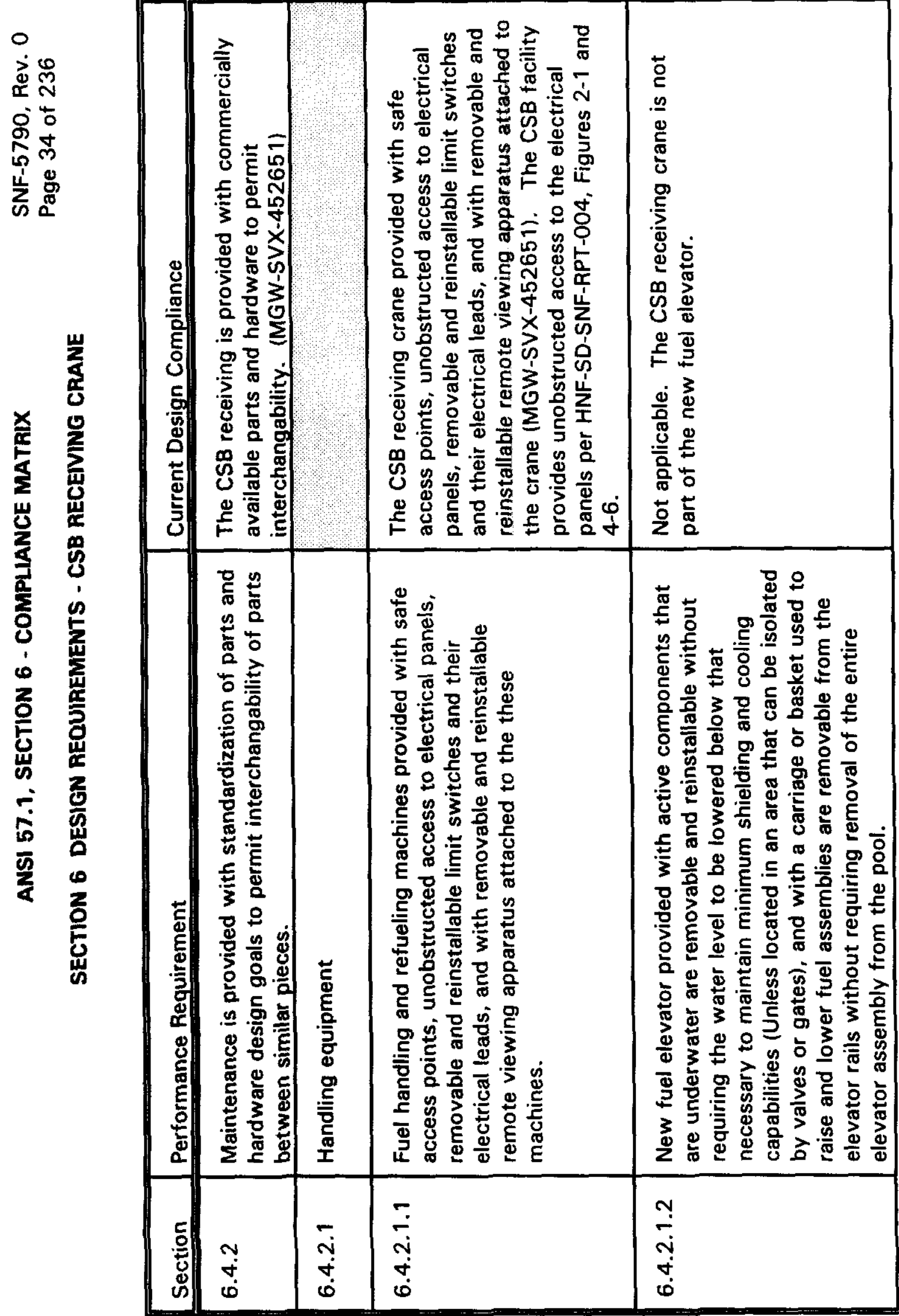




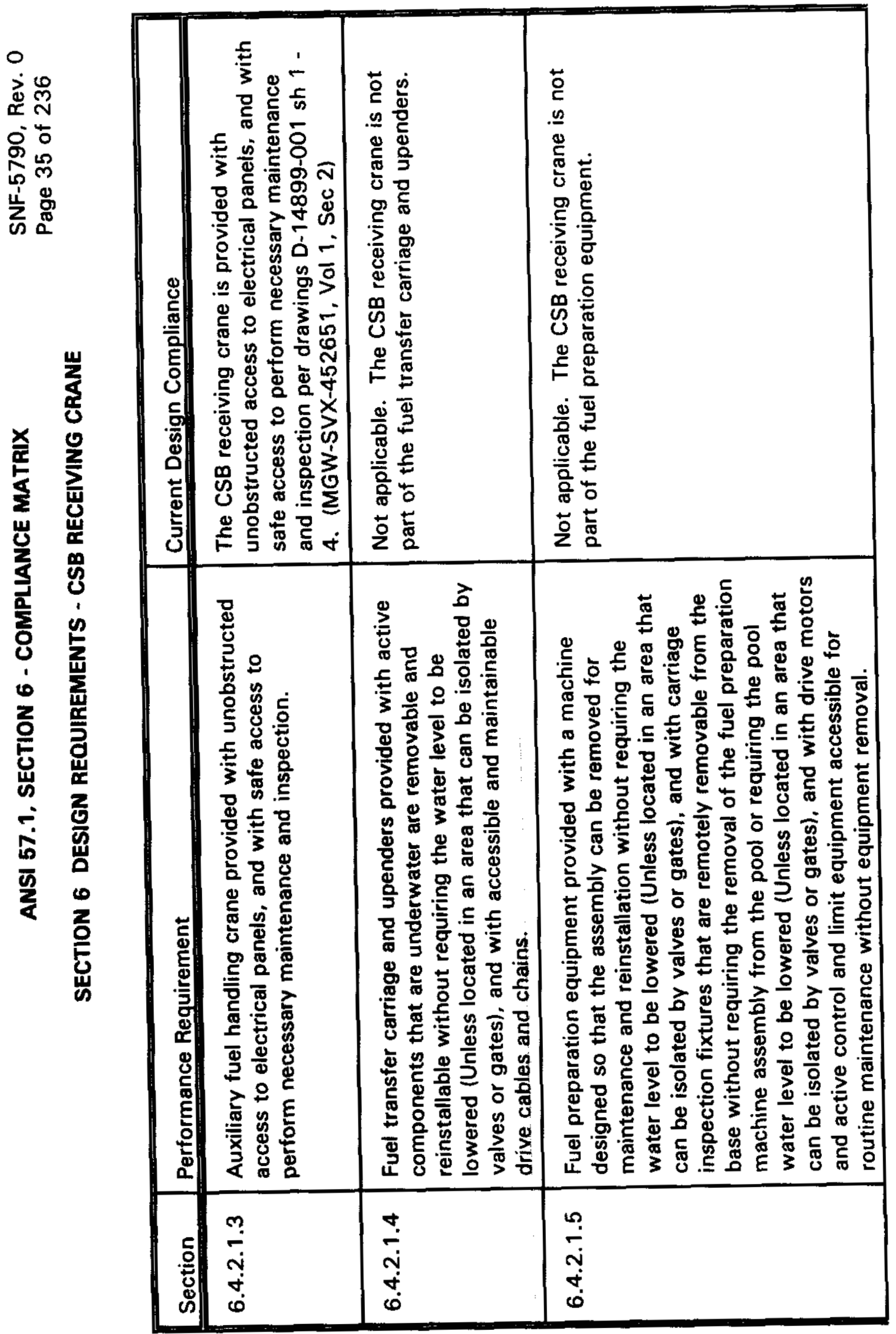




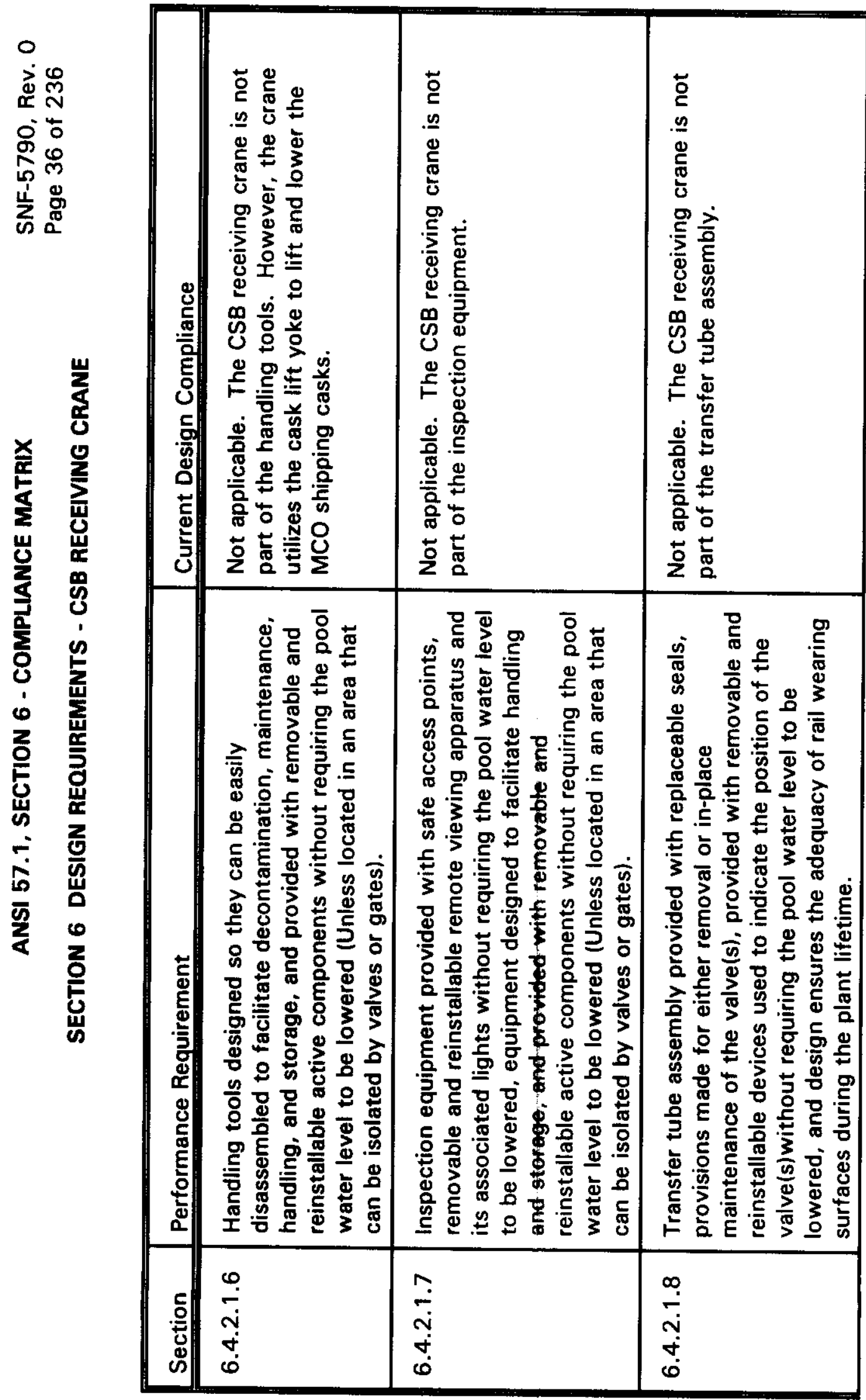




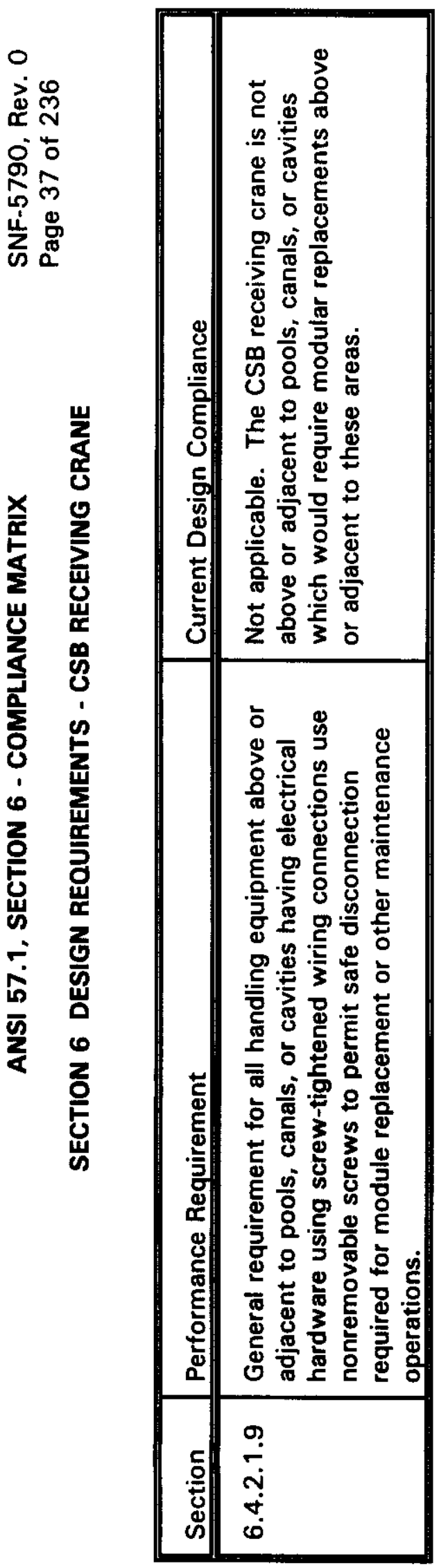


SNF-5790 Rev. 0

Page 38 of 236

\title{
ATTACHMENT 3
}

\author{
ANSI $\mathbf{5 7 . 2}$
}

COMPLIANCE MATRIX 


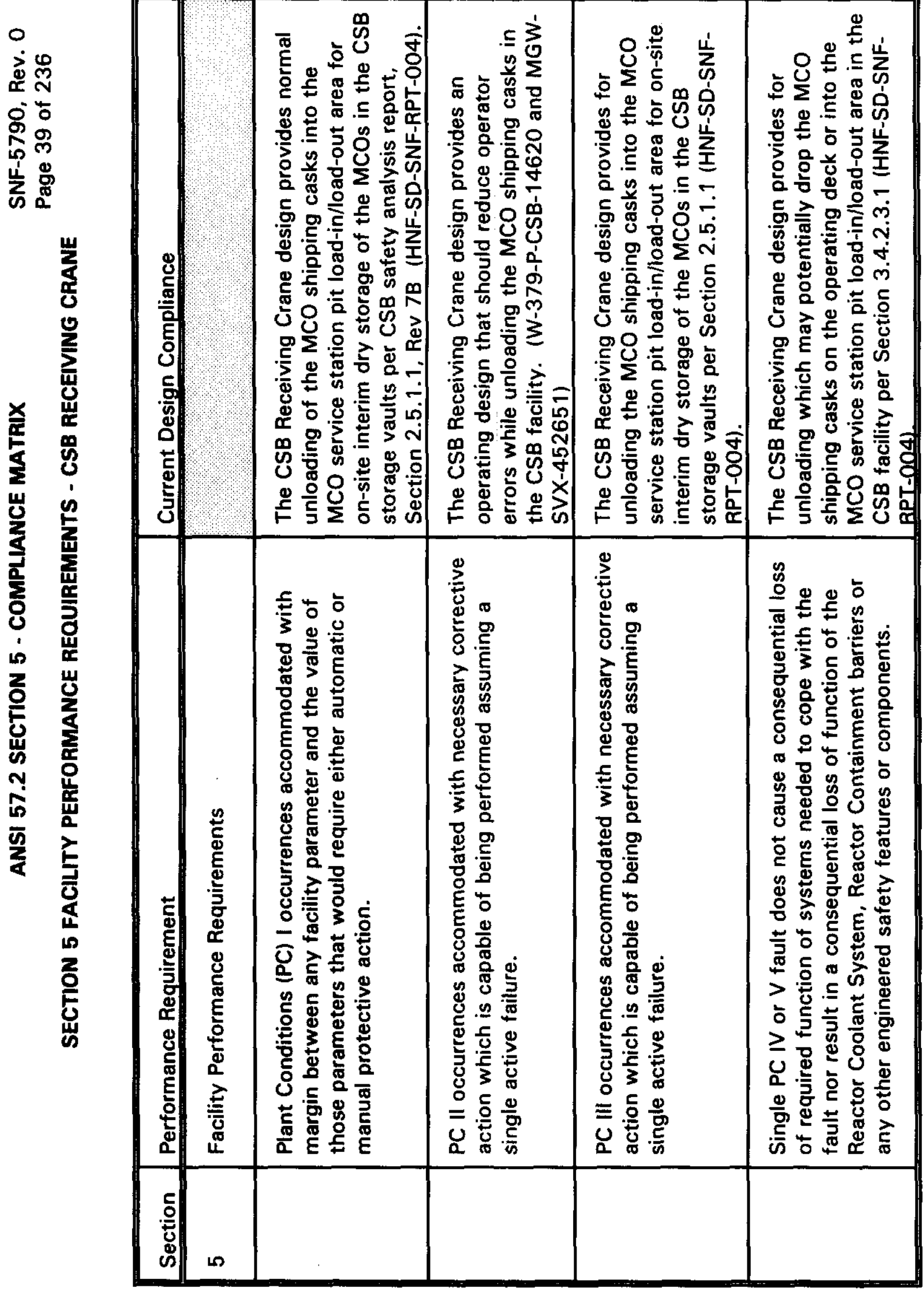


0

$>\varphi$

远

능

웅

㟔

แ

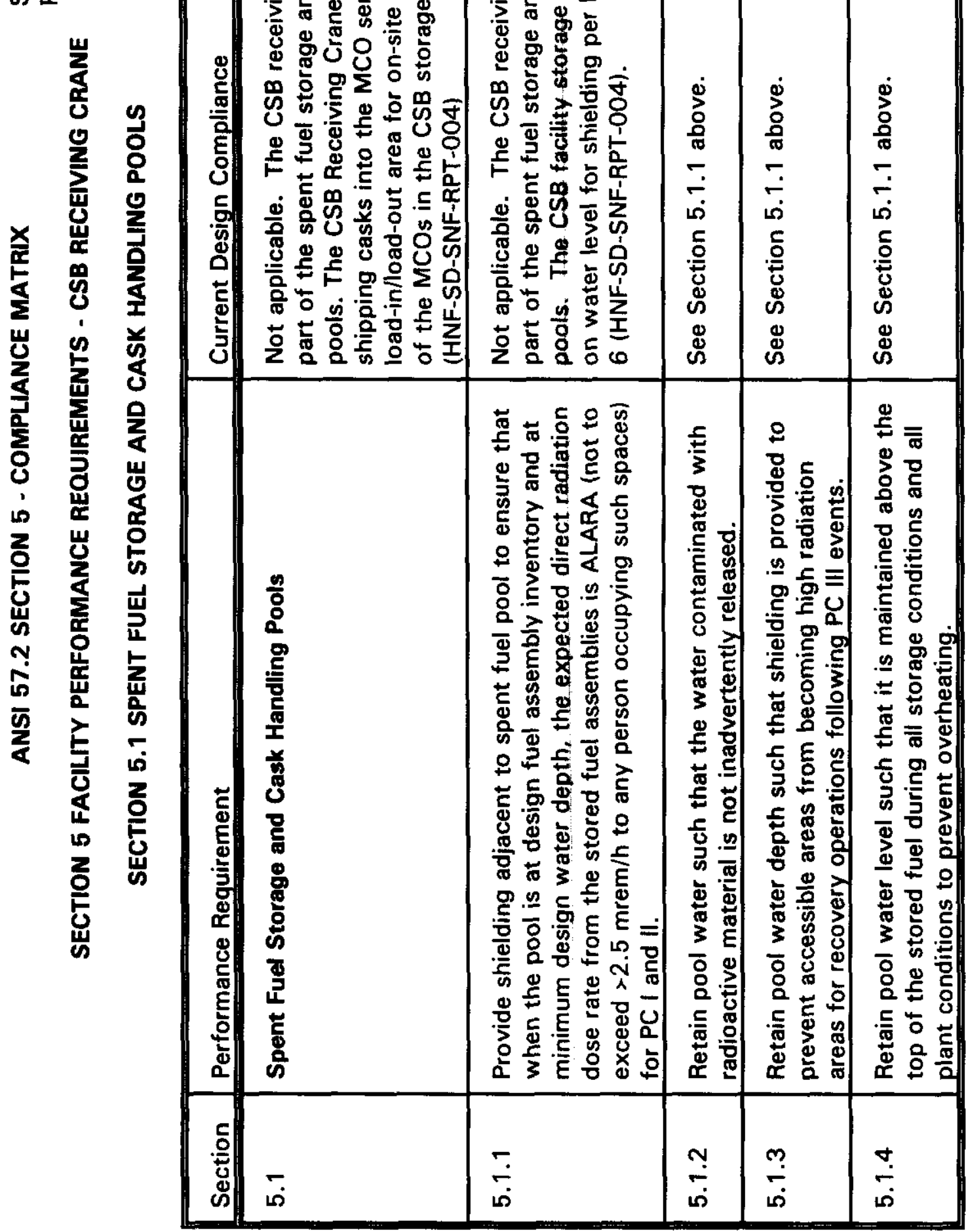




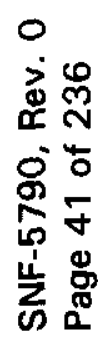

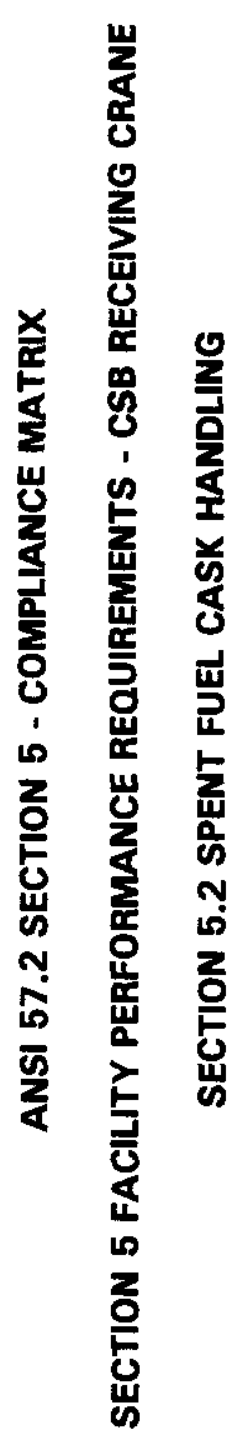

\begin{tabular}{|c|c|c|c|c|c|c|}
\hline 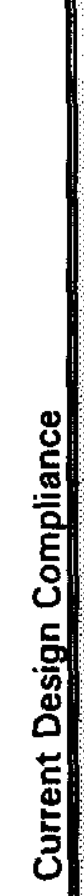 & & 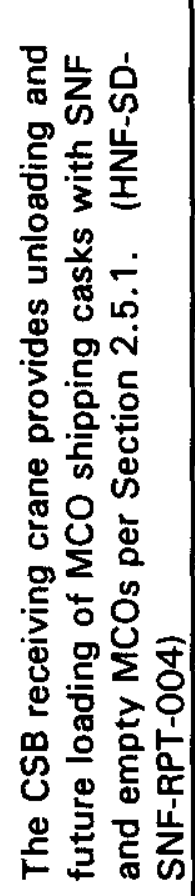 & 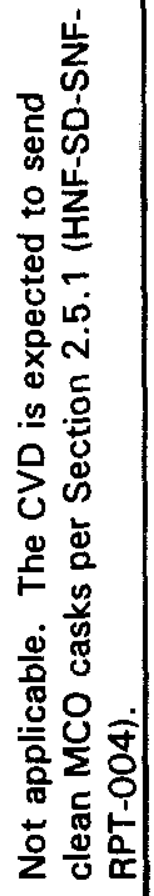 & 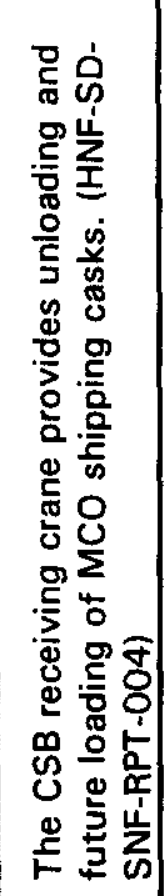 & 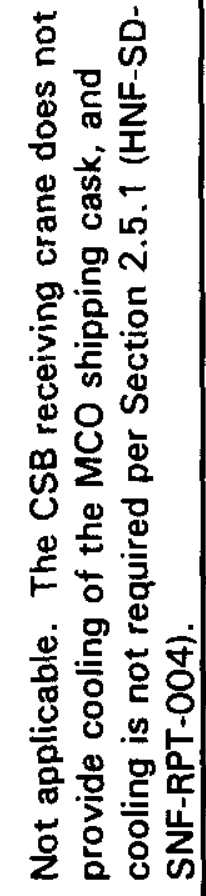 & 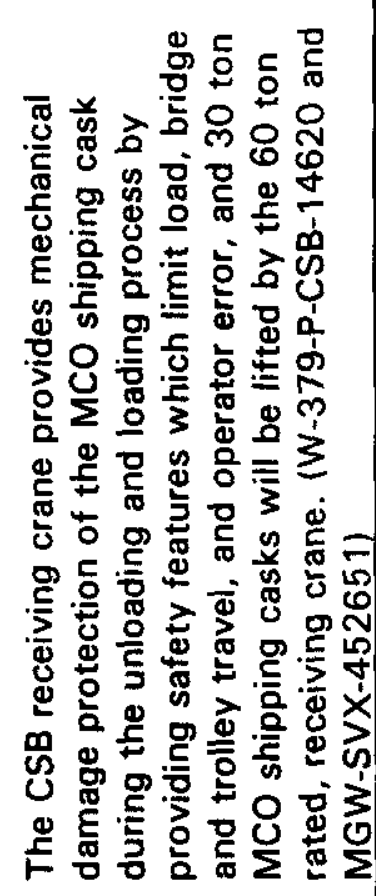 \\
\hline 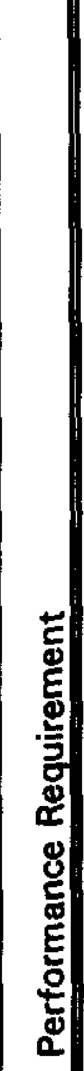 & 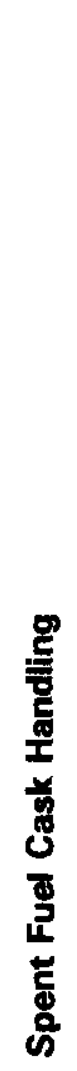 & 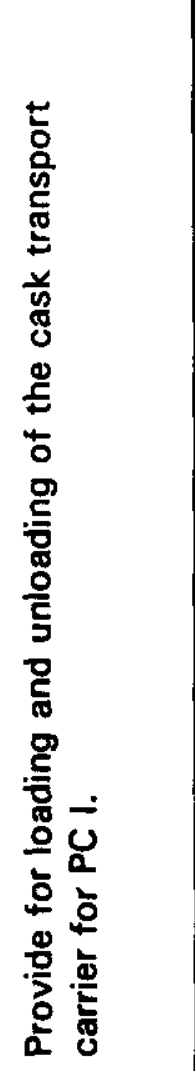 & 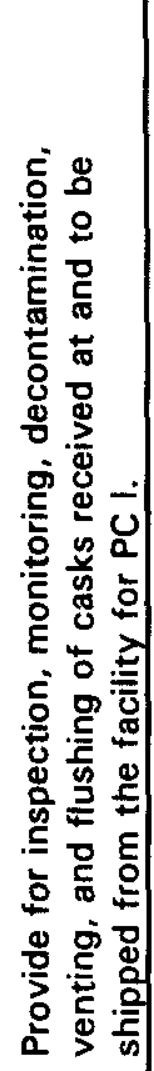 & 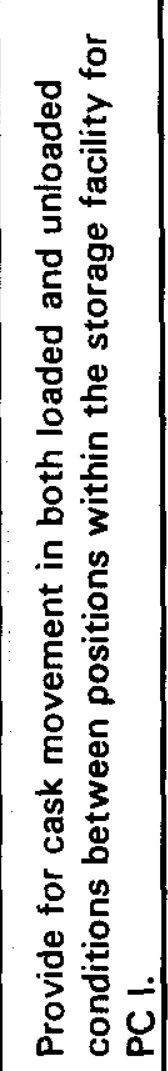 & 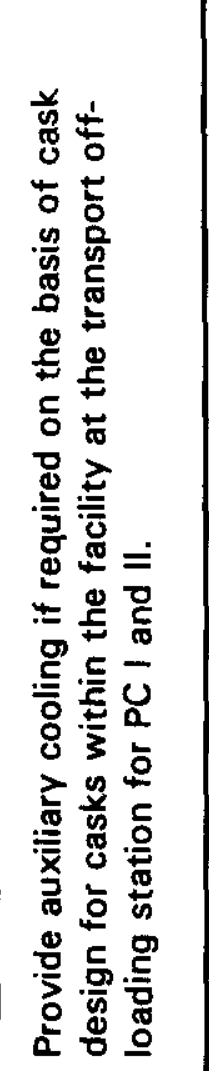 & 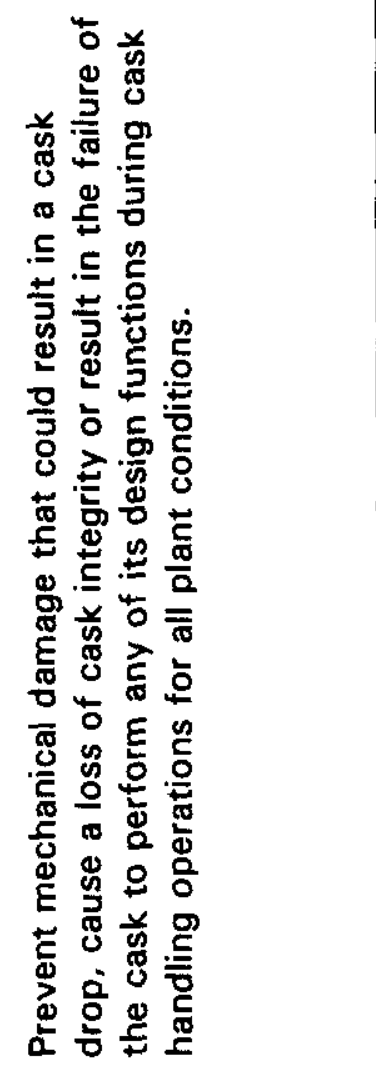 \\
\hline 寄 & مَ & ஸั & ָั & ชֶ. & ஸִ & ֶָ. \\
\hline
\end{tabular}




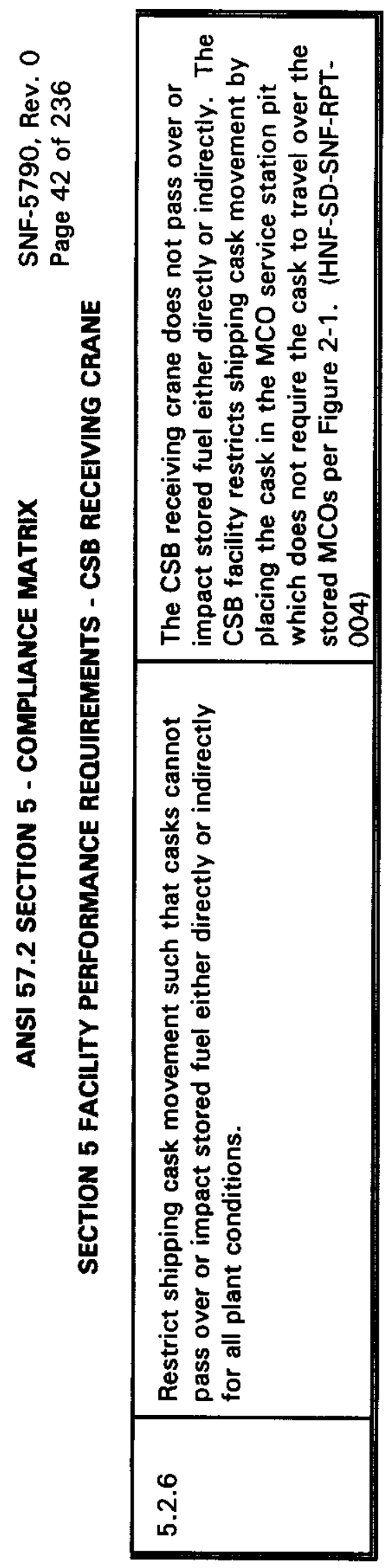




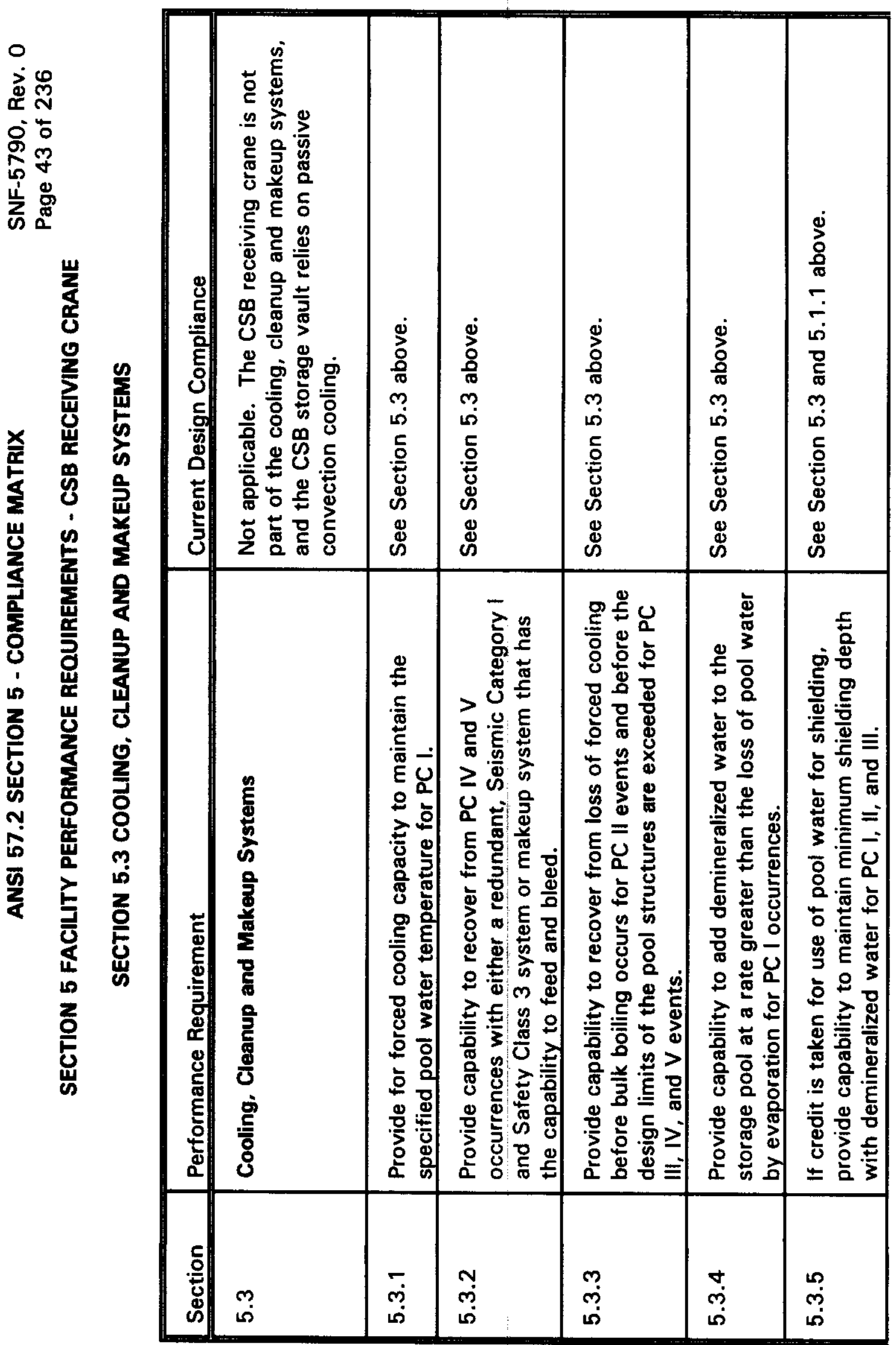




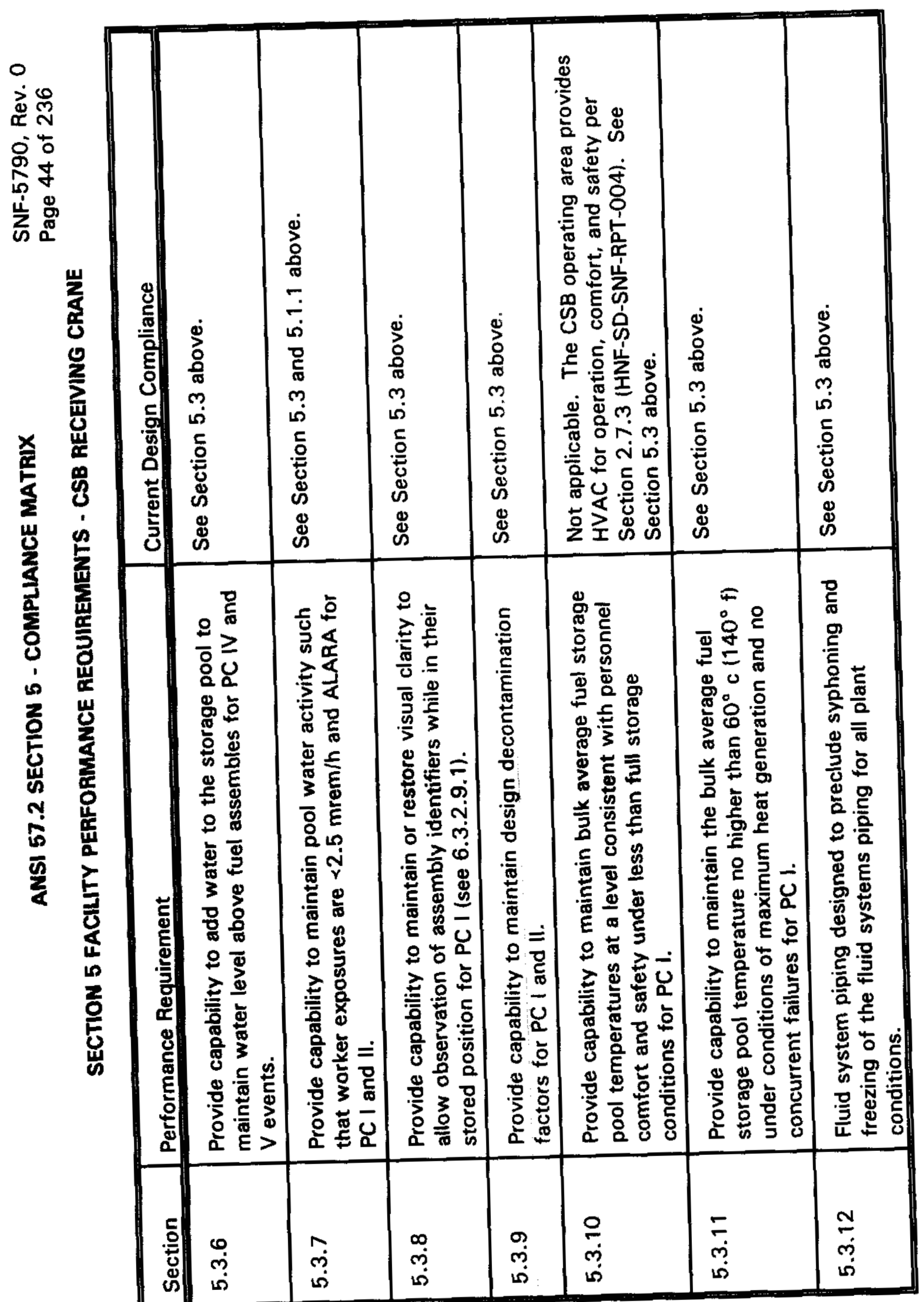




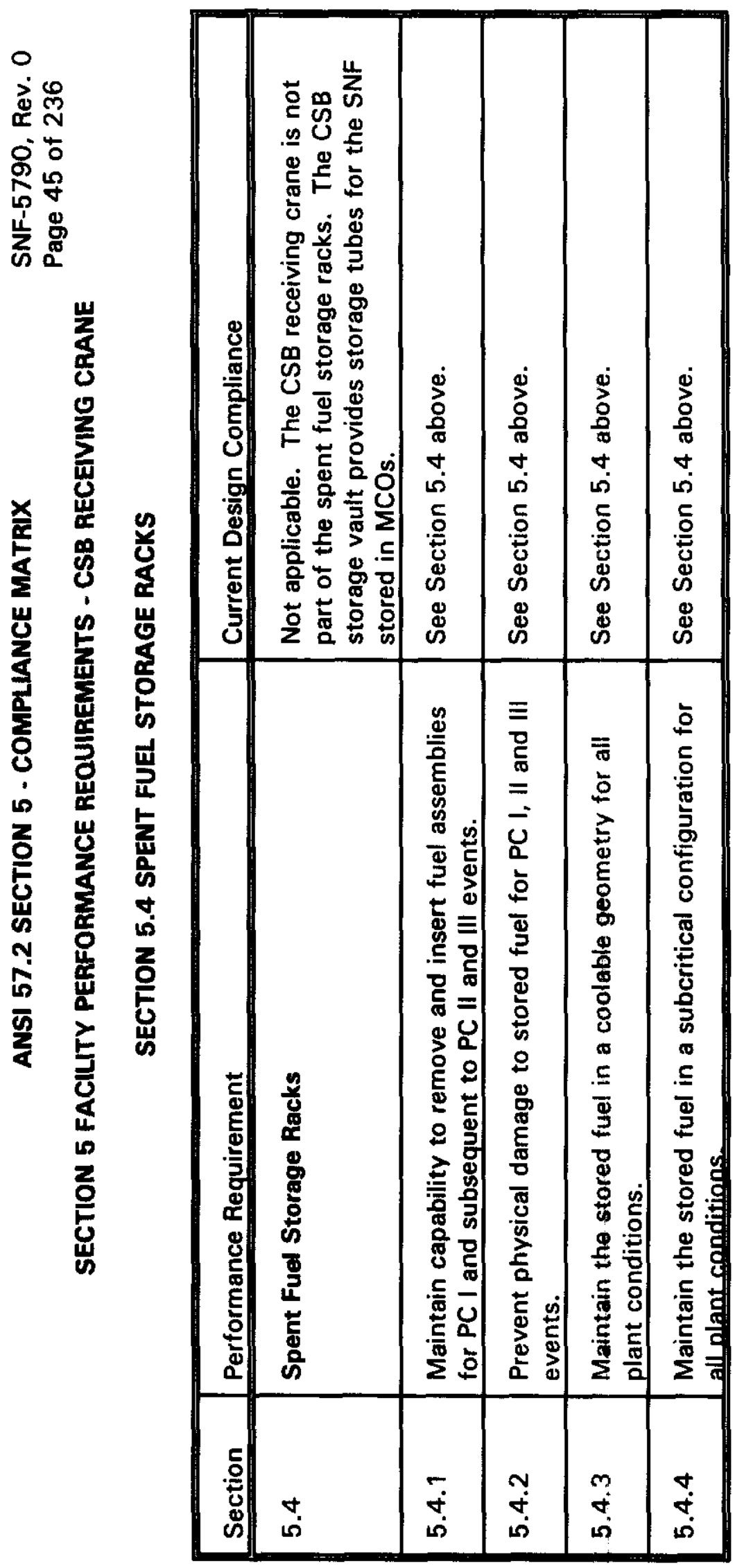




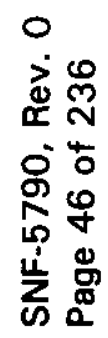

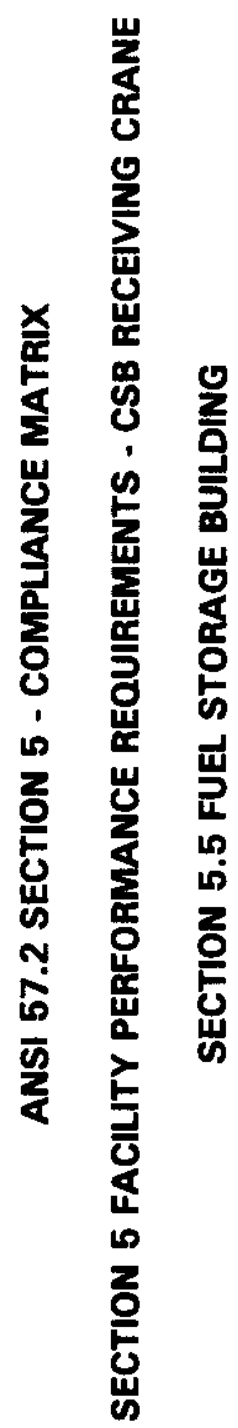

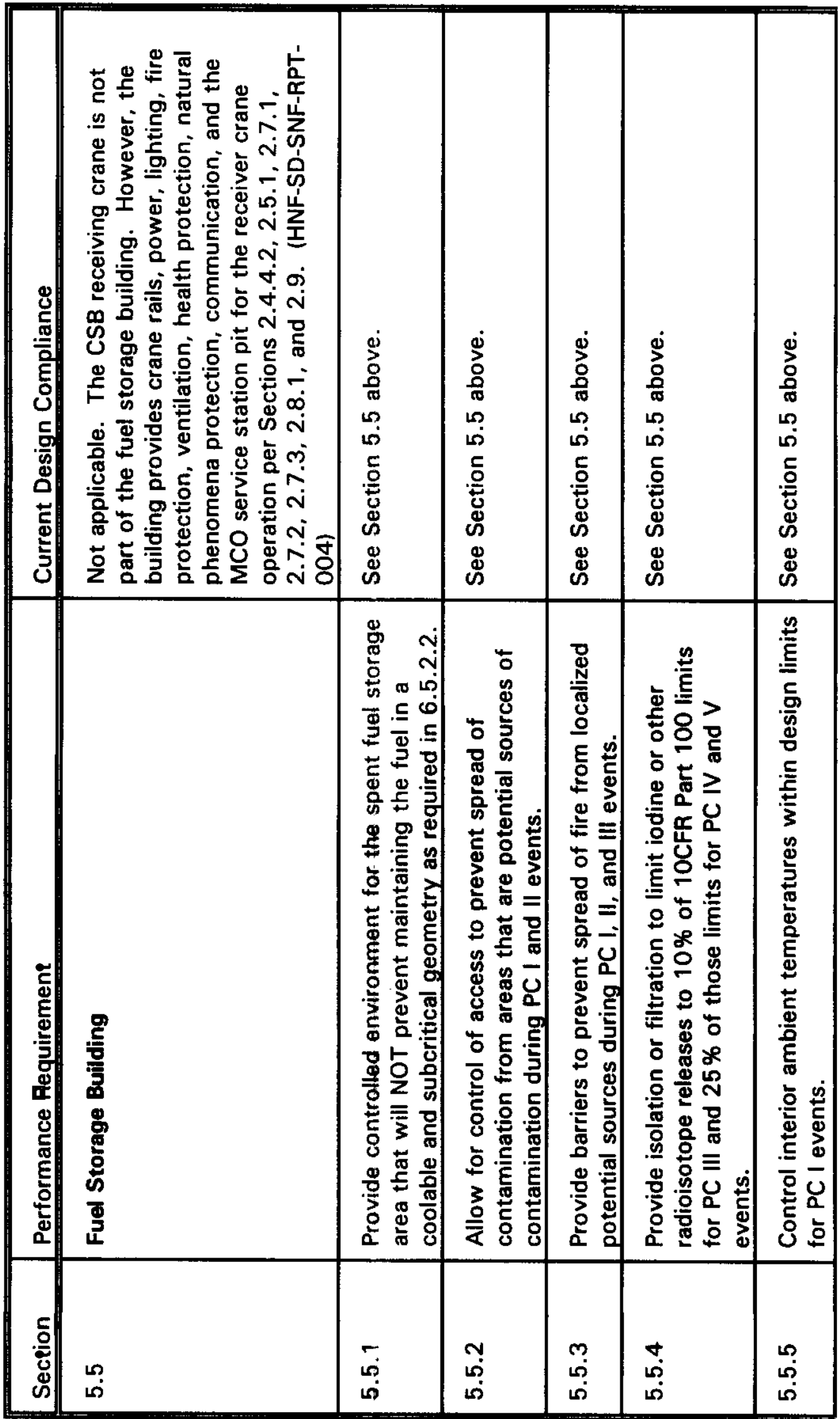




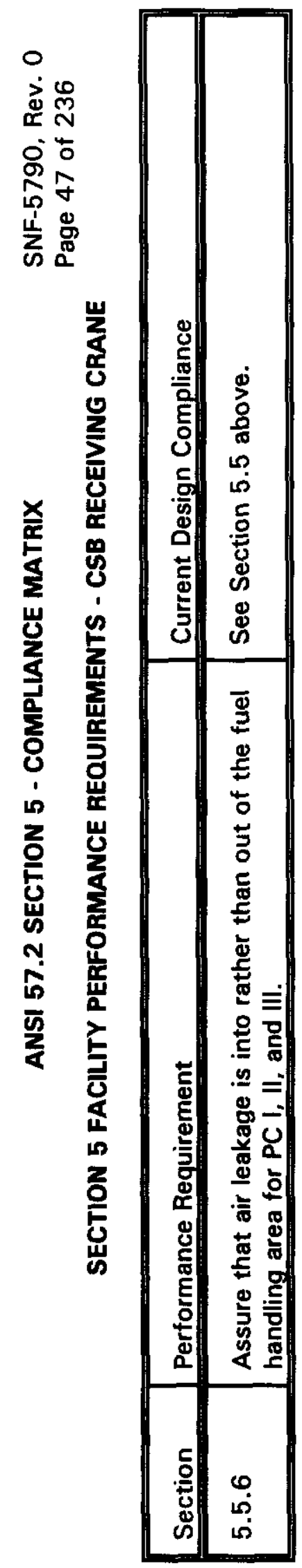




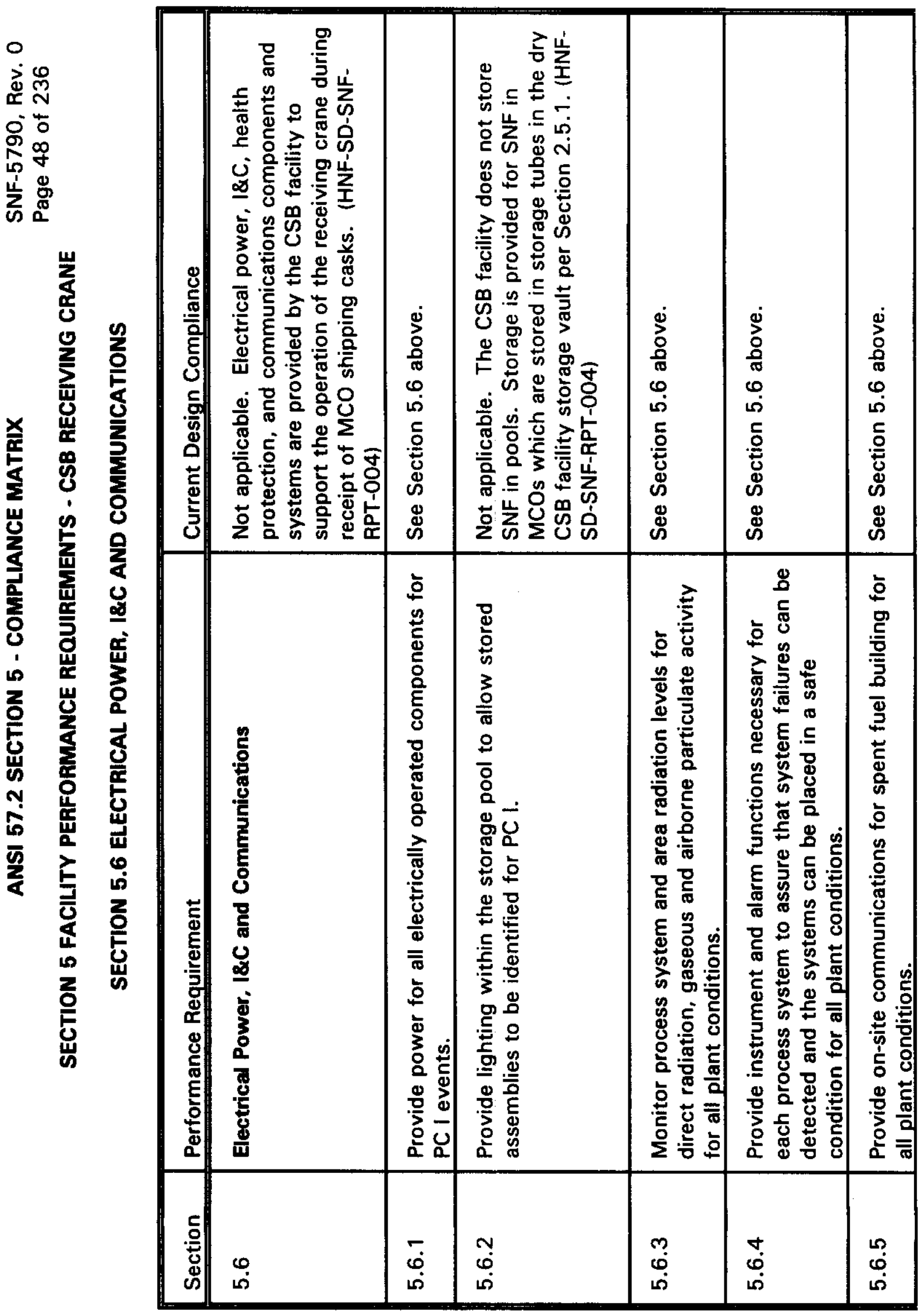




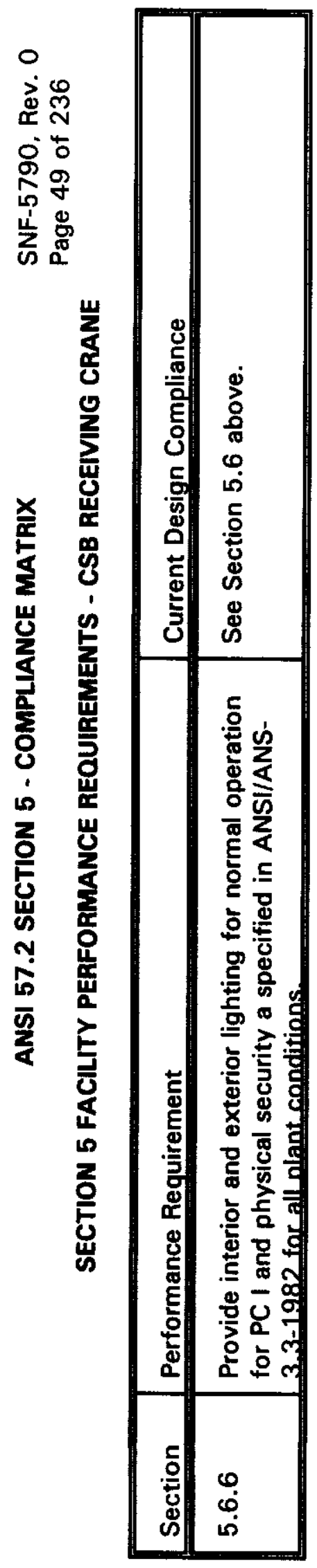


0

灾

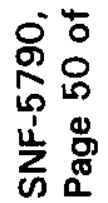

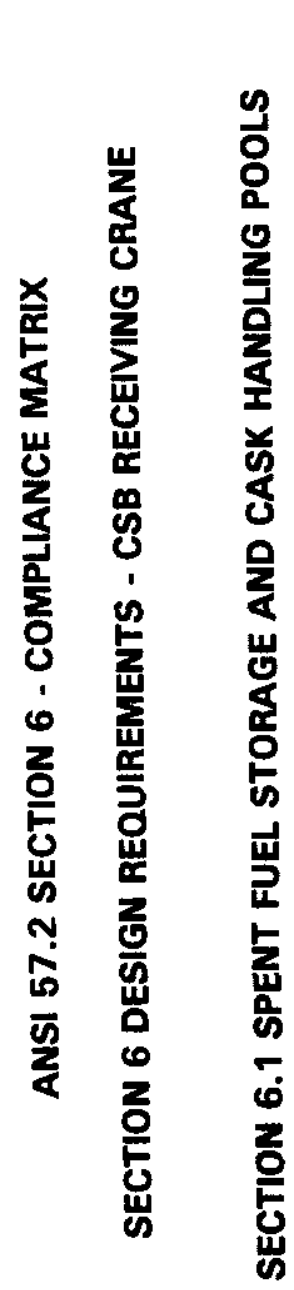

$\mid+\frac{\frac{5}{0}}{\frac{2}{2}}$ 흘흔 .․ํ요 ษ

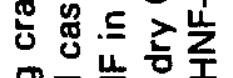

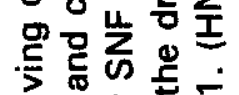
ס Ð ฮ 思点边造 흥

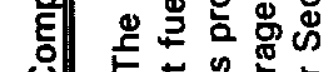
C)

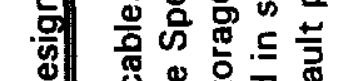

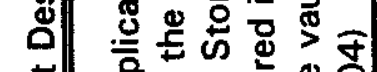

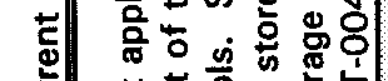

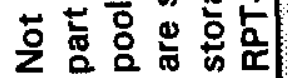

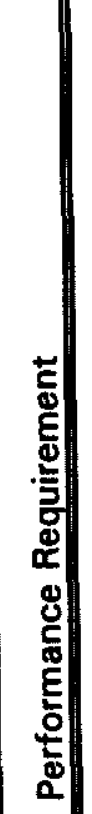

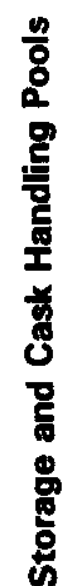

ㄹ. क

นิํㅗ

노

窎

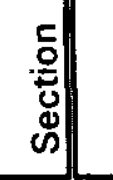

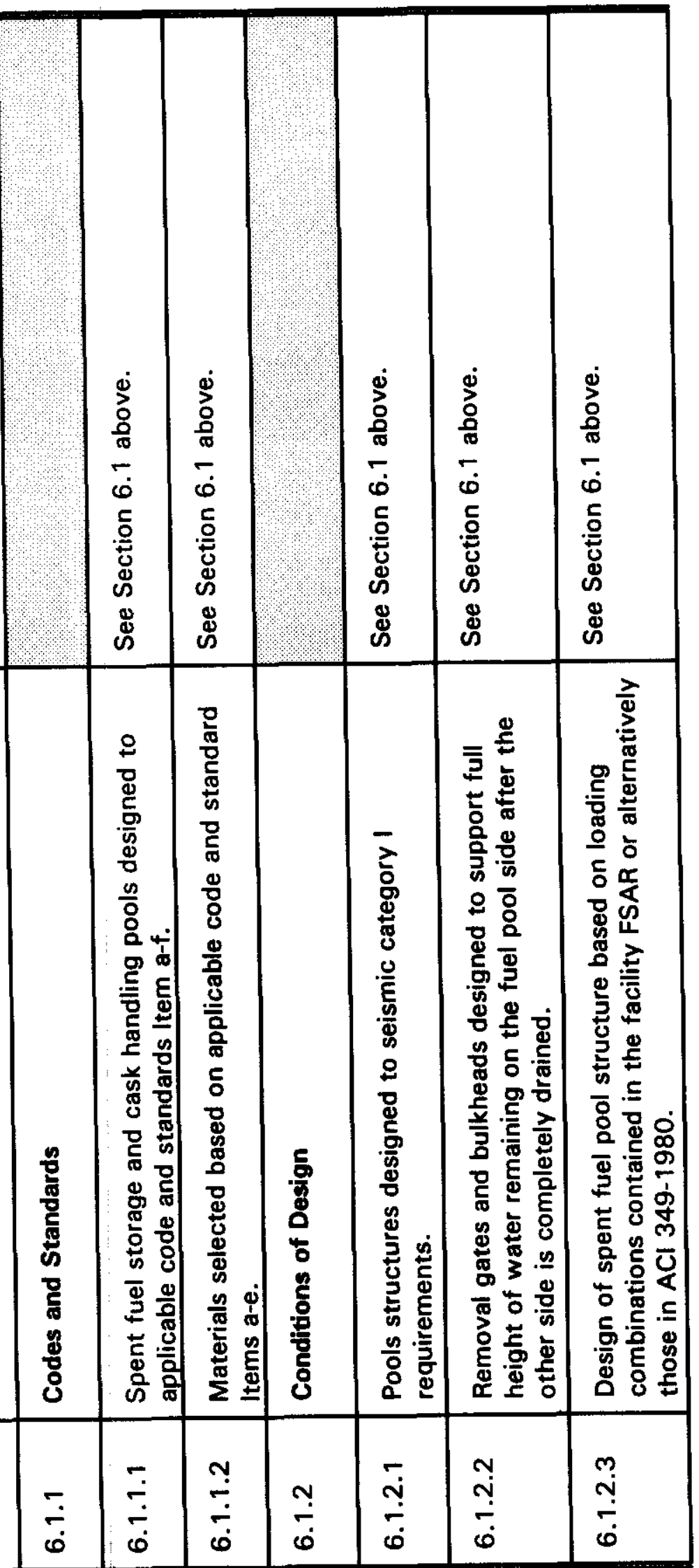




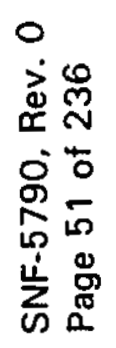

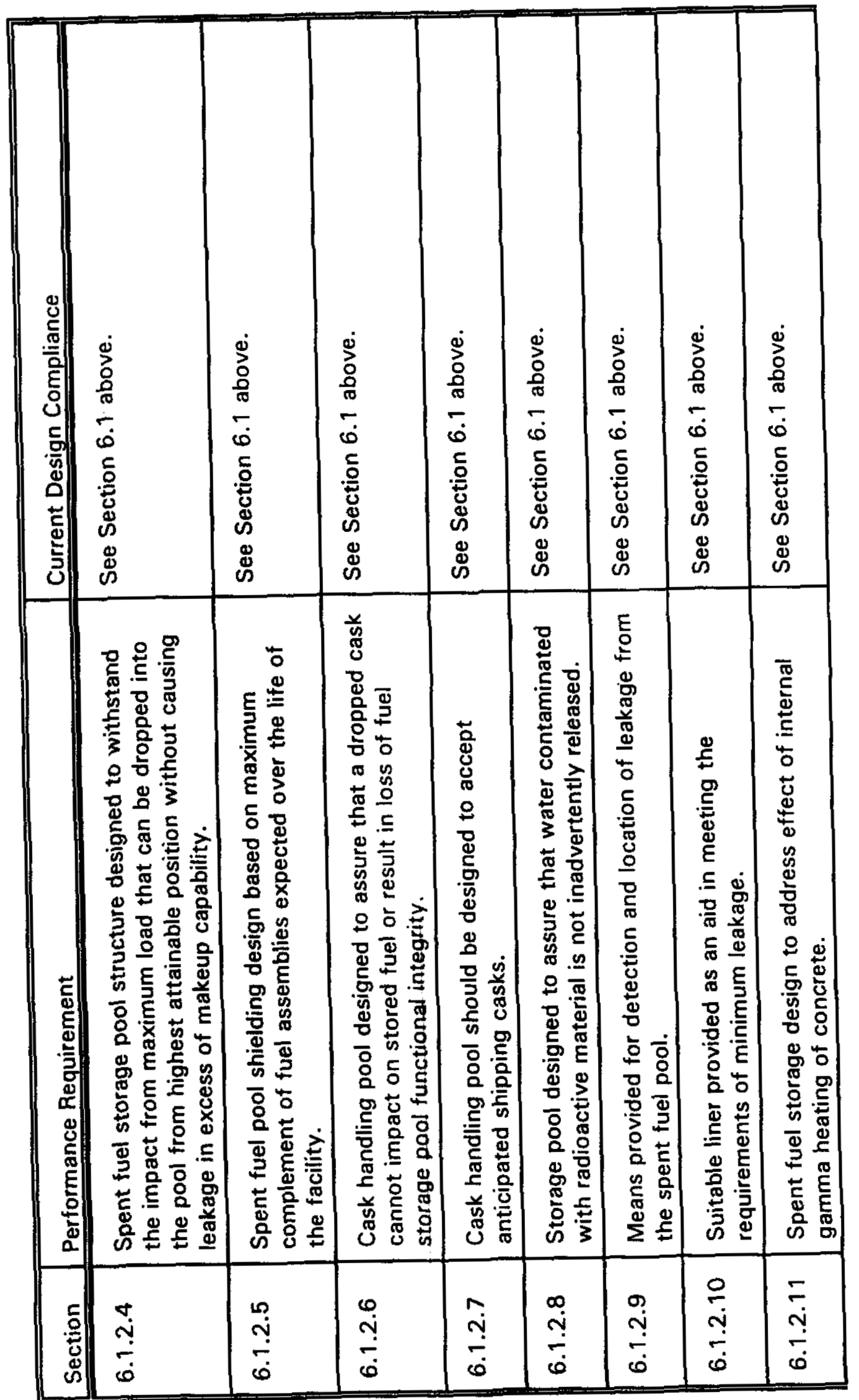




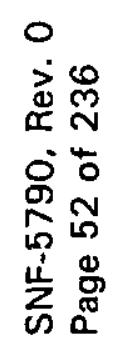

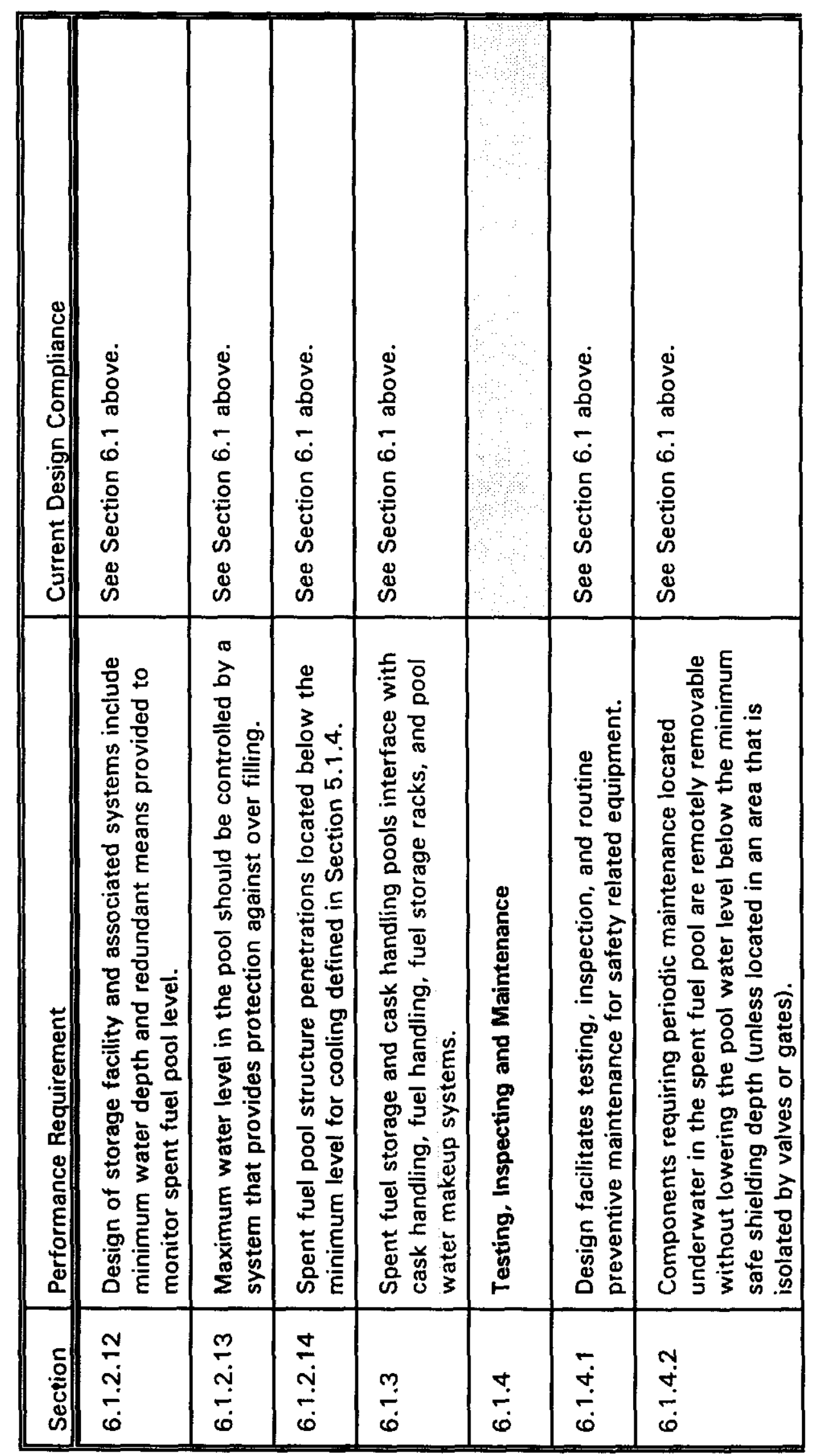




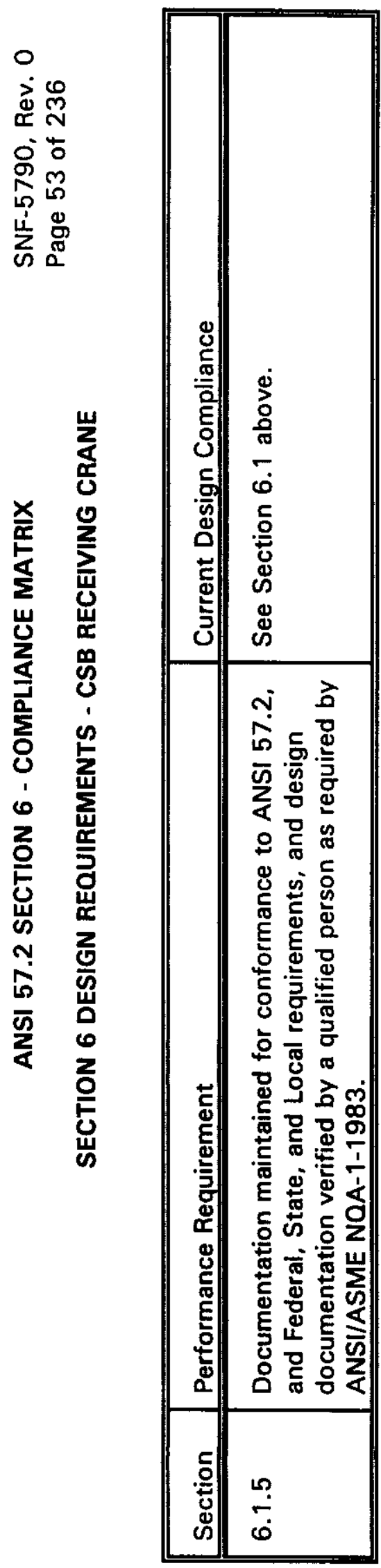




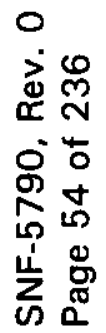

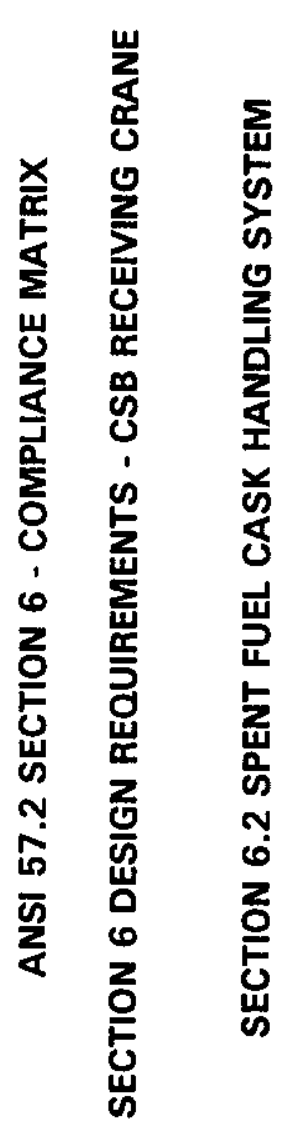

\begin{tabular}{|c|c|c|c|c|c|c|}
\hline 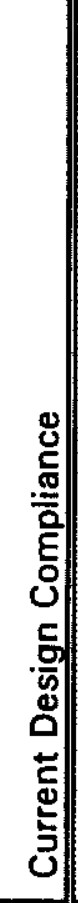 & & & 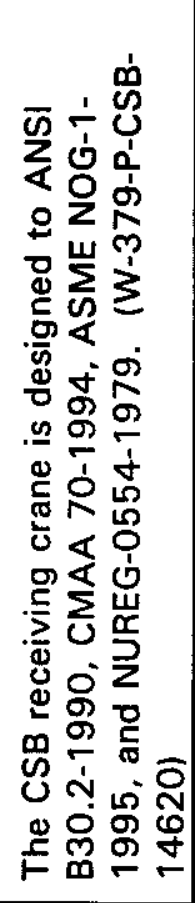 & 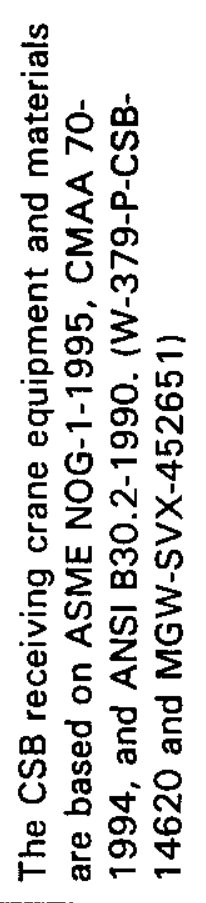 & & 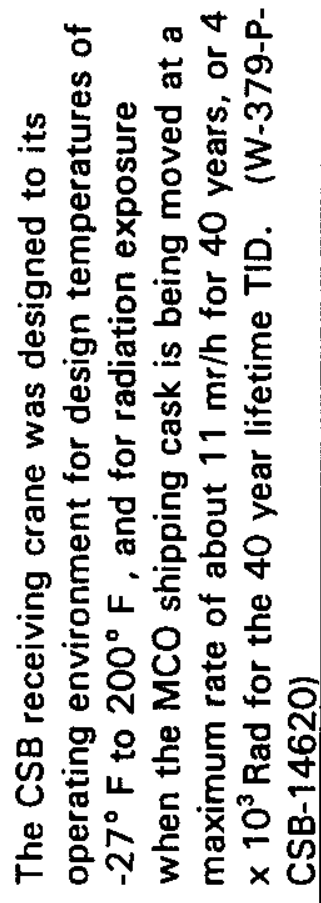 \\
\hline 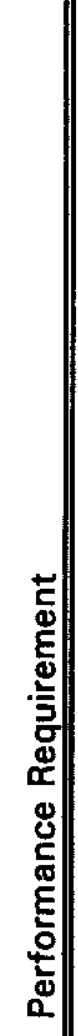 & 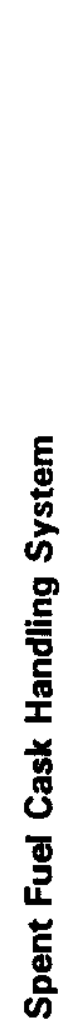 & 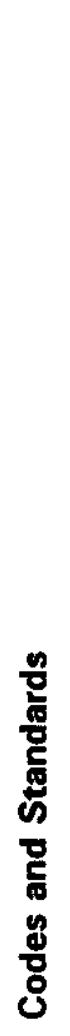 & 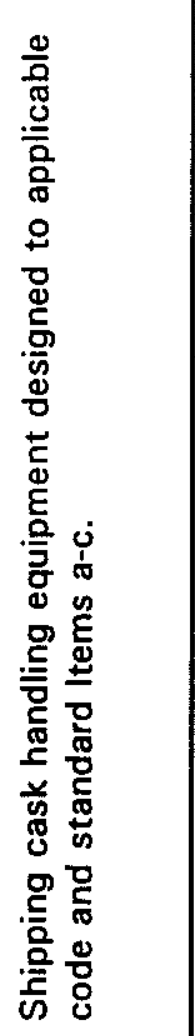 & 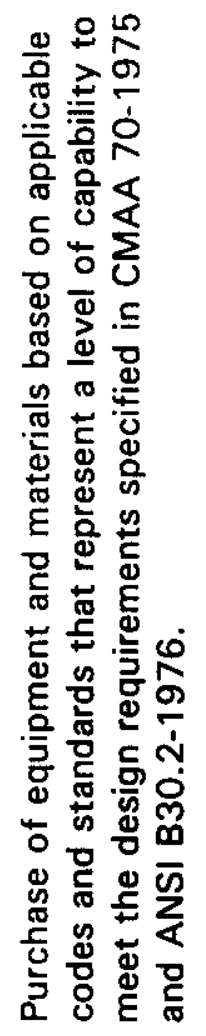 & 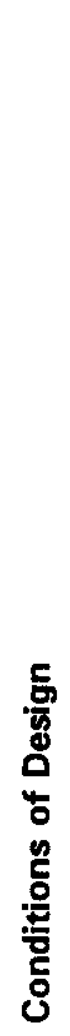 & 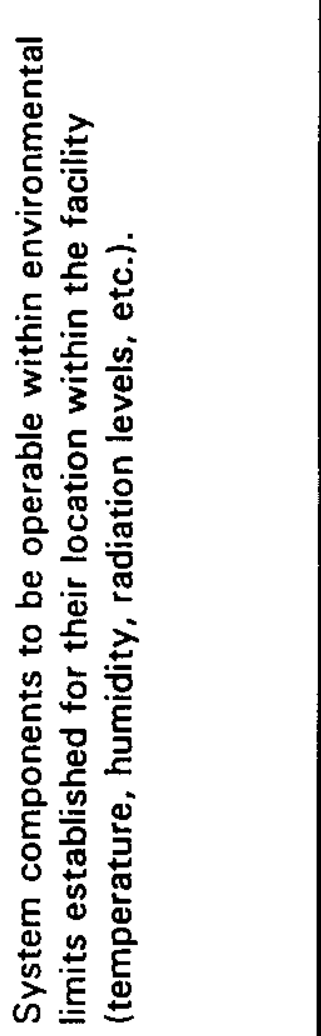 \\
\hline 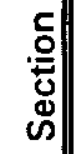 & $\underset{0}{N}$ & $\ddot{\ddot{v}}$ & $\underset{\check{\sigma}}{\check{\sigma}}$ & 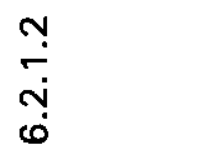 & ָָ & 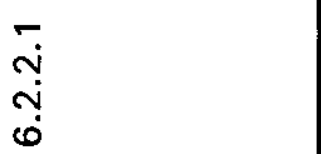 \\
\hline
\end{tabular}




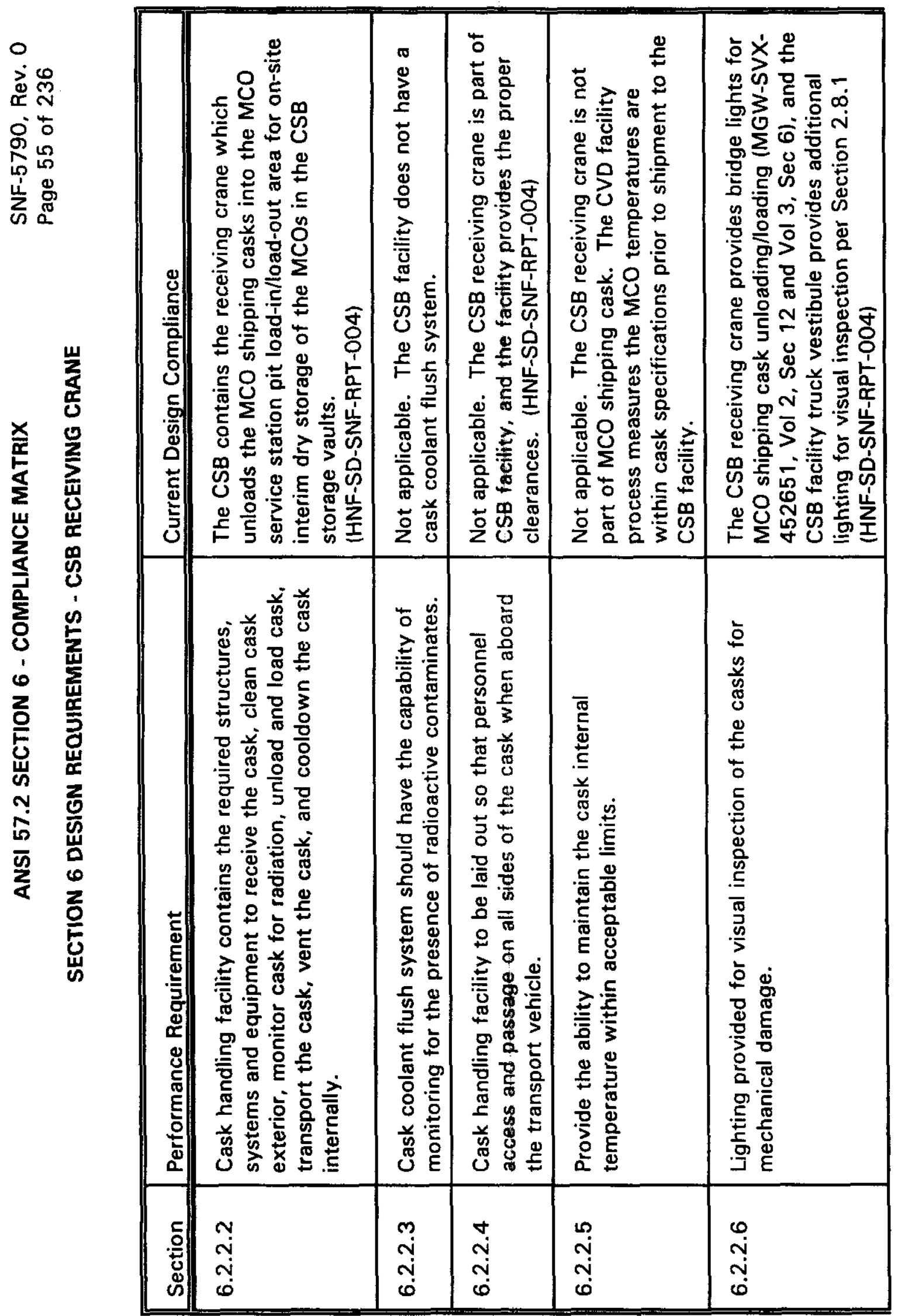




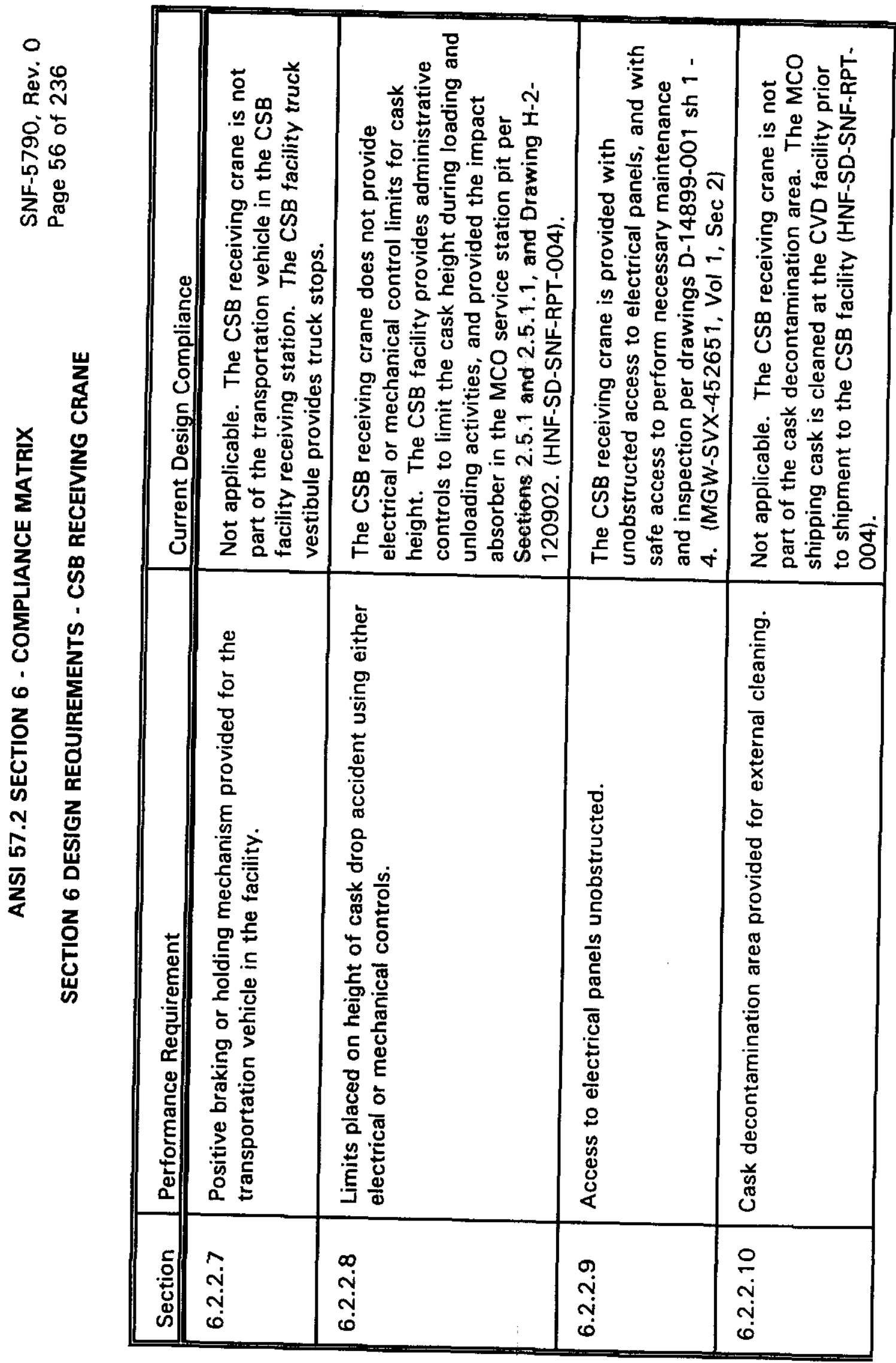




\section{0 \\ $>0$ \\ ธ \\ 융 \\ 은 \\ 㞱罚 \\ 认 ณ}

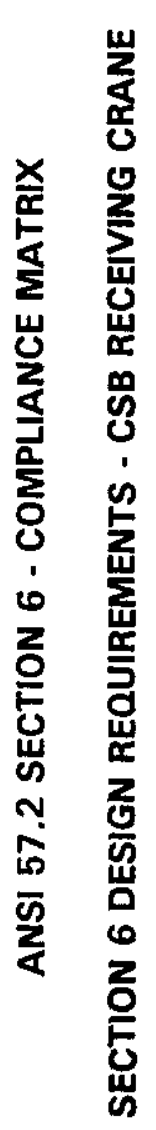

\begin{tabular}{|c|c|c|c|c|c|}
\hline 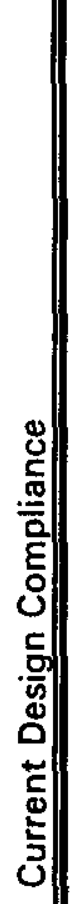 & 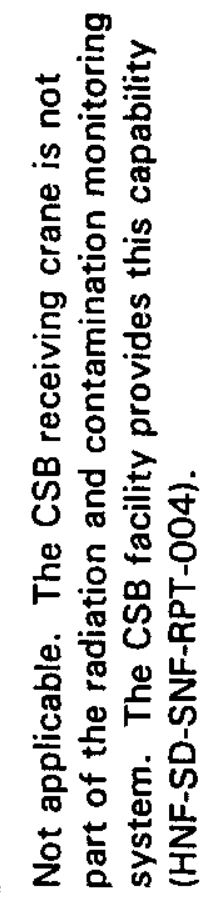 & 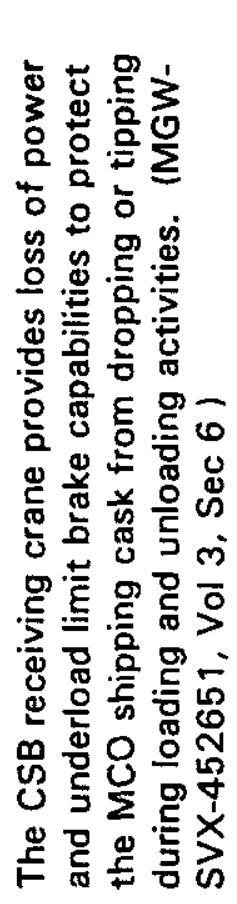 & 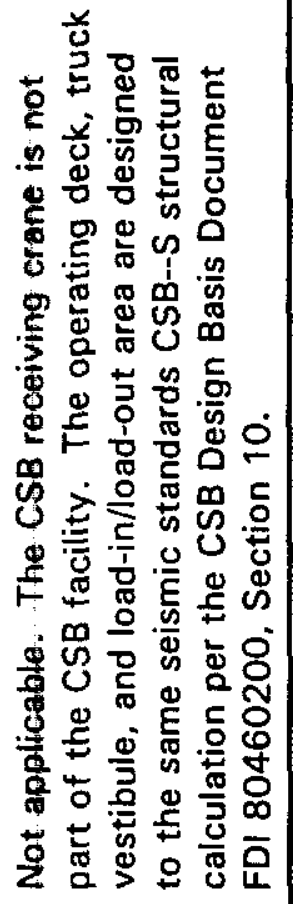 & & 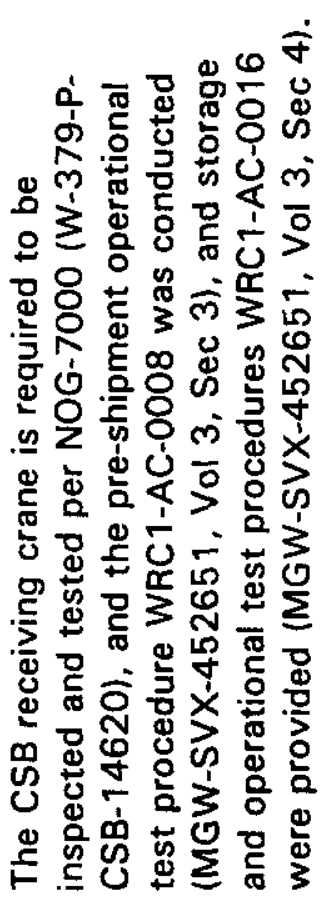 \\
\hline 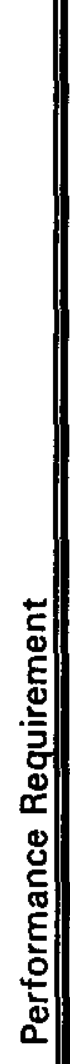 & 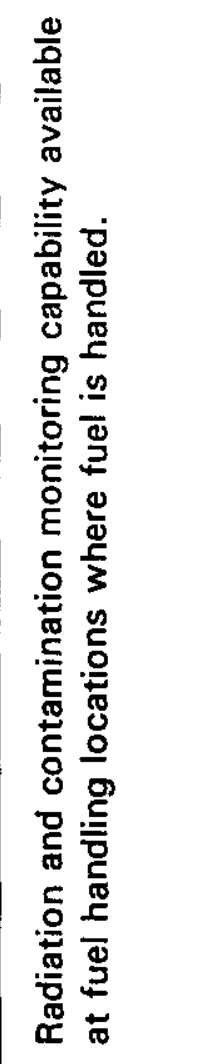 & 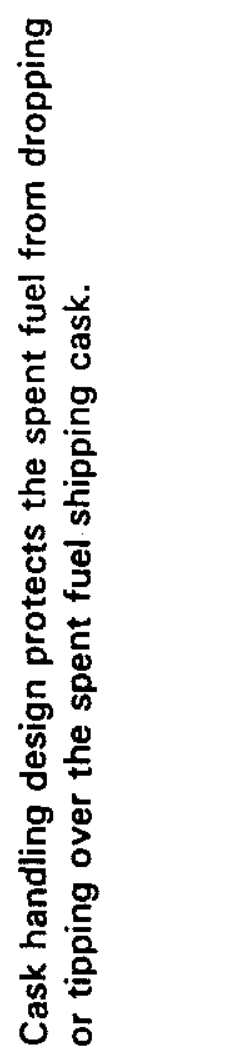 & 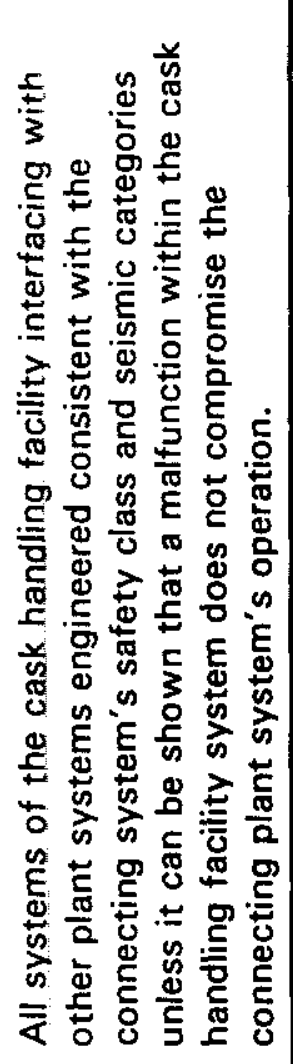 & 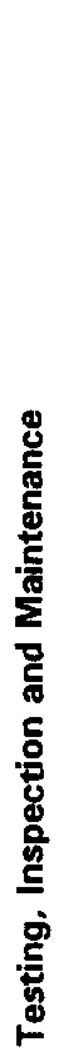 & 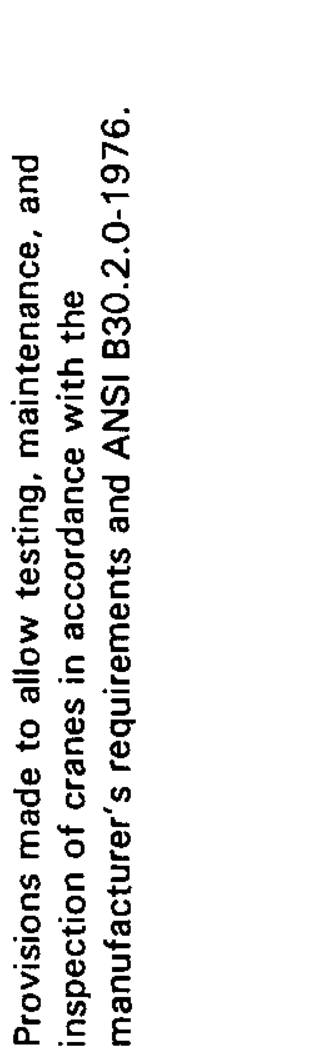 \\
\hline 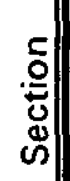 & 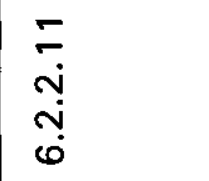 & 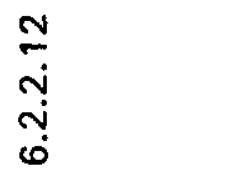 & 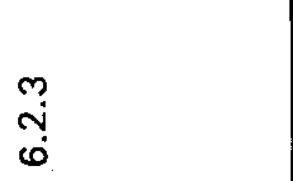 & & $\begin{array}{l}\bar{j} \\
\stackrel{+}{\dot{v}} \\
\dot{0}\end{array}$ \\
\hline
\end{tabular}




\begin{tabular}{|c|c|c|c|c|}
\hline 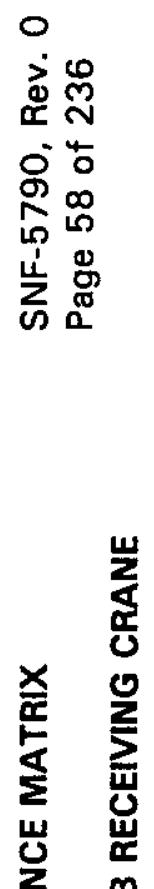 & 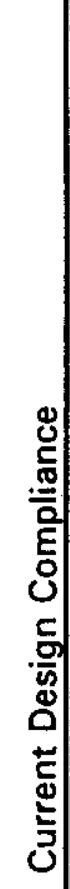 & 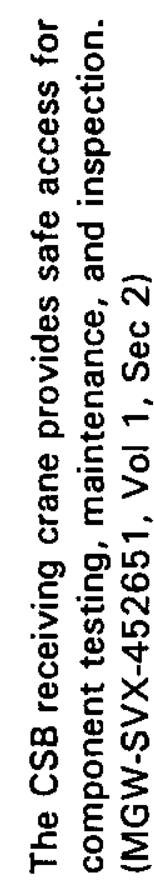 & 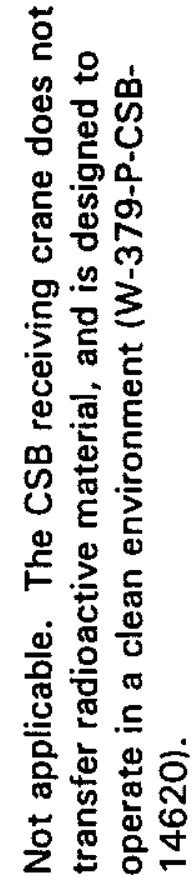 & 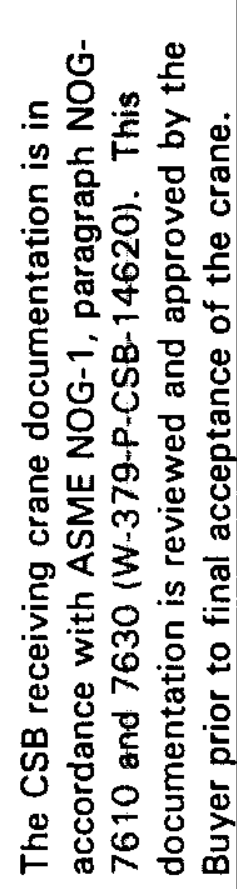 \\
\hline 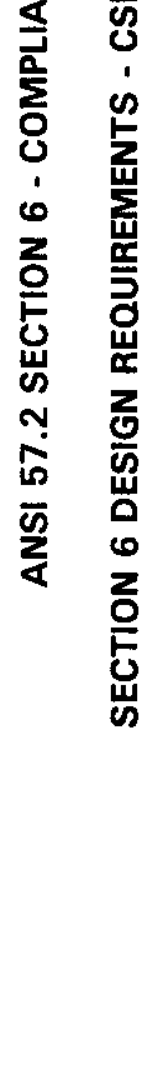 & 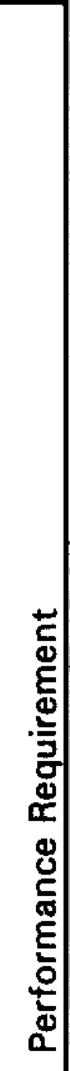 & 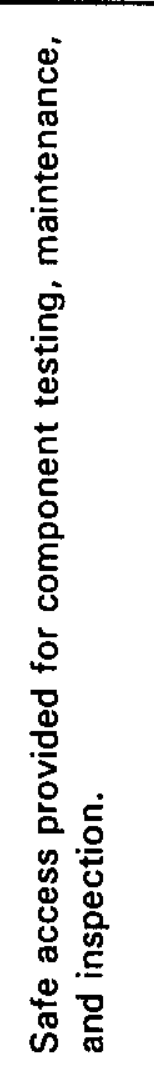 & 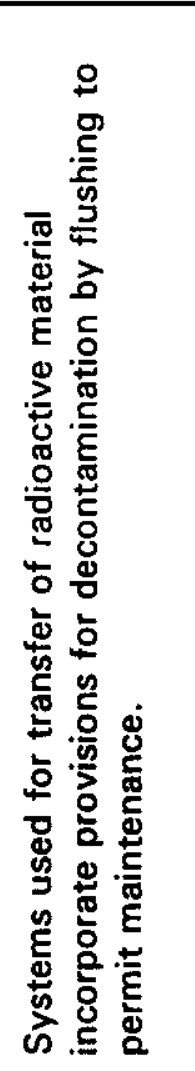 & 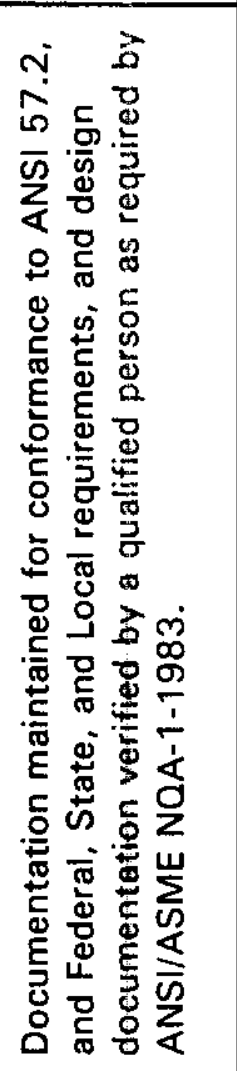 \\
\hline & 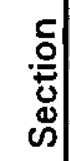 & 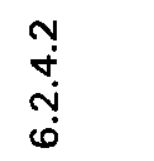 & $\begin{array}{l}m \\
\dot{v} \\
\dot{v}\end{array}$ & $\underset{0}{0}$ \\
\hline
\end{tabular}


0

¿

능

용

ᄂ

岂覂

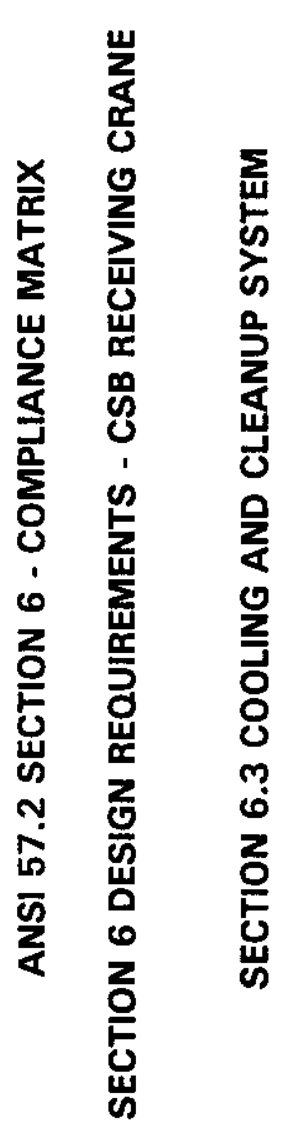

\begin{tabular}{|c|c|c|c|c|c|c|c|}
\hline 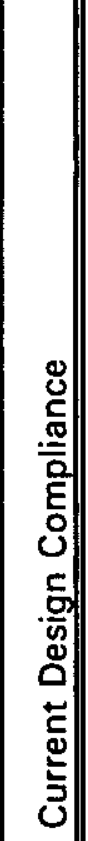 & 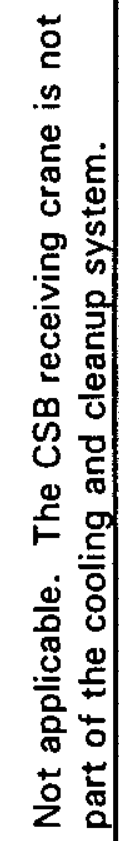 & & 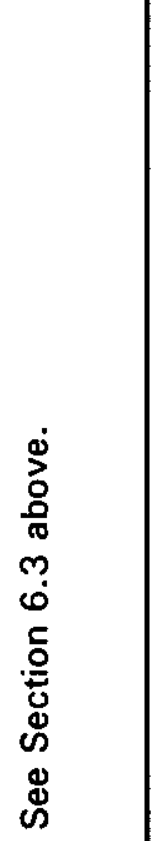 & 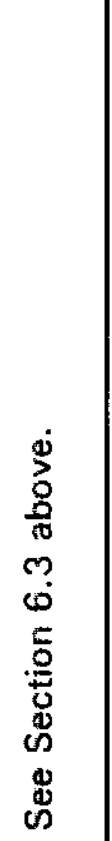 & & 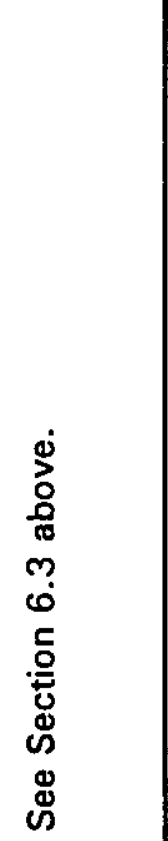 & 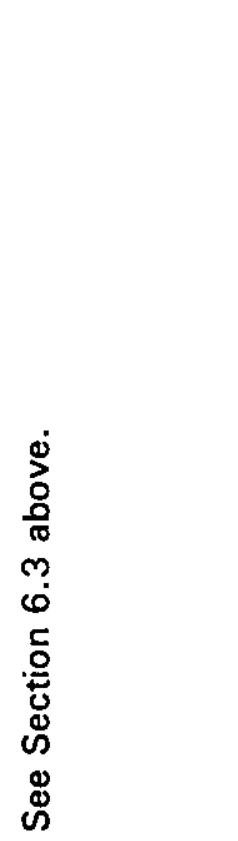 \\
\hline 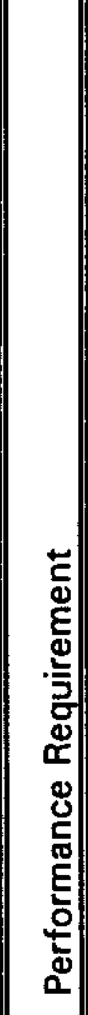 & 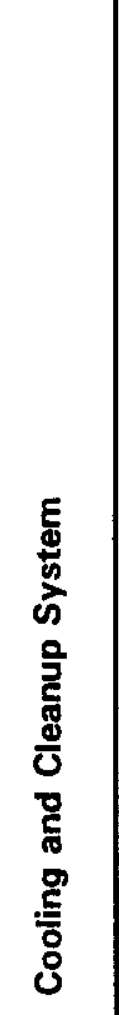 & 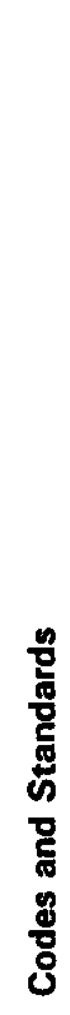 & 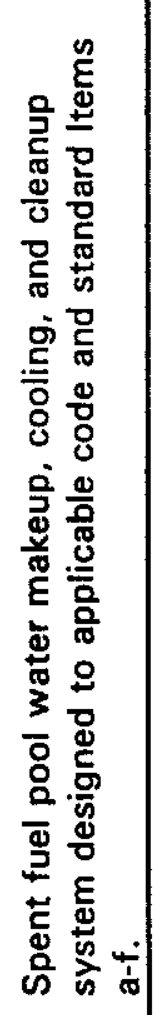 & 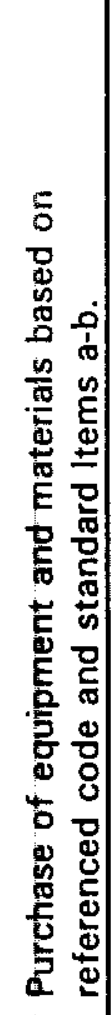 & 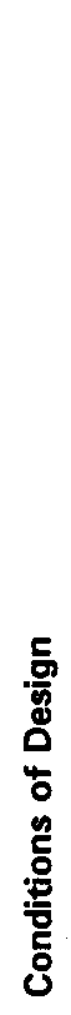 & 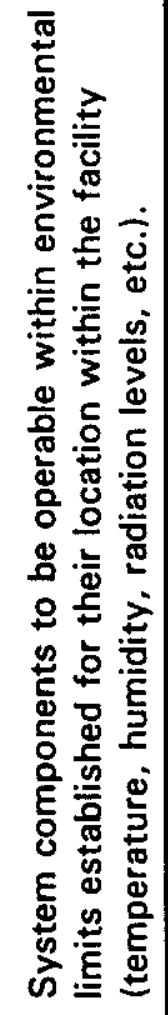 & 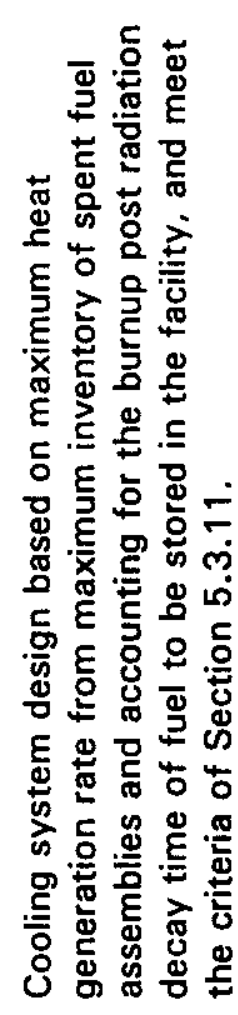 \\
\hline 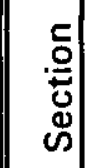 & $\stackrel{m}{0}$ & $\ddot{\circ}$ & $\begin{array}{r}\check{\sigma} \\
\ddot{0}\end{array}$ & $\begin{array}{l}\stackrel{N}{\oplus} \\
\ddot{n}\end{array}$ & ஸे & 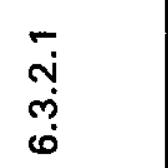 & $\begin{array}{l}\text { N } \\
\text { ஸे } \\
\text { ஸे }\end{array}$ \\
\hline
\end{tabular}




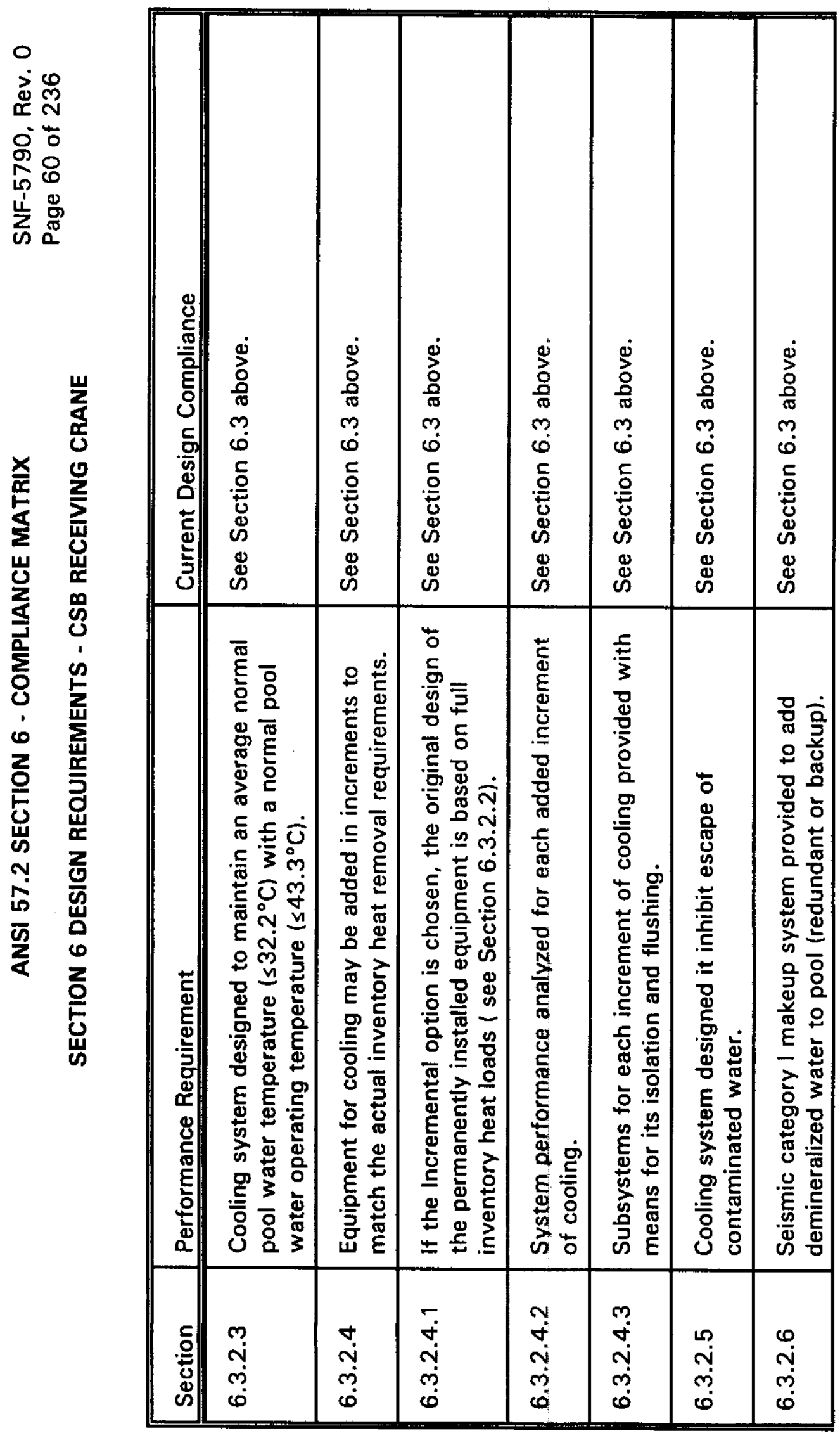




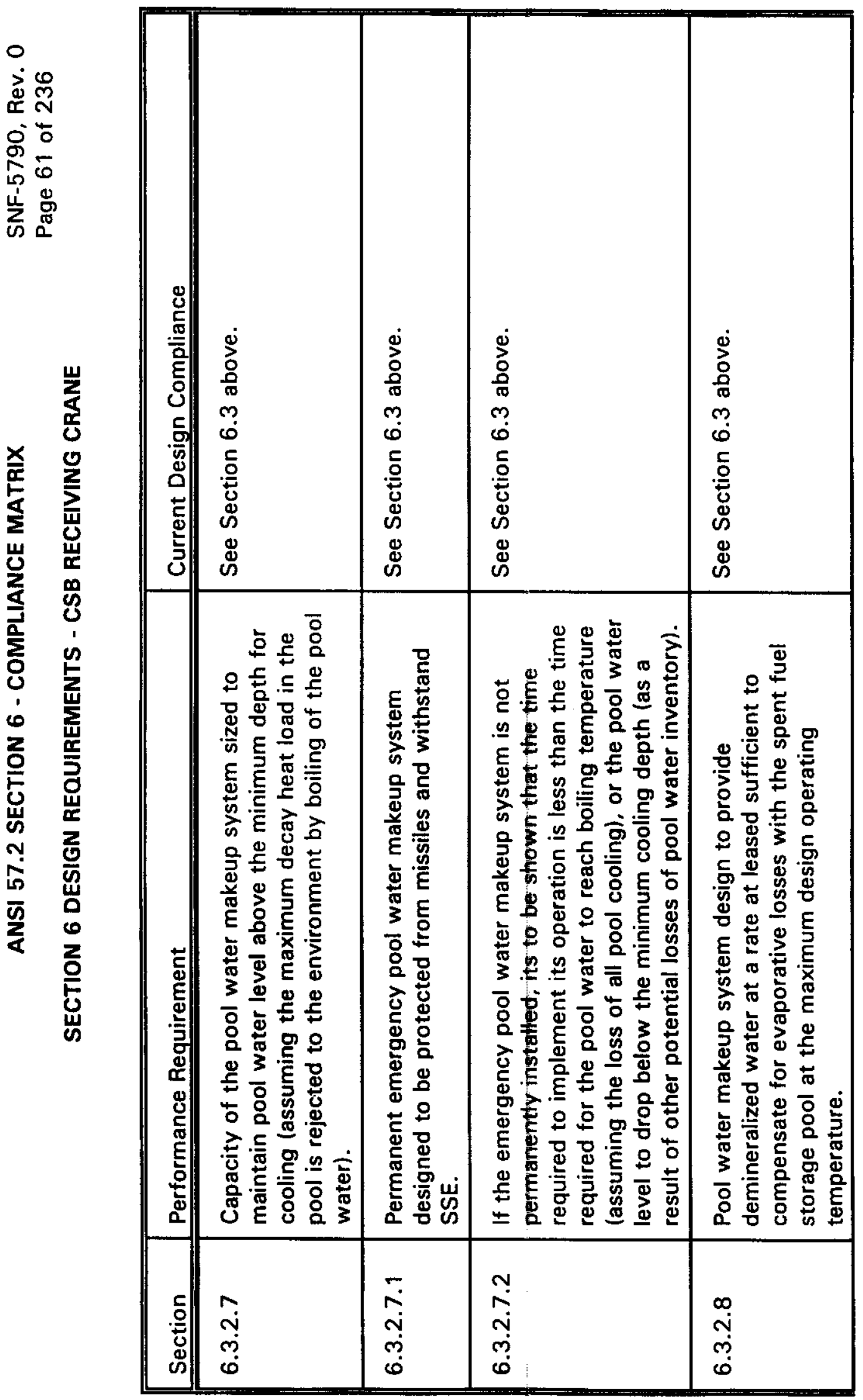




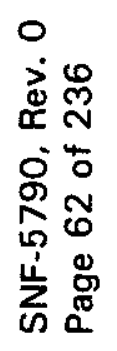

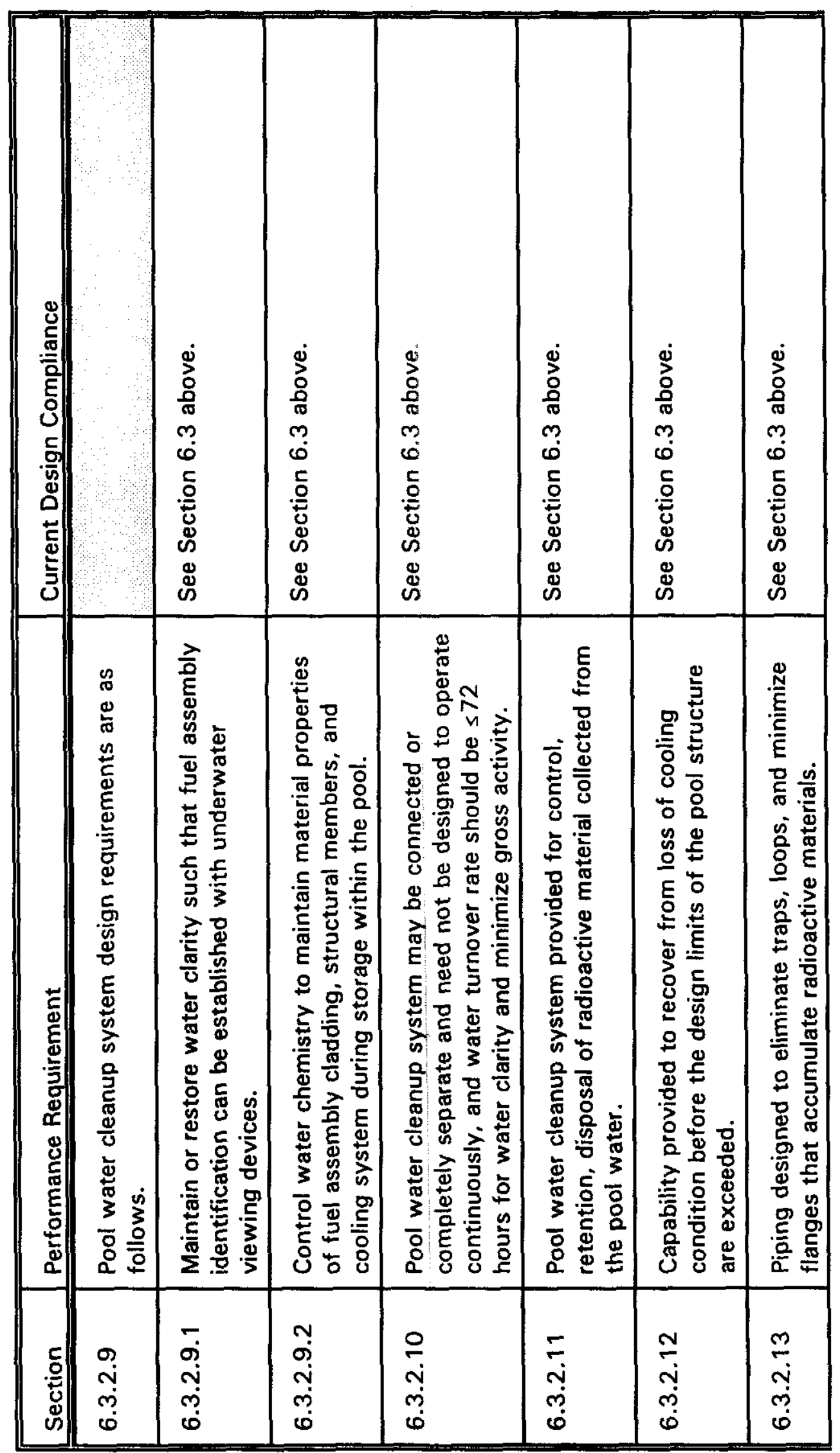




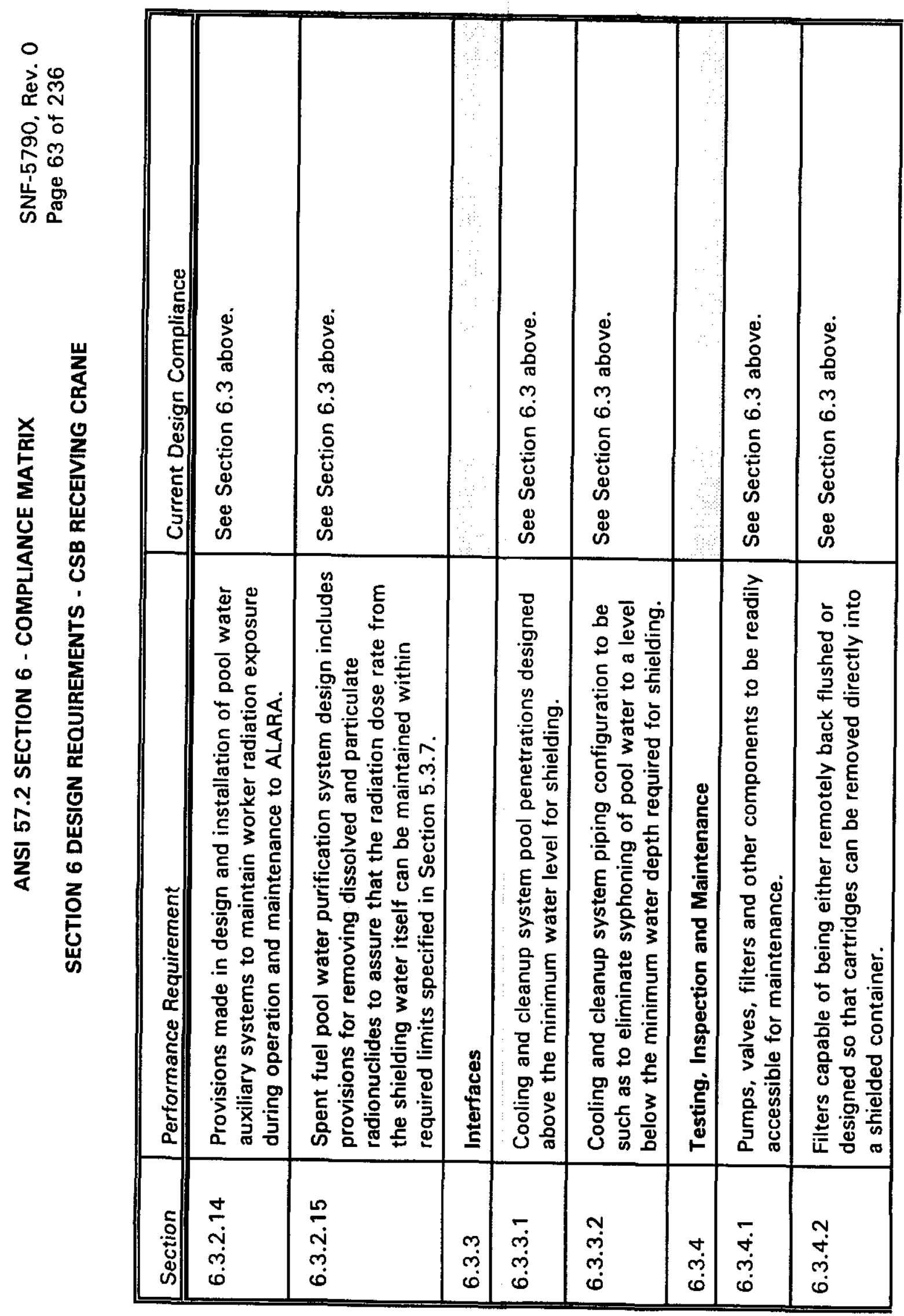




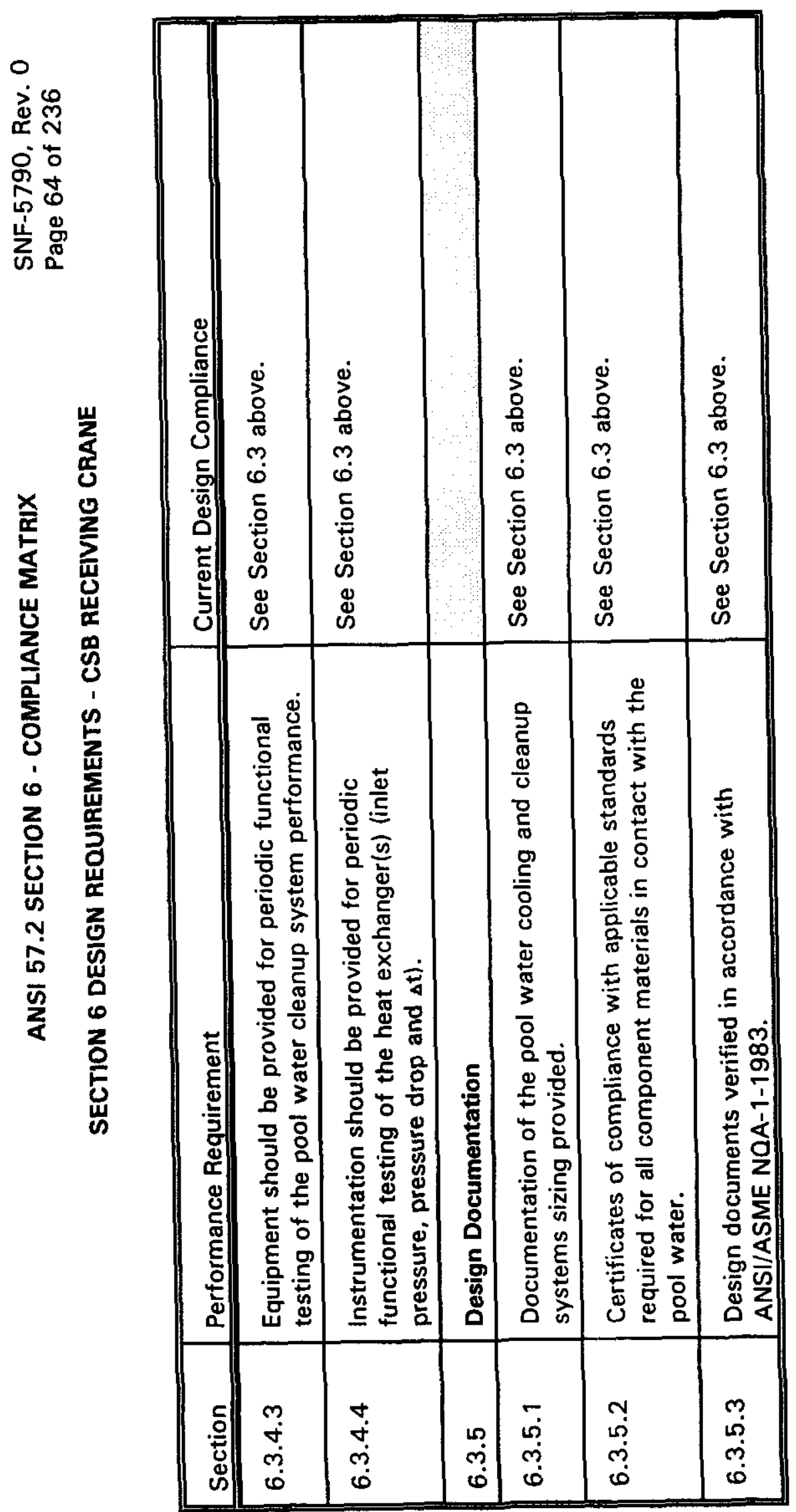




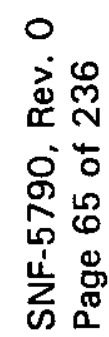

\begin{tabular}{|c|c|c|c|c|c|c|c|}
\hline 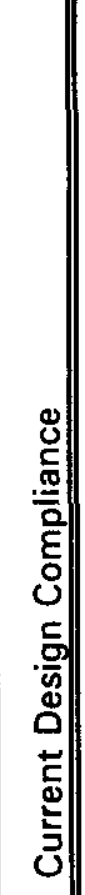 & 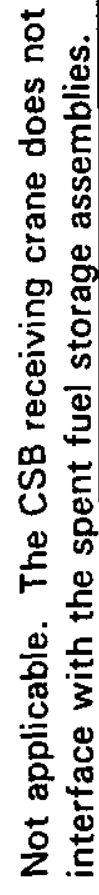 & 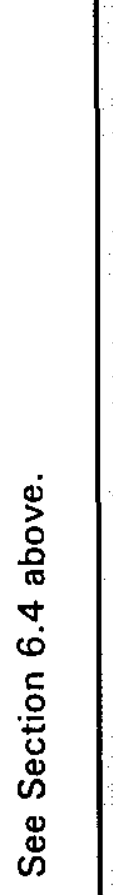 & & & 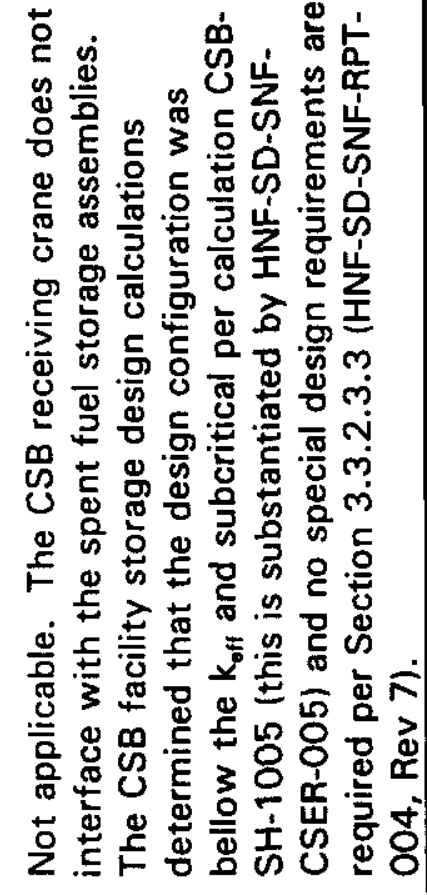 & 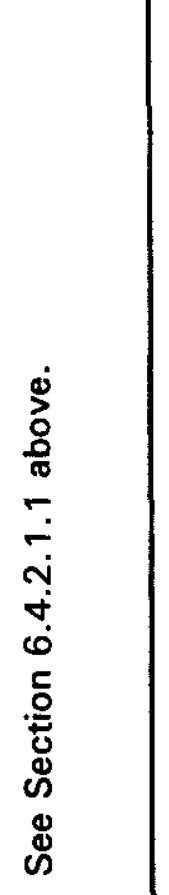 & 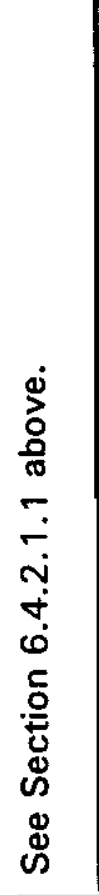 \\
\hline 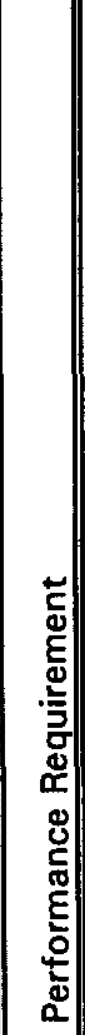 & 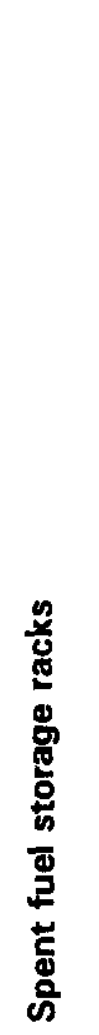 & 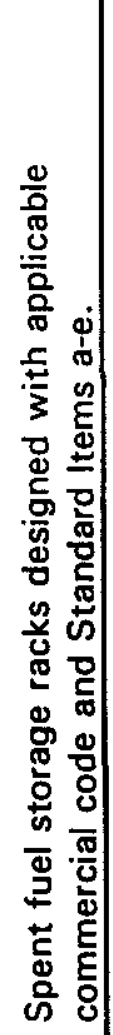 & 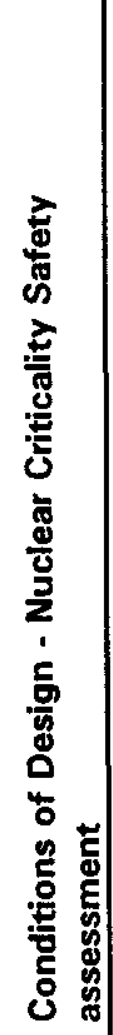 & 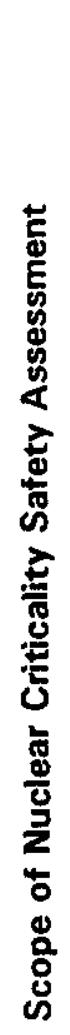 & 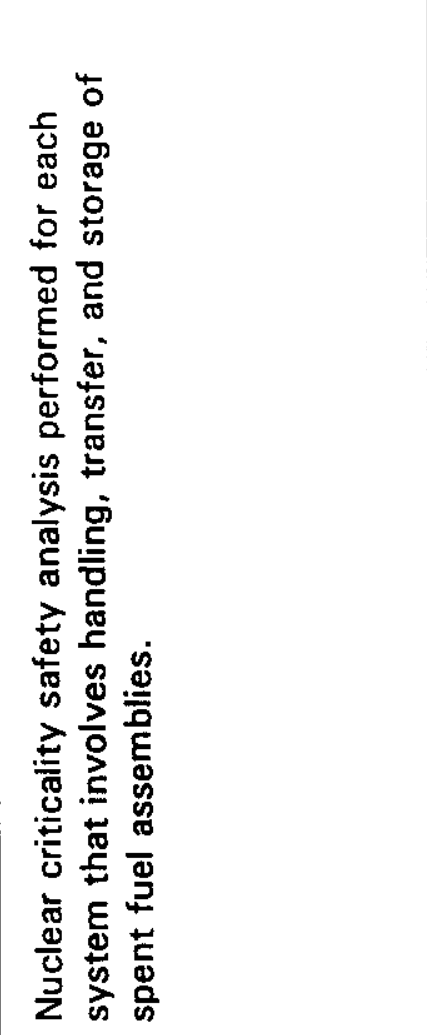 & 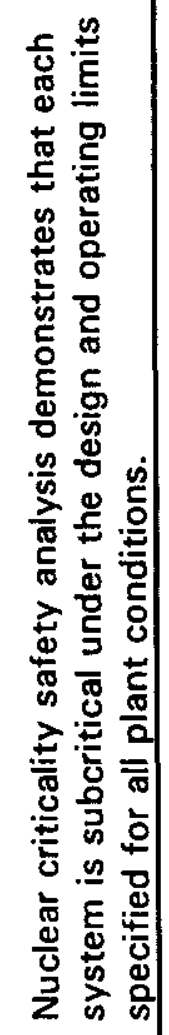 & 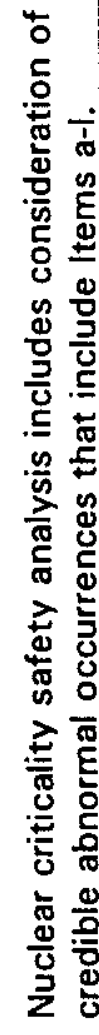 \\
\hline 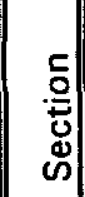 & $\stackrel{+}{\circ}$ & $\underset{\dot{\nabla}}{\square}$ & ن & $\begin{array}{l}\tilde{\sigma} \\
\dot{\sigma}\end{array}$ & 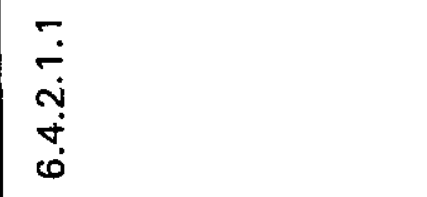 & $\begin{array}{l}\stackrel{v}{\sim} \\
\dot{\sim} \\
\dot{\sigma} \\
\dot{0}\end{array}$ & $\begin{array}{l}m \\
\stackrel{m}{~} \\
\dot{j} \\
\dot{0}\end{array}$ \\
\hline
\end{tabular}




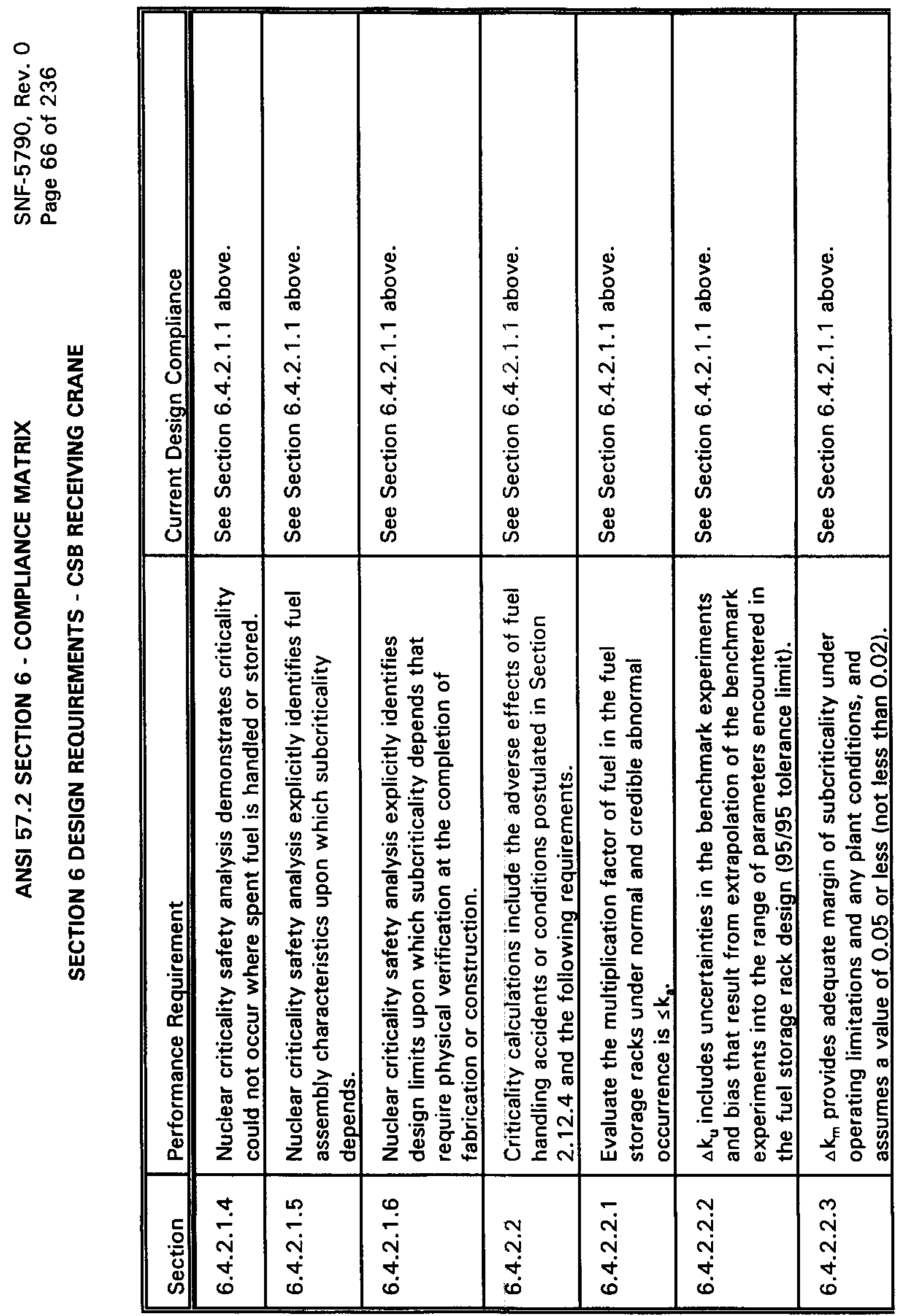




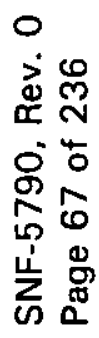

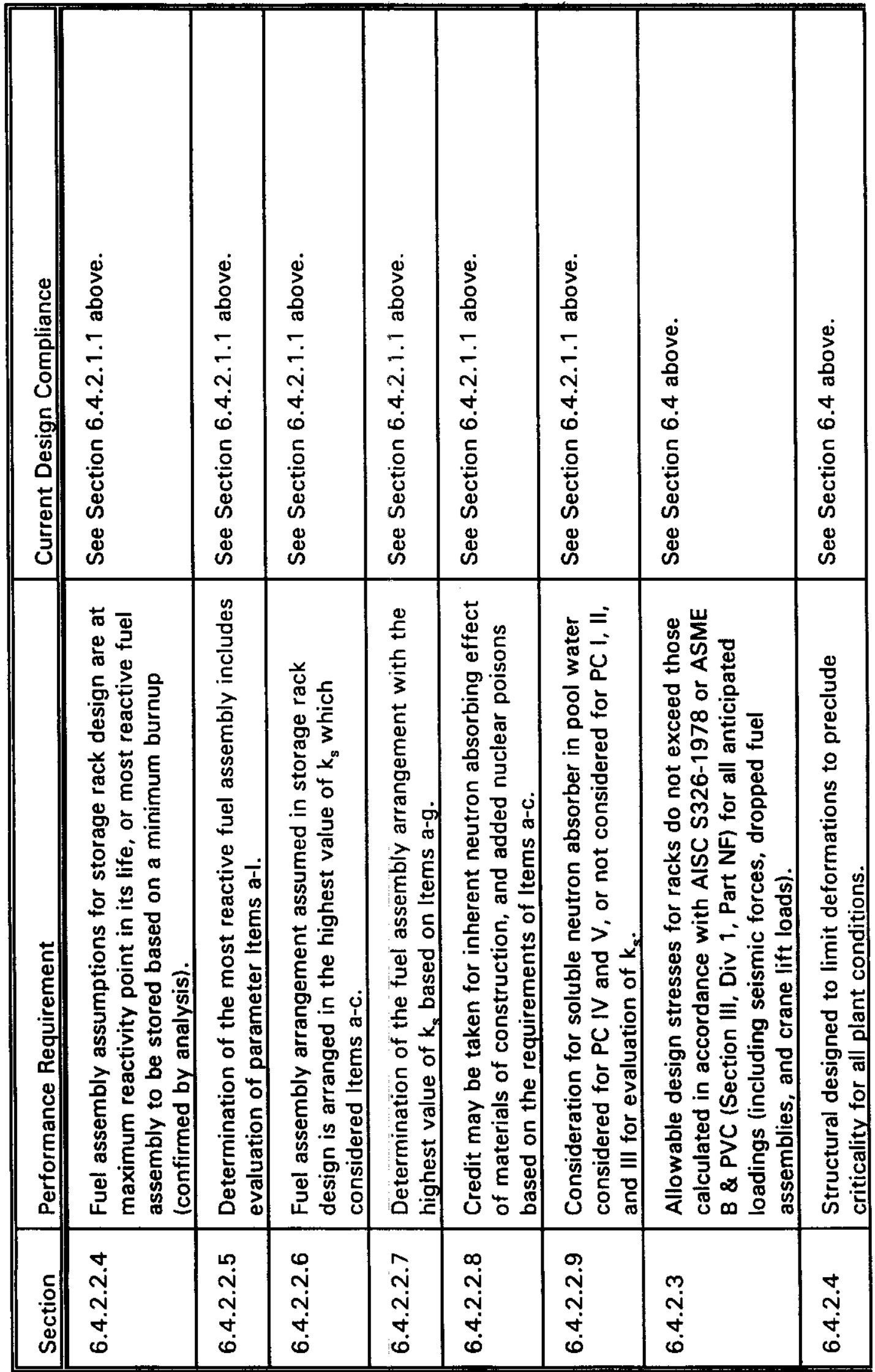




\section{0 \\ خ \\ $\llbracket$ ฟ \\ 응 \\ ค \\ 岁 \\ ง}

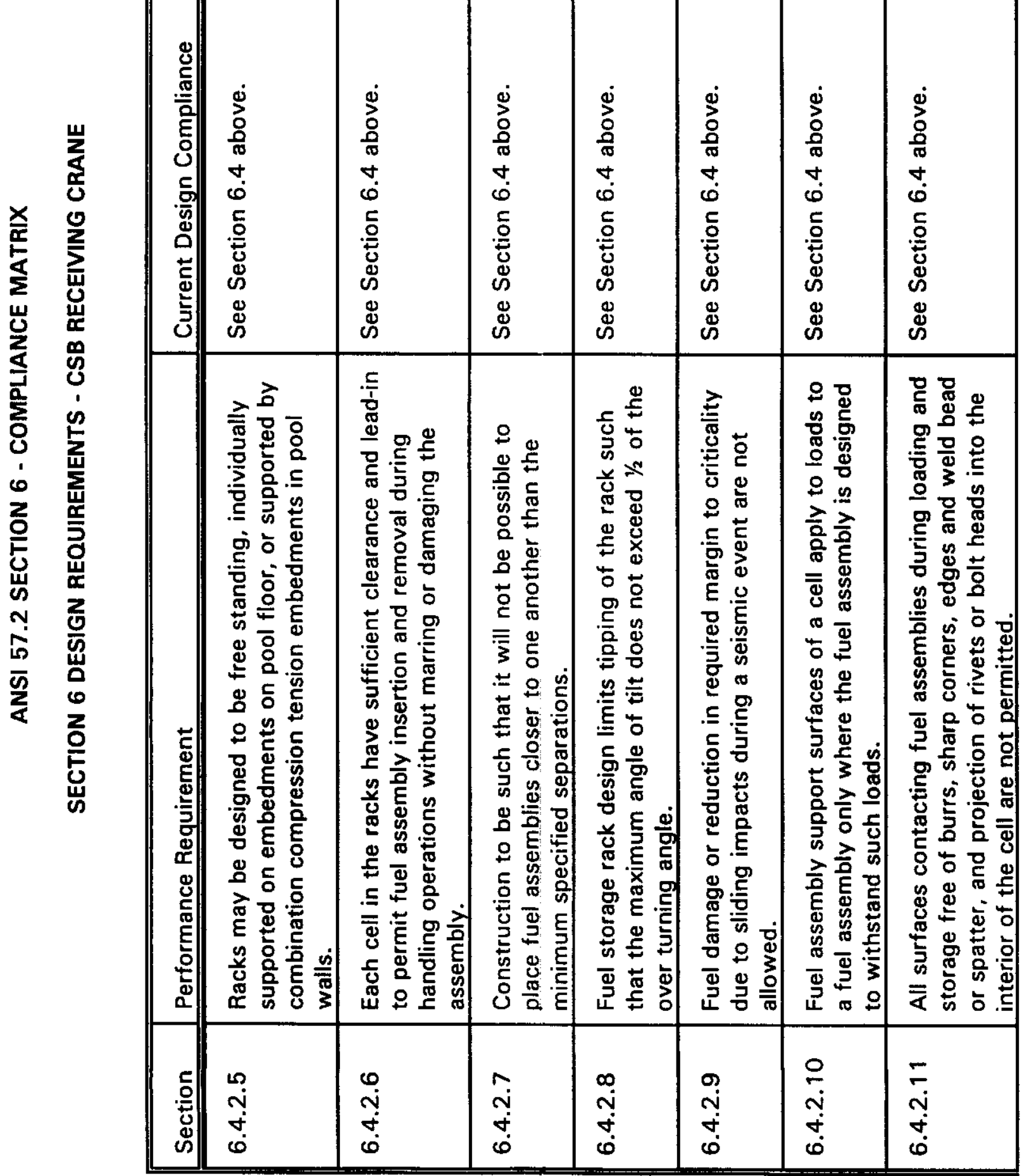




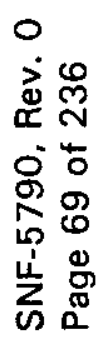

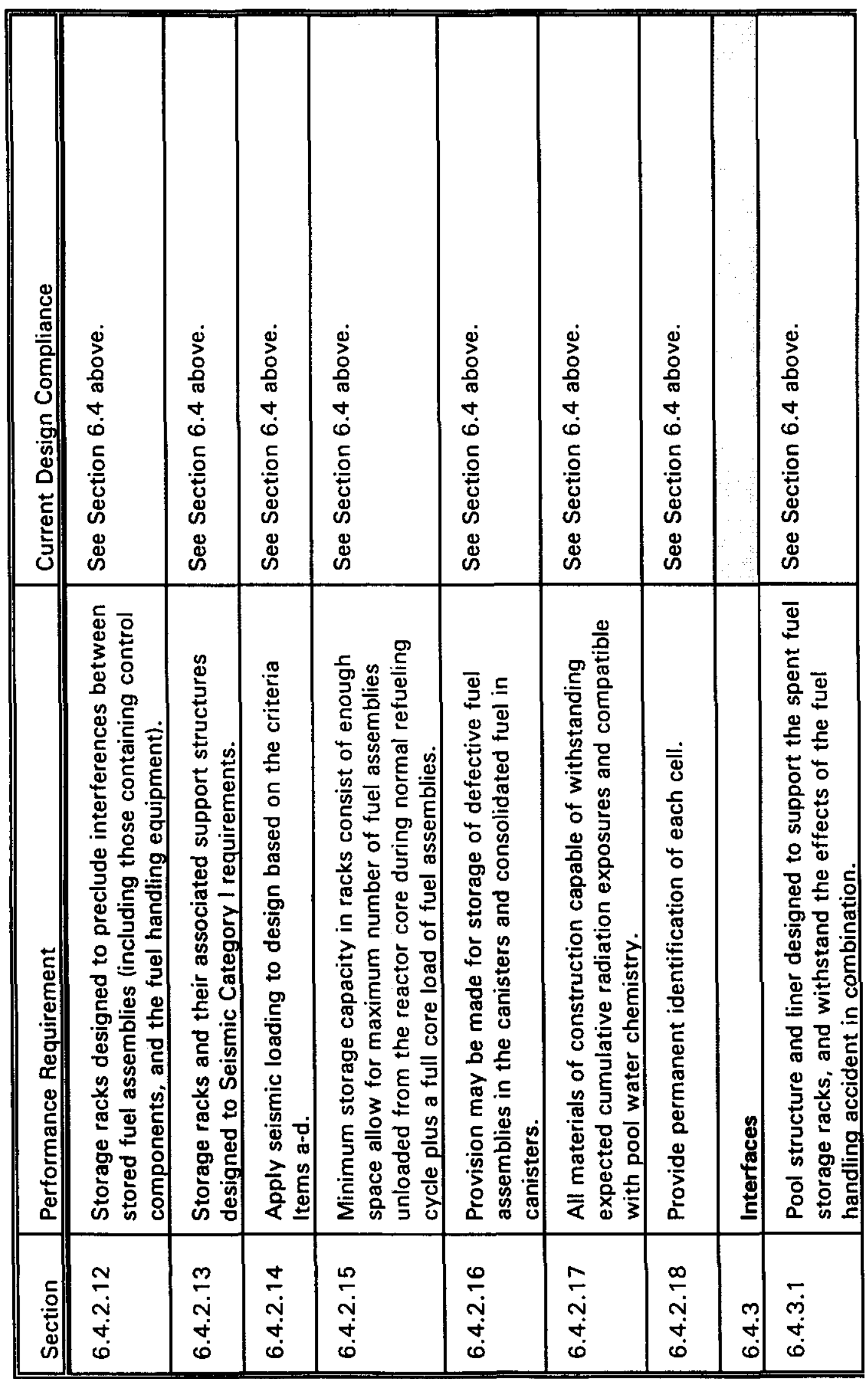



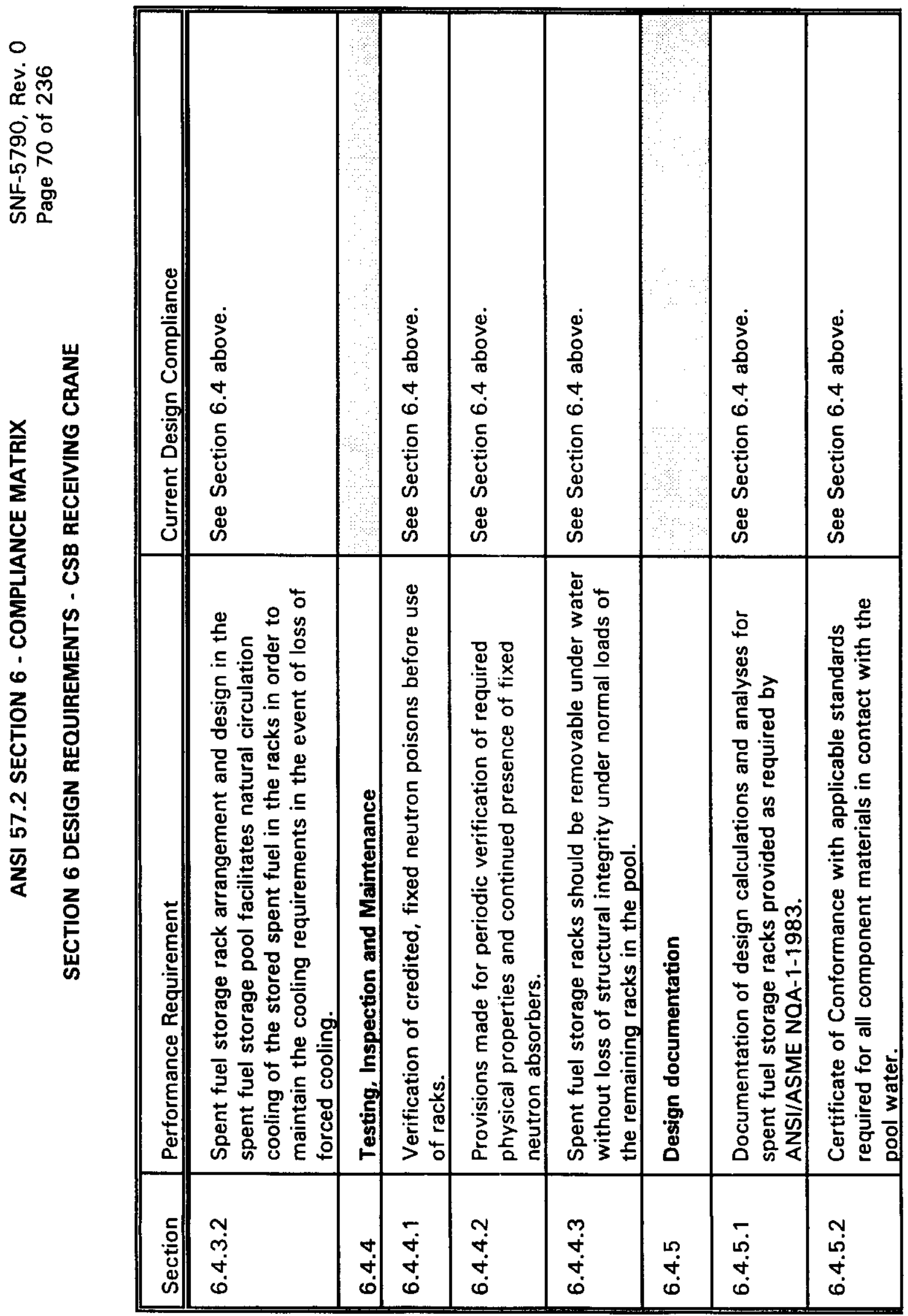


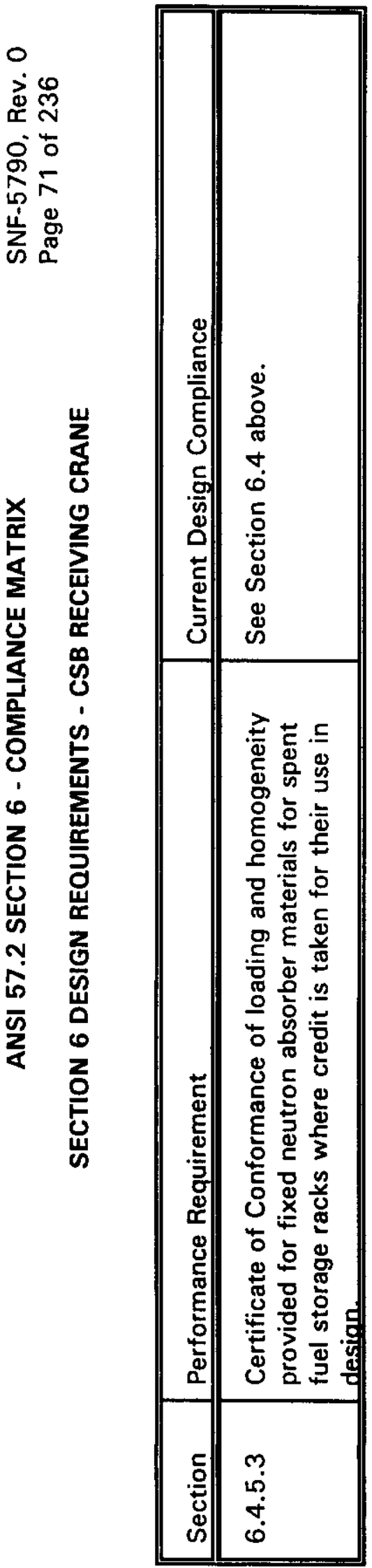




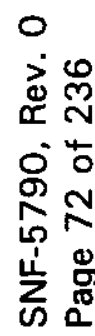

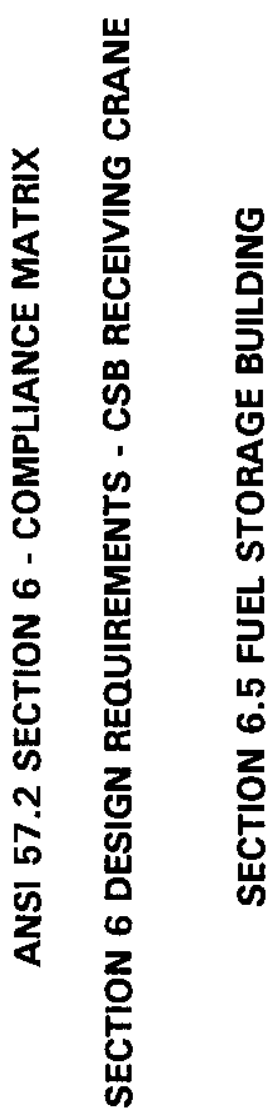

\begin{tabular}{|c|c|c|c|c|c|c|}
\hline 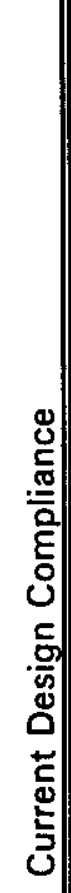 & 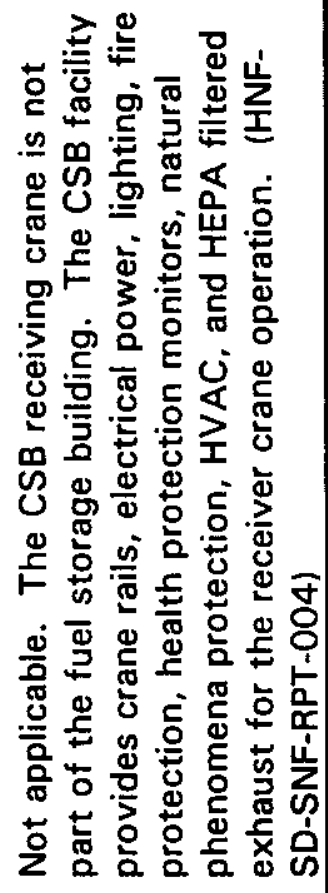 & & $\begin{array}{l}0 \\
0 \\
0 \\
0 \\
0 \\
0 \\
0 \\
0 \\
00 \\
0 \\
\infty \\
0 \\
0\end{array}$ & 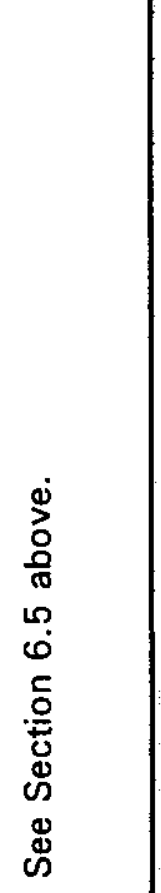 & & 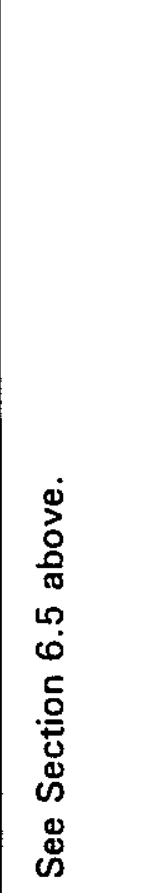 \\
\hline 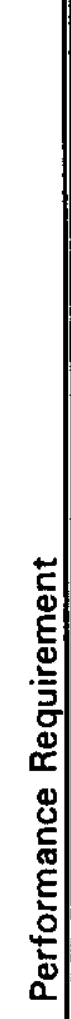 & 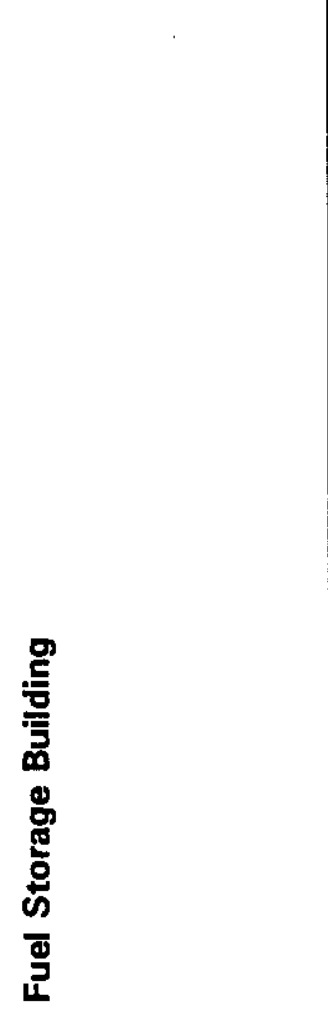 & 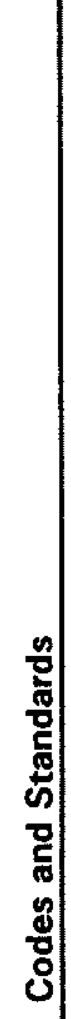 & 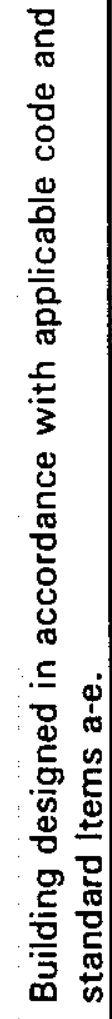 & 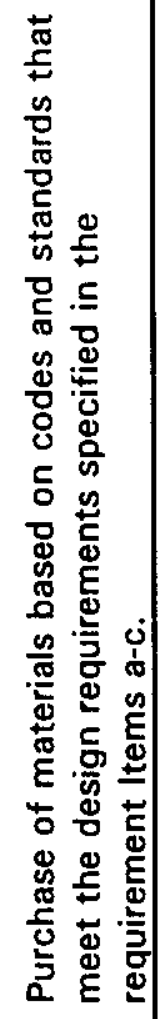 & 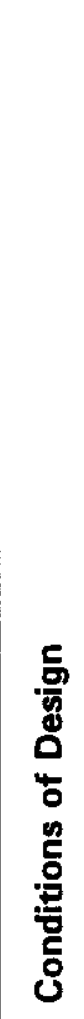 & 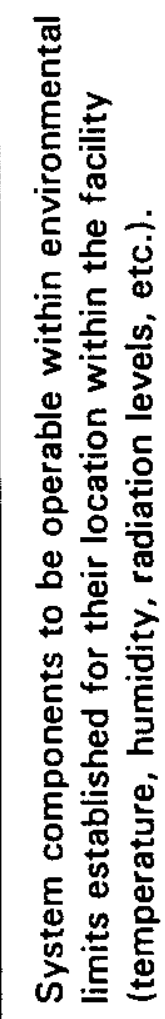 \\
\hline 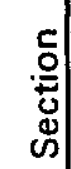 & ? & $\begin{array}{l}- \\
\dot{\omega} \\
\end{array}$ & 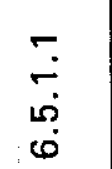 & 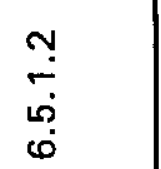 & 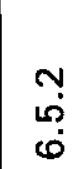 & ְִ \\
\hline
\end{tabular}




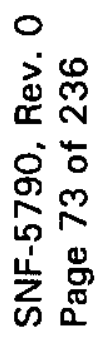

\begin{tabular}{|c|c|c|c|c|c|}
\hline 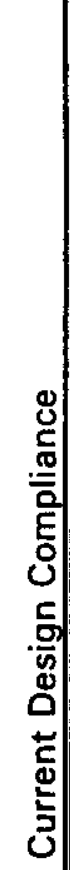 & 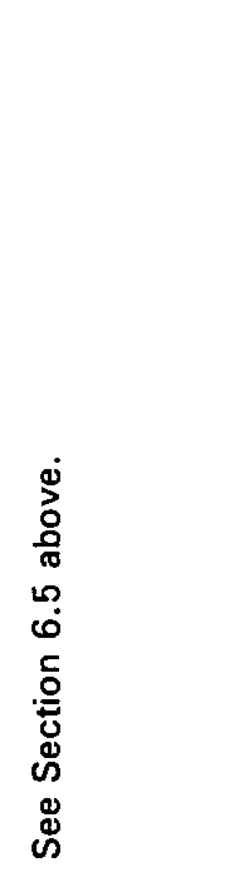 & 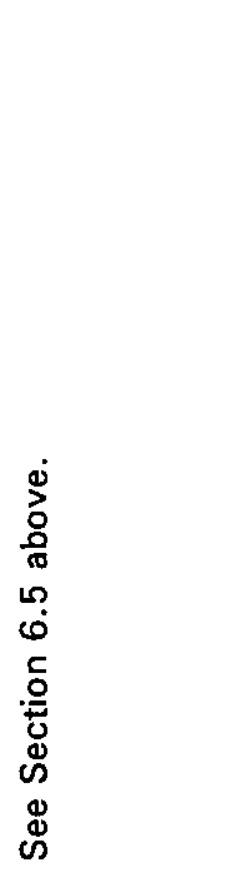 & 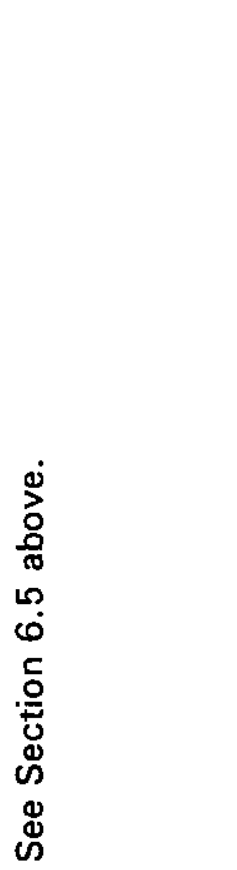 & 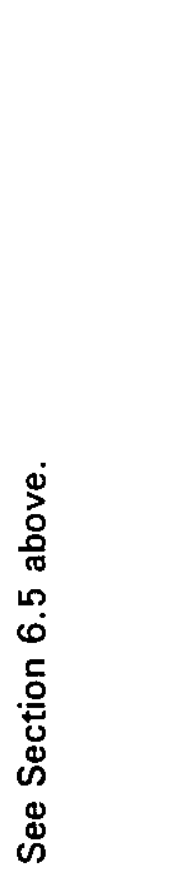 & 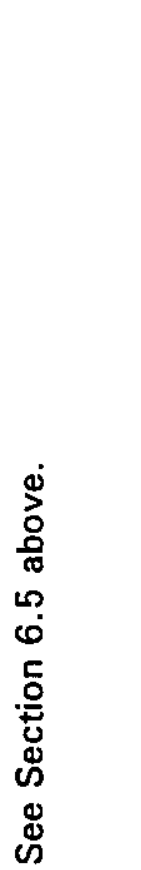 \\
\hline 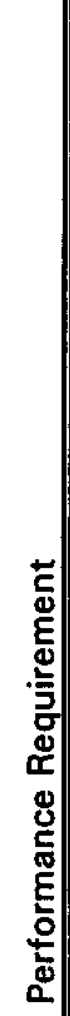 & 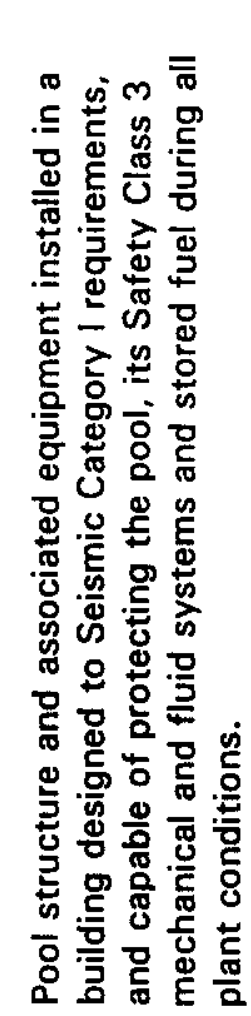 & 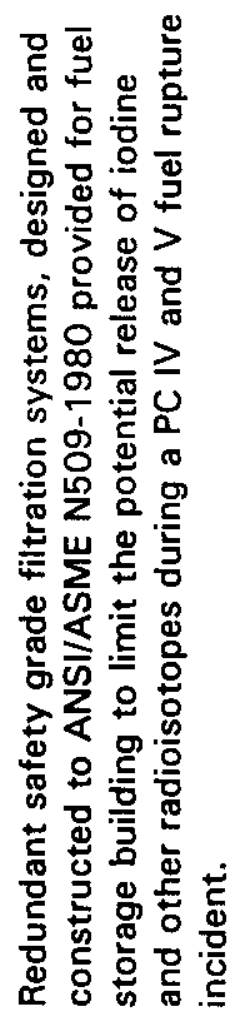 & 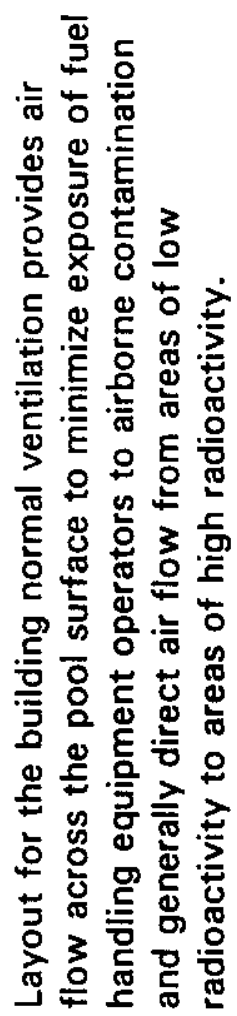 & 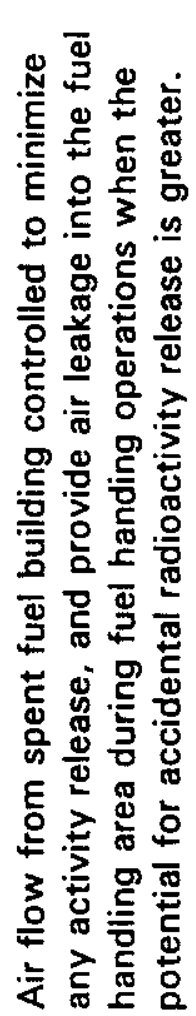 & 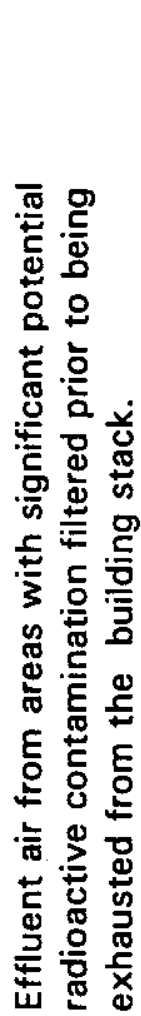 \\
\hline 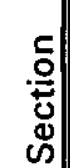 & 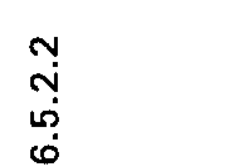 & 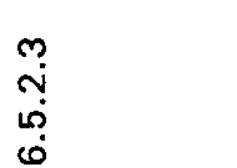 & 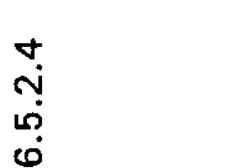 & 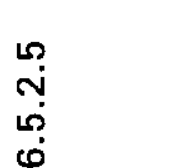 & $\begin{array}{l}0 \\
\\
\\
0\end{array}$ \\
\hline
\end{tabular}




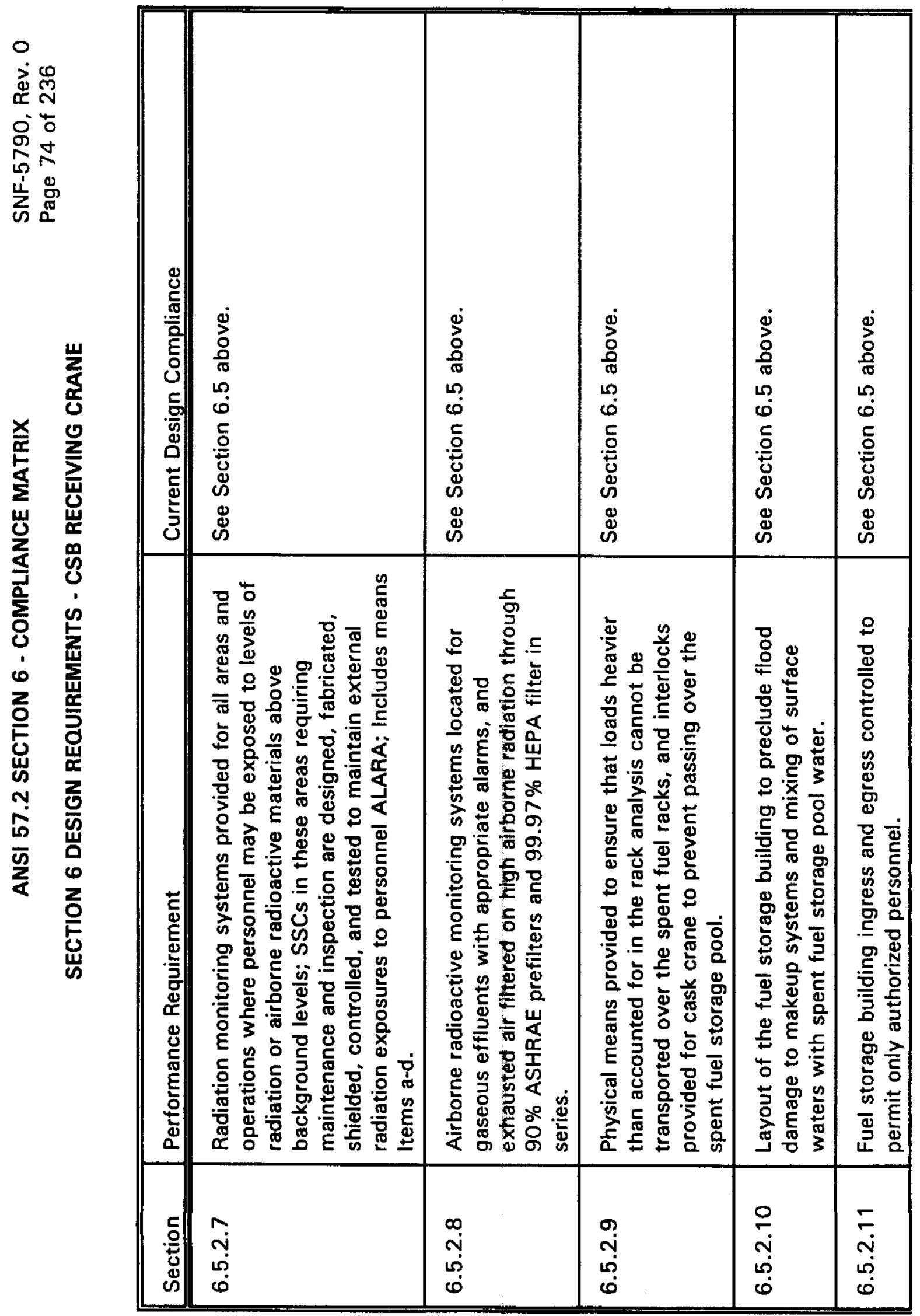




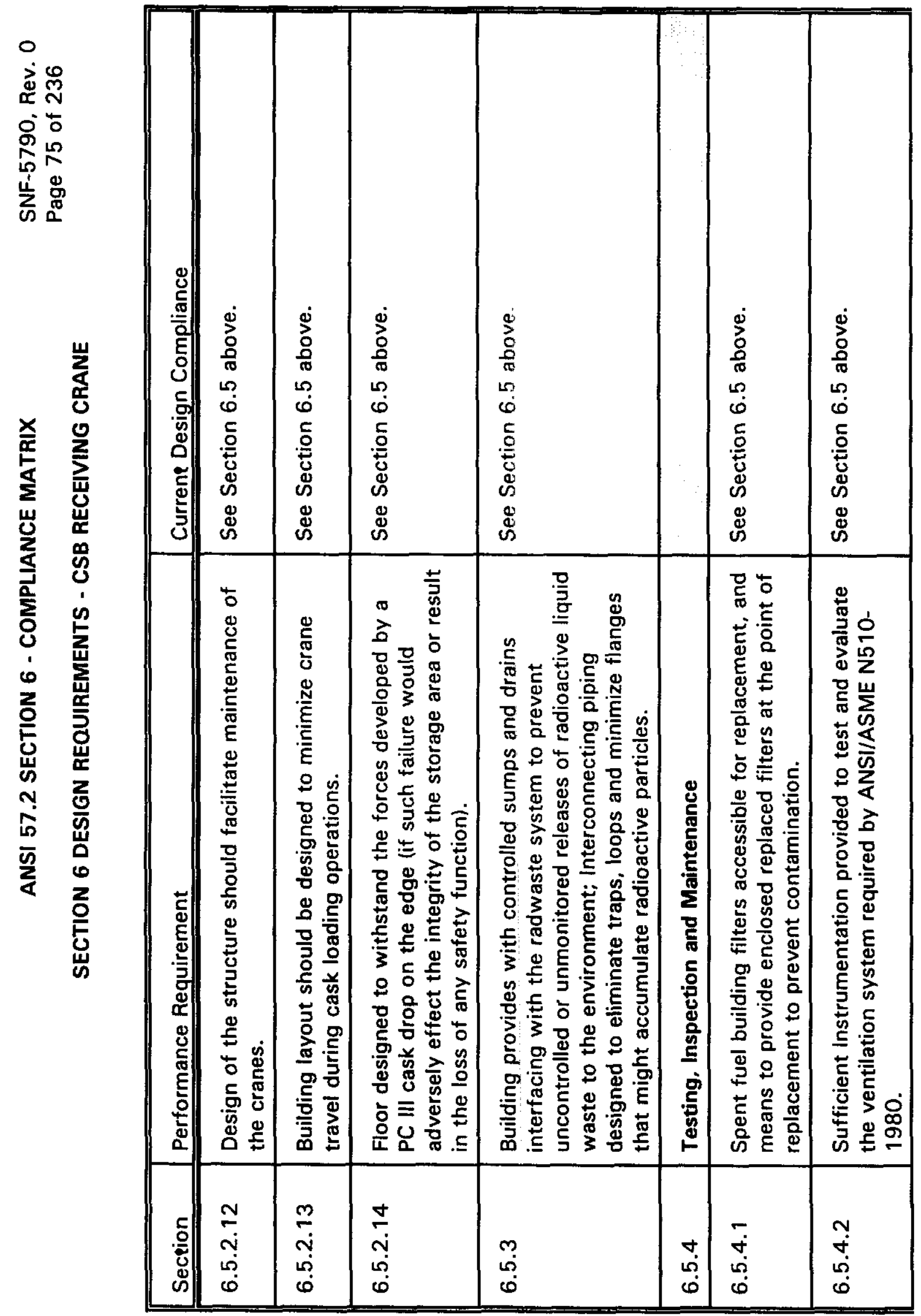




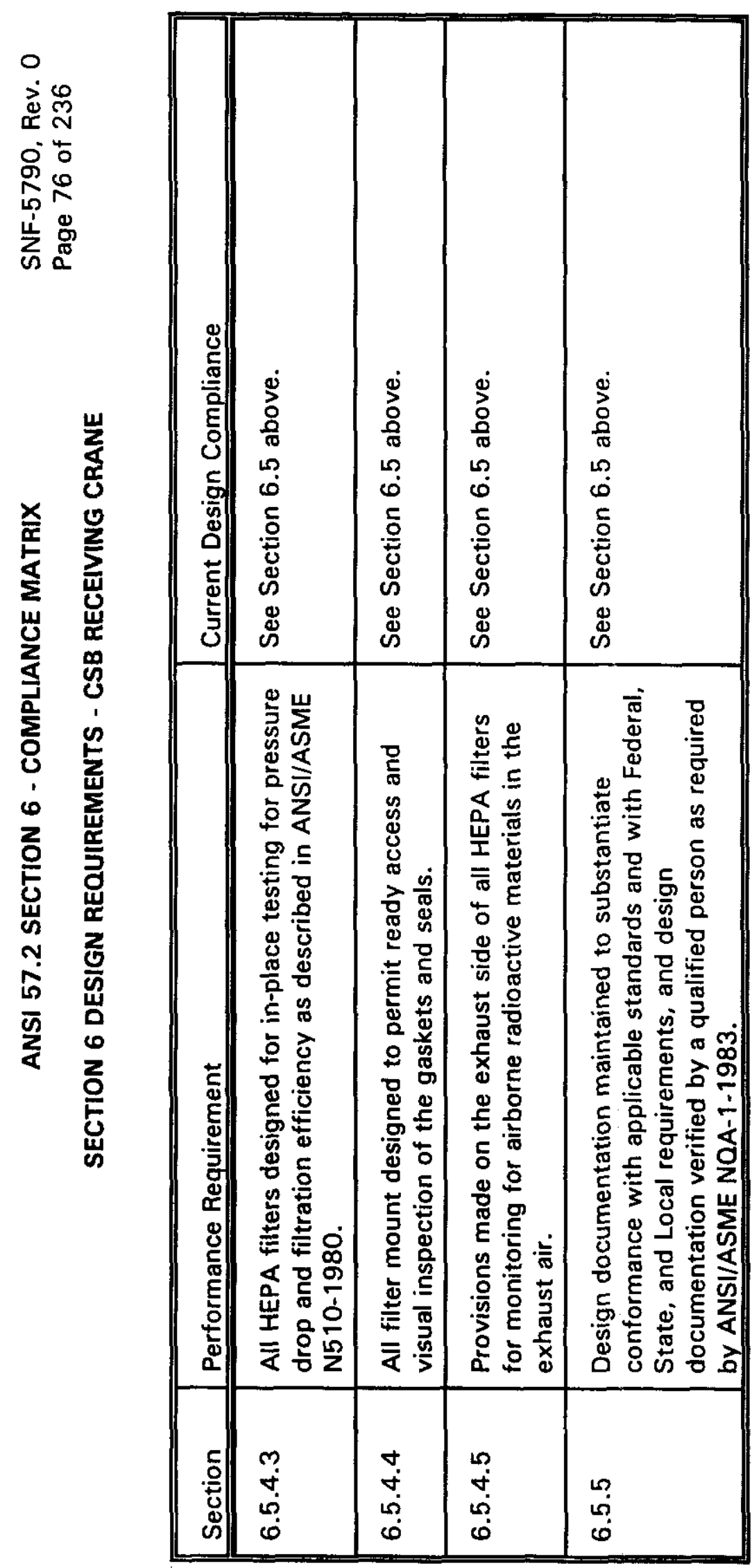




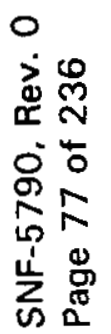

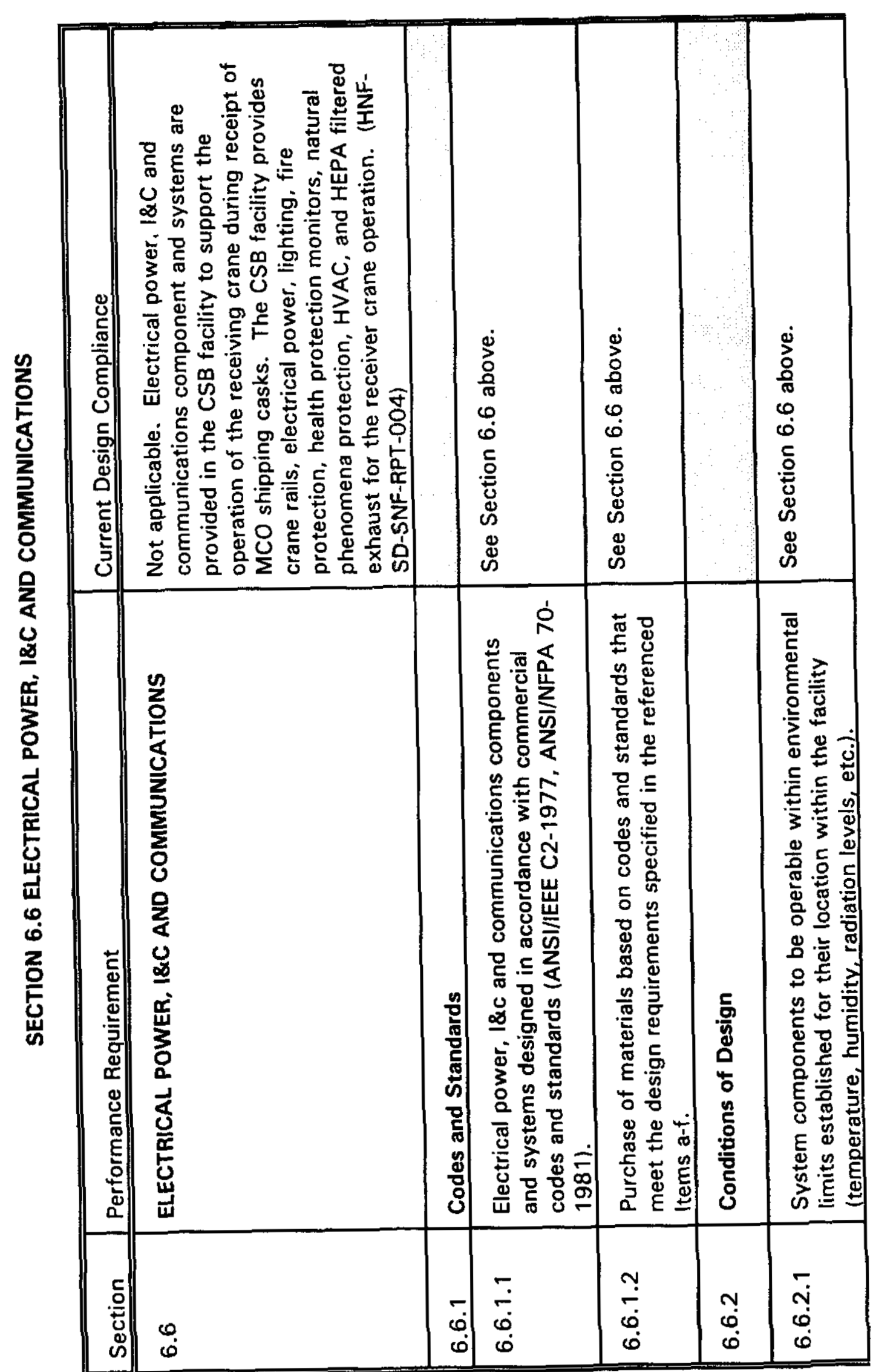




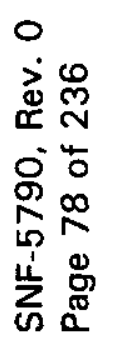

\begin{tabular}{|c|c|c|c|c|c|c|c|}
\hline 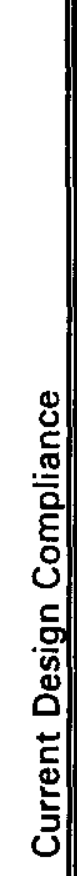 & 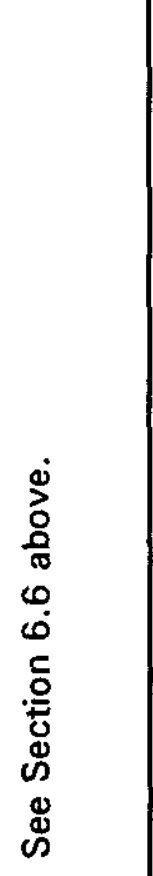 & 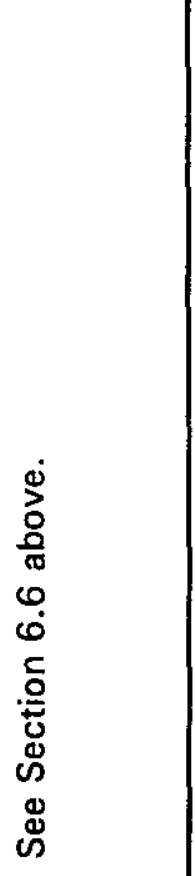 & 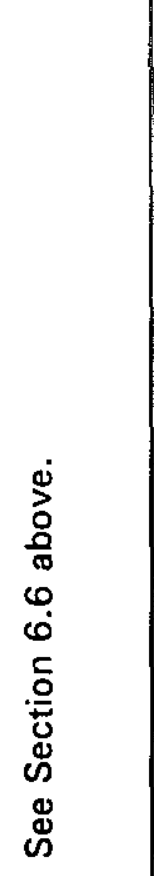 & 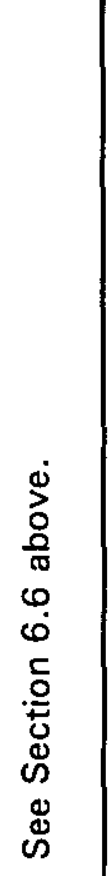 & 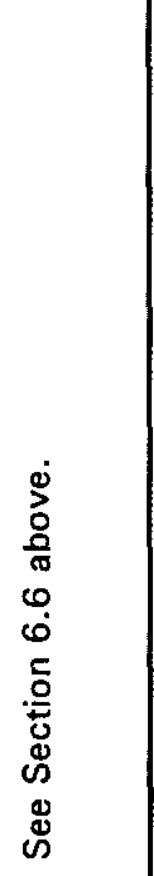 & & 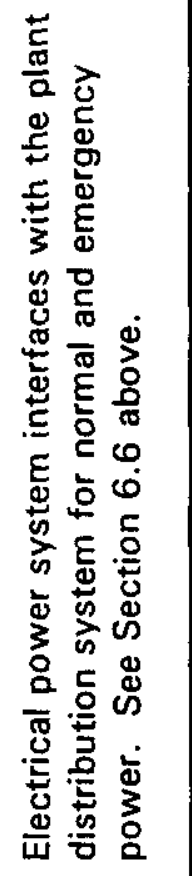 \\
\hline 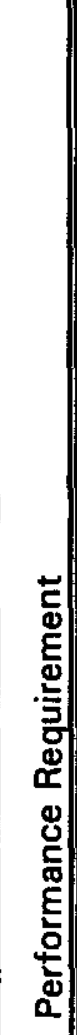 & 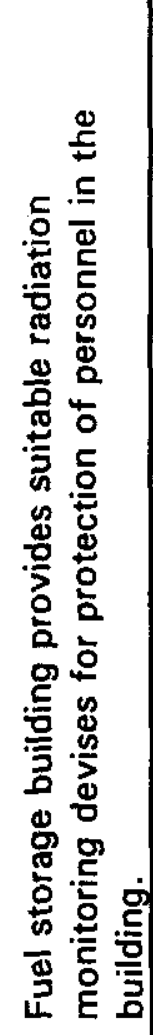 & 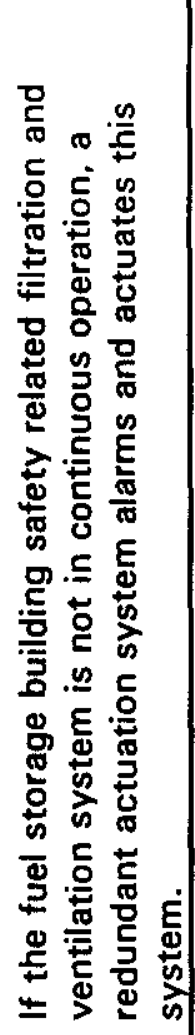 & 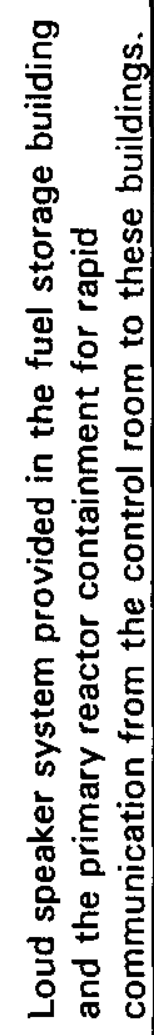 & 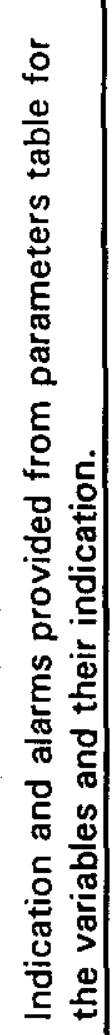 & 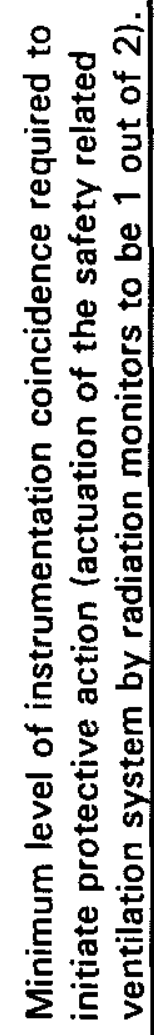 & 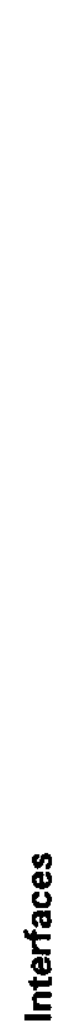 & 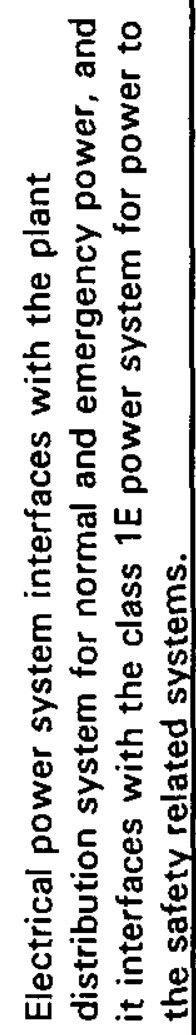 \\
\hline 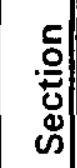 & $\begin{array}{l}N \\
\sim \\
0 \\
0\end{array}$ & 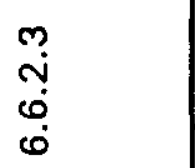 & $\begin{array}{l}+ \\
\stackrel{+}{0} \\
\dot{0} \\
\dot{0}\end{array}$ & $\begin{array}{l}\text { ס } \\
\text { ஸे } \\
0\end{array}$ & $\begin{array}{l}\infty \\
\text { ஸे } \\
\omega \\
0\end{array}$ & "ٌ & $\overrightarrow{\dot{m}}$ \\
\hline
\end{tabular}




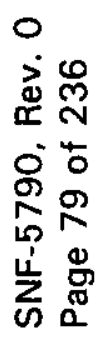

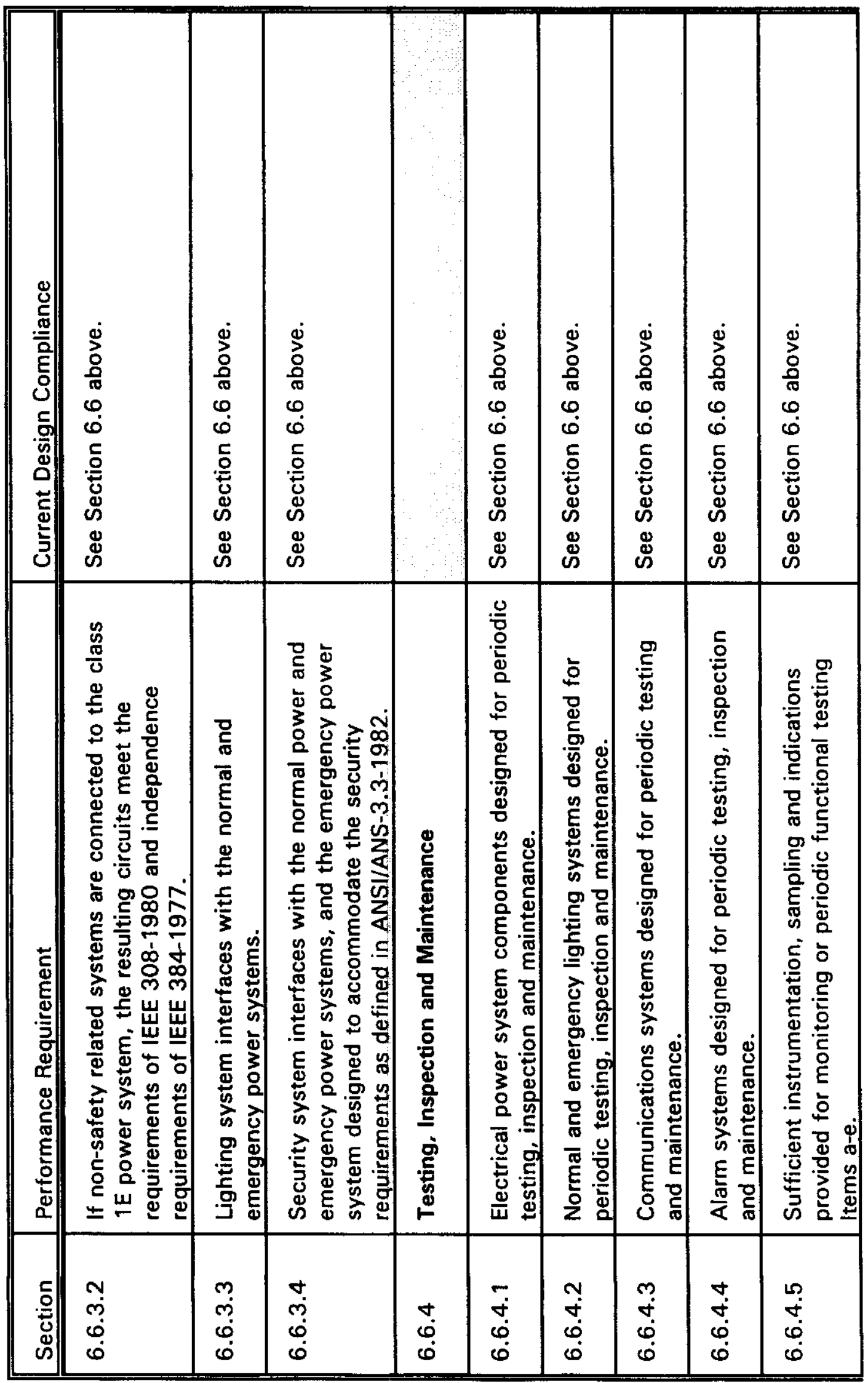




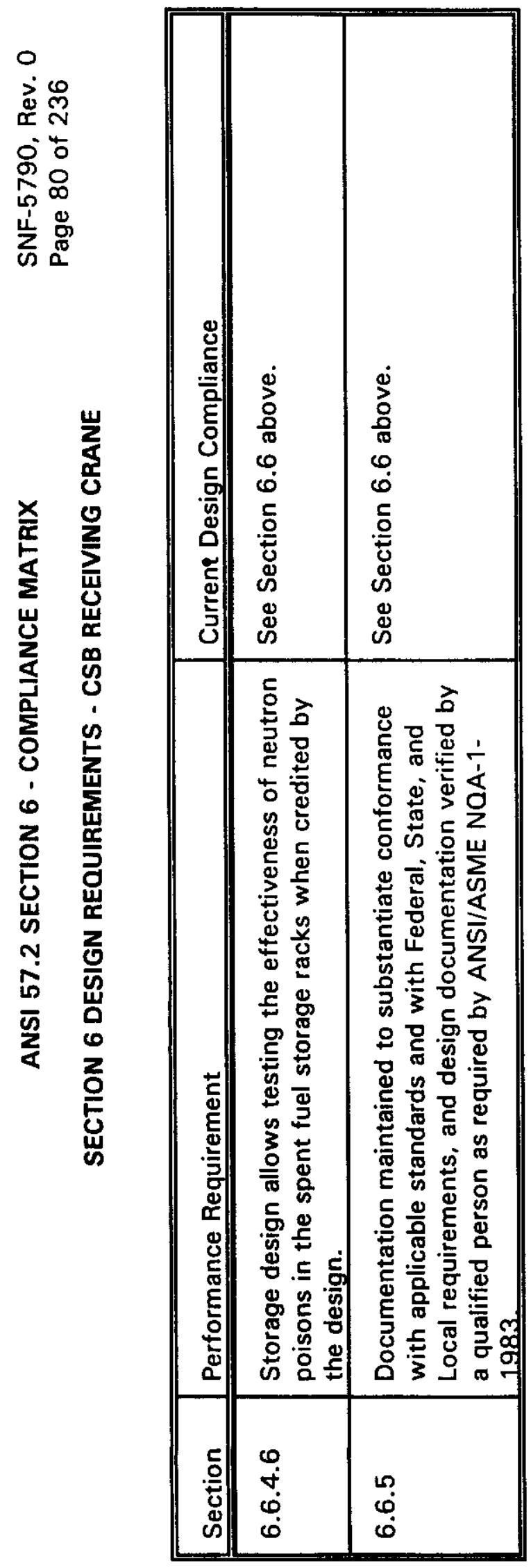


SNF-5790 Rev. 0

Page 81 of 236

\title{
ATTACHMENT 4
}

\author{
29 CFR 1910.179 \\ COMPLIANCE MATRIX
}




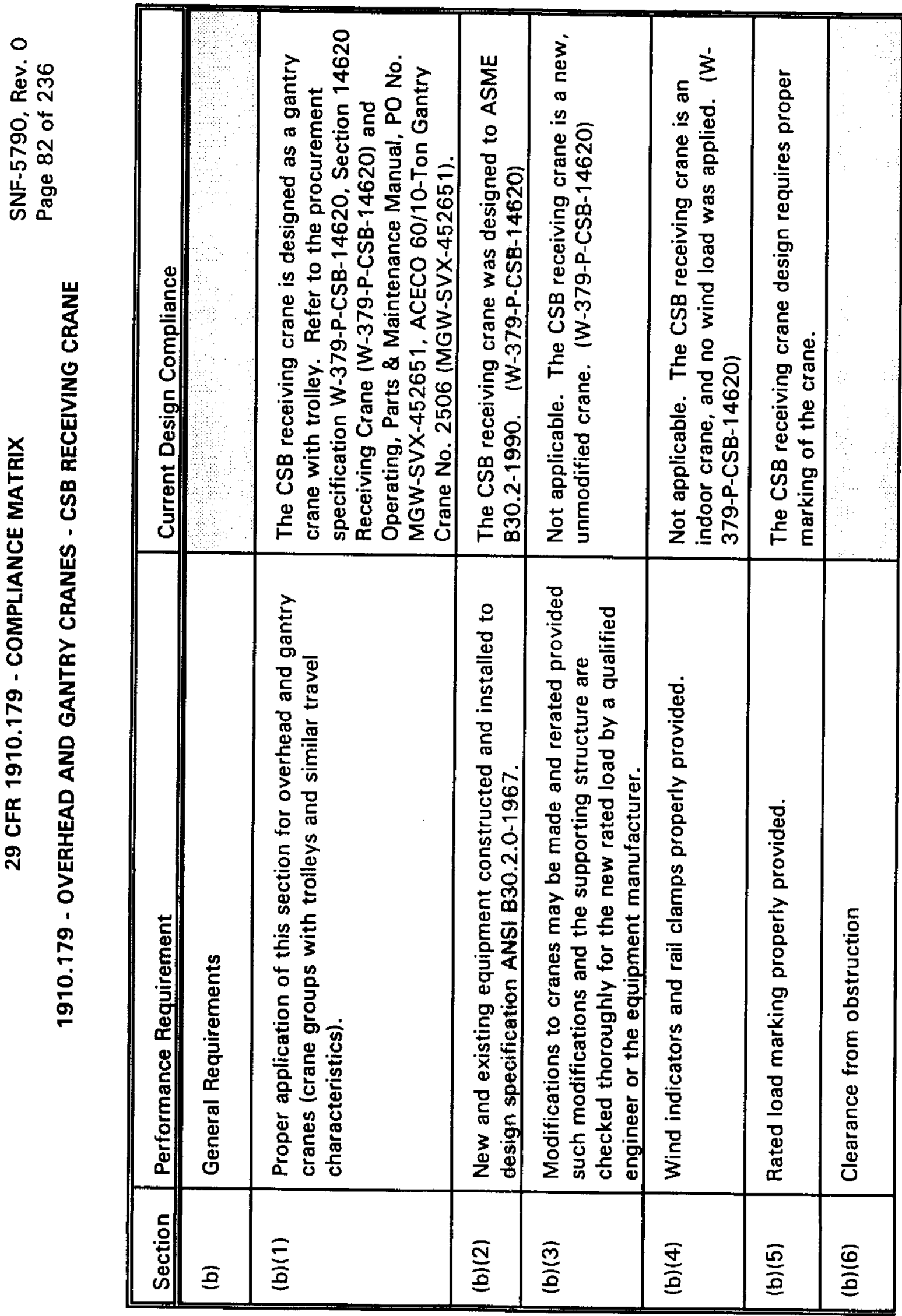




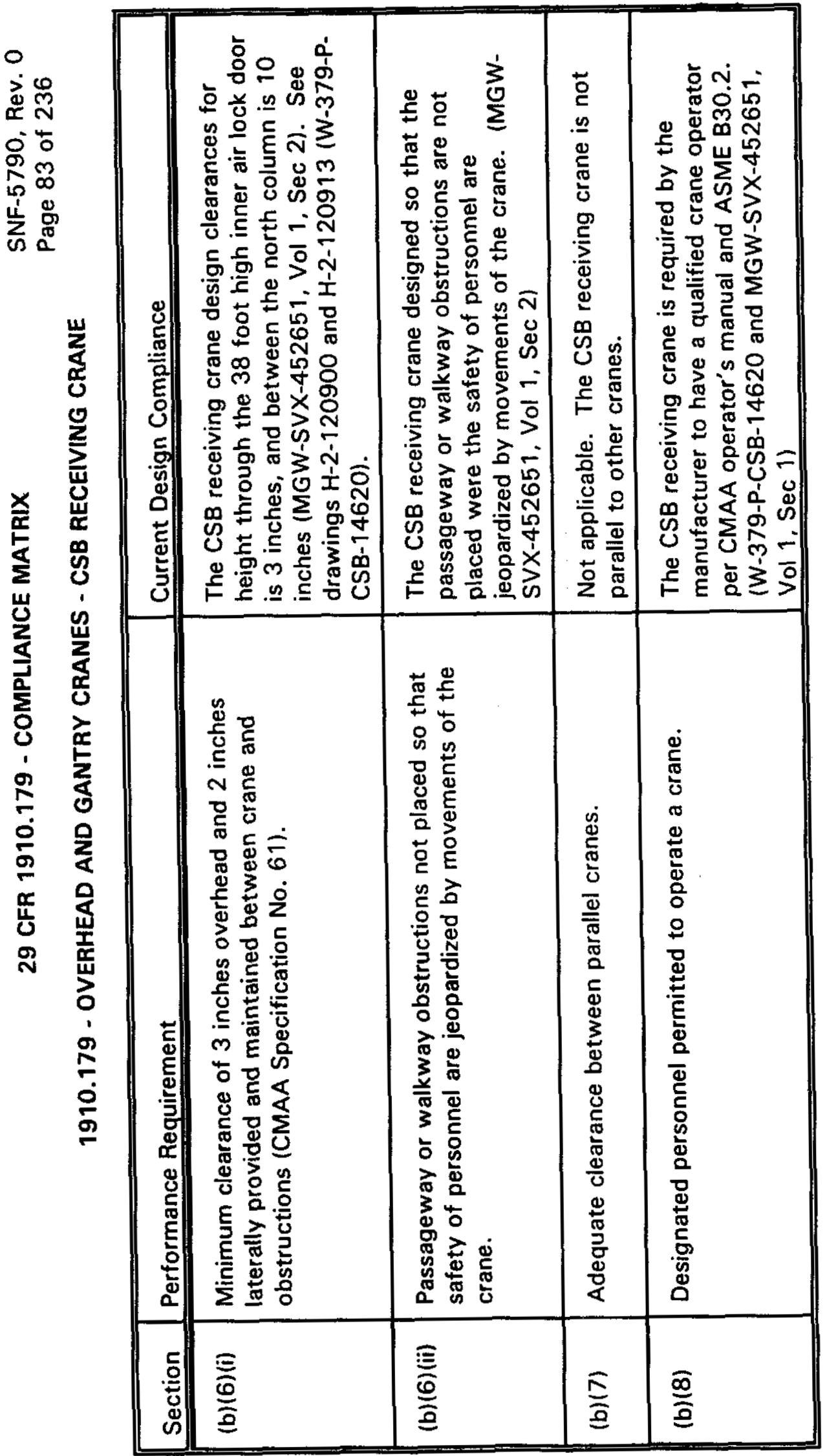




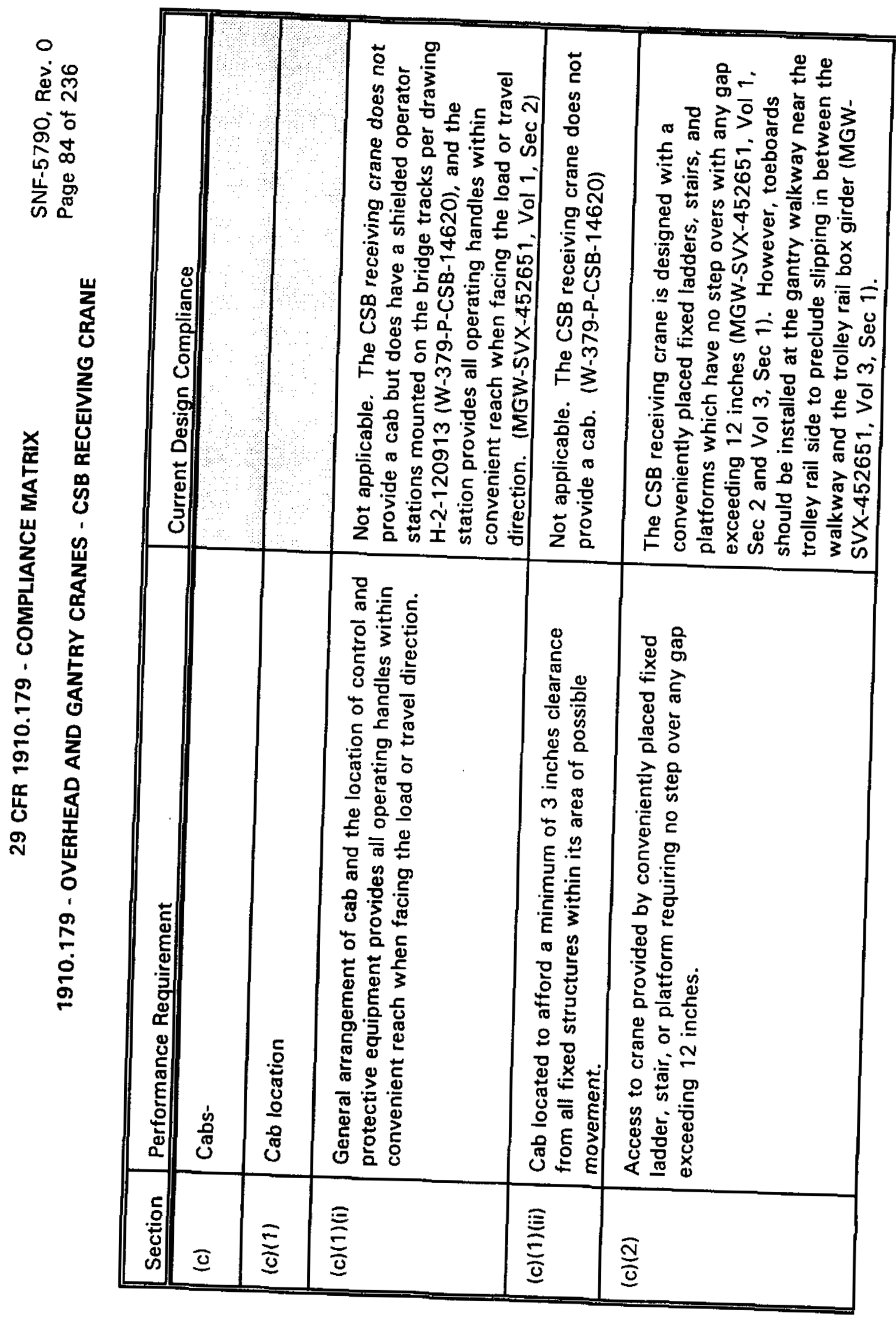




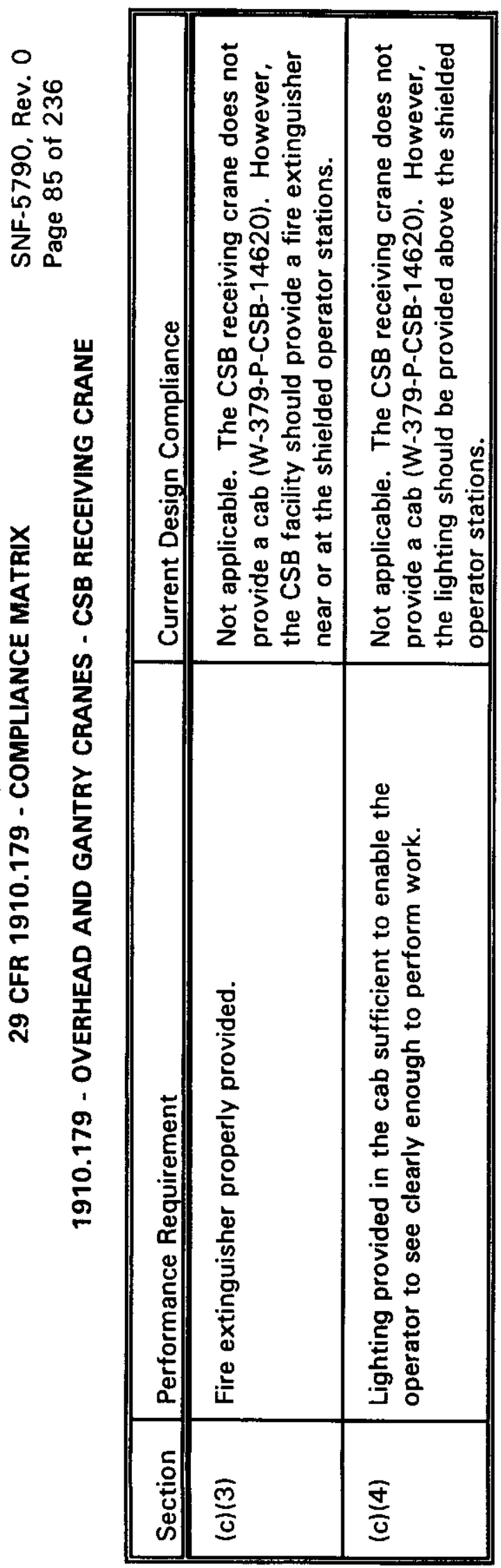




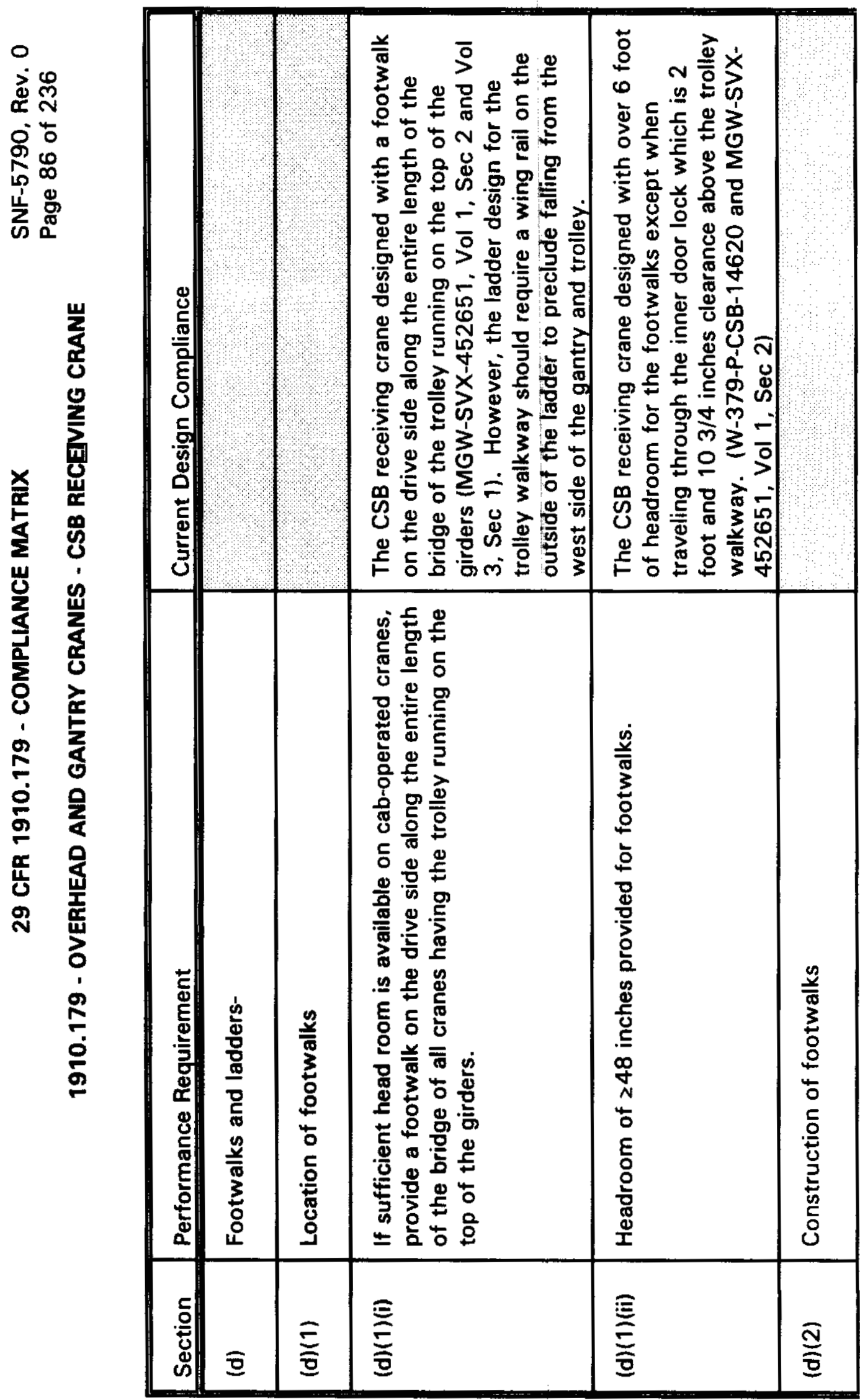




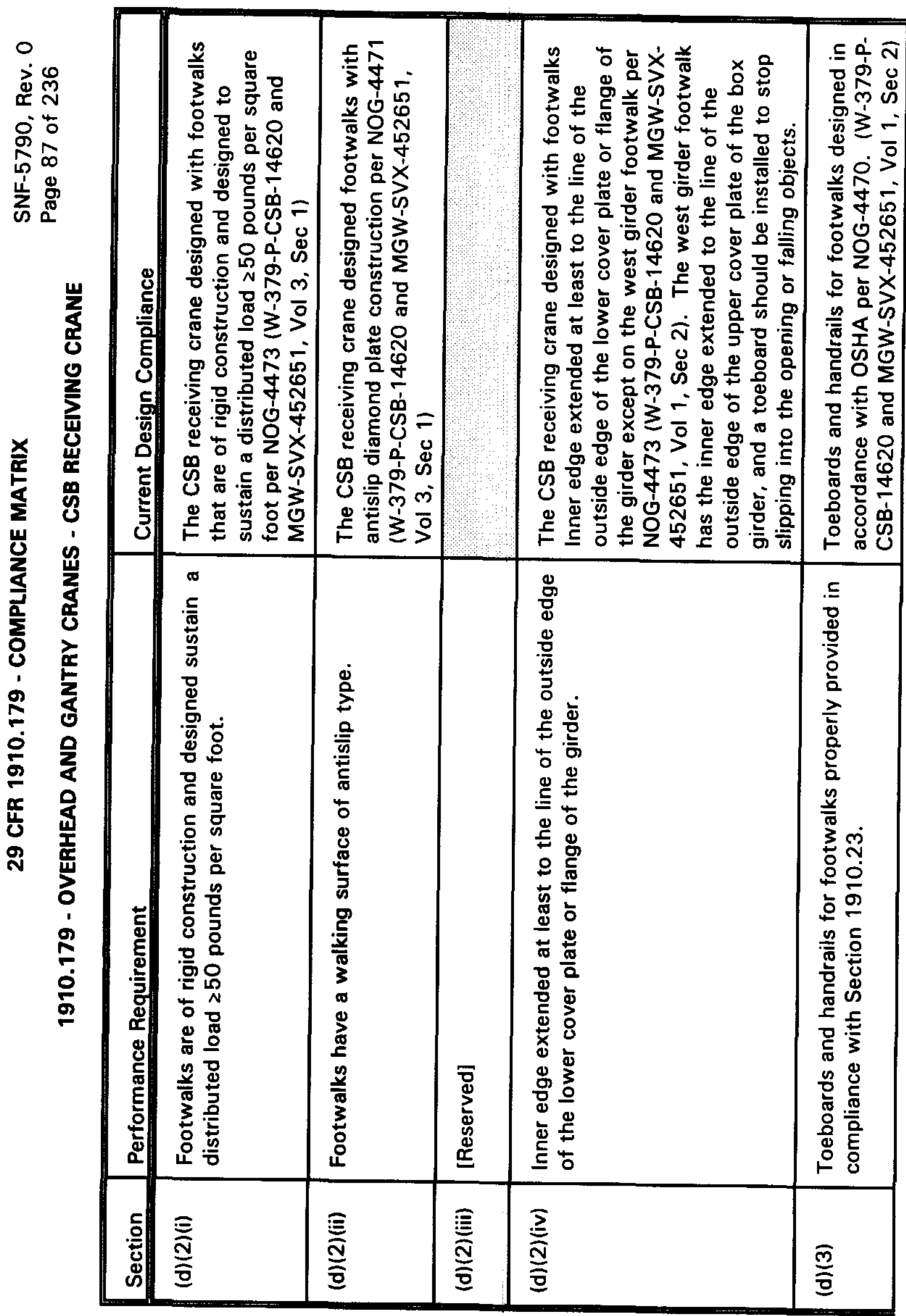




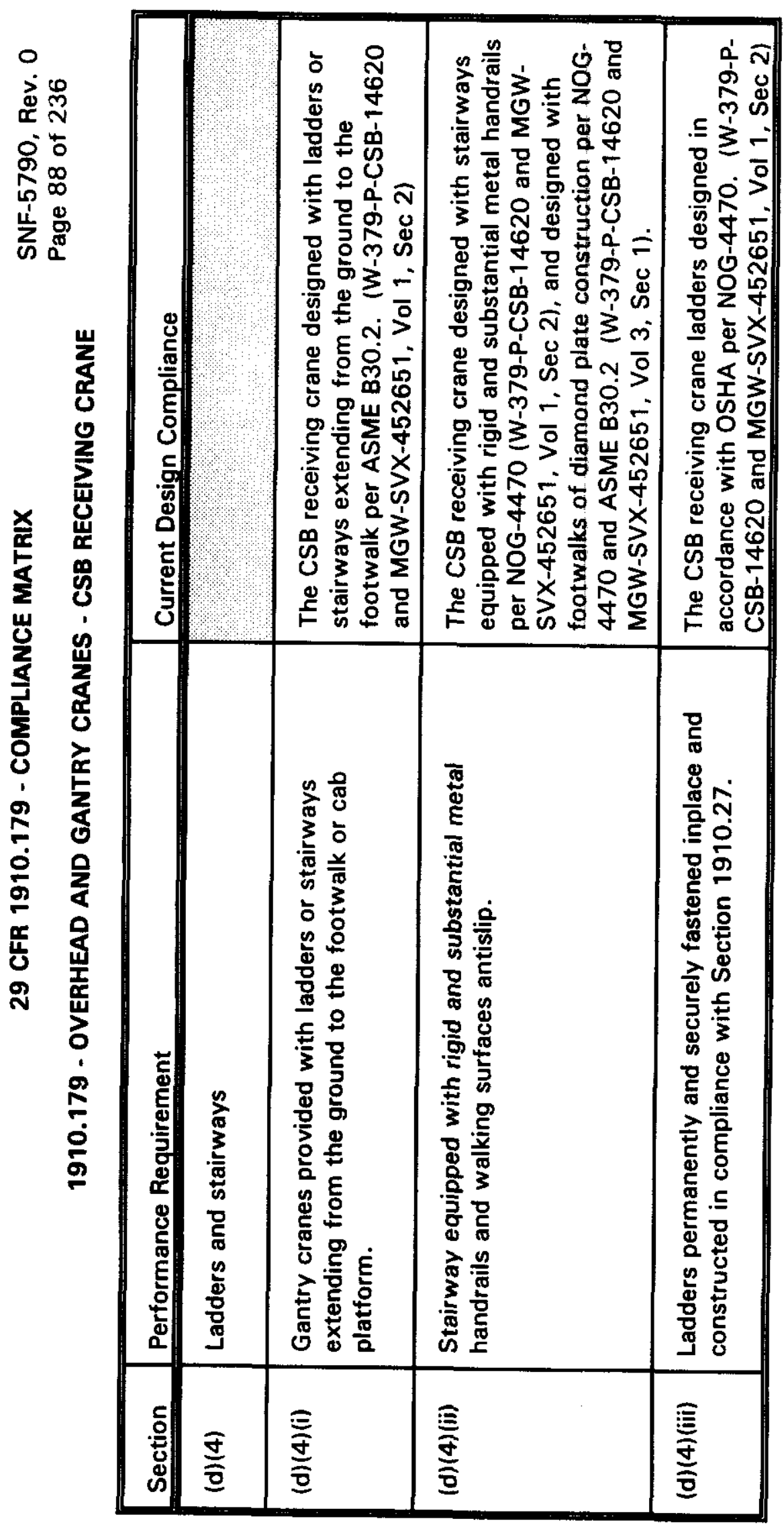




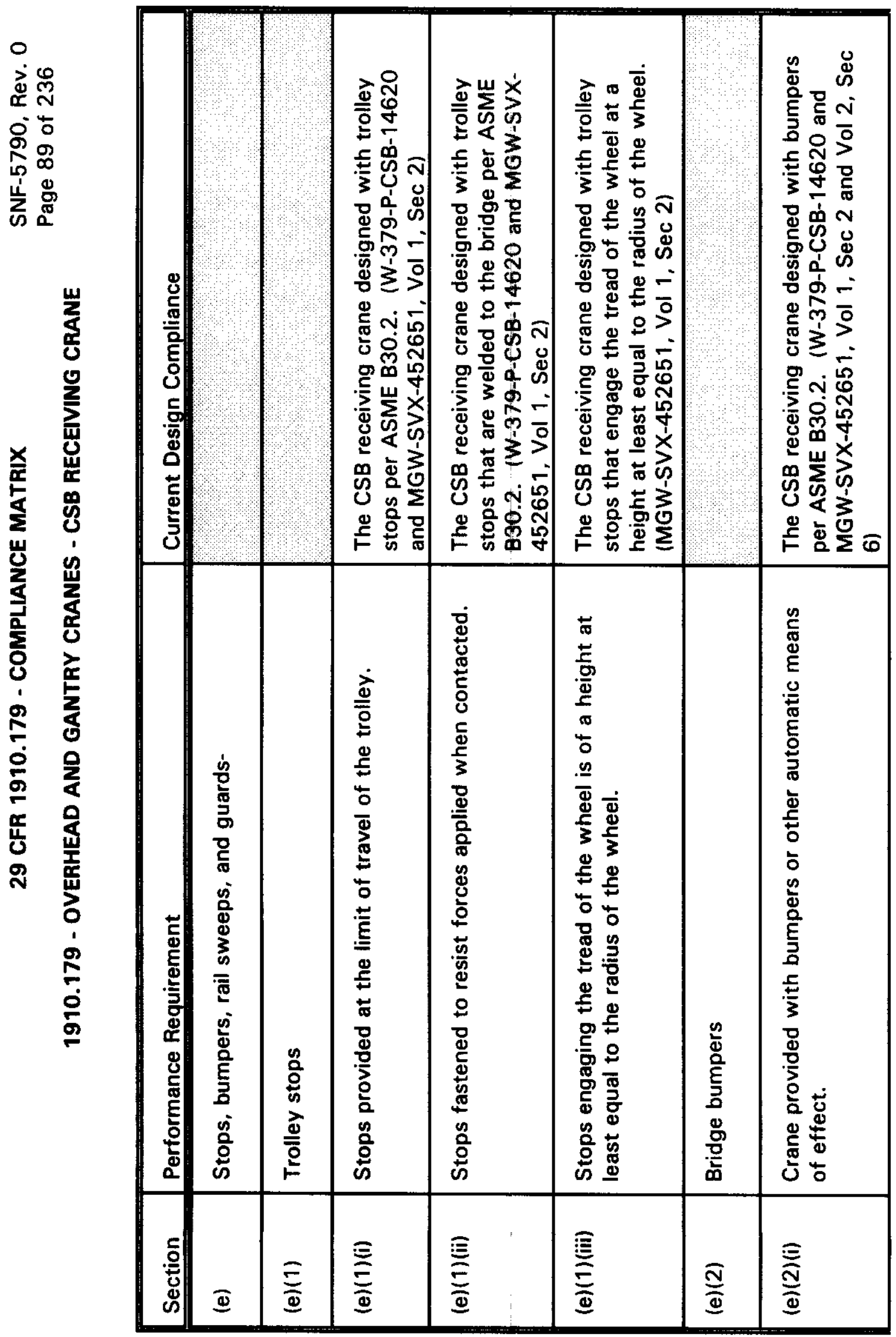




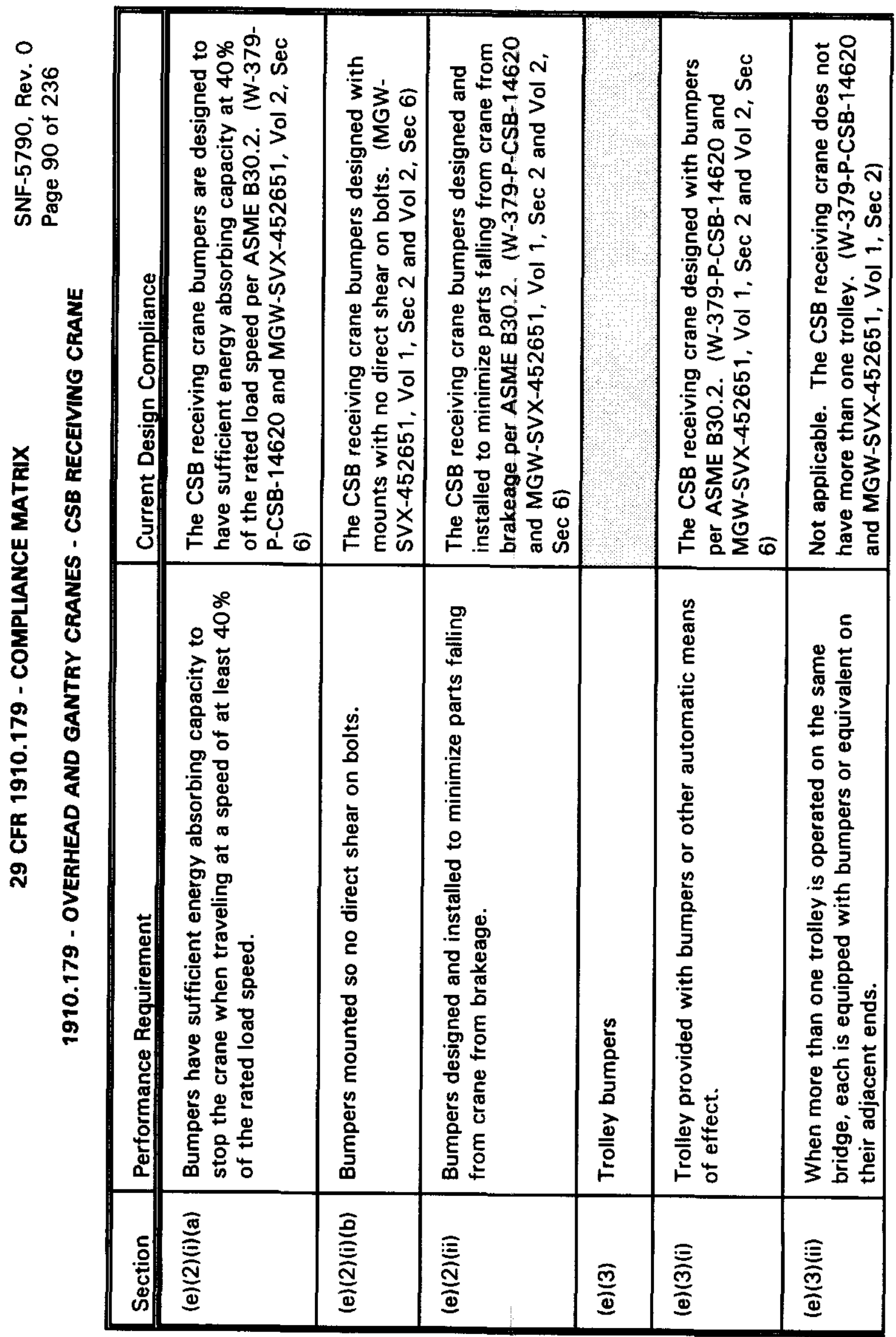




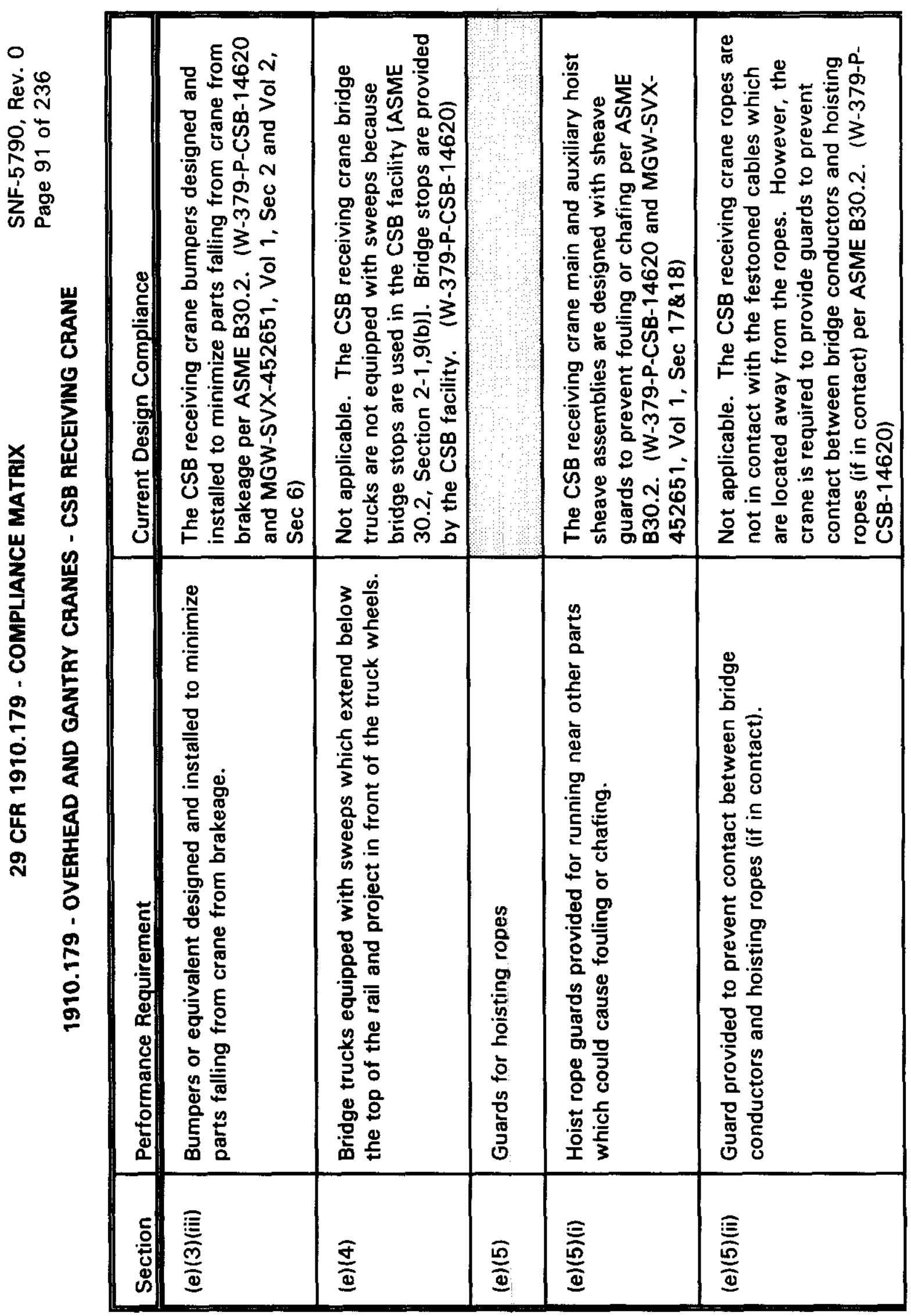




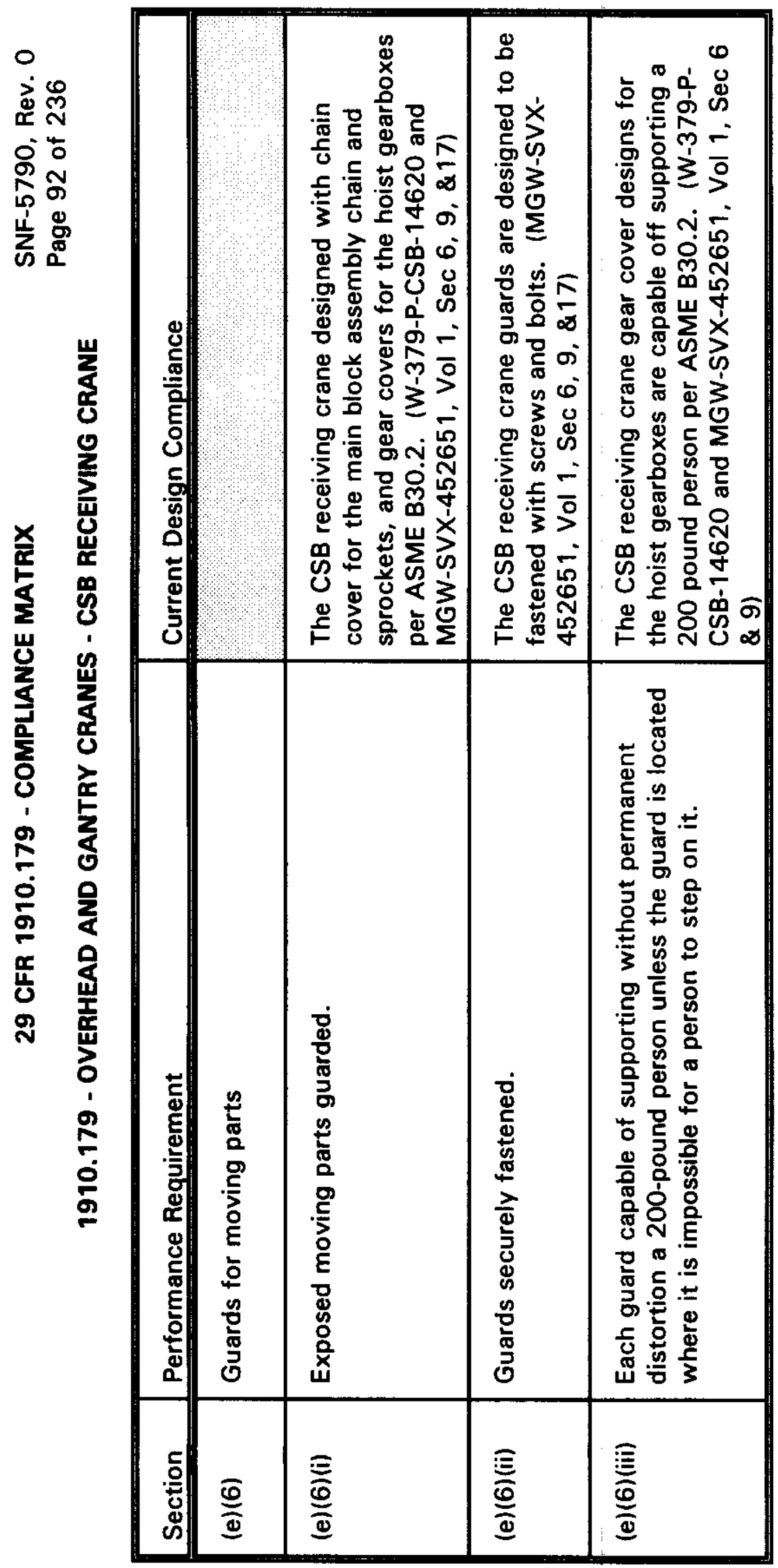




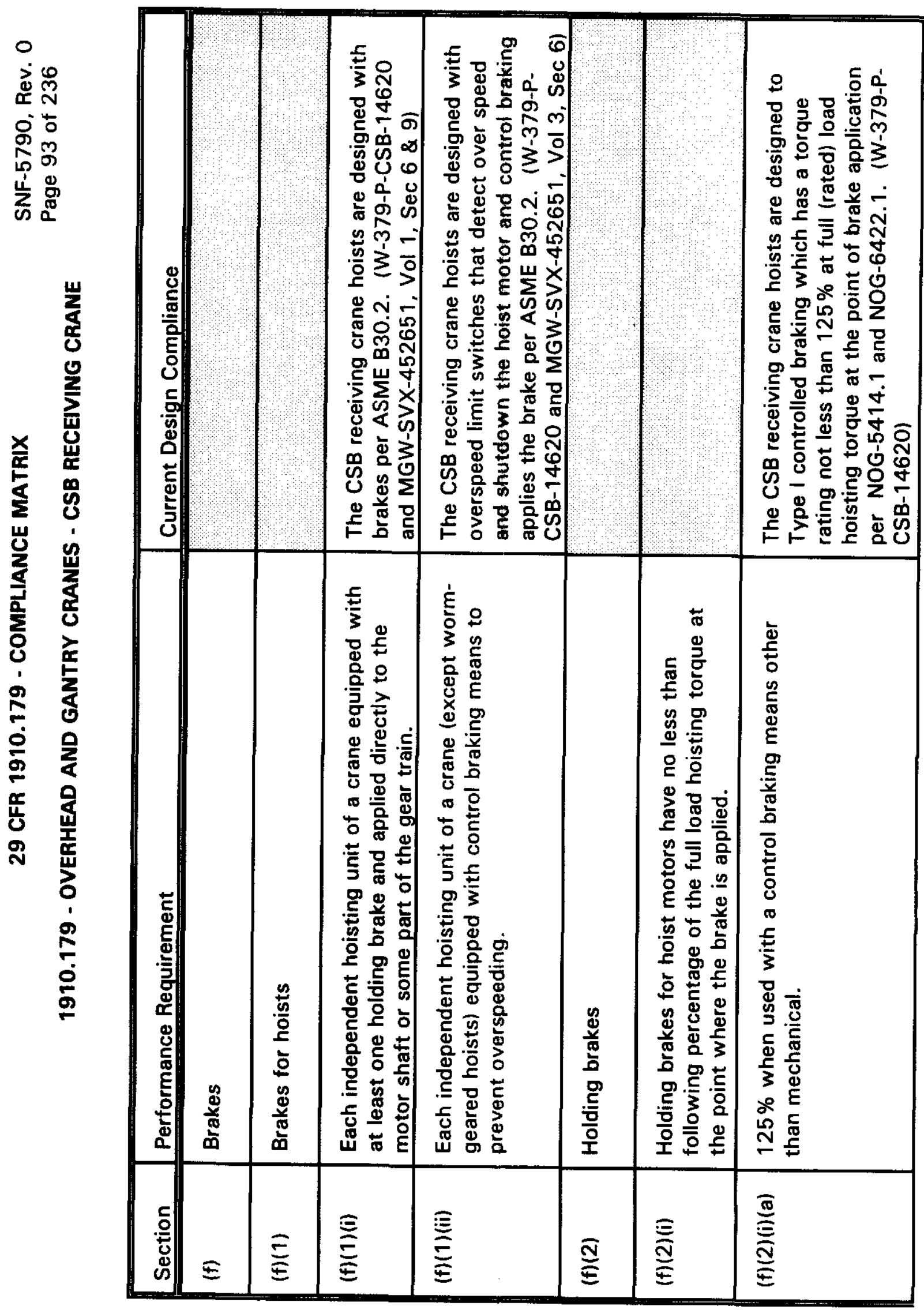




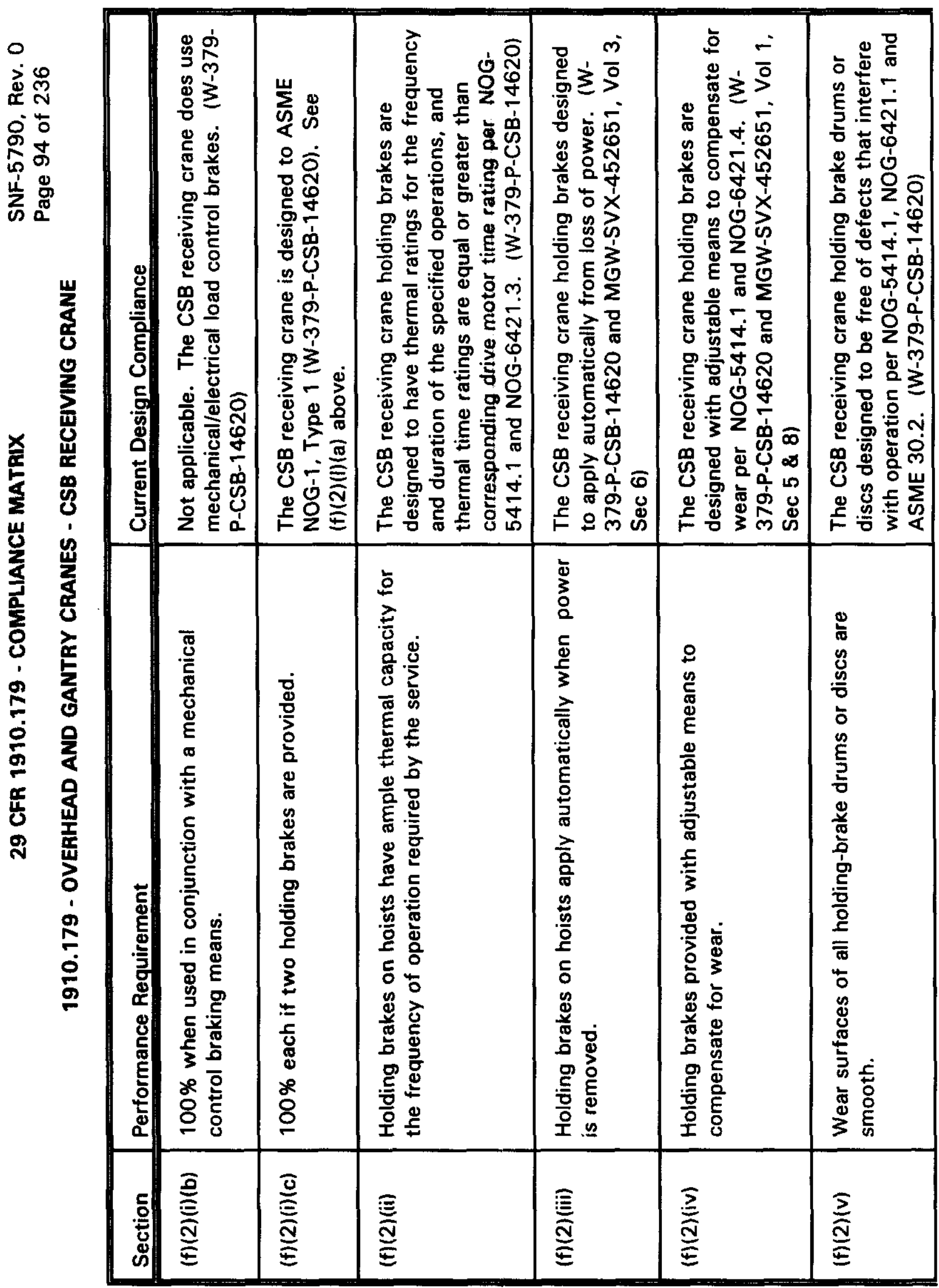




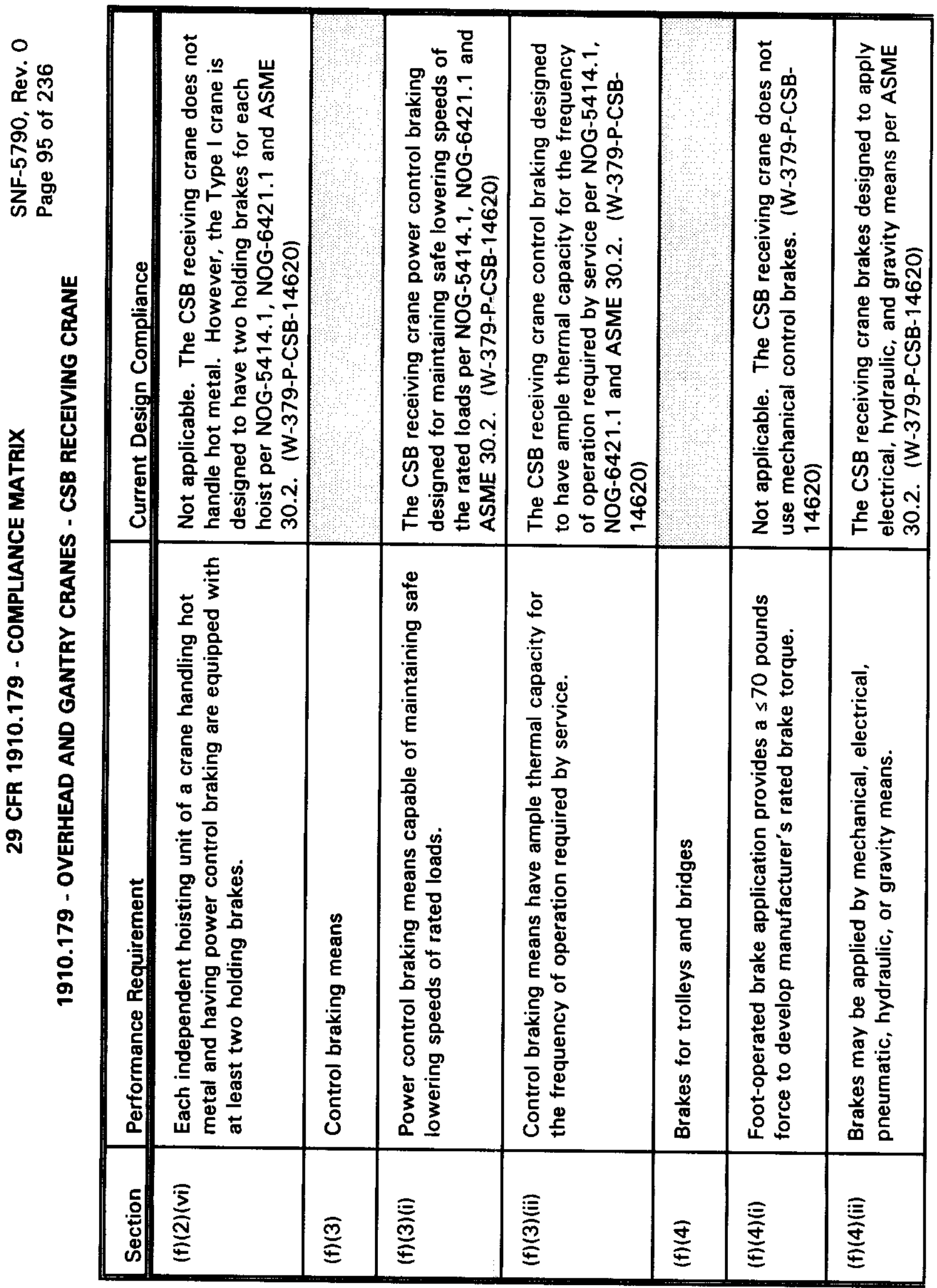




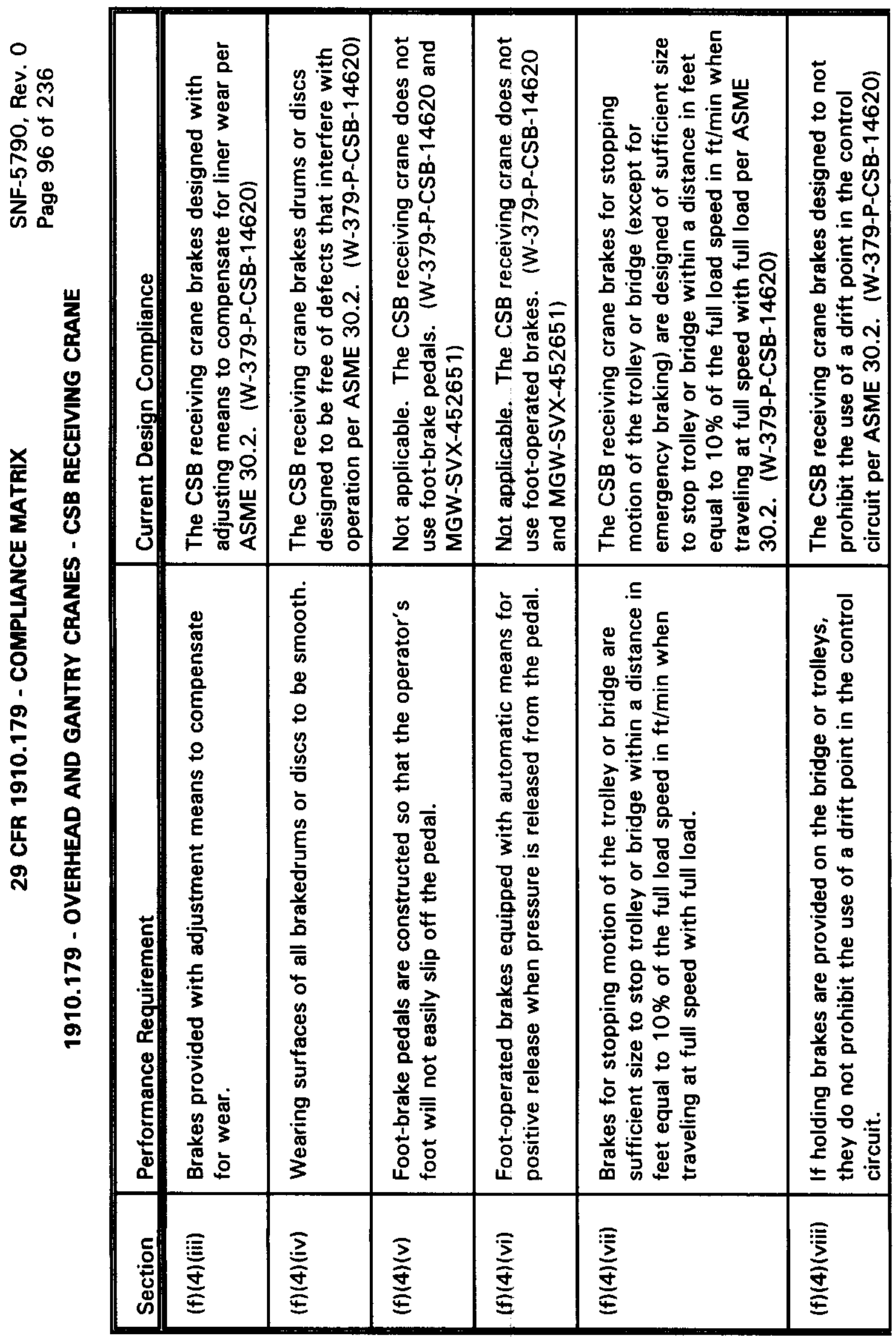




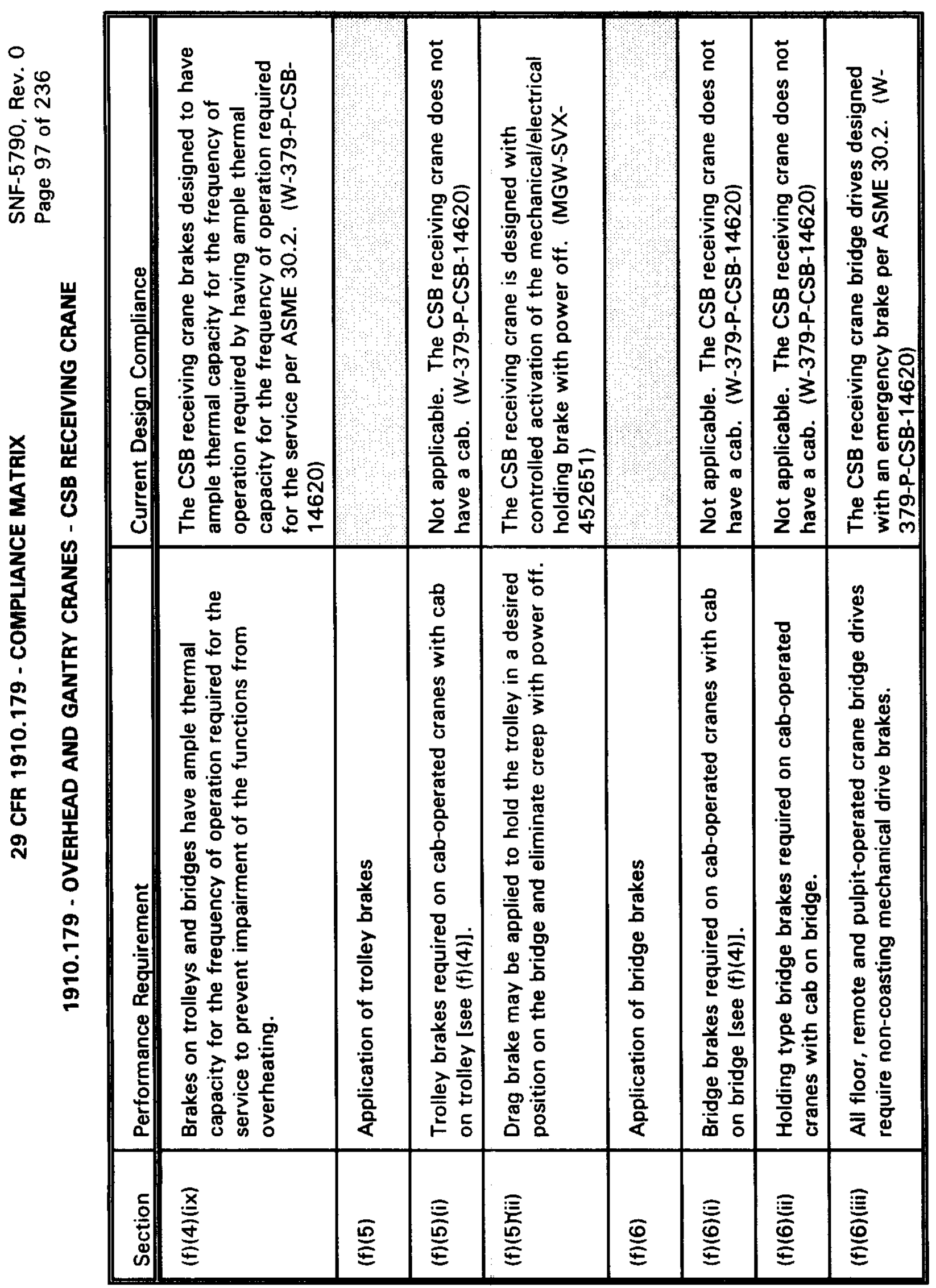




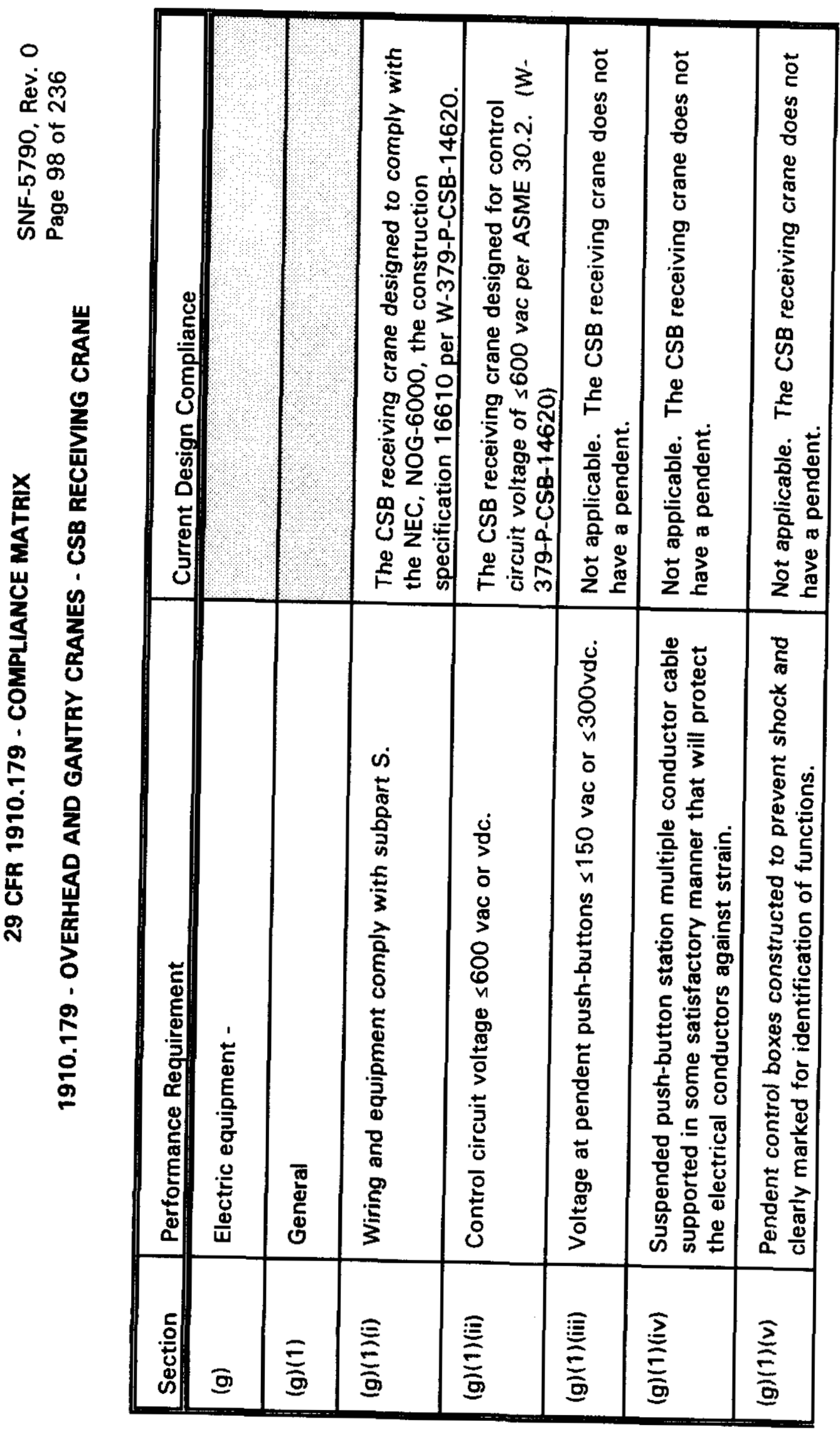




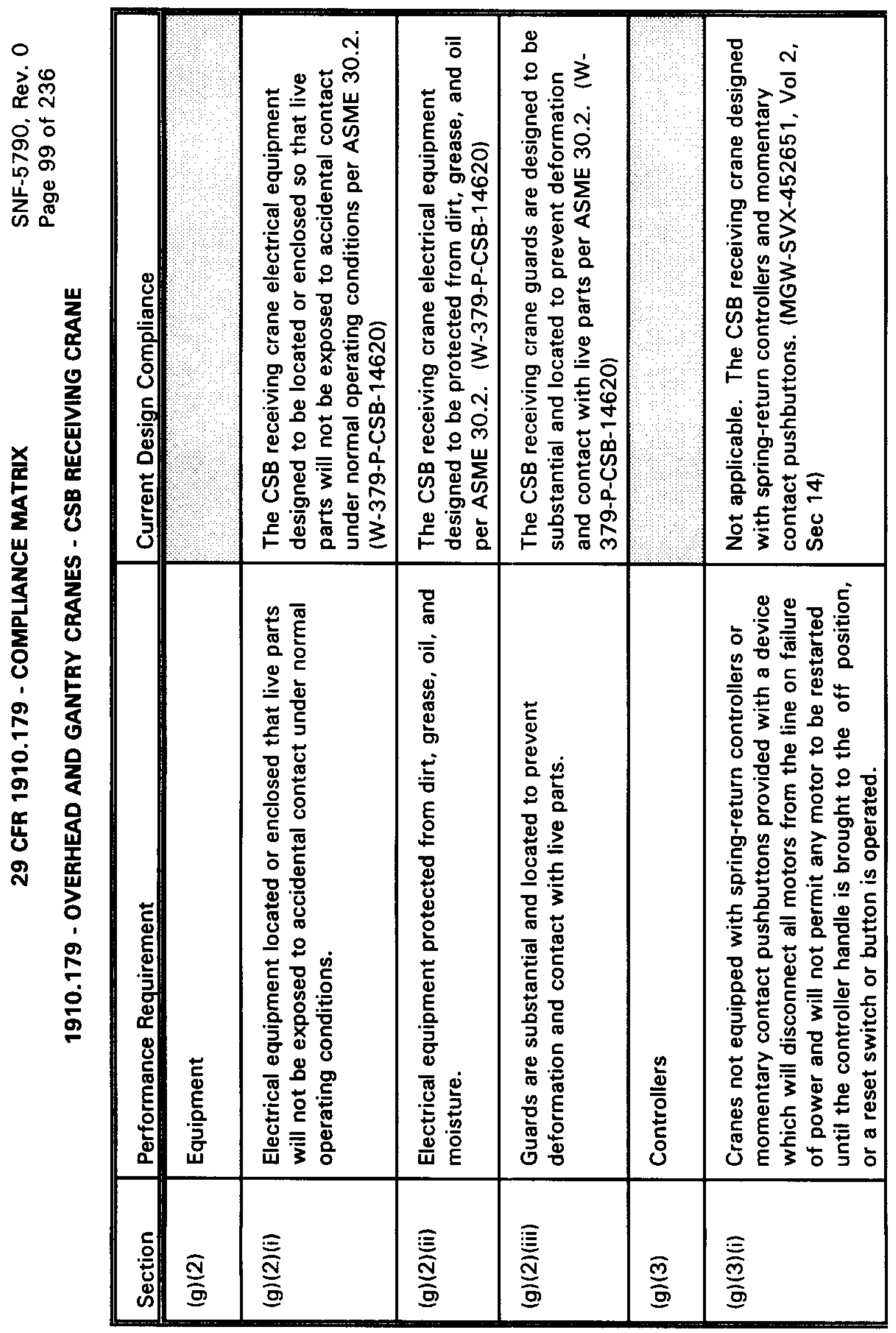




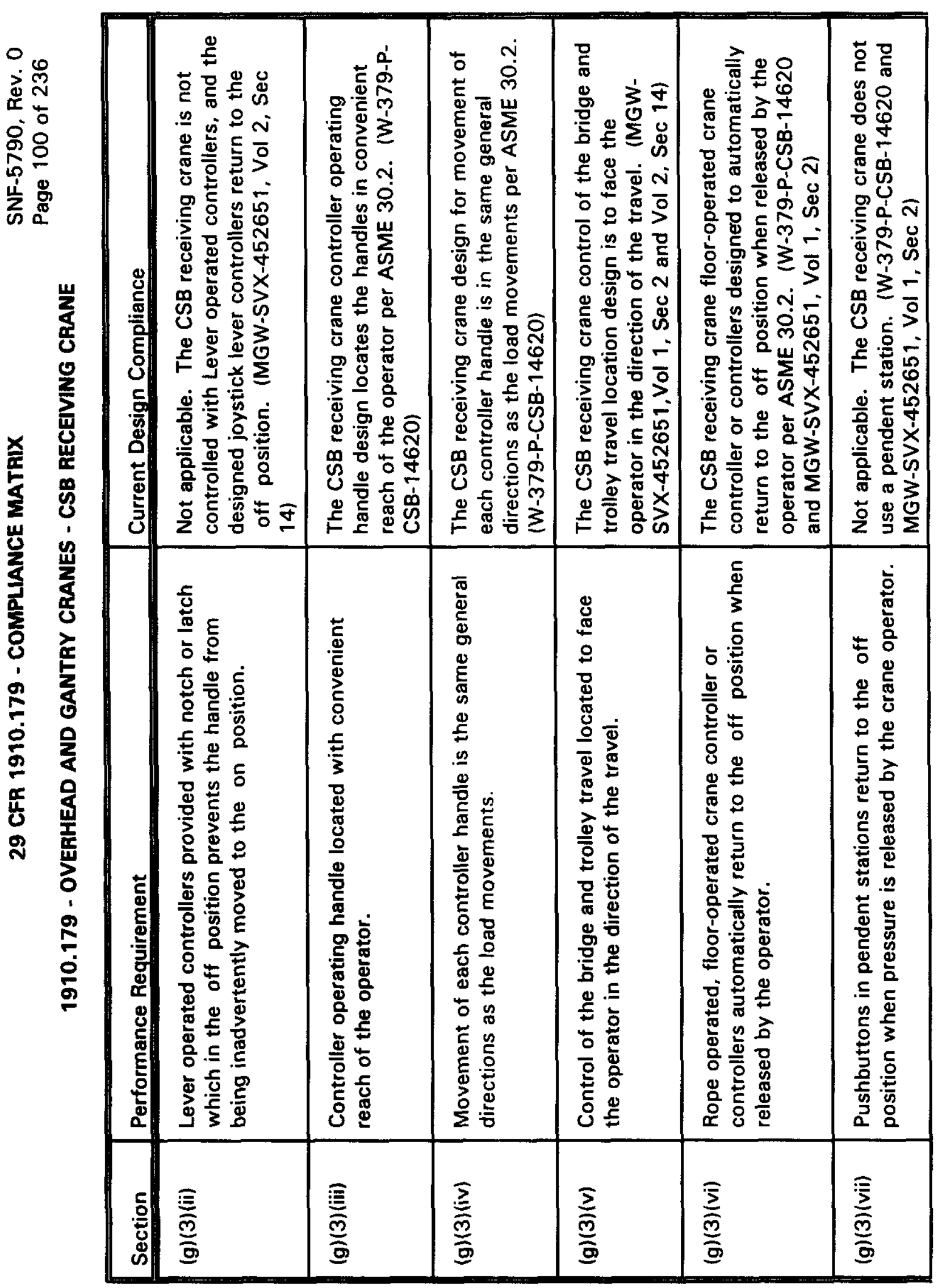




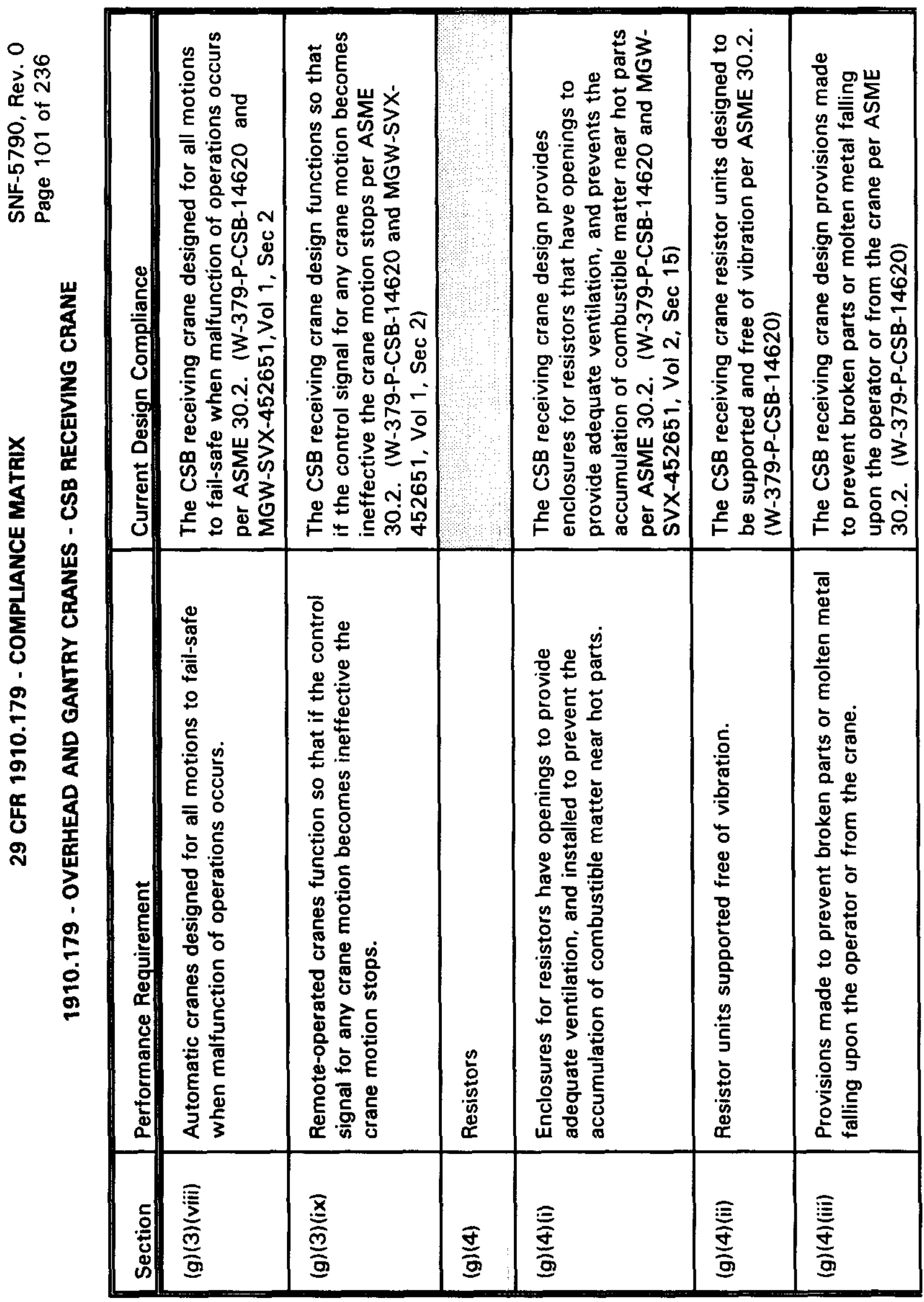




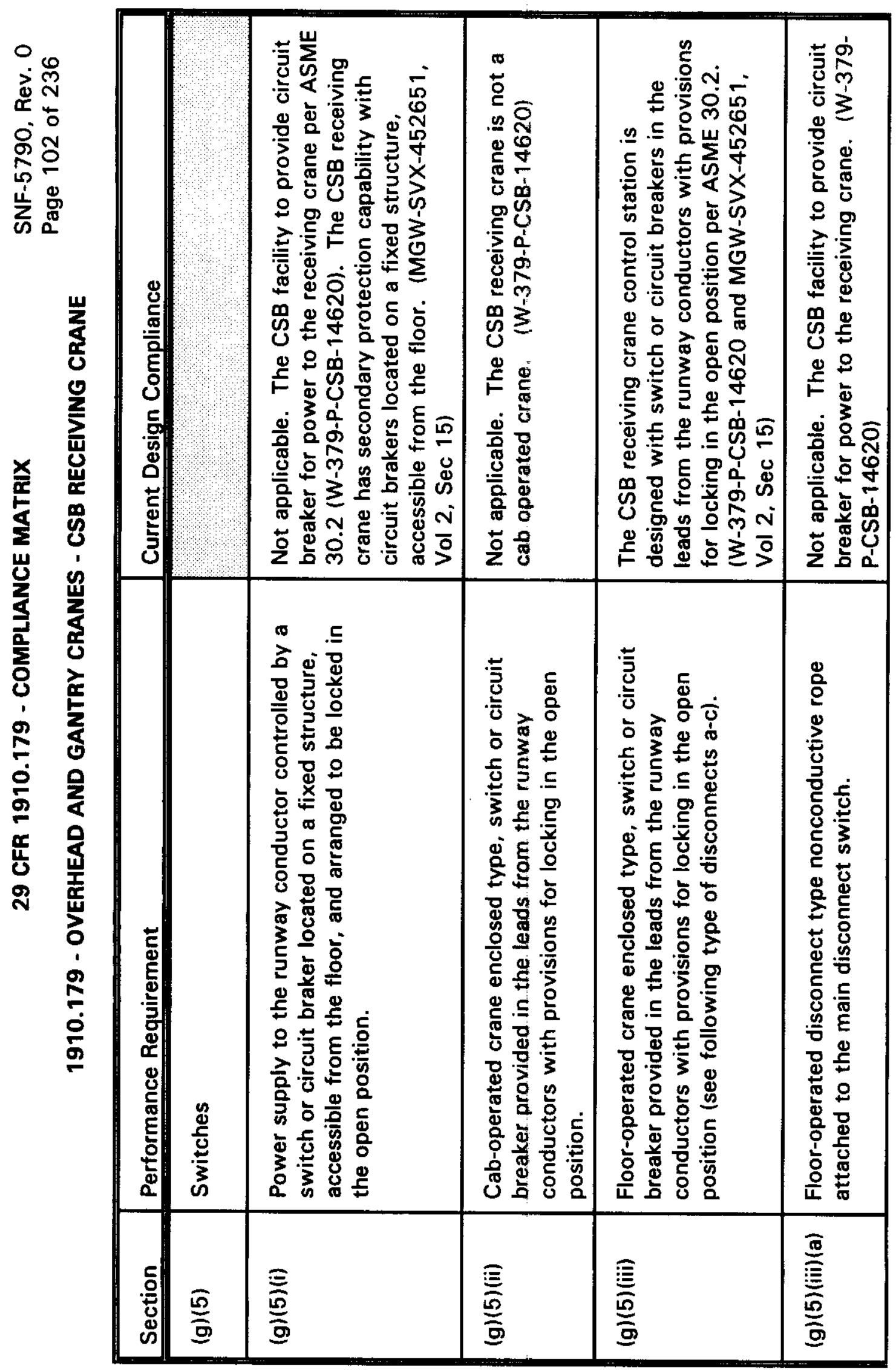




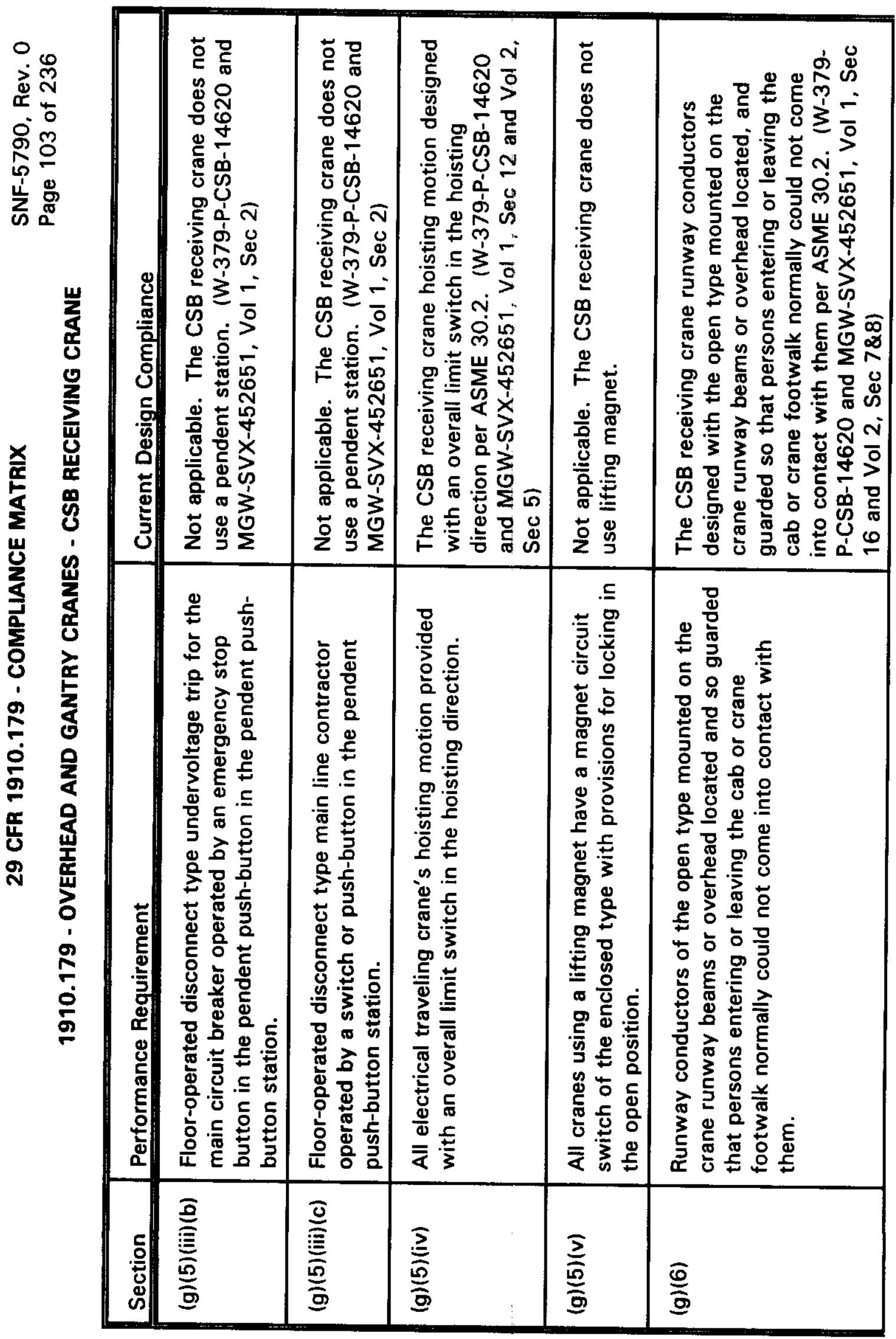




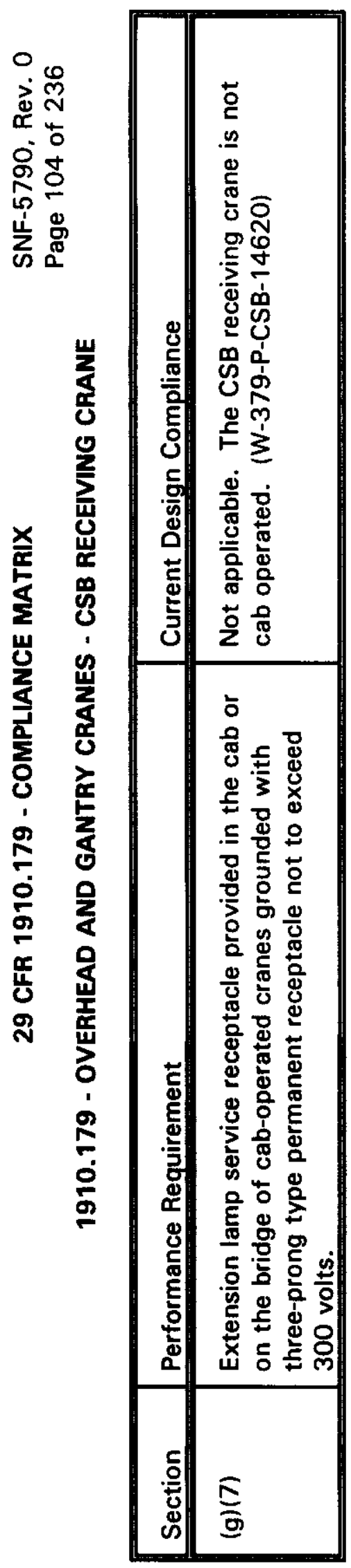




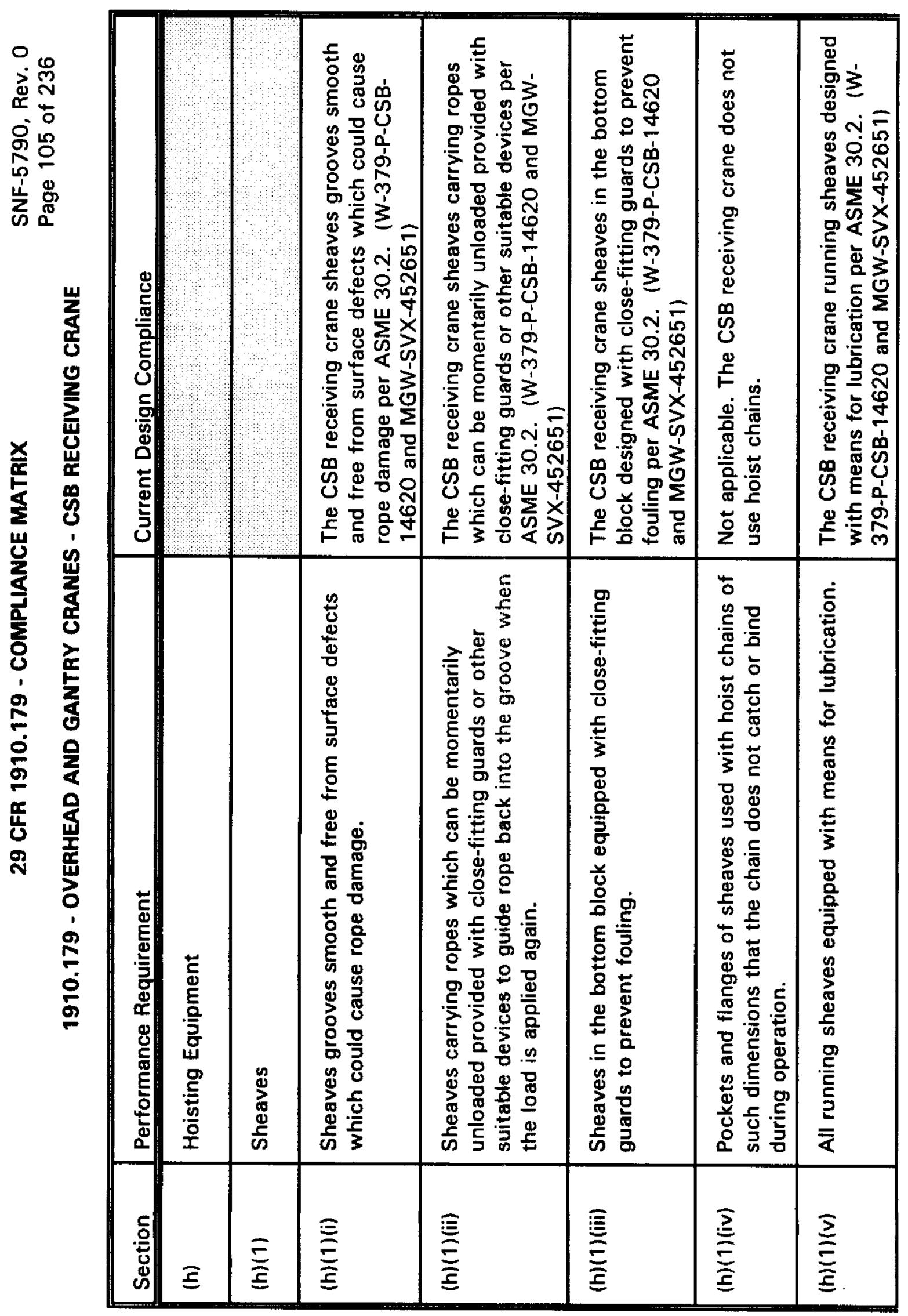




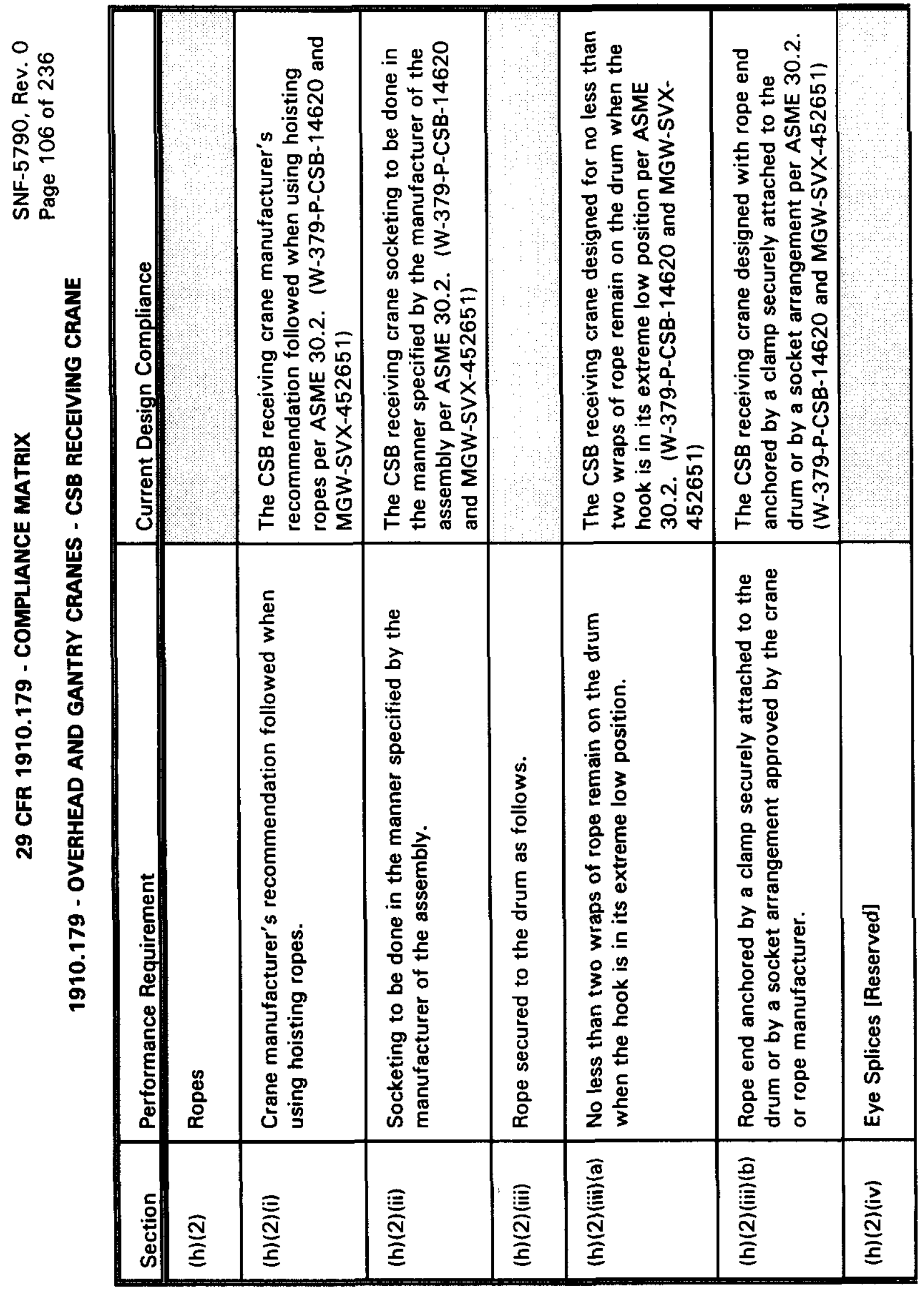




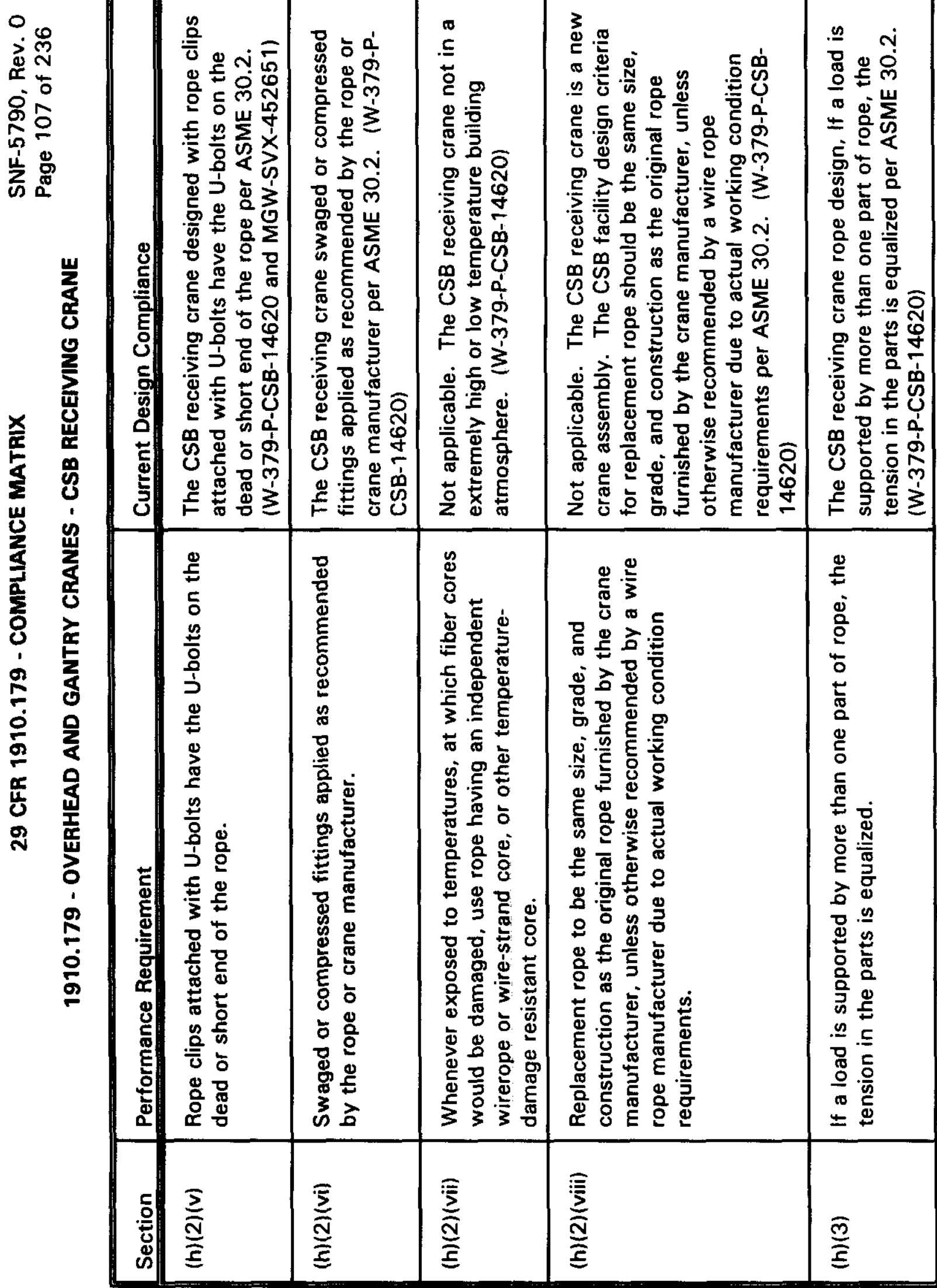




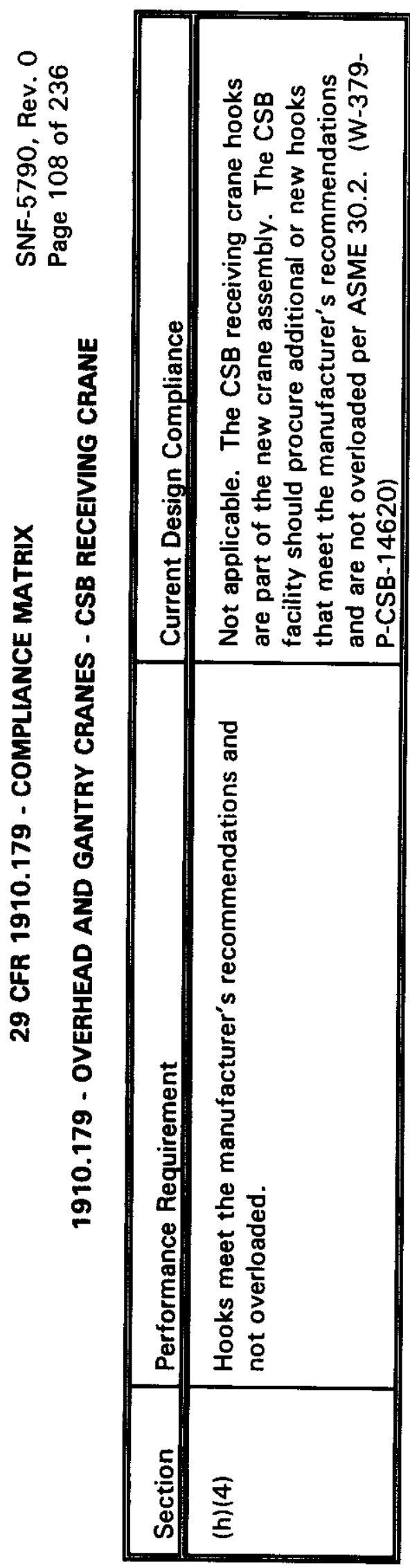




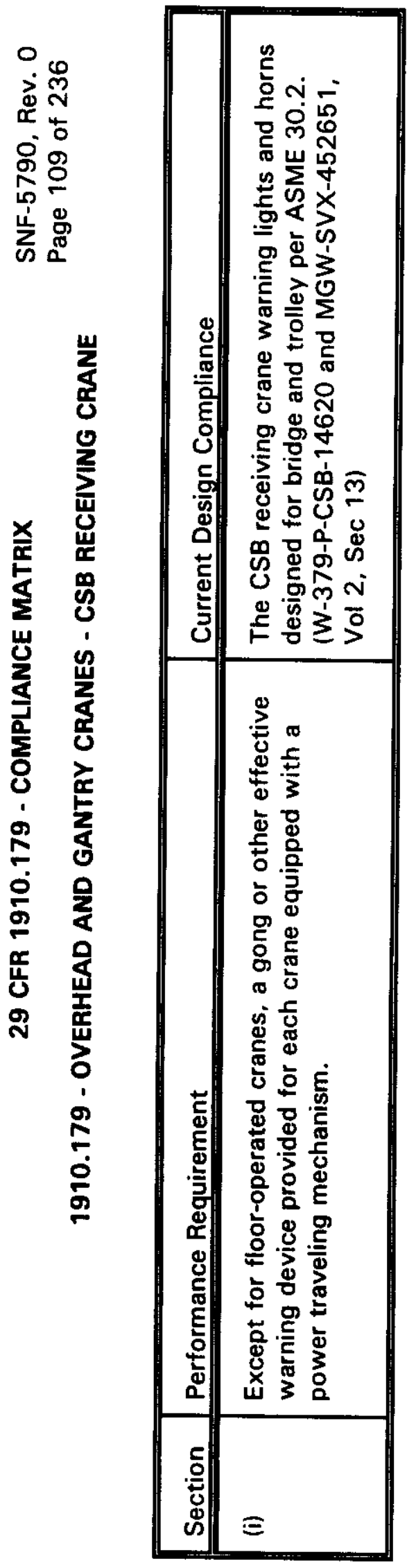




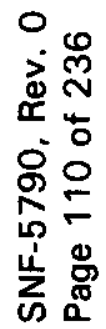

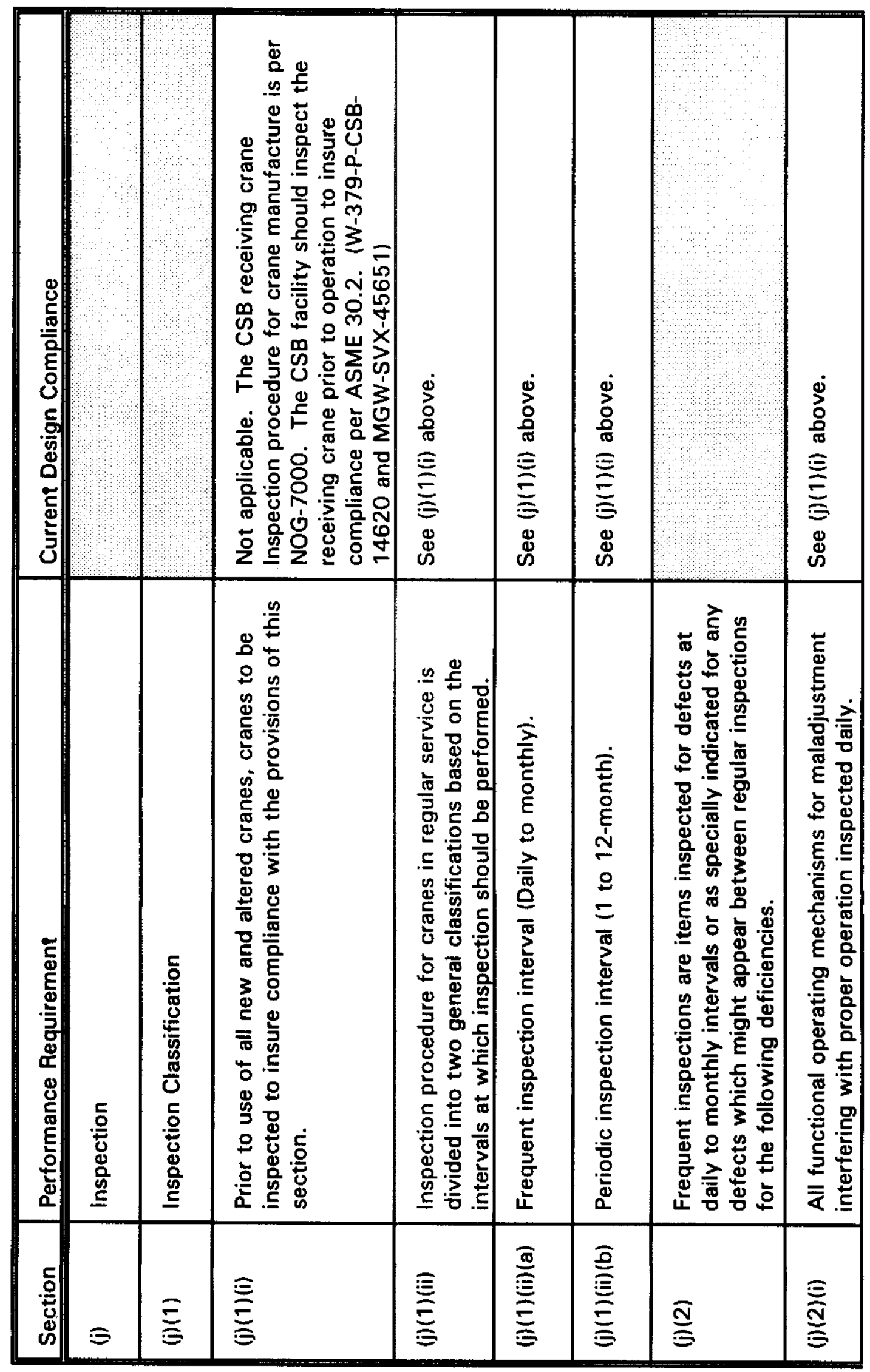




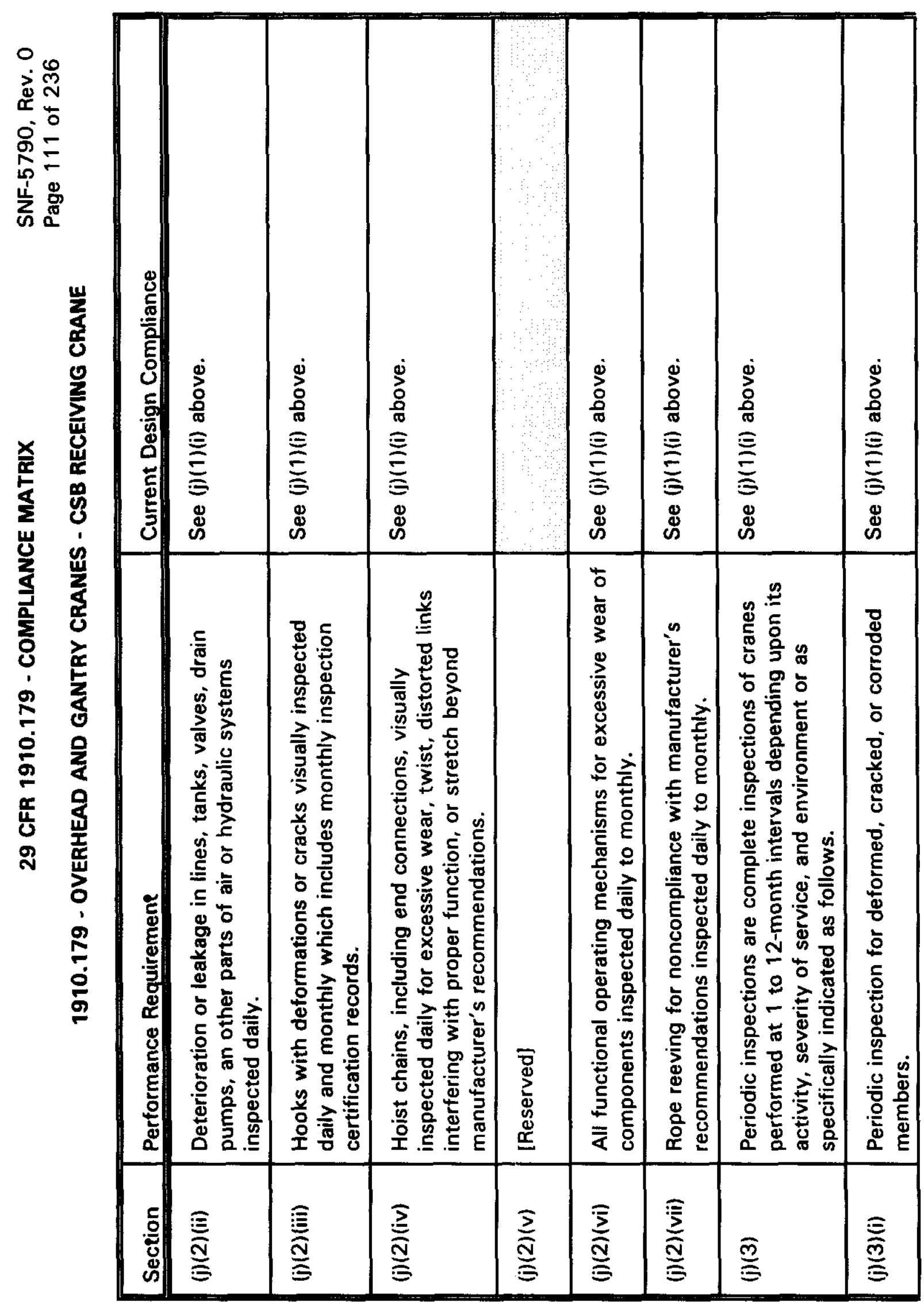




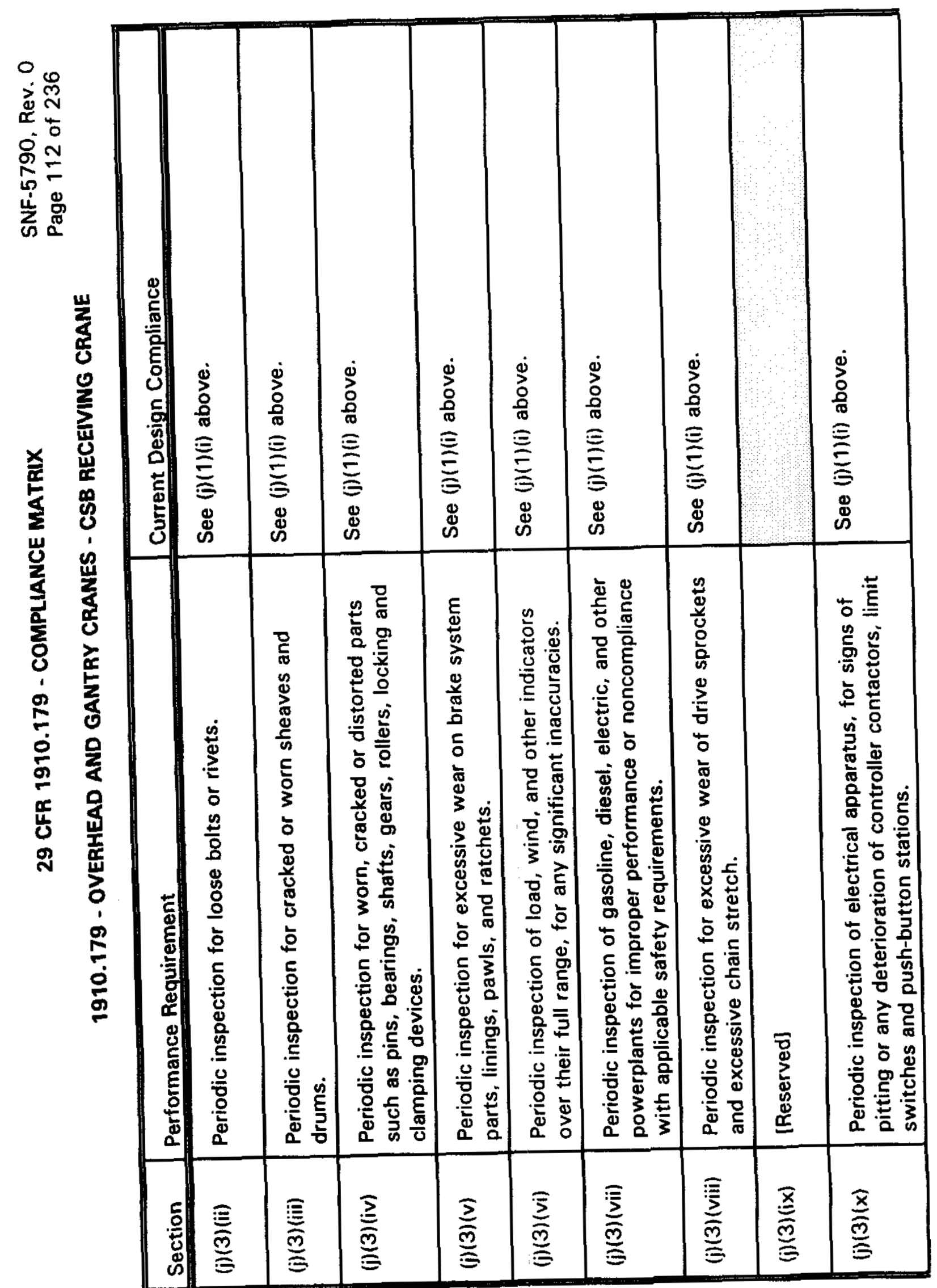




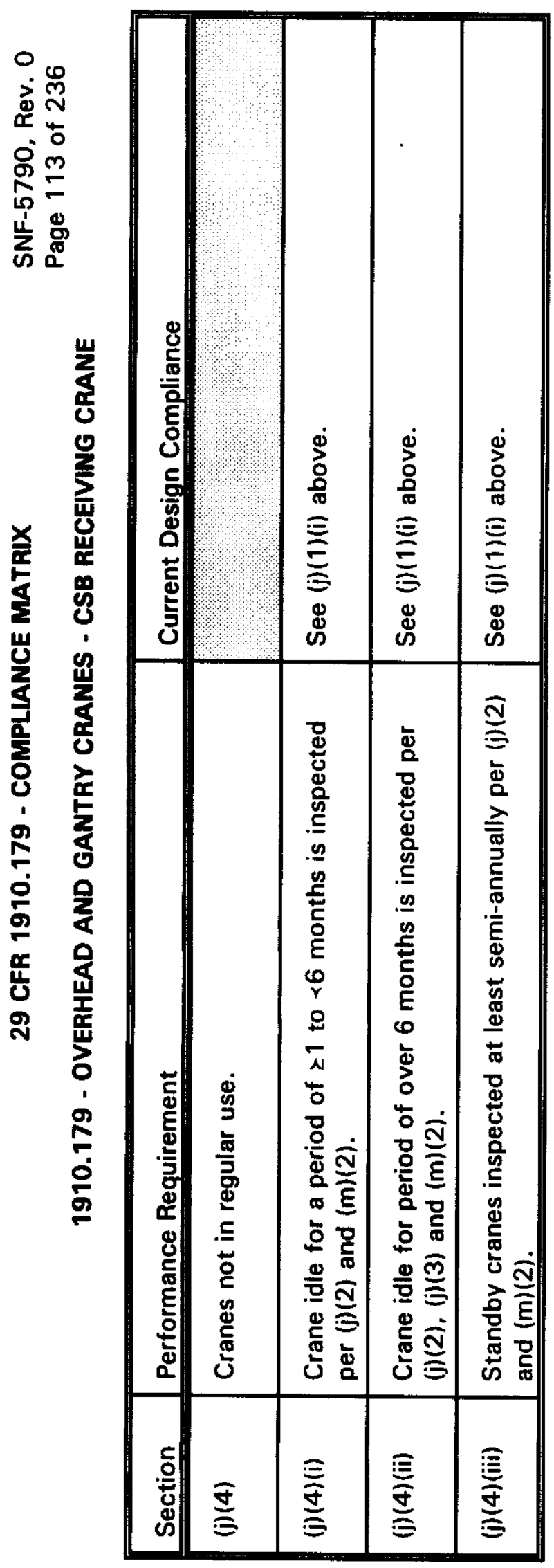




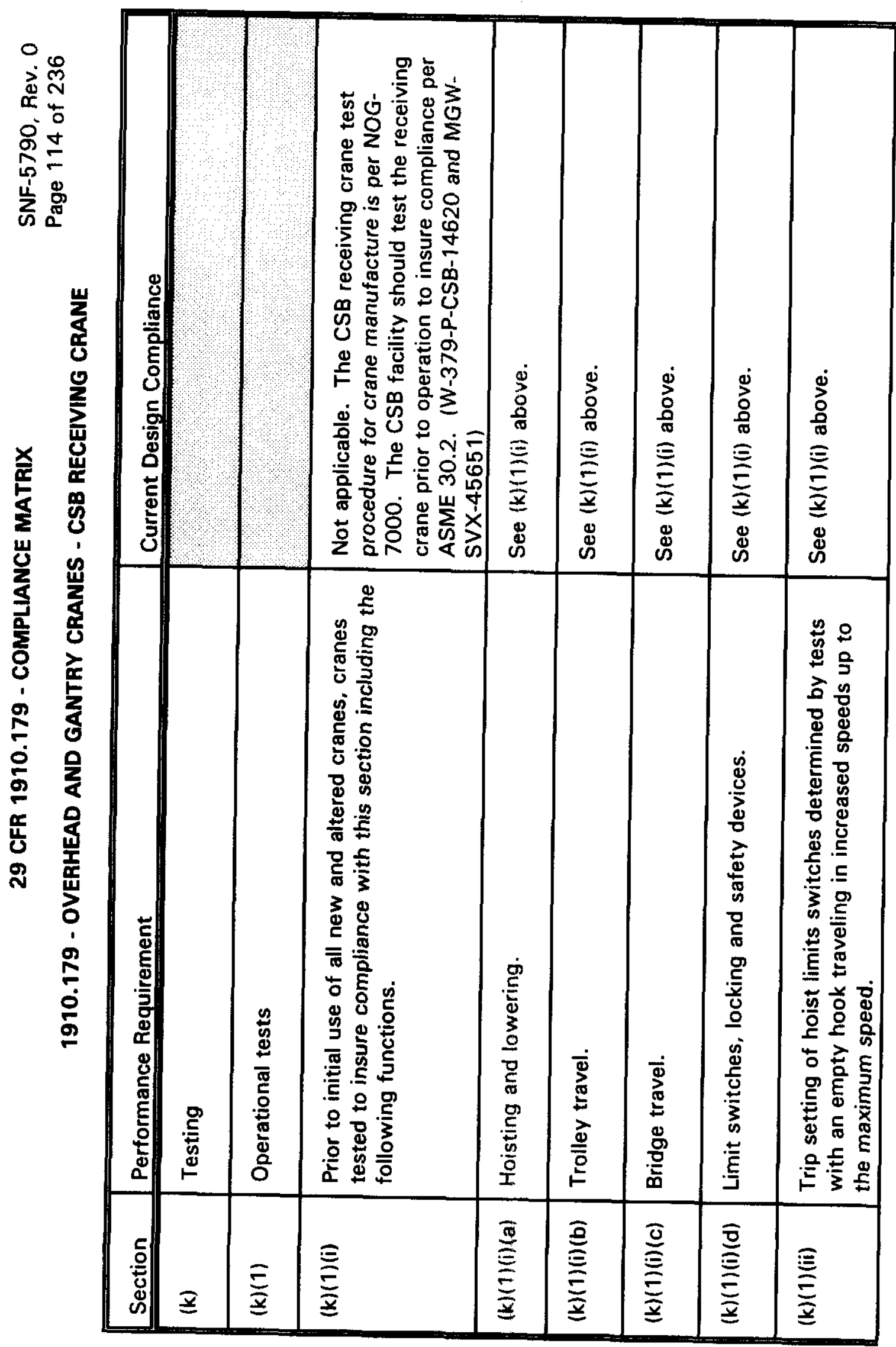




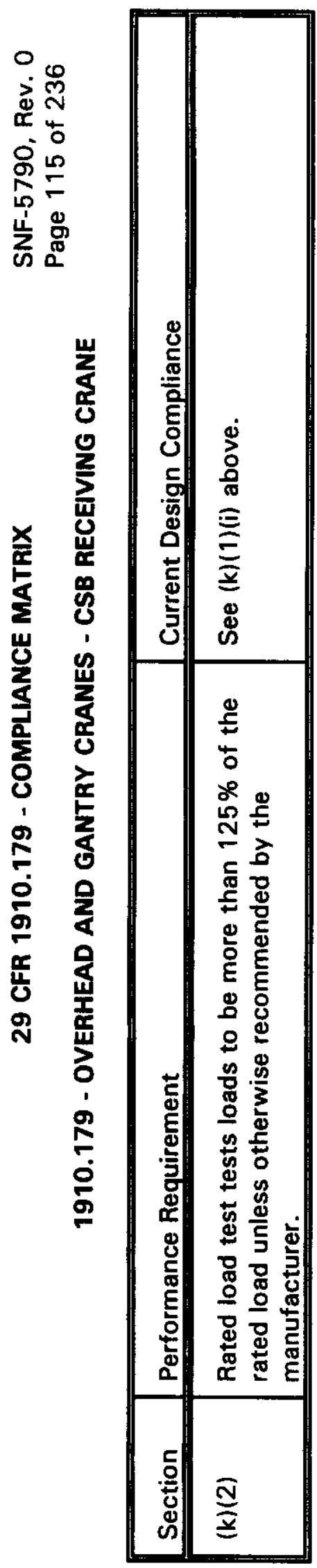




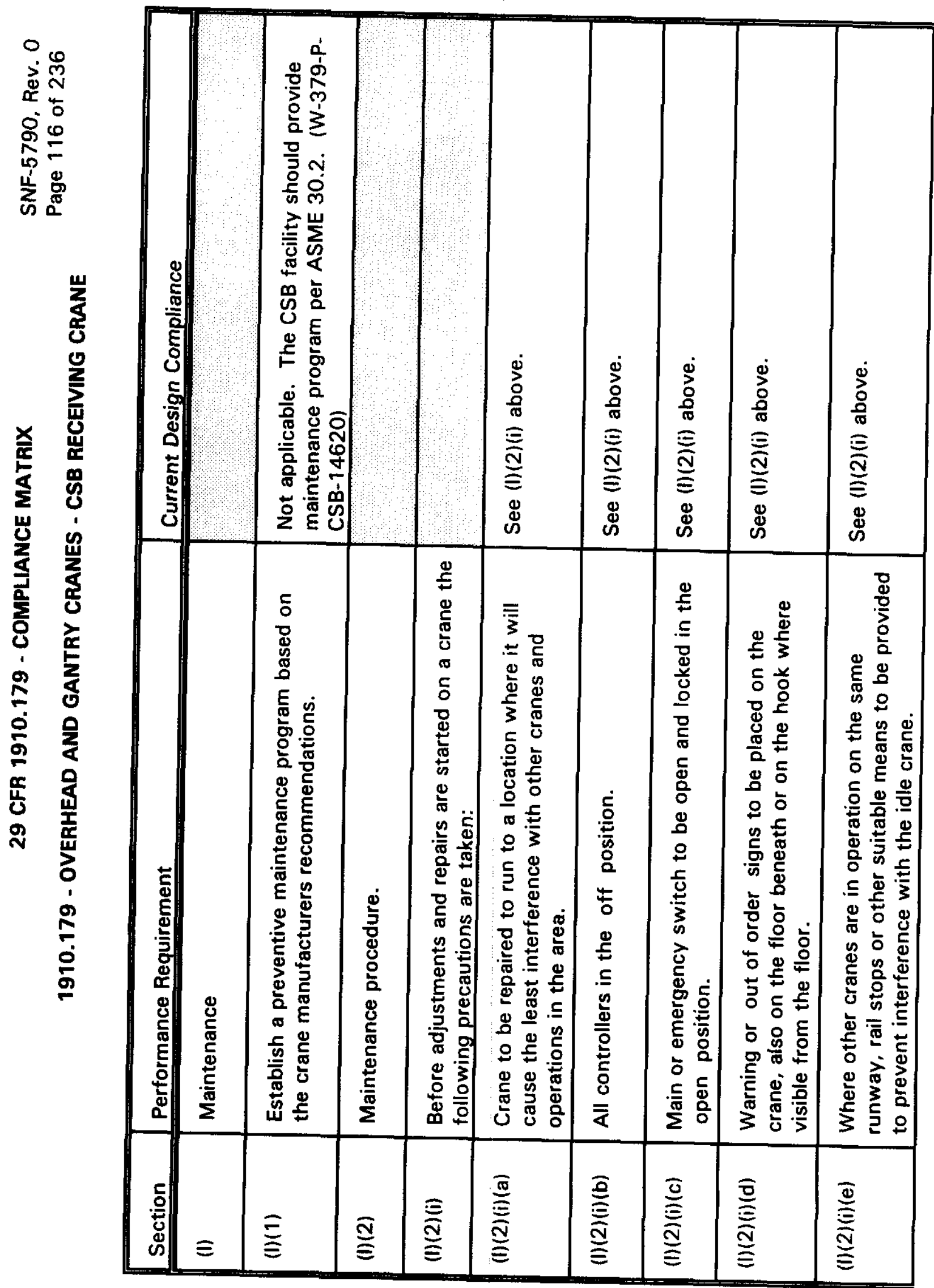




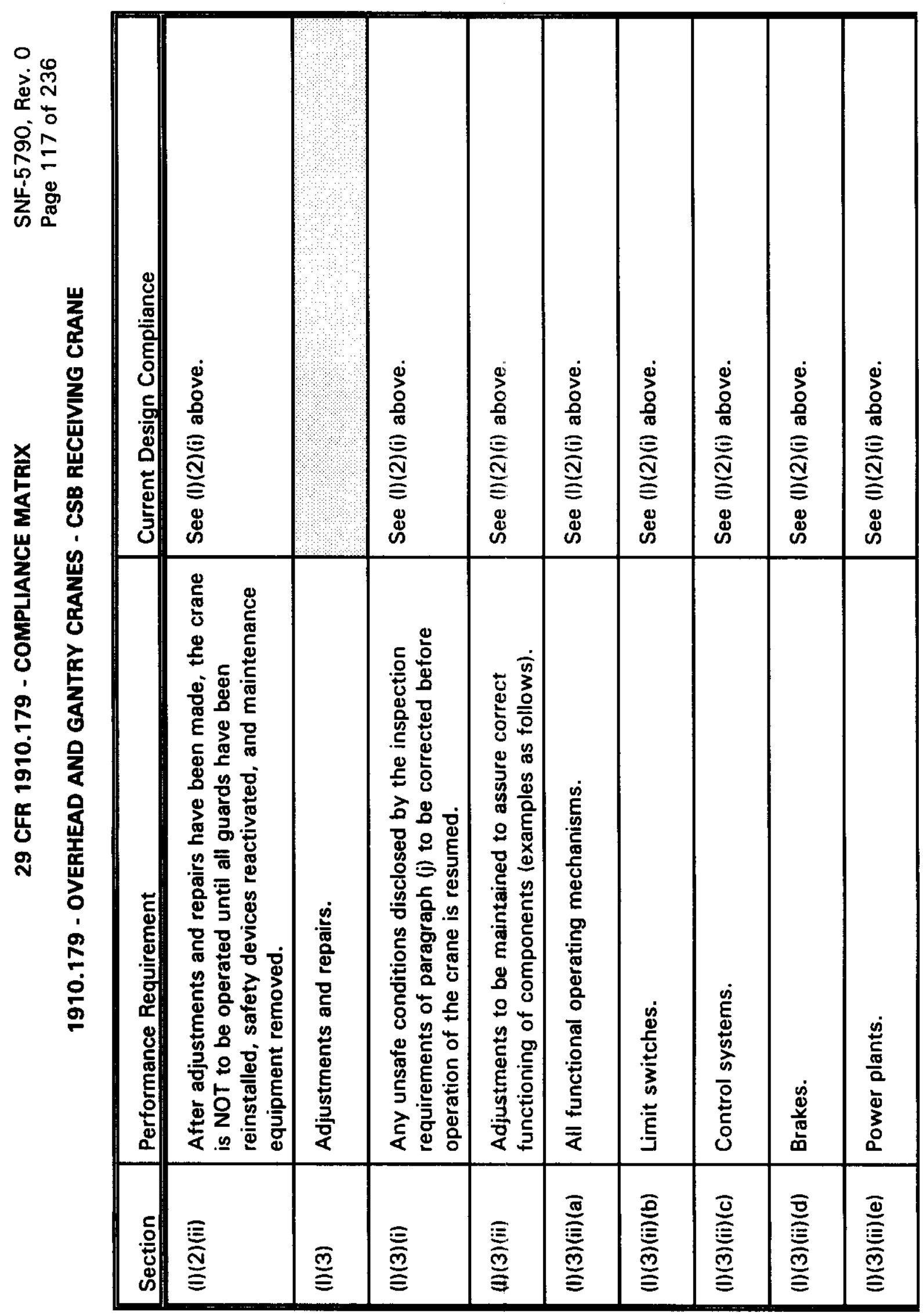




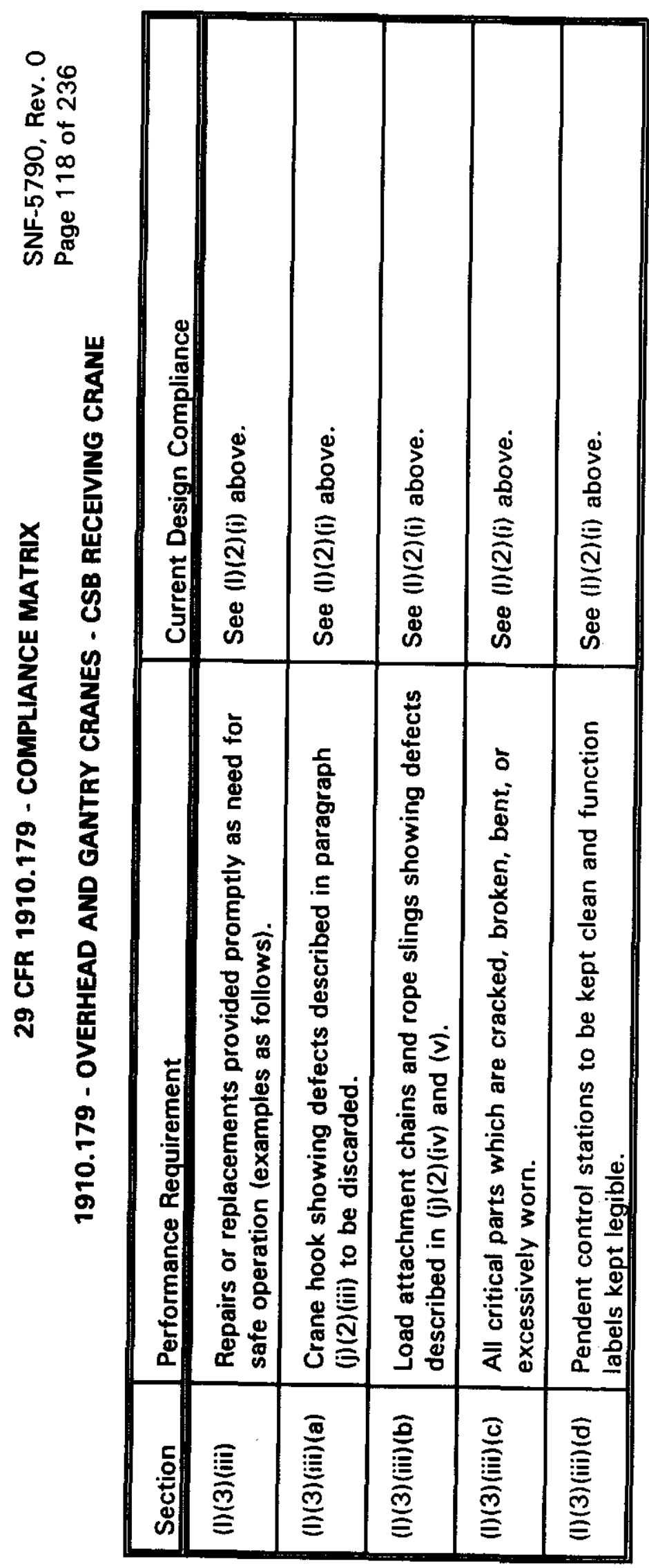




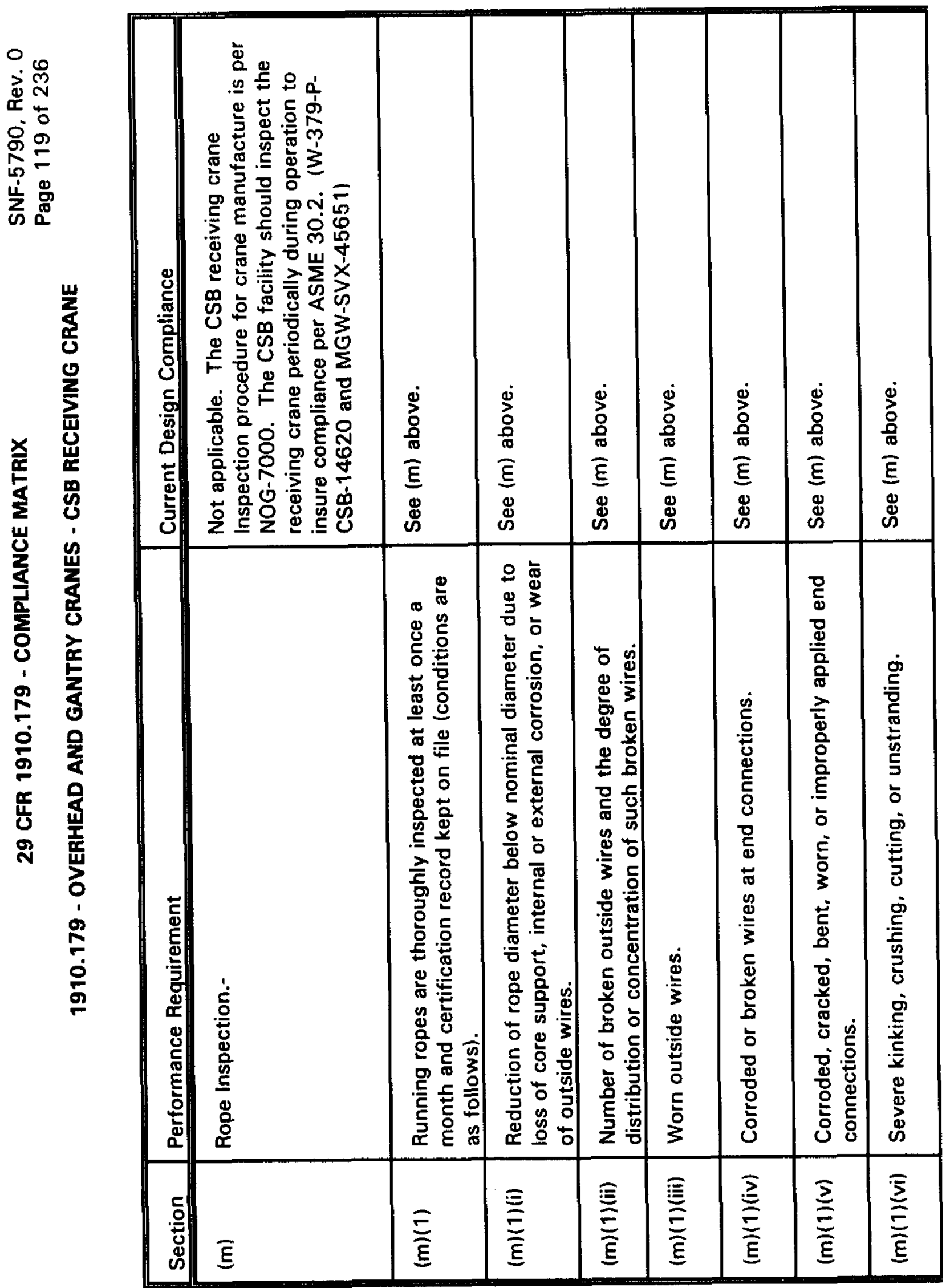




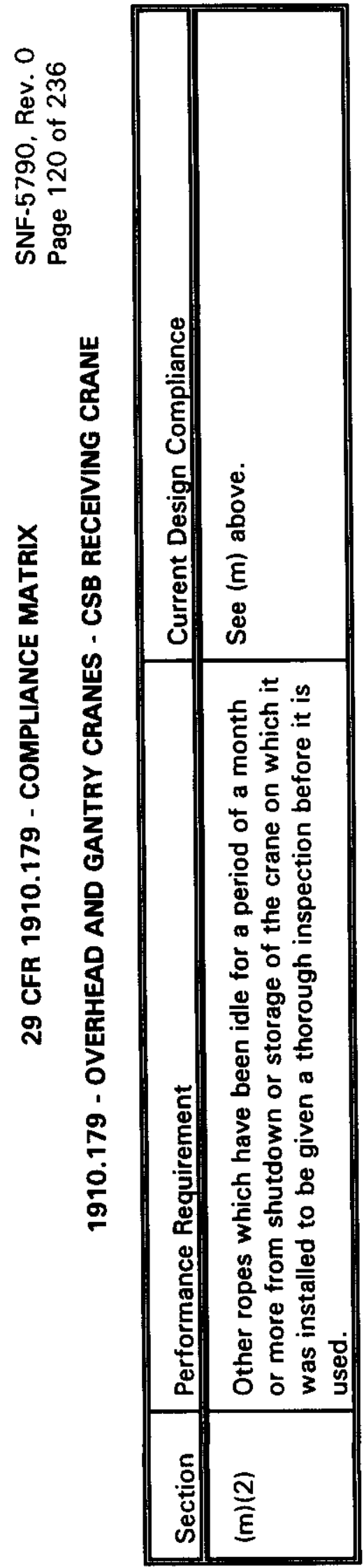




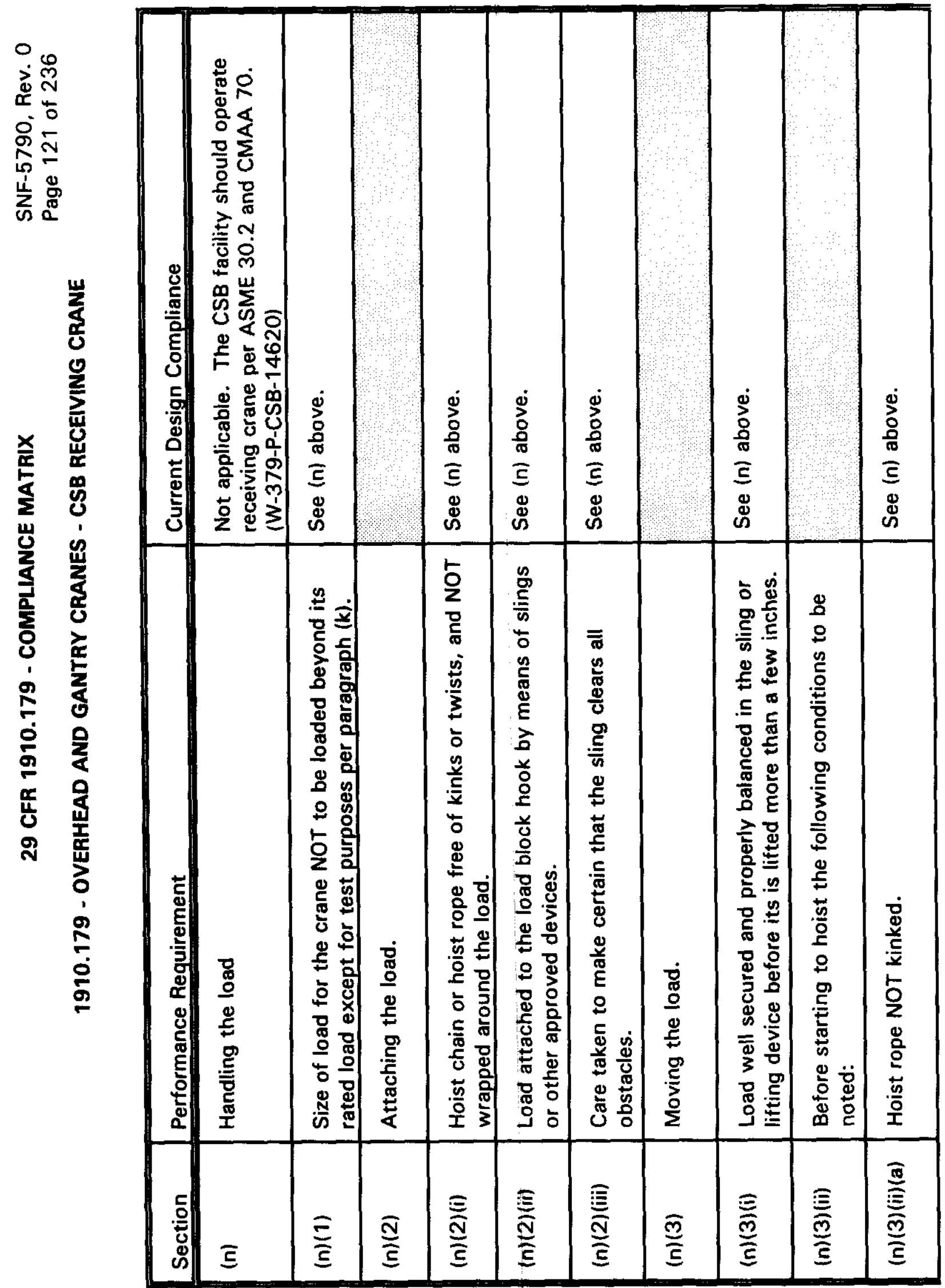




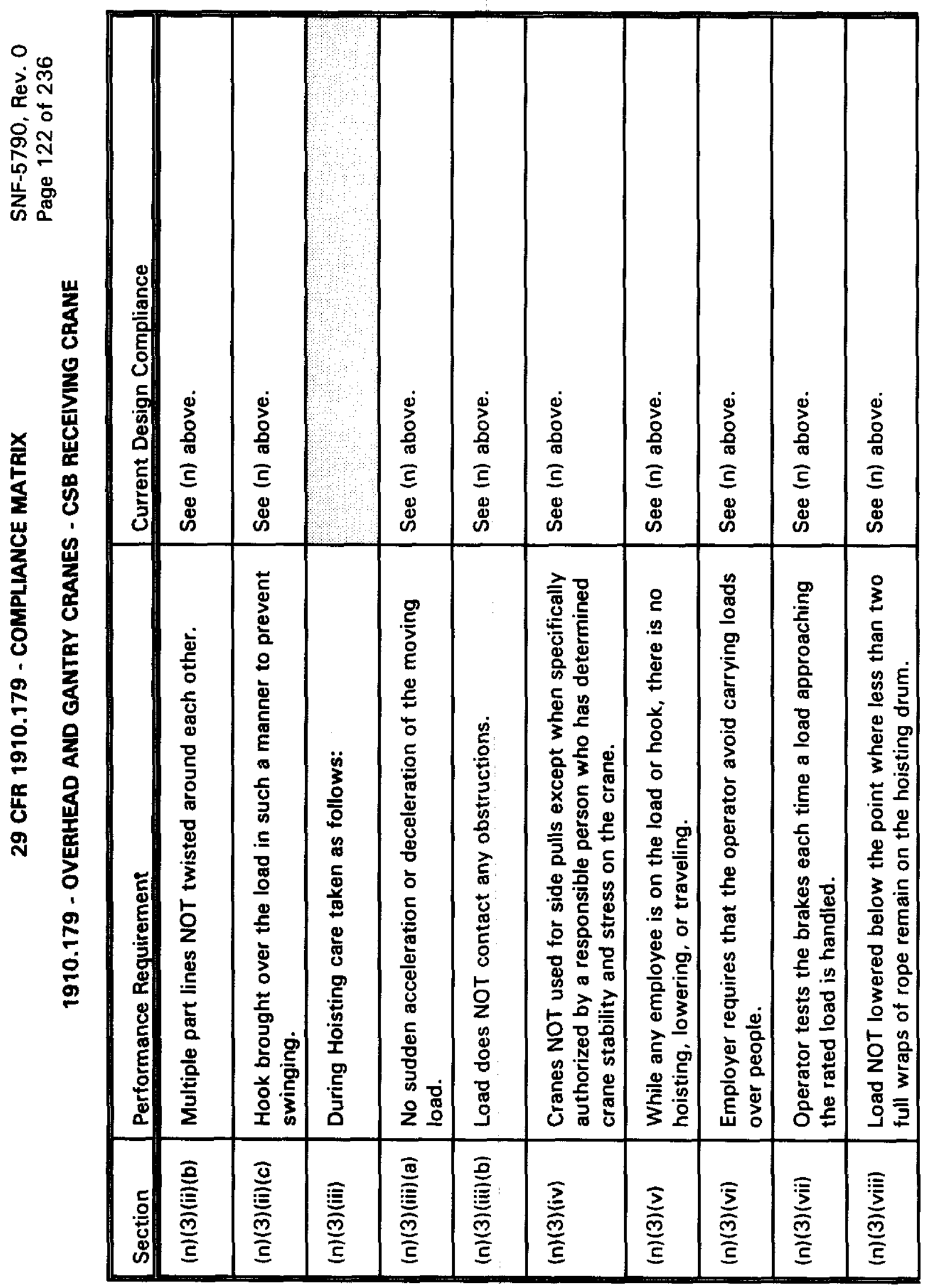




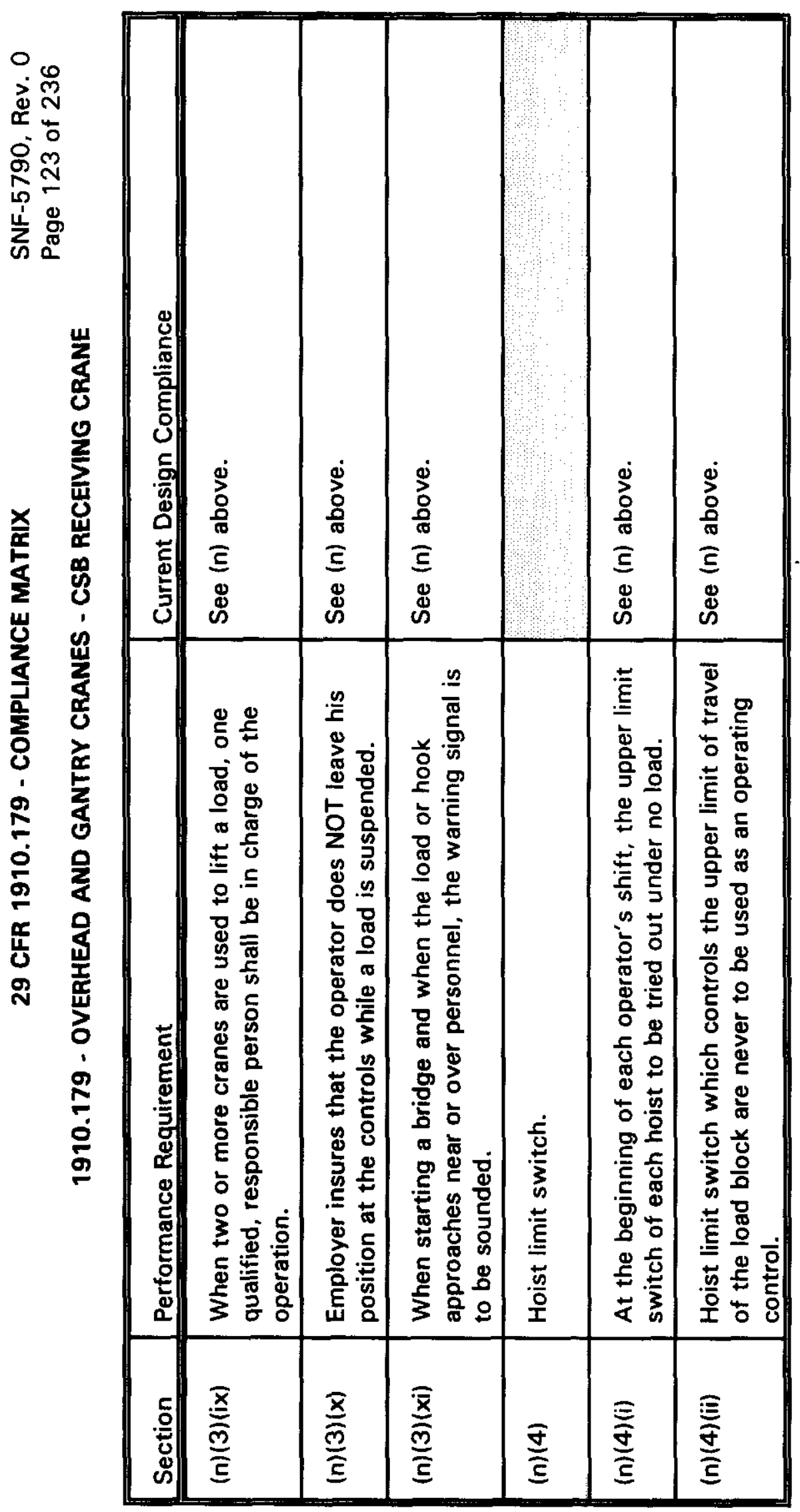




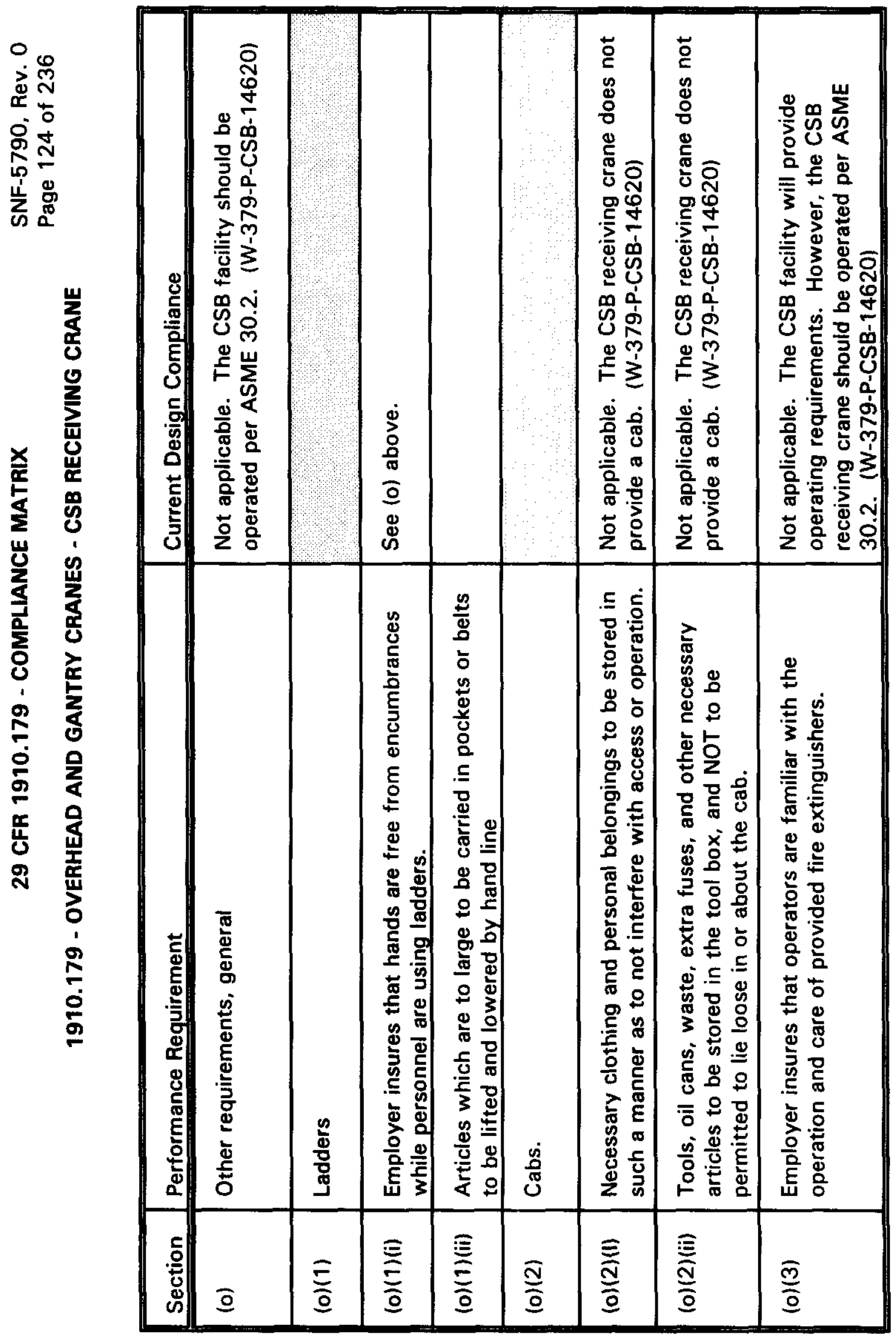


SNF-5790 Rev. 0

Page 125 of 236

\section{ATTACHMENT 5}

ANSI $\mathbf{5 7 . 9}$

COMPLIANCE MATRIX 


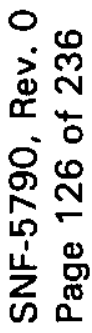

\begin{tabular}{|c|c|c|c|c|c|c|}
\hline 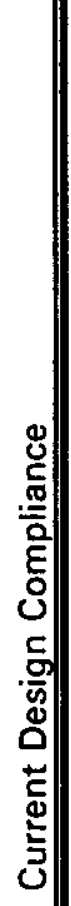 & & 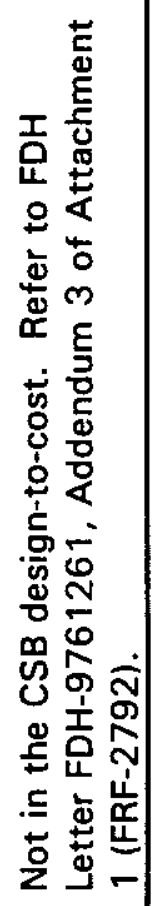 & 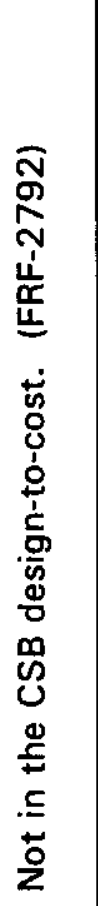 & 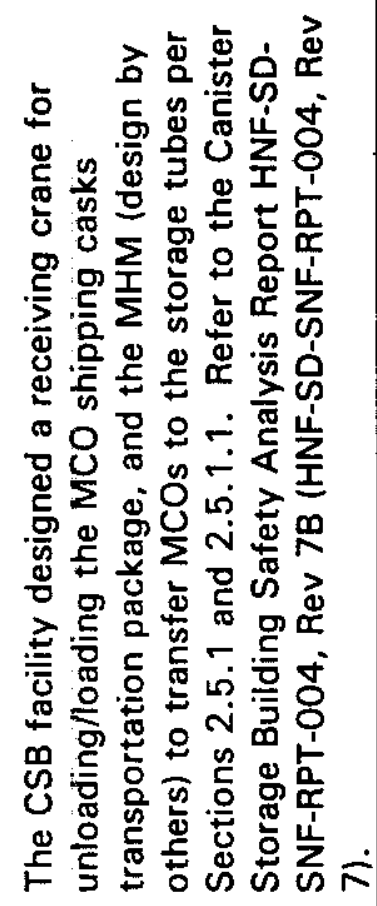 & 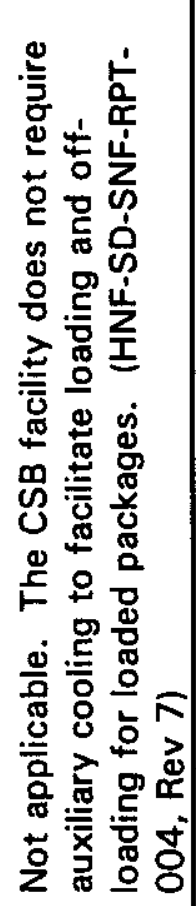 & 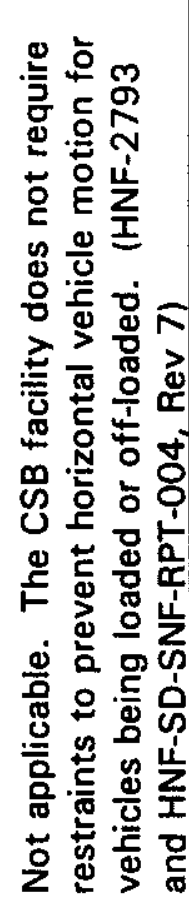 \\
\hline 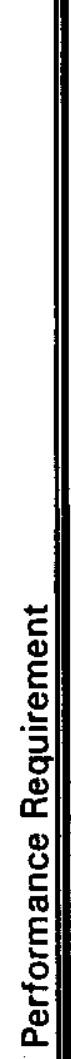 & 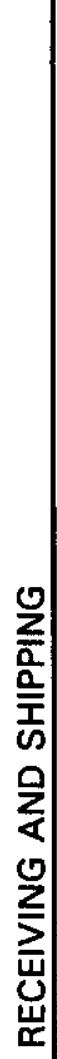 & 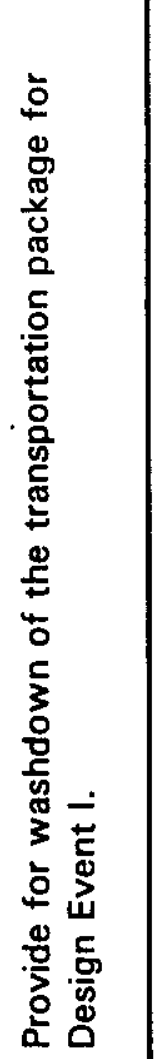 & 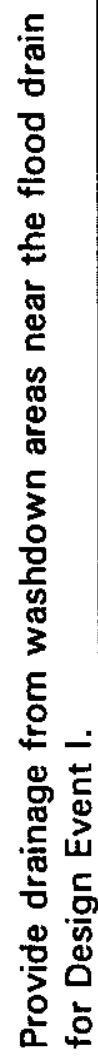 & 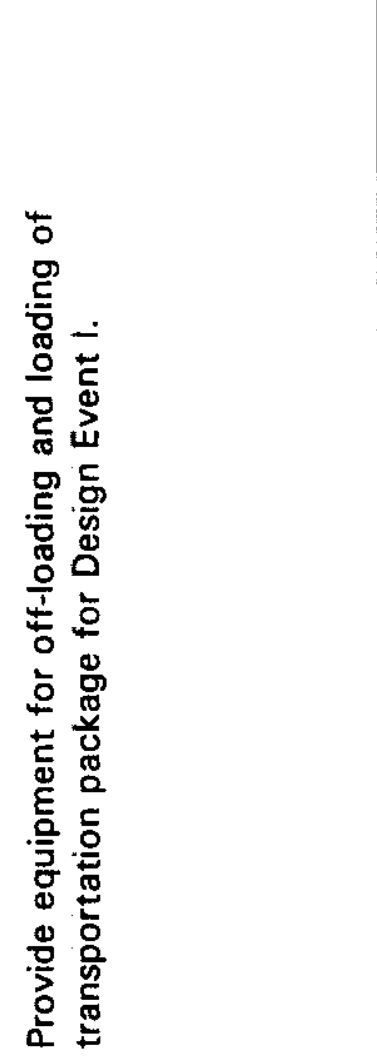 & 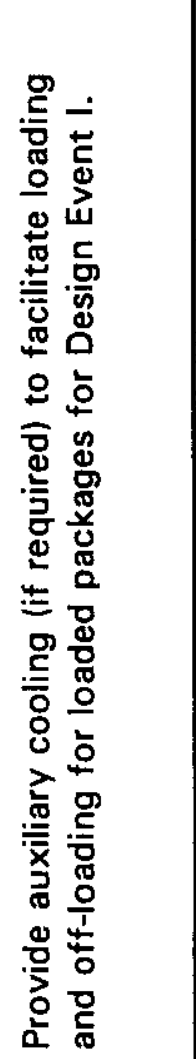 & 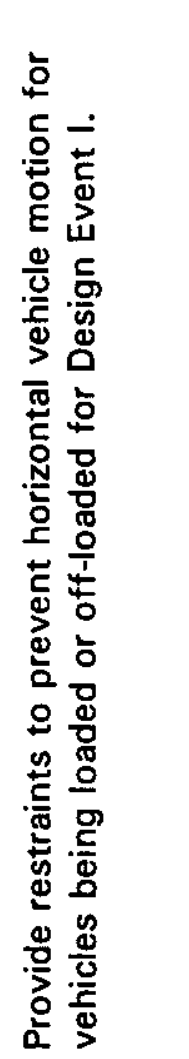 \\
\hline 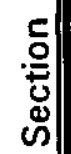 & تْ & $\underset{\sigma}{\check{\sigma}}$ & 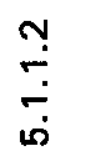 & 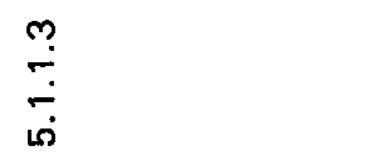 & 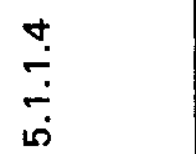 & مْ مَّا \\
\hline
\end{tabular}




\begin{tabular}{|c|c|c|c|c|c|c|c|c|c|}
\hline \multirow{3}{*}{ 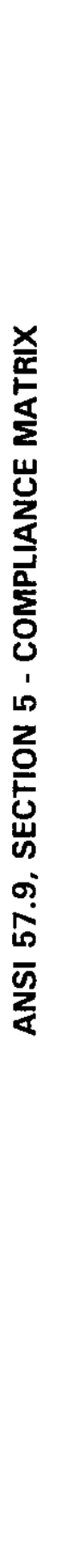 } & \multirow{3}{*}{ 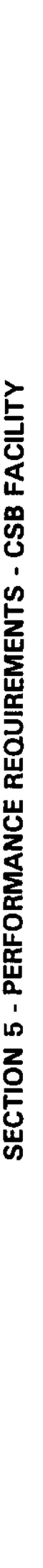 } & 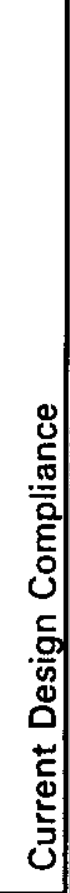 & & 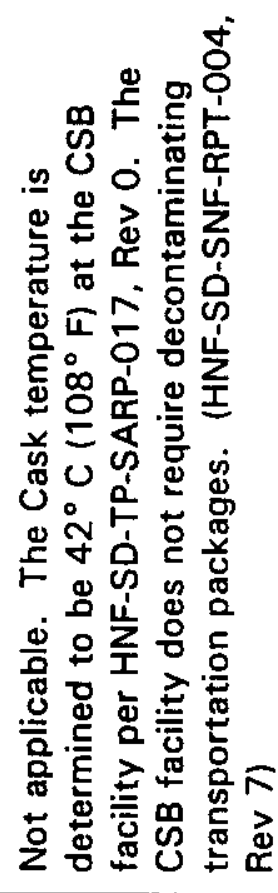 & 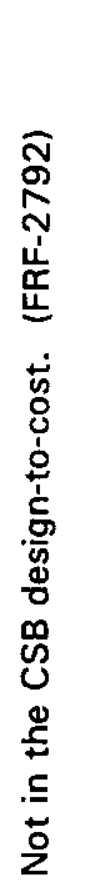 & 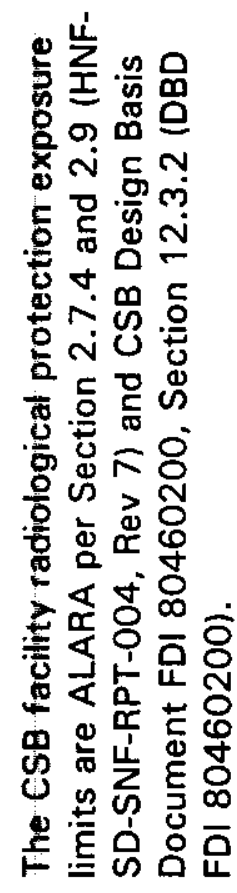 & & 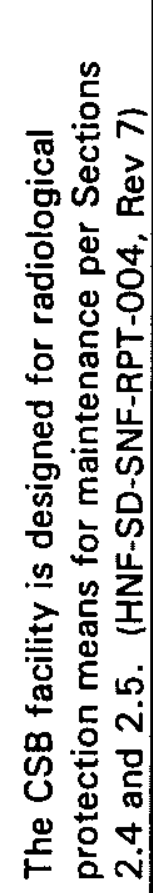 & 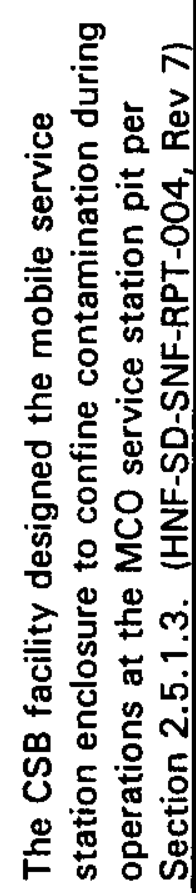 \\
\hline & & 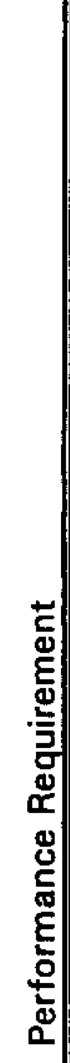 & 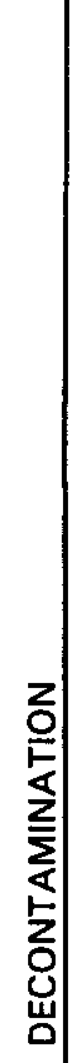 & 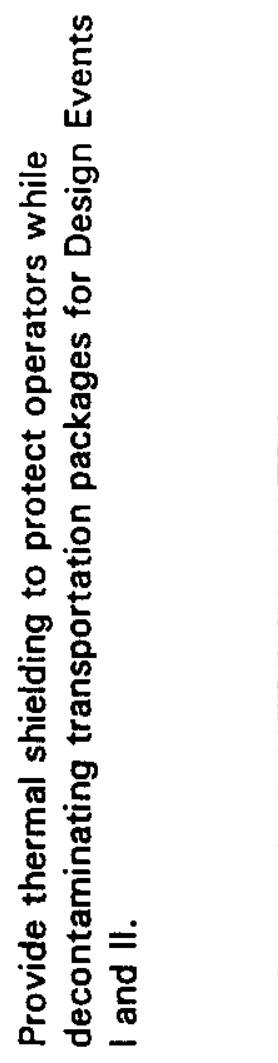 & 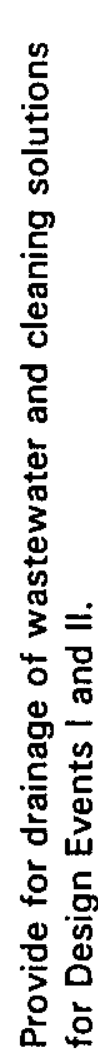 & 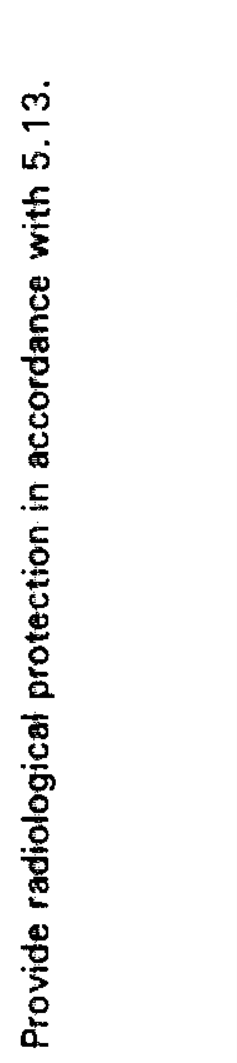 & 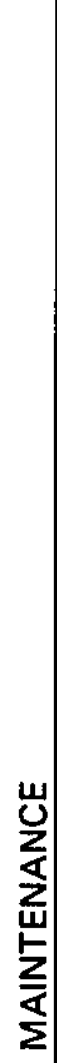 & 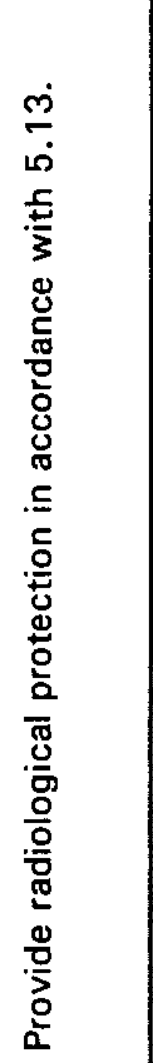 & 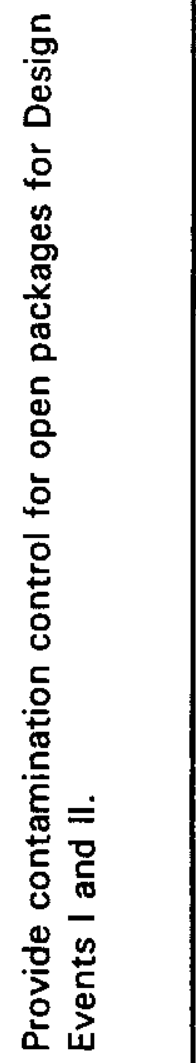 \\
\hline & & 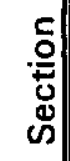 & ণָ' & $\frac{\check{r}}{\grave{i}}$ & 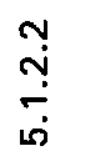 & "ri & ?ִ & $\begin{array}{r}\check{7} \\
\ddot{7} \\
\ddot{1}\end{array}$ & 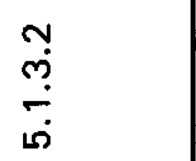 \\
\hline
\end{tabular}




\begin{tabular}{|c|c|c|c|c|c|c|c|}
\hline 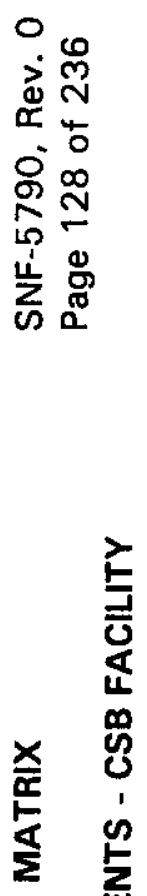 & 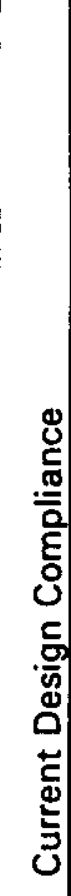 & 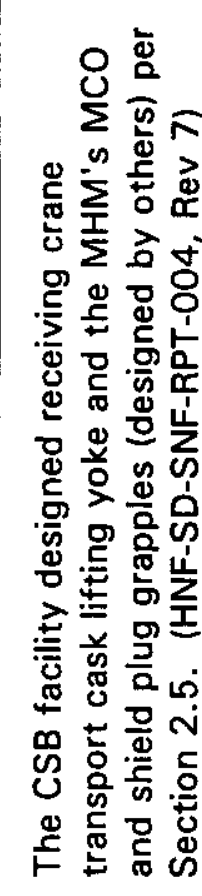 & 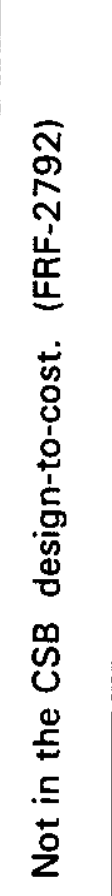 & 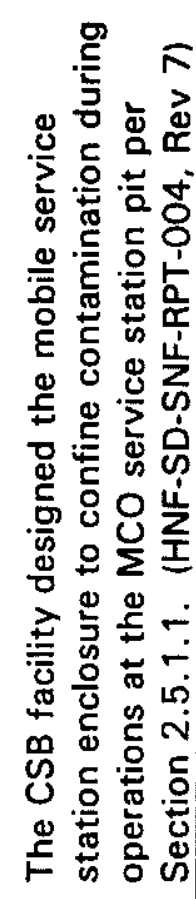 & & 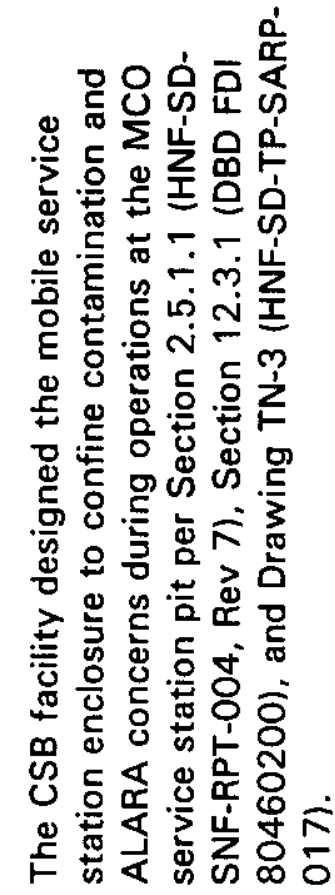 & 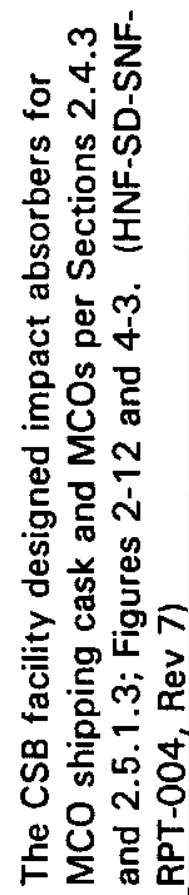 \\
\hline 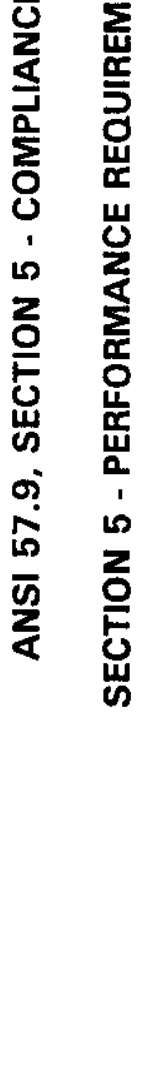 & 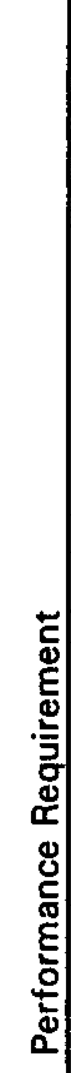 & 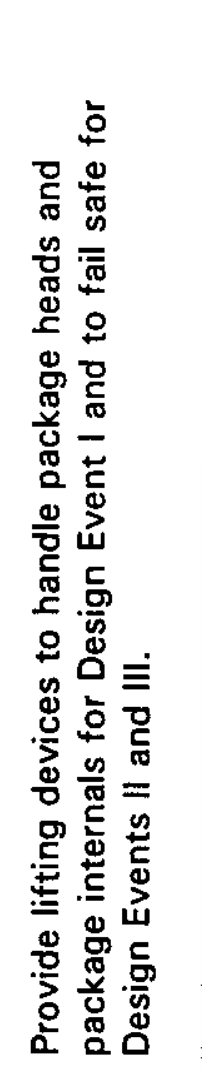 & 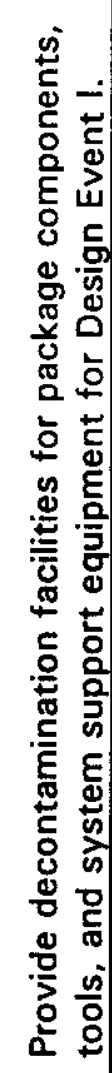 & 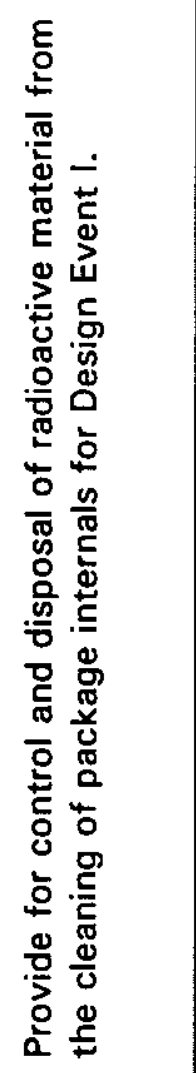 & 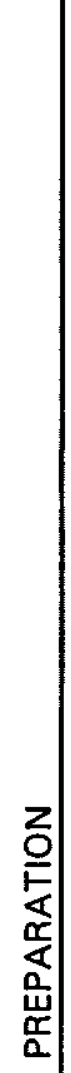 & 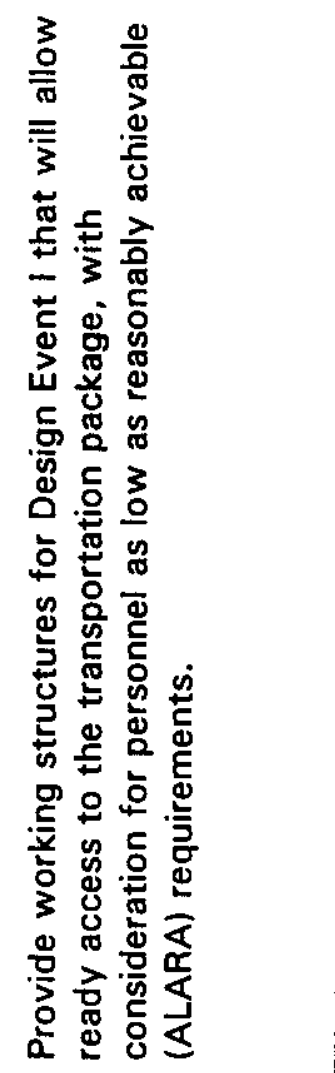 & 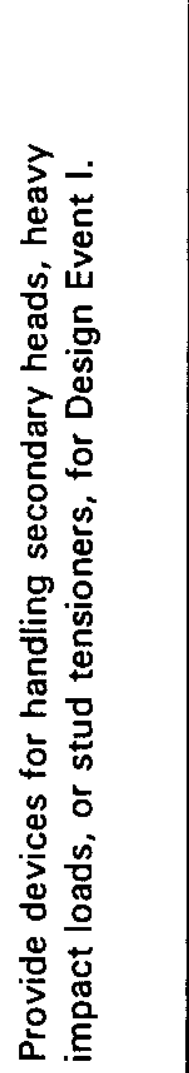 \\
\hline & 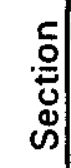 & $\begin{array}{r}m \\
\ddot{m} \\
\dot{m}\end{array}$ & $\begin{array}{l}\dot{\nabla} \\
\stackrel{\leftrightarrow}{\circ} \\
\stackrel{\circ}{\circ}\end{array}$ & $\begin{array}{l}\text { ?" } \\
\text { ?ं }\end{array}$ & $\begin{array}{l}+ \\
\div \\
\end{array}$ & $\begin{array}{l}\check{r} \\
\dot{\sigma} \\
\dot{\circ}\end{array}$ & 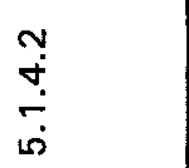 \\
\hline
\end{tabular}




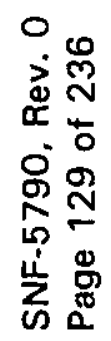

\begin{tabular}{|c|c|c|c|c|c|}
\hline 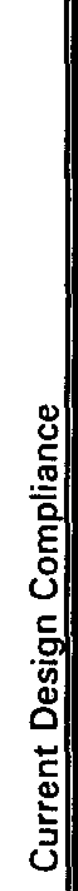 & 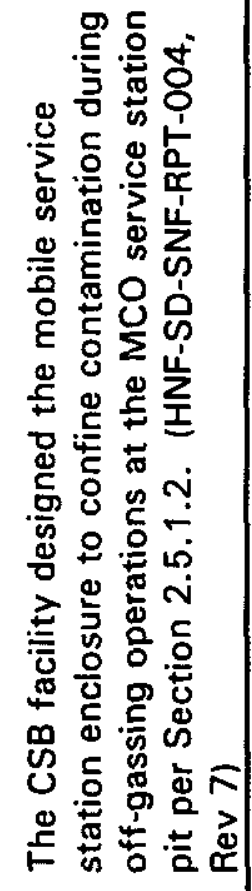 & & 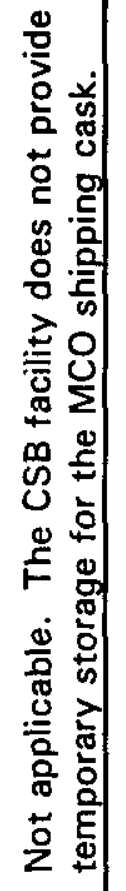 & 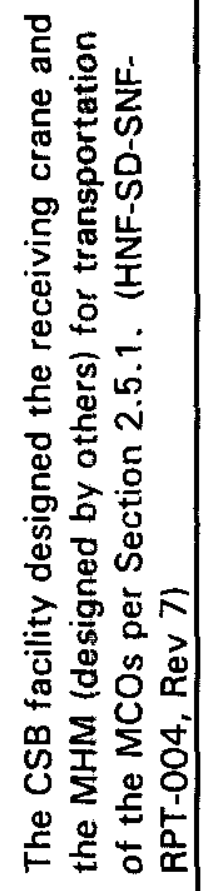 & 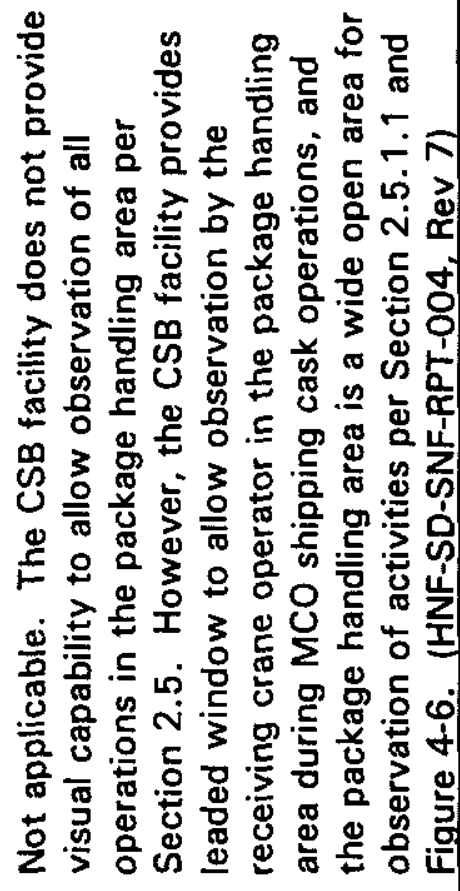 \\
\hline 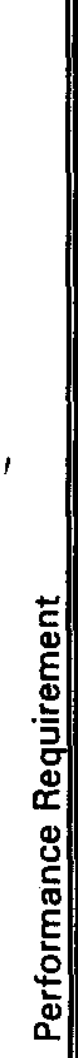 & 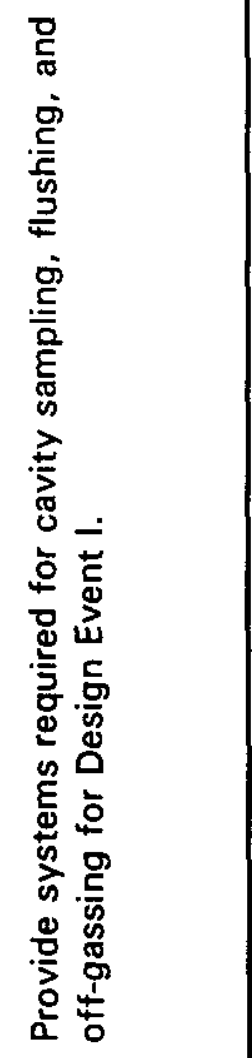 & 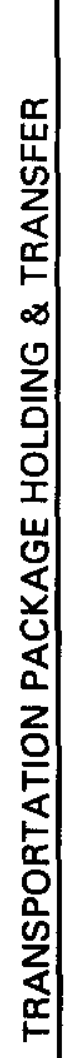 & 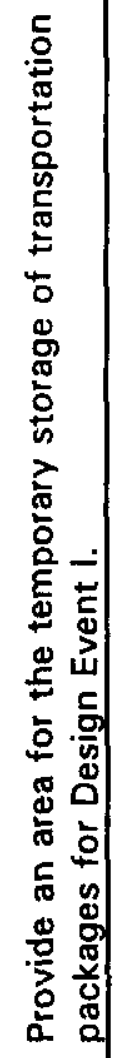 & 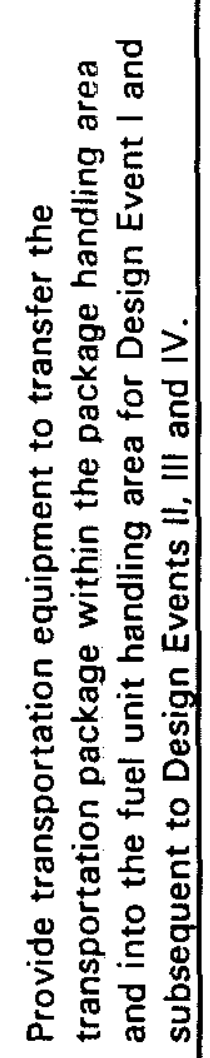 & 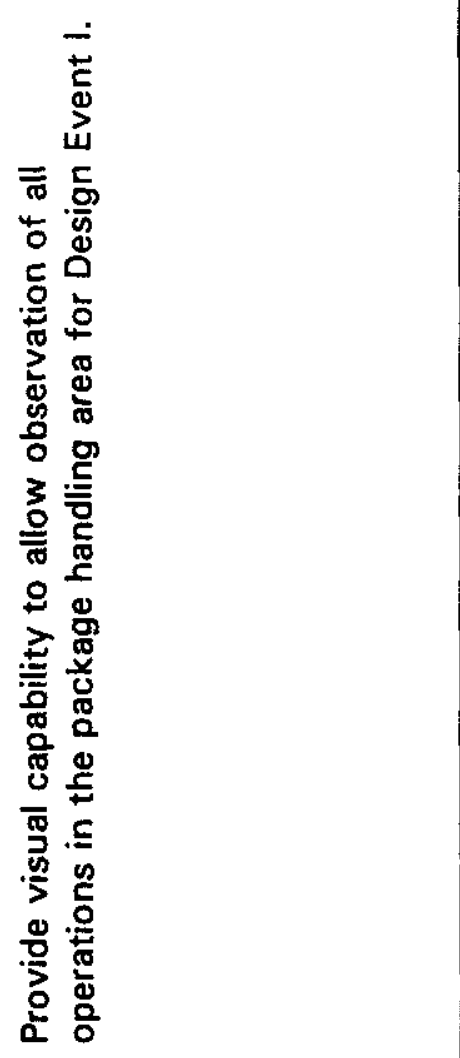 \\
\hline 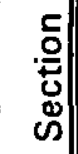 & $\begin{array}{l}m \\
\dot{+} \\
\dot{\sigma} \\
\dot{0}\end{array}$ & \begin{tabular}{c}
10 \\
\hdashline \\
10
\end{tabular} & $\frac{\ddot{\sigma}}{\dot{\sigma}}$ & 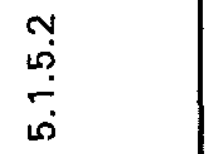 & 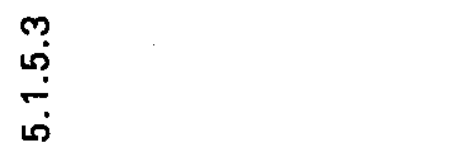 \\
\hline
\end{tabular}




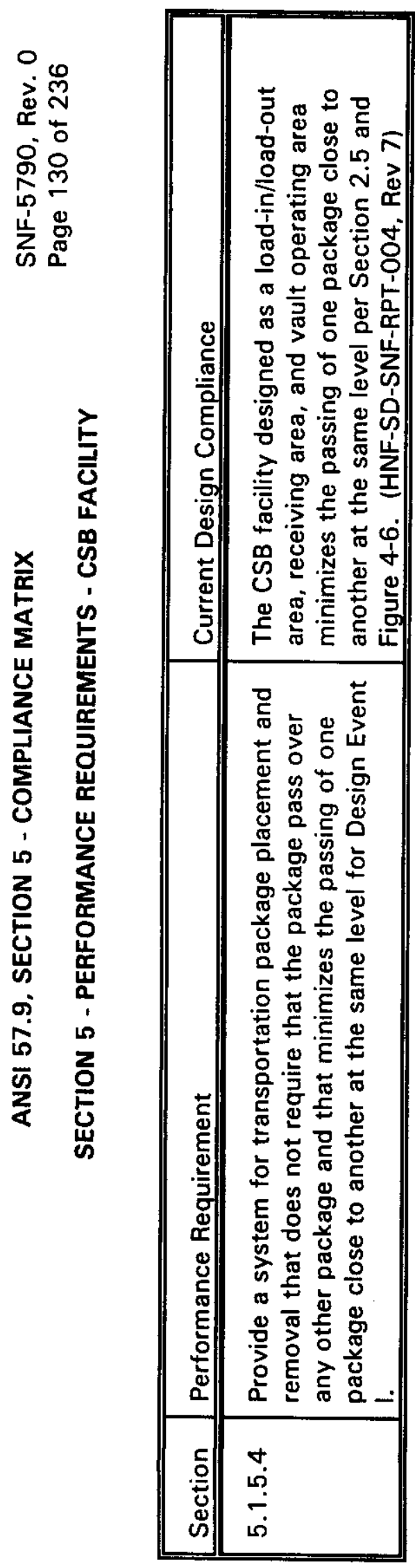




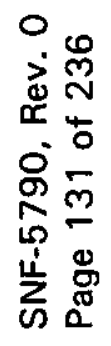

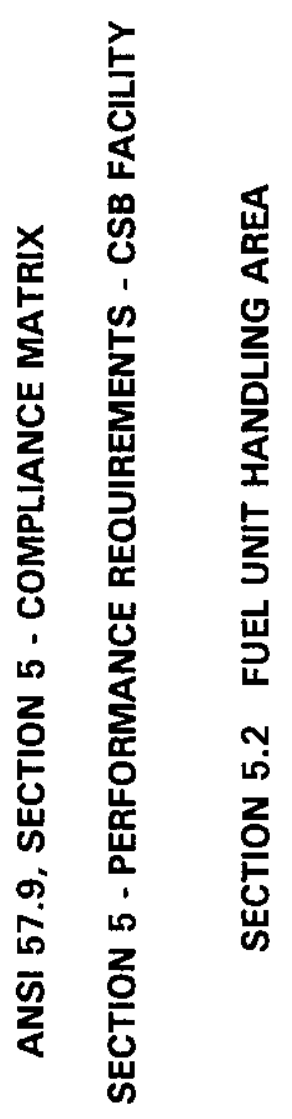

\begin{tabular}{|c|c|c|c|c|c|c|}
\hline & & 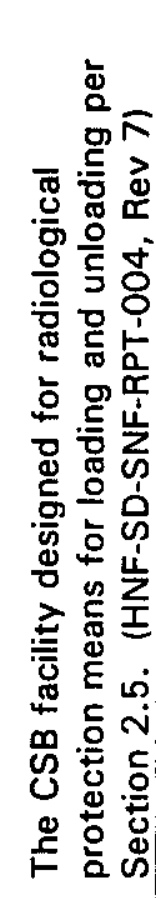 & 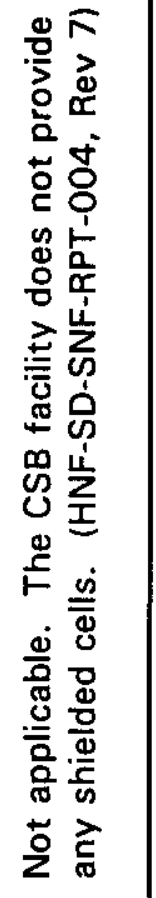 & 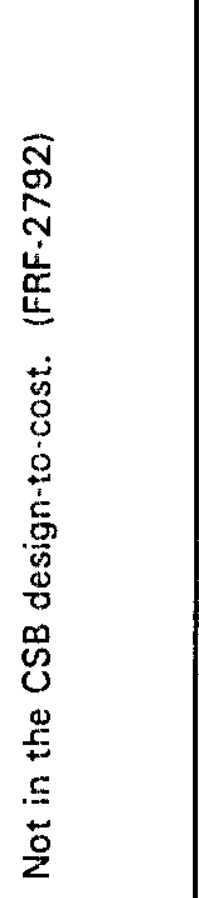 & 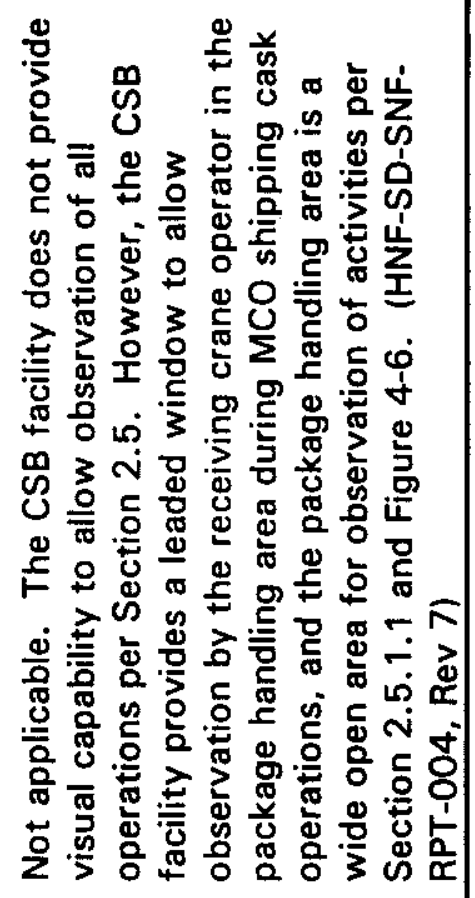 & 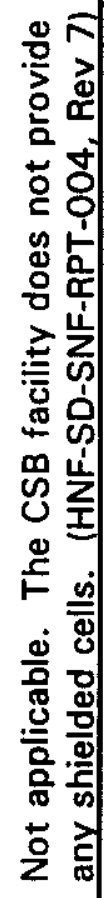 \\
\hline 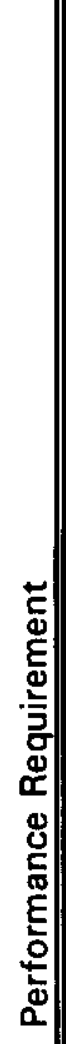 & 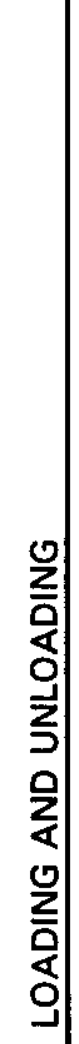 & 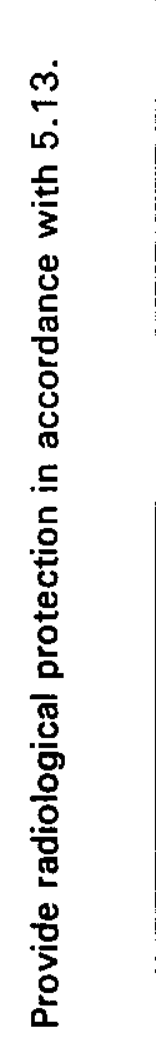 & 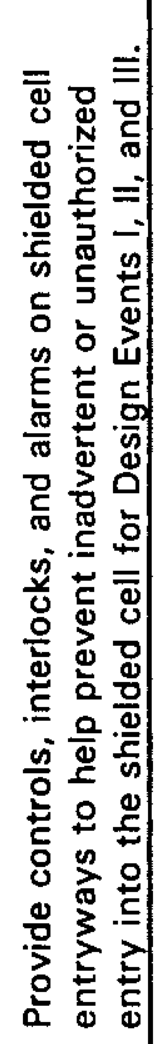 & 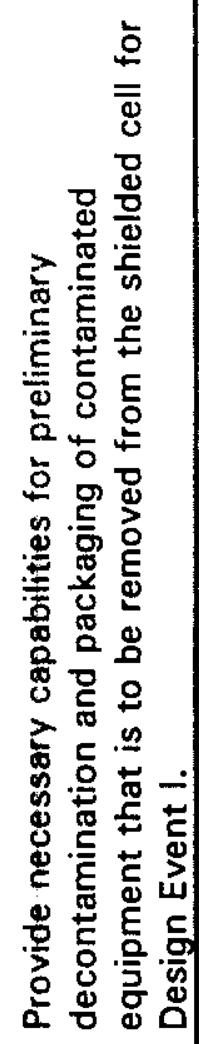 & 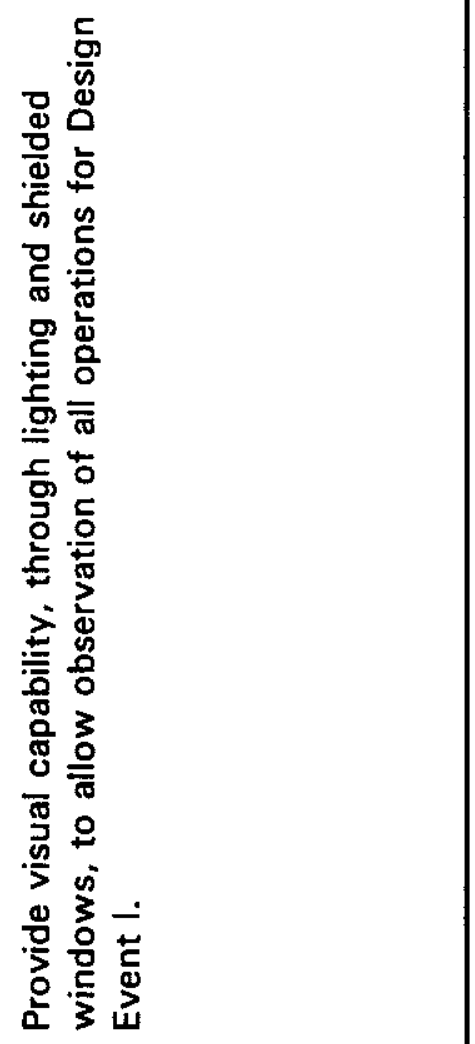 & 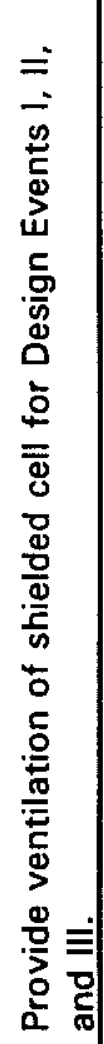 \\
\hline & సু. & סִ & ִָ & in & ه্. & هِ \\
\hline
\end{tabular}




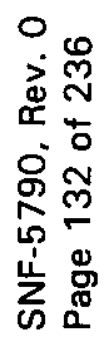

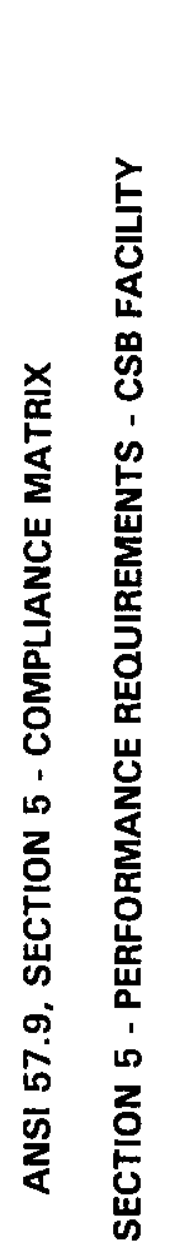

\begin{tabular}{|c|c|c|c|c|c|}
\hline 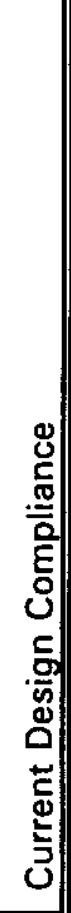 & 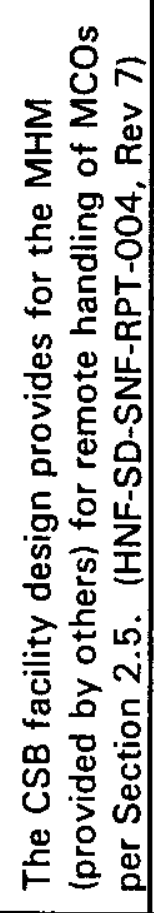 & 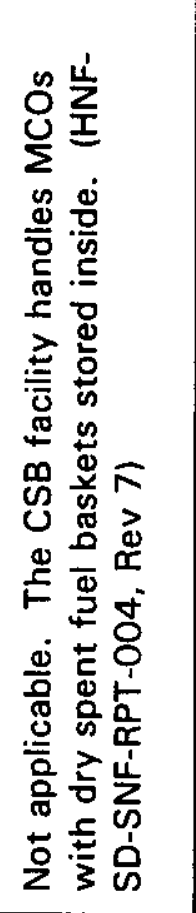 & 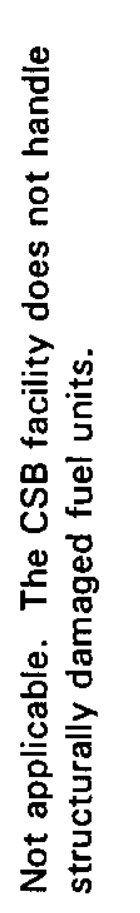 & 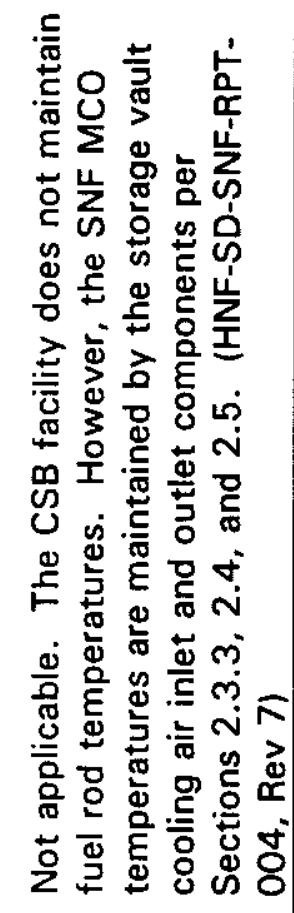 & 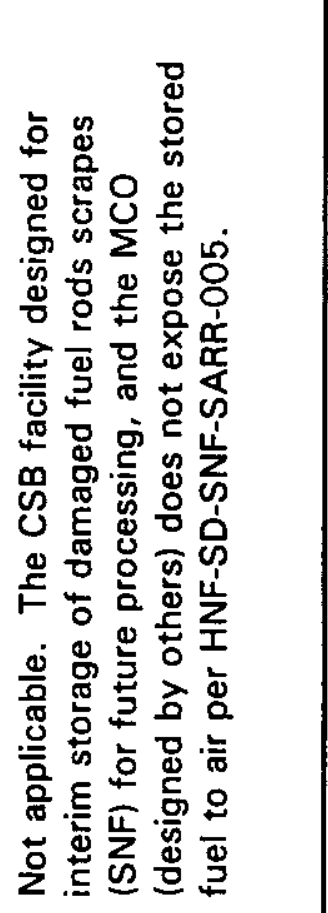 \\
\hline 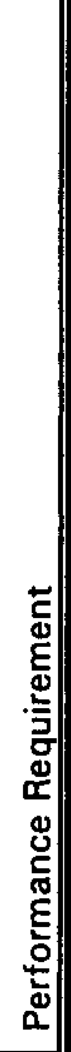 & 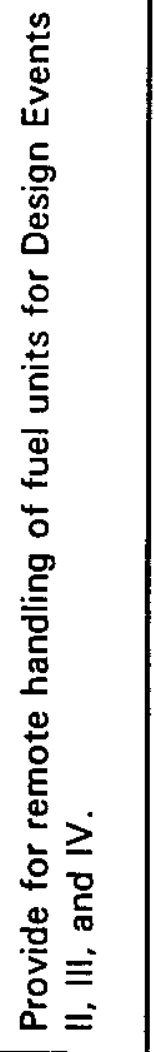 & 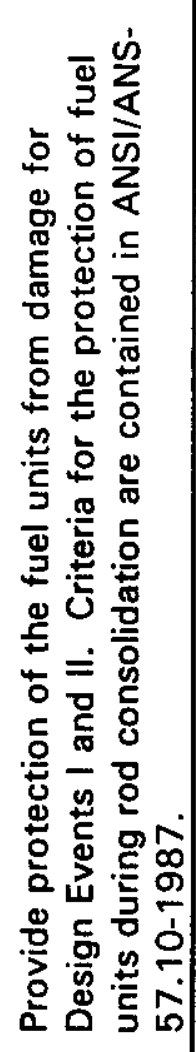 & 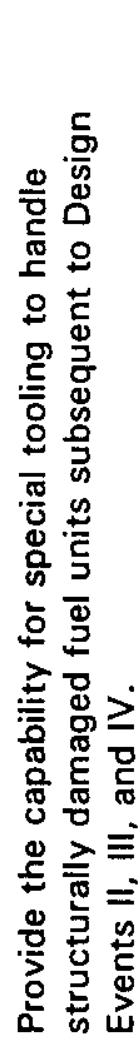 & 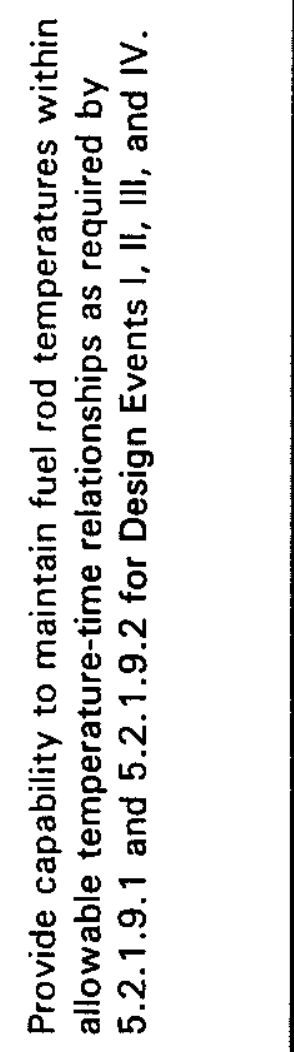 & 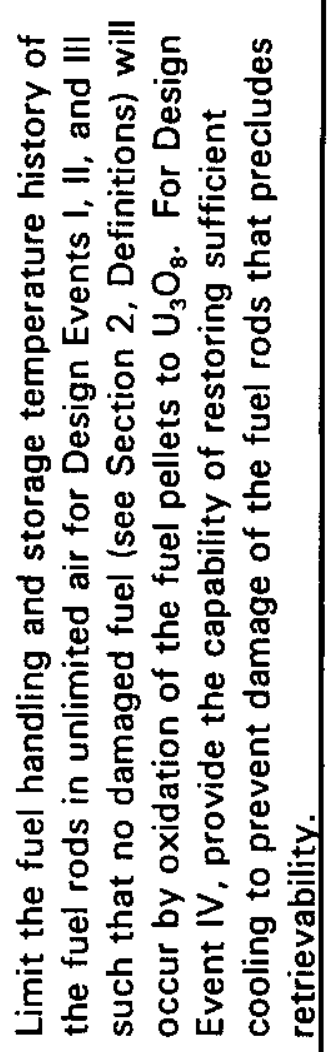 \\
\hline 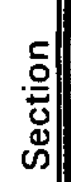 & مَّ & ڤัن & $\begin{array}{l}\infty \\
\stackrel{\infty}{\sim} \\
\stackrel{\sim}{ }\end{array}$ & 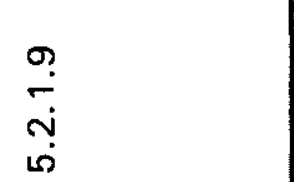 & $\begin{array}{l}\check{\sigma} \\
\dot{\sigma} \\
\dot{\sim}\end{array}$ \\
\hline
\end{tabular}




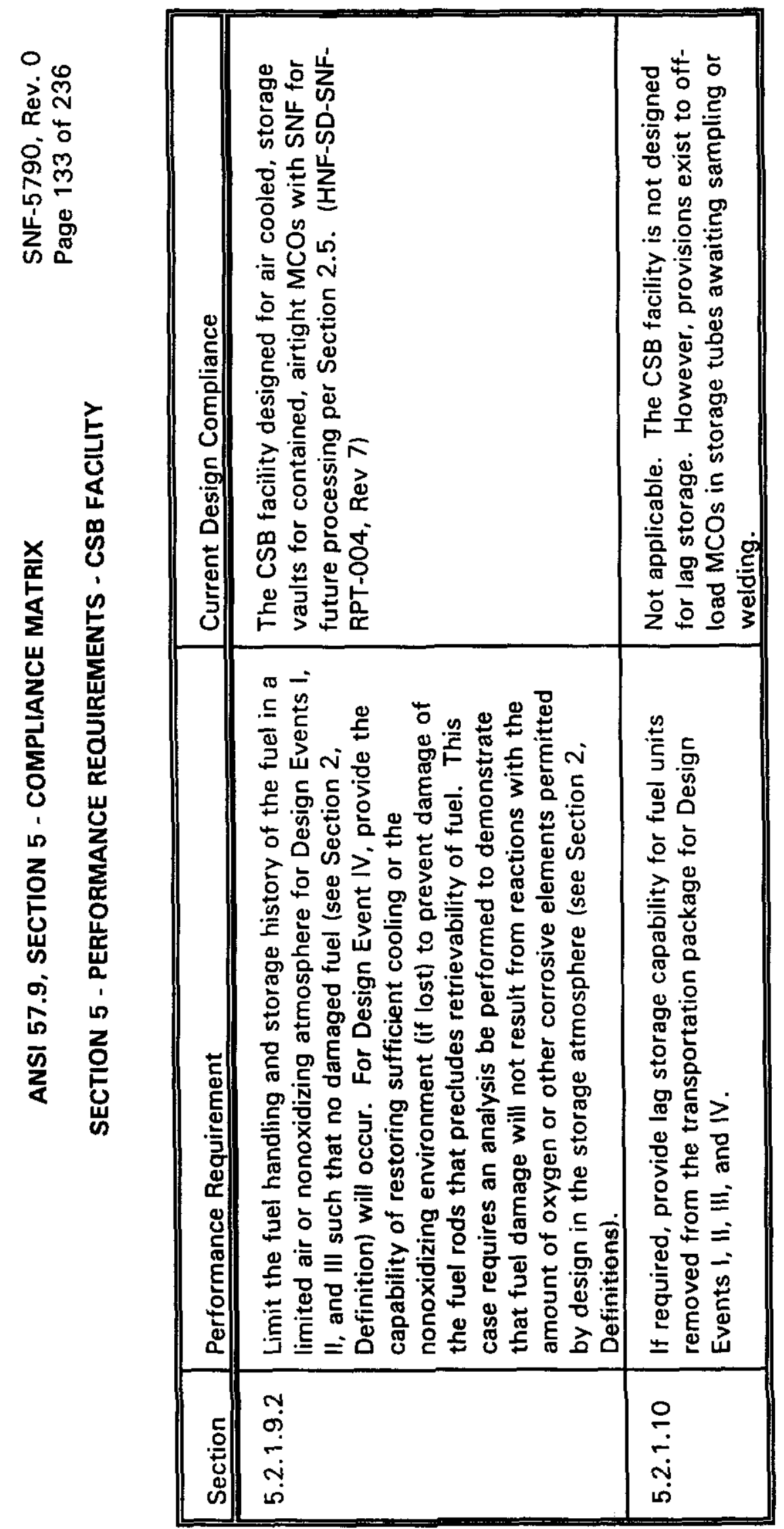




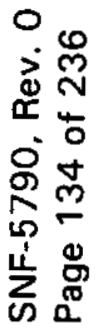

总

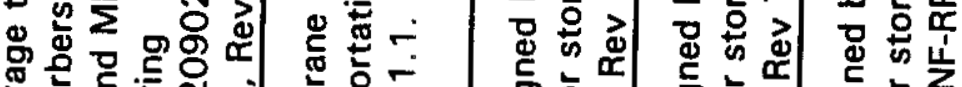

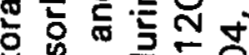

क $=0$ 守

ڤ

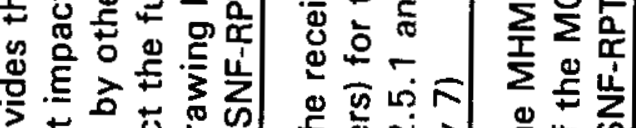

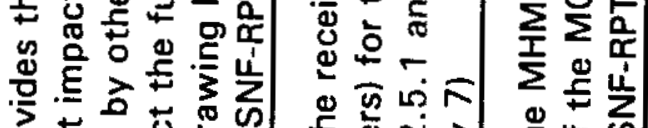

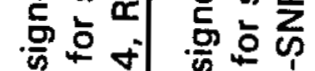

일

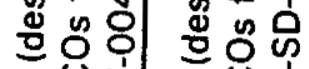

造过

응 잉

$\sum$ 占 $\sum_{\Sigma}$

등 든웡

등을 원 눈

志文文

ఫ

究苍

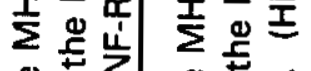

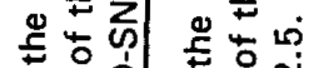

品方部

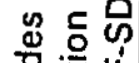

\& 5 की

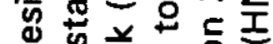

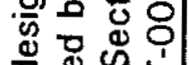

曹

눈 눈 문

出可量

هัँ

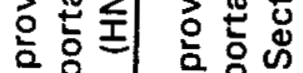

盯

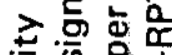

응 웅.

을

>은

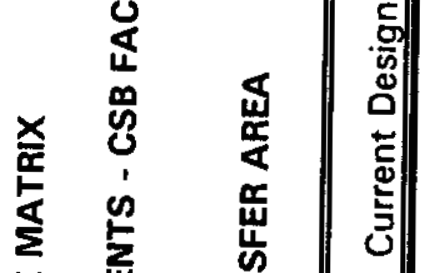

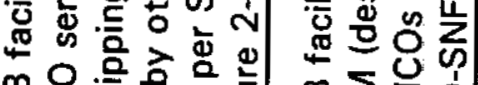

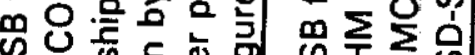

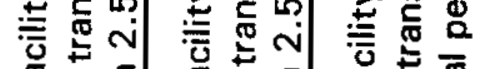

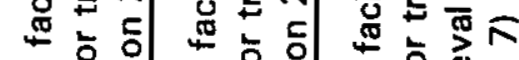

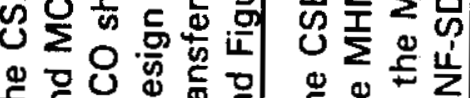

온원 운

品

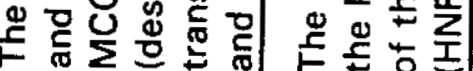

उ कूष

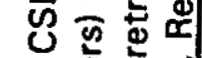

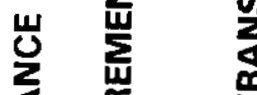

焉

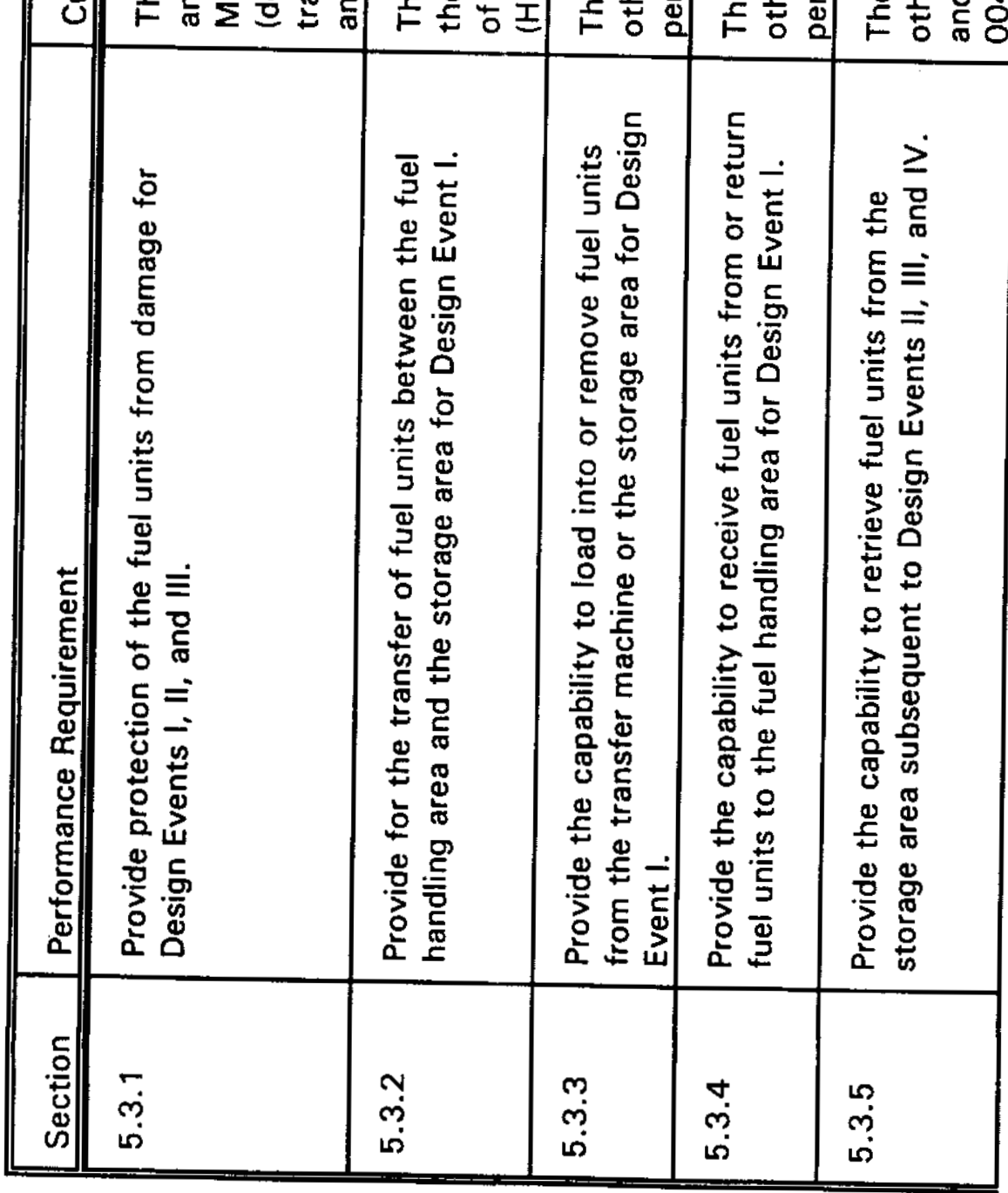




\begin{tabular}{|c|c|c|c|c|c|}
\hline 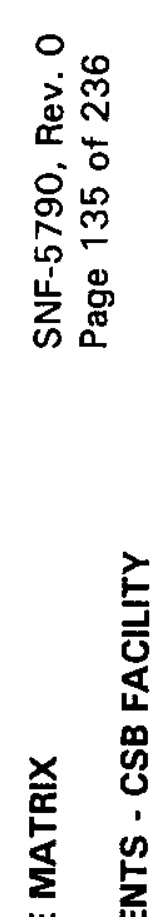 & 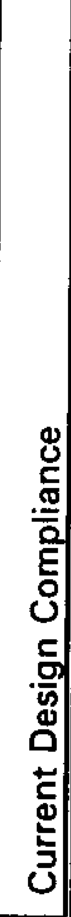 & 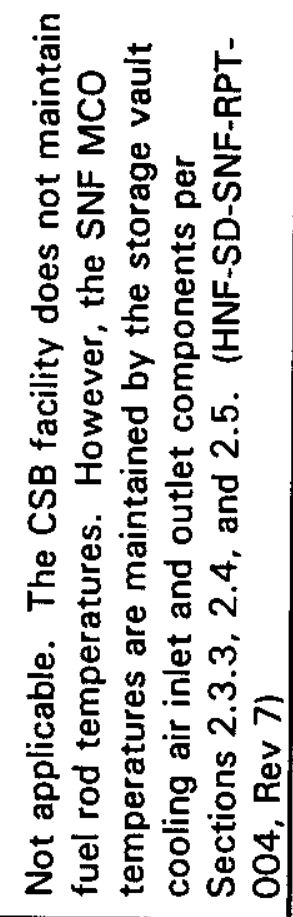 & 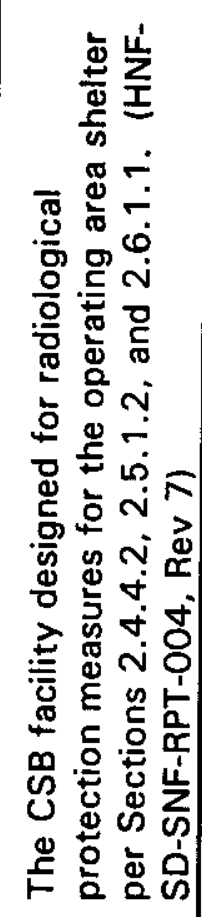 & 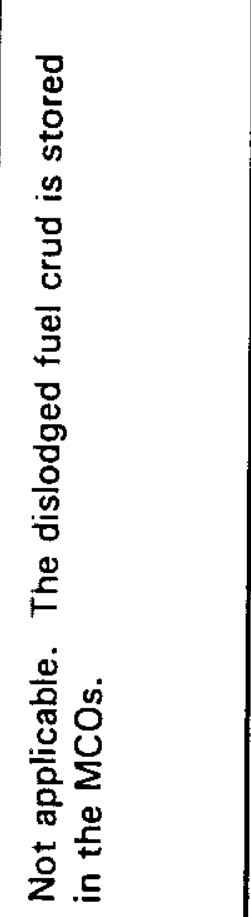 & 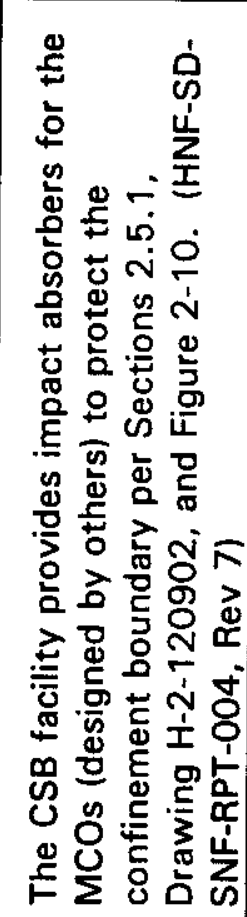 \\
\hline 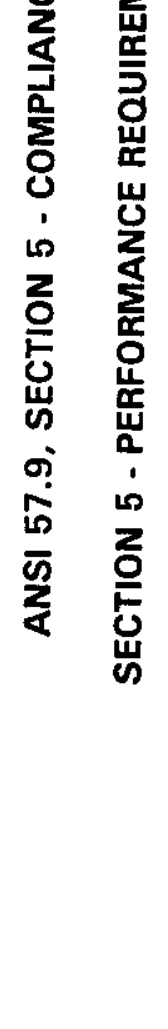 & 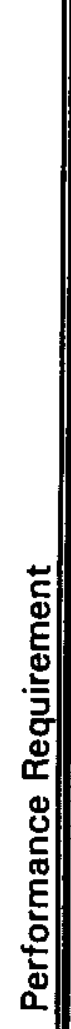 & 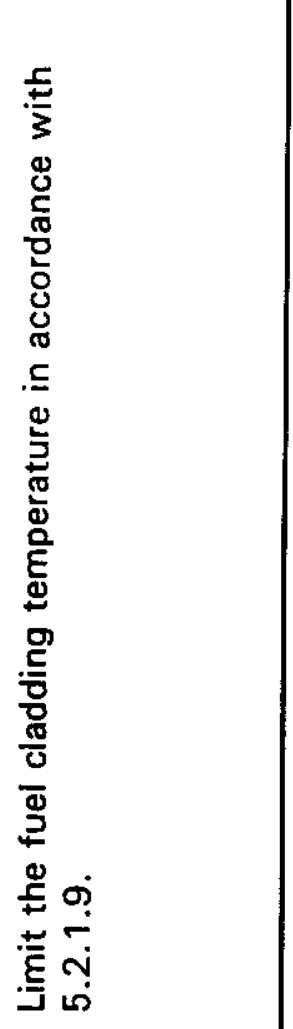 & 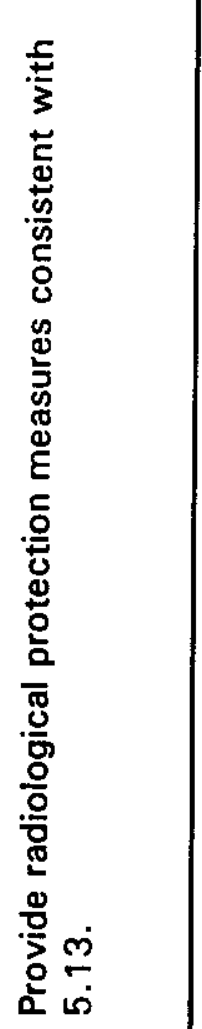 & 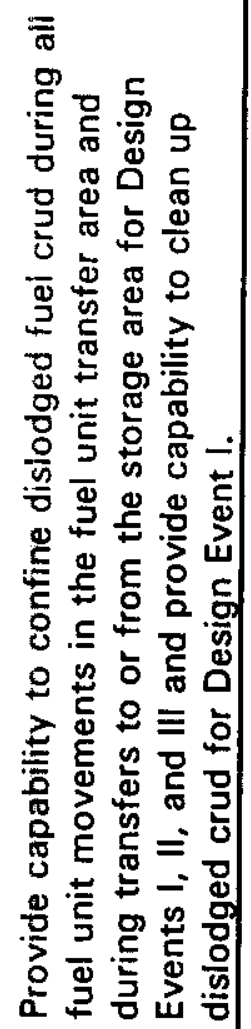 & 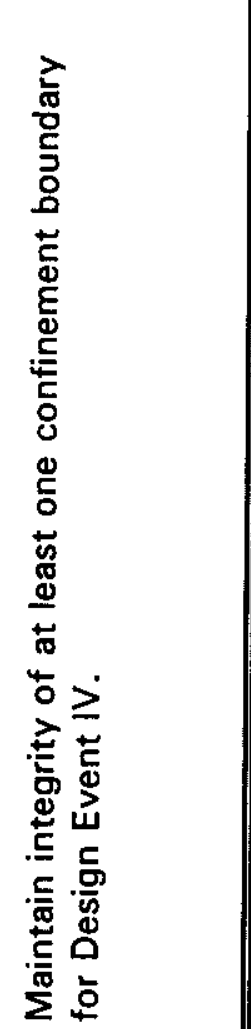 \\
\hline & 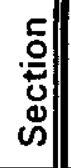 & $\begin{array}{l}\mathscr{0} \\
\dot{m} \\
\dot{\omega}\end{array}$ & ஜֶ & $\ddot{m}$ & $\begin{array}{l}\text { क) } \\
\text { مे } \\
\text { में }\end{array}$ \\
\hline
\end{tabular}




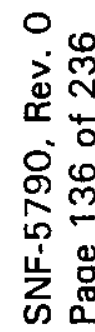

\begin{tabular}{|c|c|c|c|c|c|c|c|}
\hline 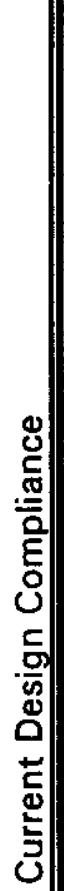 & & 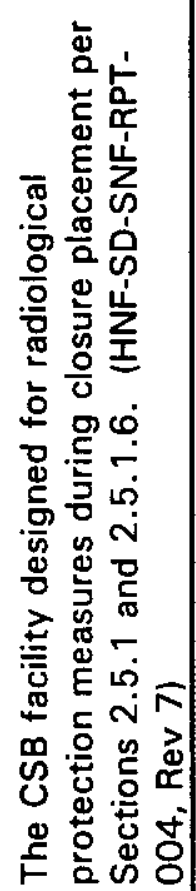 & & 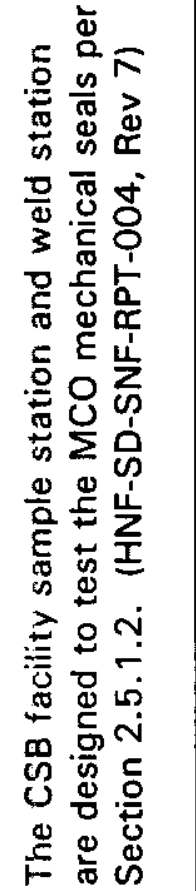 & 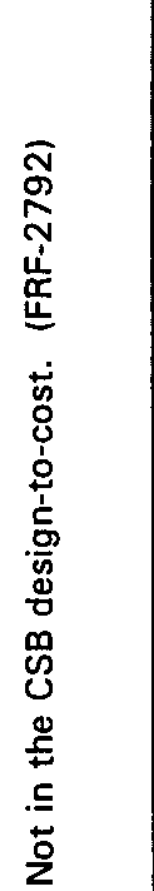 & 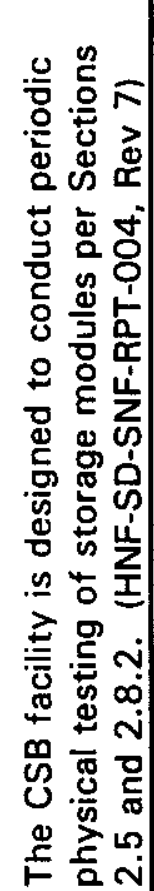 & 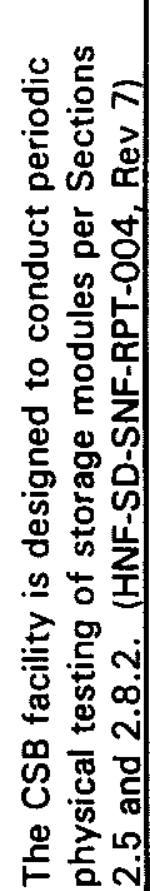 \\
\hline 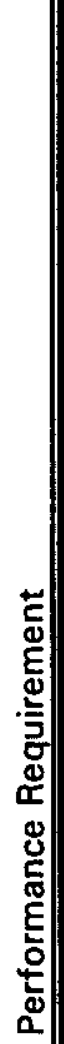 & 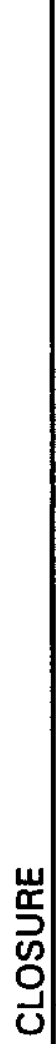 & 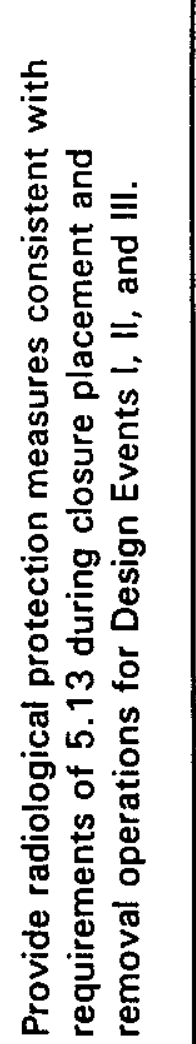 & 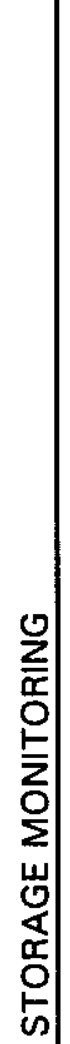 & 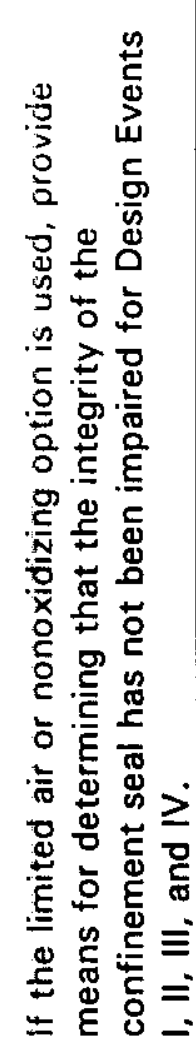 & 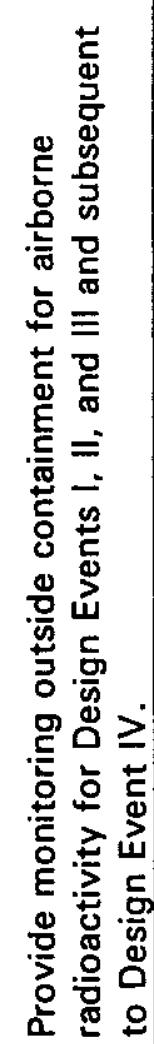 & 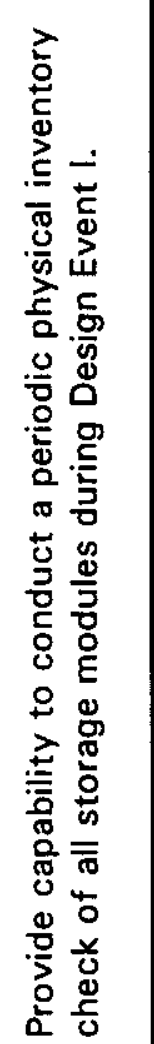 & 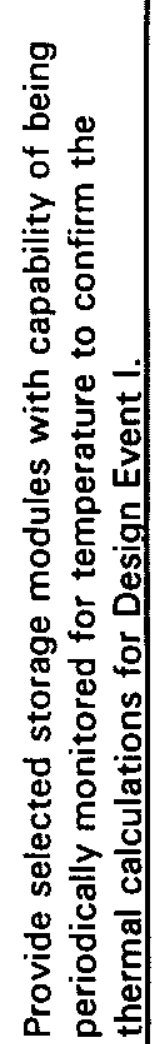 \\
\hline 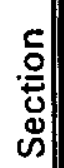 & $\begin{array}{l}5 \\
+ \\
\end{array}$ & نே & $\begin{array}{l}v y \\
\dot{y} \\
\qquad\end{array}$ & هִ & نֶ. & $\begin{array}{l}m \\
\sim \\
\dot{v} \\
\dot{N}\end{array}$ & $\begin{array}{l}\stackrel{\forall}{\sim} \\
\stackrel{+}{+} \\
\dot{\sigma}\end{array}$ \\
\hline
\end{tabular}




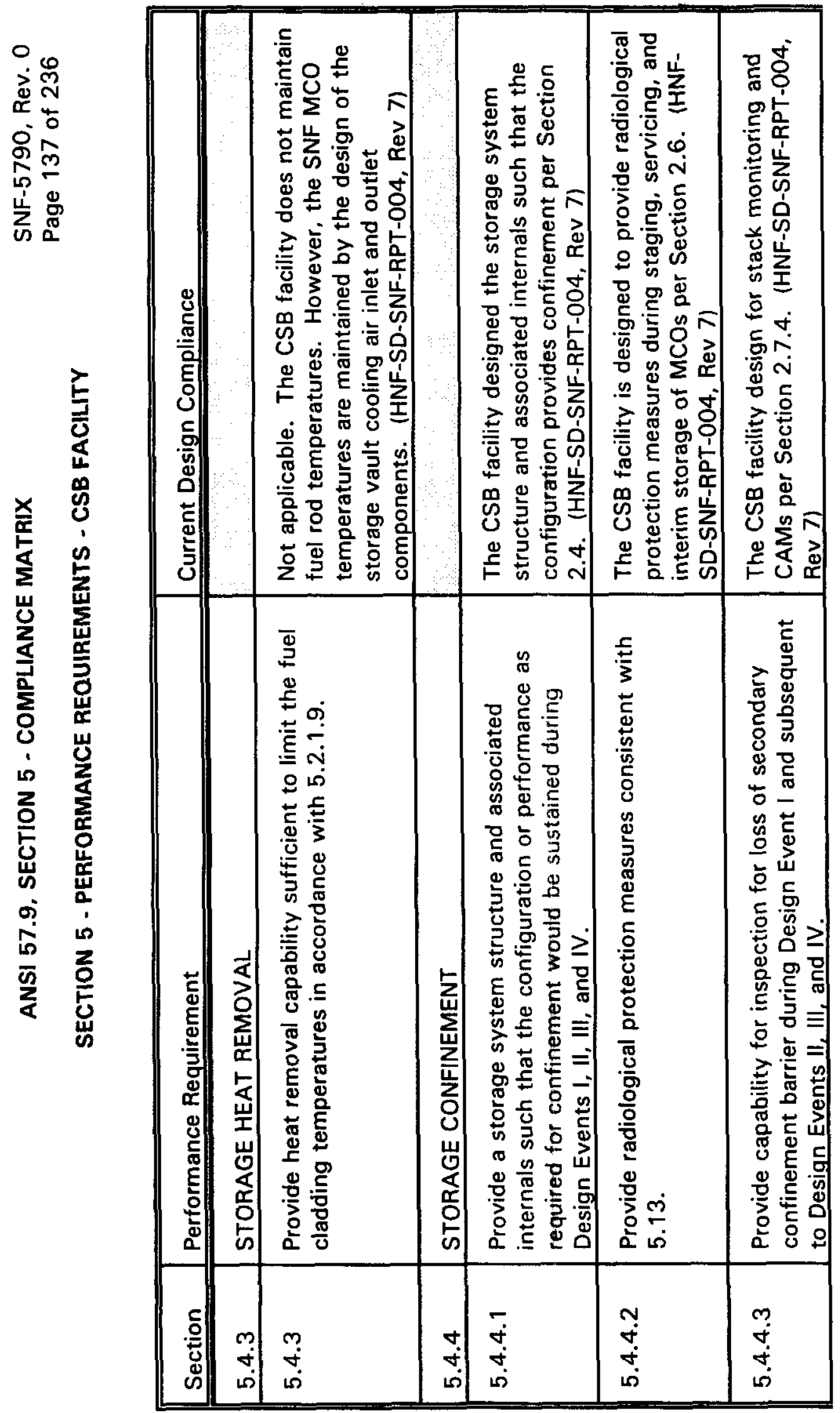




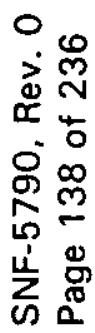

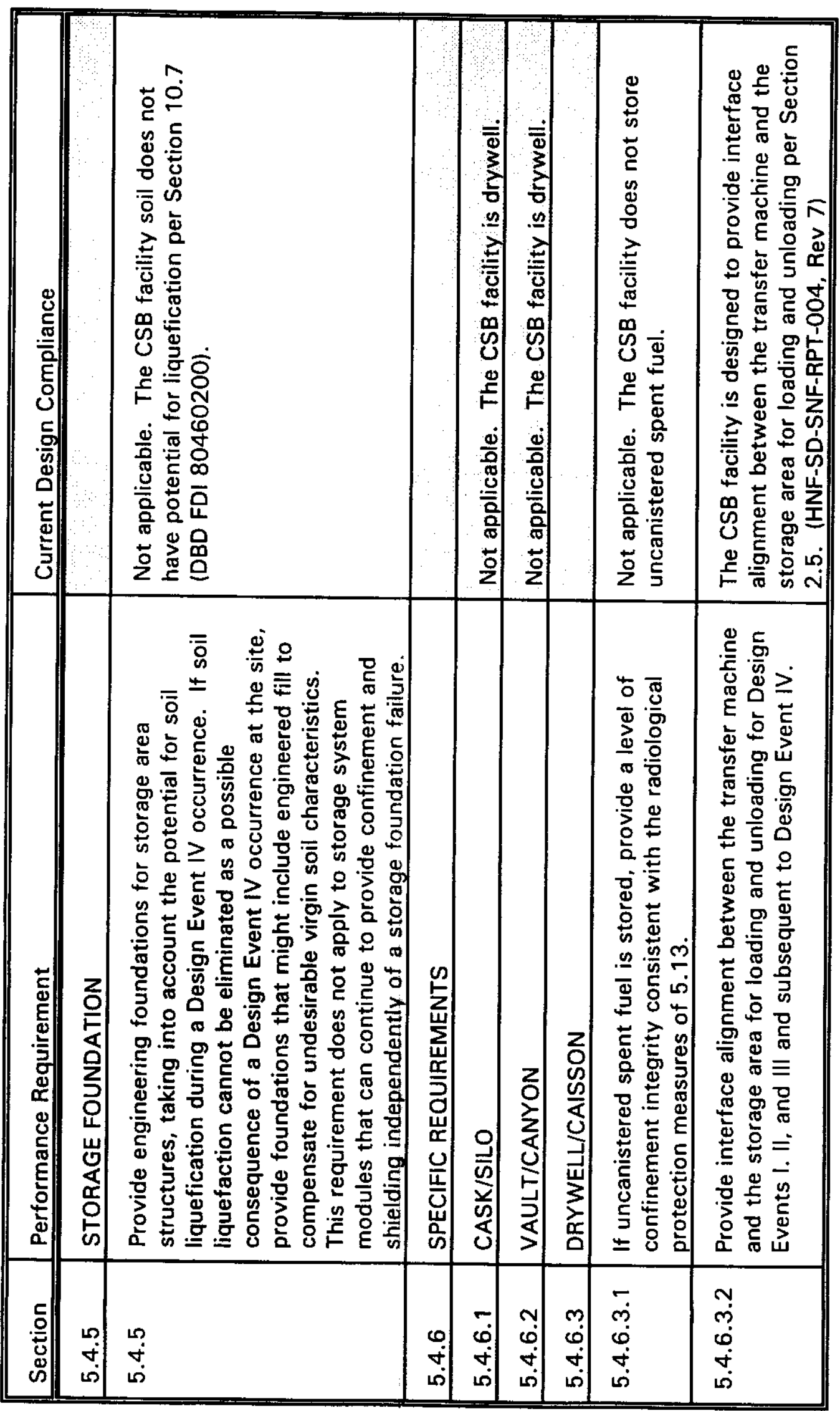




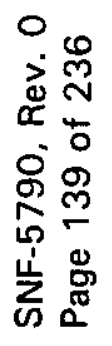

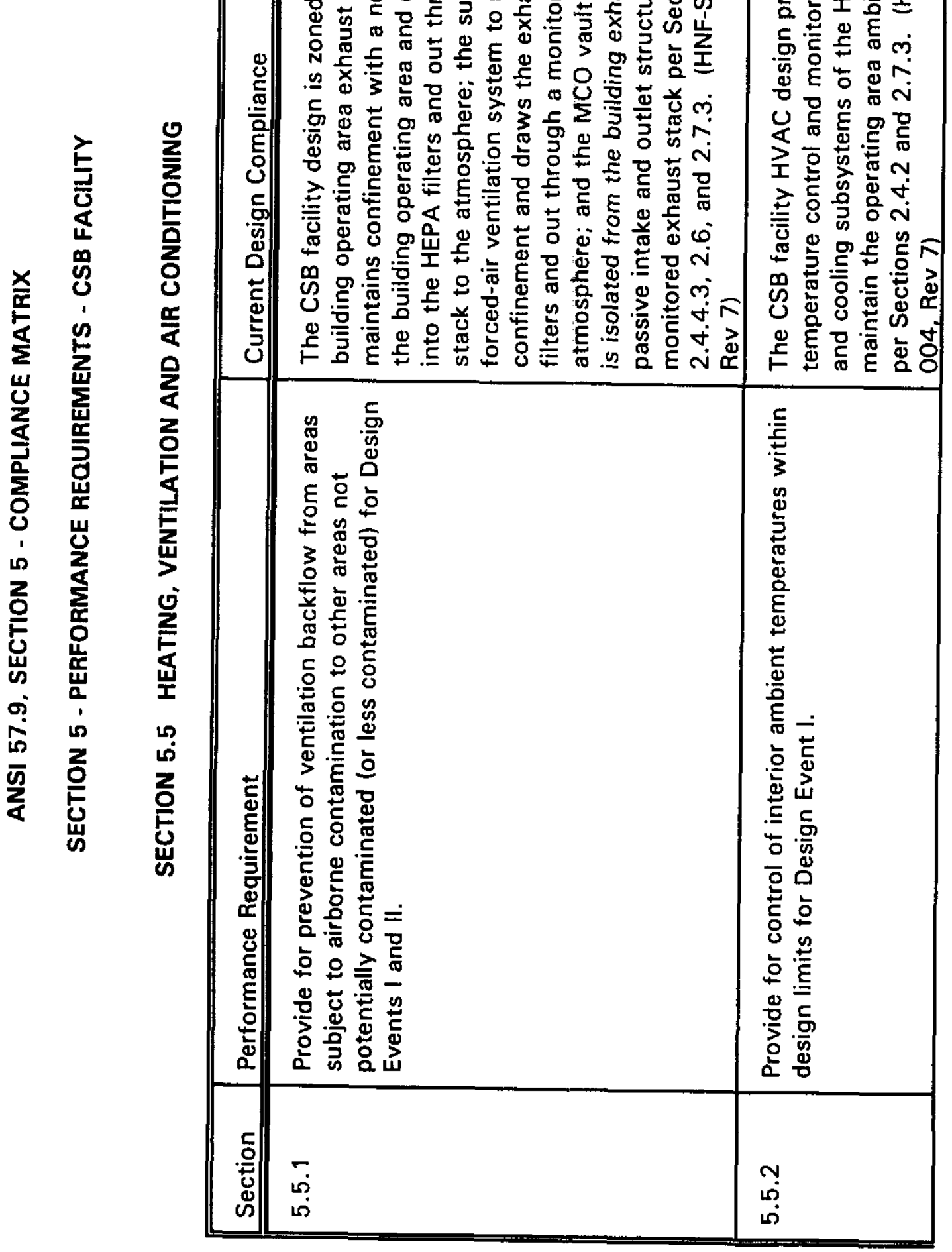




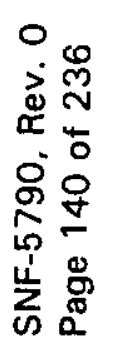

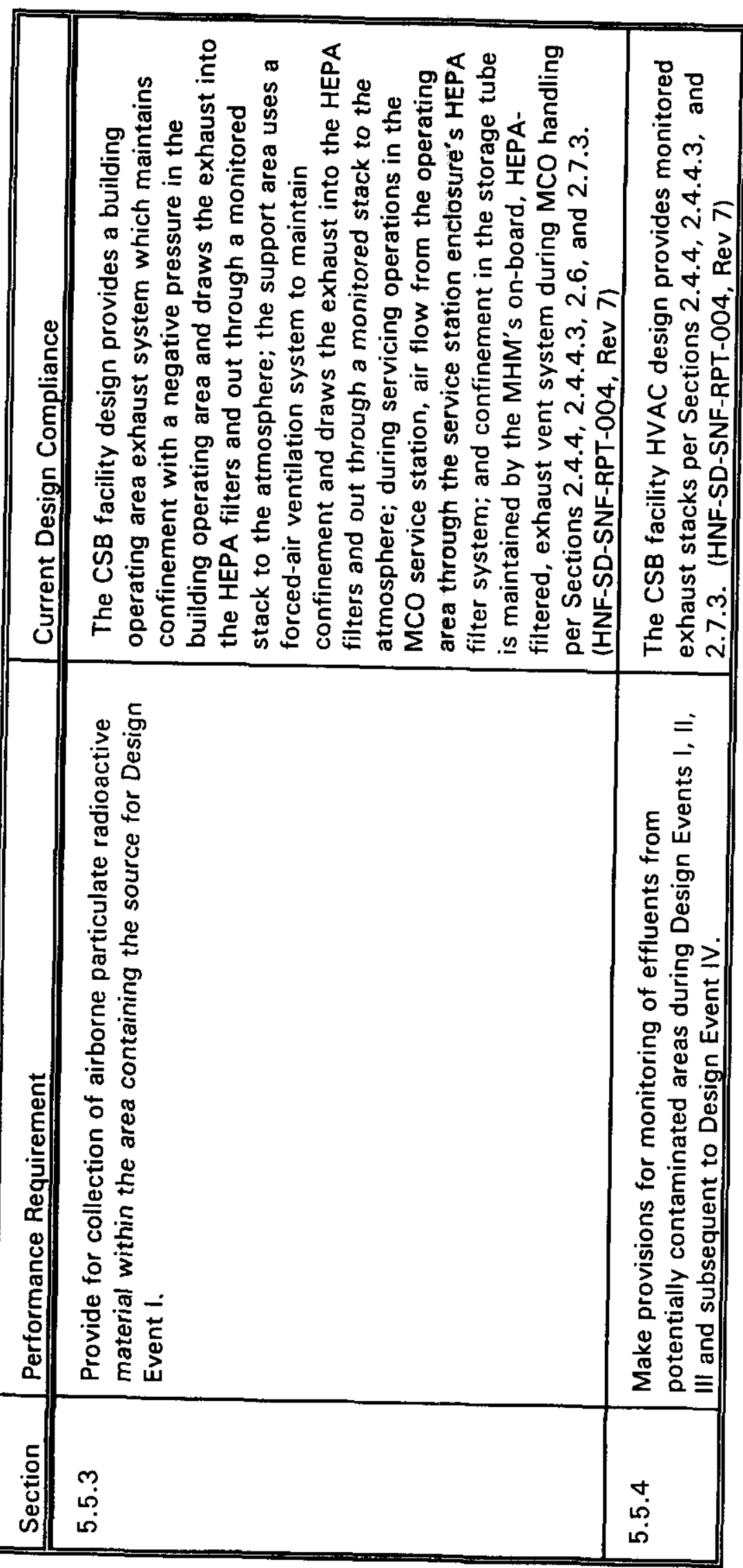




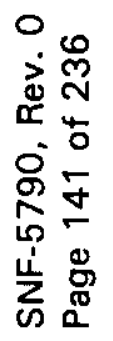

\begin{tabular}{|c|c|c|}
\hline 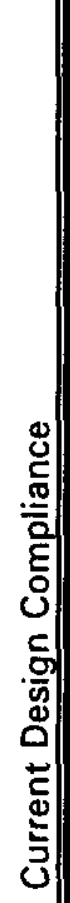 & 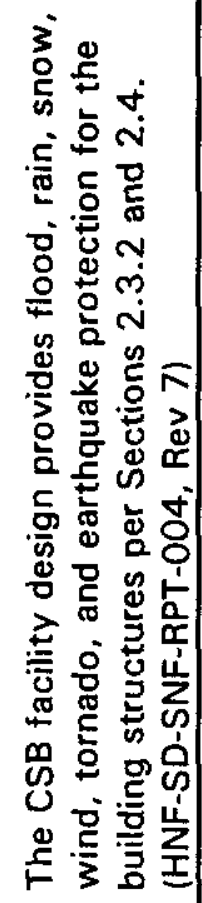 & 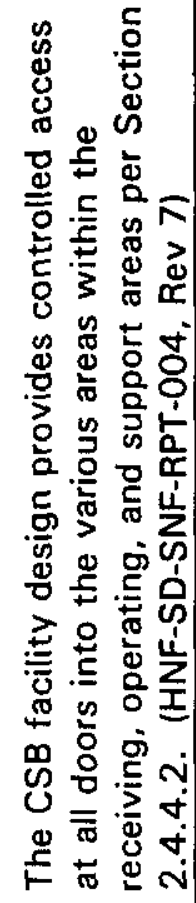 \\
\hline 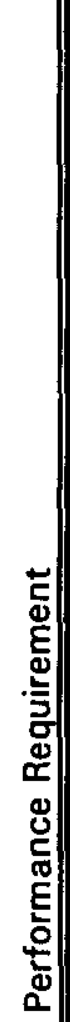 & 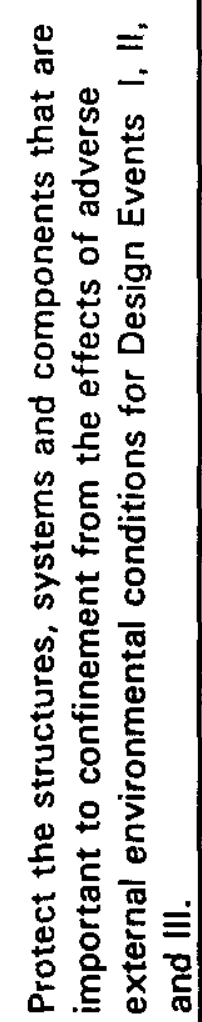 & 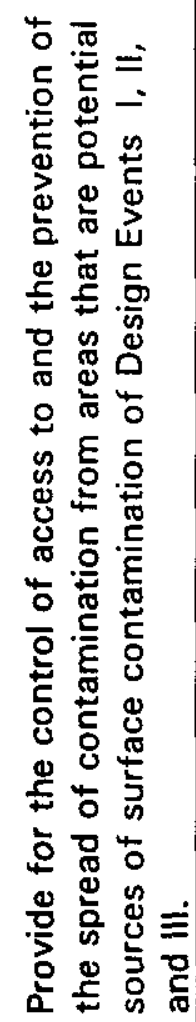 \\
\hline 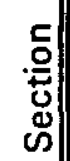 & هُ & $\begin{array}{l}7 \\
0\end{array}$ \\
\hline
\end{tabular}




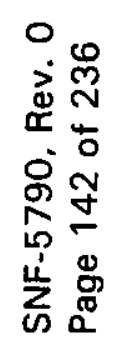

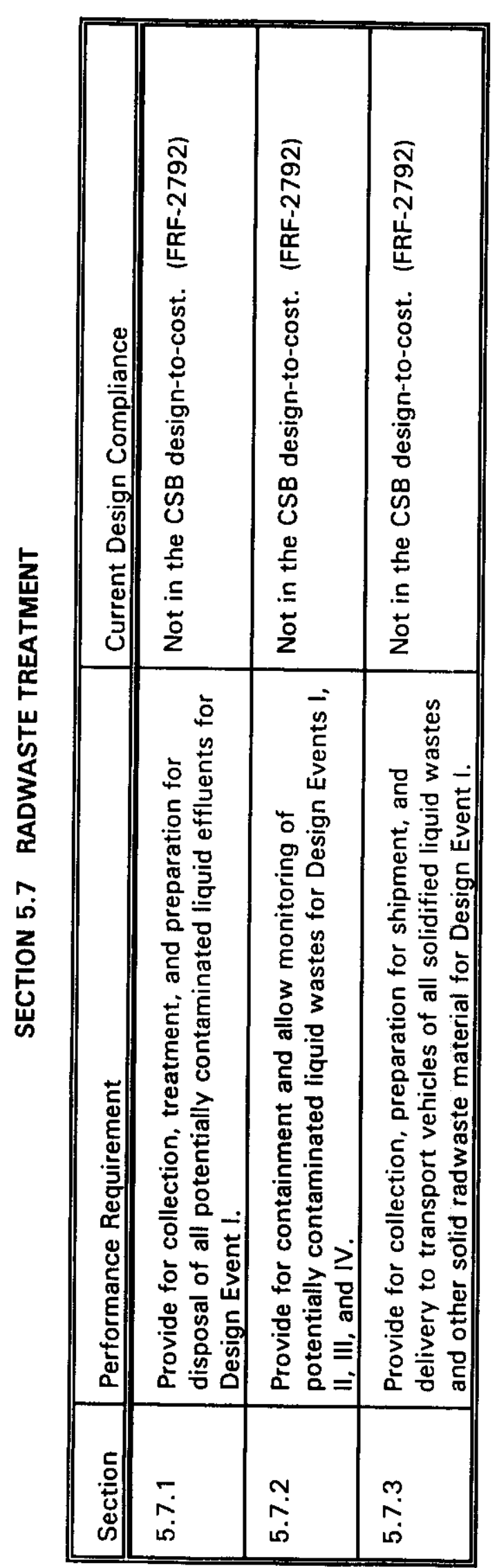




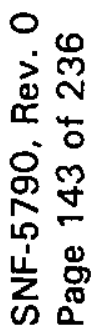

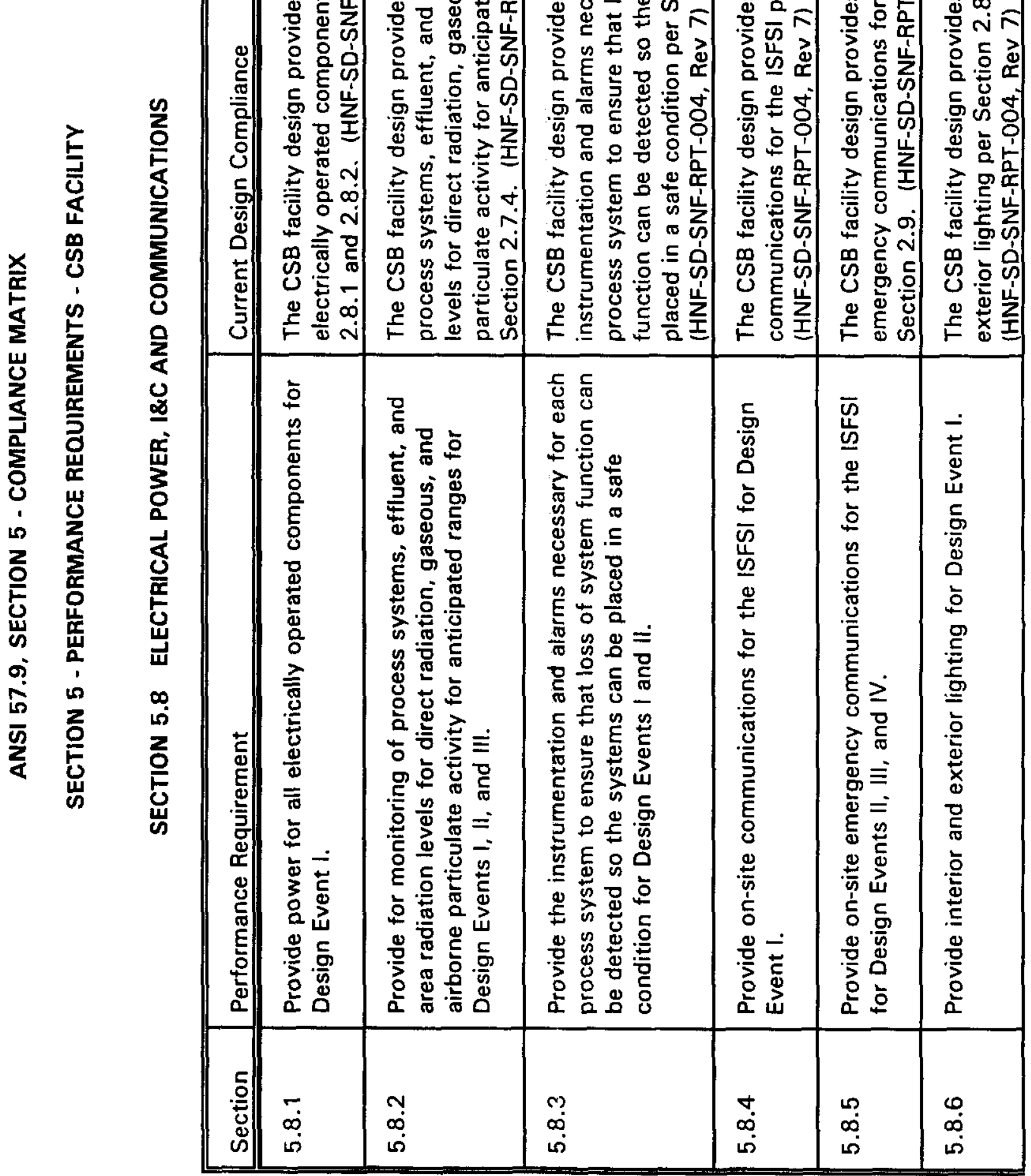




\begin{tabular}{|c|c|c|c|c|}
\hline 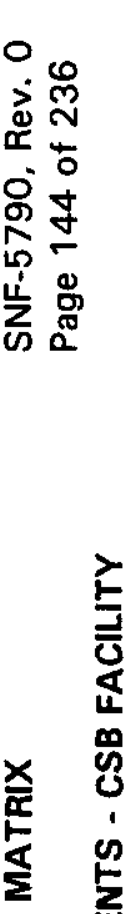 & 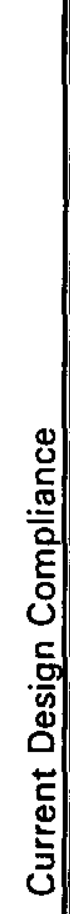 & 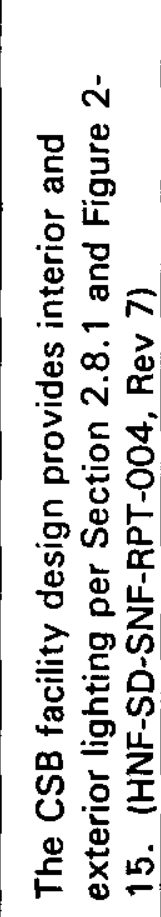 & 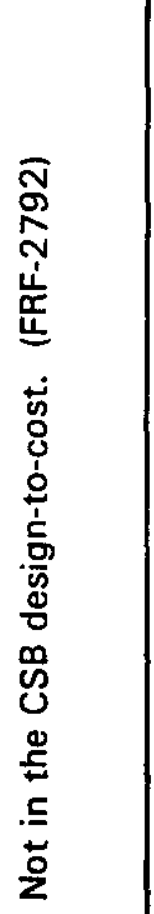 & 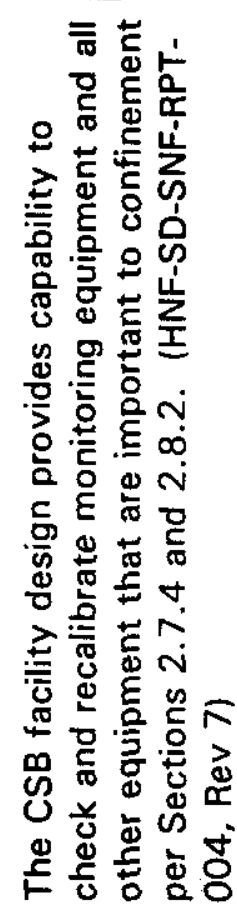 \\
\hline 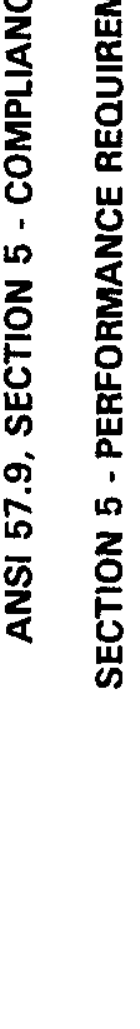 & 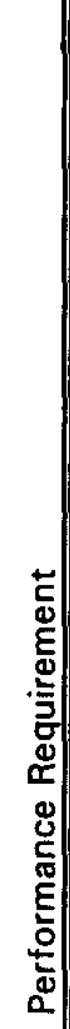 & 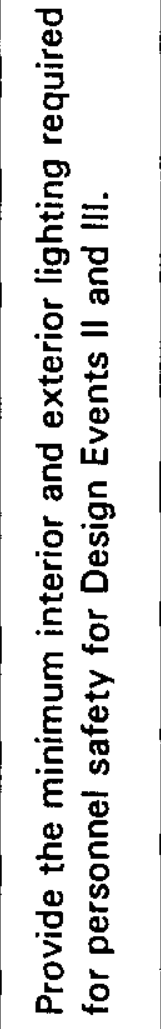 & 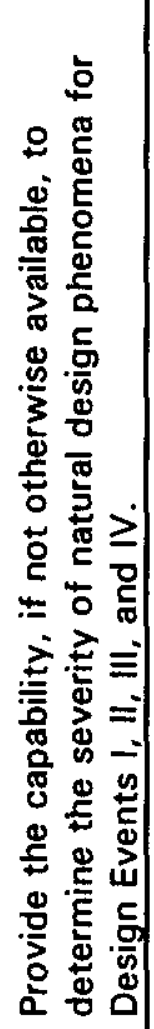 & 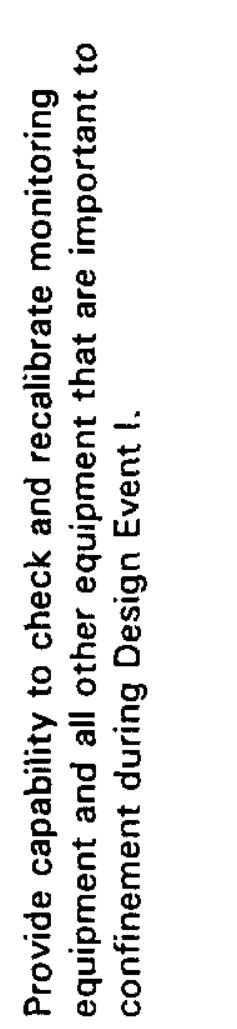 \\
\hline & $\begin{array}{l}0 \\
0 \\
0 \\
0\end{array}$ & مَ & "ద & فُ \\
\hline
\end{tabular}




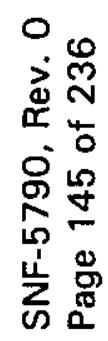

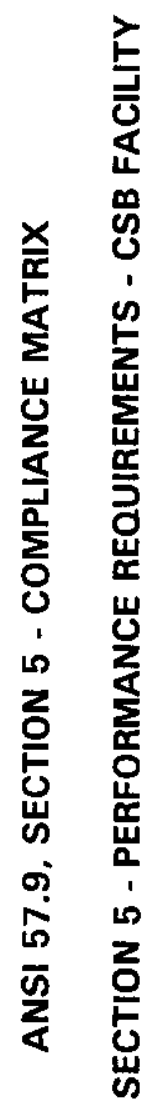

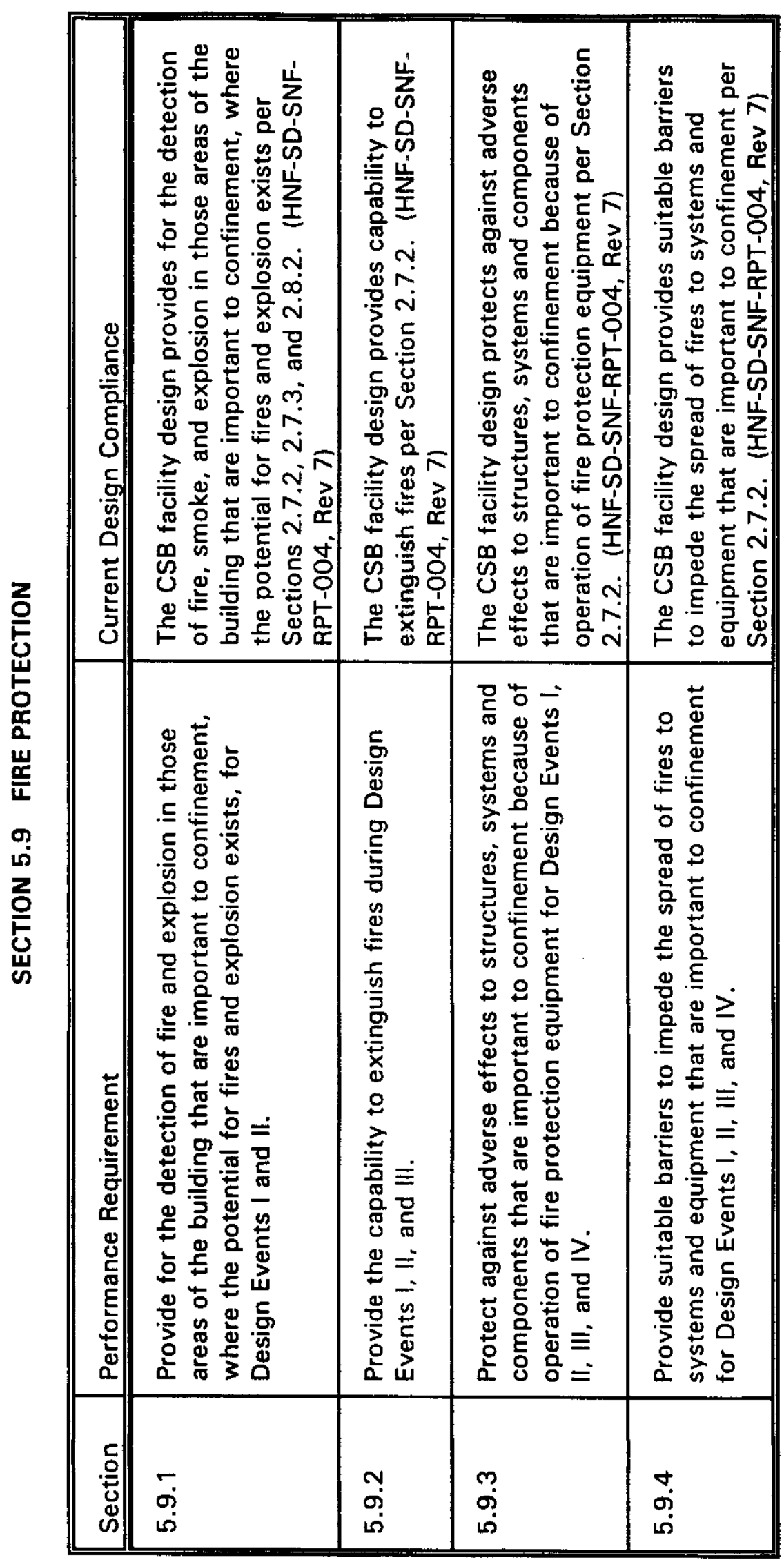




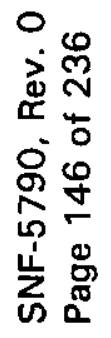

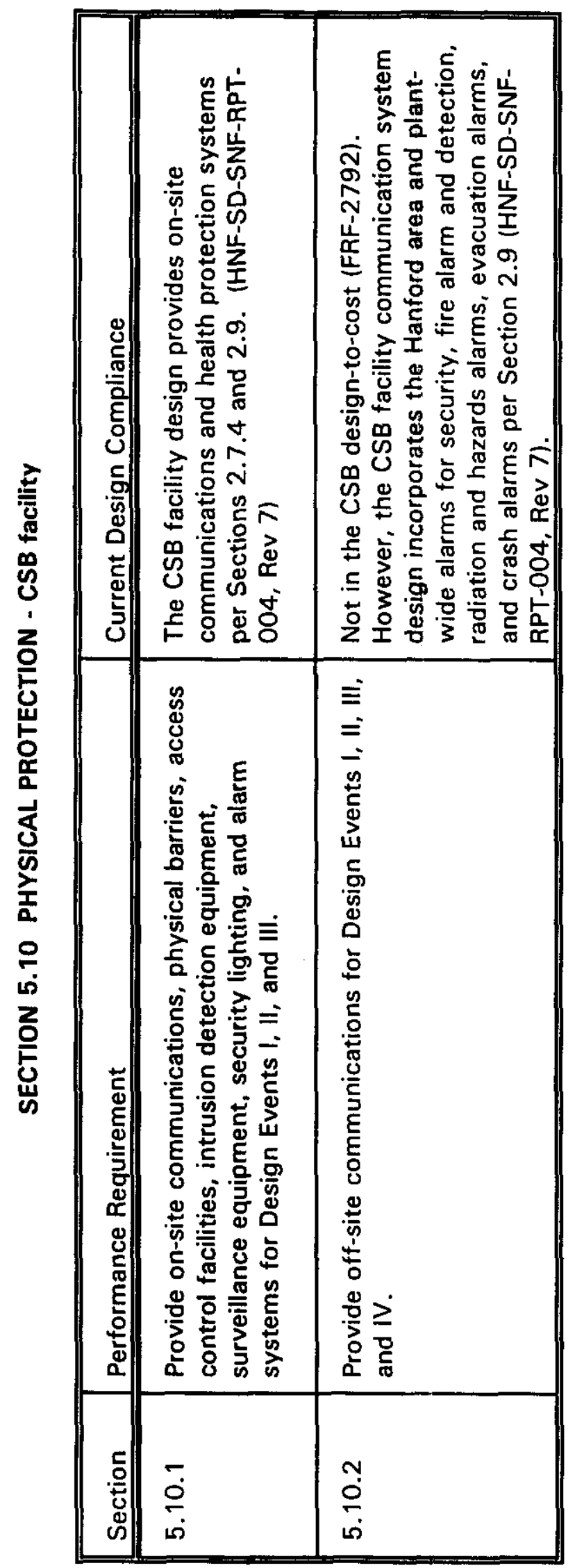




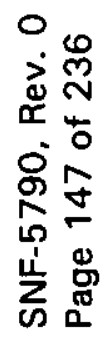

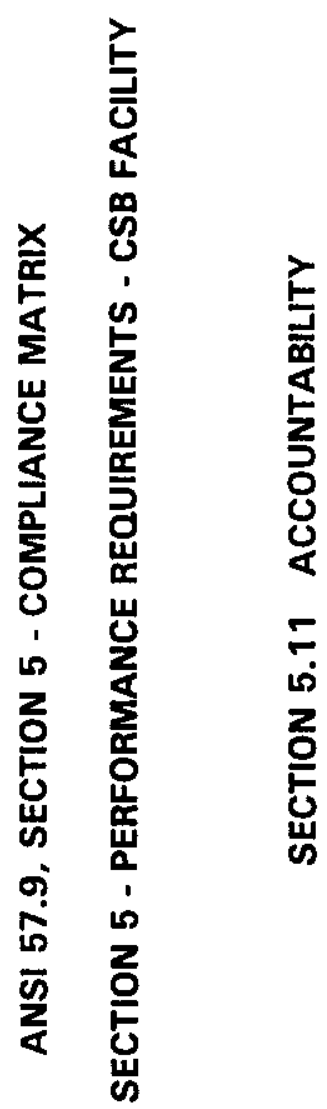

\begin{tabular}{|c|c|c|c|}
\hline 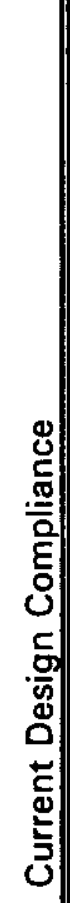 & 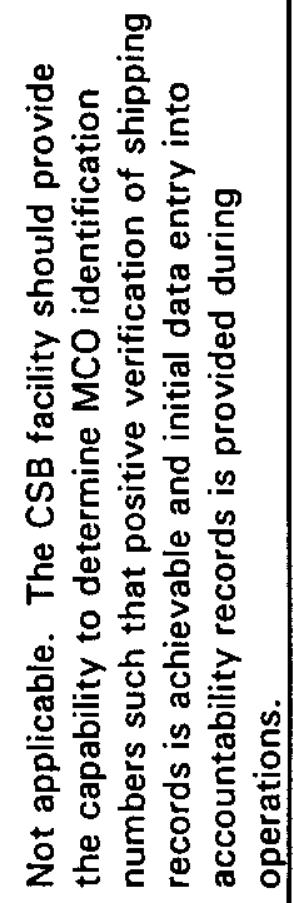 & 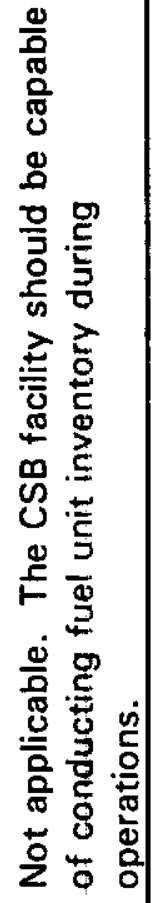 & 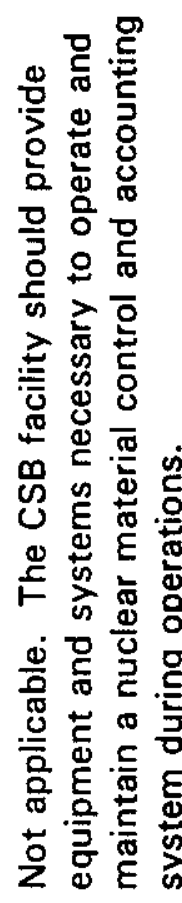 \\
\hline 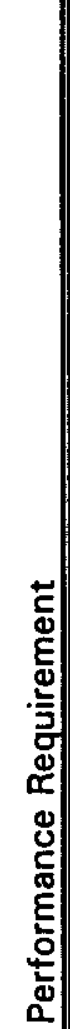 & 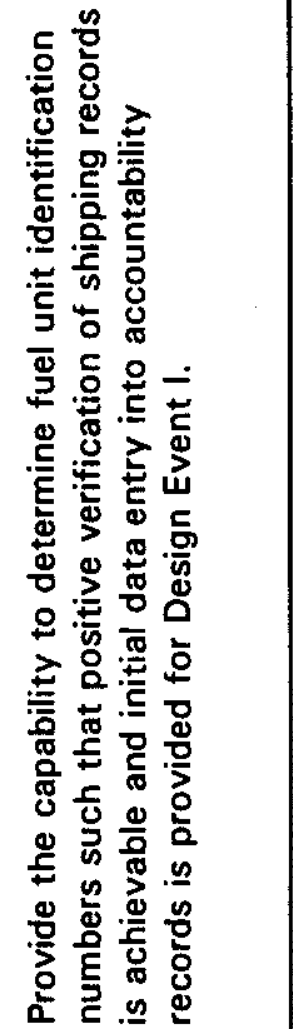 & 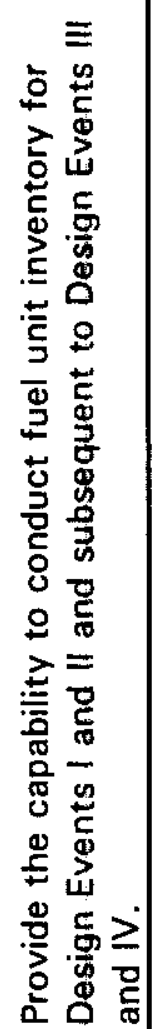 & 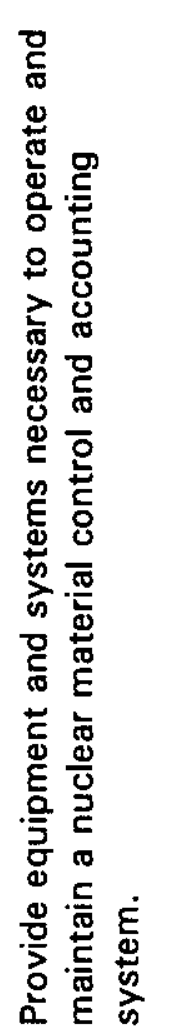 \\
\hline$\stackrel{\mathscr{N}}{\Delta}$ & 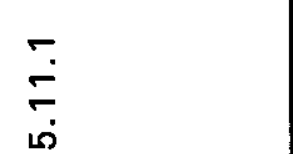 & $\frac{N}{\check{r}}$ & $\frac{m}{\check{r}}$ \\
\hline
\end{tabular}




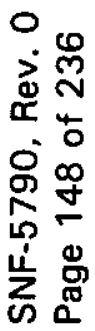

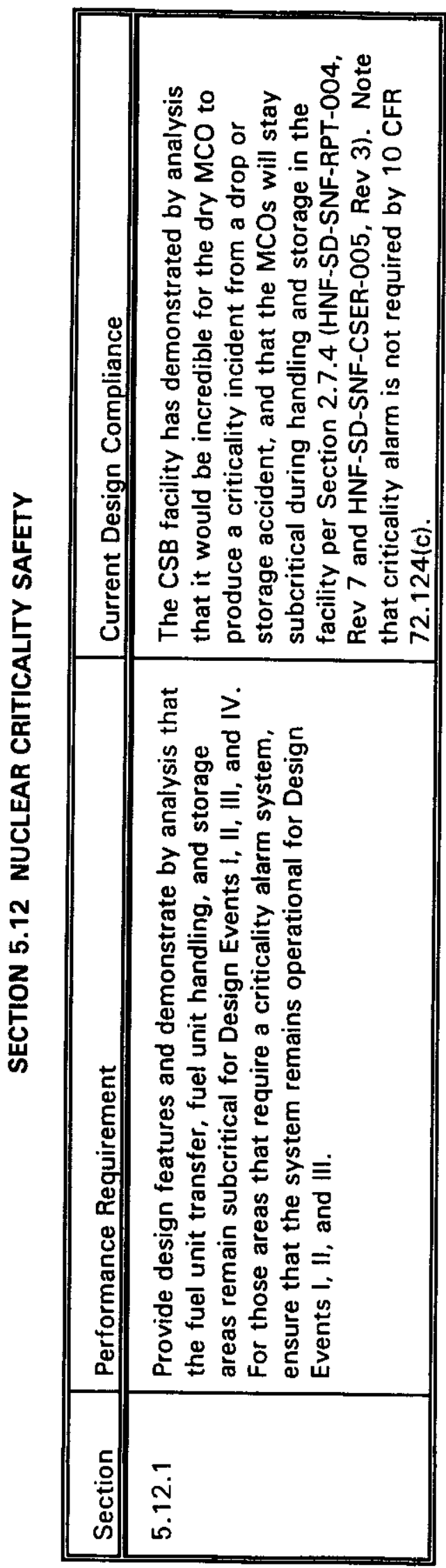




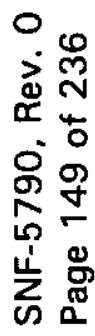

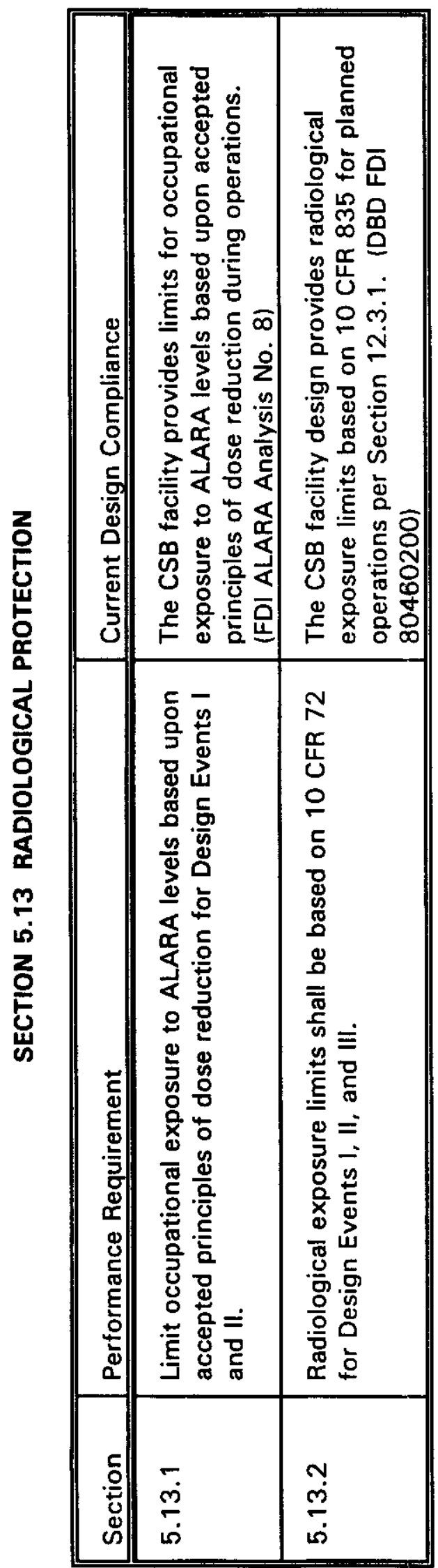




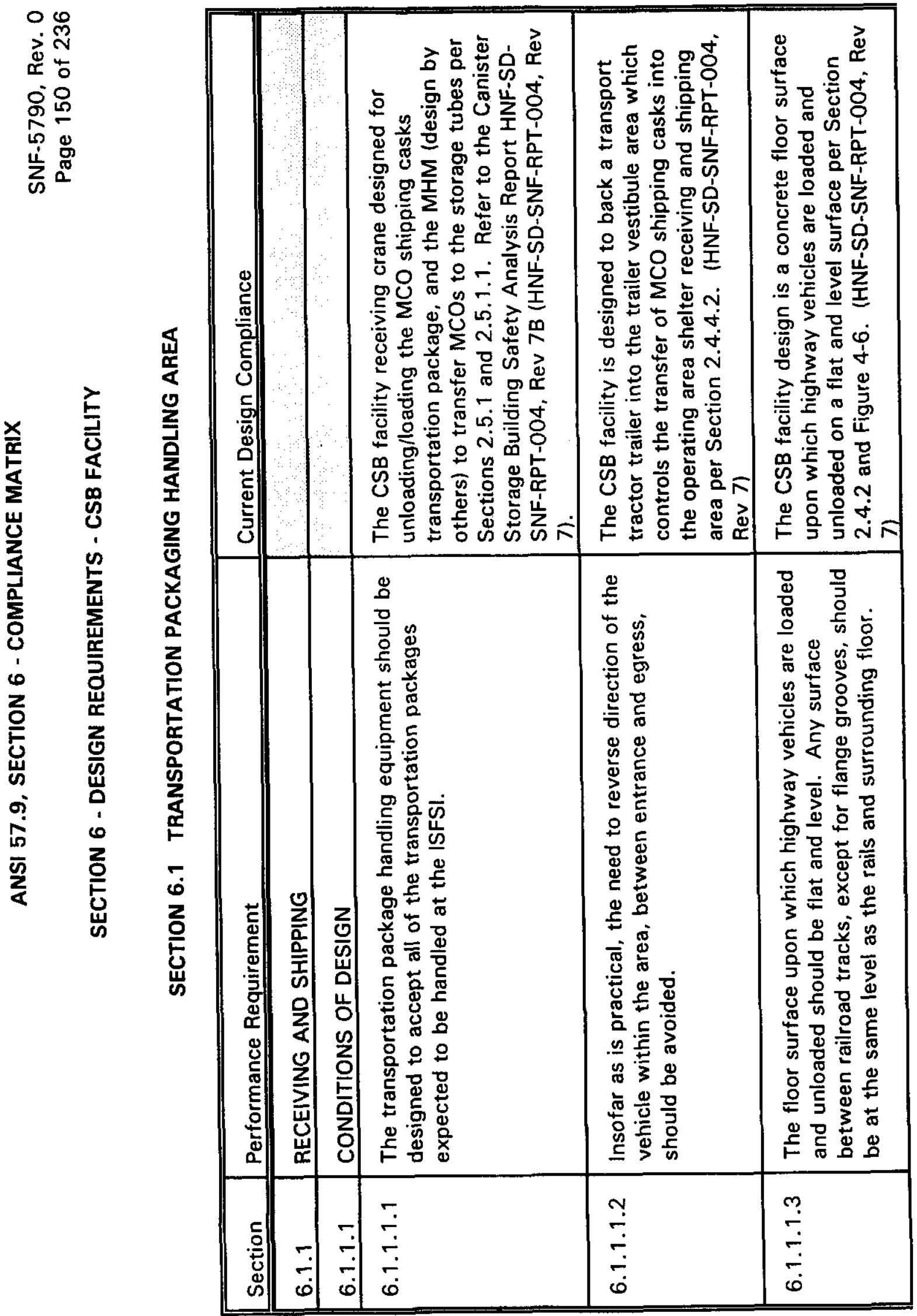




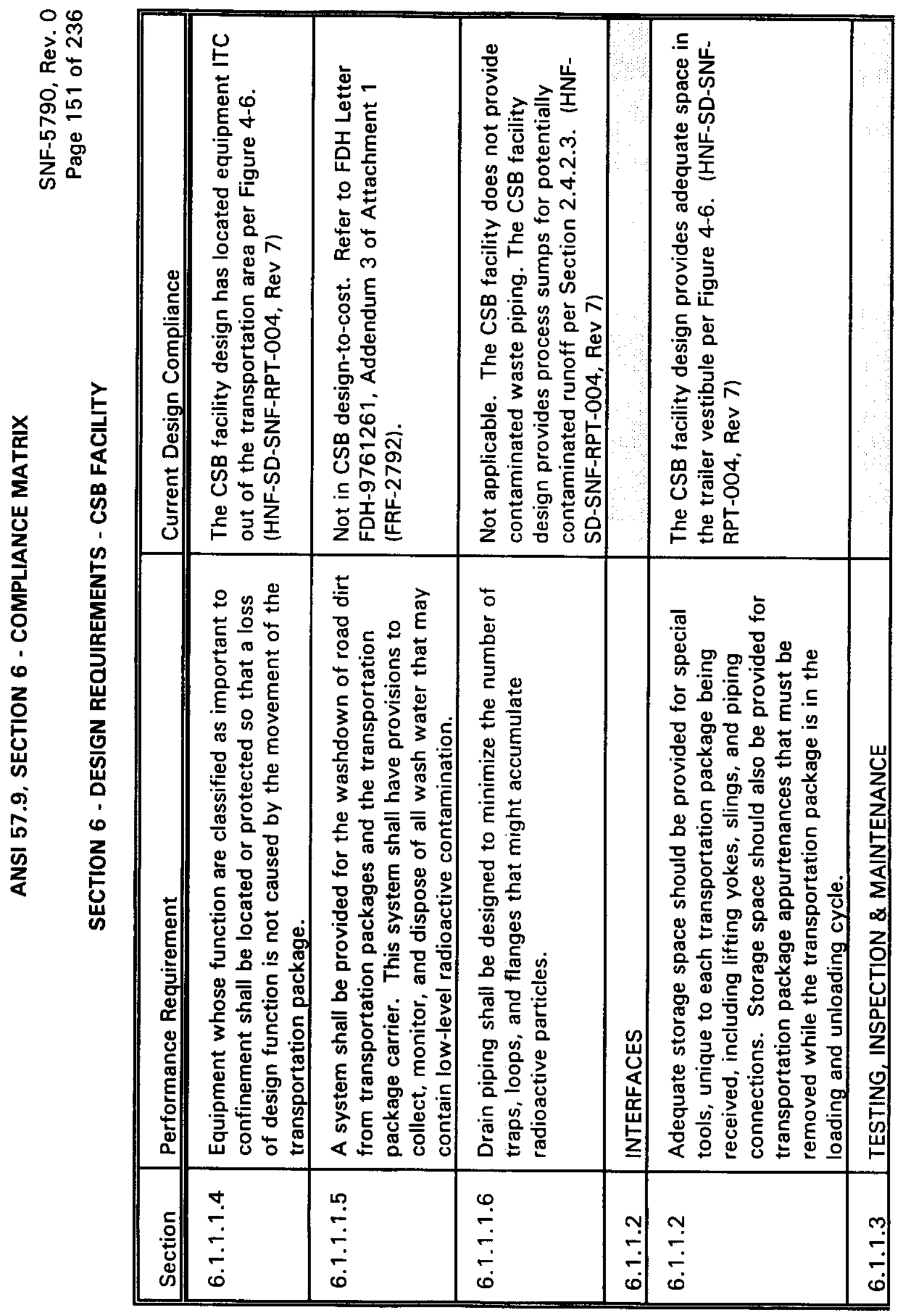




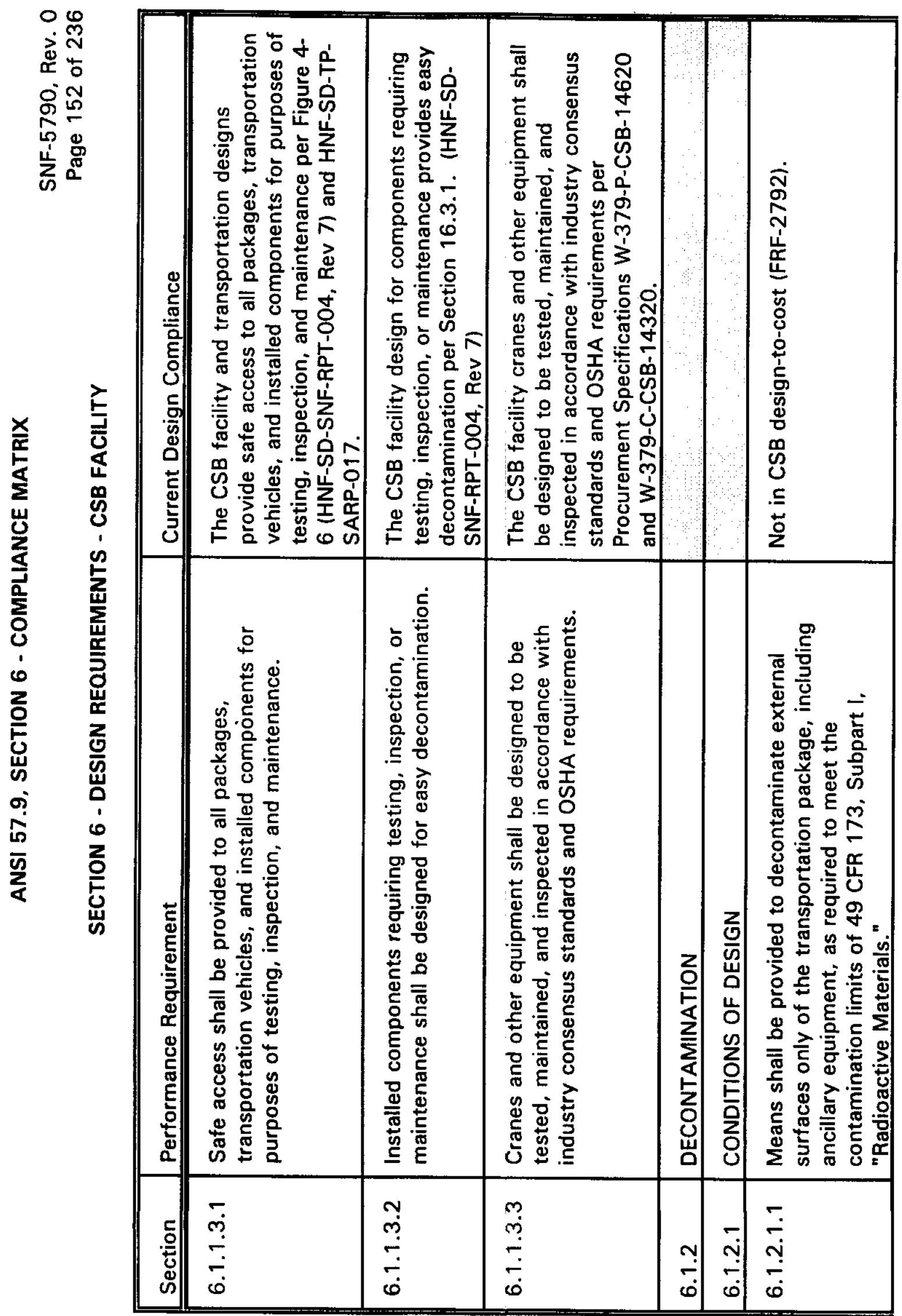




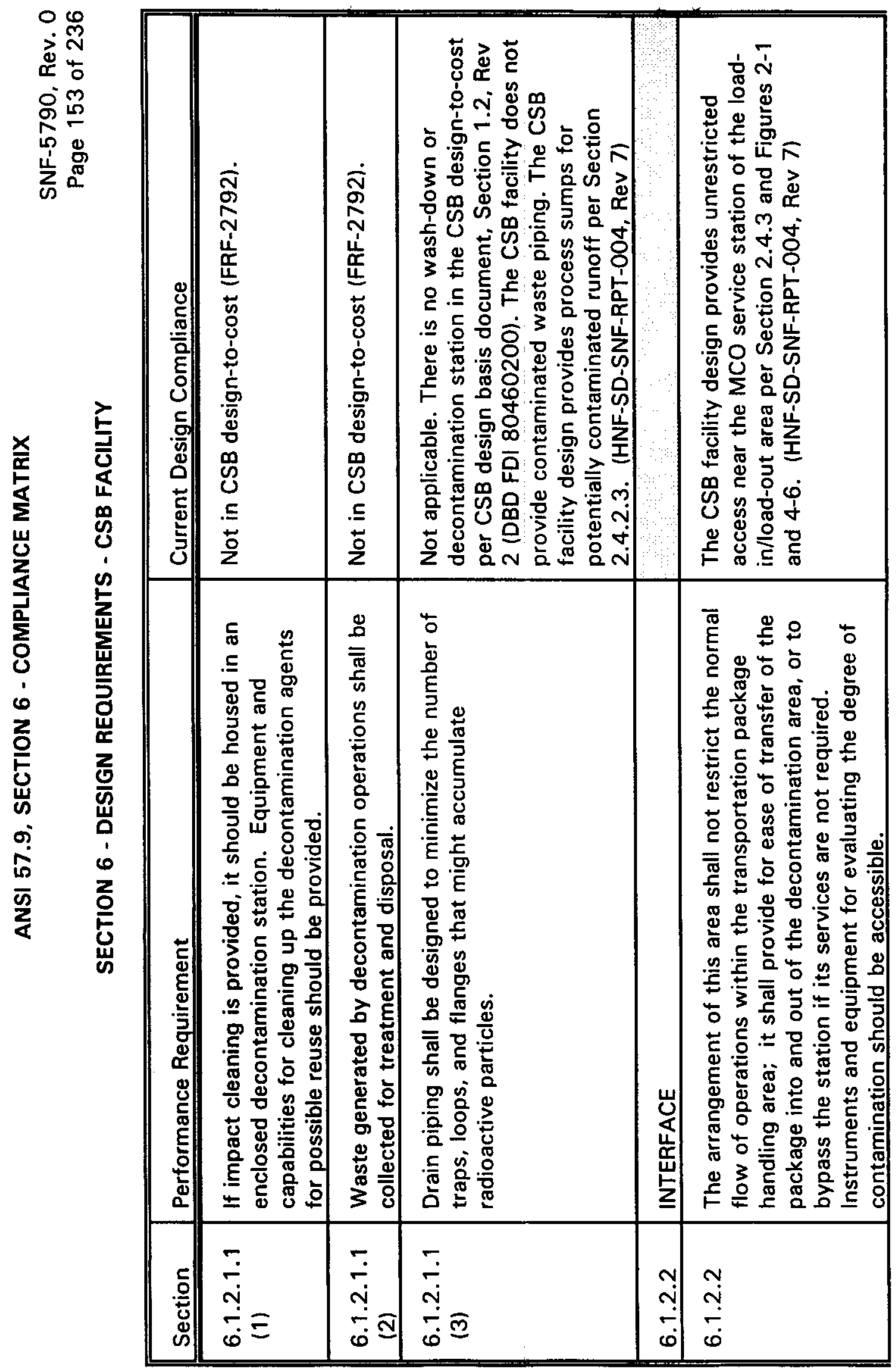




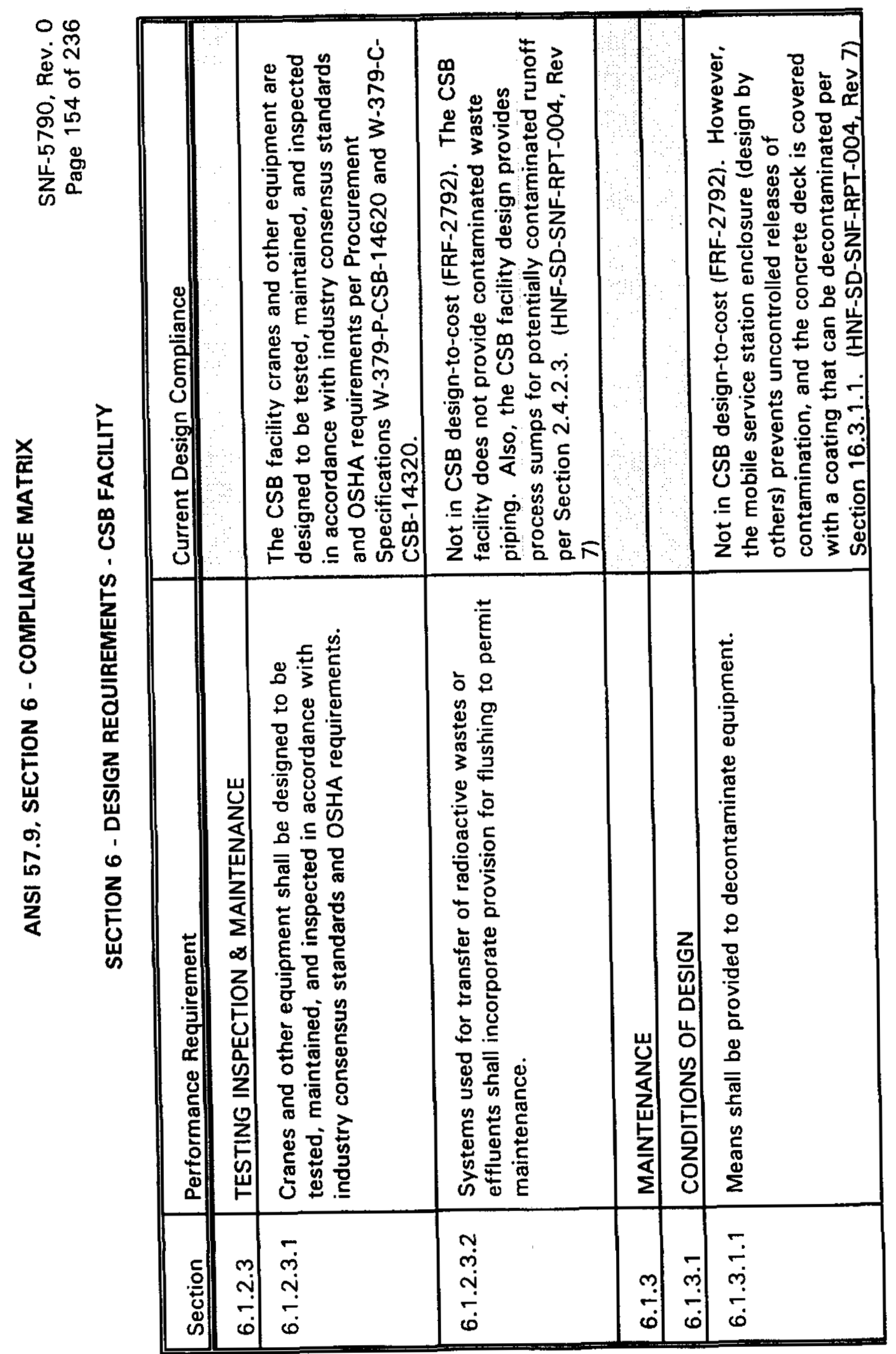




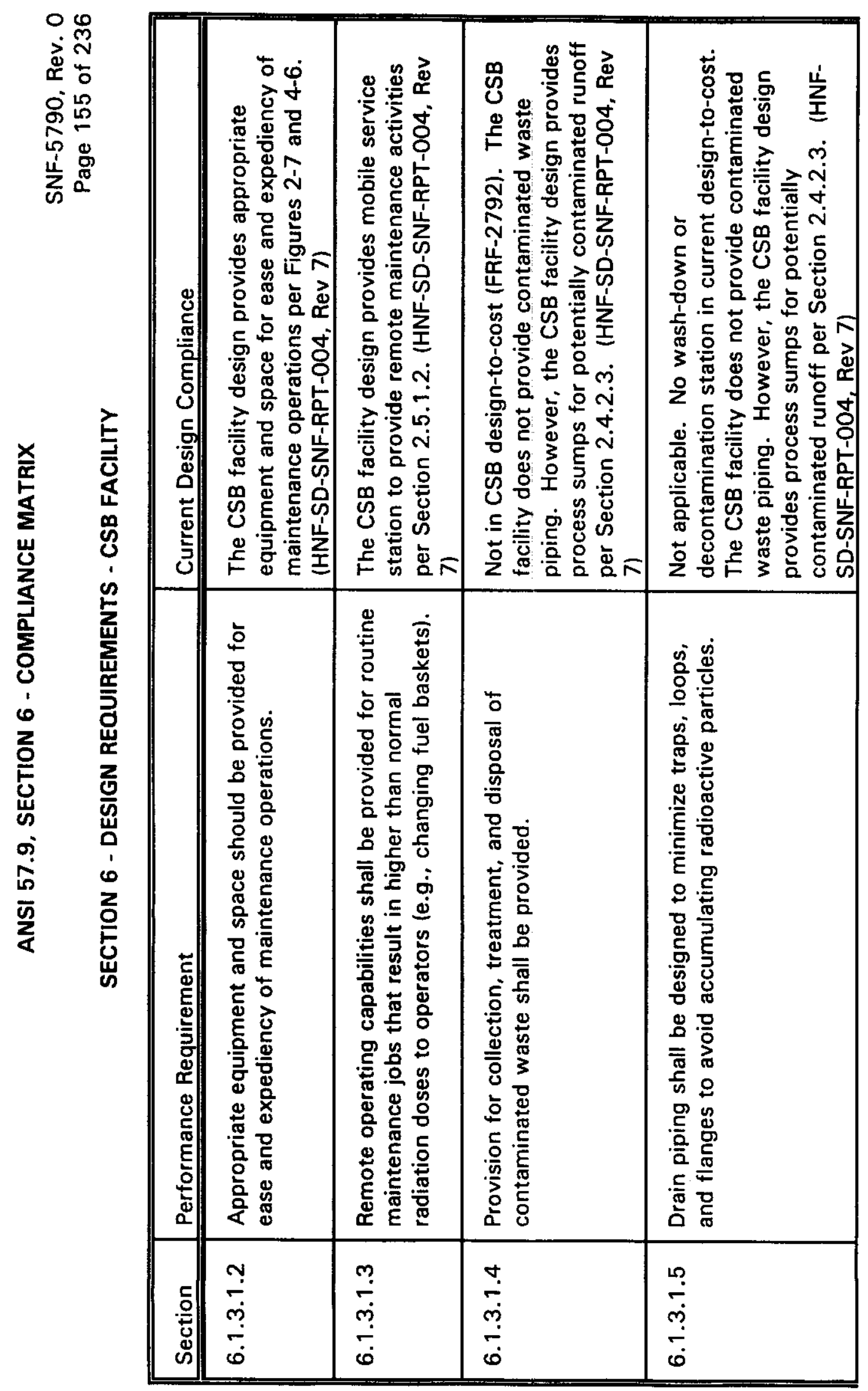




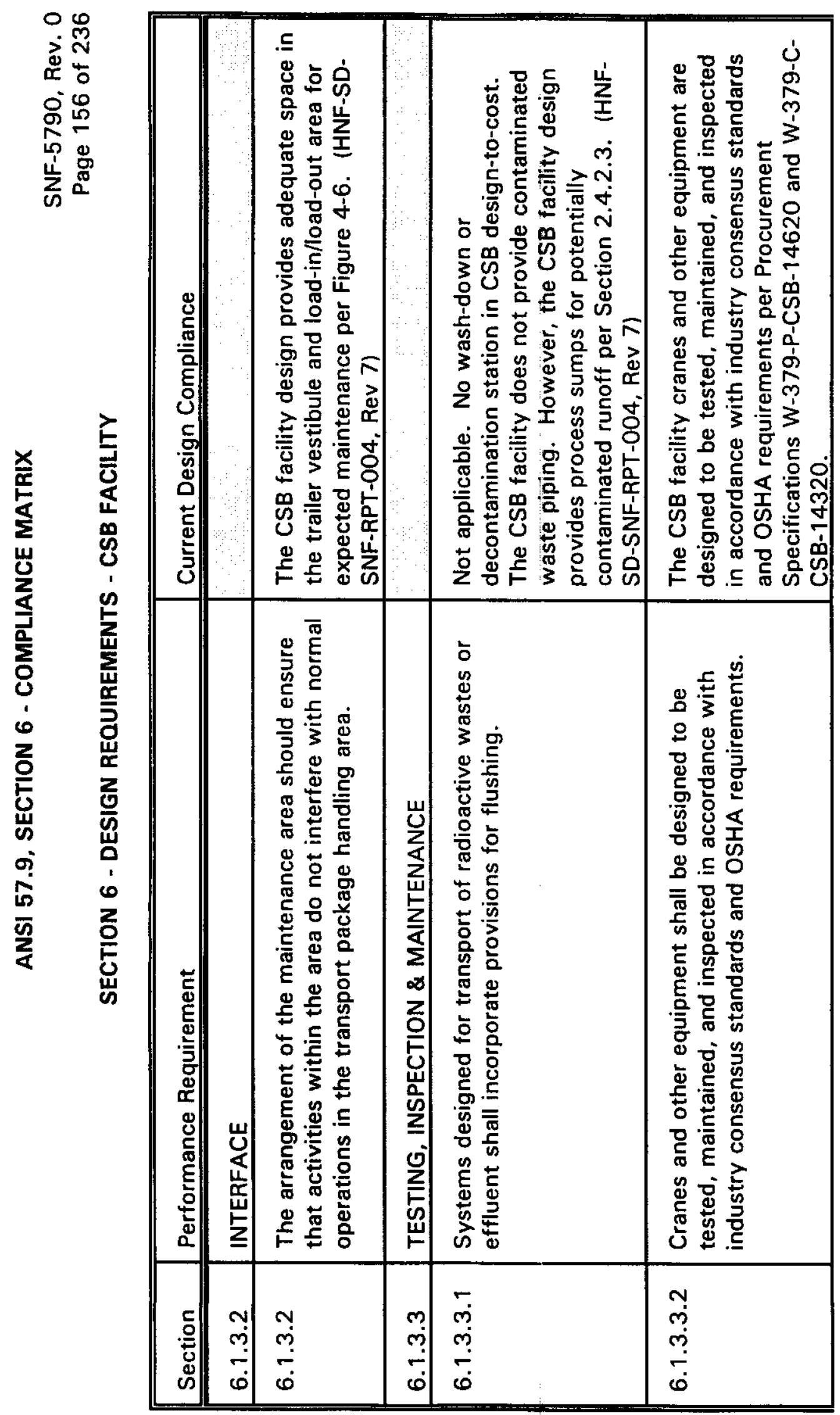




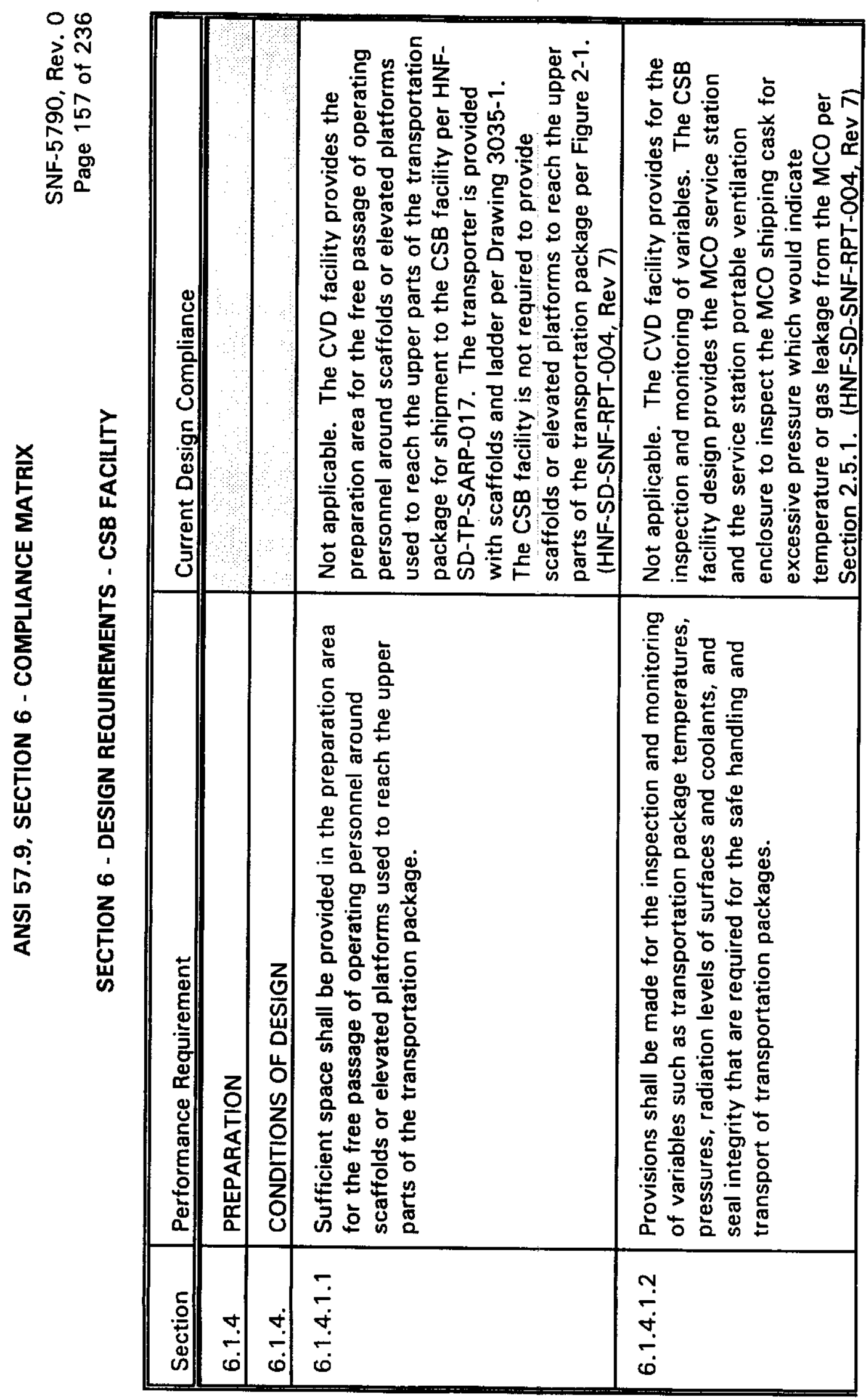




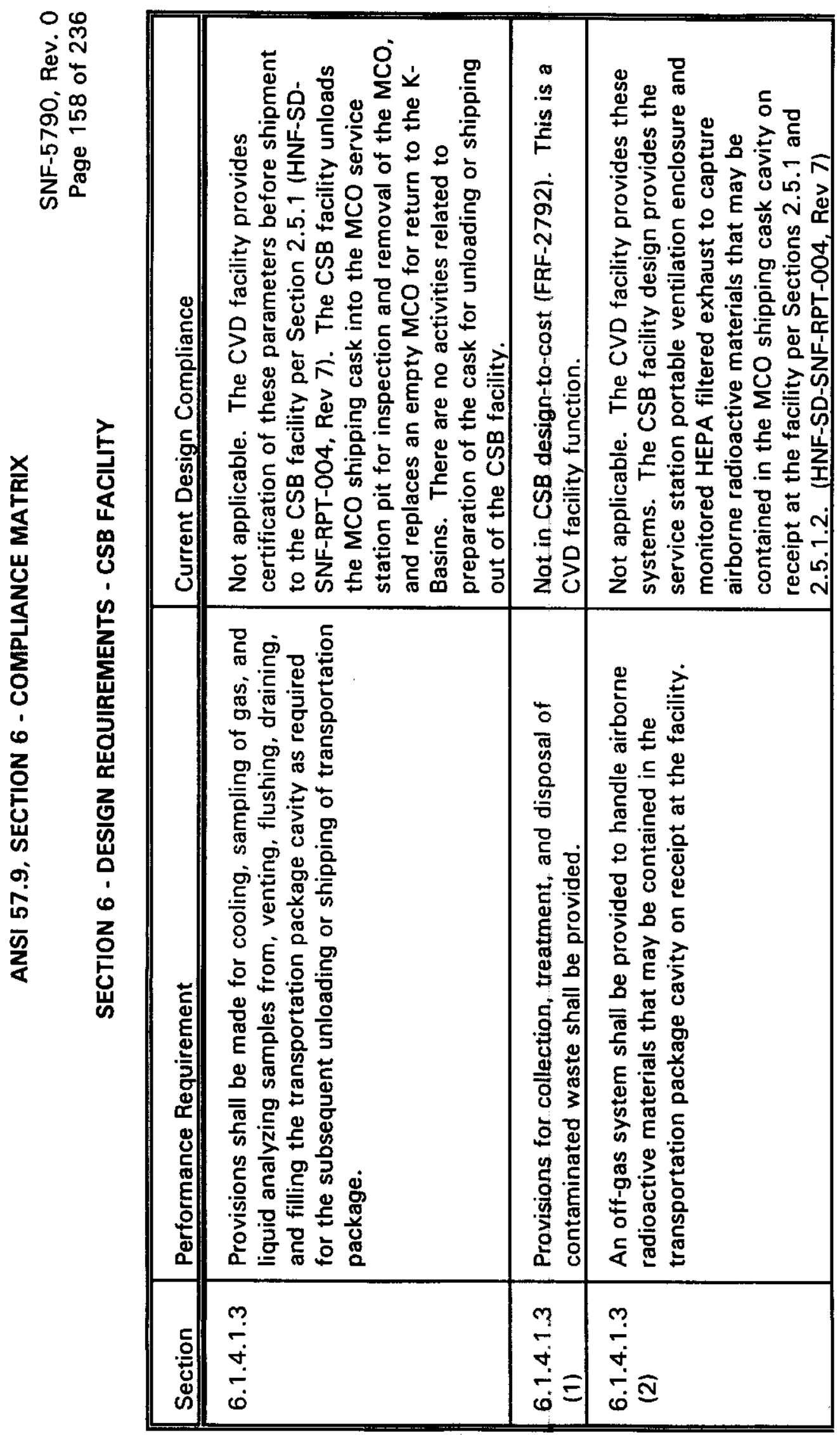




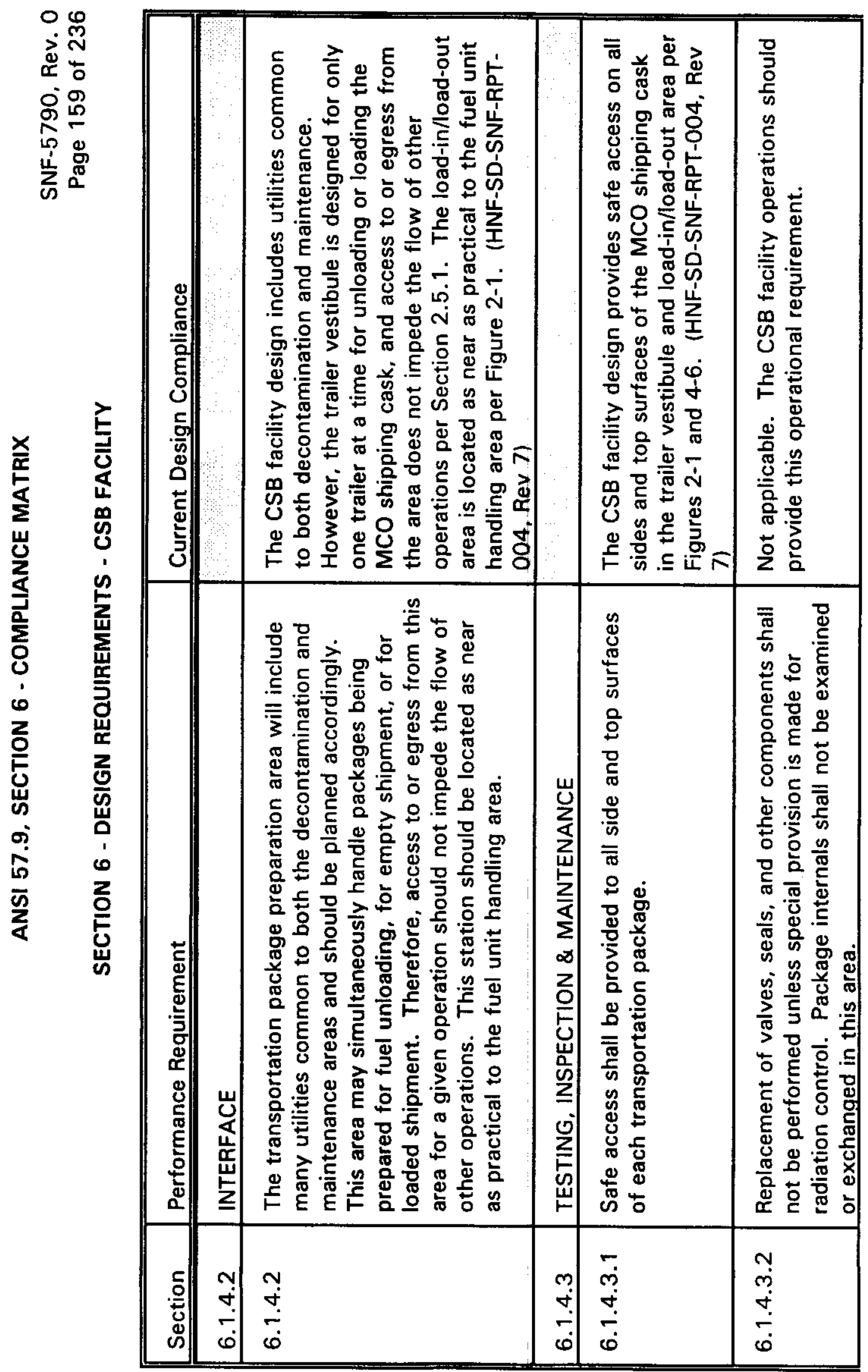




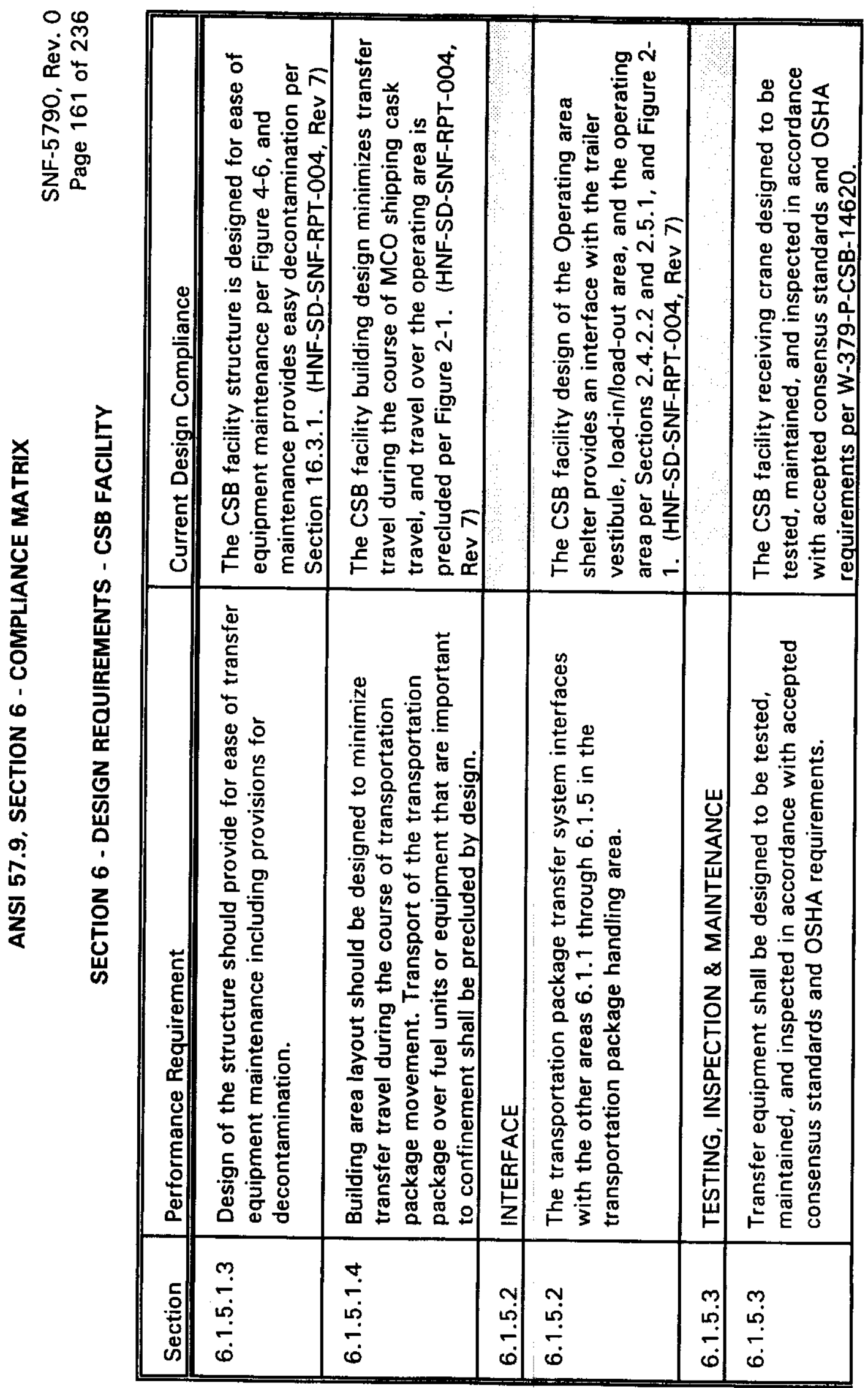




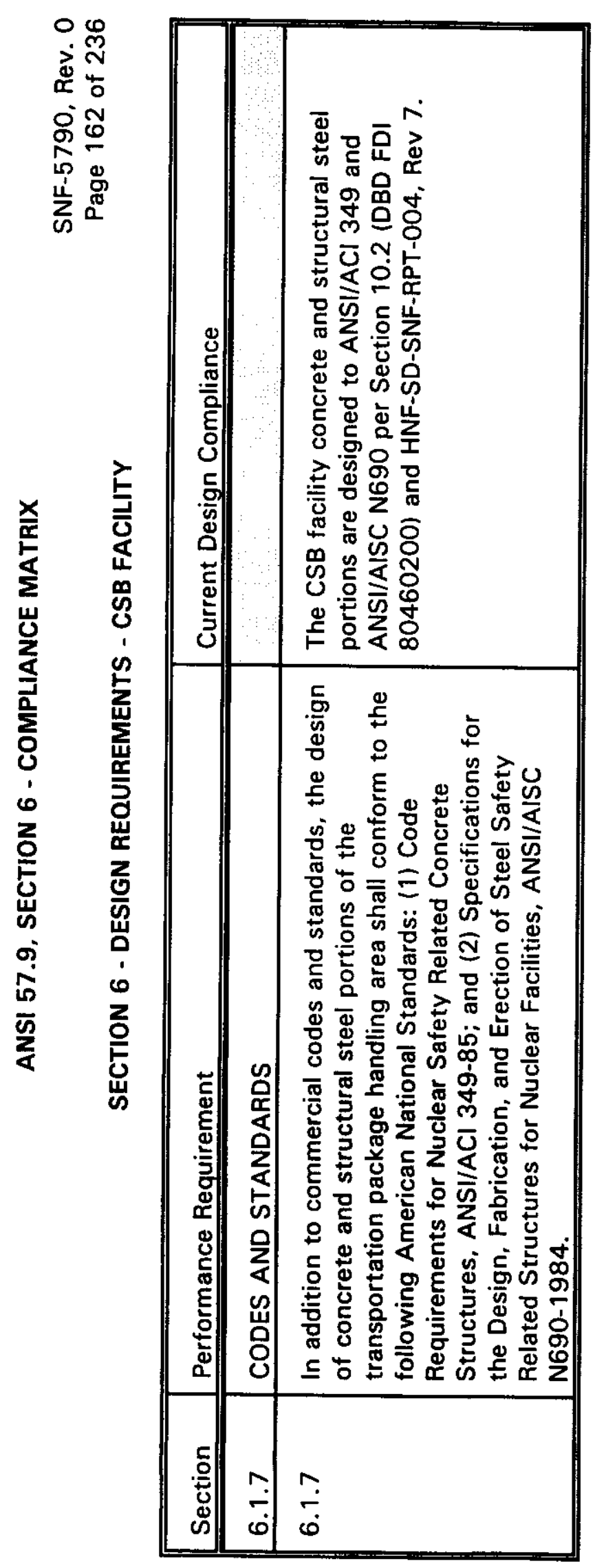




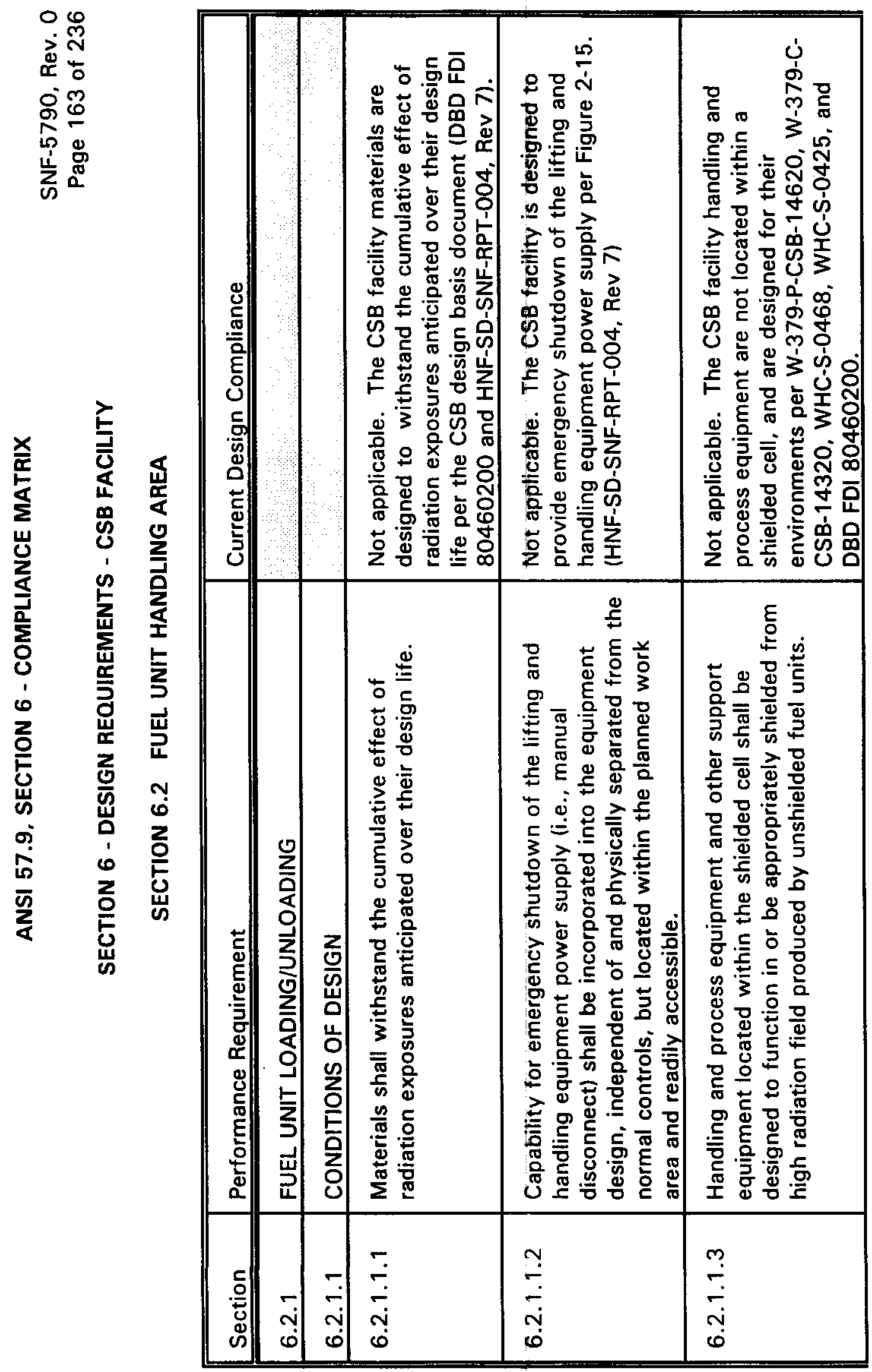




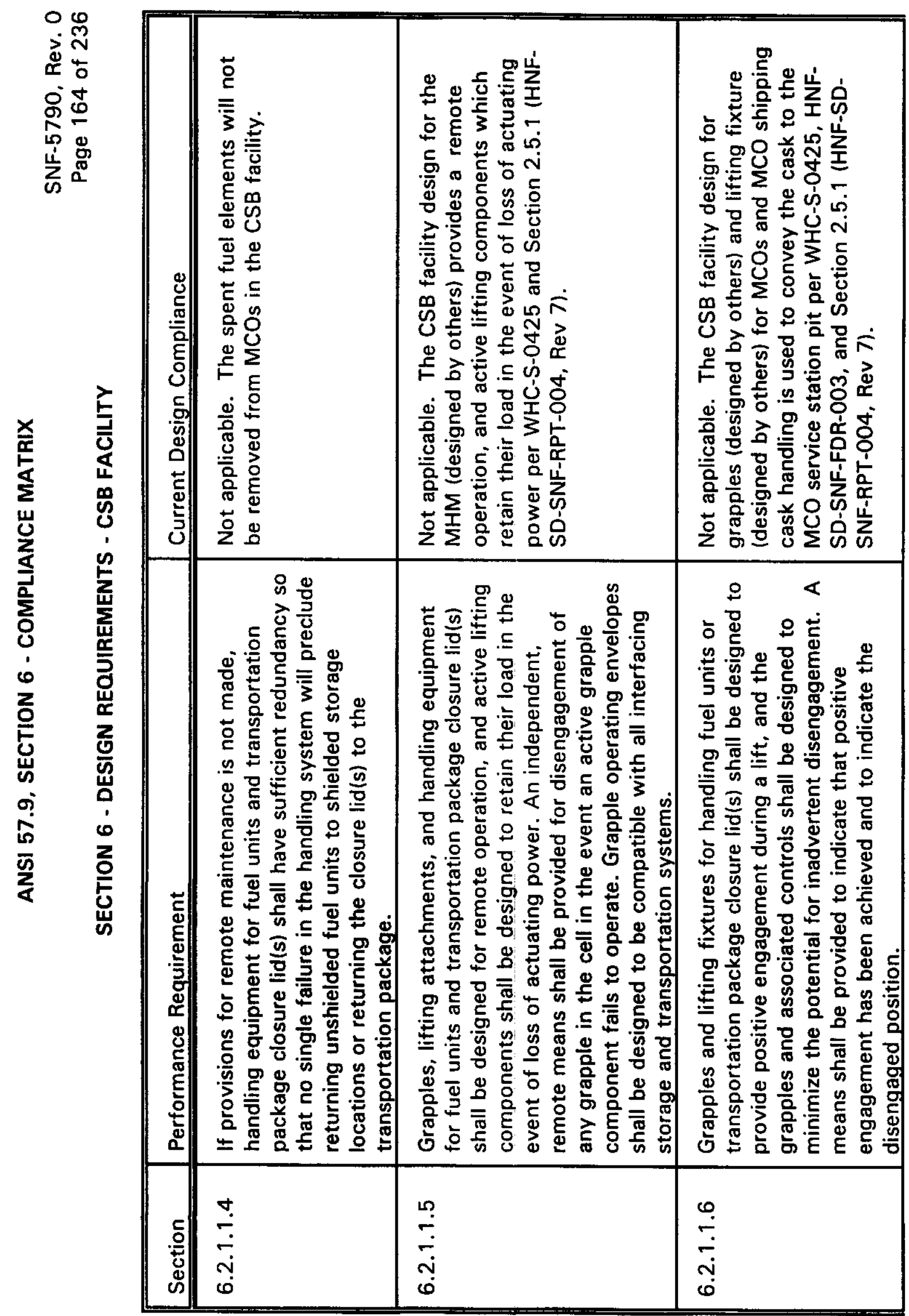




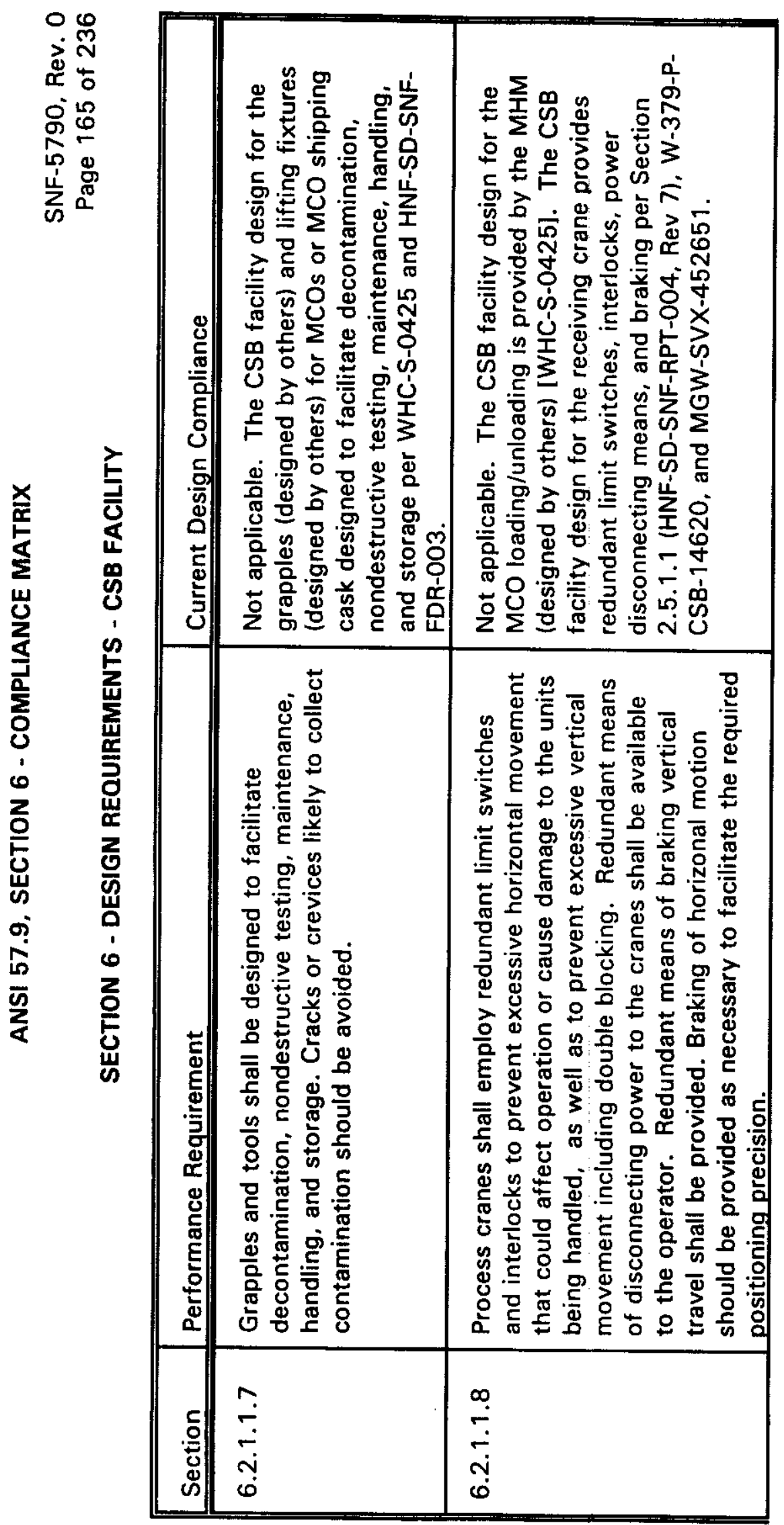




\begin{tabular}{|c|c|c|c|c|}
\hline 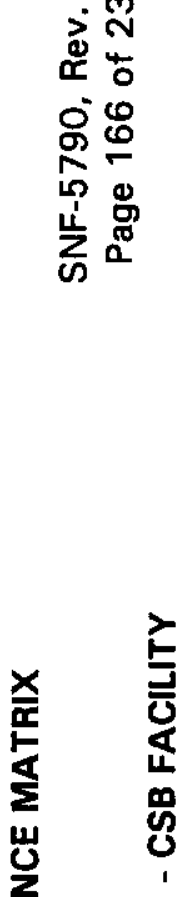 & 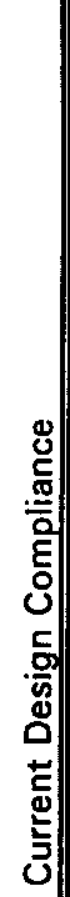 & 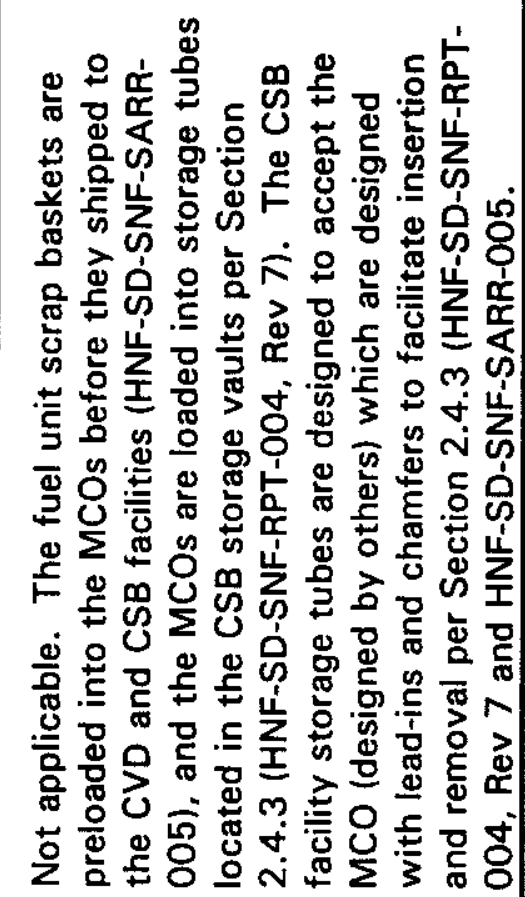 & 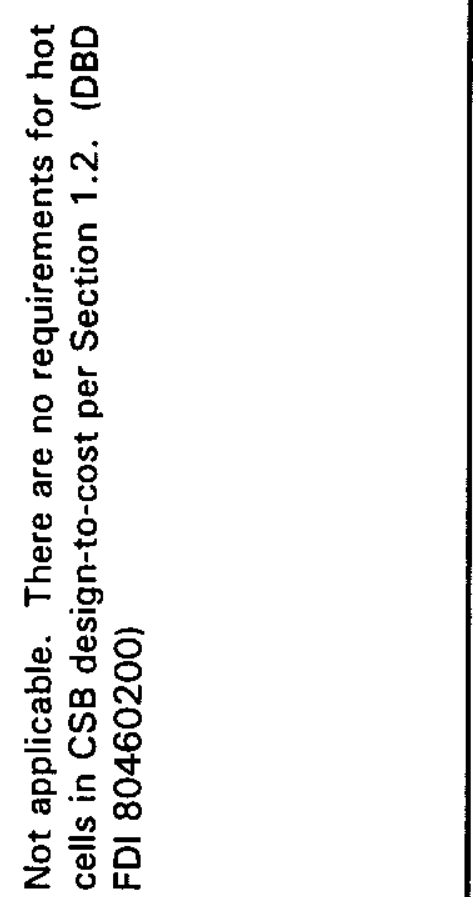 & 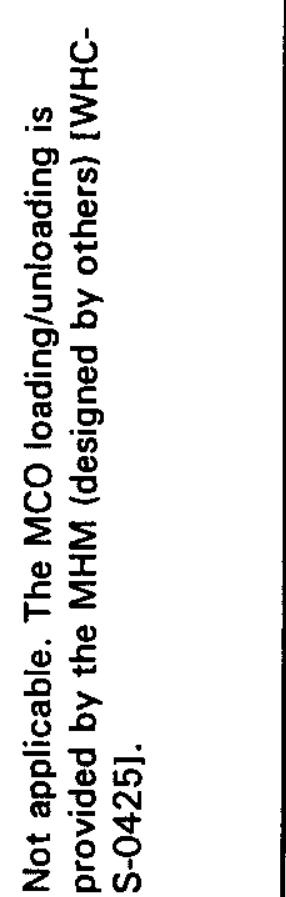 \\
\hline 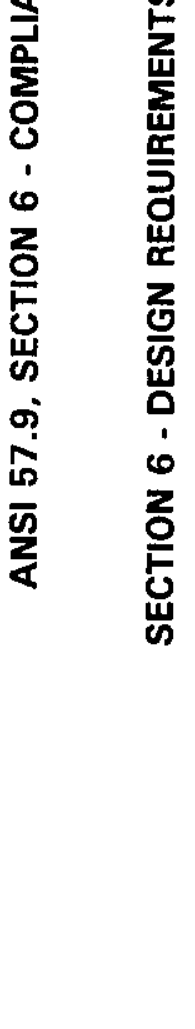 & 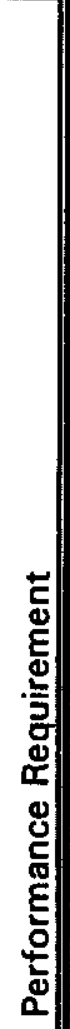 & 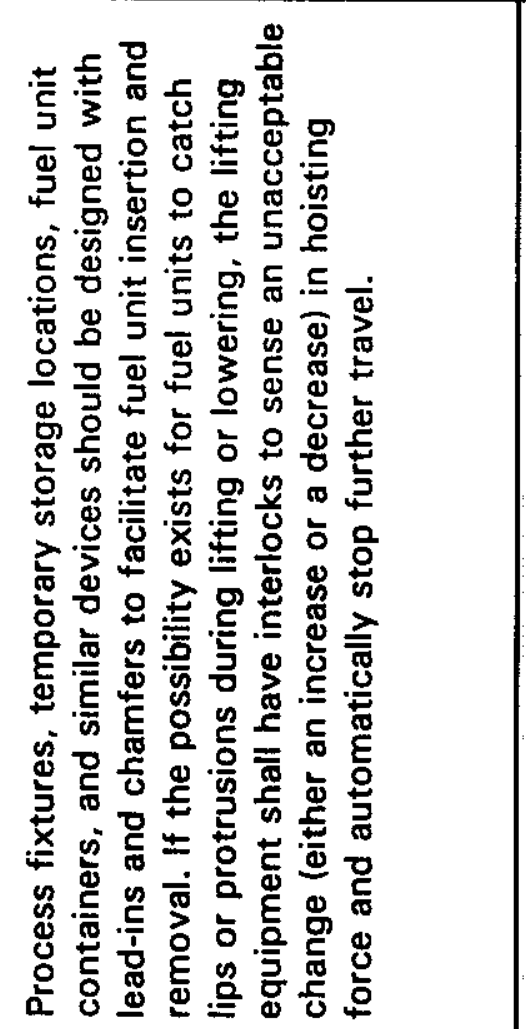 & 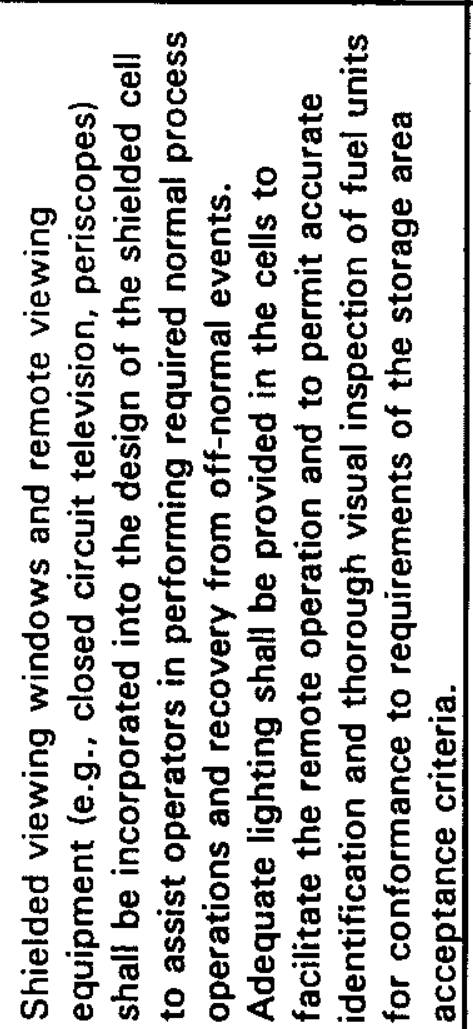 & 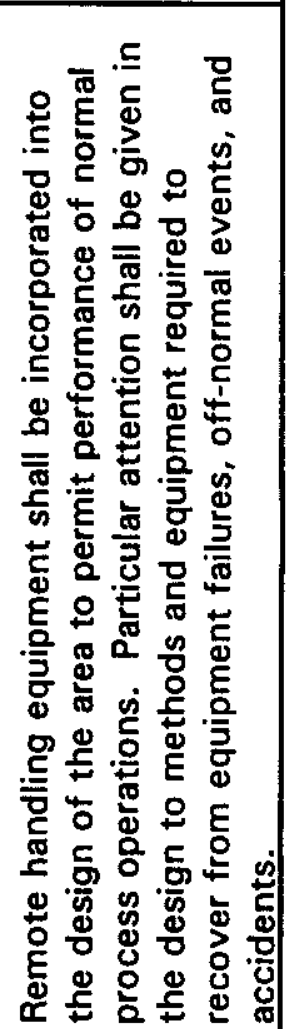 \\
\hline & 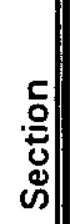 & 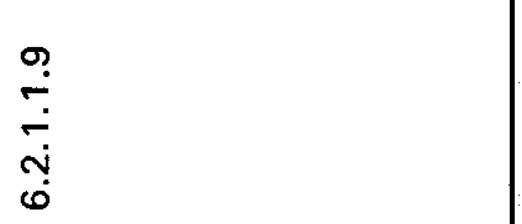 & 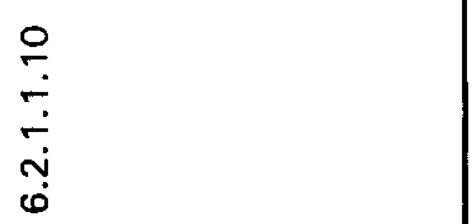 & 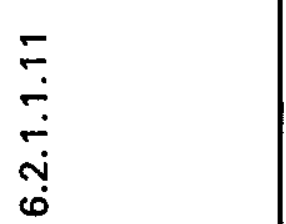 \\
\hline
\end{tabular}




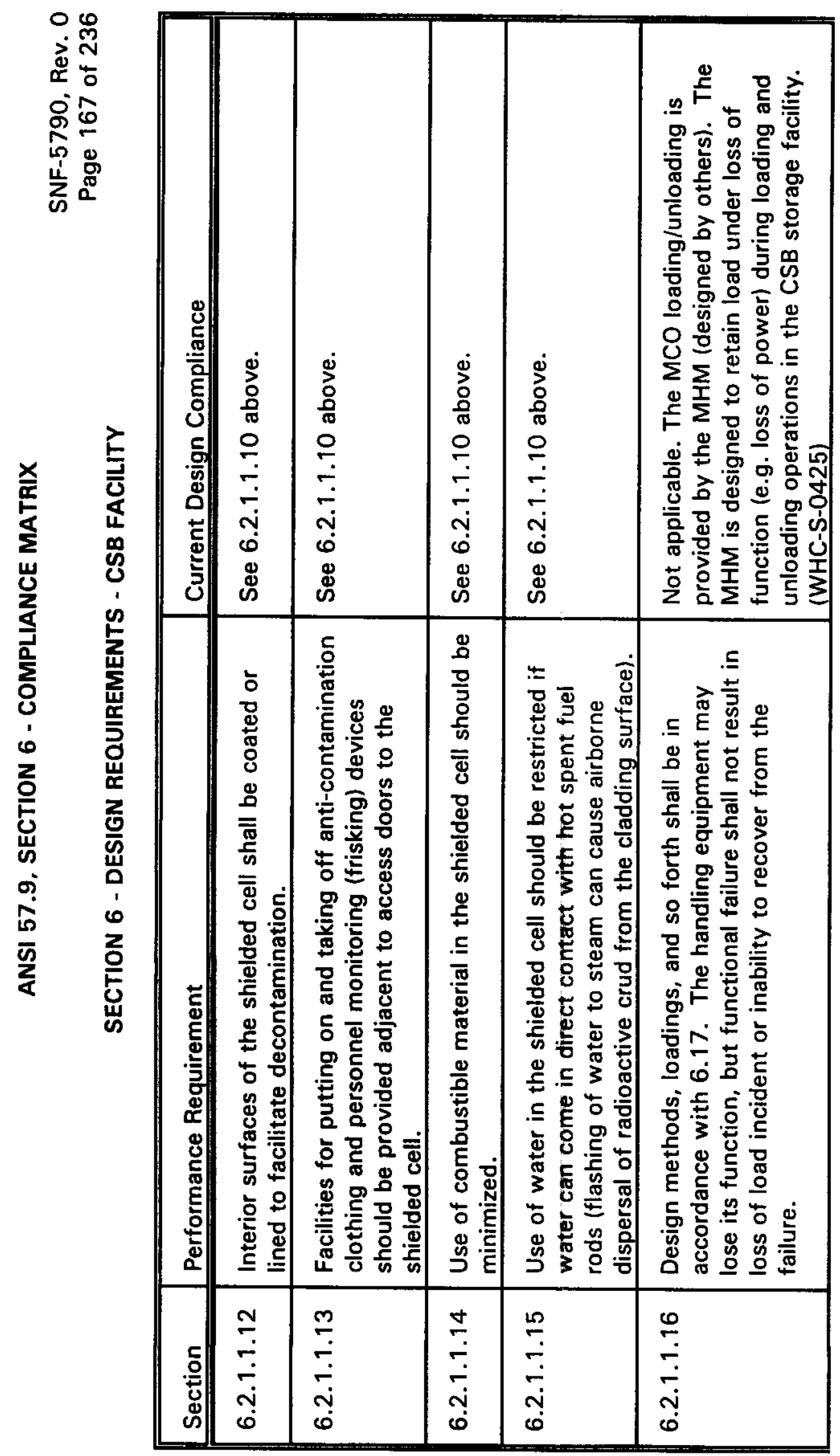




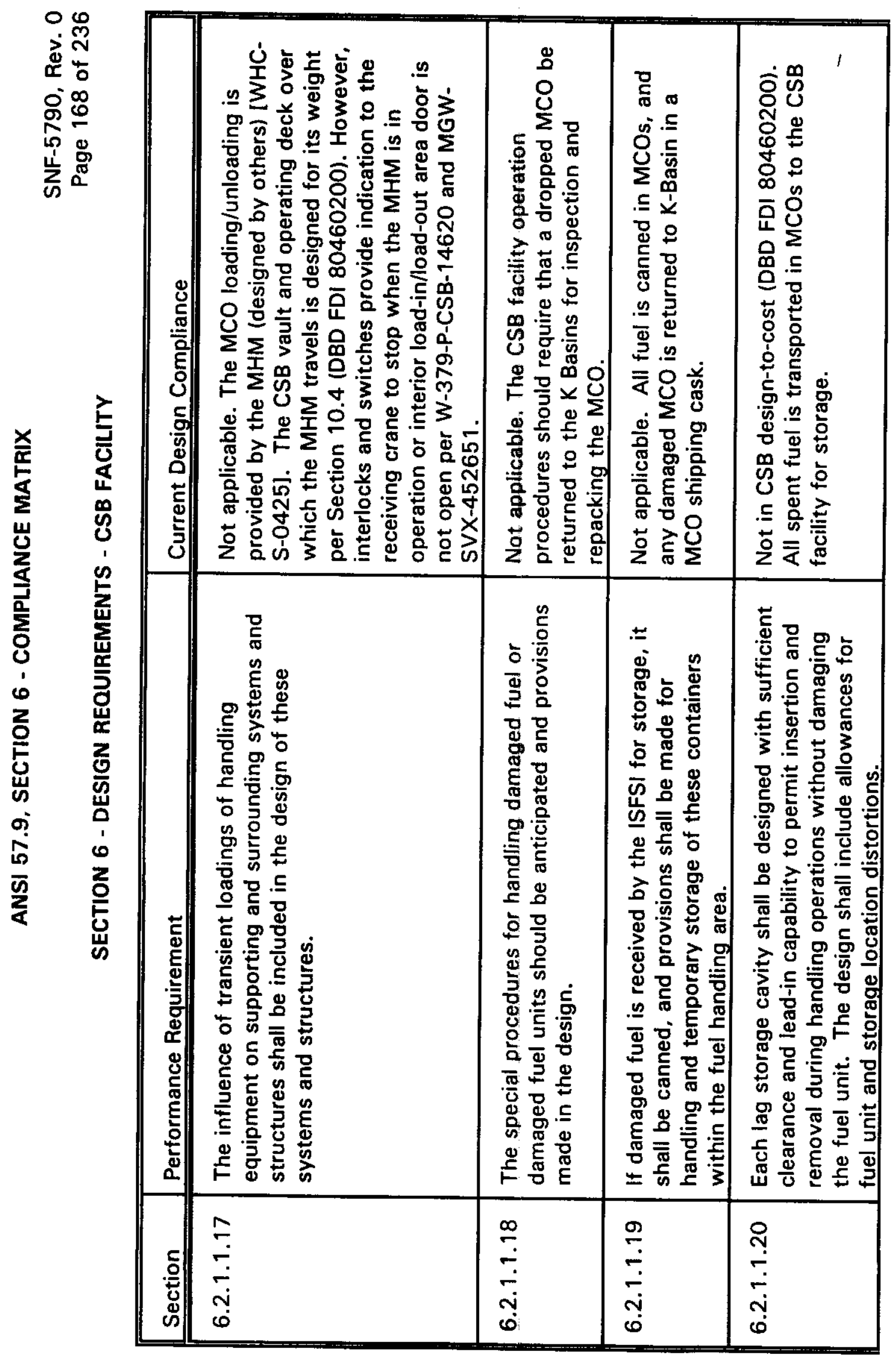




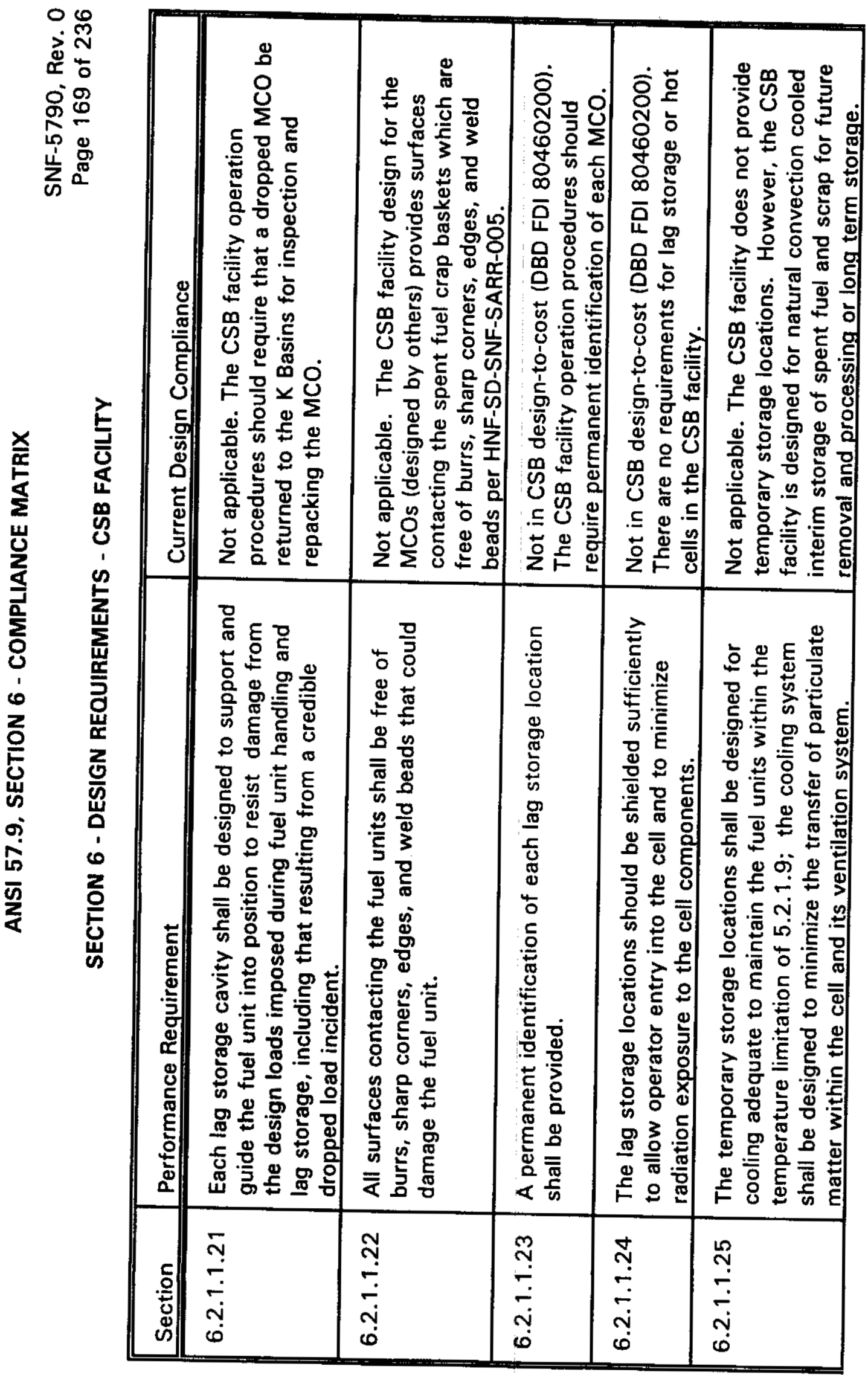




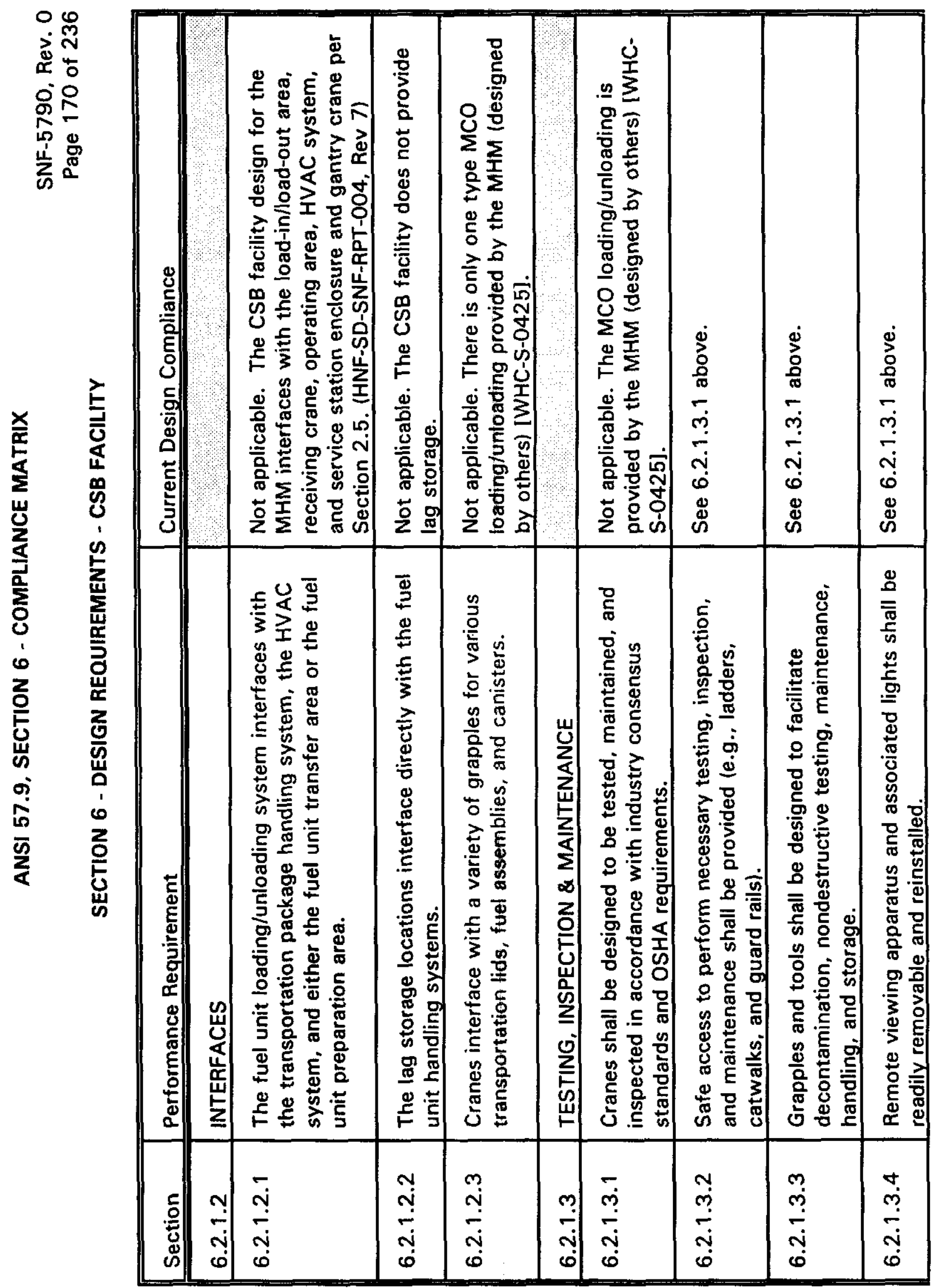




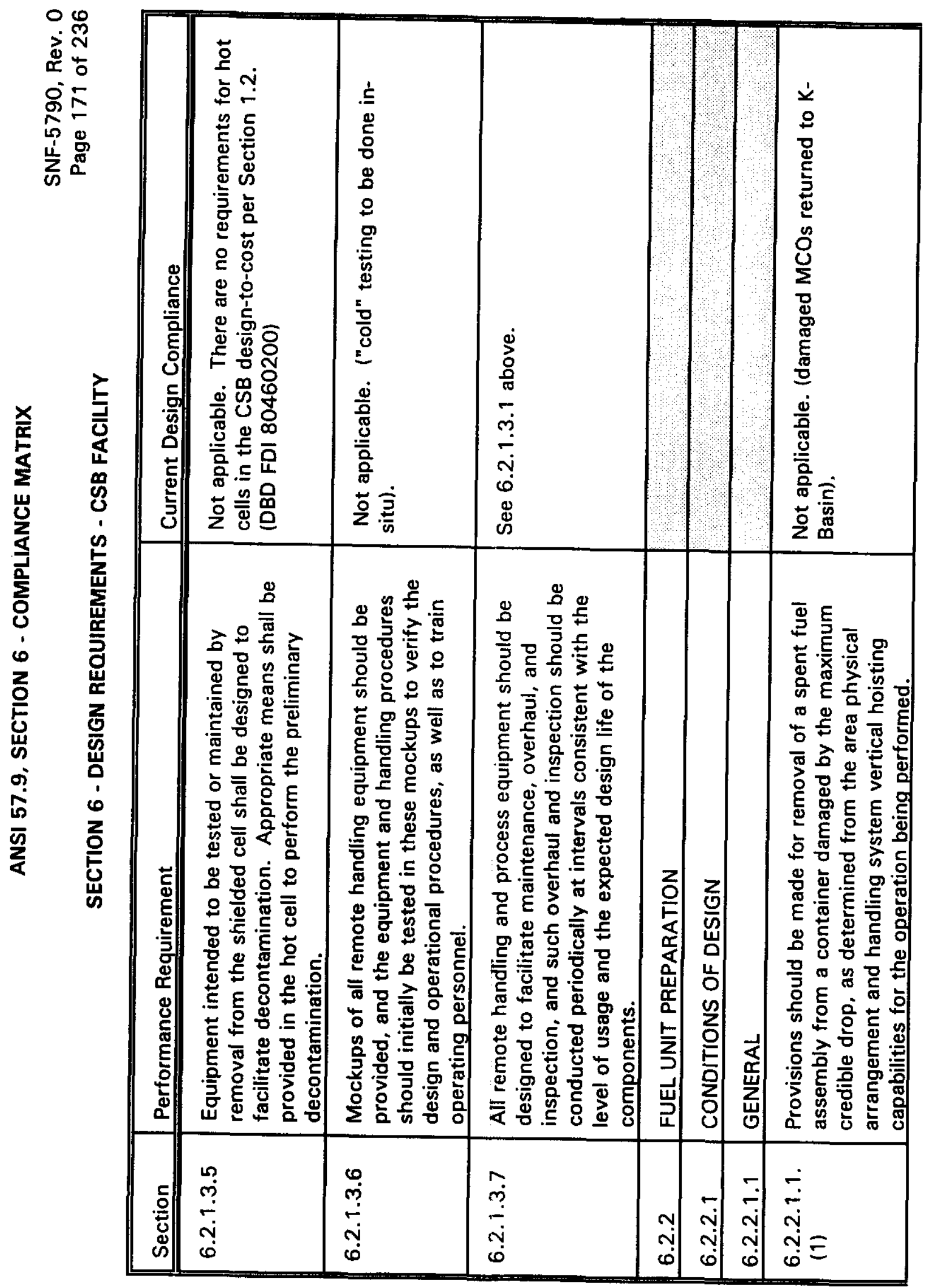




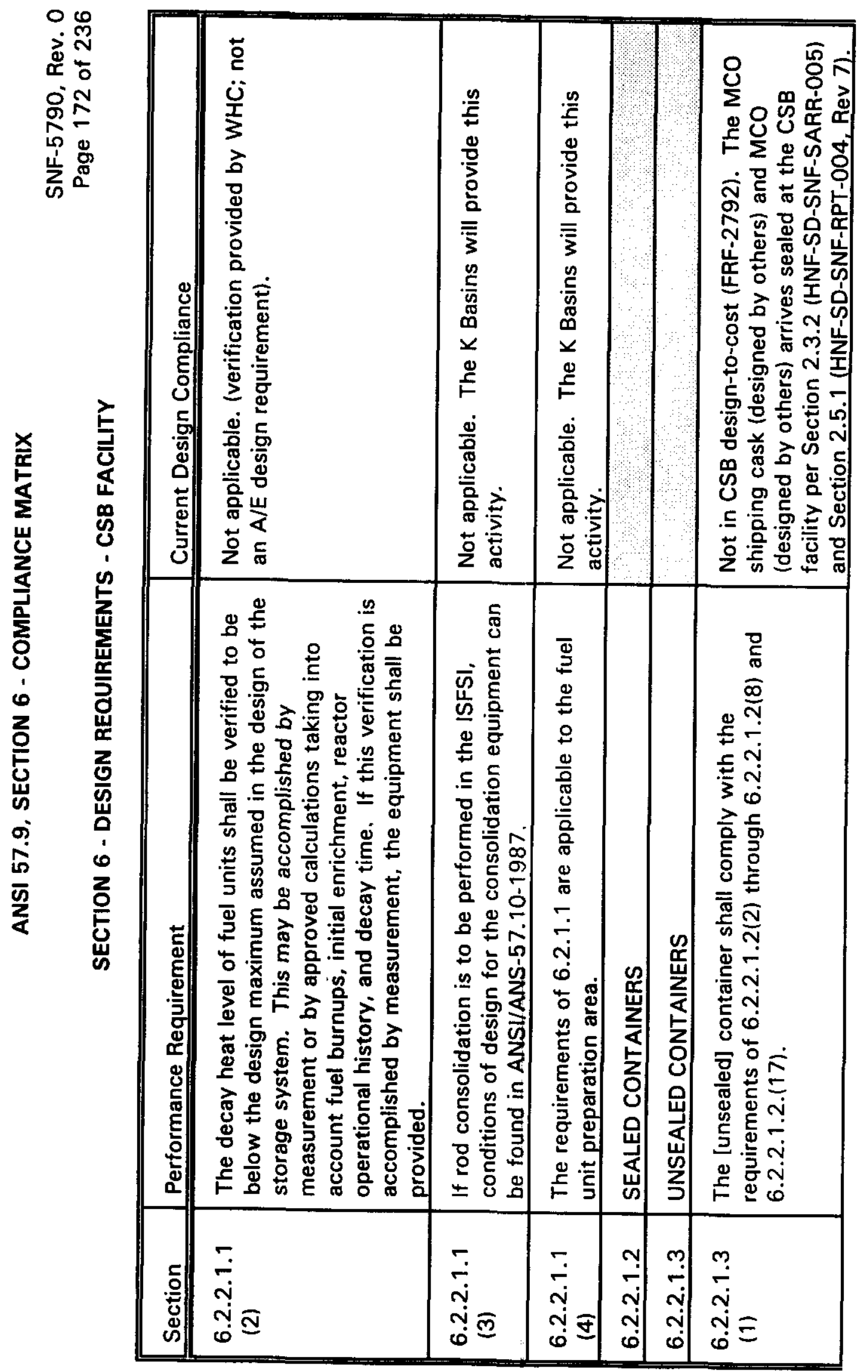




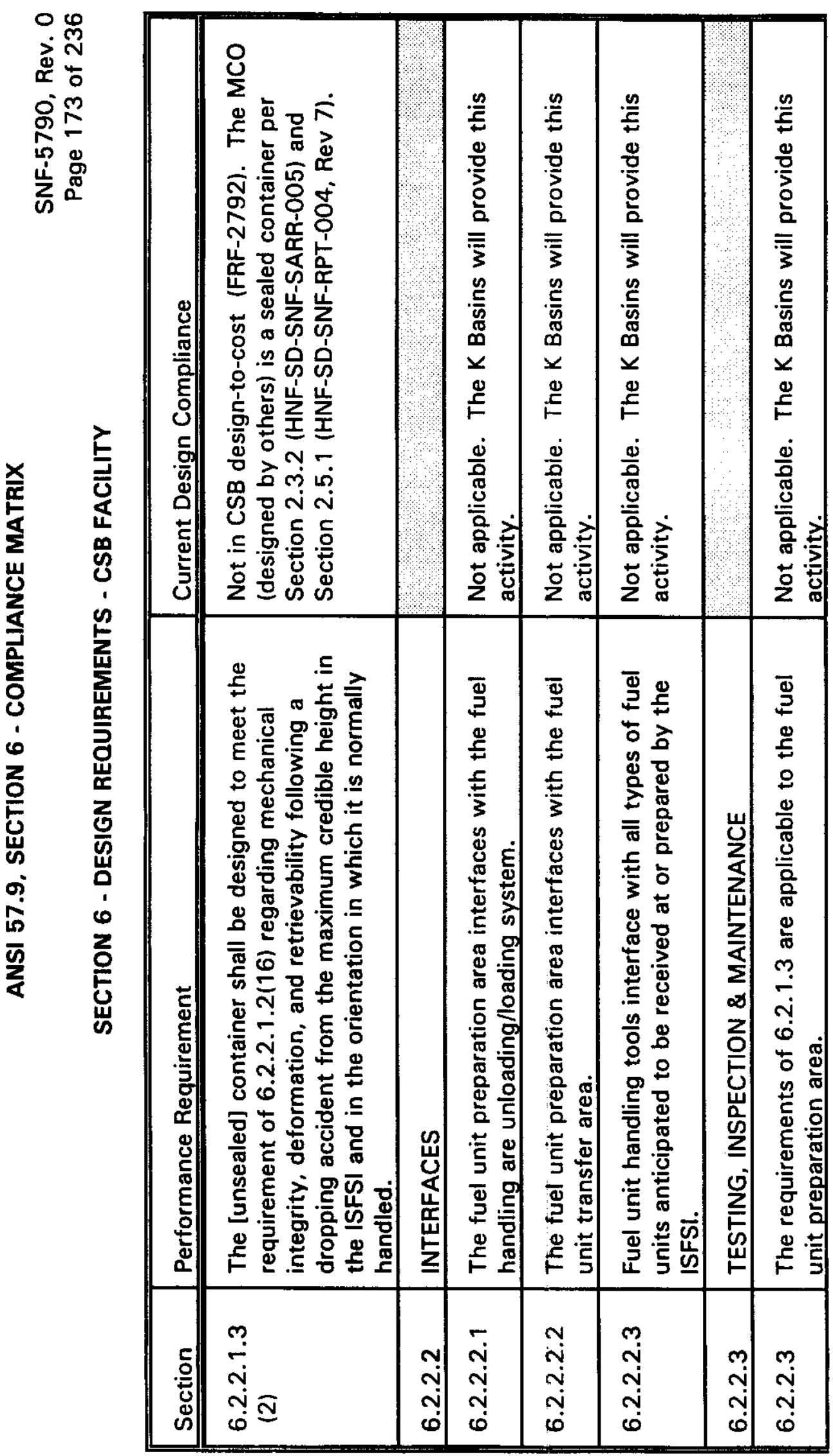




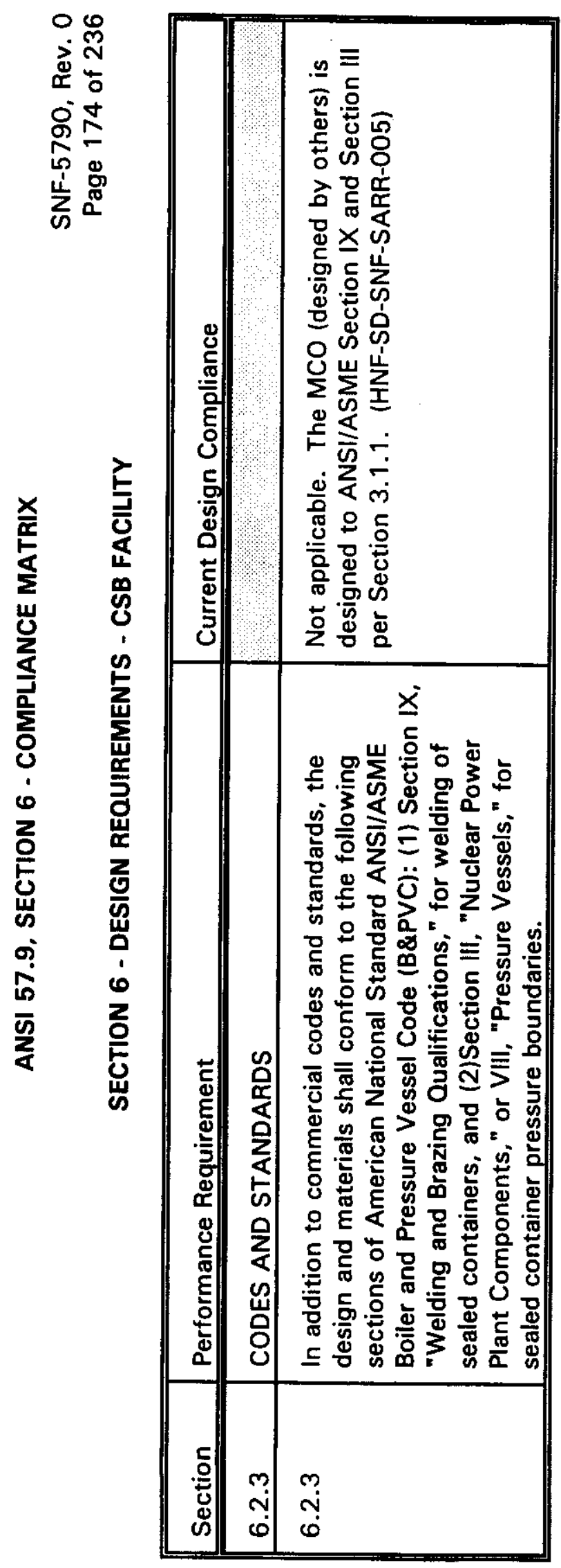




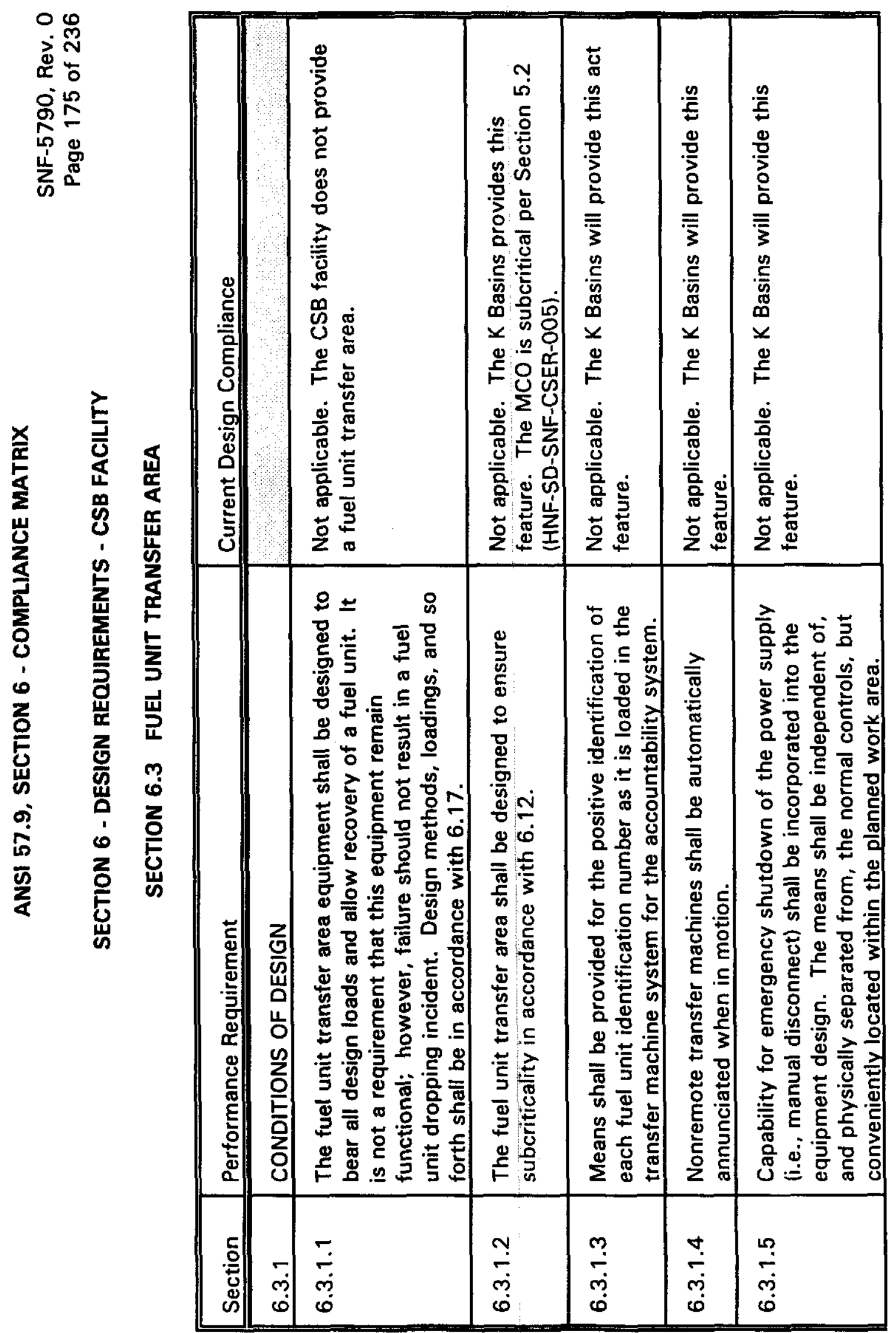




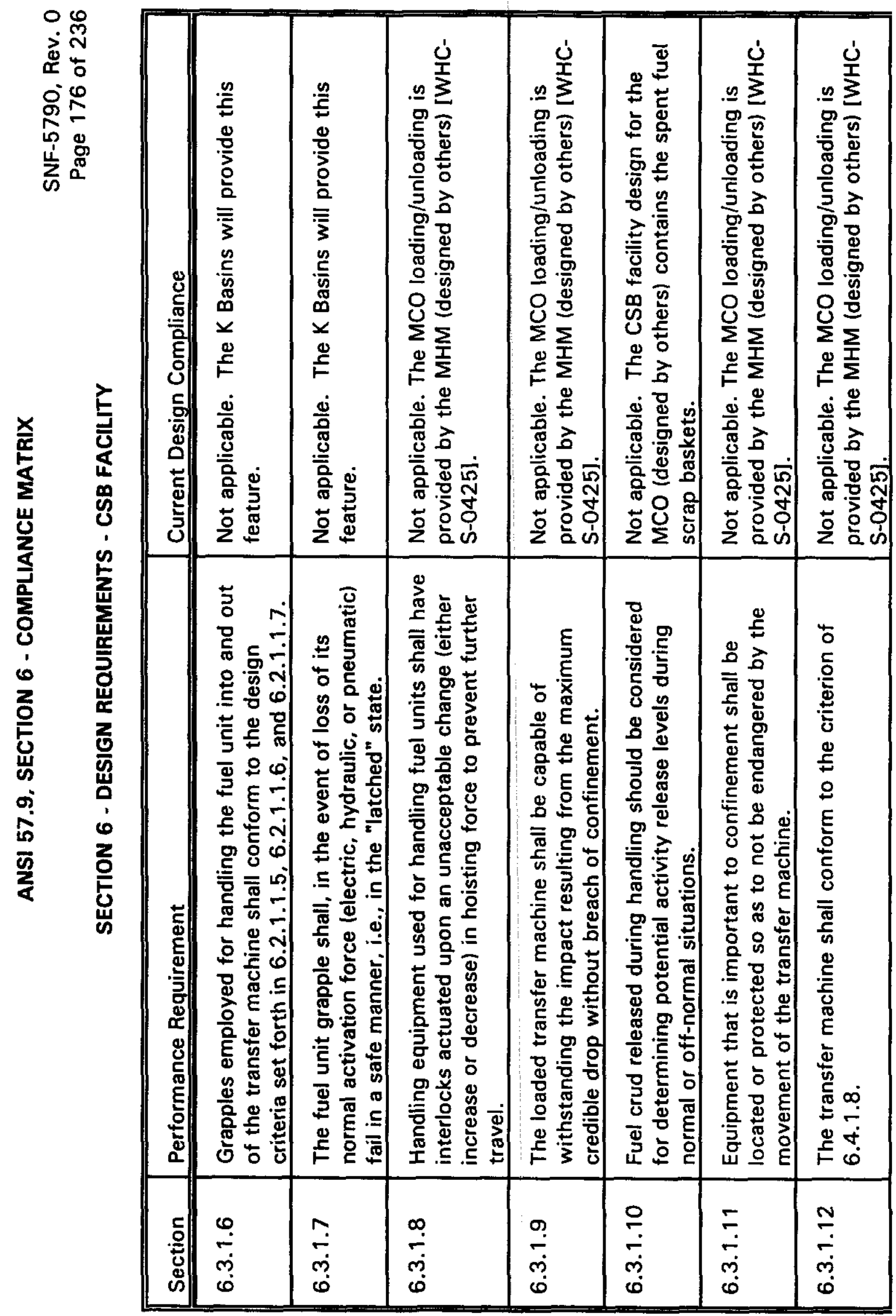




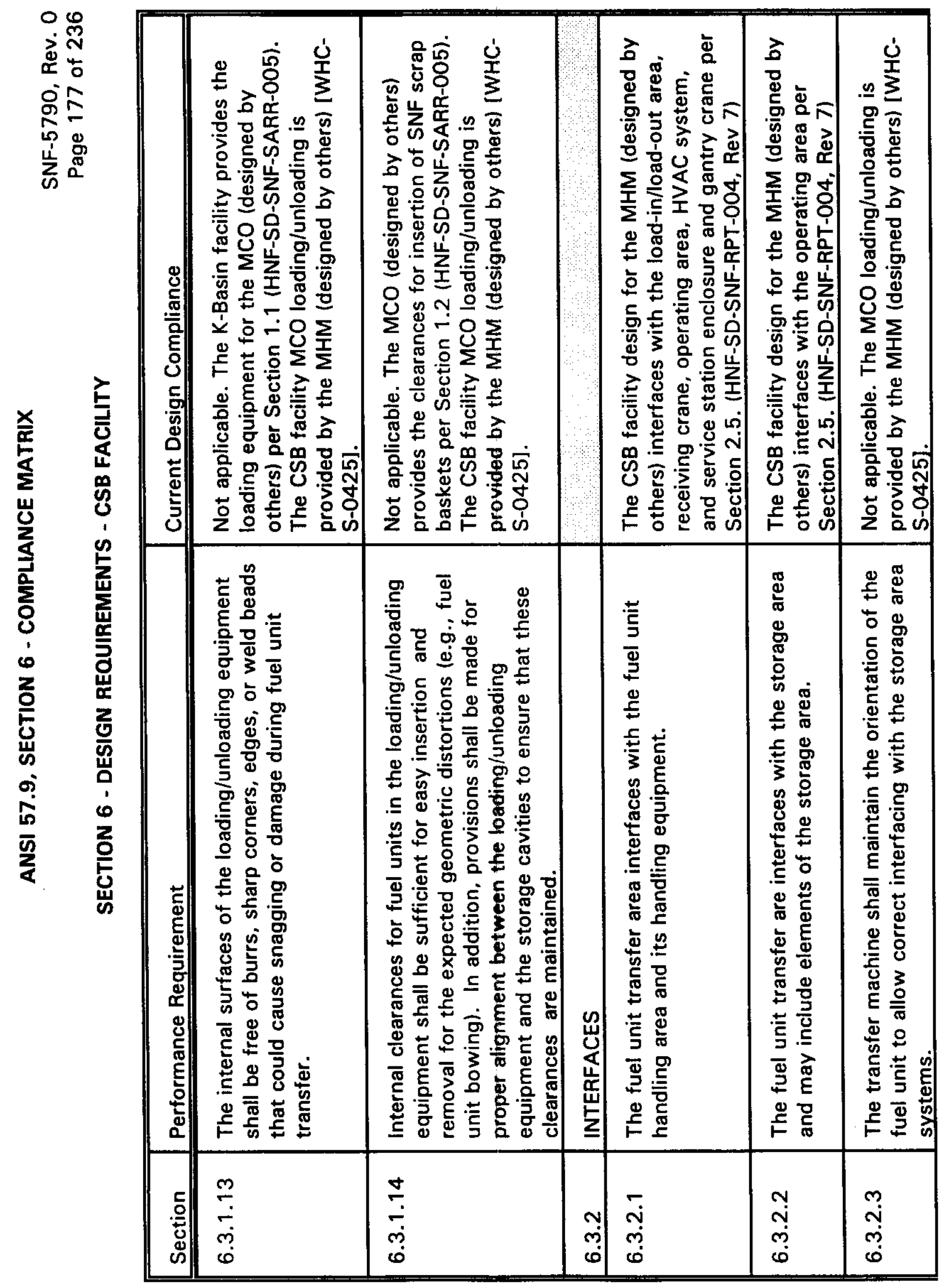




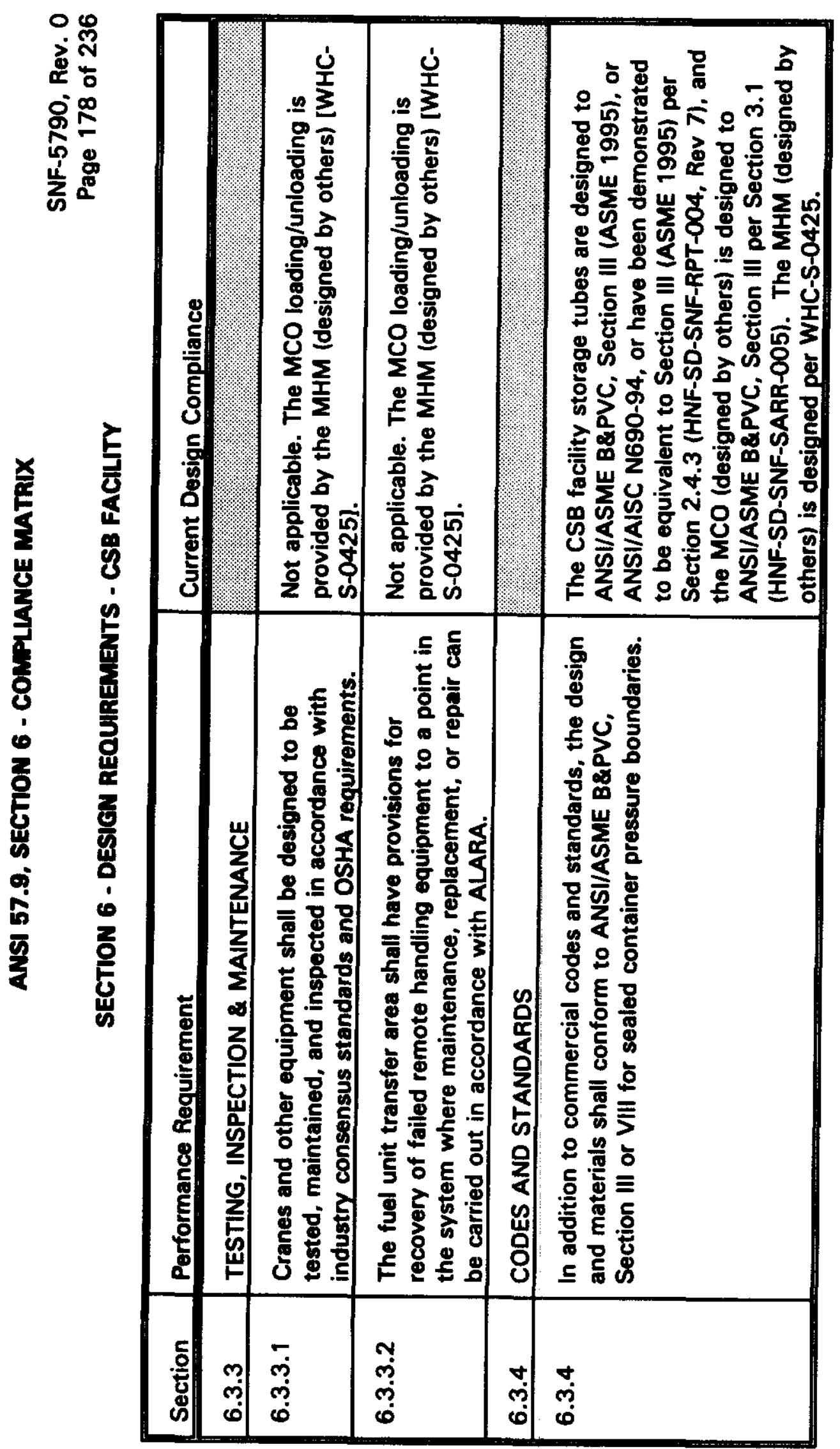




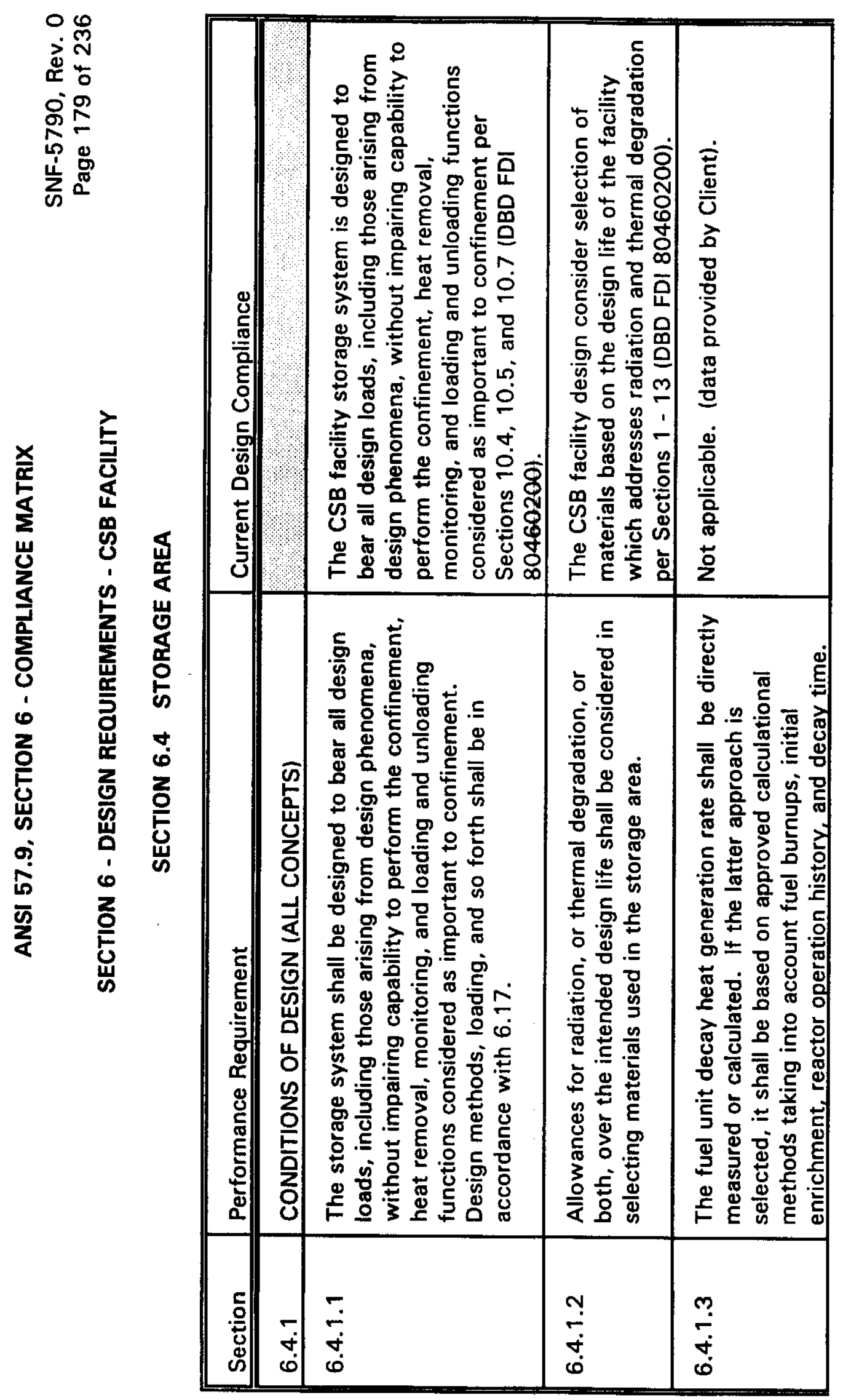




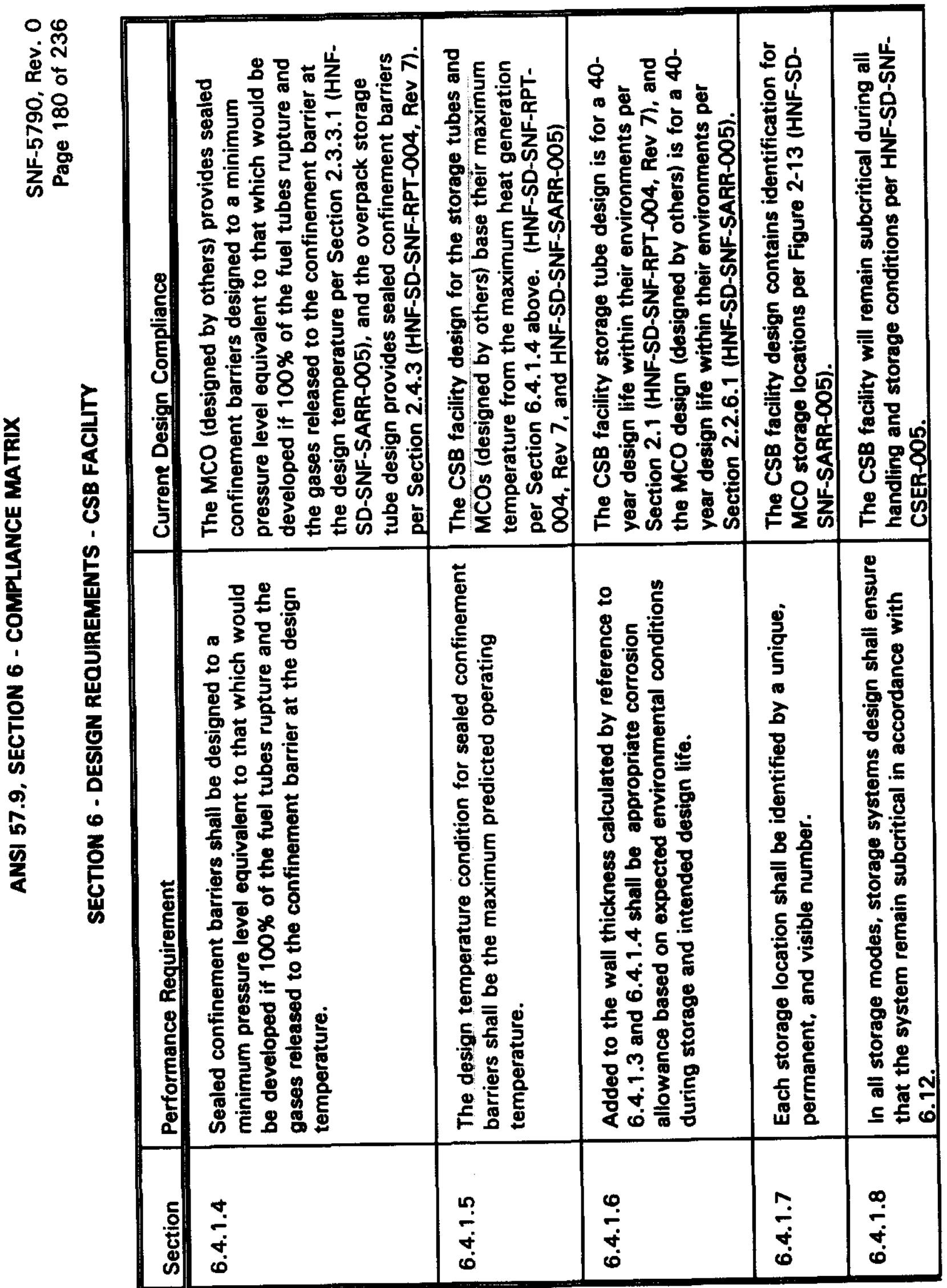




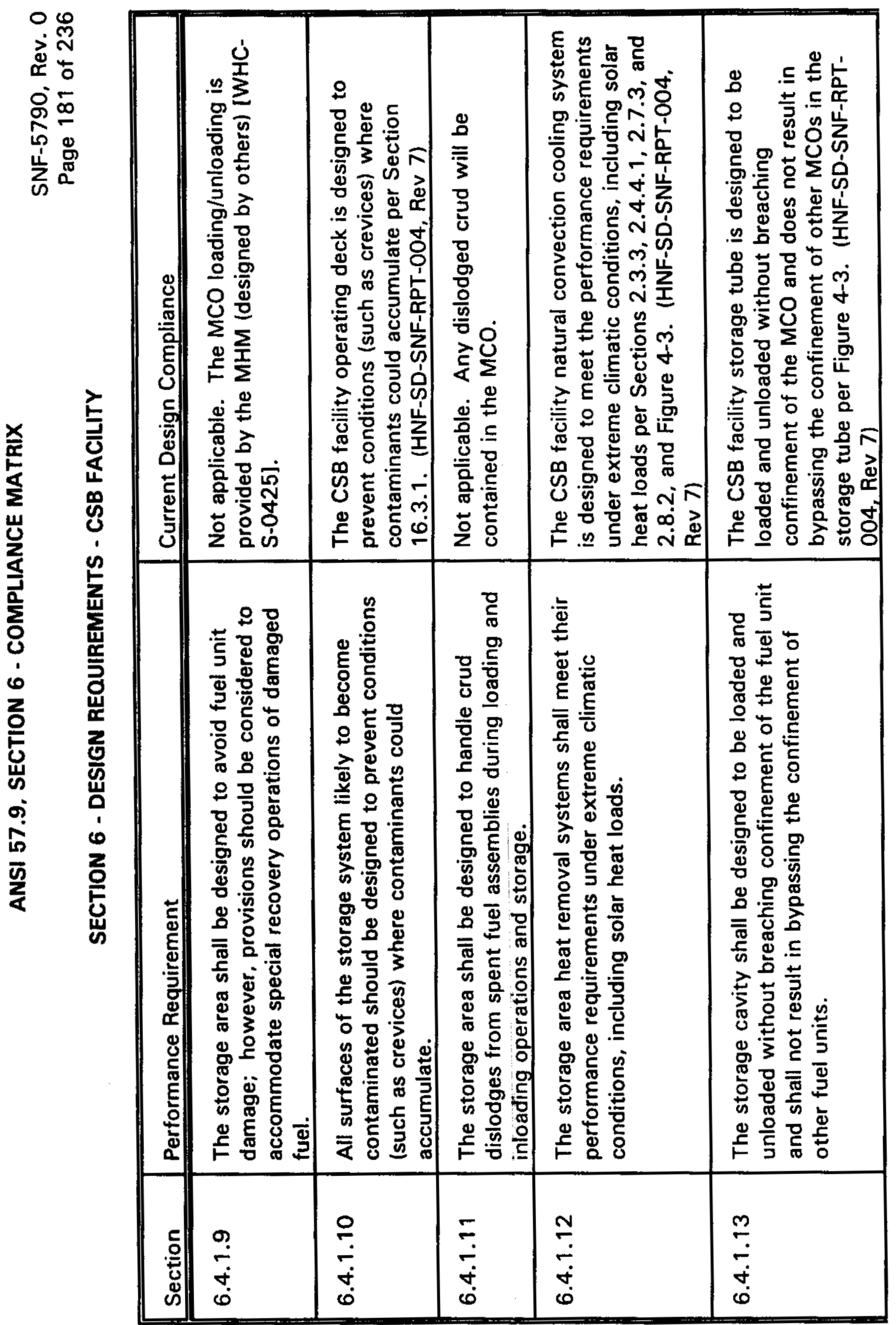




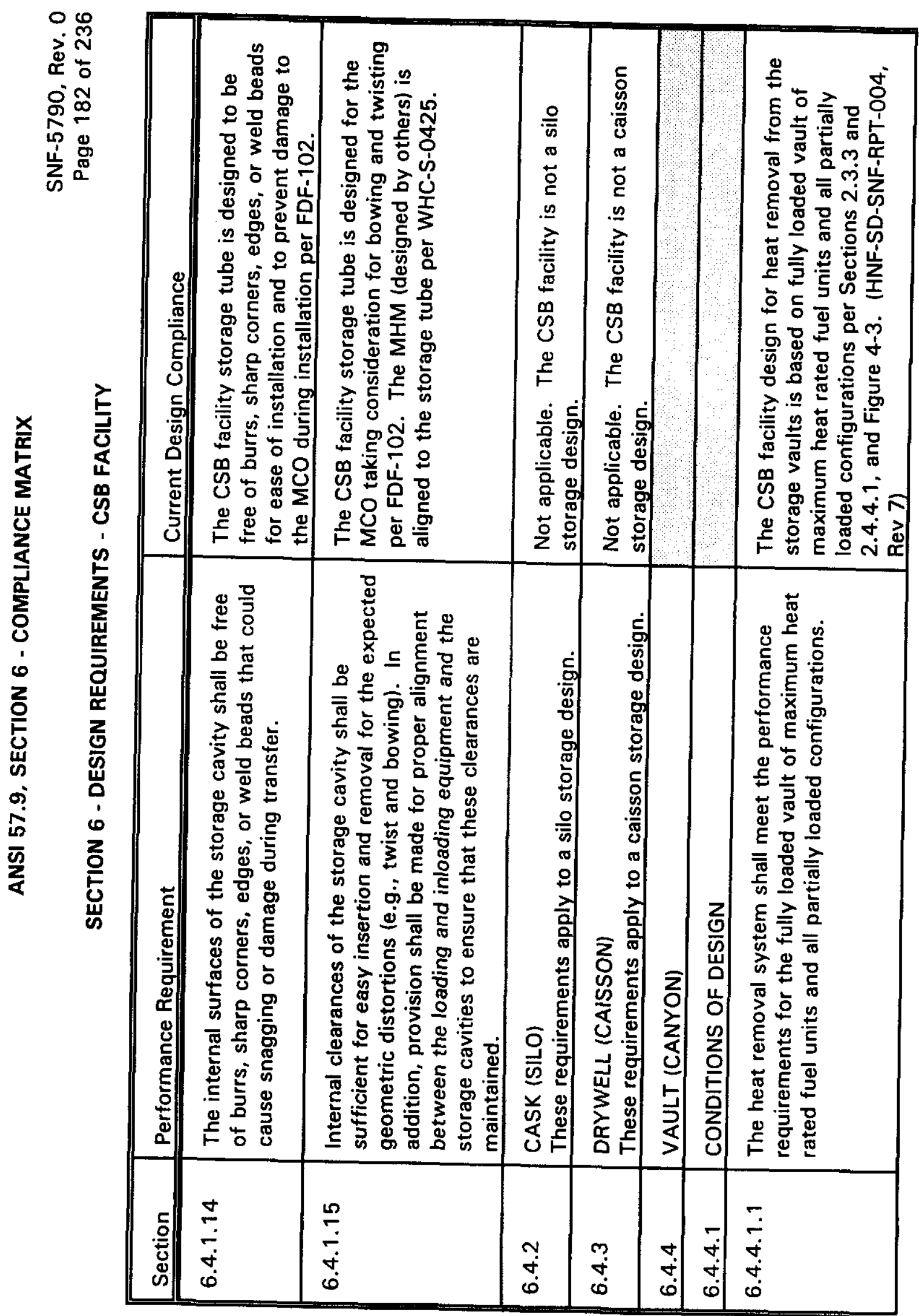




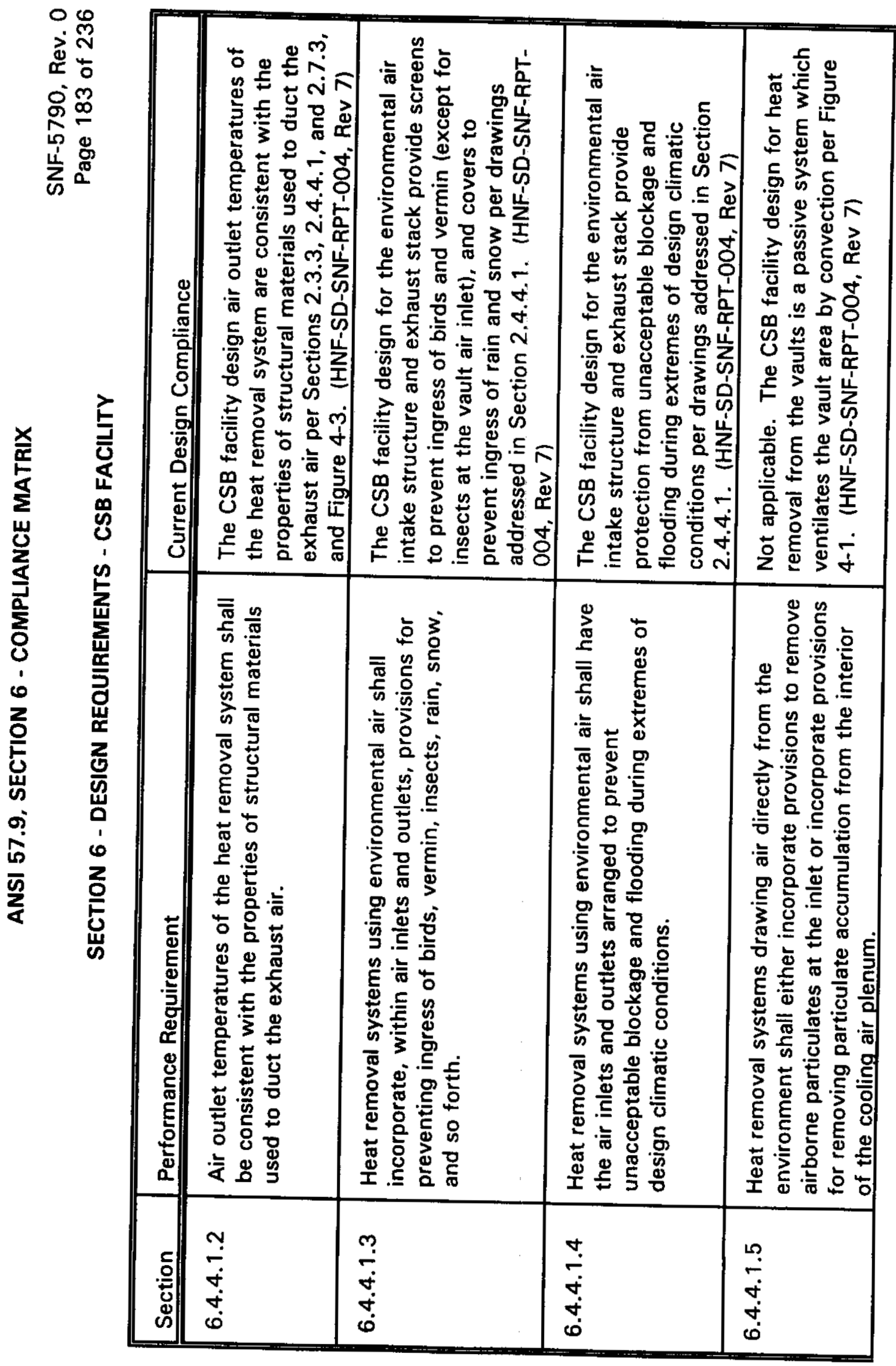




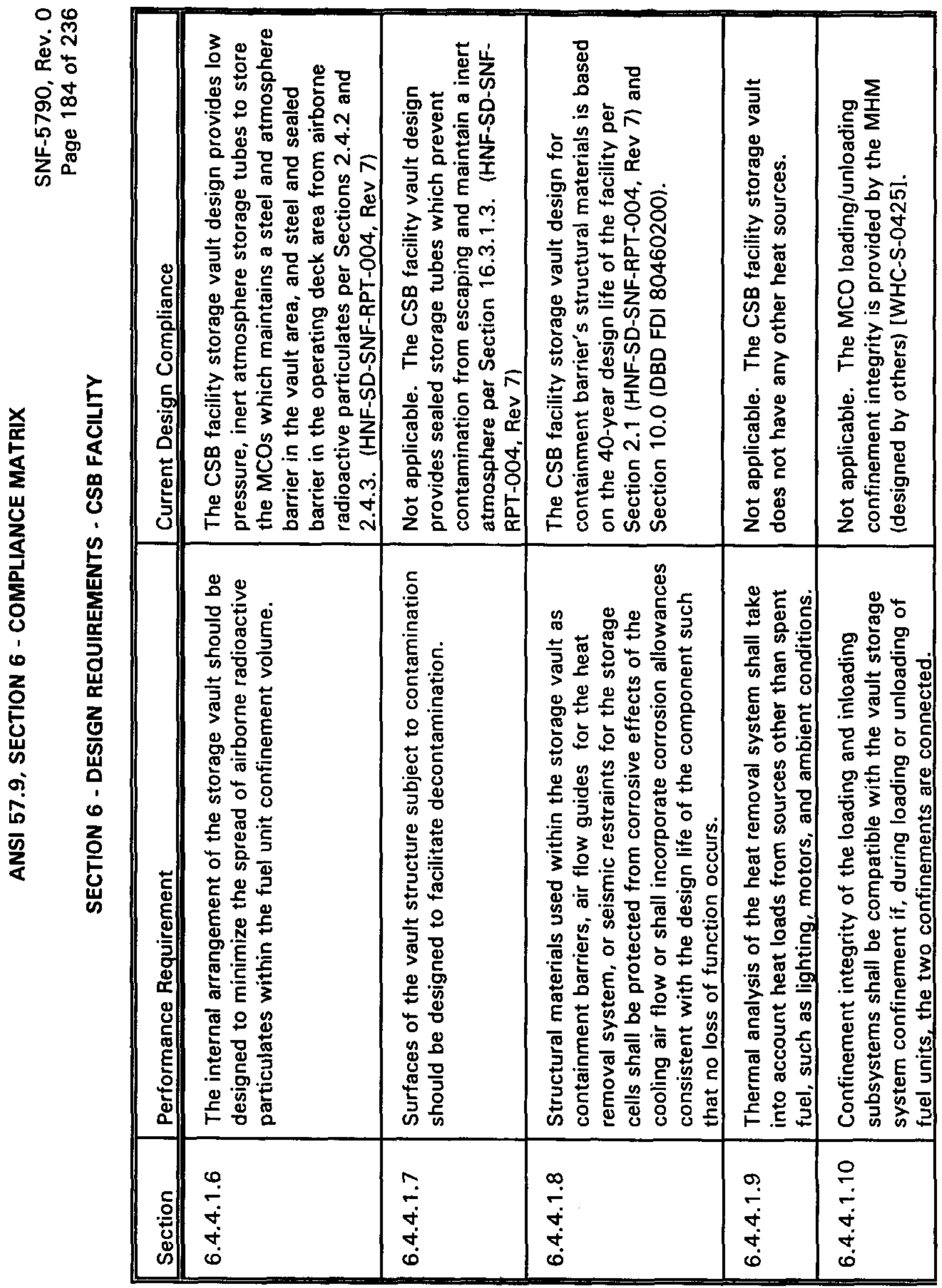




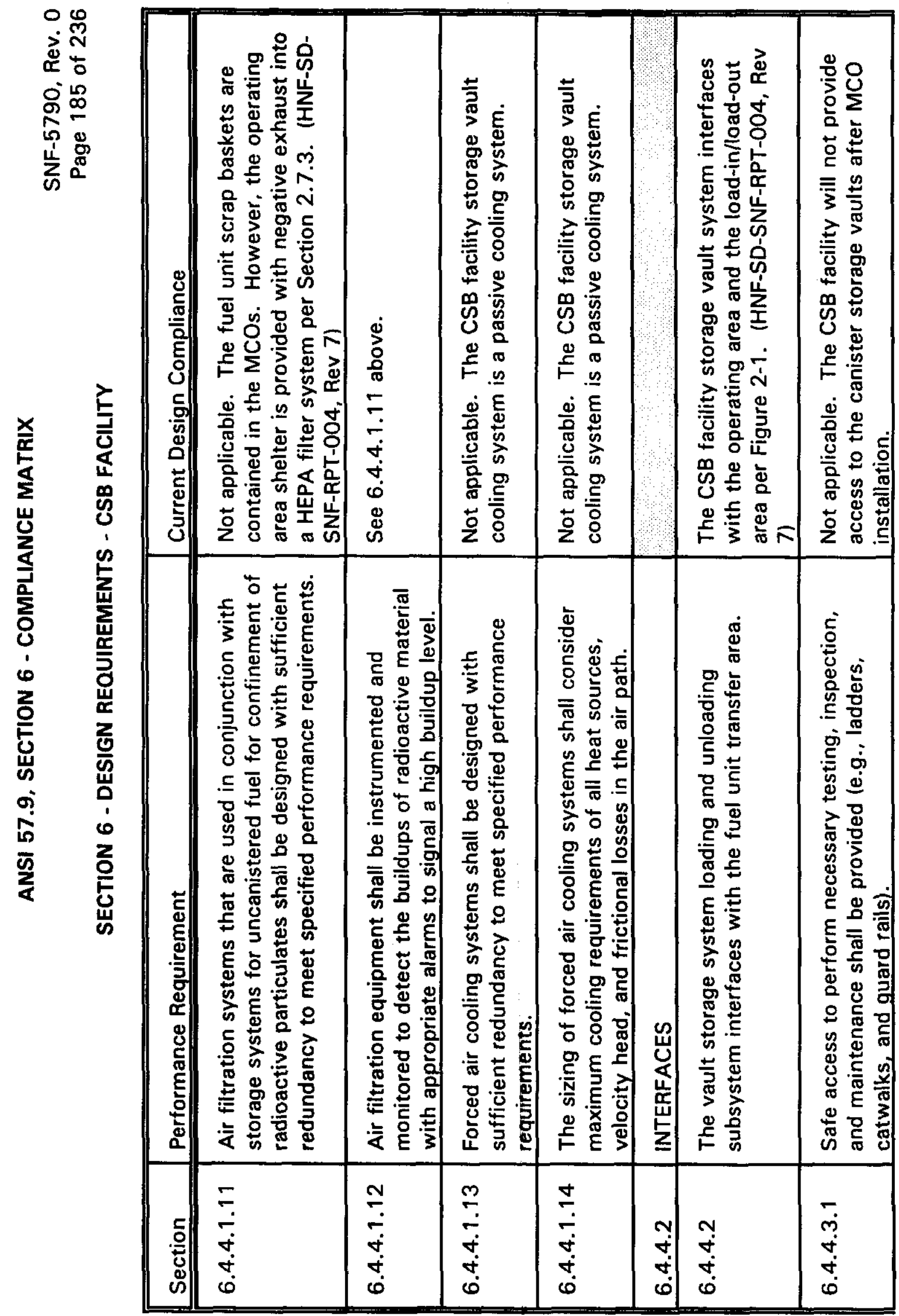




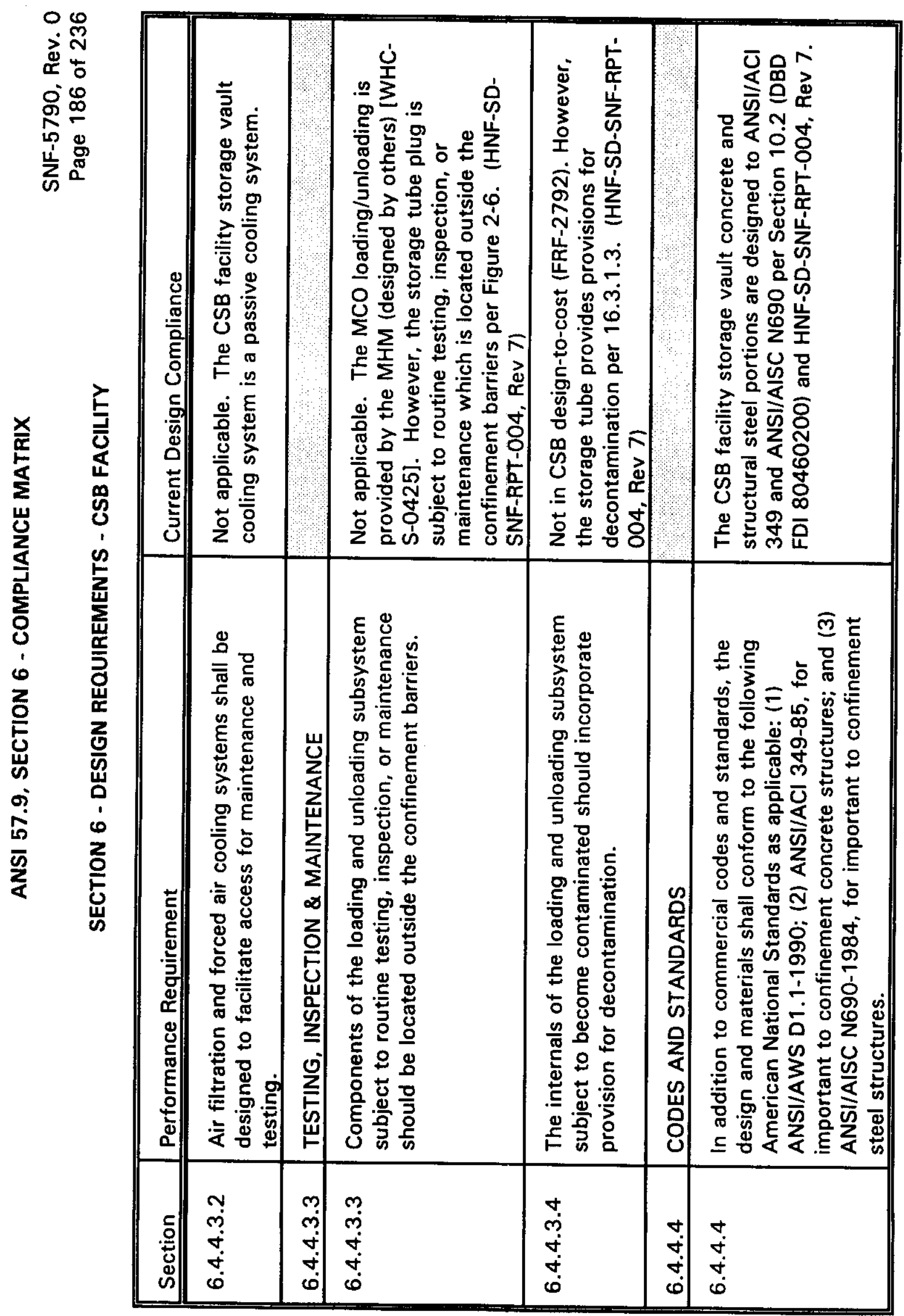




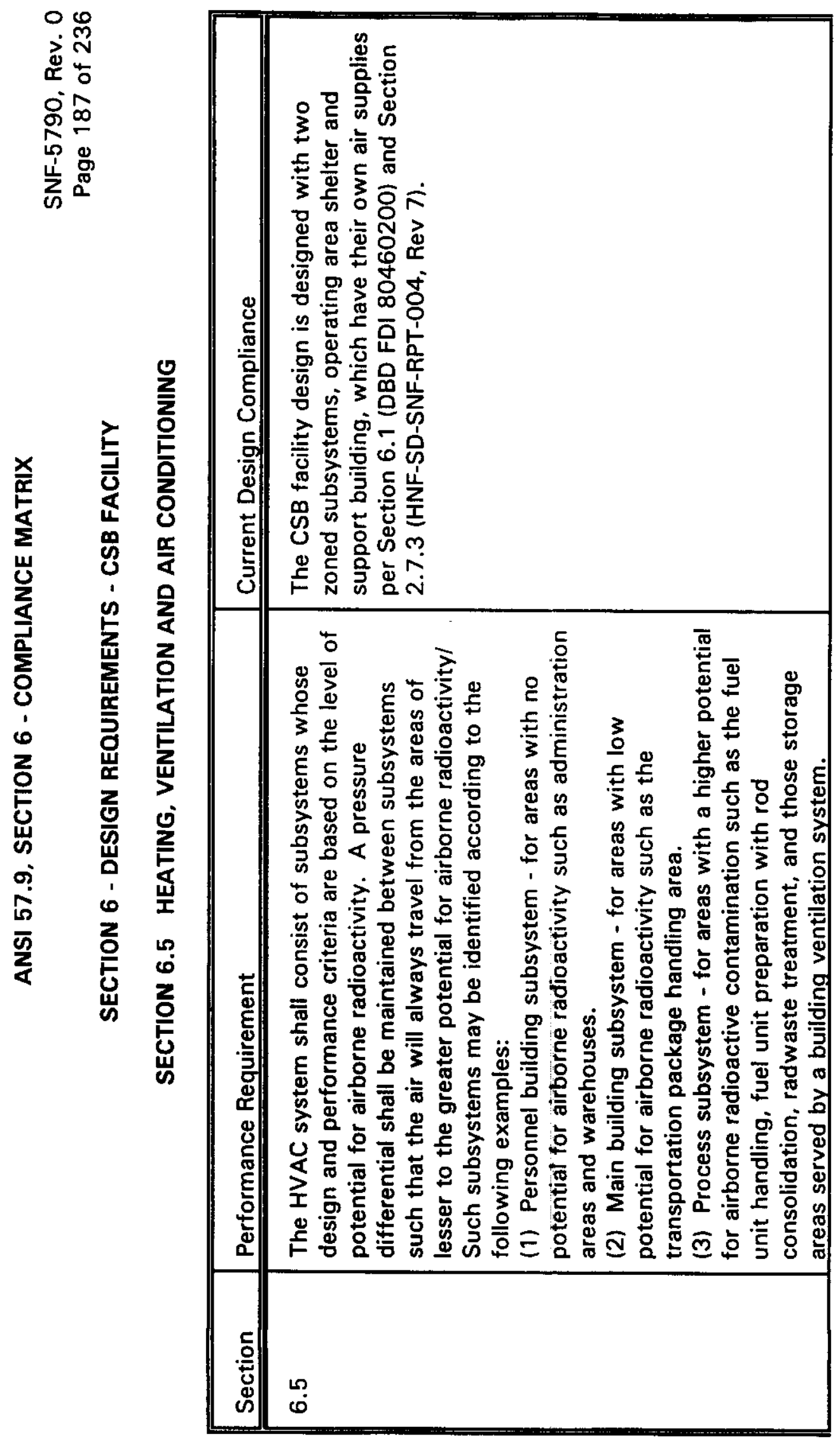




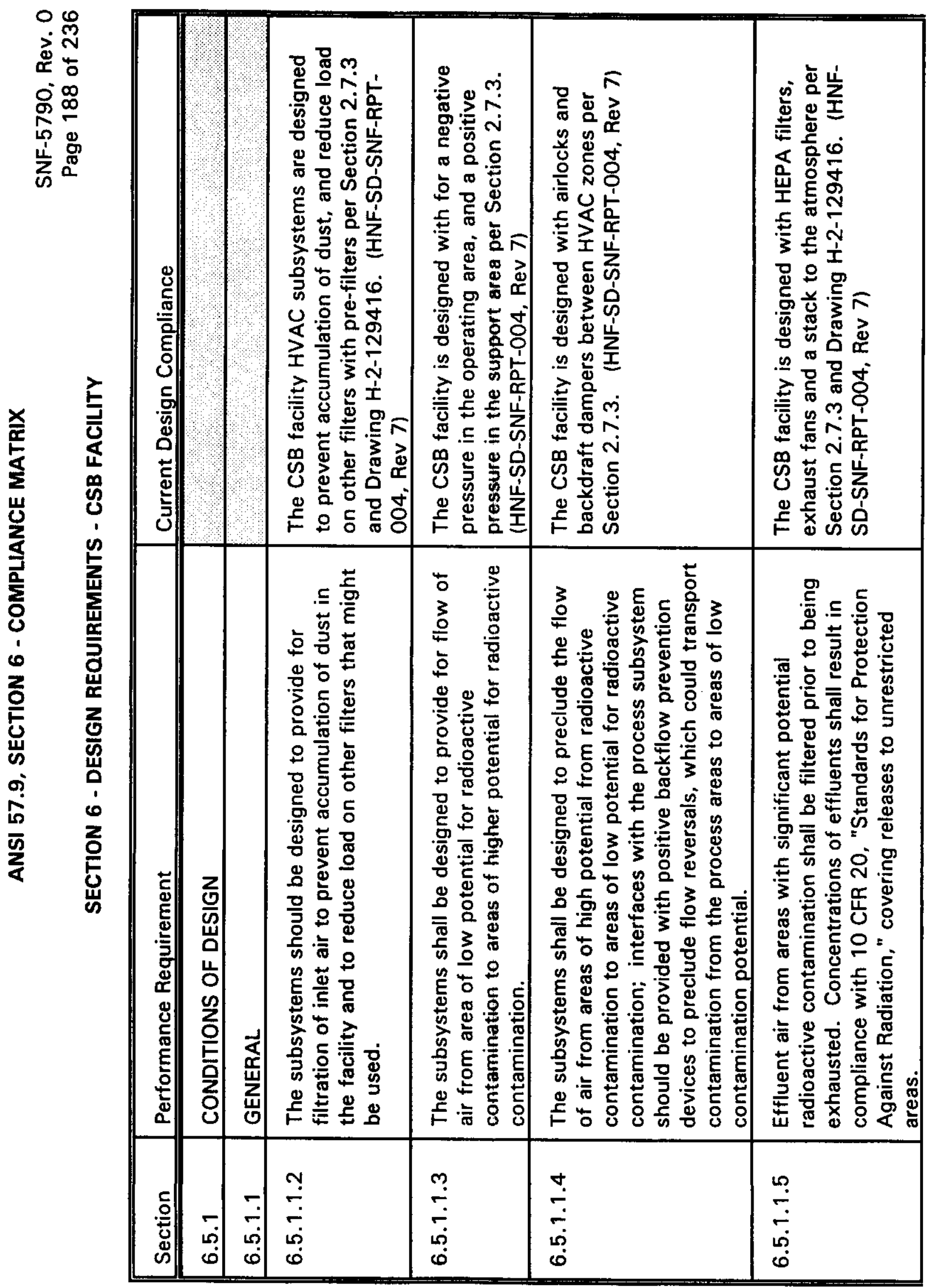




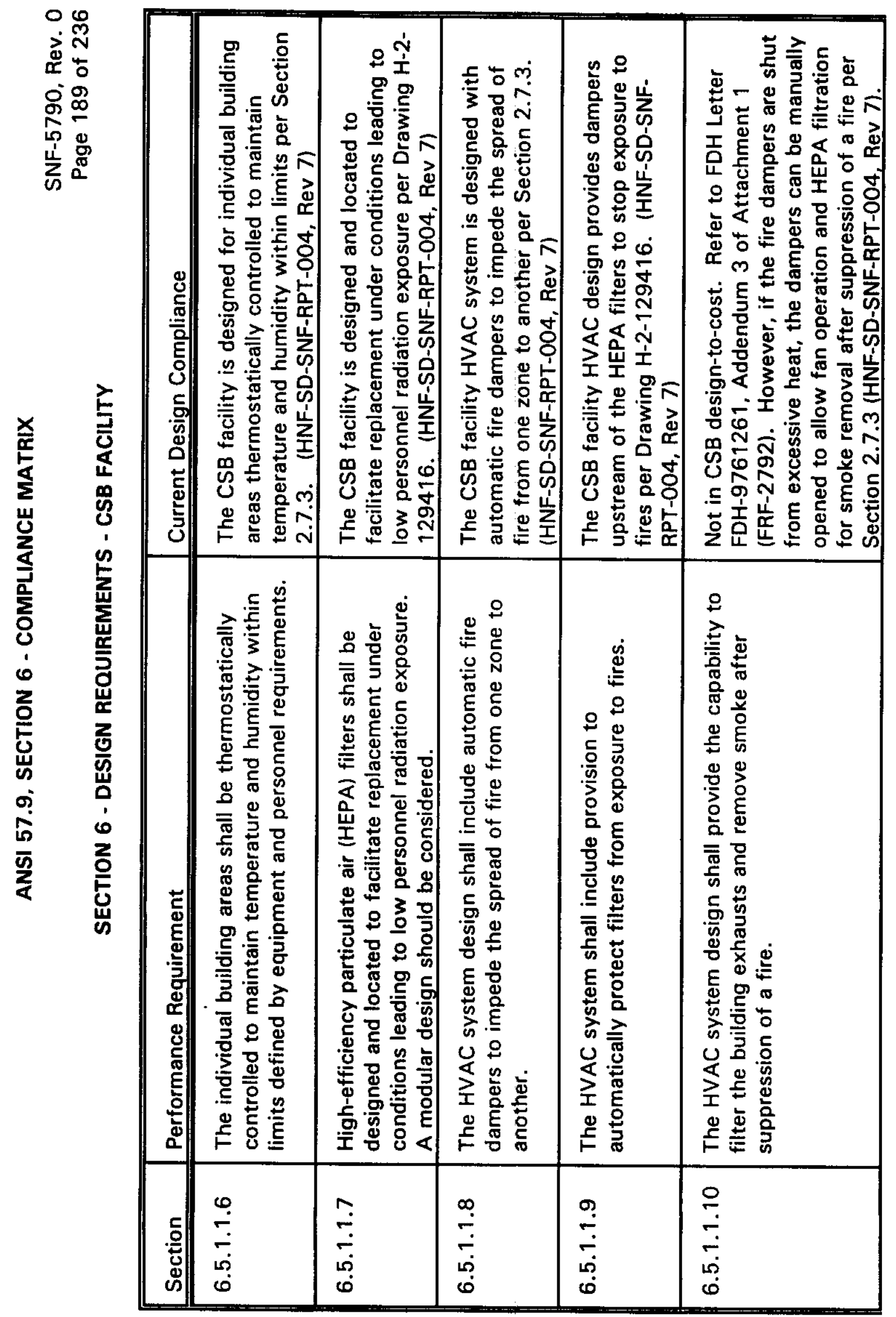




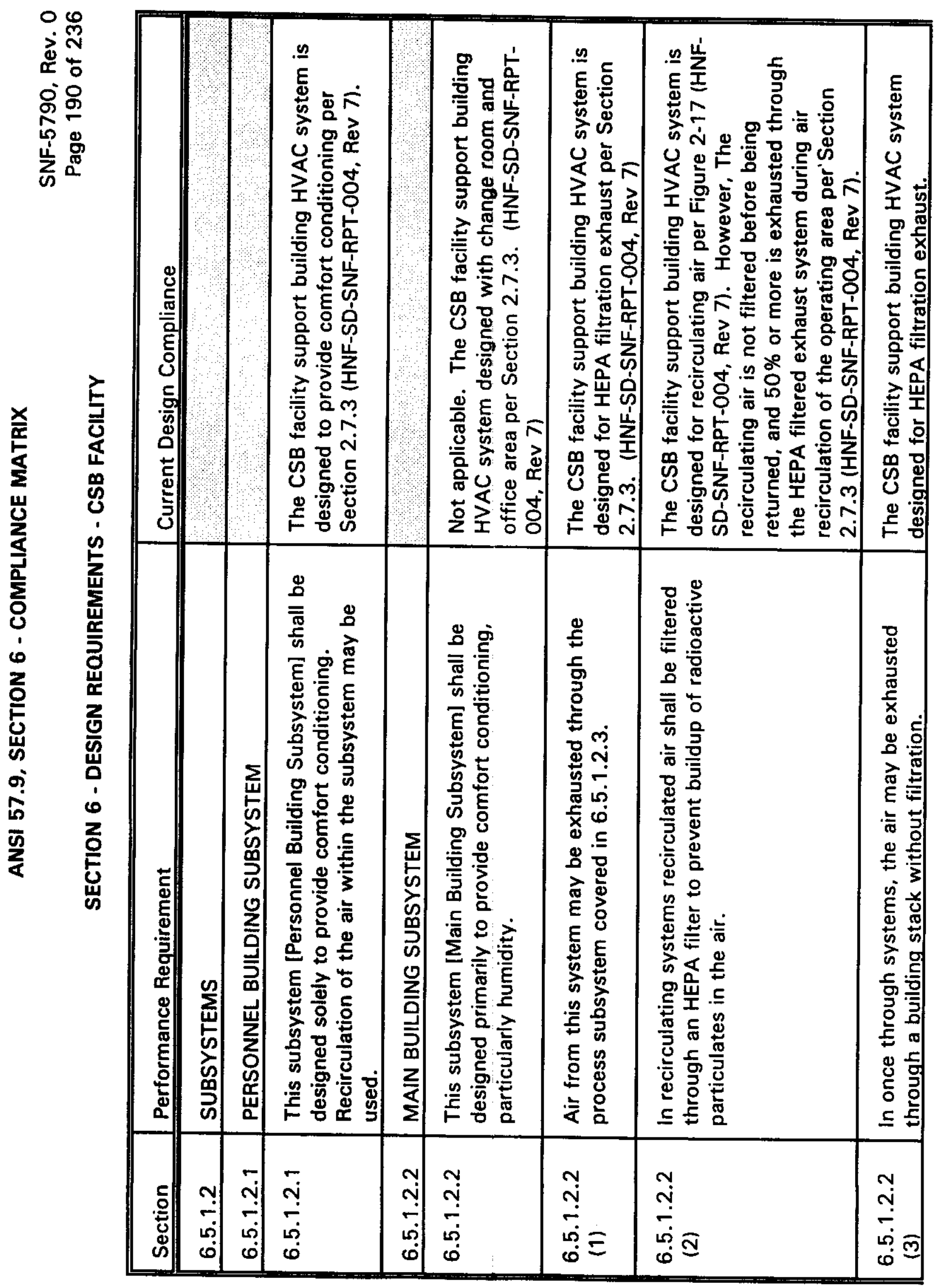




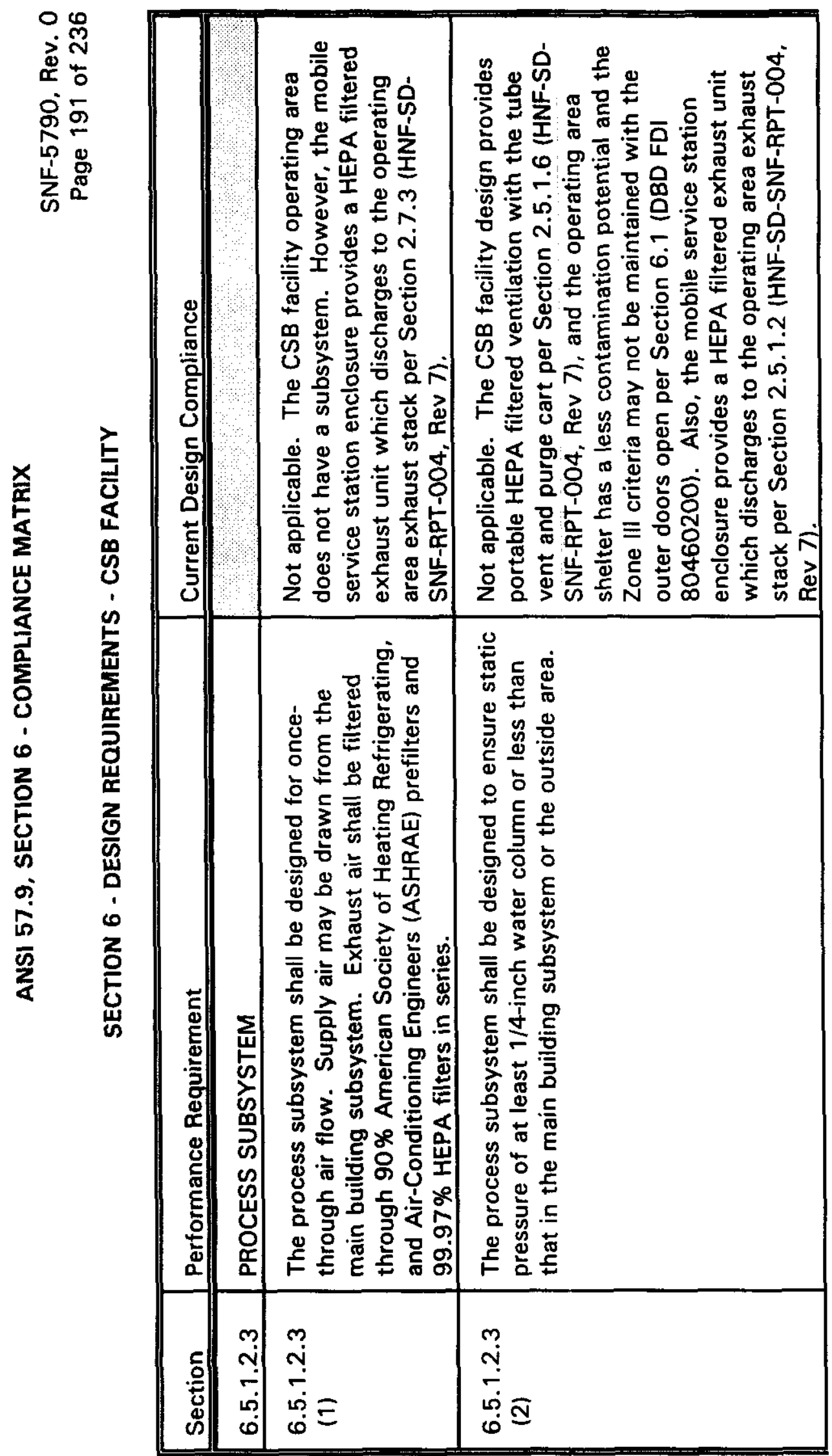




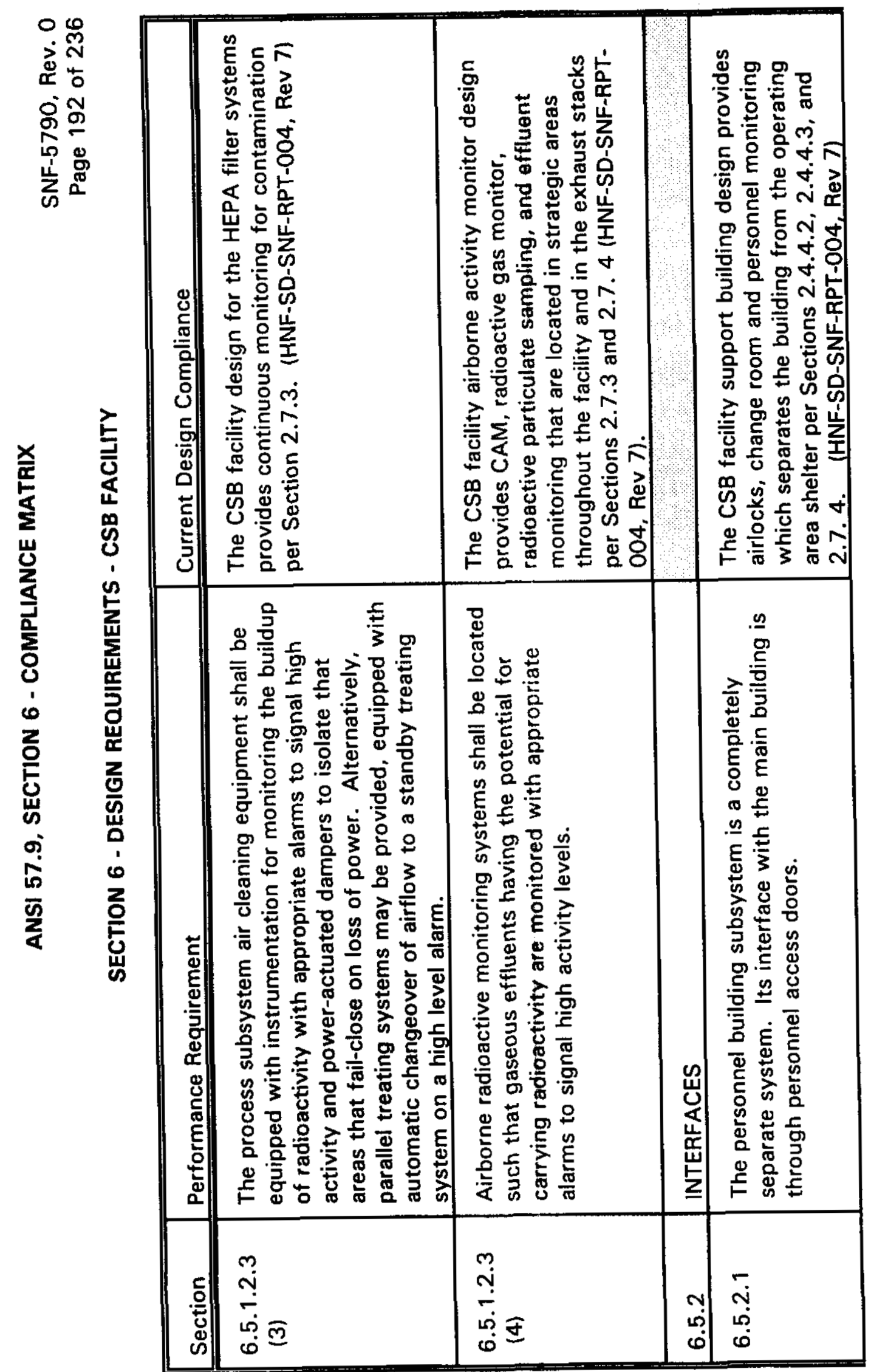




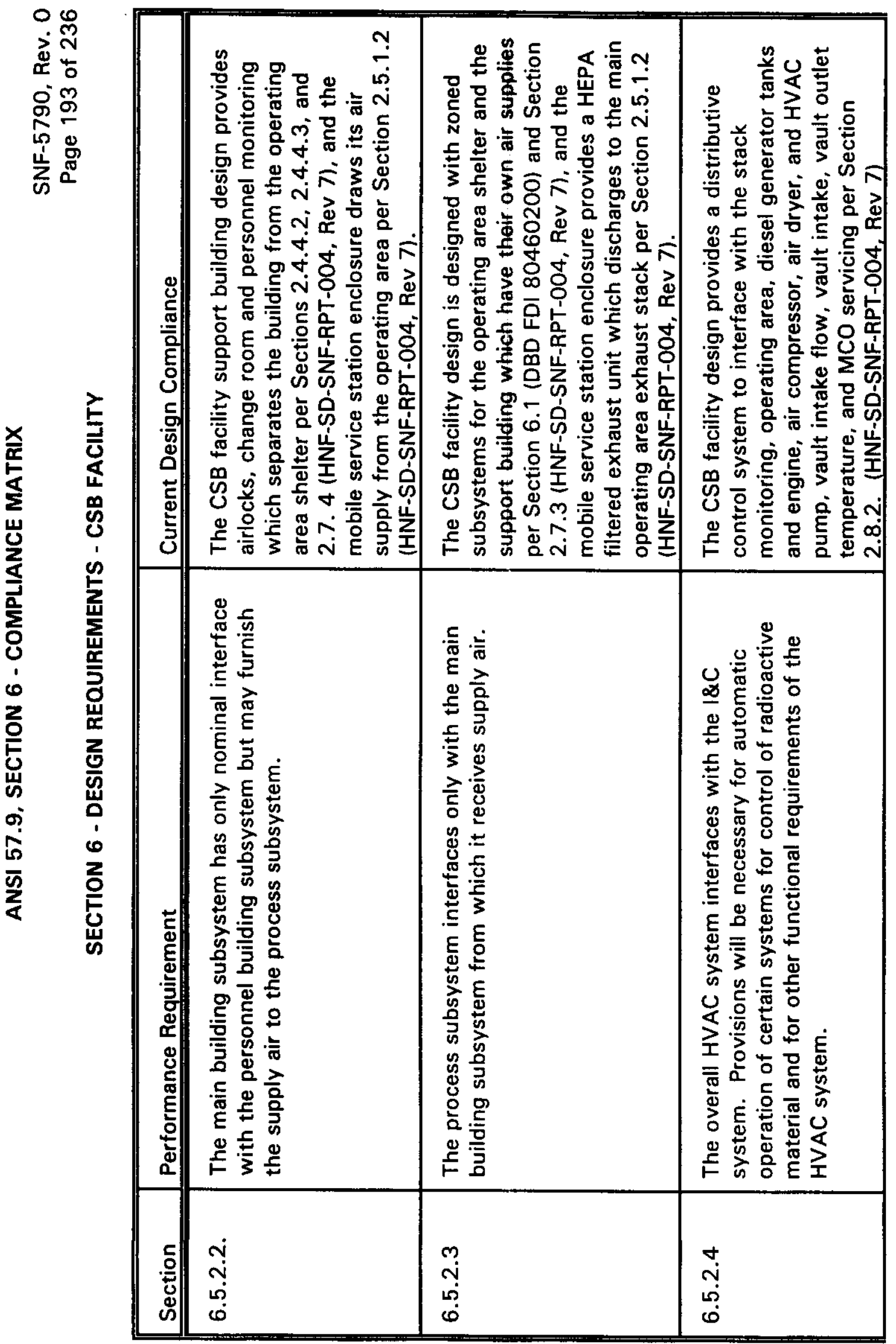




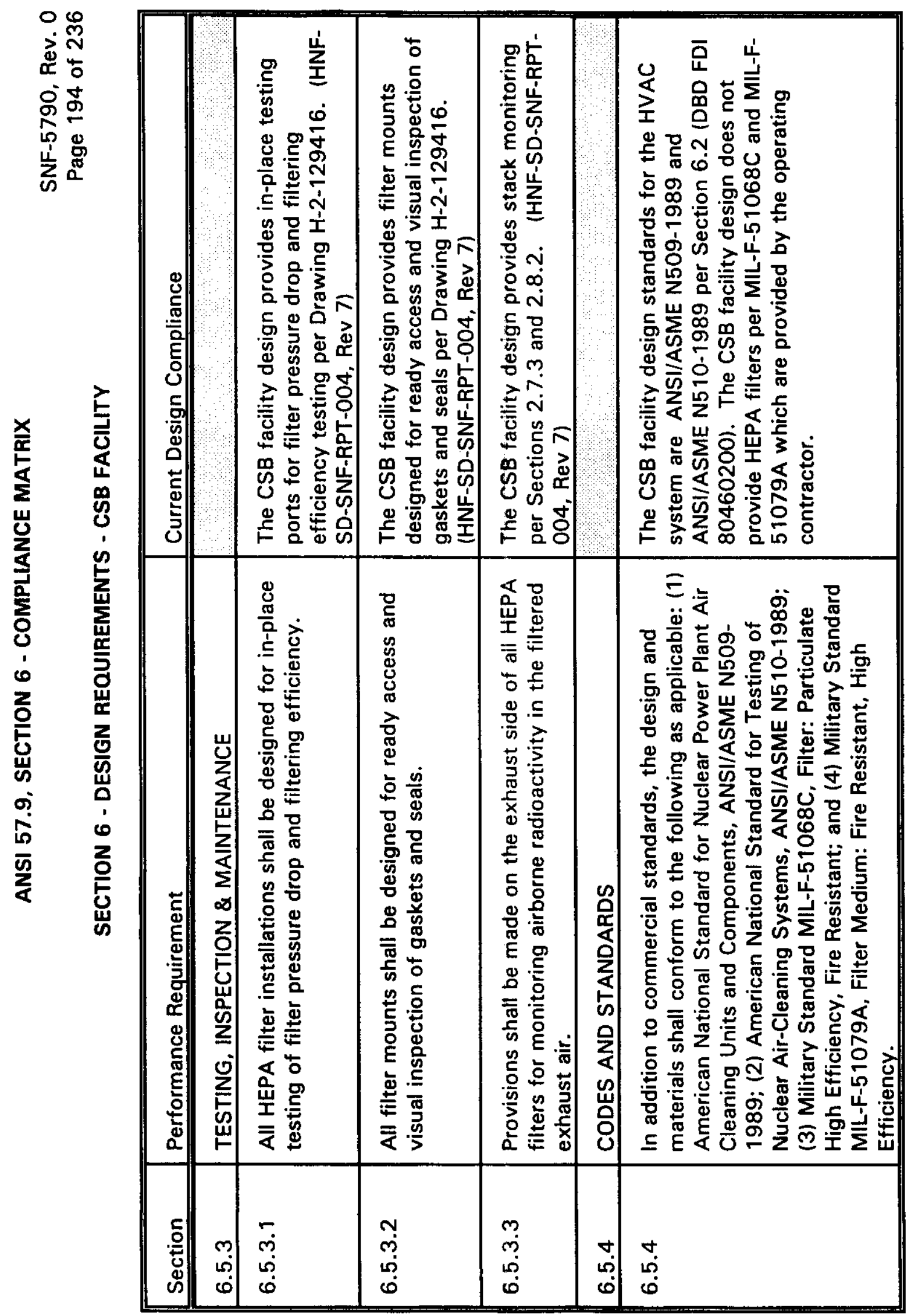




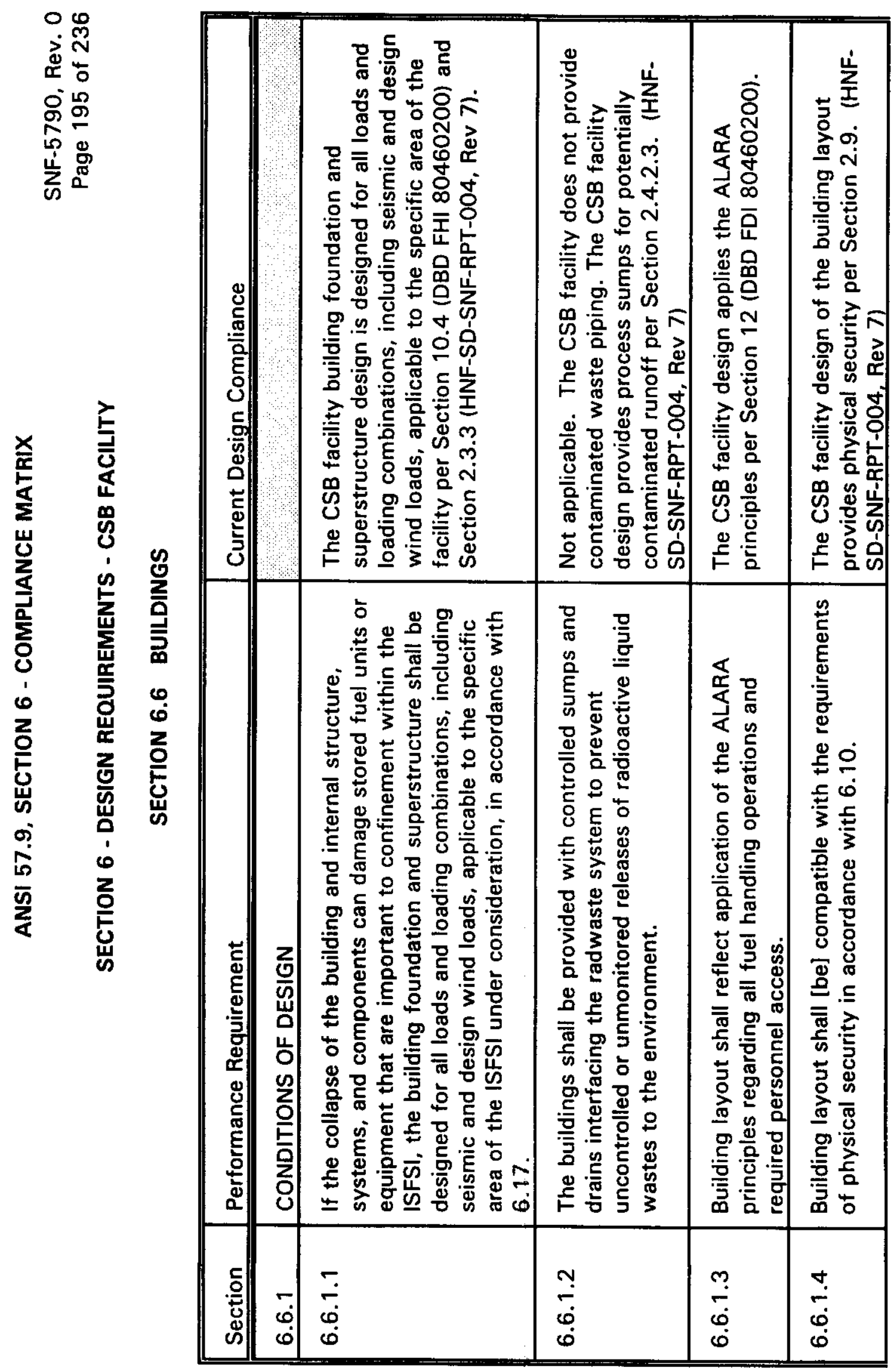




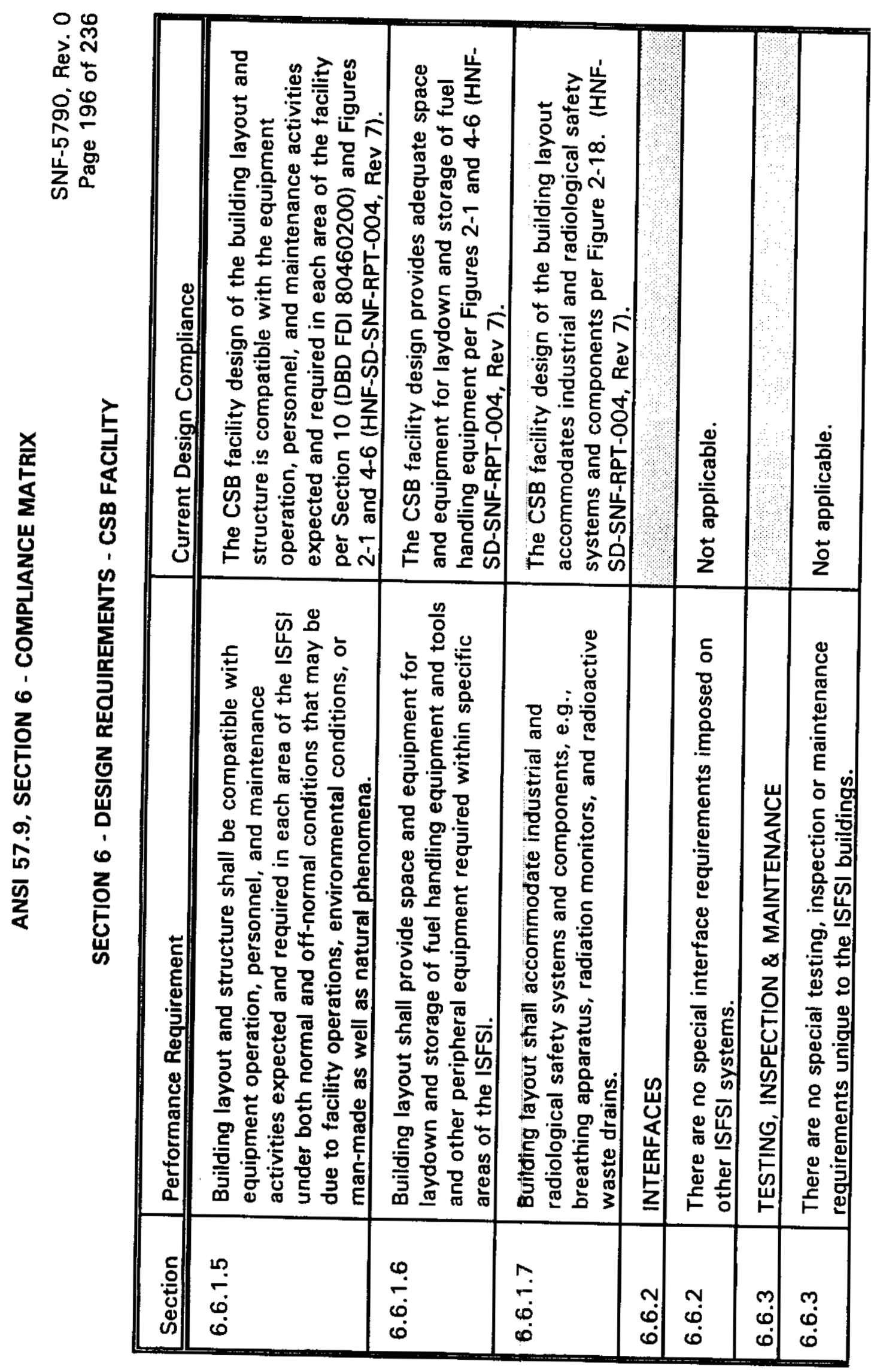




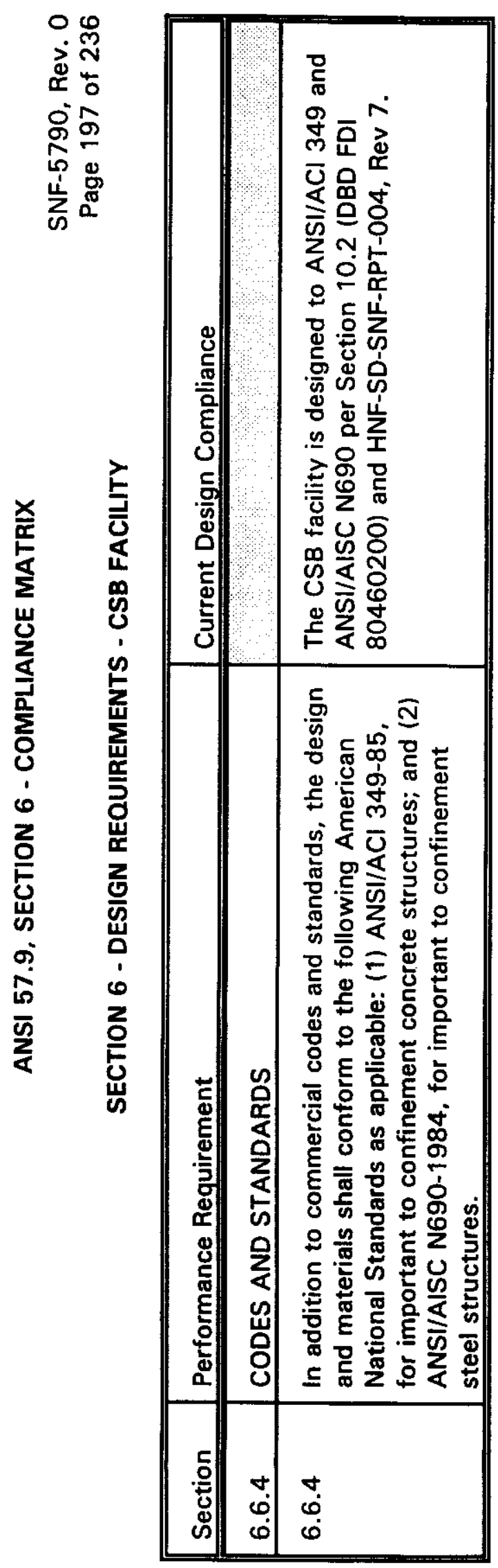




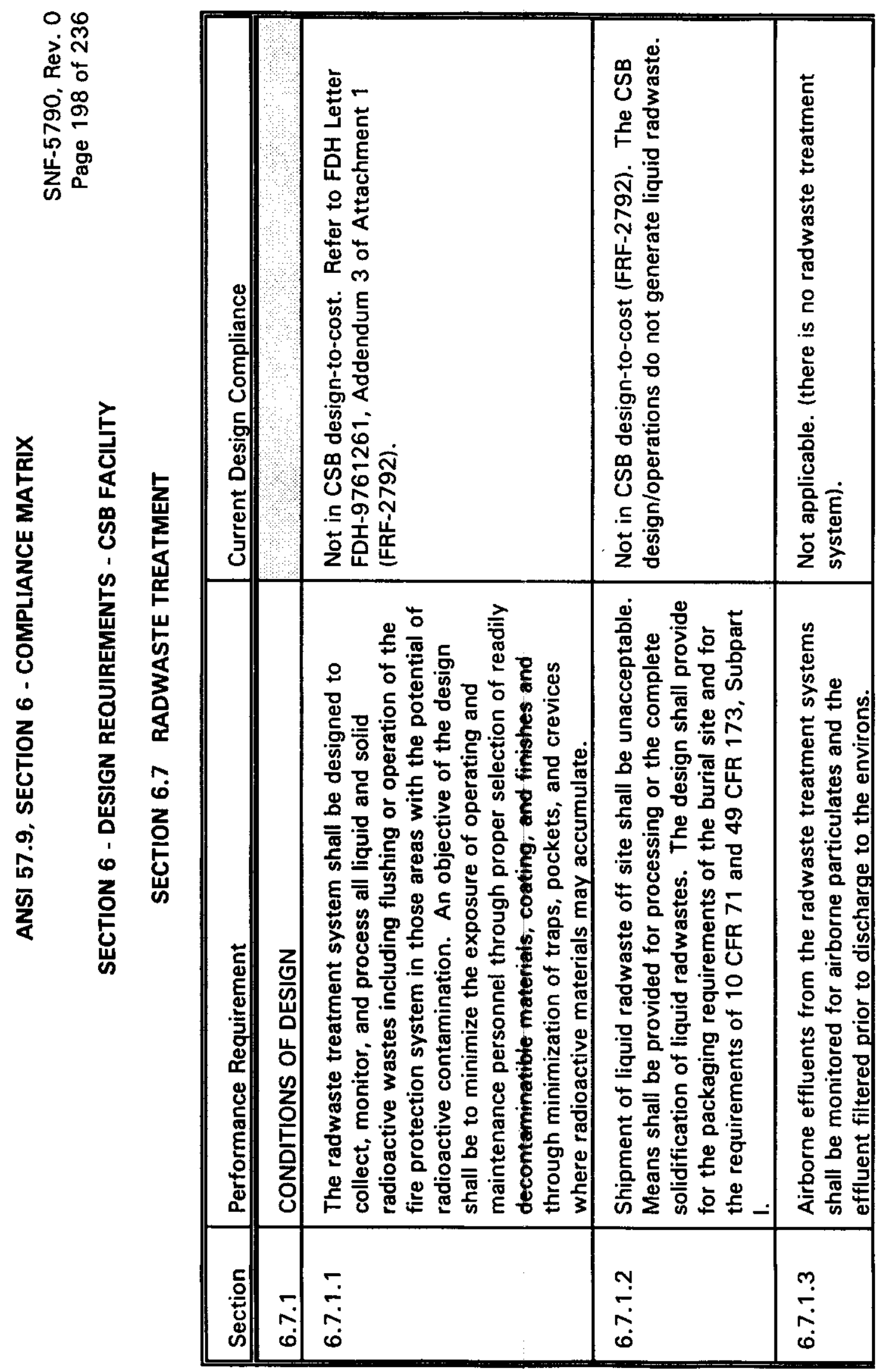




\begin{tabular}{|c|c|c|c|c|c|c|c|c|c|c|}
\hline \multirow{3}{*}{ 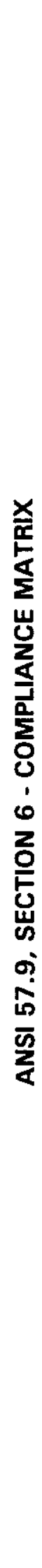 } & 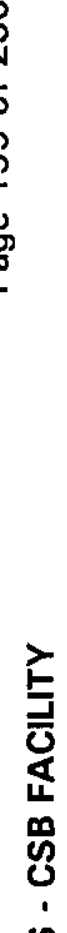 & 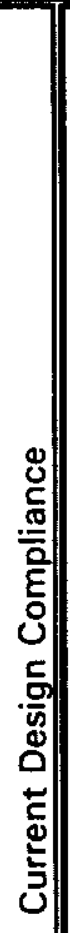 & 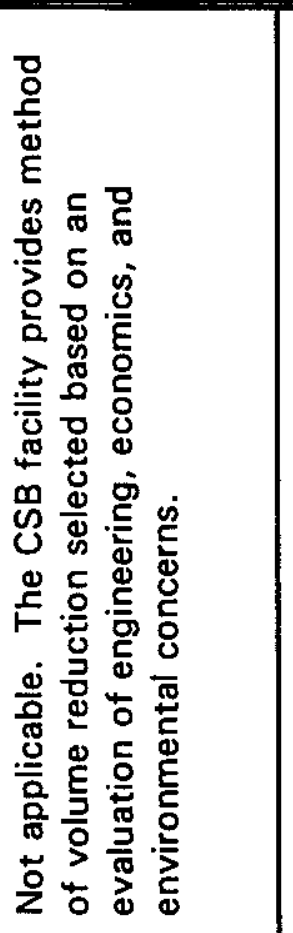 & 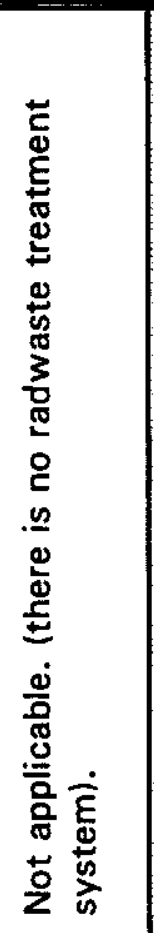 & & 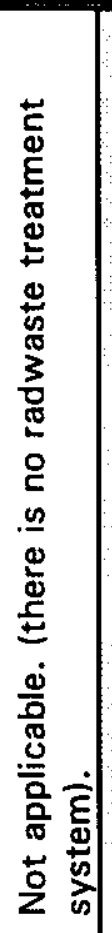 & & 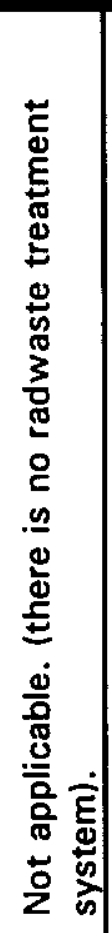 & 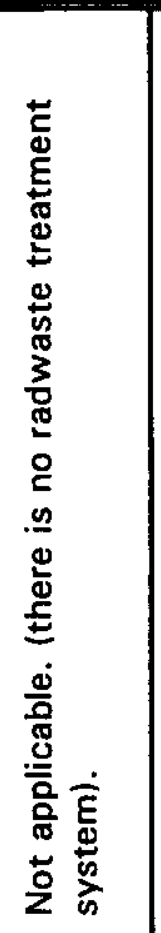 & 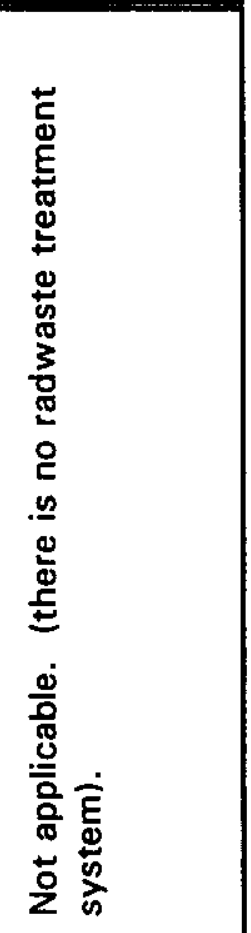 \\
\hline & 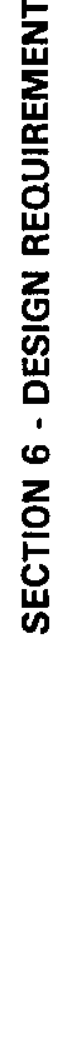 & 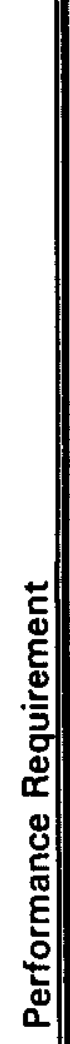 & 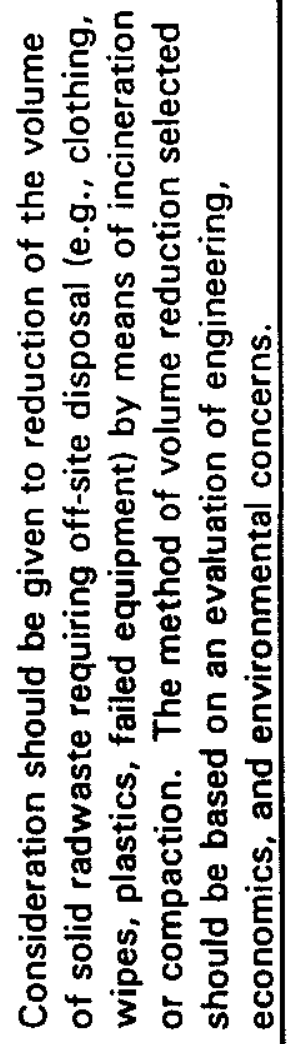 & 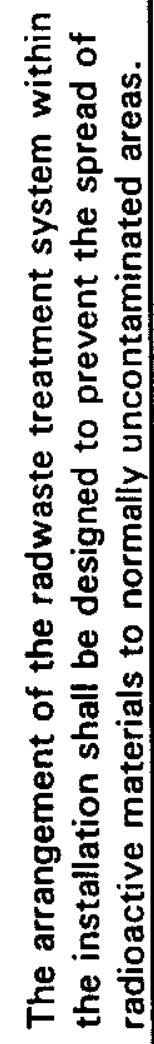 & $\begin{array}{l}u \\
\mathcal{U} \\
\mathbf{4} \\
\mathbb{\alpha} \\
\underline{w} \\
\underline{z}\end{array}$ & 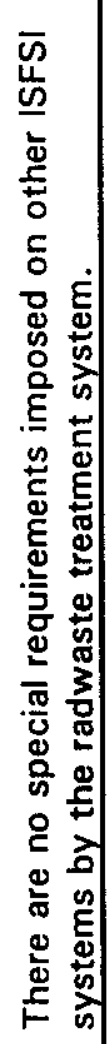 & 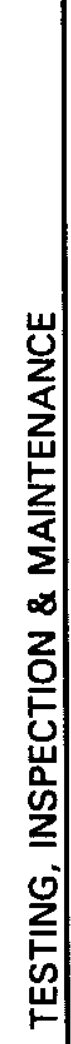 & 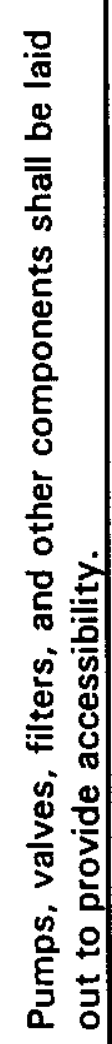 & 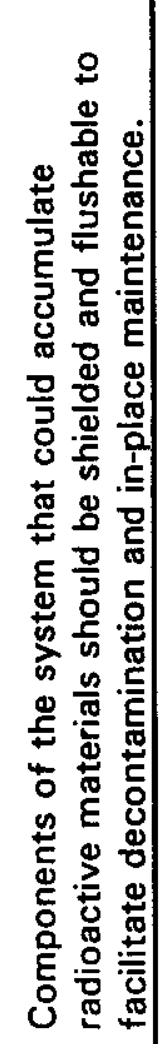 & 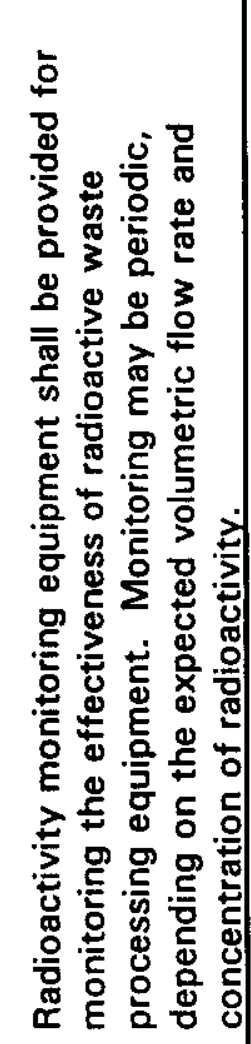 \\
\hline & & 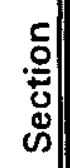 & ت. & 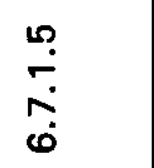 & $\begin{array}{l}N \\
N\end{array}$ & $\stackrel{N}{N}$ & ri & $\stackrel{\ddot{\oplus}}{\stackrel{\oplus}{0}}$ & 亗 & $\stackrel{m}{\stackrel{m}{~}}$ \\
\hline
\end{tabular}




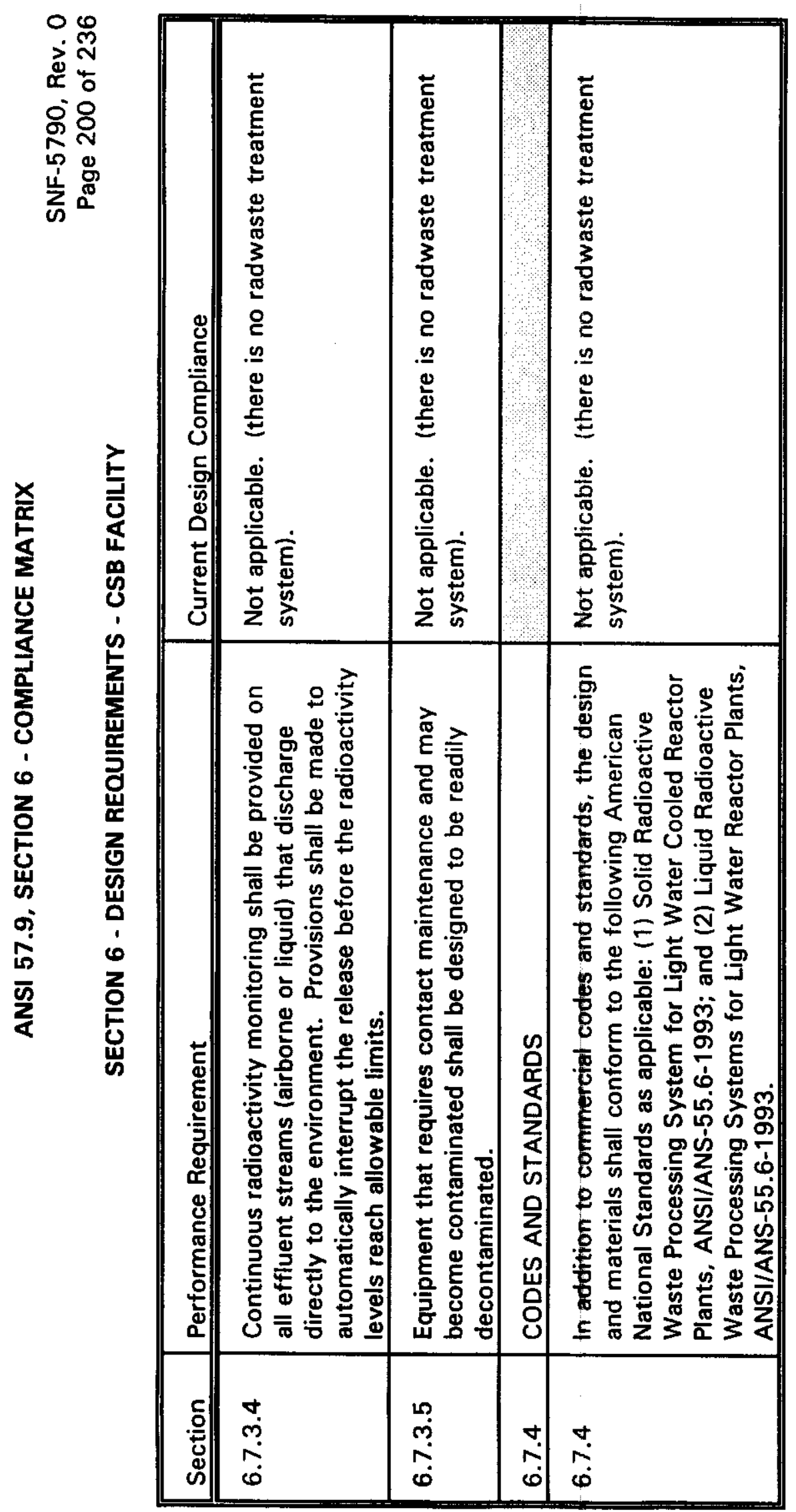




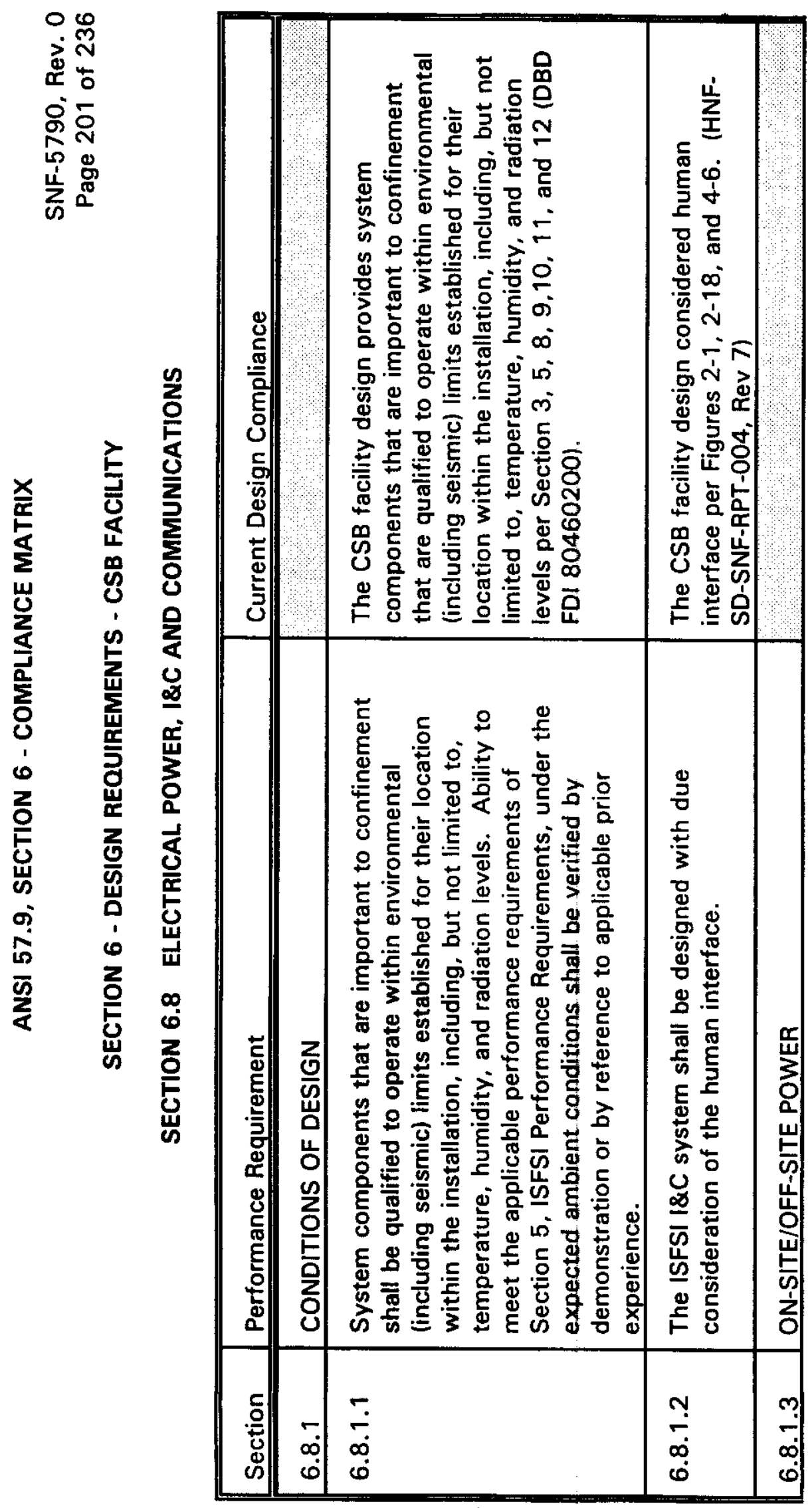




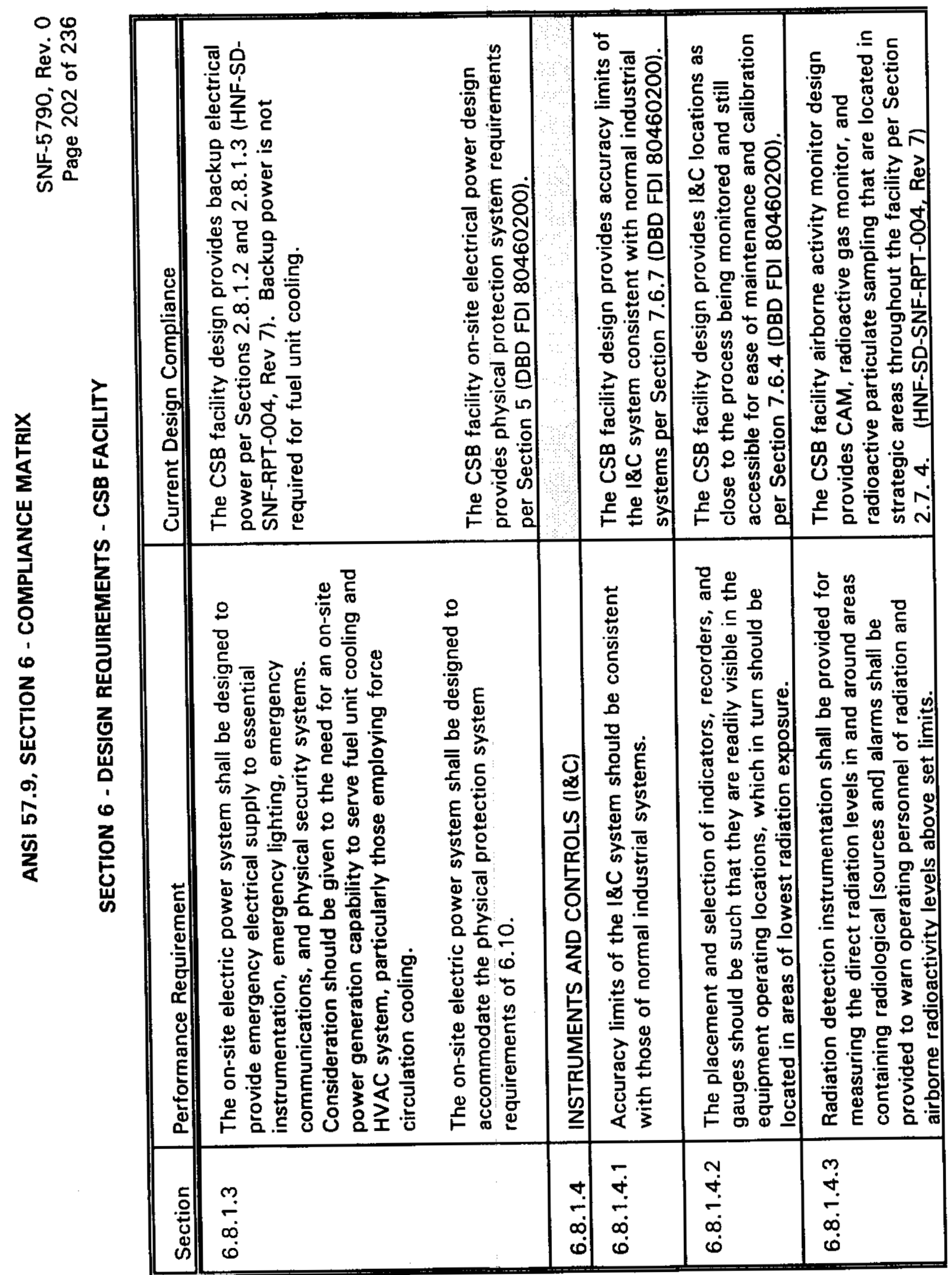




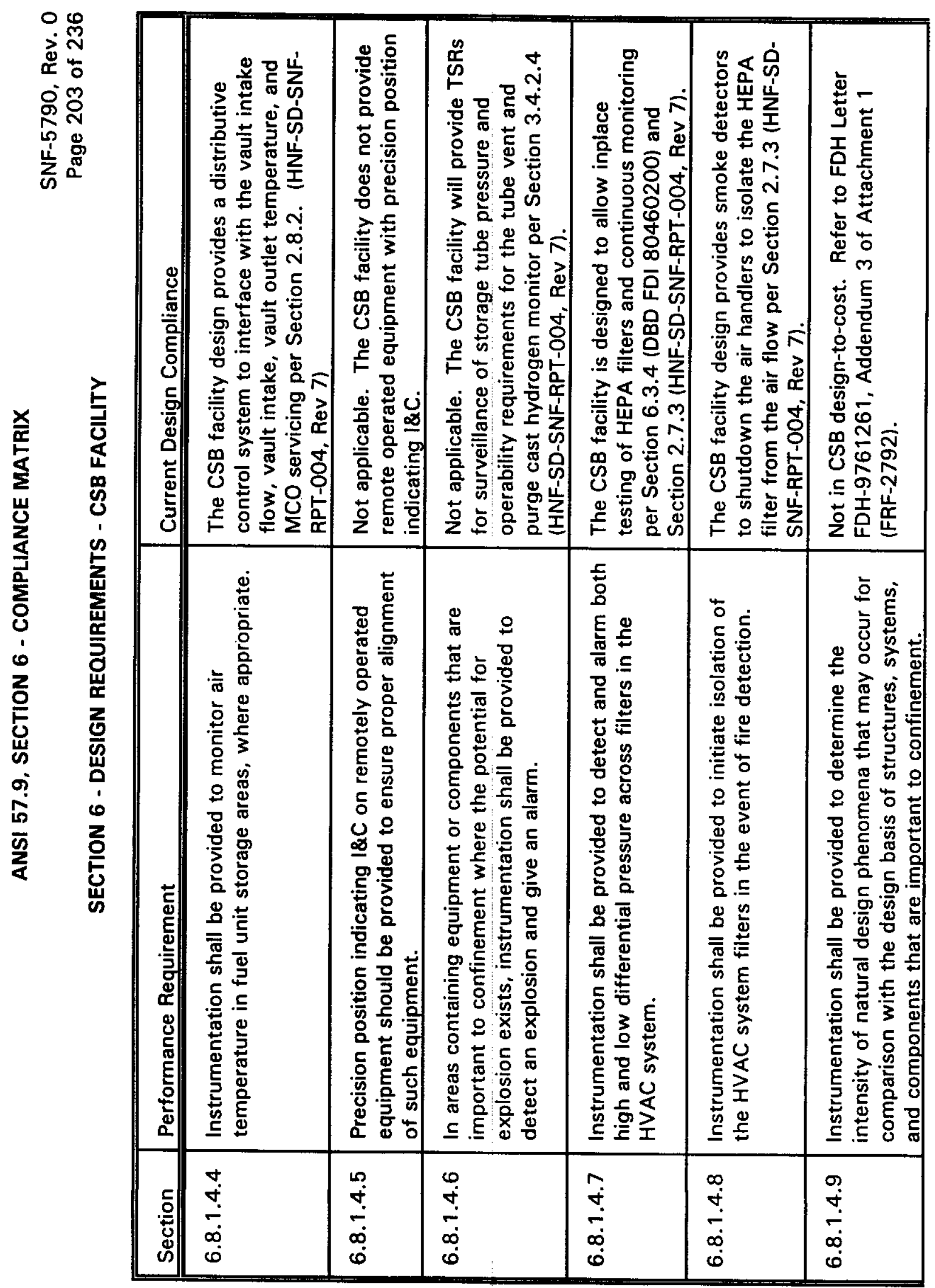




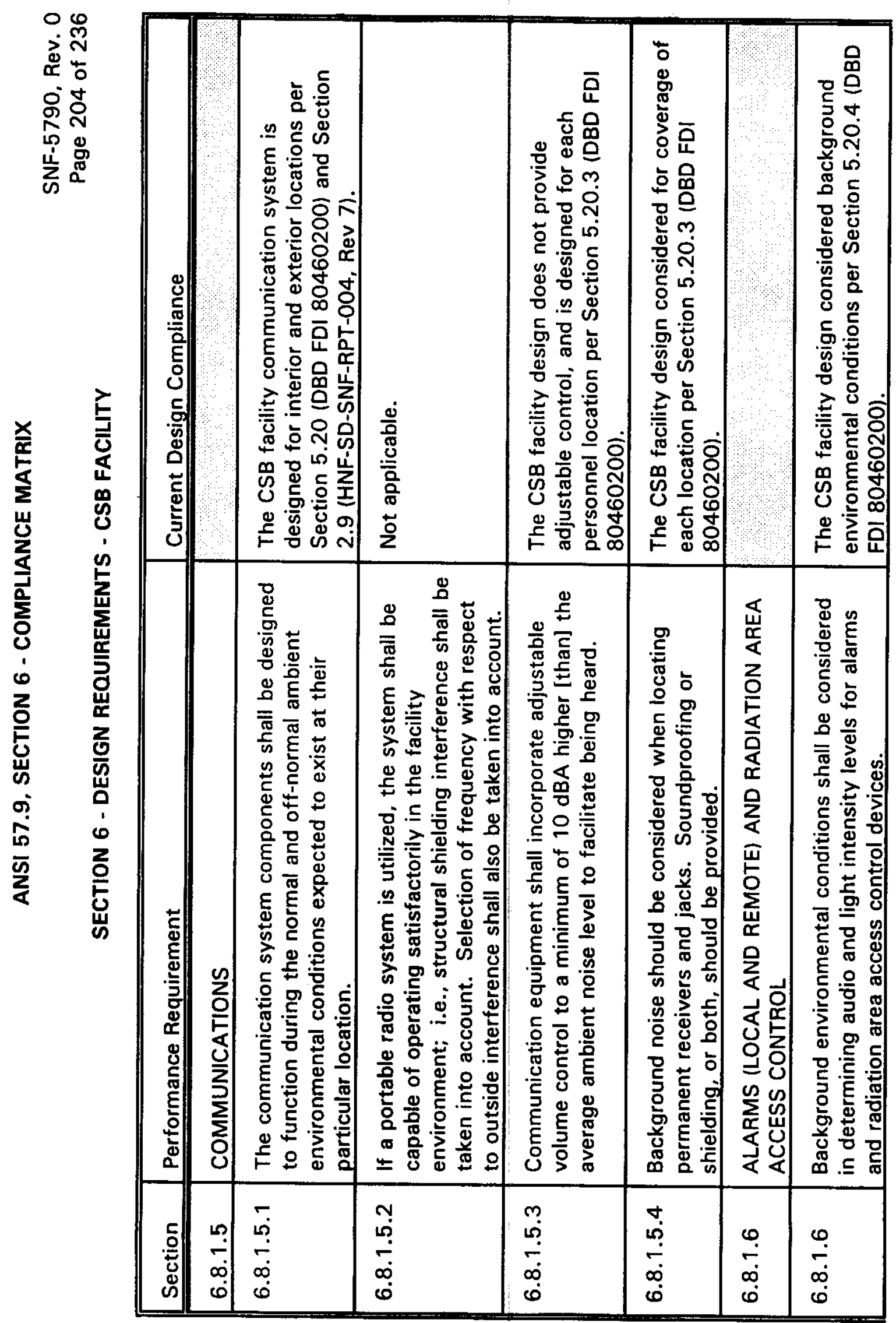




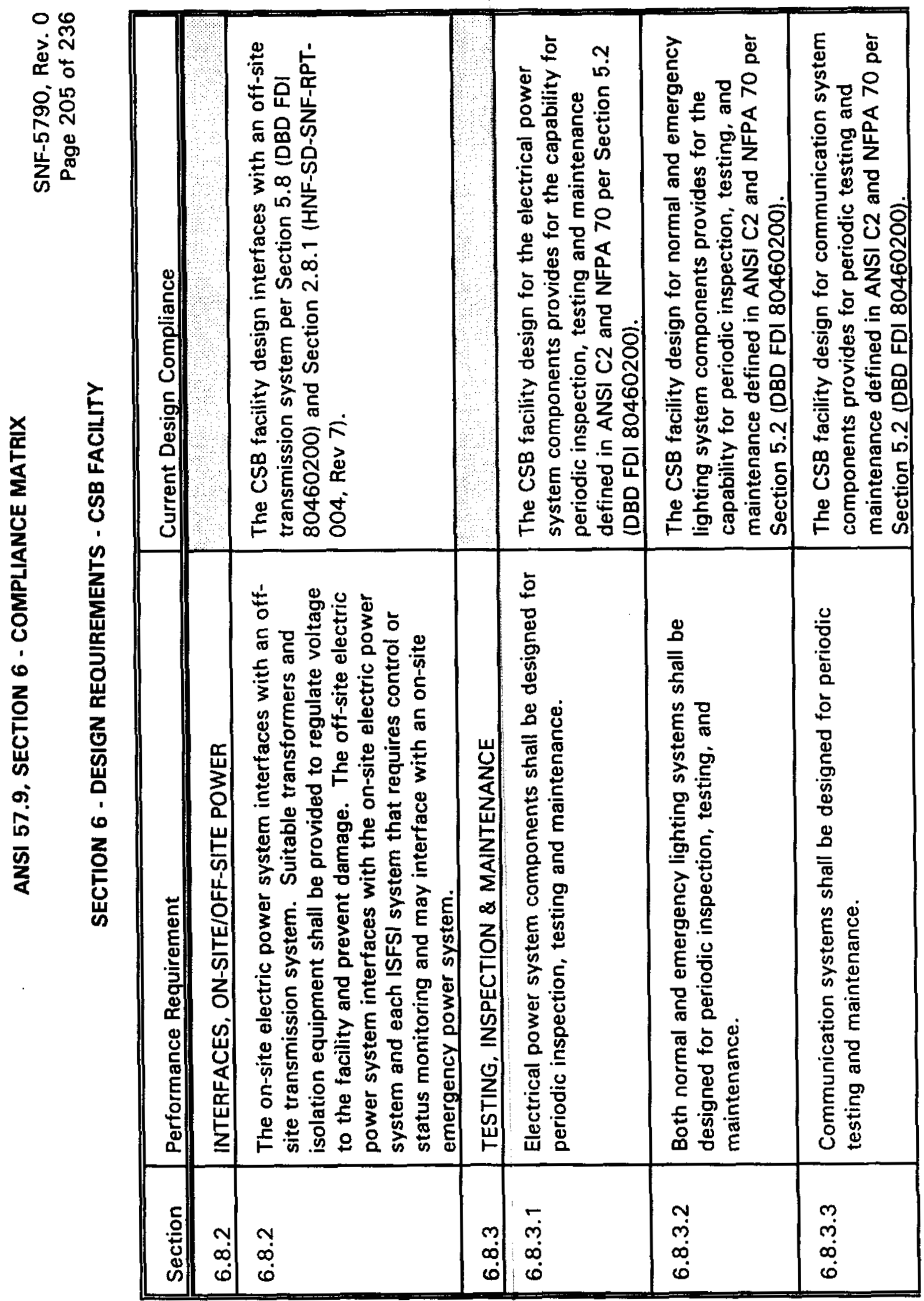




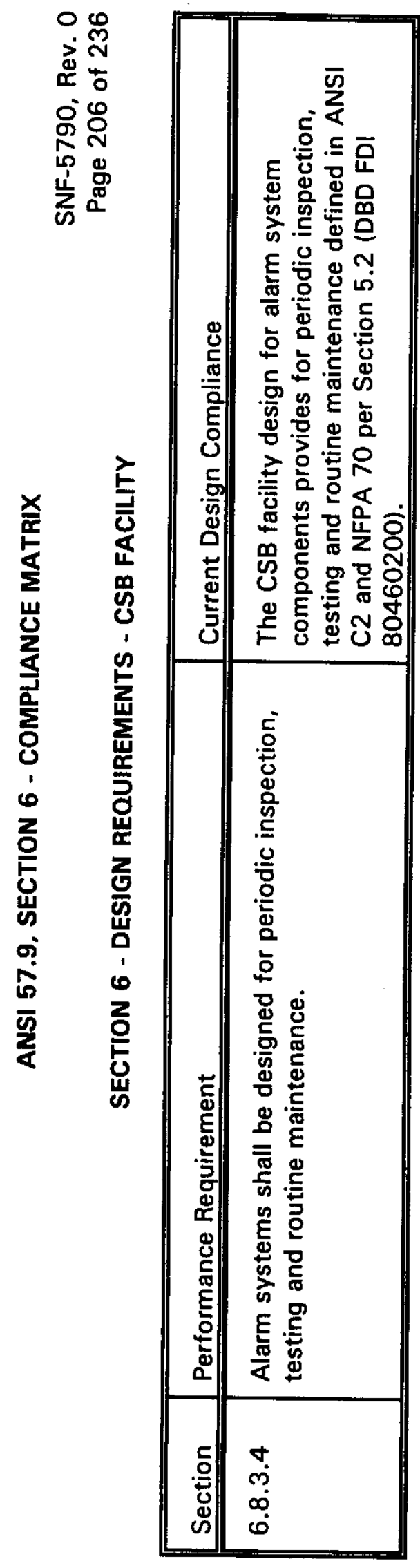




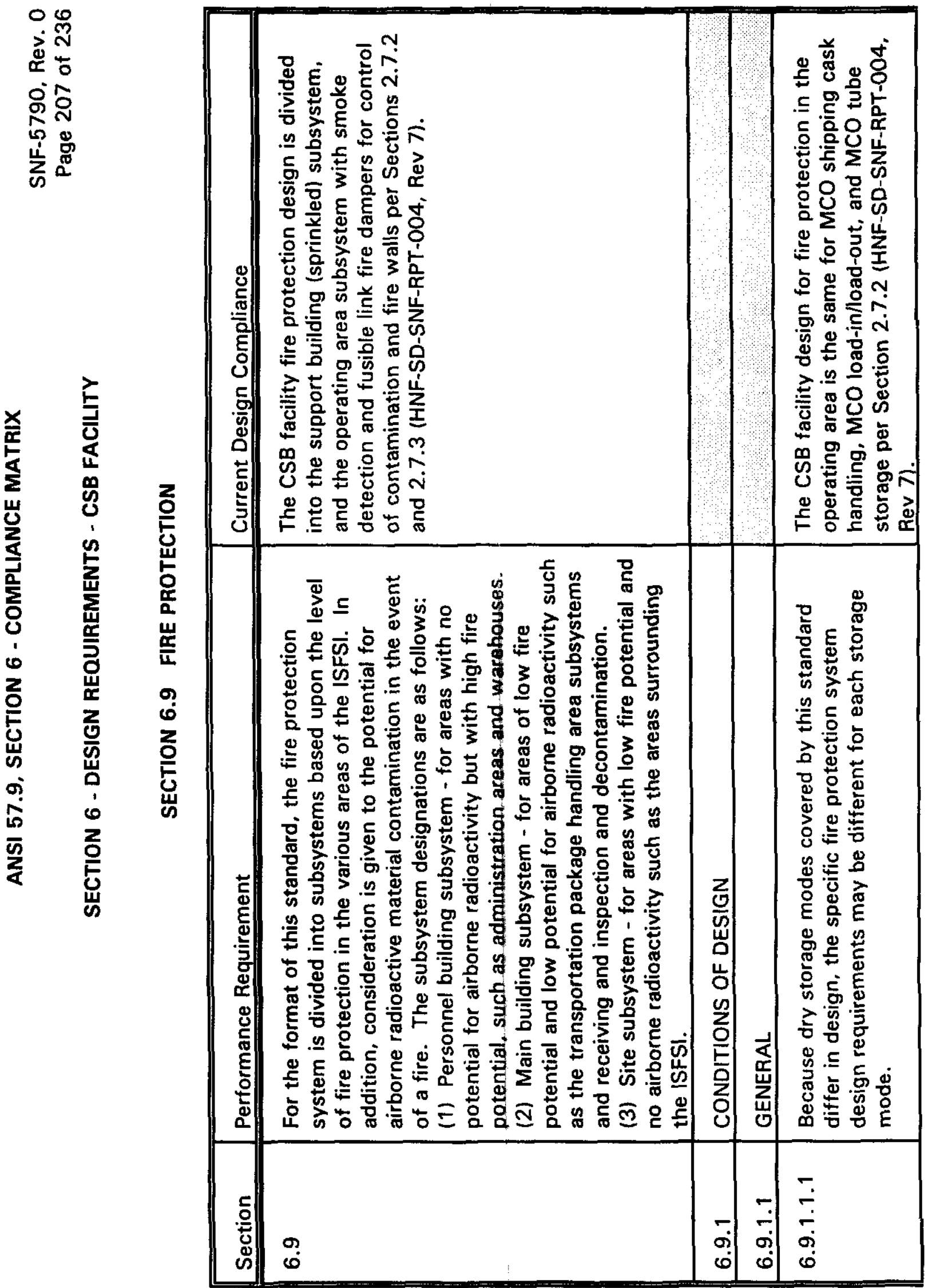




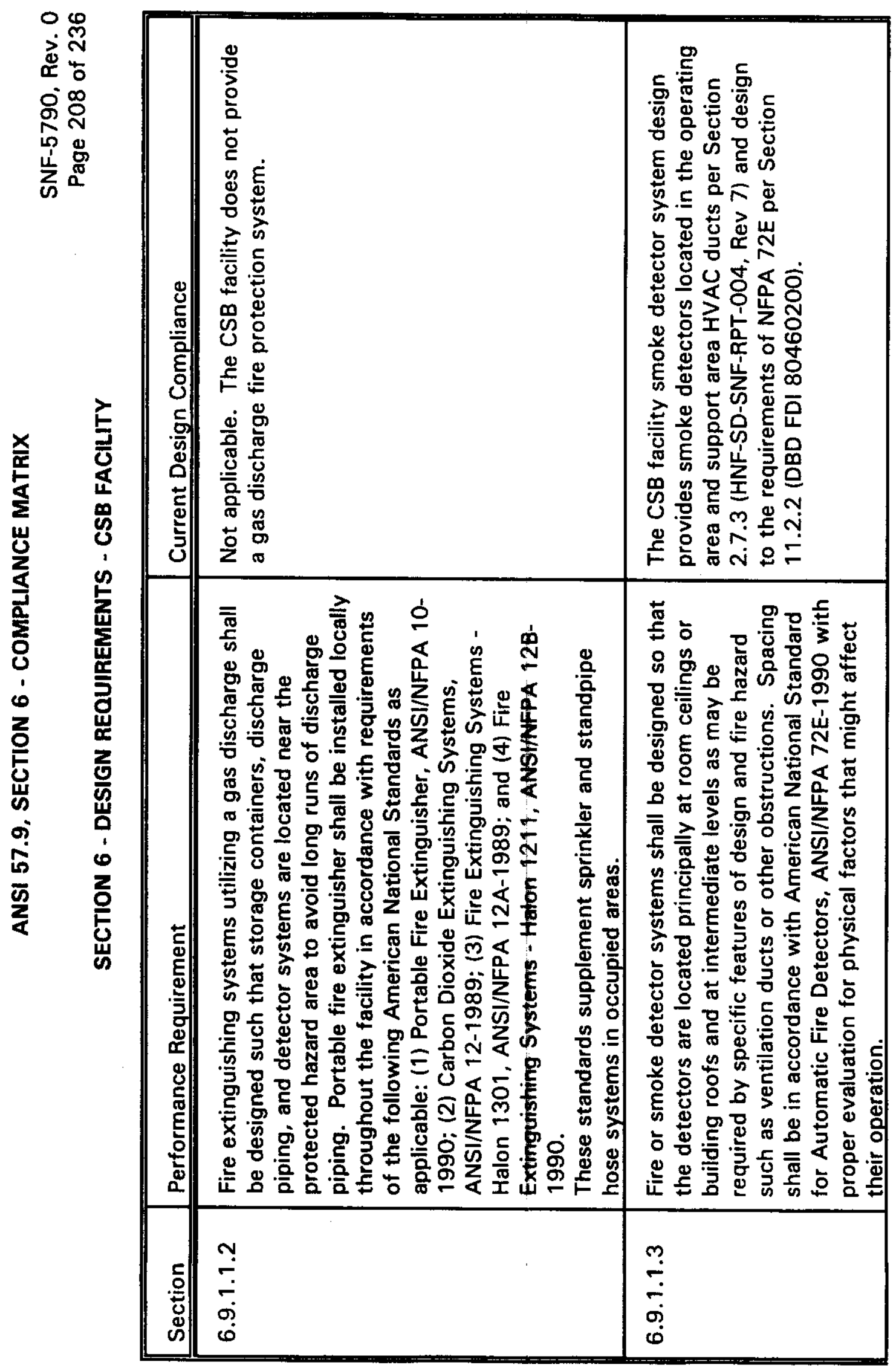




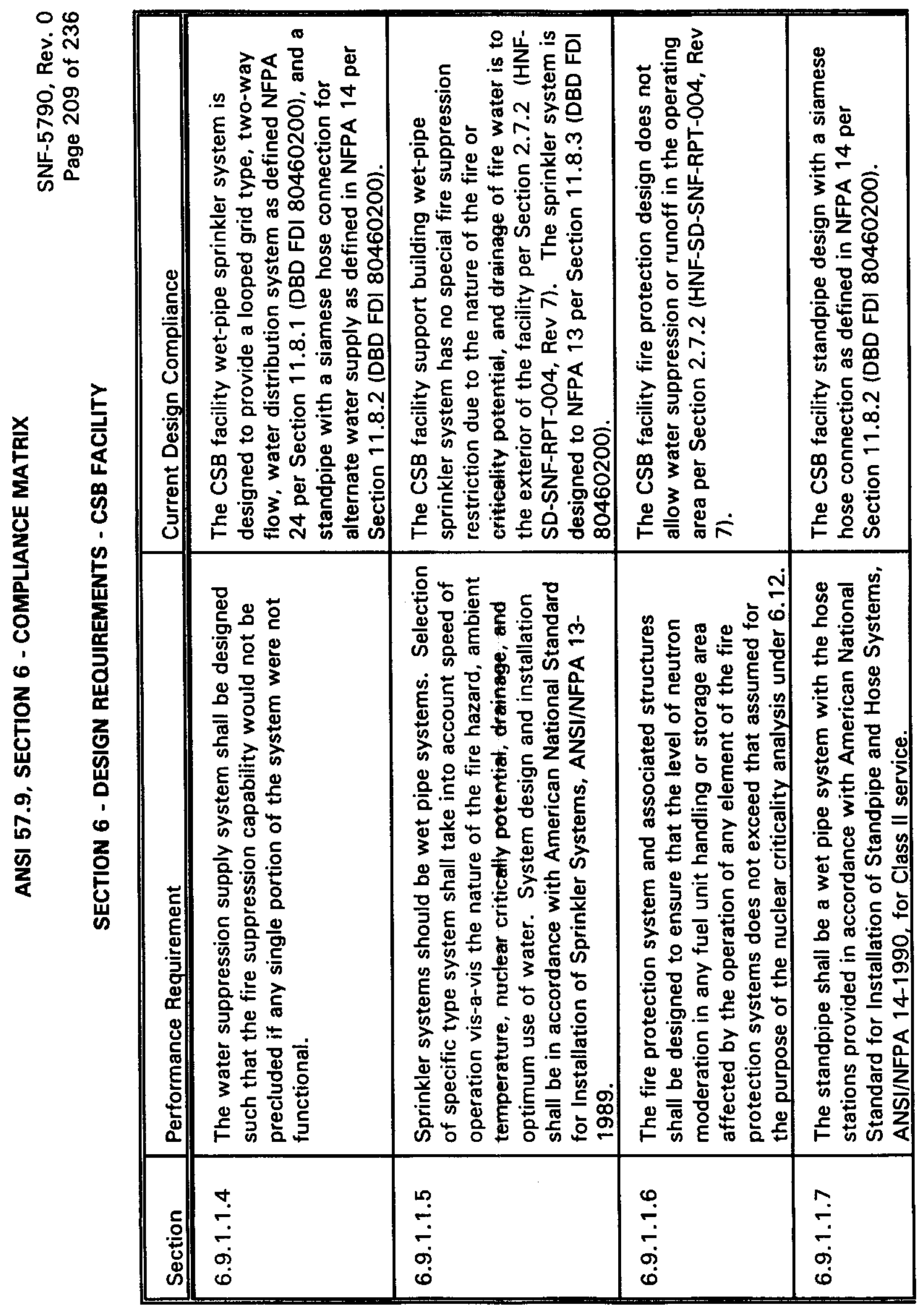




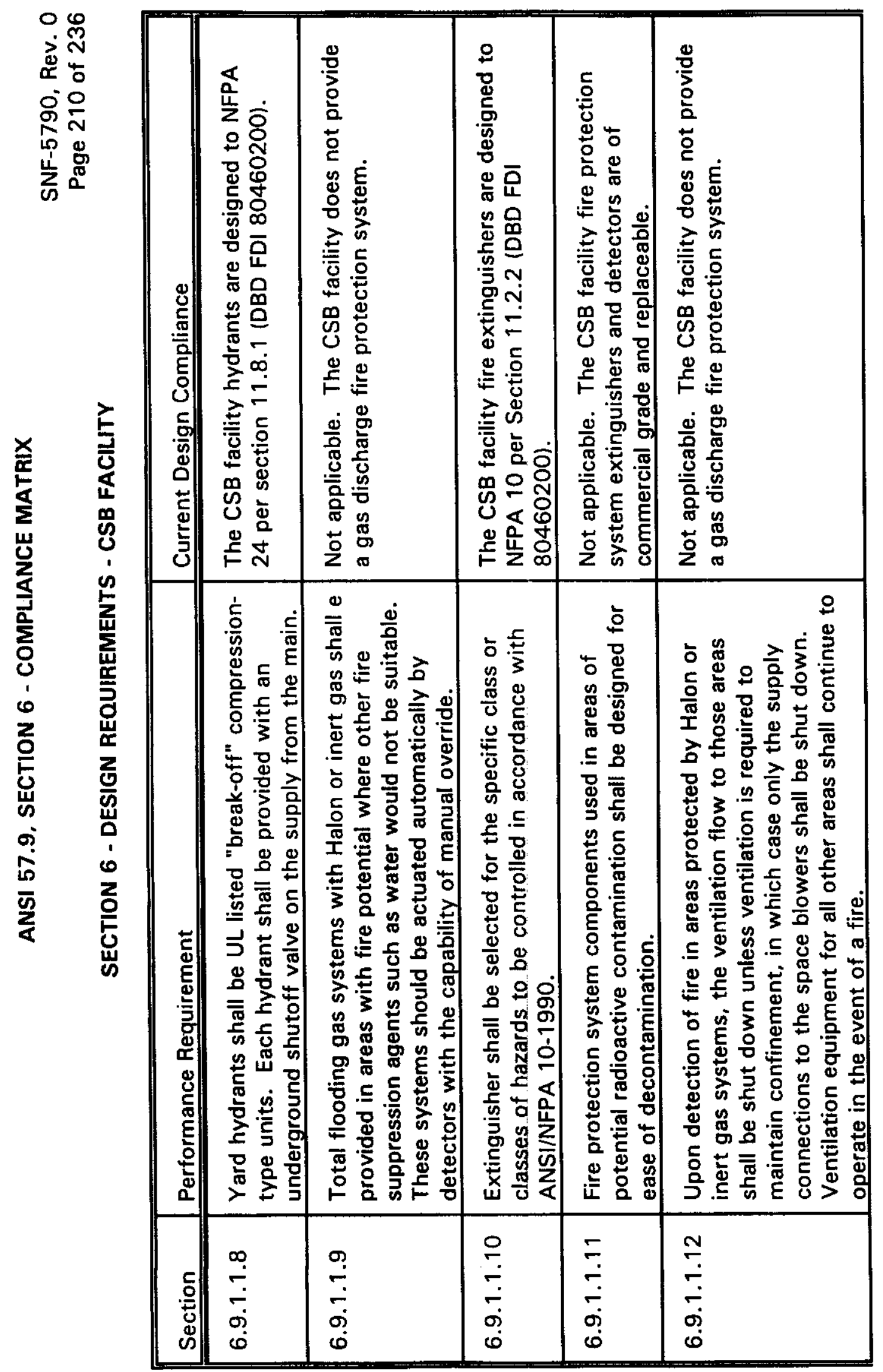




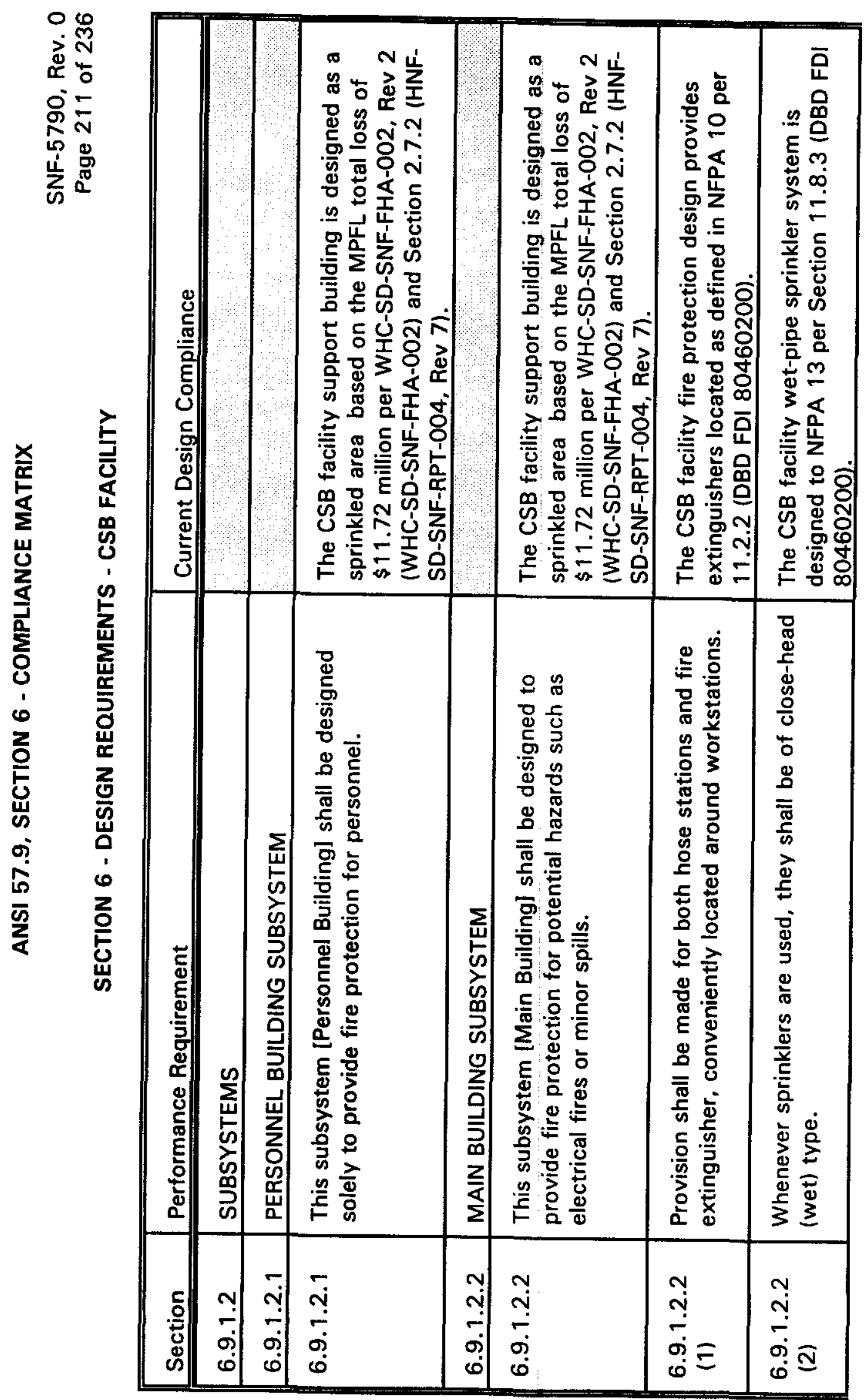




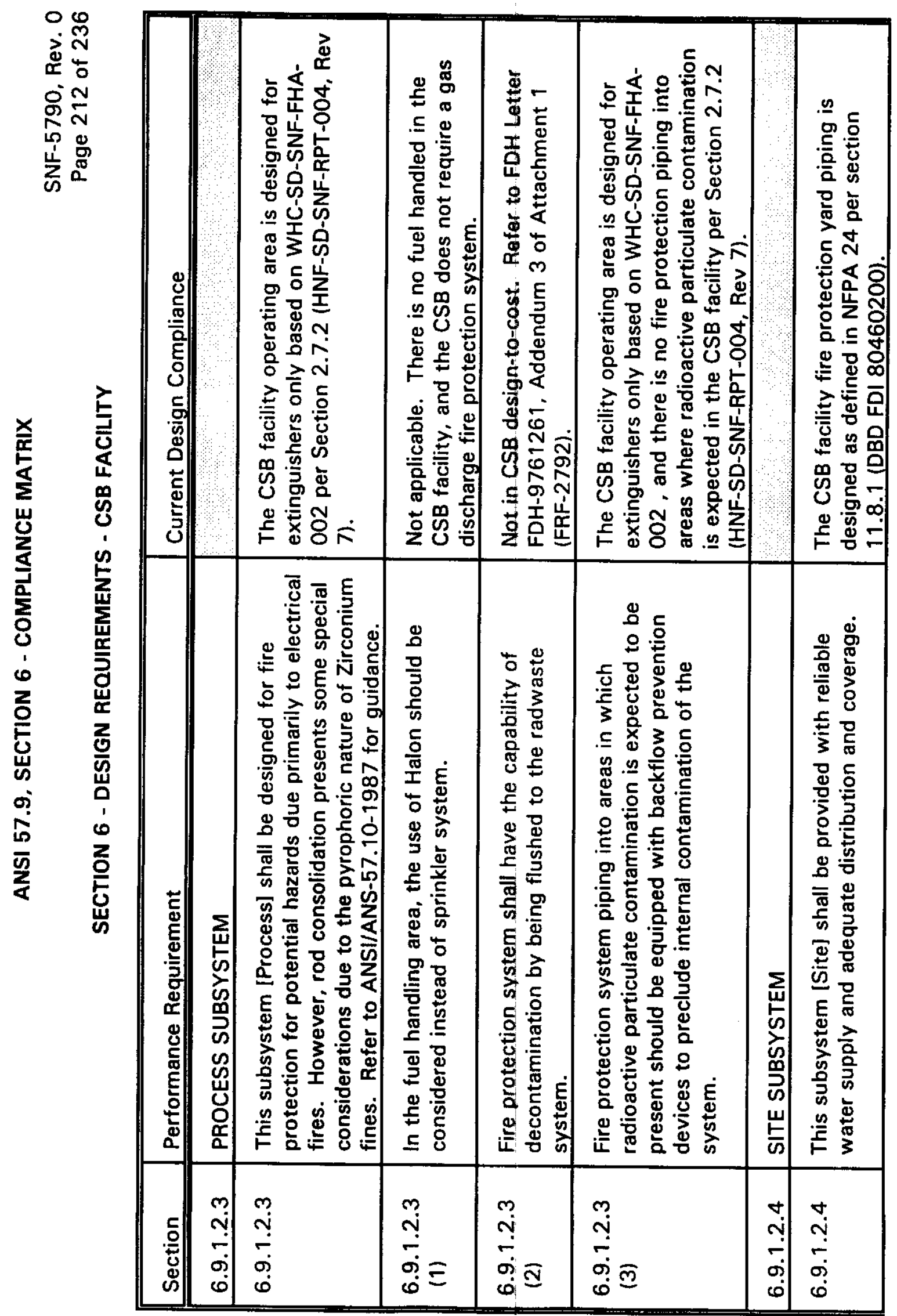




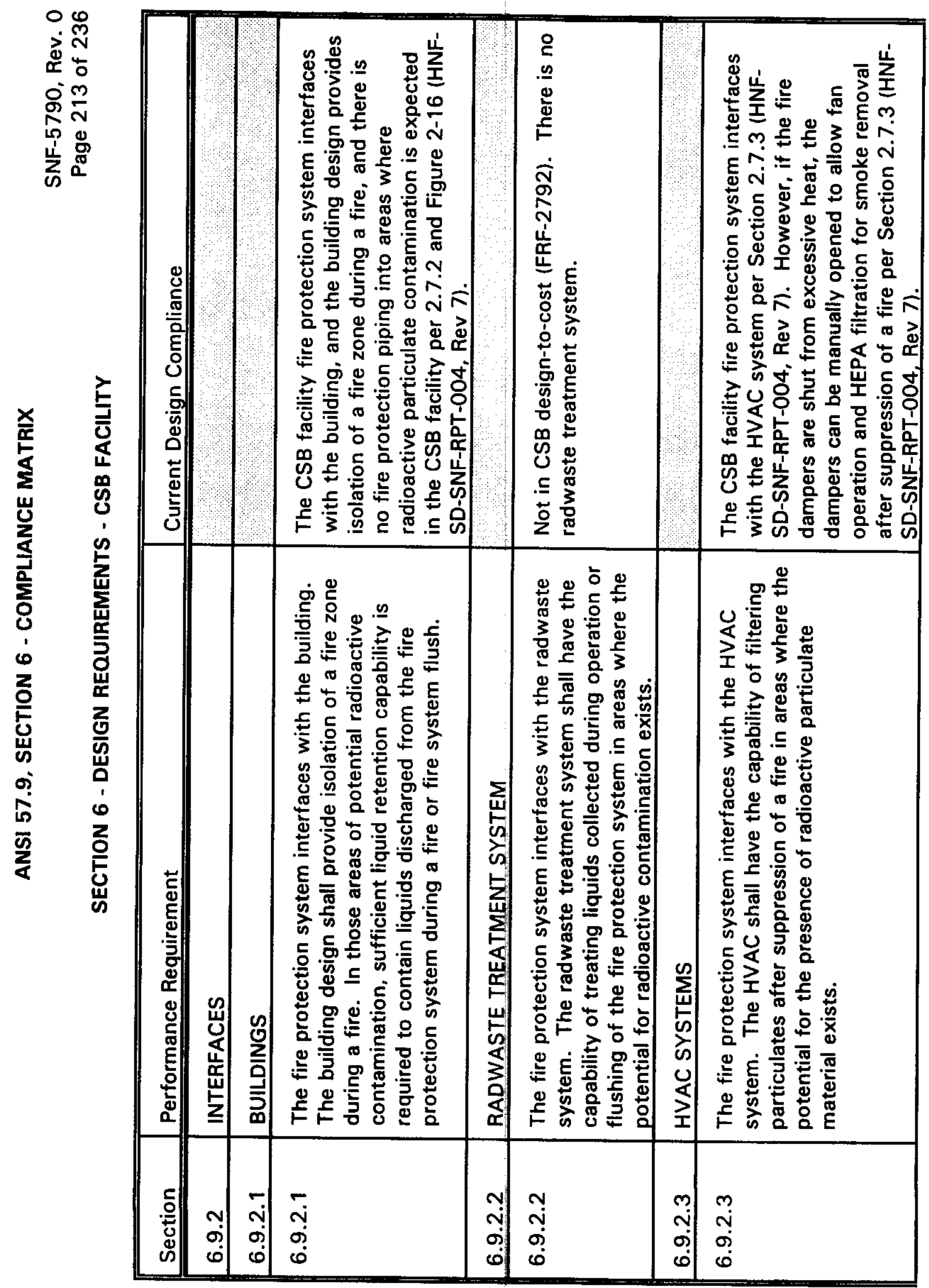




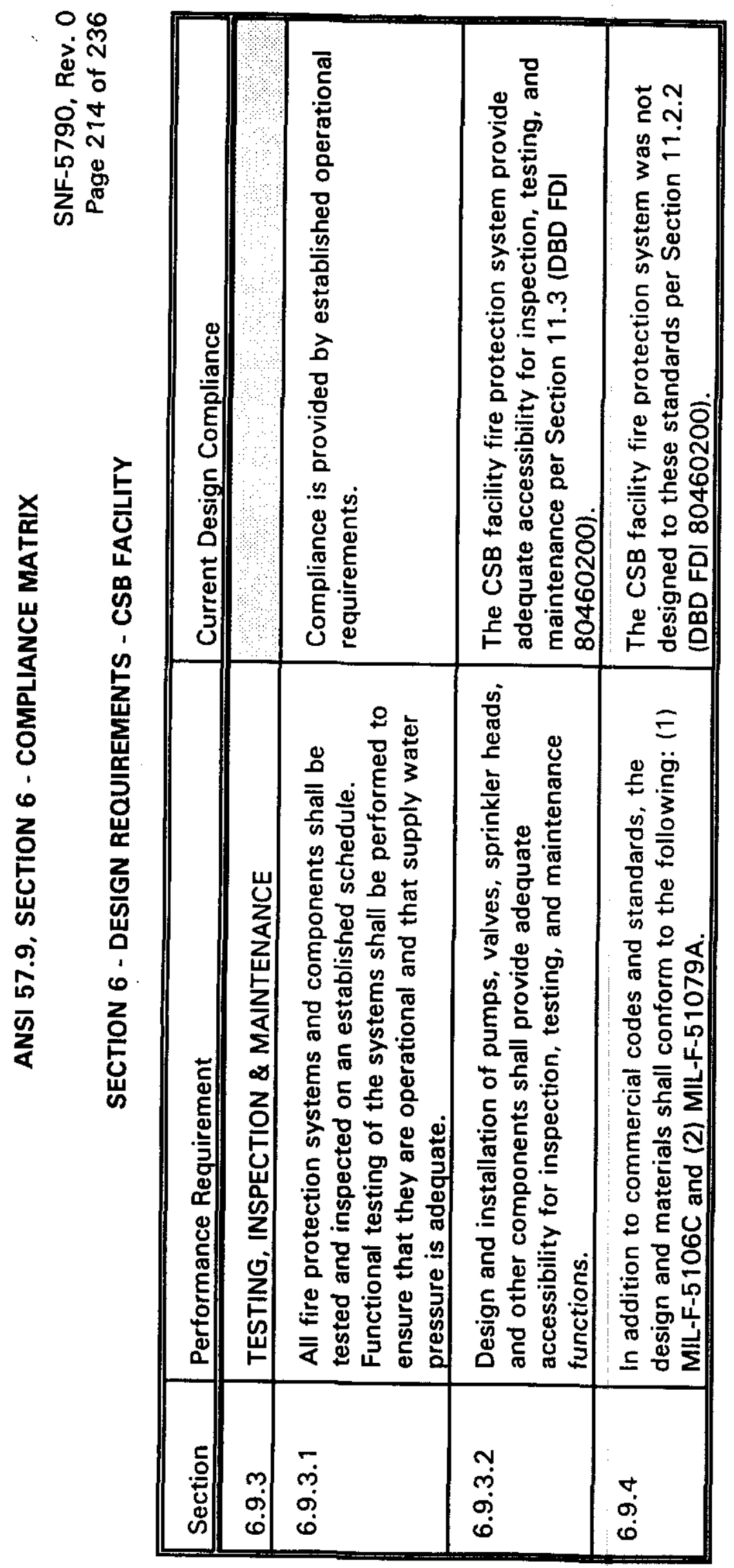




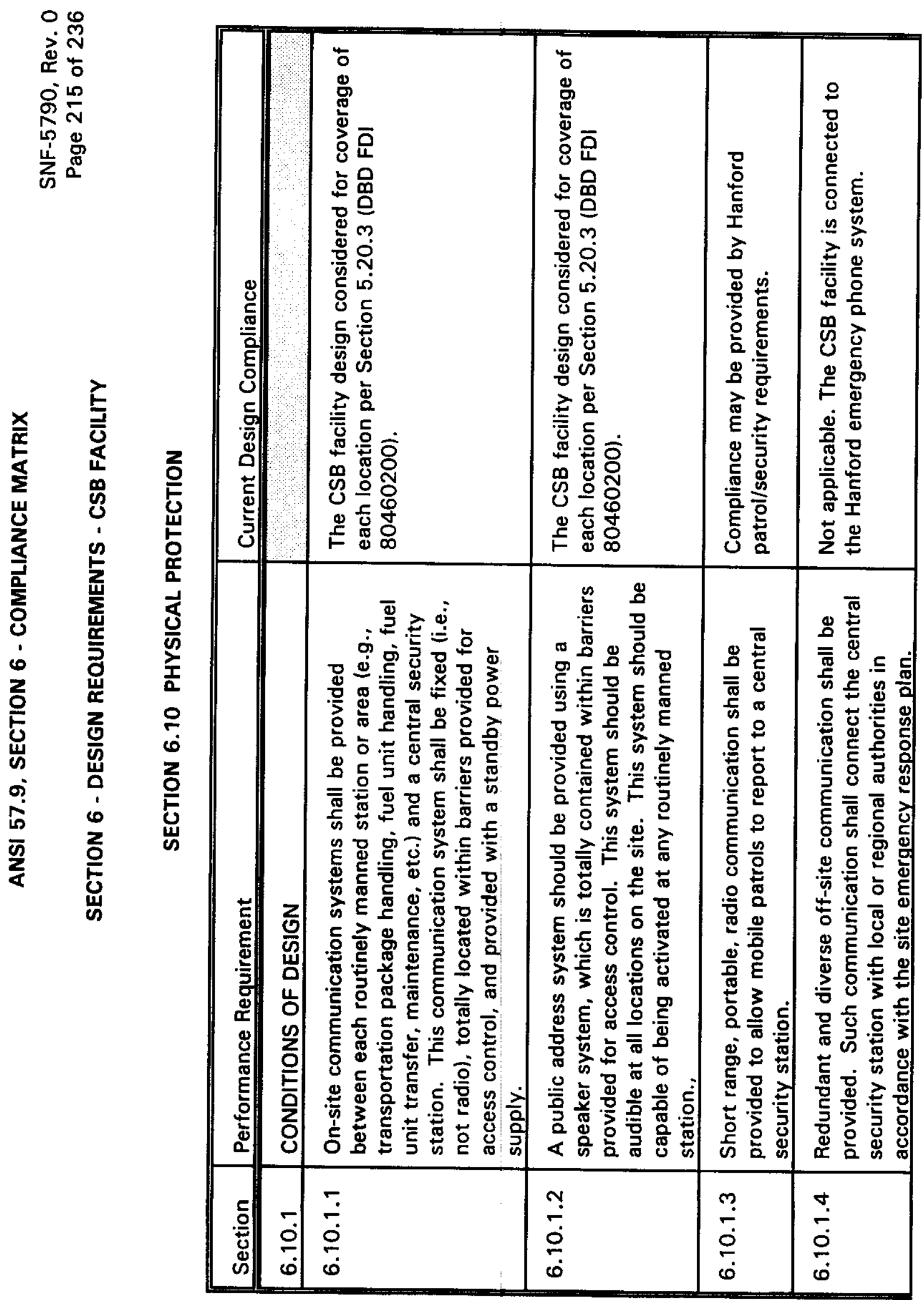




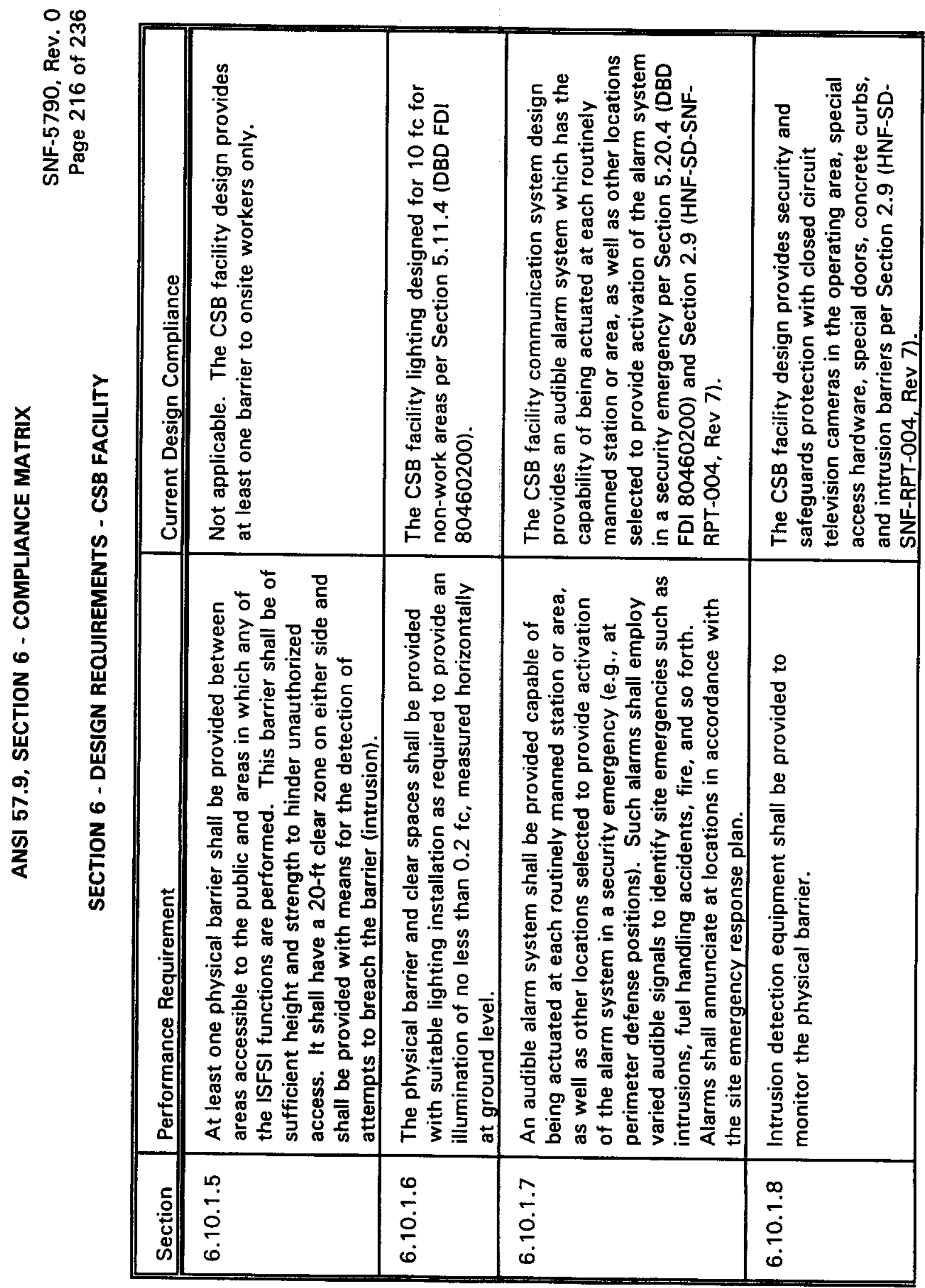




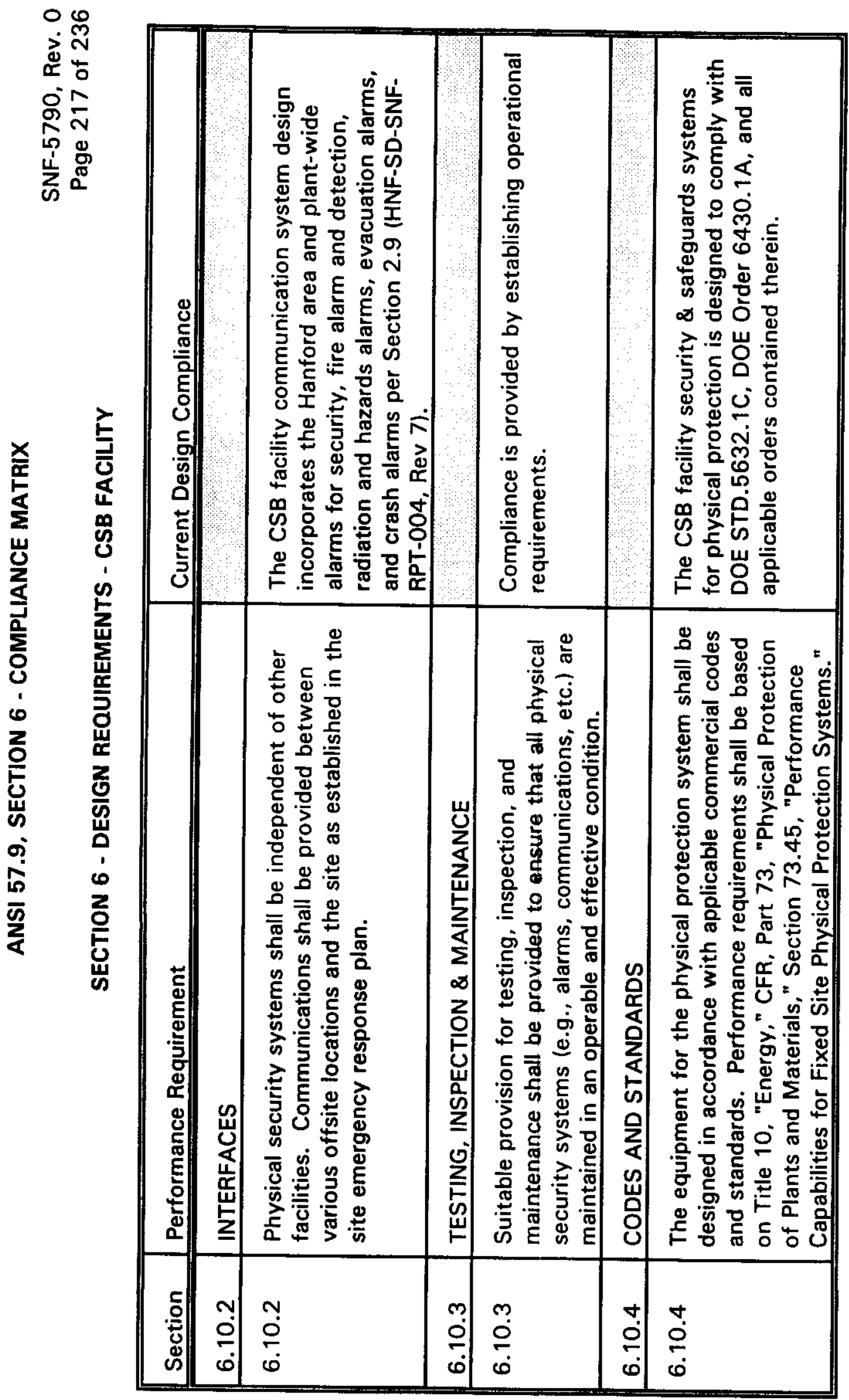




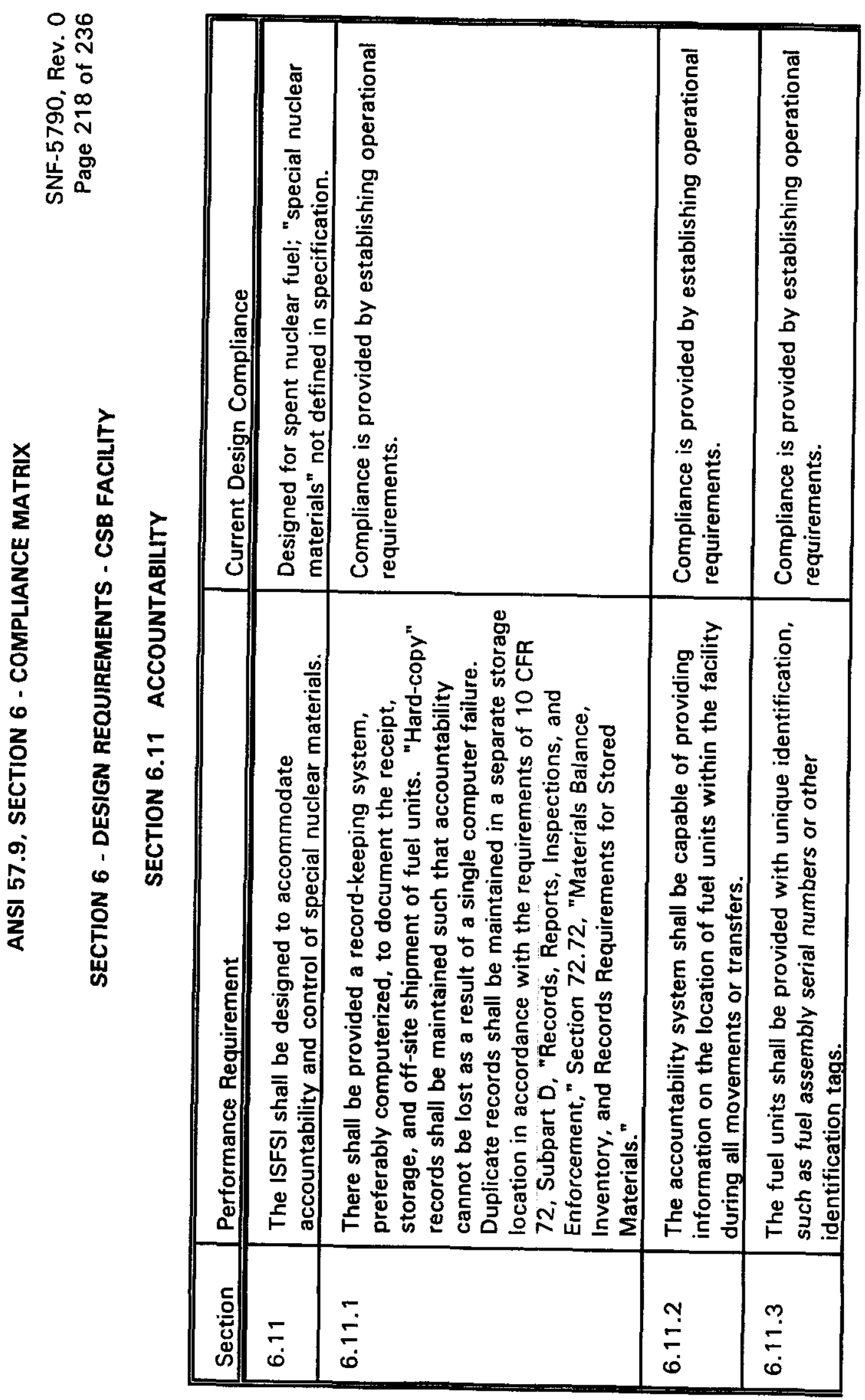




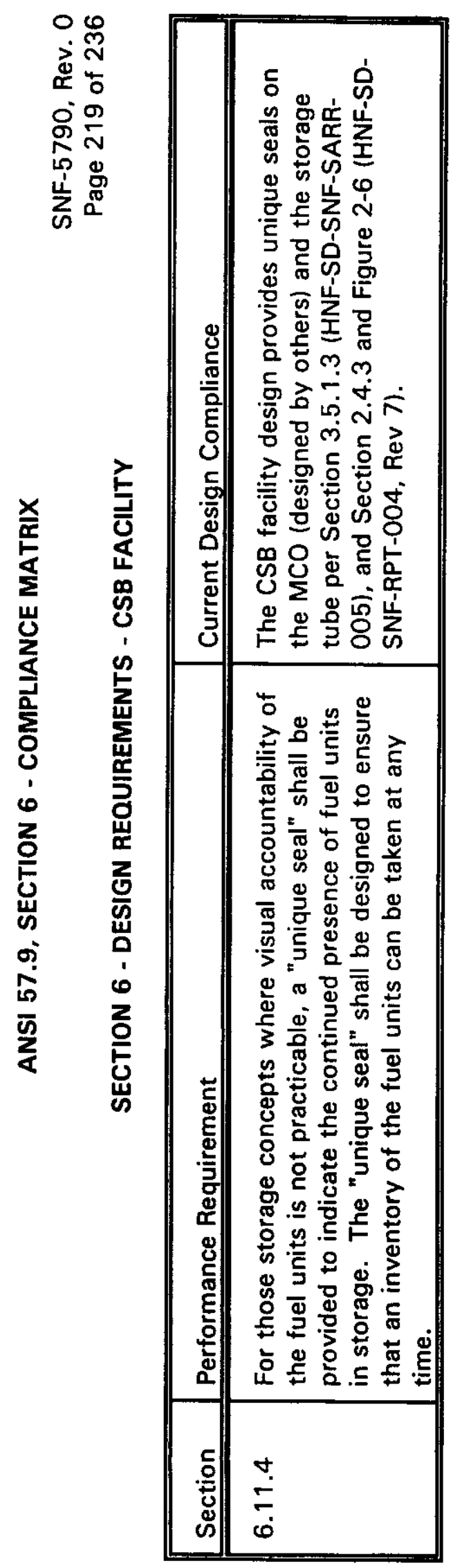




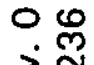 \\ 这金 \\ 윳ํํ \\ นิ \\ 份}

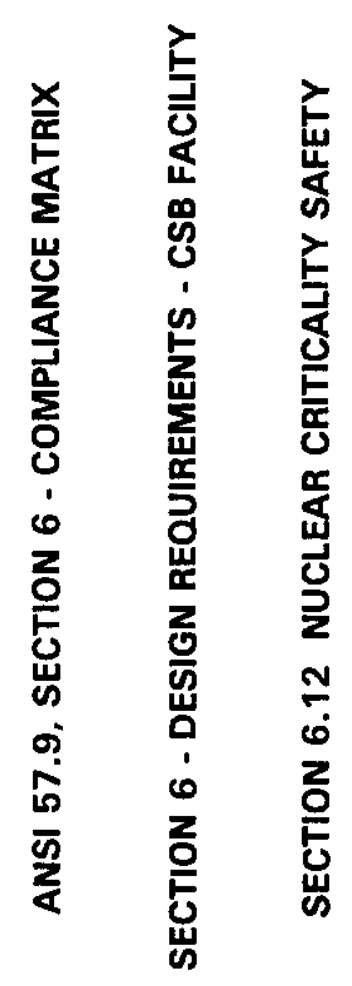

\begin{tabular}{|c|c|c|c|c|}
\hline 0 & & 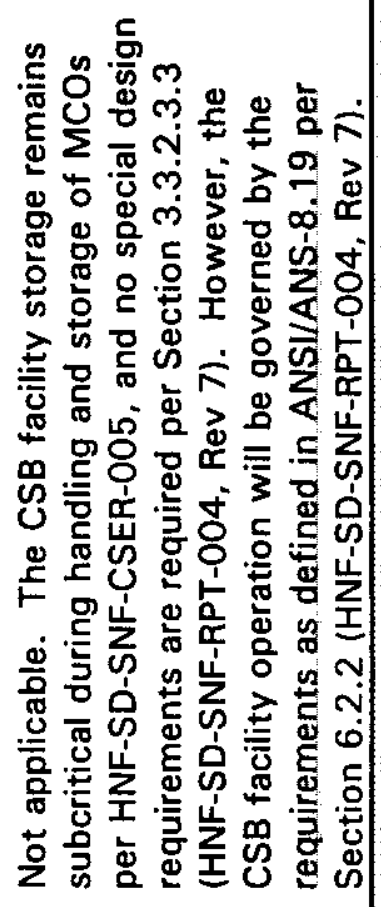 & & 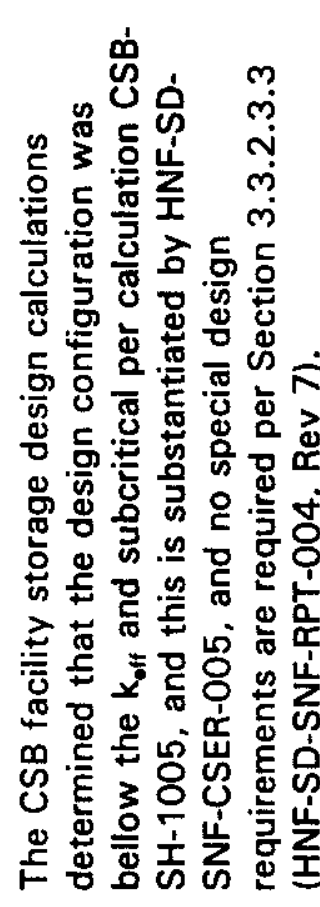 \\
\hline 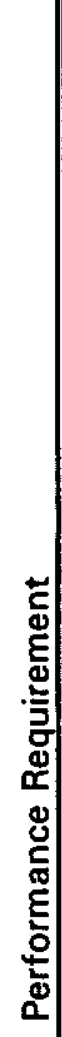 & 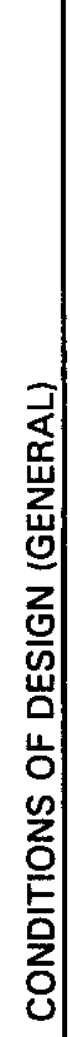 & 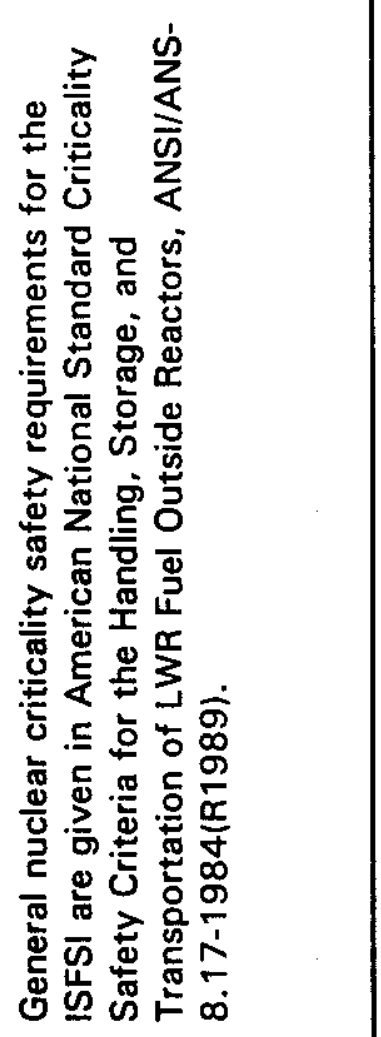 & 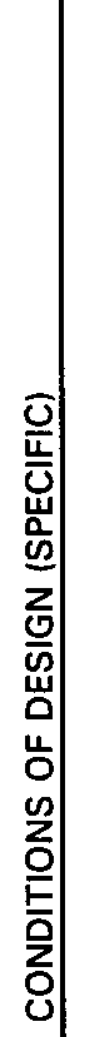 & 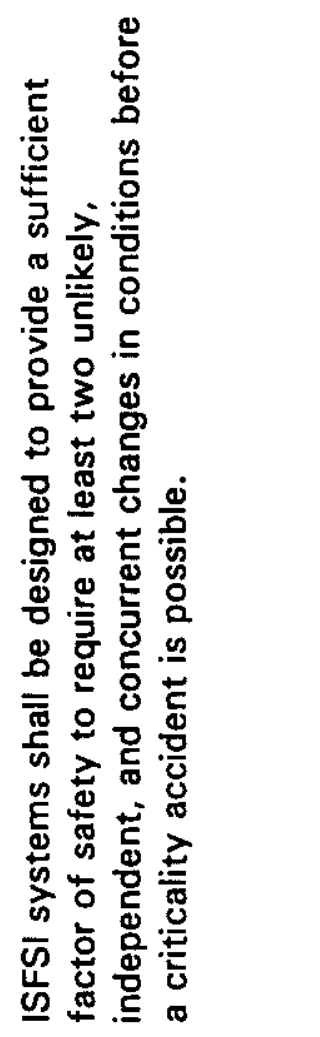 \\
\hline & $\bar{c}$ & $\frac{\bar{i}}{\grave{0}}$ & $\begin{array}{l}\stackrel{N}{*} \\
\stackrel{1}{0}\end{array}$ & 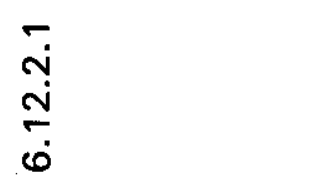 \\
\hline
\end{tabular}




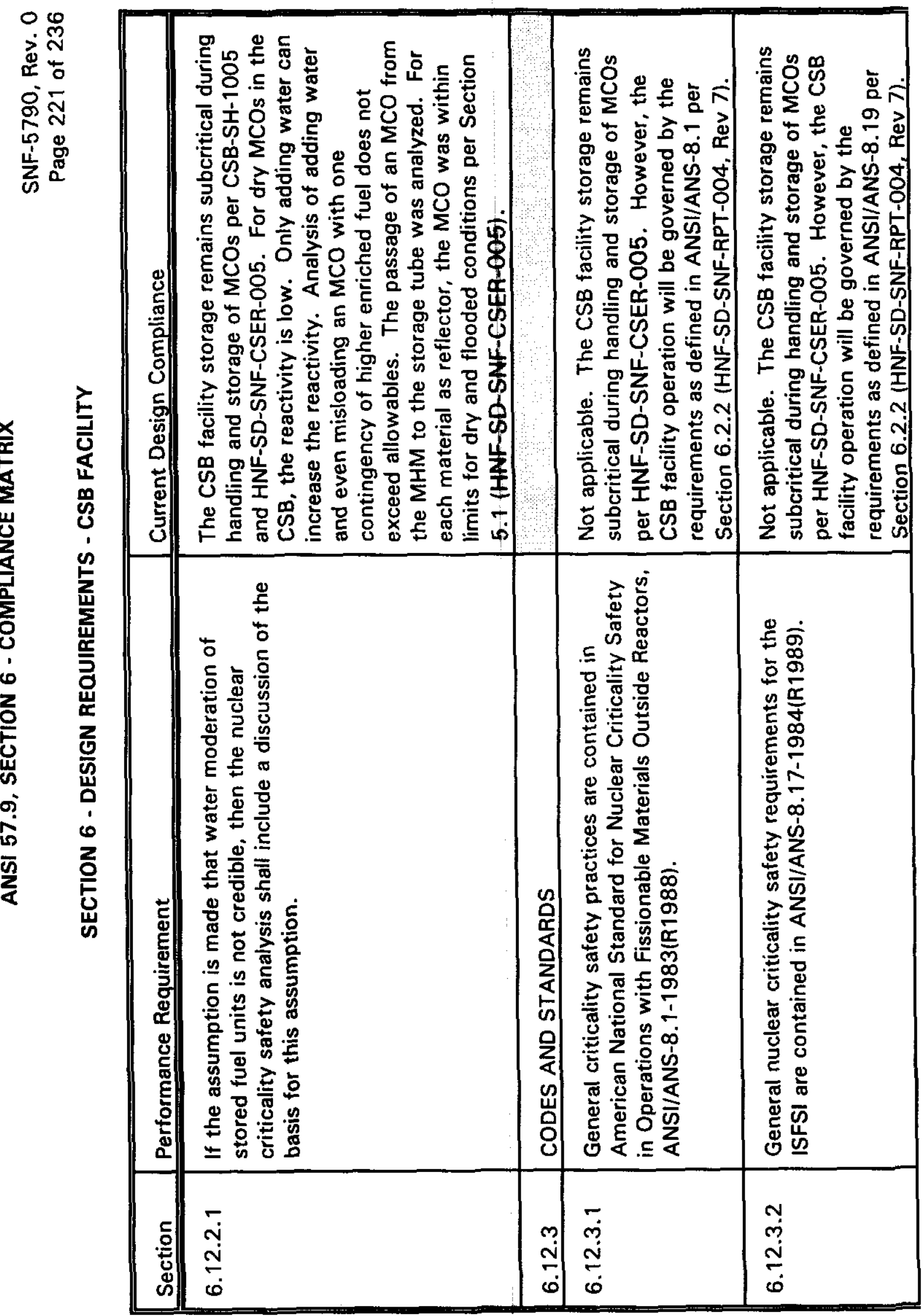




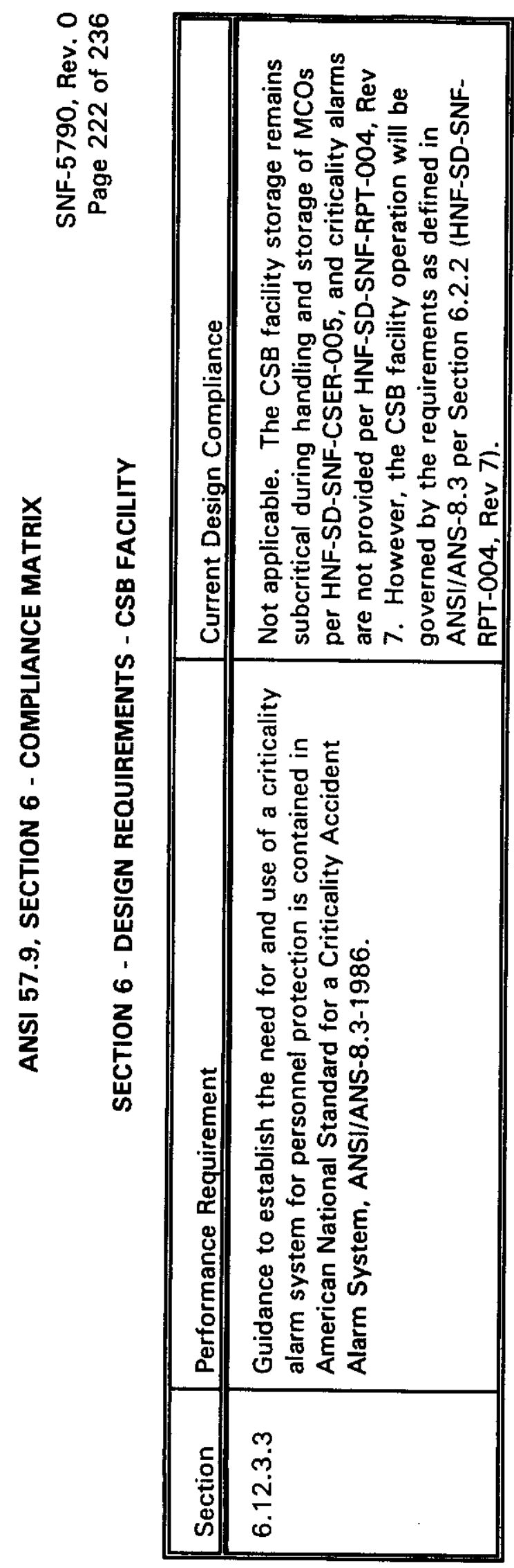




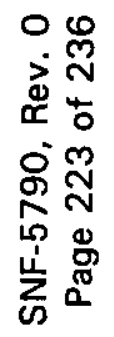

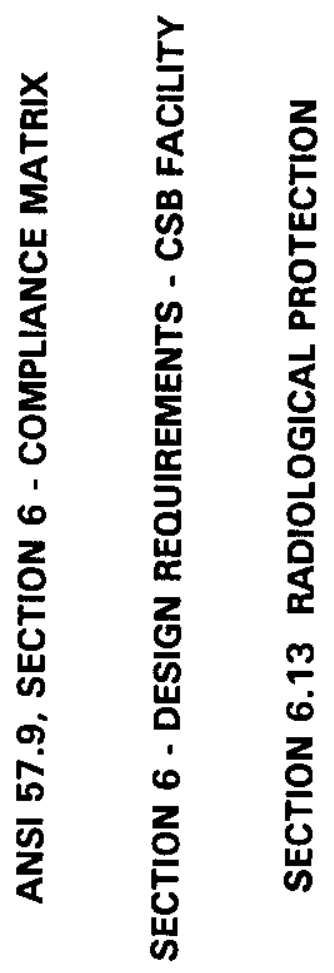

\begin{tabular}{|c|c|c|c|c|}
\hline 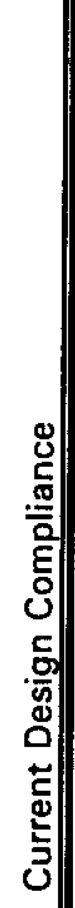 & 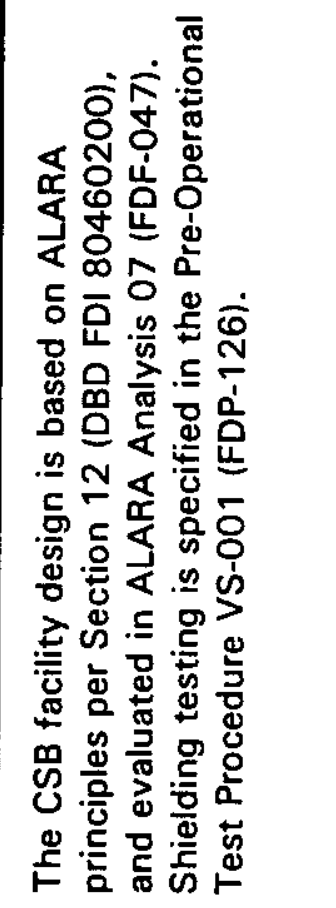 & 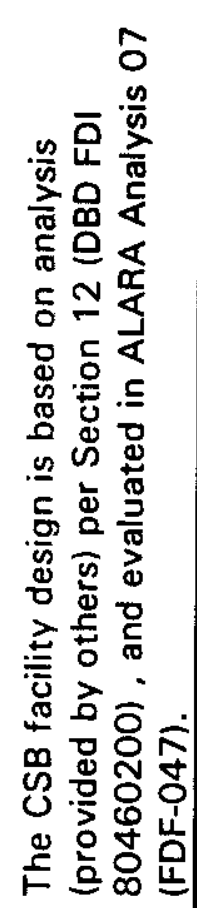 & 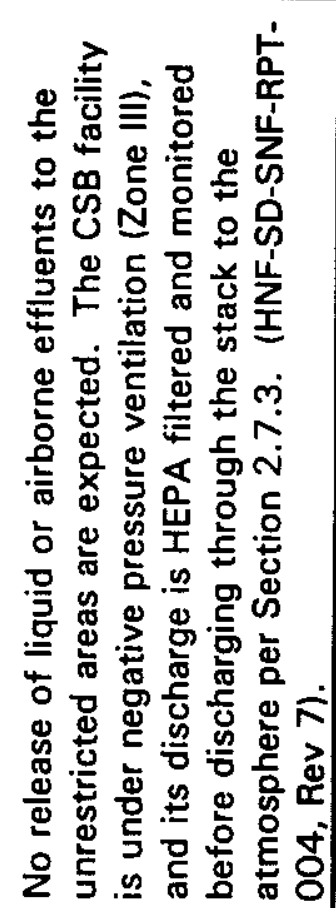 & 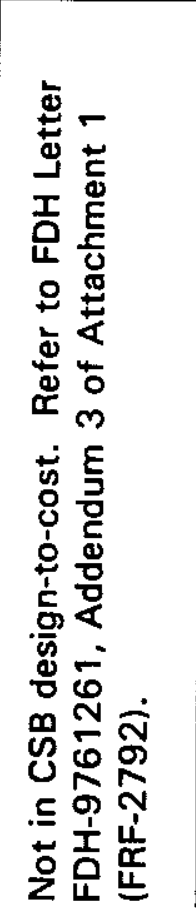 \\
\hline 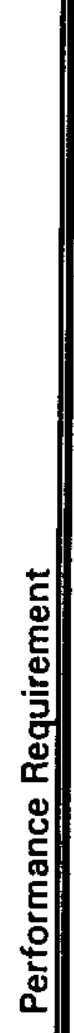 & 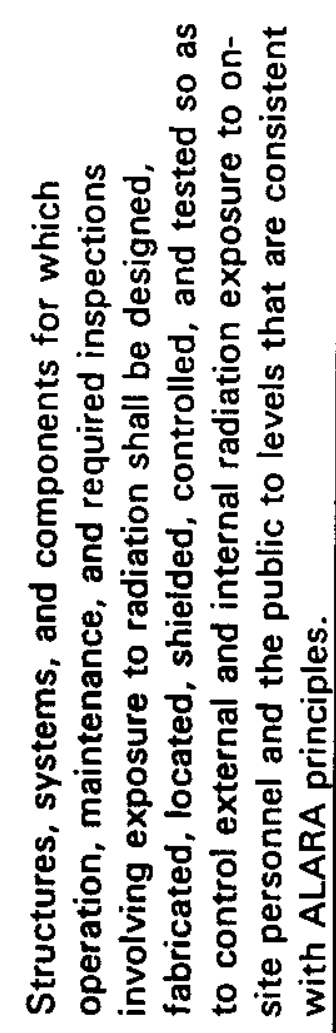 & 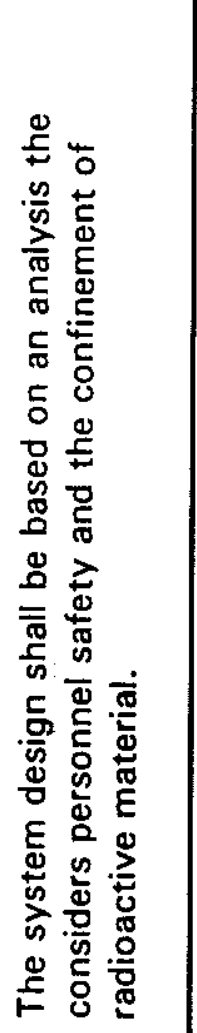 & 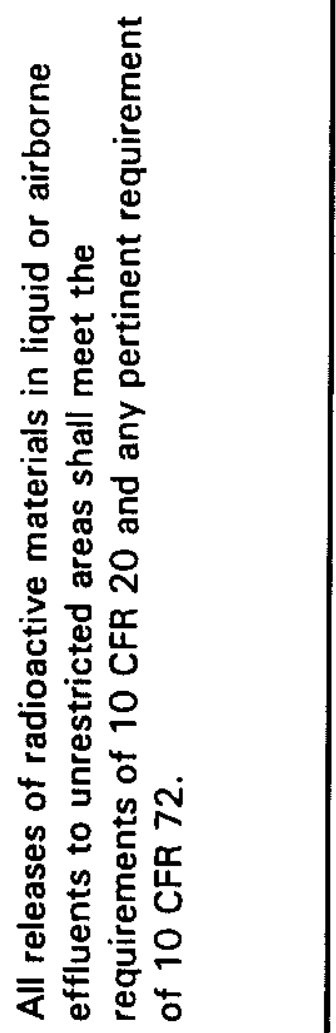 & 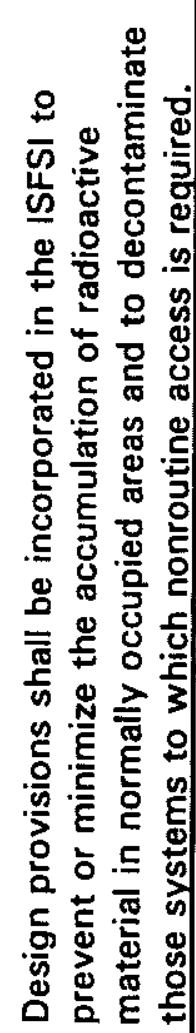 \\
\hline $\begin{array}{c}5 \\
.0 \\
0 \\
\Delta \\
5\end{array}$ & $\frac{\dot{m}}{\dot{\omega}}$ & $\frac{\underset{n}{n}}{\dot{\varphi}}$ & $\frac{\ddot{m}}{\dot{m}}$ & $\frac{m}{6}$ \\
\hline
\end{tabular}




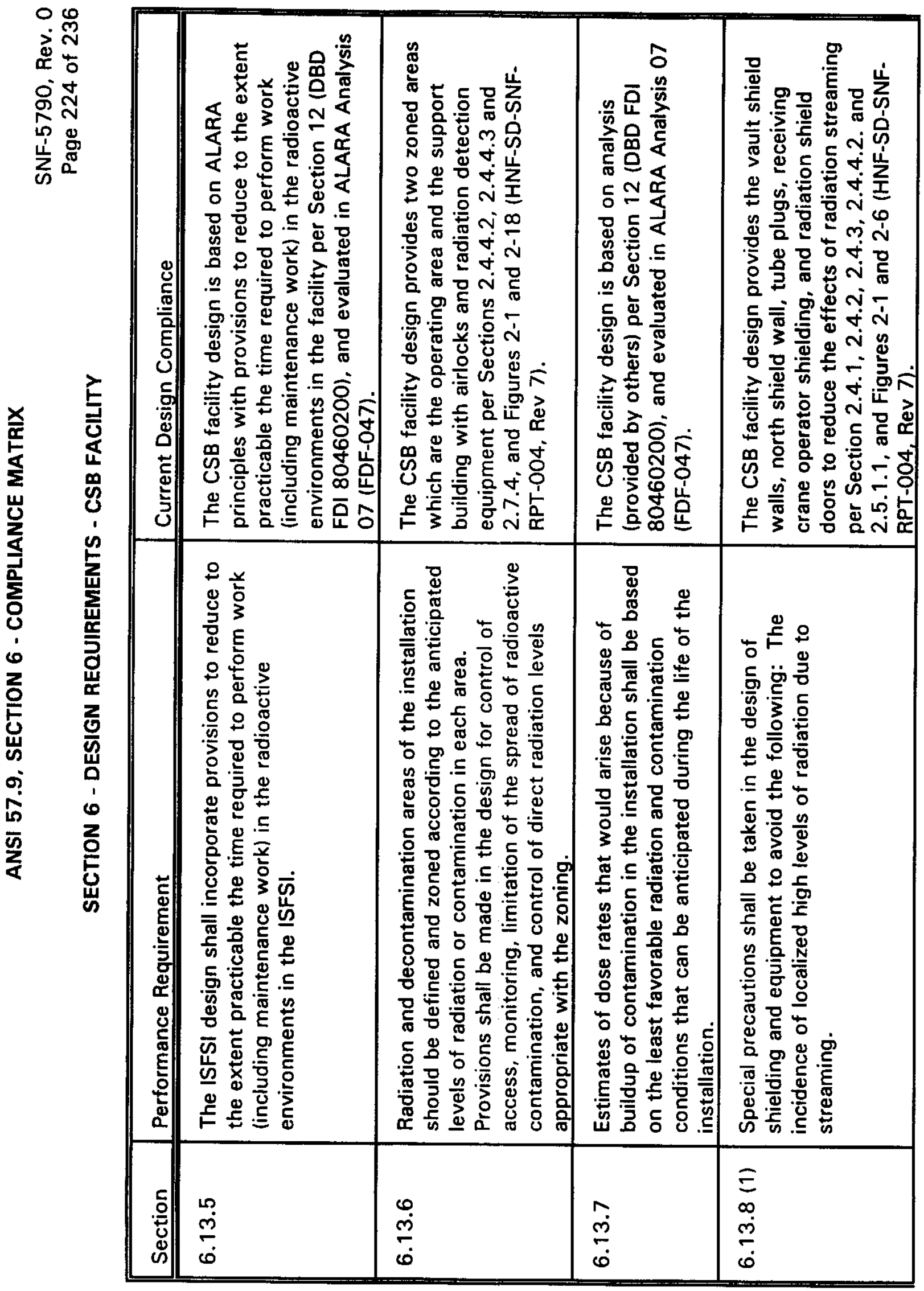




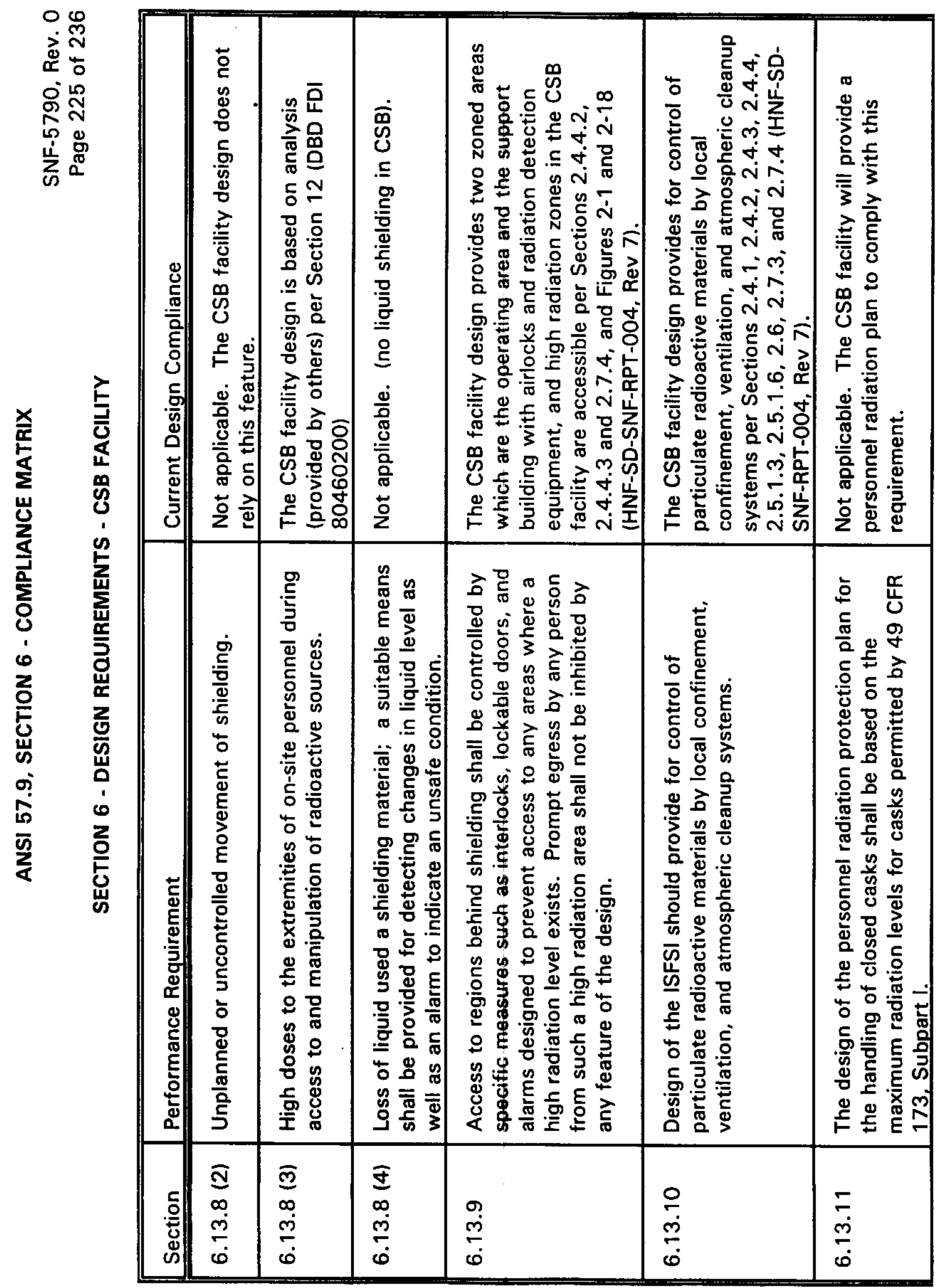




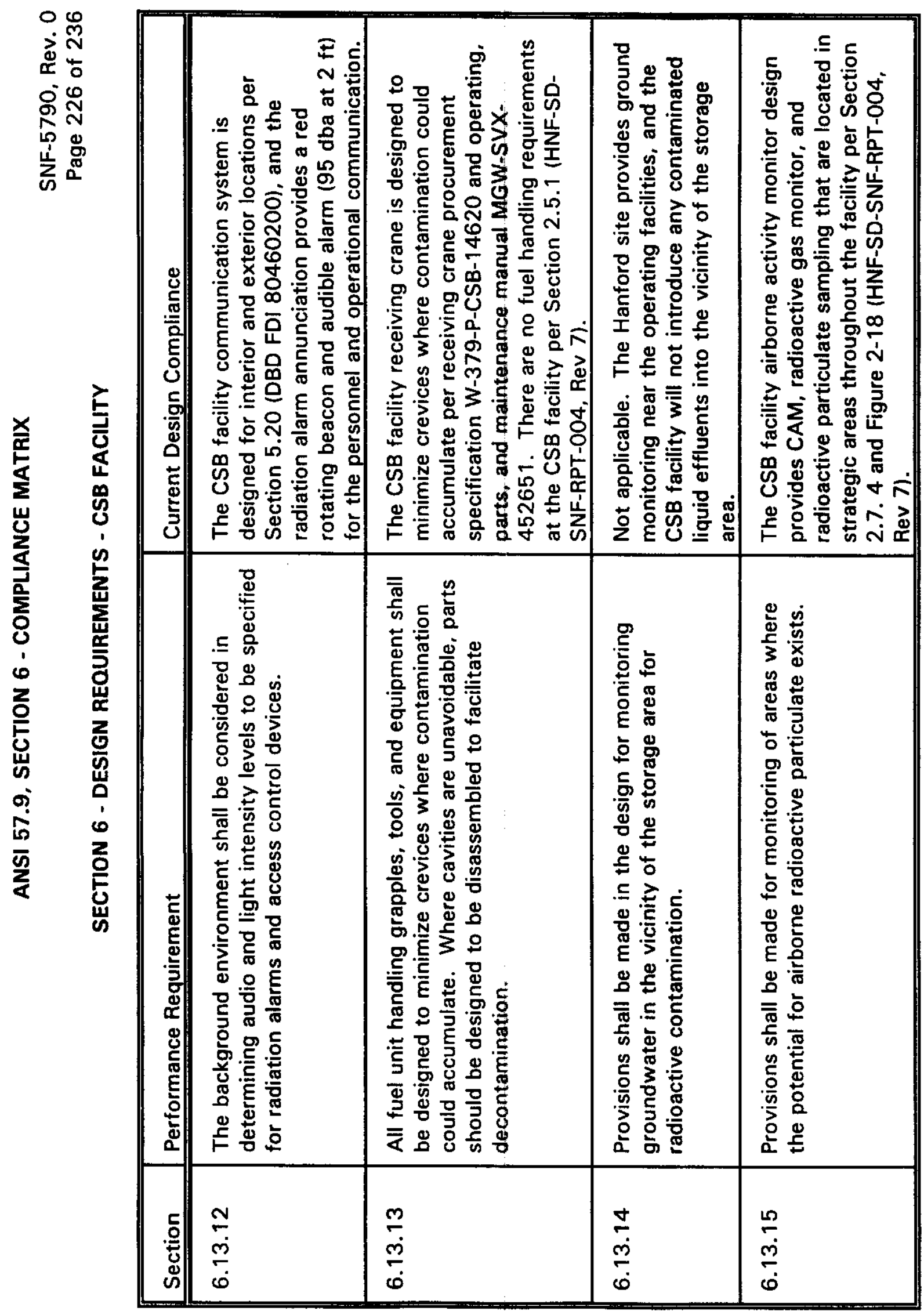




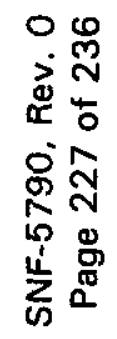

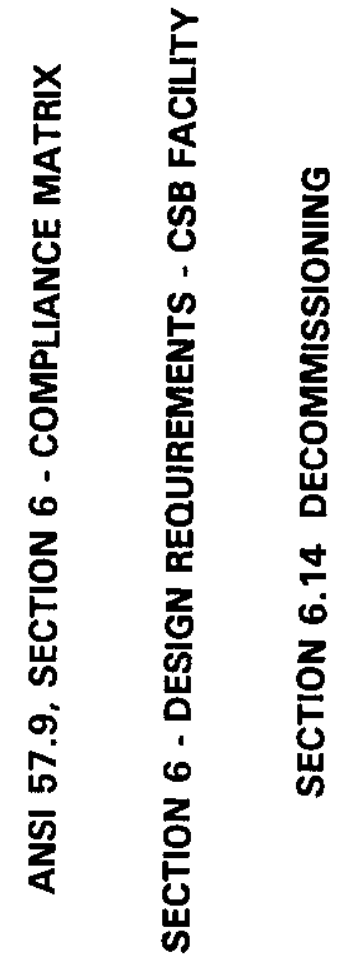

\begin{tabular}{|c|c|c|c|}
\hline 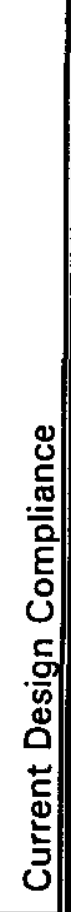 & 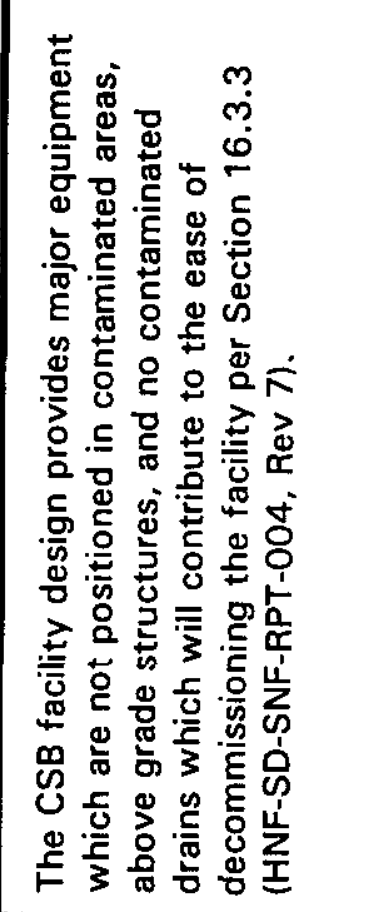 & 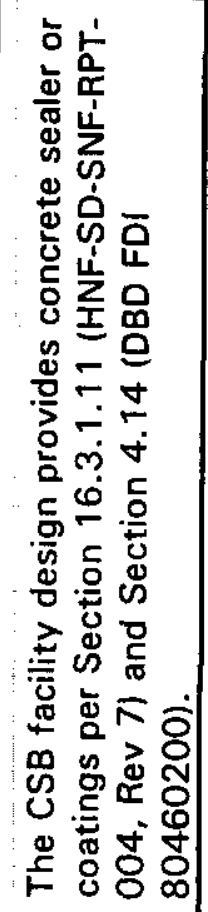 & 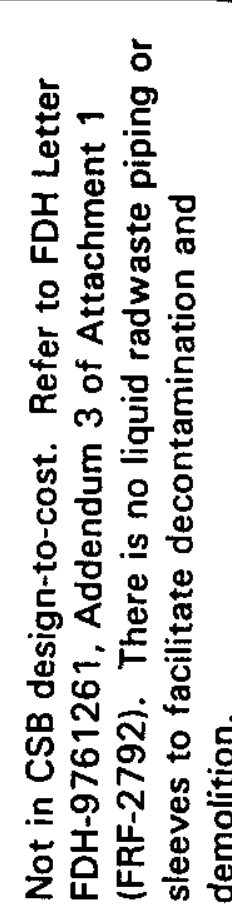 \\
\hline 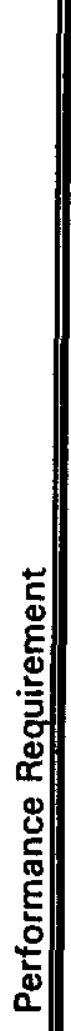 & 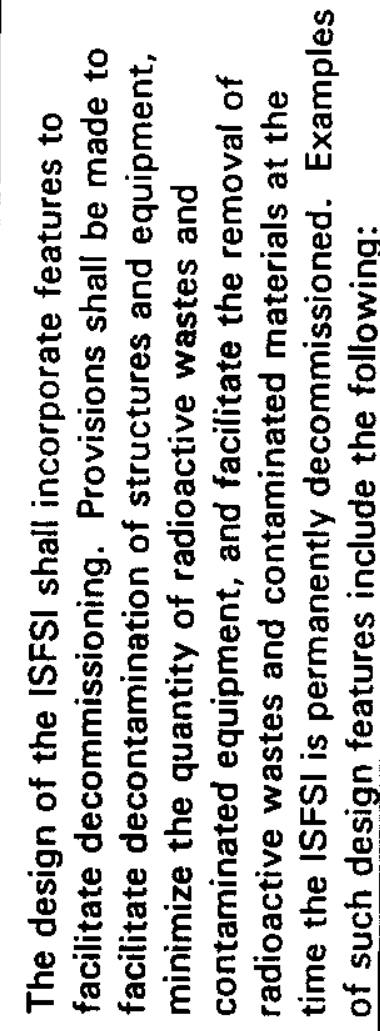 & 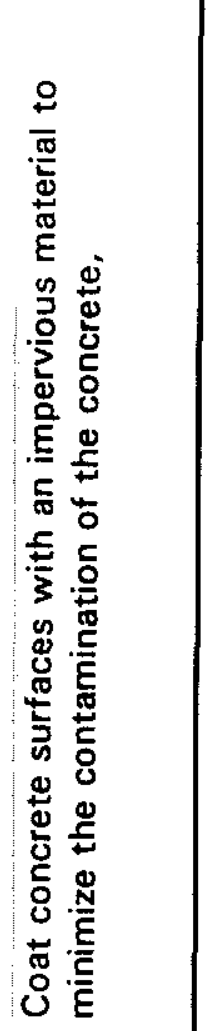 & 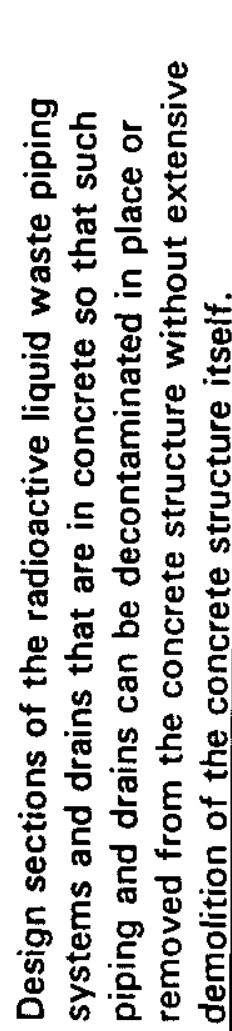 \\
\hline 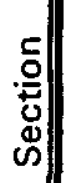 & & $\begin{array}{l}E \\
\dot{\sigma}\end{array}$ & $\begin{array}{l}\widetilde{\Xi} \\
\stackrel{\nabla}{\sigma}\end{array}$ \\
\hline
\end{tabular}




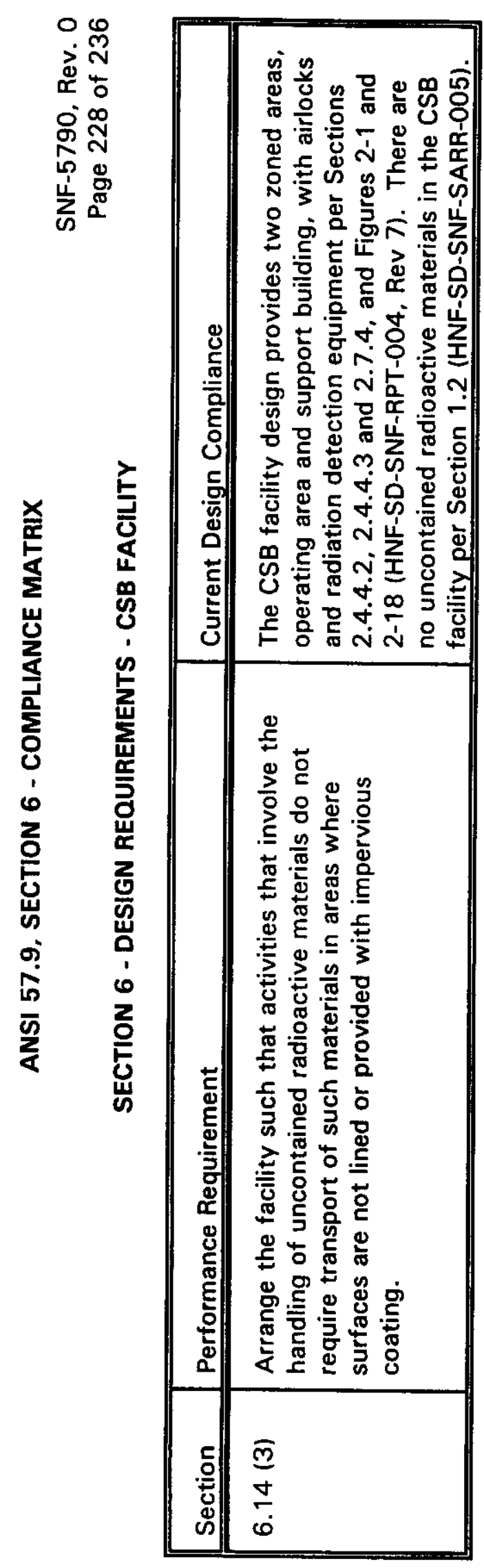




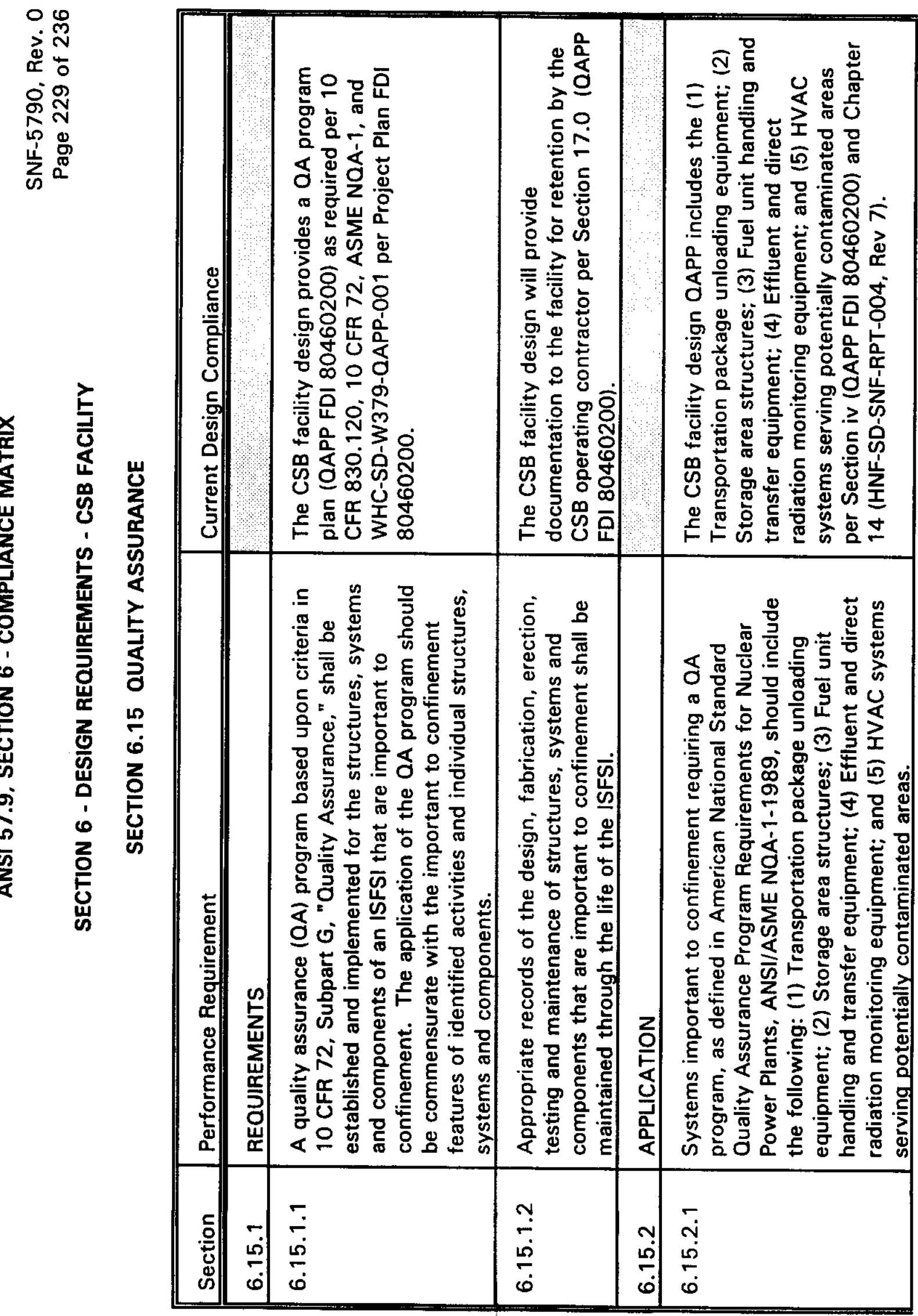




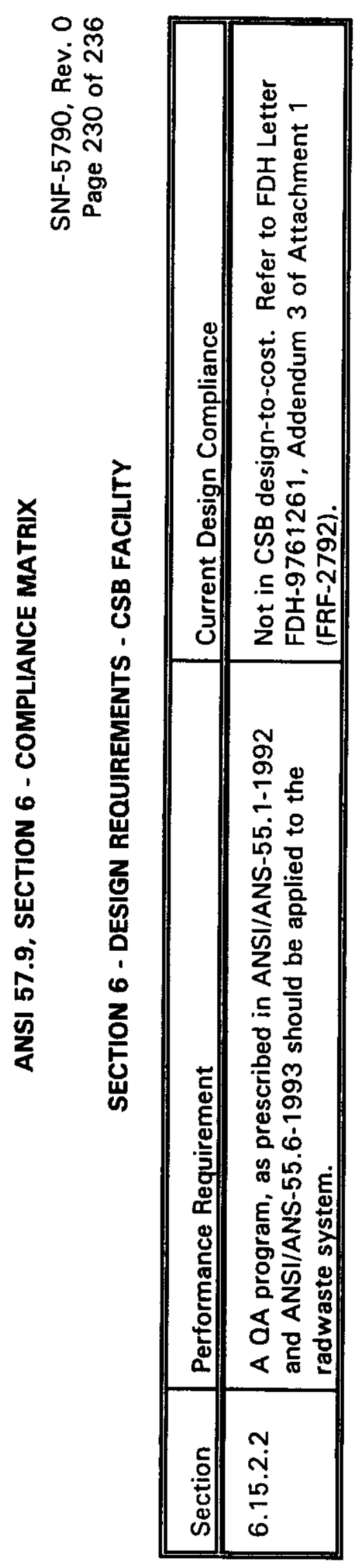




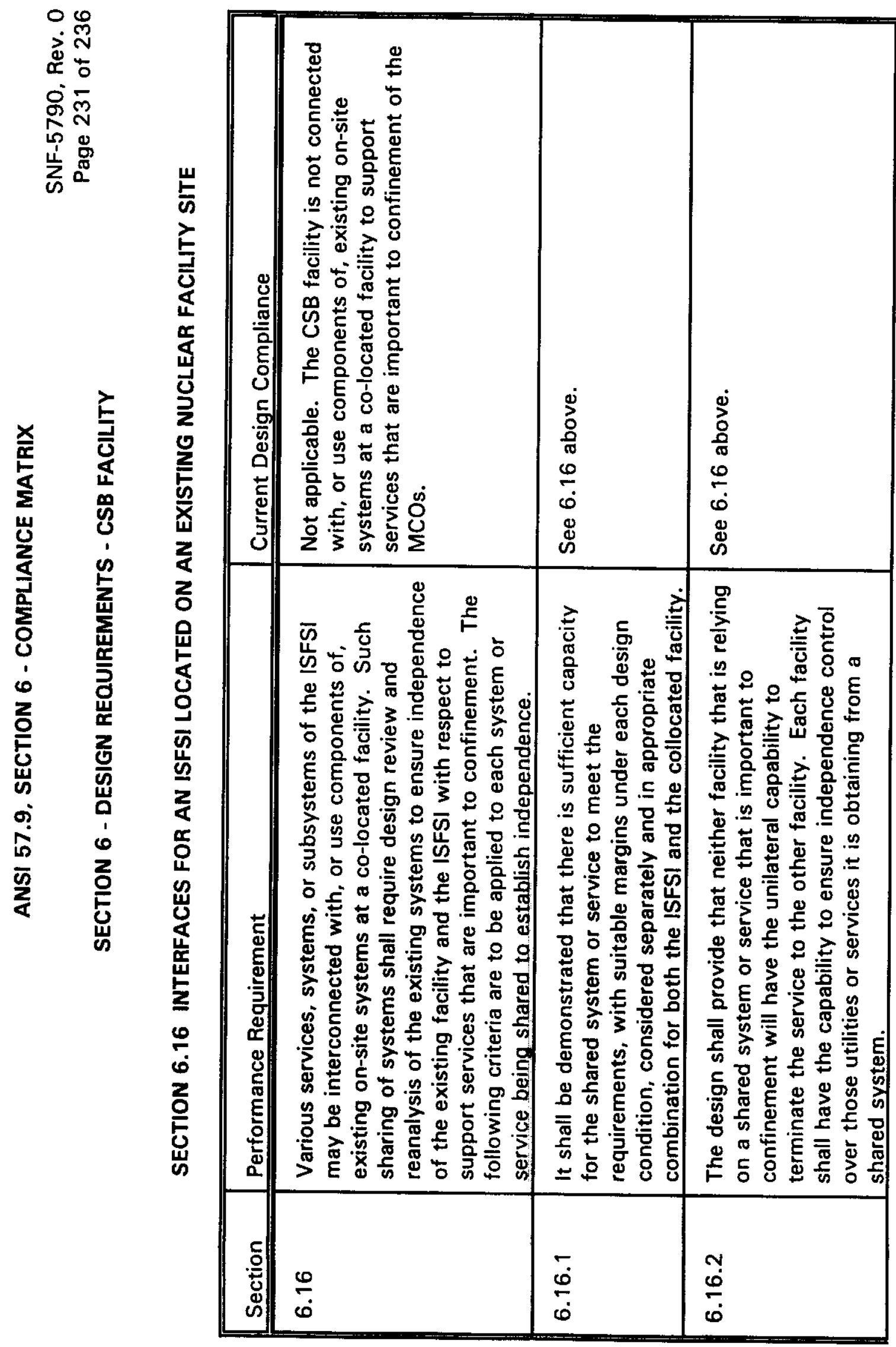




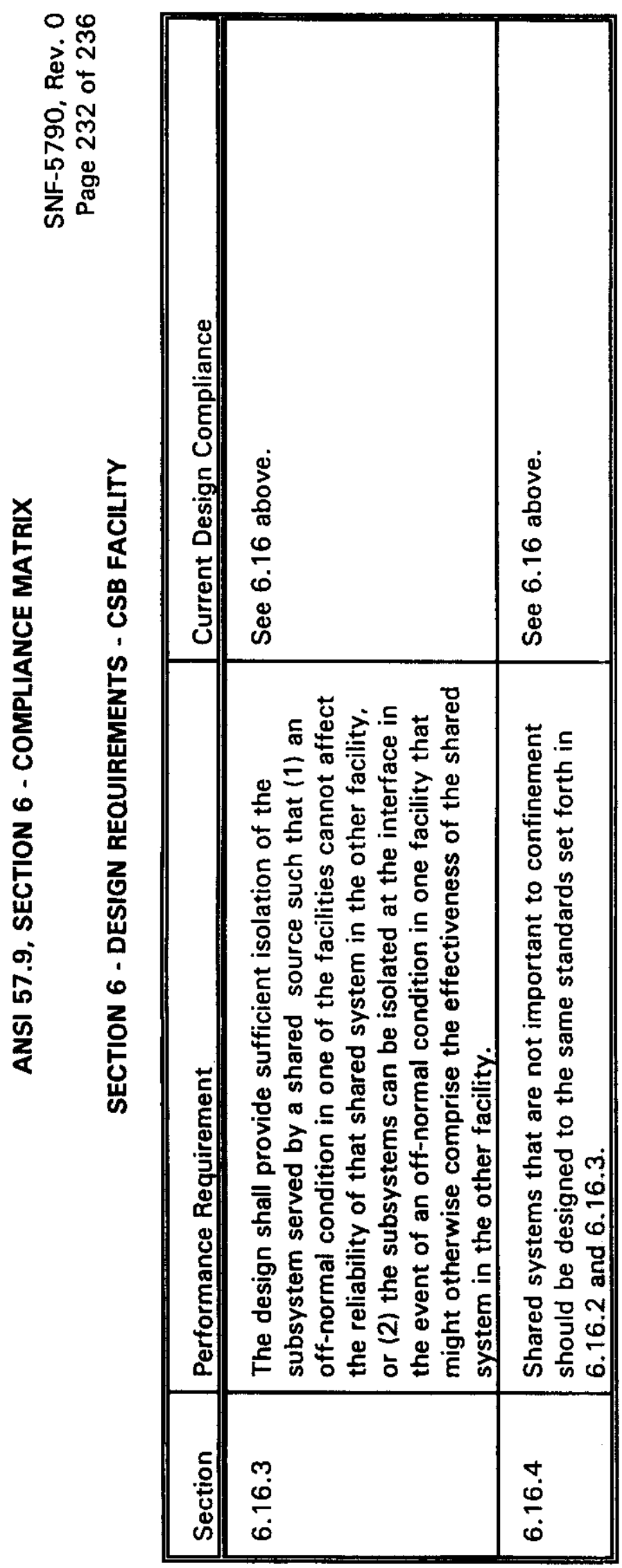




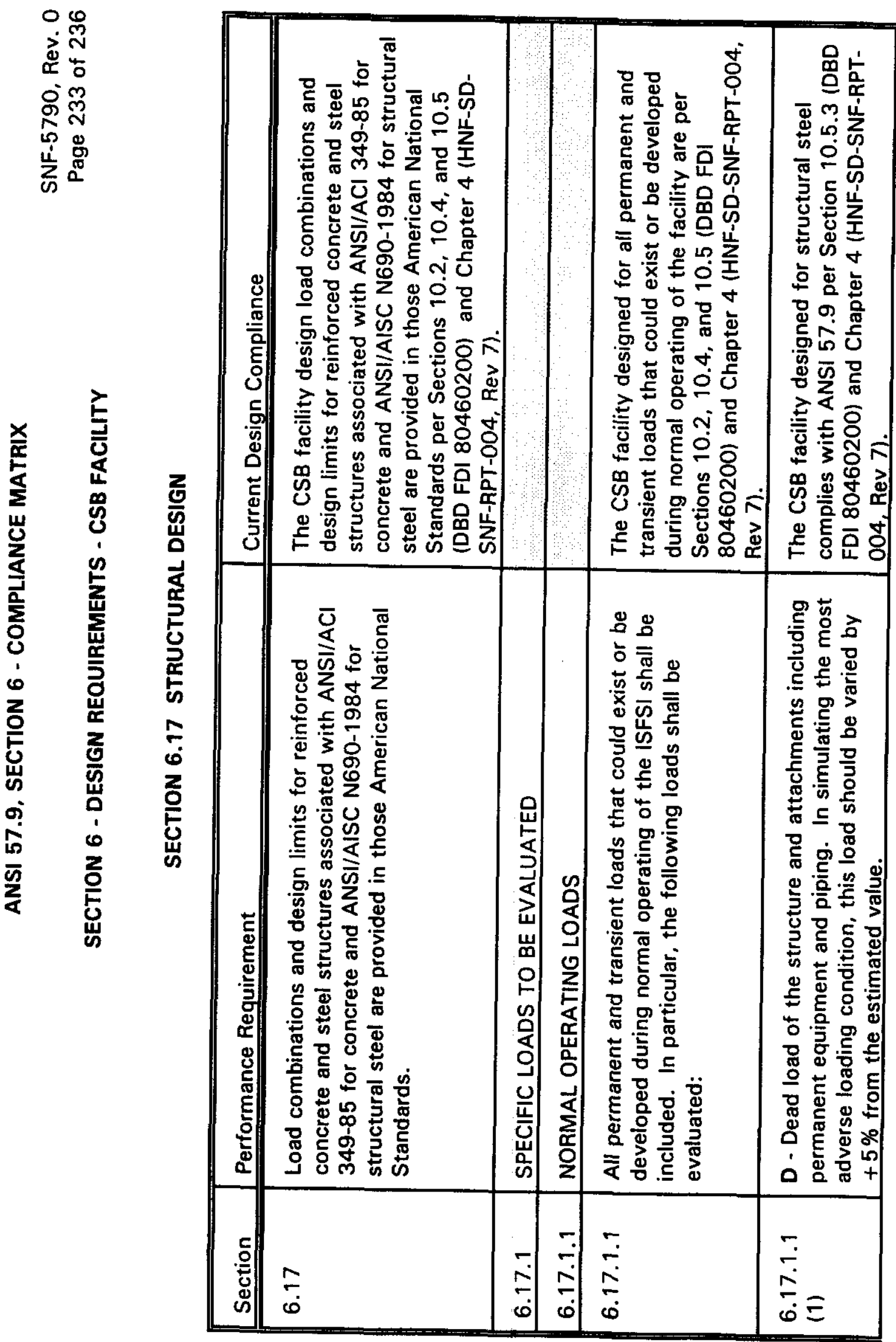




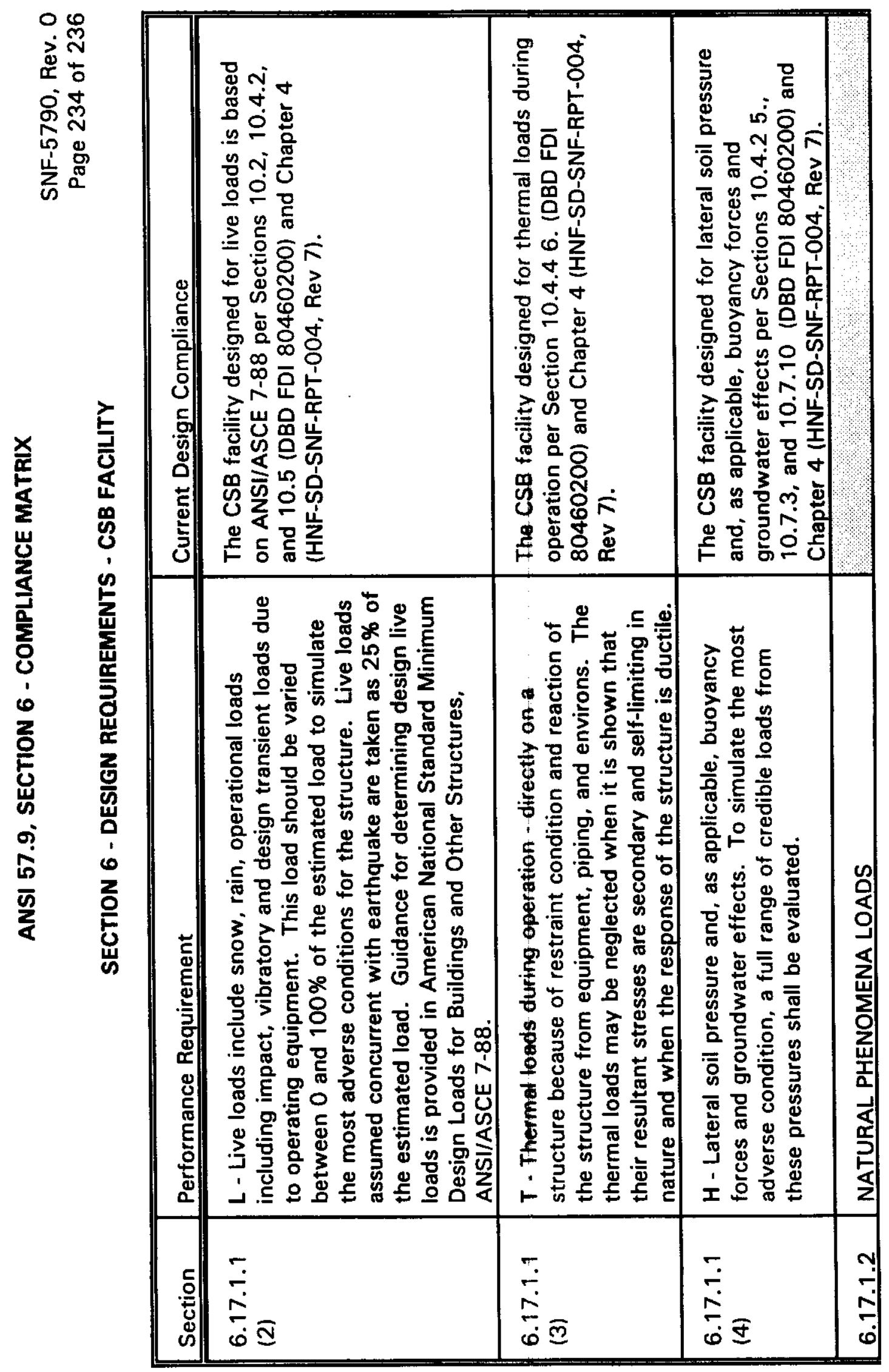




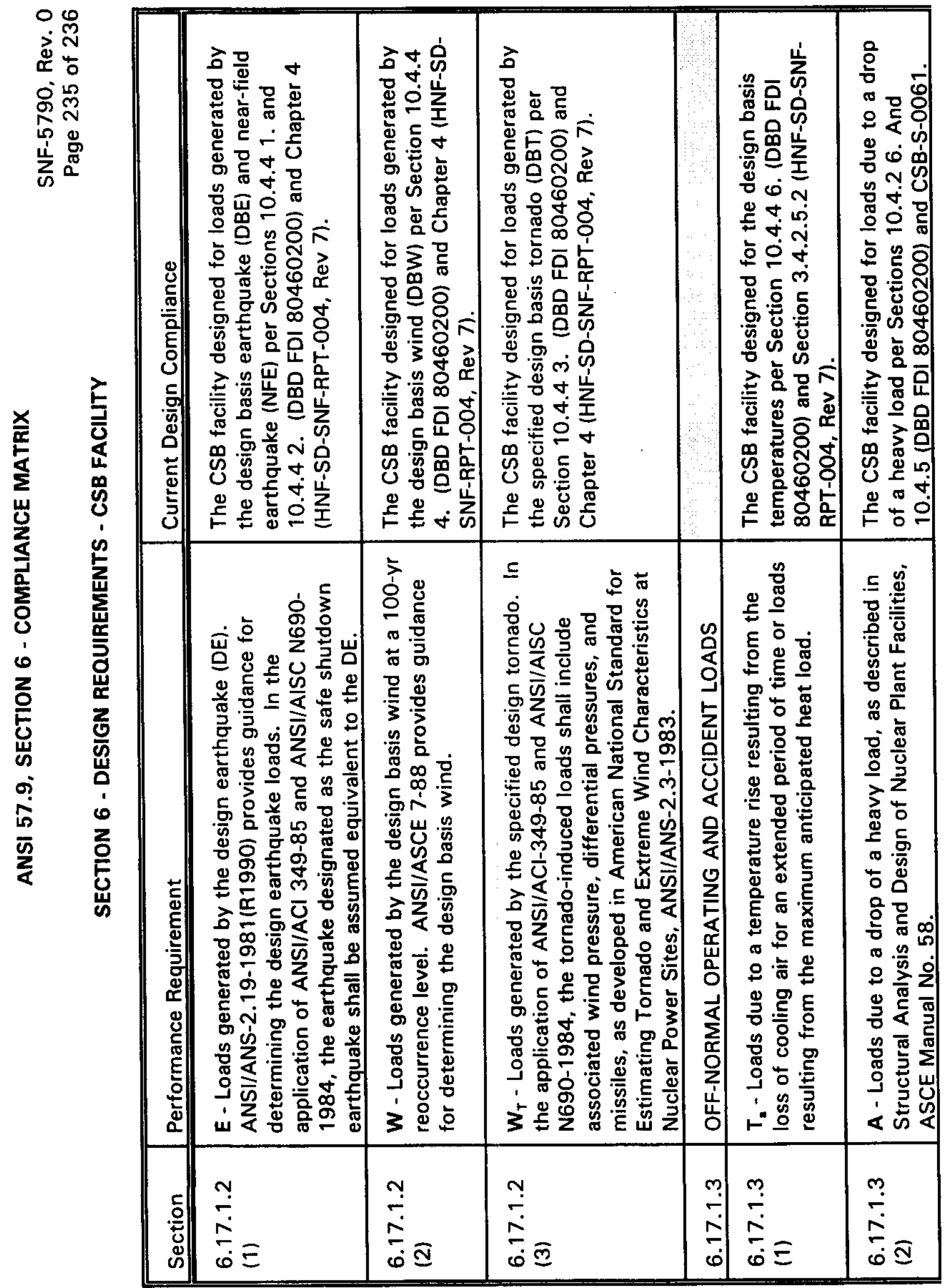




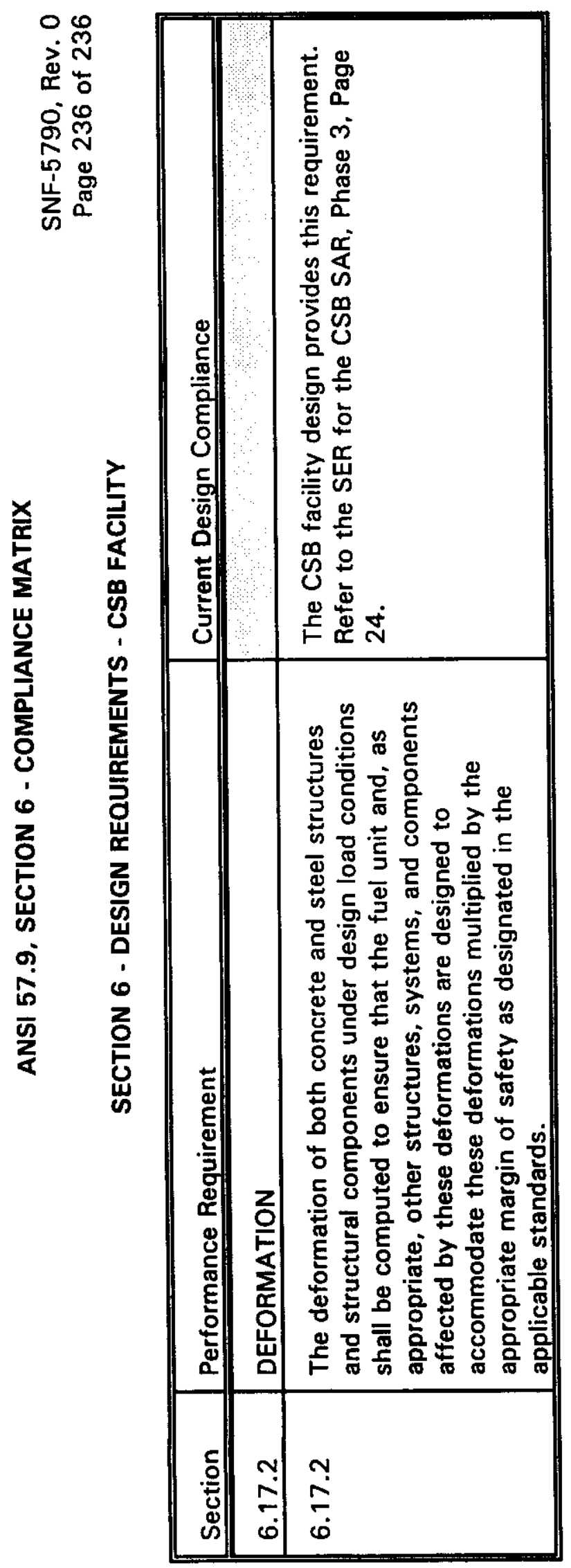

